University of Louisville

ThinkIR: The University of Louisville's Institutional Repository

Electronic Theses and Dissertations

8-2014

\title{
Analysis of contrast-enhanced medical images.
}

Fahmi Abdallah Fahmi Mohammed Khalifa

University of Louisville

Follow this and additional works at: https://ir.library.louisville.edu/etd

Part of the Electrical and Computer Engineering Commons

\section{Recommended Citation}

Khalifa, Fahmi Abdallah Fahmi Mohammed, "Analysis of contrast-enhanced medical images." (2014).

Electronic Theses and Dissertations. Paper 741.

https://doi.org/10.18297/etd/741

This Doctoral Dissertation is brought to you for free and open access by ThinkIR: The University of Louisville's Institutional Repository. It has been accepted for inclusion in Electronic Theses and Dissertations by an authorized administrator of ThinkIR: The University of Louisville's Institutional Repository. This title appears here courtesy of the author, who has retained all other copyrights. For more information, please contact thinkir@louisville.edu. 


\title{
ANALYSIS OF CONTRAST-ENHANCED MEDICAL IMAGES
}

\section{By}

Fahmi Abdallah Fahmi Mohammed Khalifa

M.Sc., ECE, Mansoura University, Mansoura 35516, Egypt 2007

\author{
A Dissertation \\ Submitted to the Faculty of the \\ J. B. Speed School of the University of Louisville \\ in Partial Fulfillment of the Requirements \\ for the Degree of \\ Doctor of Philosophy \\ Department of Electrical and Computer Engineering \\ University of Louisville \\ Louisville, Kentucky
}

May 2014 



\section{ANALYSIS OF CONTRAST-ENHANCED MEDICAL IMAGES}

$$
\text { By }
$$

Fahmi Abdallah Fahmi Mohammed Khalifa

M.Sc., ECE, Mansoura University, Mansoura 35516, Egypt 2007

A Dissertation Approved on

March, 28th, 2014

by the Following Reading and Examination Committee:

Ayman El-Baz, Ph.D., Dissertation Director

Karla Conn Welch, Ph.D., Co-advisor

Tamer Inanc, Ph.D.

Olfa Nasraoui, Ph.D.

Cindy Harnett, Ph.D.

Jake Wildstrom, Ph.D. 


\section{DEDICATION}

This dissertation is dedicated to my mother. Words cannot express how much I wish that she was still with me to see the completion of this endeavor. 


\section{ACKNOWLEDGMENTS}

In the name of Allah the most merciful, the most compassionate. All deepest thanks are due to Almighty Allah for the uncountable gifts given to me.

I would like to thank my dissertation advisor, Dr. Ayman El-Baz, for his continuous encouragement, guidance, and support over the course of my Ph.D. study. I thank him for giving me the opportunity to work in his research group and providing me the opportunity to work on promising applications and meet interesting people. Without his expertise, guidance, and commitment, my research would not have been possible. I truly appreciate his optimism whenever things looked impossible.

I also express my deepest gratitude to Dr. Karla Conn Welch, my dissertation coadvisor, and Dr. Tamer Inanc, Dr. Cindy Harnett, Dr. Olfa Nasraoui, and Dr. Jake Wildstrom for being on my dissertation committee with enthusiasm and taking interest in my research in the midst of many other responsibilities and commitments.

I also have to thank Dr. Georgy Gimel'farb for his useful discussions and valuable comments and feedback. He has never hesitated to share his experience in Markov-Gibbs random field and the field of image processing. I also would like to thank Dr. Mohamed Abou El-Ghar of the radiology department, Urology and Nephrology Center, University of Mansoura, Egypt; and Dr. Garth M. Beache of the department of radiology at the University of Louisville hospital. They spent much of their valuable time providing advices for my work in the analysis of perfusion MR images and commenting on its quality.

I also express my deepest thanks to all members of the research group in the BioImag- 
ing Laboratory at the University of Louisville. They have been a tremendous source of support and a lot of fun to work with. Special thanks to Dr. Ahmed Elnakib, Ahmed Firjani, Matthew Nitzken, Ahmed Soliman, Hisham Sliman, Amir Alansary, and Mahmoud Mostapha whose encouragement during hard times was especially invaluable.

Last but not least, I would like to sincerely thank all my family members: my father Mr. Abdallah Khalifa, my mother Mrs. Sakina Abdelaziz, my wife Mrs. Amira Abdelhady, my brother Mr. Mohammed Khalifa, my sisters Mrs. Abeer Khalifa and Mrs. Hanan Khalifa, and my sons Ahmed Khalifa and Omar Khalifa, for their great patience and encouragement with me all these years. Completing my Ph.D. would not have been possible without their unconditional support and love. 


\section{ABSTRACT \\ ANALYSIS OF CONTRAST-ENHANCED MEDICAL IMAGES}

Fahmi Abdallah Khalifa

March, 28th, 2014

Early detection of human organ diseases is of great importance for the accurate diagnosis and institution of appropriate therapies. This can potentially prevent progression to end-stage disease by detecting precursors that evaluate organ functionality. In addition, it also assists the clinicians for therapy evaluation, tracking diseases progression, and surgery operations. Advances in functional and contrast-enhanced (CE) medical images enabled accurate noninvasive evaluation of organ functionality due to their ability to provide superior anatomical and functional information about the tissue-of-interest. The main objective of this dissertation is to develop a computer-aided diagnostic (CAD) system for analyzing complex data from CE magnetic resonance imaging (MRI). The developed CAD system has been tested in three case studies: $(i)$ early detection of acute renal transplant rejection, (ii) evaluation of myocardial perfusion in patients with ischemic heart disease after heart attack; and (iii), early detection of prostate cancer.

However, developing a noninvasive CAD system for the analysis of CE medical images is subject to multiple challenges, including, but are not limited to, image noise and inhomogeneity, nonlinear signal intensity changes of the images over the time course of data acquisition, appearances and shape changes (deformations) of the organ-of-interest during data acquisition, determination of the best features (indexes) that describe the perfu- 
sion of a contrast agent (CA) into the tissue. To address these challenges, this dissertation focuses on building new mathematical models and learning techniques that facilitate accurate analysis of CAs perfusion in living organs and include: (i) accurate mathematical models for the segmentation of the object-of-interest, which integrate object shape and appearance features in terms of pixel/voxel-wise image intensities and their spatial interactions; (ii) motion correction techniques that combine both global and local models, which exploit geometric features, rather than image intensities to avoid problems associated with nonlinear intensity variations of the CE images; (iii) fusion of multiple features using the genetic algorithm. The proposed techniques have been integrated into CAD systems that have been tested in, but not limited to, three clinical studies.

First, a noninvasive CAD system is proposed for the early and accurate diagnosis of acute renal transplant rejection using dynamic contrast-enhanced MRI (DCE-MRI). Acute rejection-the immunological response of the human immune system to a foreign kidney-is the most sever cause of renal dysfunction among other diagnostic possibilities, including acute tubular necrosis and immune drug toxicity. In the U.S., approximately 17,736 renal transplants are performed annually, and given the limited number of donors, transplanted kidney salvage is an important medical concern. Thus far, biopsy remains the gold standard for the assessment of renal transplant dysfunction, but only as the last resort because of its invasive nature, high cost, and potential morbidity rates. The diagnostic results of the proposed CAD system, based on the analysis of 50 independent in-vivo cases were $96 \%$ with a $95 \%$ confidence interval. These results clearly demonstrate the promise of the proposed image-based diagnostic CAD system as a supplement to the current technologies, such as nuclear imaging and ultrasonography, to determine the type of kidney dysfunction.

Second, a comprehensive CAD system is developed for the characterization of myocardial perfusion and clinical status in heart failure and novel myoregeneration therapy using cardiac first-pass MRI (FP-MRI). Heart failure is considered the most important cause of morbidity and mortality in cardiovascular disease, which affects approximately 6 million 
U.S. patients annually. Ischemic heart disease is considered the most common underlying cause of heart failure. Therefore, the detection of the heart failure in its earliest forms is essential to prevent its relentless progression to premature death. While current medical studies focus on detecting pathological tissue and assessing contractile function of the diseased heart, this dissertation address the key issue of the effects of the myoregeneration therapy on the associated blood nutrient supply. Quantitative and qualitative assessment in a cohort of 24 perfusion data sets demonstrated the ability of the proposed framework to reveal regional perfusion improvements with therapy, and transmural perfusion differences across the myocardial wall; thus, it can aid in follow-up on treatment for patients undergoing the myoregeneration therapy.

Finally, an image-based CAD system for early detection of prostate cancer using DCE-MRI is introduced. Prostate cancer is the most frequently diagnosed malignancy among men and remains the second leading cause of cancer-related death in the USA with more than 238,000 new cases and a mortality rate of about 30,000 in 2013 . Therefore, early diagnosis of prostate cancer can improve the effectiveness of treatment and increase the patient's chance of survival. Currently, needle biopsy is the gold standard for the diagnosis of prostate cancer. However, it is an invasive procedure with high costs and potential morbidity rates. Additionally, it has a higher possibility of producing false positive diagnosis due to relatively small needle biopsy samples. Application of the proposed CAD yield promising results in a cohort of 30 patients that would, in the near future, represent a supplement of the current technologies to determine prostate cancer type.

The developed techniques have been compared to the state-of-the-art methods and demonstrated higher accuracy as shown in this dissertation. The proposed models (higherorder spatial interaction models, shape models, motion correction models, and perfusion analysis models) can be used in many of today's CAD applications for early detection of a variety of diseases and medical conditions, and are expected to notably amplify the accuracy of CAD decisions based on the automated analysis of CE images. 


\section{TABLE OF CONTENTS}

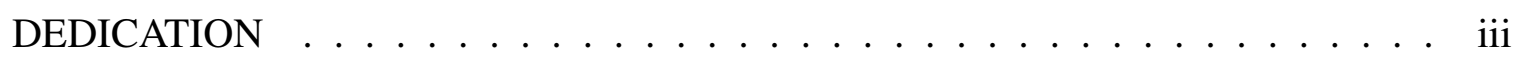

ACKNOWLEDGMENTS . . . . . . . . . . . . . . . . . iv

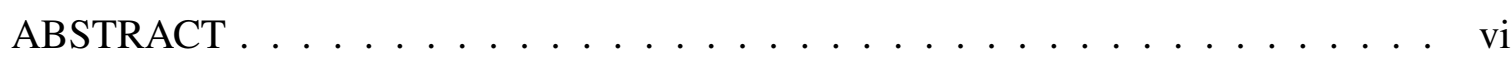

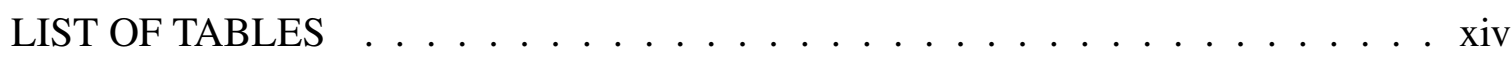

LIST OF FIGURES . . . . . . . . . . . . . . . . . . . xvi

LIST OF ALGORITHMS . . . . . . . . . . . . . . . . . . xxvi

\section{CHAPTER}

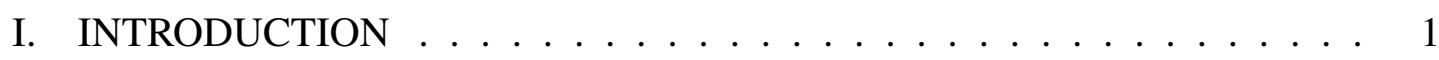

A. MAGNETIC RESONANCE IMAGING . . . . . . . . . . . . . . 3

1. Structural MRI . . . . . . . . . . . . . . . . 4

2. Dynamic Contrast-Enhanced MRI (DCE-MRI) . . . . . . . . 7

3. Diffusion MRI . . . . . . . . . . . . . . . . 9

a. Diffusion-weighted imaging (DWI) . . . . . . . . . 10

b. Diffusion tensor imaging (DTI) . . . . . . . . . 10

c. Diffusion spectrum imaging (DSI) . . . . . . . . . . . 11

4. Functional Magnetic Resonance Imaging (fMRI) . . . . . . . . . 11

5. Tagged Magnetic Resonance Imaging . . . . . . . . . . . . . 12

6. Perfusion-Weighted Imaging (PWI) . . . . . . . . . . . 13

B. QUANTIFICATION OF ORGAN FUNCTIONALITY USING CE IM-

AGES . . . . . . . . . . . . . . . . . . . 14 
1. Early Detection of Renal Transplanted Rejection . . . . . . . . 15

2. Functional Assessment of Myocardial Perfusion . . . . . . . 18

3. Early Diagnosis of Prostate Cancer . . . . . . . . . . . . . 20

C. LIMITATION OF EXISTING WORK AND THE INNOVATION OF THIS WORK . . . . . . . . . . . . . . . . 21

D. BROADER IMPACT OF THE PROPOSED RESEARCH IN THE U.S. AND WORLDWIDE . . . . . . . . . . . . . . . . 25

E. DISSERTATION ORGANIZATION . . . . . . . . . . . . . . 26

II. MODELS AND METHODS FOR ANALYZING DCE-MRI: A SURVEY . . 27

A. INTRODUCTION . . . . . . . . . . . . . . . 27

B. TECHNIQUES FOR ANALYZING DYNAMIC MRI . . . . . . . . 28

C. NONPARAMETRIC DCE-MRI ANALYSIS . . . . . . . . . . 31

1. Clinical Applications of Nonparametric Approaches . . . . . . 33

D. PARAMETRIC DCE-MRI ANALYSIS . . . . . . . . . . . . . . . 41

1. Arterial Input Function (AIF) . . . . . . . . . . . . 43

2. Estimation of Contrast Agent Concentration . . . . . . . . 46

3. Compartment Models . . . . . . . . . . . . . . . . . . 49

a. The Larsson model $(\mathrm{LM}) \quad \ldots . . . . . . . . . .50$

b. The Brix model $(\mathrm{BM}) \ldots \ldots \ldots \ldots 1$

c. The Tofts and Kermode (TK) model . . . . . . . . . . . . . 53

d. Extended Tofts and Kermode (ETK) model . . . . . . . . 55

e. Patlak model $(\mathrm{PM}) \ldots \ldots \ldots$. . . . . . . . . . 56

f. Two-compartment exchange model $(2 \mathrm{CXM}) \ldots . . . . .56$

4. Distributed Models . . . . . . . . . . . . . . . . . . . . 59

a. Distributed-parameter (DP) model . . . . . . . . . . . . 59 
b. Tissue homogeneity $(\mathrm{TH})$ model . . . . . . . . . . . . . . 61

5. Clinical Applications of Parametric Models . . . . . . . . . . . 62

E. SUMMARY AND DISCUSSION . . . . . . . . . . . . 73

III. EARLY DETECTION OF ACUTE RENAL TRANSPLANT REJECTION 76

A. KIDNEY: ANATOMY, FUNCTION, AND RENAL TRANSPLANT

DISEASES . . . . . . . . . . . . . . . 77

B. ACUTE RENAL TRANSPLANT REJECTION . . . . . . . . . . . . . 81

1. Related Work on Image Analysis for Acute Renal Rejection . . . 84

C. METHODS ........................... 90

1. Kidney Segmentation Using a Joint Bi-Level MGRF Model . . . 91

a. Weighted shape prior . . . . . . . . . . . 92

b. MGRF model with second and higher-order spatial interaction 93

c. Intensity model . . . . . . . . . . . . . . . 95

d. Deformable model evolution . . . . . . . . . . . . . 96

2. Iso-contours Based Nonrigid Registration . . . . . . . . . . . 97

3. Cortex Segmentation and Generation of Physiological Data . . . 101

4. Classification, Performance Analysis, and Visualization of Perfusion Indexes . . . . . . . . . . . . . . . . 102

D. PERFORMANCE EVALUATION AND VALIDATION . . . . . . . . 103

1. Evaluating Segmentation Accuracy Using the Dice Metric . . . . 103

2. Evaluating and Validating Accuracy of Nonrigid Registration . . 104

E. PATIENT DATA AND MRI ACQUISITION PROTOCOL . . . . . . . 105

F. EXPERIMENTAL RESULTS . . . . . . . . . . . . . . . 106

1. Kidney Segmentation . . . . . . . . . . . . . . 106

2. Nonrigid Registration . . . . . . . . . . . . . . 110 
3. Cortex Segmentation . . . . . . . . . . . . . 120

4. Perfusion Indexing and Diagnostic Results . . . . . . . . . . . 121

5. ROC Analysis and Regional Perfusion Displays . . . . . . . . . 124

G. DISCUSSIONS . . . . . . . . . . . . . . . . 126

H. SUMMARY . . . . . . . . . . . . . . . 128

IV. FUNCTIONAL ASSESSMENT OF MYOCARDIAL PERFUSION . . . . . 129

A. HEART: ANATOMY, FUNCTION, AND DISEASES . . . . . . . . 130

B. ANALYSIS OF MYOCARDIAL PERFUSION . . . . . . . . . . . 133

1. Related Work on the Segmentation of Cardiac Perfusion MRI . . 135

2. Related Work on Motion Correction of Cardiac Perfusion MRI . 136

C. MYOCARDIAL BORDERS SEGMENTATION . . . . . . . . . . 139

1. Similarity Function . . . . . . . . . . . . . . . . . . 140

a. Estimation of the first-order NMI . . . . . . . . . . . . 141

b. Estimation of the second-order NMI . . . . . . . . . . 142

2. Level Set Evolution . . . . . . . . . . . . . . . . . . 144

D. ISO-CONTOURS REFINEMENT . . . . . . . . . . . . . . . . . . 145

1. Centerline Extraction of the LV Wall . . . . . . . . . . . . 146

2. Evolution of the Generated Iso-contours . . . . . . . . . . . . . 147

E. PERFUSION TIME SERIES AND PARAMETERS . . . . . . . . . . 148

F. PERFORMANCE EVALUATION . . . . . . . . . . . . . . . . . . 149

G. EXPERIMENTAL RESULTS . . . . . . . . . . . . . . . . 150

1. Clinical Data . . . . . . . . . . . . . . . . 150

2. Registration Results and Evaluation . . . . . . . . . . . 150

3. Segmentation Results and Evaluation . . . . . . . . . . . 153 
4. Iso-Contours Refinement . . . . . . . . . . . . . . . . 158

5. Perfusion Indexing and Physiological Assessment . . . . . . . 159

H. SUMMARY . . . . . . . . . . . . . . . . 162

V. EARLY DETECTION OF PROSTATE CANCER . . . . . . . . . . . . . 163

A. PROSTATE: ANATOMY, FUNCTION, AND DISEASES . . . . . . . 164

B. CURRENT DIAGNOSTIC METHODS OF PROSTATE CANCER . . 167

1. Related Work in Prostate Segmentation and Registration . . . . . 169

2. Related Work in CAD Systems for Prostate Cancer . . . . . . . . 175

C. DELINEATION OF THE PROSTATE BORDERS . . . . . . . . . . . 182

1. Nonrigid Registration . . . . . . . . . . . . . 185

2. Perfusion Characterization and Tumor Boundary Determination . 187

D. EXPERIMENTAL RESULTS . . . . . . . . . . . . . . . . . . 189

1. Patients and Data Acquisition . . . . . . . . . . . . . 189

2. Segmentation Results . . . . . . . . . . . . . . 190

3. Diagnostic Results . . . . . . . . . . . . . . . . . 191

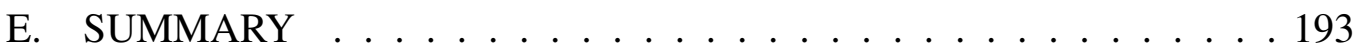

VI. CONCLUSIONS AND FUTURE WORK . . . . . . . . . . . . . . . . 197

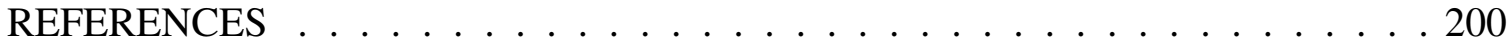

APPENDIX

I. ANALYTICAL ESTIMATION OF GIBBS POTENTIALS . . . . . . . . . 244

II. NOMENCLATURE . . . . . . . . . . . . . . . . . . . . . . 247

III. LIST OF ABBREVIATIONS . . . . . . . . . . . . . . . . 252

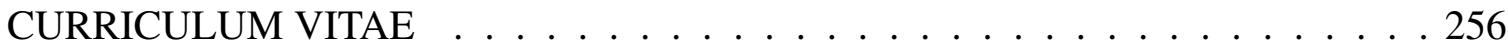




\section{LIST OF TABLES}

TABLE.

1. Recent studies for nonparametric analysis of DCE-MRI. Note that DIM and AL stand for data dimension (i.e., 2D, 3D, or 4D) and the automation level (i.e., automated or semi-automated), respectively . . . . . . . . . . . . 38

2. Recent parametric DCE-MRI studies in different clinical applications. . . . . . 64

3. Summary of kidney motion correction and segmentation techniques using magnetic resonance imaging $(\mathrm{MRI}) \ldots \ldots \ldots$. . . . . . . . . . . . 88

4. Kidney segmentation accuracy for the proposed level set segmentation (combined shape prior, higher-order MGRF, and intensity features) versus the level set segmentation based on the shape prior only or the combined shape prior and intensity information ("SD" stands for standard deviation). . . . . . . . . . . . . 110

5. Accuracy of the proposed level-set segmentation of the kidney versus the shapebased [1], the vector level set [2], and the second-order MGRF [3] approaches in comparison to the expert's ground truth using the Dice metric ("SD" - standard deviation)

6. Comparison of inter-observer variability in annotating corresponding anatomical landmarks for the proposed nonrigid registration and the B-splines approach [4], for real data using 30 data points ("SD" - standard deviation and "OB"-observer). . . . . . . . . . . . . . . . . . . . 113

7. Comparison of the proposed nonrigid approach versus B-splines approach [4] for landmarks identified by three independent observers ("SD" - standard deviation).

8. Alignment errors (in $\mathrm{mm}$ ) of the proposed nonrigid registration for the expertidentified landmarks at different phases of contrast enhancement. . . . . . . . 116

9. Accuracy of the proposed nonrigid registration approach (PA) versus the Bsplines (BS) approach for displacements simulated with cubic B-splines, for real data using 30 data points ("SD" - standard deviation). . . . . . . . . . . . 120 
10. Diagnostic accuracy of the $k_{n}$-nearest neighbor classification using individual perfusion-related renal indexes. . . . . . . . . . . . . . . 123

11. Sensitivity to a training set using a 4-fold cross-validation. . . . . . . . . . . . 124

12. Accuracy of the proposed nonrigid registration (in $\mathrm{mm}$ ) on the realistic synthetic phantoms [5]. Note that the total number of points is 460 ("SD" - standard deviation). . . . . . . . . . . . . . . . . . 153

13. Performance of the proposed segmentation versus the shape-based approach [1] and the vector level set approach [2] in terms of the DSC compared with the expert manual ground truth. Note that "SD" stands for standard deviation. . . . . 155

14. Inter-observer performance of the segmentation of 50 agent-enhanced image frames. DSC between the segmented myocardium and expert ground truth obtained using both intra- and inter-observers. Note that "SD" stands for standard deviation. . . . . . . . . . . . . . . . . . . 156

15. Sensitivity to the selection of the training data, divided into three groups. Note that "SD" stands for standard deviation. . . . . . . . . . . . . . . . . 157

16. Average perfusion parameter values for the mid-cavity level sectors of the 17 segment model [6] for the results shown in Figure 80.

17. Summary of the discussed prostate segmentation and registration techniques and their experimental performance. Note that DIM and AL stand for data dimension (i.e., 2D, 3D, or 4D) and the automation level (i.e., automated or semi-automated), respectively. . . . . . . . . . . . . . 172

18. Summary of MRI-based CAD systems for prostate cancer detection and diagnosis, including segmentation method, selected features, classifier, and experimental performance. . . . . . . . . . . . . . . . . 178

19. Error statistics of the proposed segmentation approach. Note that "SD", stands for standard deviation. . . . . . . . . . . . . . . . . . . 190

20. Comparative segmentation accuracies of the proposed prostate segmentation against the shape-based (SB) approach [1] in comparison to the expert's ground truth ("SD" - standard deviation) . . . . . . . . . . . . . . . . . . 193

21. Label combinations and their marginal probabilities for second-, third-, and fourth-order cliques. Here, $p$ denote the probability, "eq" and "ne" denote two equal or non-equal labels for a second-order clique, and "eq $i$ " denote $i$ equal labels for a third- or fourth-order clique. 


\section{LIST OF FIGURES}

FIGURE.

1. Different types of medical images: magnetic resonance imaging (MRI) of $(a)$ the kidney and $(b)$ the heart, $(c)$ computed tomography (CT) image of the lung, $(d)$ ultrasound image of the kidney [7], $(e)$ positron emission tomography image of the lung, and $(f)$ single photon emission computed tomography image of the heart (Courtesy of [8]). . . . . . . . . . . . . . . . . .

2. Schematic illustration of different classes of medical image modalities. . . . . . 3

3. Categories of medical image modalities based on the type of information that they provide about the organ being imaged, i.e., structural or functional imaging.

4. 2D cine MR image of the heart. . . . . . . . . . . . . . . . 4

5. 3D $T_{2}$-weighted MRI of the prostate, captured in the axial $(a)$, coronal $(b)$, and sagittal $(c)$ planes. Courtesy of [9] . . . . . . . . . . . . . . . .

6. 4D (3D + time) cardiac MRI data. Images are acquired at different sections covering the heart (from basal to apical) and each section consists of a time series of 25 image frames over the cardiac cycle $\left(t_{1} \longrightarrow t_{25}\right)$. The inner and the outer borders of the heart wall are shown in red and green, respectively. . . . . .

7. Different specialized MRI acquisition techniques. . . . . . . . . . . . 6

8. Examples of axial MRI scans of the osteochondroma underlying the pseudoaneurysm: (a) $T_{1}$-weighted, (b) proton density-weighted, and (c) $T_{2}$-weighted images. The images have different contrast that reveal specific information about the structures in the knee. Courtesy of $[10] . \ldots$. . . . . . . . . . . . .

9. Dynamic MR images taken at different time points post the adminstration of the contrast agent (CA) showing the change of the contrast as the CA perfuse into the tissue for the heart, kidney, and prostate. . . . . . . . . . . 8

10. Different types of contrast agents used in MRI medical scans. . . . . . . . . . . 9

11. Diffusion MR images for the prostate at $(a)$ b-value of $0 \mathrm{~s} / \mathrm{mm}^{2}$ and $(b)$ b-value of $800 \mathrm{~s} / \mathrm{mm}^{2}$ 
12. A $2 \mathrm{D}$ projection of the tractographic color map estimated from a DTI imaging data set. Color codes have been assigned based on the mean orientation of the fibers. Note that the data set may be rotated continuously into various planes to provide better tractographic assessments of the brain.

13. A lateral view showing the active human brain areas of a subject viewed moving and static figures. Orange regions are the activated brain areas when the subject viewed moving figures, while green and blue regions responded similarly to both moving and static figures. Courtesy of $[11] . \ldots$. . . . . . . . . . . . 12

14. A example of the tagged MRI time-series for the heart. . . . . . . . . . . . . . . 13

15. An illustration of taking biopsy samples from the kidney. A thin needle is inserted through the skin to perform the operation, and the procedure is usually directed by an imaging device (e.g., ultrasound) to help in placing the needle into the right location [12].

16. A typical CAD system for the detection of acute renal transplant rejection. The motion correction step is used to handle global and/or local motions of the input DCE-MRI data. Then, the kidney and its functional unit (cortex) are extracted from the surrounding abdomen tissue. Next, agent kinetic curves are constructed and perfusion parameters are extracted and are used for diagnosis.

17. A typical time-intensity curve of the average intensity of the kidney measured before and after contrast agent adminstration. The curve illustrates typical perfusion-related indexes that can be estimated and used for diagnosis of the kidney status. . . . . . . . . . . . . . . . . . . . . .

18. Pixel-wise perfusion maps of the perfusion indexes shown in Figure 17: $(a)$ peak signal intensity, $(b)$ initial up-slope, $(c)$ average plateau, and $(d)$ time-topeak; for one normal (upper row) and one abnormal (lower row) kidney. The red and blue hues of each color scale correspond to the highest and lowest values, respectively. Note all indexes show worsening of perfusion with pathology. . . .

19. A schematic diagram of the proposed CAD system for the analysis of myocardial perfusion. The input to the system is the cardiac FP-MRI data. The motion correction step is used to handle the global and/or the local motions of the heart. Then, the left ventricle (LV) wall is segmented, the agent kinetic curve is constructed, and perfusion parameters are estimated and used to depict regional and transmural effects across the myocardial wall. . . . . . . . . . . . . .

20. Pixel-wise parametric map display of the peak signal intensity demonstrating local LV areas that may be damaged due to the heart attack. The red and blue hues of each color scale correspond to enhanced and reduced perfusion, respectively. . . . . . . . . . . . . . . . . . . . 
21. A block diagram of the proposed CAD system for the early diagnosis of prostate cancer using DCE-MRI. The time series data is first corrected for motion artifacts. Then, the prostatic tissue is segmented and the agent kinetic curves are constructed. Finally, perfusion-related parameters are extracted from the kinetic curves and are used for the classification of both malignant and benign cases. .

22. Typical $S(t)$ curve showing the time points that quantifies the CA's dynamics and results in different metrics that qualitatively characterize the agent's perfusion: the onset time $\left(T_{o}\right)$, time-to-peak $\left(T_{P}\right)$, peak signal intensity, wash-in slope (initial up-slope), wash-out slope (down-slope), area under the curve (AUC), and initial area under the curve (IAUC). Note that $S_{0}$ is the intensity before the adminstration of CA and $T_{\max }$ is the time period of the MR experiment. . . . .

23. Different enhancement patterns: Type I - a progressive signal intensity $(S(t))$ increase during the experiment; Type II - an initial peak followed by a relatively constant enhancement (no wash-out), and Type III - a sharp uptake followed by an enhancement decrease over time (wash-out). . . . . . . . . . . . . 30

24. The population-based arterial input functions (AIF) proposed by (a) Tofts and Kermode [13] with $D=0.1 \mathrm{mM} \mathrm{kg}^{-1}$; and (b) Parker et al. [14]. Different time scales are used to visualize better the CA uptake and wash-out phases in each AIF.

25. Two-compartment model: the function $C_{\mathrm{a}}(t)$ quantifies the arterial input into the plasma compartment; the peripheral compartment receives the contrast agent (CA) from and returns it to the plasma compartment at the rates $k_{12}$ and $k_{21}$, respectively, and the rate $k_{\mathrm{el}}$ specifies the CA loss from the system. . . . . . .

26. The Larsson model (LM) for a capillary-tissue system: the blood plasma flows in the capillary at a rate of $F_{\mathrm{p}}$ and exchanges the contrast agent (CA) with the extracellular and extravascular space $(\mathrm{EES})$ at a rate of $k_{\mathrm{ep}} \ldots \ldots \ldots$

27. The Brix model (BM): the $\mathrm{CA}$ is administered at a constant rate of $k_{\text {in }}$ into the plasma compartment; exchanged between the two compartments at rates of $k_{\mathrm{ep}}$ and $k_{\mathrm{pe}}$, respectively, and eliminated (cleared) from the plasma at a rate of $k_{\mathrm{el}}$. .

28. Schematic illustration of the CA transfer in the Tofts and Kermode (TK) model between the central (plasma) compartment and the EES space with the $K^{\text {trans }}$ and $k_{\text {ep }}$ rates, respectively. . . . . . . . . . . . . . . .

29. Schematic illustration of the two-compartment exchange model (2CXM). The CA delivered via arteries to the plasma compartment at an $F_{\mathrm{P}}$ rate, is exchanged between the intravascular plasma and EES compartments at a symmetric rate of $P S$, and is eliminated subsequently from the plasma compartment. . . . . . . 
30. Schematic illustration of the Distributed-parameter (DP) model. The CA concentration within the capillary decreases with position $(x)$ along the capillary length $(L)$, producing concentration gradients between the arterial $(x=0)$ and venous $(x=L)$ capillary ends. During the CA passage, some amounts diffuse between the plasma and EES at a controlled $P S$ rate, so that the plasma, $C_{\mathrm{p}}(x, t)$, and EES, $C_{\mathrm{e}}(x, t)$, concentrations show both the spatial and temporal

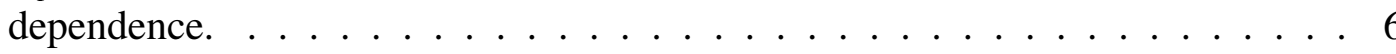

31. The abdominal area of the human body showing the kidney location [15]. . . . .

32. A schematic illustration of a corronal cross-section of the human right kidney showing its internal structures $[15] . \ldots \ldots \ldots$. . . . . . . . . . 78

33. A schematic illustration of the detailed structure of a nephron [16]. . . . . . . 79

34. Illustration of the basic processes of urine formulation [17]. . . . . . . . . . . 80

35. Different types of renal transplant complications. . . . . . . . . . . . . . 80

36. The proposed computer-aided diagnostic (CAD) system for noninvasive evaluation of acute renal transplant rejection. . . . . . . . . . . . . . . . . 9

37. Forming kidney shape prior: $(a)$ training samples, $(b)$ their affine alignment, and (c) manually segmented kidney objects . Note that the registration enhances the overlap between the kidney objects and thus reduce the variability of the final estimated shape. . . . . . . . . . . . . . . . . . . . 93

38. Gray-coded shape prior of the kidney before $(a)$ and after $(b)$ the affine mutual information (MI) based registration and the color-coded visualization of the shape prior $(c) \ldots \ldots \ldots \ldots \ldots$. . . . . . . . . . . . . 94

39. Second- (a), third- (b), and fourth-order (c) cliques for the 8-neighborhood. . . . 94

40. Schematic illustration of establishing point-to-point correspondences between two boundaries by a potential field.

41. Generation of iso-contours: the reference and target images $(a, d)$, their distance maps $(b, e)$, and the generated iso-contours $(c, f)$.

42. Co-allocation of point-to-point correspondences for two possible scenarios of kidney misregistration: the reference (green) and target (red) contours before $(a, d)$, and after $(b, e)$ affine alignment, and streamlines (yellow) linking the corresponding points found by solving the Laplace equation $(c, f)$. . . . . . . . 100

43. Measuring segmentation errors between the ground truth $\mathbf{G}$ and automatic segmentation S. . . . . . . . . . . . . . . . . . . . . . . . . . 104 
44. Illustration of the relative error $e=100 \cdot\left(d_{1} / d_{2}\right) \%$ measurement for the evaluation of nonrigid registration. . . . . . . . . . . . . . . . . 105

45. A typical DCE-MRI kidney image ( $a$ ); and estimated density $(b)$ using only two dominant Gaussian components $(c)$, deviation between empirical and estimated densities $(d)$, estimated density of absolute deviation (e) LCDG components $(f)$, final estimated density $(g)$, and the final estimated marginal density for each class $(h) \ldots \ldots \ldots$. . . . . . . . . . . . . . . . . 107

46. Segmentation results of the proposed level set-based approach $(a)$ in comparison with the shape-based (SB) approach $(b)$ by Tsai et al. [1], and the vector level set (VLS) approach (c) by Abdelmunim and Farag [2]. The segmented kidney boundary is in red, and the manual expert's segmentation is in green. . . 108

47. (a) Typical MRI slice to be segmented; and the segmentation results using (b) the shape prior information only, $(c)$ the combined intensity and shape prior information, and $(d)$ the proposed approach. The segmented kidney boundary is in red, and the manual expert's segmentation is in green. . . . . . . . . . . . 109

48. Superimposed reference (green) and target (red) kidney borders (A); and checkerboard displays before (B) and after (C) nonrigid registration for four independent subjects with $(a, b)$ and without $(c, d)$ overlapping edges. . . . . . . . . . 111

49. Typical time-intensity curve for one data set before and after registration. . . . . 112

50. Validating the proposed nonrigid registration and the B-Splines (BS) approach [4] using the corresponding anatomical landmarks identified by an expert in both the reference $($ red; $\bullet)$ and target $($ green; $\diamond)$ images. . . . . . . . . . . . . . . 113

51. Bland-Altman plots for alignment errors with respect to annotations of the three observers (OB1, OB2, and OB3). Each plot shows the difference errors ( $y$-axis) versus their average ( $x$-axis) and the $95 \%$ confidence interval $(95 \% \mathrm{CI} ; \pm 1.96$ $\mathrm{SD}$, dotted lines) for inter-observer comparison for OB1 and OB2 $(a, b), \mathrm{OB} 2$ and $\mathrm{OB} 3(d, e)$, and $\mathrm{OB} 1$ and $\mathrm{OB} 3(g, h)$. The comparison between the proposed nonrigid registration and the B-splines (BS) approach [4], for OB1, OB2, and OB3 annotations are shown in $(c),(f)$, and $(i)$, respectively. For close agreement the bias (mean, solid lines) should be near zero, while the data points should fall within the $95 \%$ CI. These plots document that while there is good interobserver agreement for both the proposed approach and the BS approach, it also demonstrates the significant differences in performance between both methods. . 114

52. Validating the proposed nonrigid registration with the anatomical landmarks identified by an expert for different phases of contrast-enhancement. Top row: the reference $(a)$, baseline $(b)$, peak-intensity $(c)$, and tissue distribution $(d)$ images. Middle row: superimposed expert-identified correspondences for both the reference $($ red; $\bullet$ ) and target (green; $\diamond$ ) images. Bottom row: the registration results obtained by the proposed approach. . . . . . . . . . . . . . 115 
53. Validation of the proposed nonrigid registration on synthetic kidney-shaped phantom with sharp edges: ground truth (white lines) versus estimated (black lines) correspondences.

54. Validation of the proposed nonrigid alignment (top row) versus affine alignment (bottom row) on three realistic simulated kidney-shaped phantoms at different contrast-enhancement stages (from left to right: baseline, peak intensity, and tissue distribution phase). Blue and green circles represent ground truth correspondences on the reference and target objects, respectively. Laplace and affinedetermined correspondences are represented by red diamonds. The ground truth and correspondences estimated on the target object are connected to the reference ground truth points by green and red lines, respectively. Small $(a)$, moderate $(b)$, and large $(c)$ deformations of a manually segmented kidney (black) were generated by using the 2D cubic B-spline transformation model [18]. . . . 118

55. Checkerboard display for the proposed (top row) and the affine registration (bottom row) for small $(a)$, moderate $(b)$, and large $(c)$ deformations of the phantom shown in Figure 54. The reference object is in light gray, and target objects are in dark gray. . . . . . . . . . . . . . . . . . . . .

56. Step-by-step cortex segmentation for one subject: (a) kidney object after nonrigid alignment; $(b)$ automatically-determined bounding box (yellow) that yields the seed point (red) for level set initialization; $(c)$ initial contour (yellow); $(d)$ final contour (red); $(e)$ the extracted cortex mask; and $(f)-(j)$ the propagation of the mask over the registered time series images at representative time instants $6,42,120,180$, and $234 \mathrm{sec}$, respectively. . . . . . . . . . . . . . . . . 121

57. Normalized signal intensity, averaged over the entire cortex, with respect to timing of agent delivery. Standard deviations (error bars) for the non-rejection (black; •) and acute rejection (red; o) subjects indicate the clear separation between the two groups by the transient phase kinetic parameters (peak signal, time-to-peak, and initial up-slope), and the average signal magnitude at the more slowly varying tissue distribution plateau phase. Note that the time-topeak index has the least selectivity, and the area under the signal intensity time curve is related to the kinetic parameter blood volume. . . . . . . . . . . . . . . 122

58. Radiologist clinical method for the estimation of the perfusion parameters for the classification of the transplanted kidney status. . . . . . . . . . . . . . 12

59. ROC curves for the $k_{n}$ diagnostic classification based on the individual perfusion indexes extracted from the cortex - the peak signal intensity (green; $\times$ ); time-to-peak (black; $\mathbf{\square}$ ); initial up-slope (pink; •), the average plateau (blue; $\diamond)$, and the combined classifier (red; ○). Note that the weighted combination of the four indexes has almost the ideal performance, as evidence by the area under the ROC curve approaching unity $\left(A_{z} \approx 1.0\right) \ldots \ldots . \ldots 125$ 
60. Perfusion maps for the four perfusion indexes, using the normalized signal intensity: peak signal intensity $(a)$; time-to-peak $(b)$; initial up-slope $(c)$, and average plateau $(d)$ for a representative non-rejection subject (upper row) and acute rejection subject (lower row). The red and blue hues of each color scale correspond to highest and lowest values, respectively. Note all indexes show worsening of perfusion with pathology. . . . . . . . . . . . . 126

61. Illustration of the location of the heart in the body [19]. . . . . . . . . 130

62. A schematic illustration of a long-axis cross-section of the heart showing the four chambers of the heart and the atrioventricular valves that controls blood

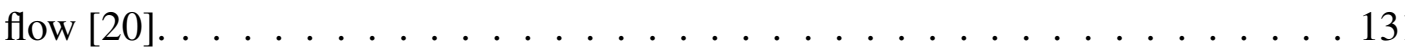

63. A short-axis MR image taken at the mid-cavity level of the heart showing different layers of the cardiac wall. . . . . . . . . . . . . . . . 131

64. The proposed framework for the analysis of cardiac first-pass perfusion MRI. . . 139

65. Building the LV wall probabilistic shape prior: (a) training samples, $(b)$ their affine-based alignment, $(c)$ their B-splines based nonrigid registration, $(d)$ the deformation fields used for the B-splines registration, and $(e)$ the manually segmented myocardium. . . . . . . . . . . . . . . . . . 141

66. Neighborhood systems for the estimation of the second-order marginal (a), and joint (b) MGRF probabilities. . . . . . . . . . . . . . . . . . . 142

67. Overlaps of the training maps before $(a)$ and after $(b)$ affine registration; $(c)$ after the two-step registration; and $(d)$ color-coded visualization of shape prior. . 144

68. Illustration of the centerline extraction: (a) the distance map of a typical LV wall, $(b)$ the streamlines found by solving Laplace equation between the inner and the outer borders of the LV wall, $(c)$ the identified centerline points (blue), and $(d)$ the extracted centerline overlaid on the binary mask of the LV wall. . . . 146

69. A typical example of the generated iso-contours for the reference $(a)$ and target (b) LV walls. The extracted centerline of the LV wall is shown in yellow. . . . . 148

70. A typical agent kinetic curve showing the time points within the cardiac perfusion MRI data used to estimate the perfusion indexes. Image frames are acquired at an interval of approximately one second. . . . . . . . . . . . . . . 149

71. The realistic synthetic phantom images [5] at different myocardial signal intensities: (a) baseline, (b) peak intensity, and (c) tissue distribution phases. The images were generated based on the integration of the Gibbs sampler and the MAP estimates [21] . . . . . . . . . . . . . . . . . . . . 151 
72. Three different types of deformations of the realistic synthetic phantom [5] for the tissue distribution phase: (a) reference image; and target images with $(b)$ small, $(c)$ moderate, and $(d)$ large deformations.

73. Registration accuracy of the synthetic phantom shown in Figure $71(c)$ for the (A) small, (B) moderate, and (C) large deformations: (a) reference image, $(b)$ deformed image, $(c)$ registered image, and $(d)$ checkerboard visualization of the superimposed reference and target images after alignment using (I) the Bsplines model with first-order NMI only $\left(\mathrm{BS}_{1}\right)$, (II) B-splines model with the proposed first- and second-order NMI $\left(\mathrm{BS}_{2}\right)$, and (III) the proposed approach $\mathrm{BS}_{2}$ followed by iso-contours registration $\left(\mathrm{BS}_{2}+\mathrm{ISO}\right) \ldots \ldots . \ldots 152$

74. Typical time-intensity curves, averaged over the entire myocardium, for one patient before and after registration. . . . . . . . . . . . . . . . 153

75. Step-by-step segmentation: (a) reference image, $(b)$ target image, $(c)$ affine registration, $(d) \mathrm{B}$-splines registration, $(e)$ deformation field used for B-splines registration, and $(f)$ final borders' segmentation (red) with respect to the ground truth (green) . . . . . . . . . . . . . . . . . . . 154

76. More segmentation results of the proposed segmentation approach for one image frame in three different patients showing reliable determination of the wall borders (red) with respect to the ground truth (green), at different myocardial signal intensities. . . . . . . . . . . . . . . . . . 154

77. Comparative segmentation results for $(a)$ the proposed approach, $(b)$ the shapebased approach [1], and $(c)$ the vector level set approach [2] for different image frames from different data sets. . . . . . . . . . . . . . . . 156

78. The ROC curves for different feature combinations for level set guidance: the proposed approach (blue); intensity feature only (red), combined intensity and spatial interactions features (green), and combined intensity and shape features (black). . . . . . . . . . . . . . . . . . . . 157

79. An example of the final iso-contours-based adjustment: (a) reference image, (b) target image, (c) checkerboard visualization of the superimposed target and reference walls before iso-contours registration, $(d)$ Laplace-based registration, and $(e)$ checkerboard visualization after iso-contours registration showing nearly ideal match of the boundaries. . . . . . . . . . . . . . . . . . . 158

80. Parametric maps for the perfusion indexes: $(a)$ peak signal intensity, $(b)$ time-topeak, $(c)$ initial up-slope, and $(d)$ average of tissue distribution (plateau) phase for one subject. The red and blue hues of the color scale relate to highest and lowest values, respectively. The top and bottom rows represent pre- and posttherapy, respectively. Note that there is an apparent improvement in the myocardial wall after therapy. . . . . . . . . . . . . . . . . . . . . . . . 159 
81. Corresponding segment locations for the mid-cavity image section of the myocardial 17-segment model [6]. The segment numbering starts counter-clockwise from the anatomical landmark indicated by the white arrow. Please note that the coloring is simply to indicate different segments and provide visibility of the segment numbering. . . . . . . . . . . . . . . . . . . . . 160

82. Box plots of the perfusion indexes (average over all heart sectors) for the pretreatment $(\mathrm{PR}, \mathrm{red})$ and post-treatment $(\mathrm{PO}, \mathrm{blue})$ for all patients enrolled in this study: peak signal intensity $(a)$, time-to-peak $(b)$, initial up-slope $(c)$, and average plateau $(d) \ldots \ldots \ldots \ldots$. . . . . . . . . . . . . . . . . . . . . . . . . .

83. Schematic illustration of an anatomical view of the lower abdomen area that contains the prostate [22].

84. Schematic illustration of the front and side cross-sectional views of the prostate showing different prostate zones [23]. . . . . . . . . . . . . 165

85. The proposed CAD system for early detection of prostate cancer. . . . . . . . 182

86. Illustration of the Joint Markov-Gibbs random field (MGRF) image model of the prostate DCE-MRI. . . . . . . . . . . . . . . . . . 183

87. 3D second-order MGRF neighborhood system. Note that the reference voxel is shown in red and $a$ represents the absolute distance between two voxels in the same and adjacent MRI slices, or cross-sections. . . . . . . . . . . . . . . . 184

88. Gray-coded $(a)$ and color-coded $(b)$ axial view of the prostate shape prior. $\quad \ldots 185$

89. Generation of the iso-contours: the reference and target distance maps $(a, b)$, and their iso-contours $(c, d) \ldots \ldots \ldots \ldots$. . . . . . . . . . . . . . . . . .

90. Estimating the changes in image signals $\Delta$ from the kinetic curve as the difference between the peak $\left(I_{p}\right)$ and initial $\left(I_{0}\right)$ signals of the image sequences. . . 188

91. Enhanced perfusion estimation and continuity analysis using the $3 \mathrm{D}$ GGMRF image model. . . . . . . . . . . . . . . . . . . . . . . . . . . 189

92. Sample segmentation results of the proposed segmentation approach for different subjects at different cross-sections. The false negative (FN) and false positive (FP) error referenced to the ground truth $\mathrm{G}$ are shown in yellow and red, respectively. . . . . . . . . . . . . . . . . . . . . . . . 191

93. Comparative segmentation results: (a) different prostate dynamic MR images, $(b)$ proposed approach segmentation, and $(c)$ segmentation with [1]. The false negative $(\mathrm{FN})$ and false positive (FP) segmentation errors referenced to the ground truth G are shown in yellow and red, respectively . . . . . . . . . . . . 192 
94. Selected normalized signal intensity, averaged over the entire prostate, with respect to the timing of contrast agent (CA) delivery for malignant (red) and benign (blue) subject. . . . . . . . . . . . . . . . . . . . . . 194

95. Color-coded maps for three subjects before and after the 3D GGMRF smoothing using $\alpha=1, \chi=5, b=1.01, a=2$, and $\Gamma_{\mathbf{P}, \mathbf{P}^{\prime}}=\sqrt{2}$. The red and blue ends of the color scale relate to the maximum and minimum changes, respectively. 195

96. Color-coded maps of local tumor progression overlaid on anatomic DCE-MRI data for one benign $(a)$ and one malignant $(b)$ subject. The determined tumor contours are shown in green. . . . . . . . . . . . . . . . . 196 


\section{LIST OF ALGORITHMS}

ALGORITHM.

PAGE

1. Key Steps for Level Set Segmentation of the Kidney . . . . . . . . . . . . . 98

2. Laplace-Based Iso-Contours Nonrigid Registration . . . . . . . . . . . . . 101

3. Left Ventricle Wall Borders Segmentation . . . . . . . . . . . . . . 145

4. Centerline Determination of the Left Ventricle Wall . . . . . . . . . . . 147

5. Prostate Segmentation Approach . . . . . . . . . . . . . . . . . . 186 


\section{CHAPTER I INTRODUCTION}

Early detection of human organ diseases is crucial for the accurate diagnosis and institution of appropriate medical therapies, which can increase the survival rate of the patients. Therefore, it is critical to detect precursors that evaluate human organ functionality, which can potentially prevent progression to end-stage disease. In addition, accurate evaluation of organ functionality can assist the clinicians for early diagnosis of diseases, therapy decisions and evaluations, and surgery operations. Advances in medical imaging techniques, particularly functional and contrast-enhanced (CE) medical images, enabled accurate noninvasive evaluation of human organ functionality due to their ability to provide superior anatomical and functional information about the tissue of interest. Therefore, the development of noninvasive computer-aided diagnostic (CAD) systems using CE images for the evaluation of human organ functionality is of great importance. However, this is subject to multiple challenges that include, but are not limited to, image noise and inhomogeneity, nonlinear signal intensity changes of the images over the time course of data acquisition, and shape changes (deformations) of the organ of interest during data acquisition. The goal of this dissertation is to develop a general, noninvasive, image-based framework that accounts for these challenges for the analysis of complex data from $\mathrm{CE}$ magnetic resonance imaging (MRI) for the evaluation of human organ functionality. The proposed framework is applied in three case studies: early detection of renal transplant rejection, functional assessment of myocardial perfusion in patients with ischemic heart disease, and early detection of prostate cancer.

Recent advances in medical image modalities, including magnetic resonance imaging (MRI), computed tomography (CT), ultrasound, positron emission tomography, and single photon emission computed tomography, enable the acquisition of images for almost 
all types and sizes of different structures with acceptable degrees of contrast and resolution. Nowadays, medical imaging is a basic component in current medical diagnostic tools and has been effectively used to assist clinicians and radiologists in diagnosis, therapy decisions, and surgery operations. Each image modality (see e.g., Figure 1 and Figure 2) has its own mechanism of providing relevant physiological information of the organ being imaged as well as its own advantages and limitations. Medical images can be classified based on their modalities (Figure 2) or based on the type of information that they provide (i.e., the structure or the function of the organ being imaged, see Figure 3). Since all the work presented in this dissertation mainly deals with MRI, an overview of different types of the MRI modality with the focus on functional and CE images is given in the following section.

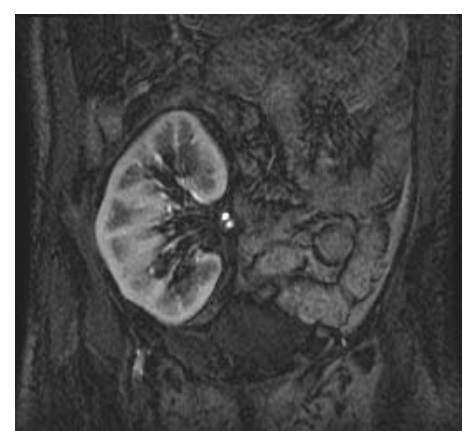

(a)

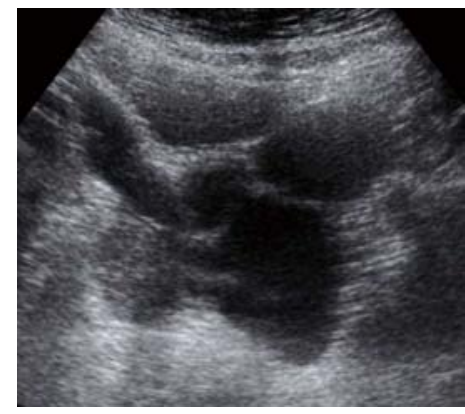

(d)

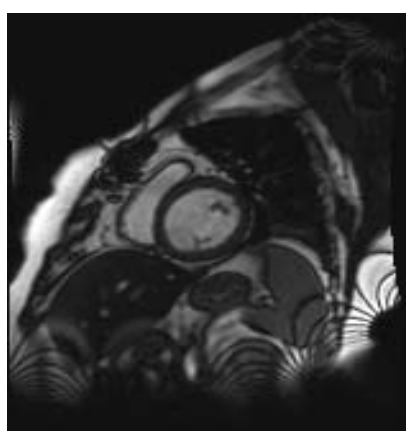

(b)

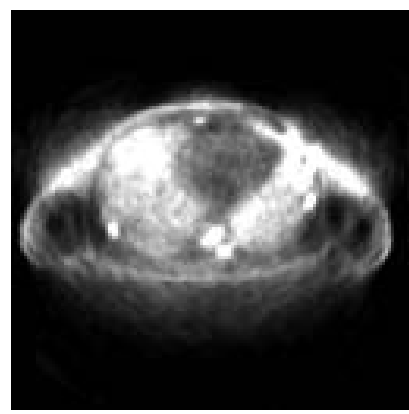

$(e)$

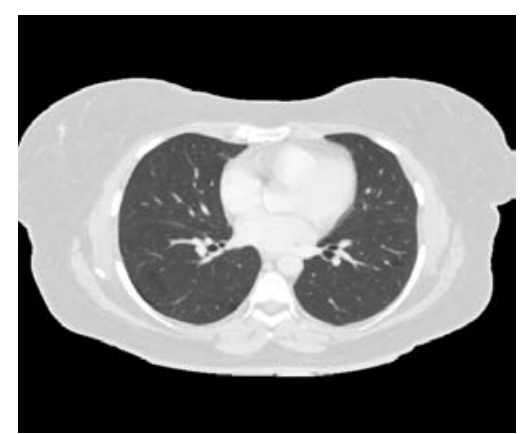

$(c)$

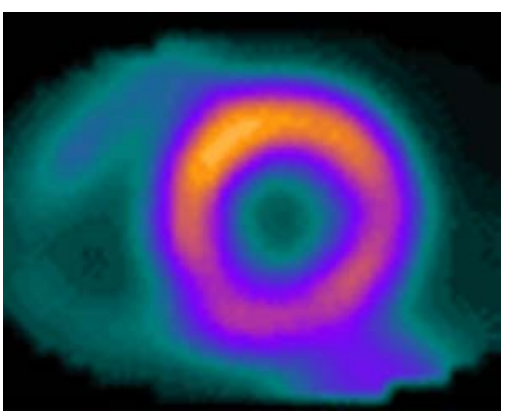

$(f)$

FIGURE 1: Different types of medical images: magnetic resonance imaging (MRI) of (a) the kidney and (b) the heart, (c) computed tomography (CT) image of the lung, $(d)$ ultrasound image of the kidney [7], (e) positron emission tomography image of the lung, and $(f)$ single photon emission computed tomography image of the heart (Courtesy of [8]). 


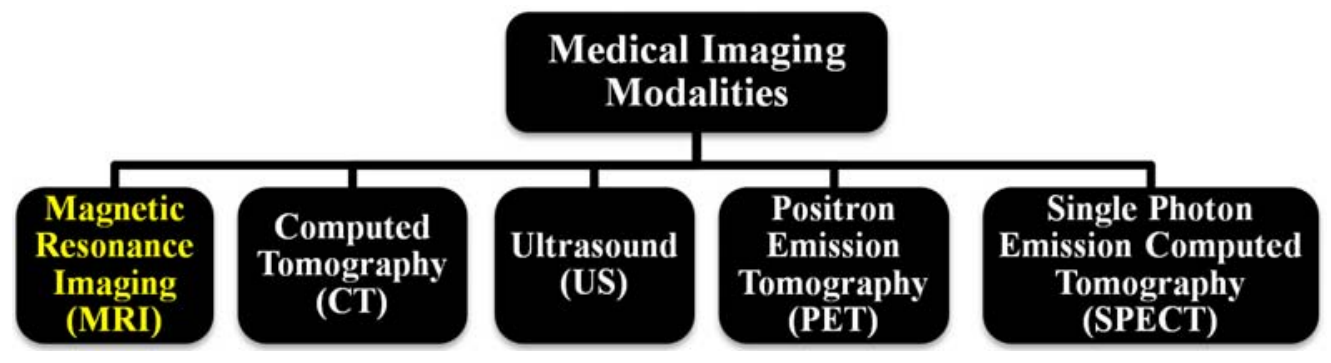

FIGURE 2: Schematic illustration of different classes of medical image modalities.

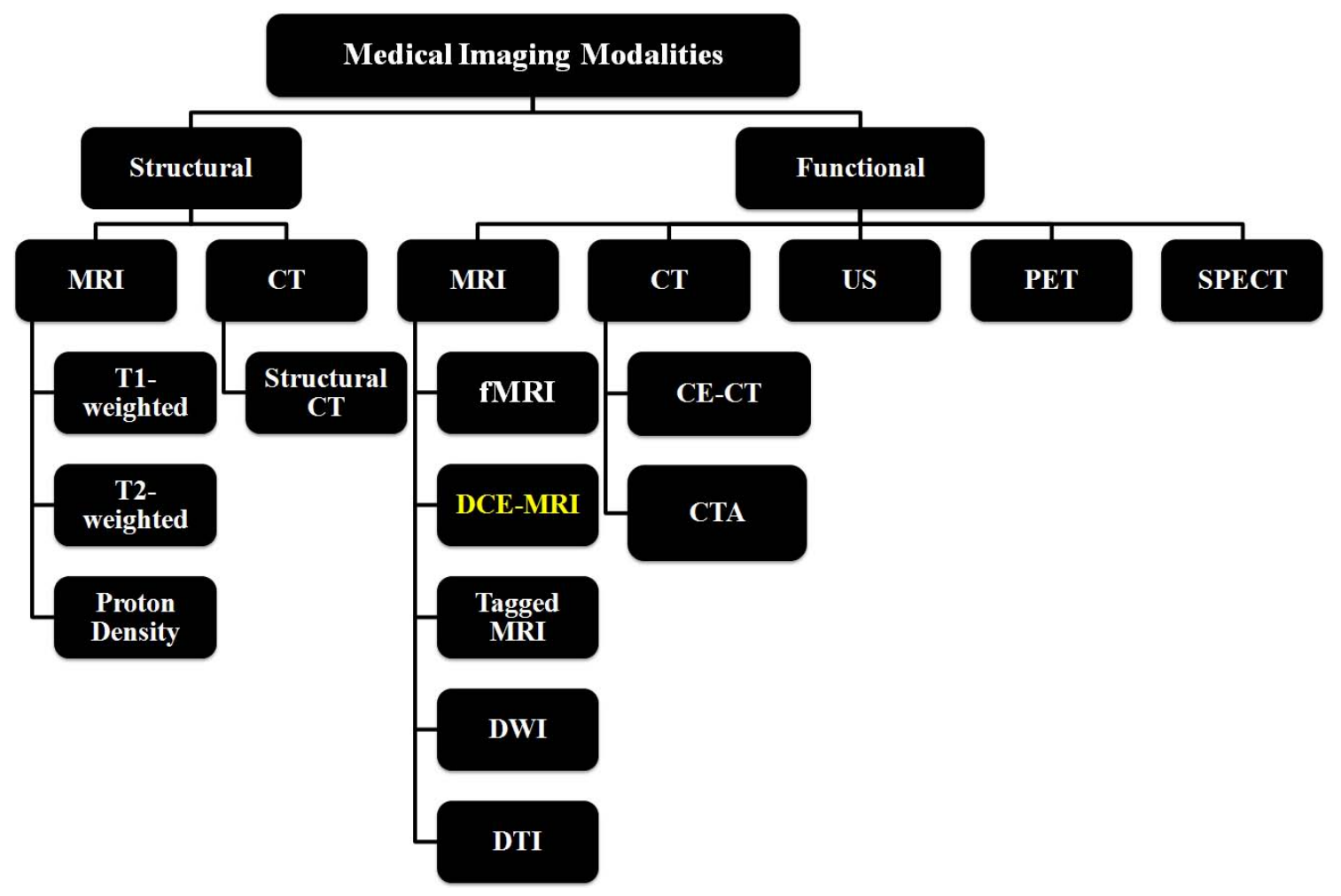

FIGURE 3: Categories of medical image modalities based on the type of information that they provide about the organ being imaged, i.e., structural or functional imaging.

\section{A. MAGNETIC RESONANCE IMAGING}

Magnetic resonance imaging (MRI) is a medical imaging modality that is based on the same principals of nuclear magnetic resonance spectroscopy [24]. MRI has become the most powerful and central noninvasive tool for clinical diagnosis of diseases [25]. The fundamental principle of MRI is based on the use of a strong static magnetic field in which the hydrogen nuclei (single proton) of water molecules in human tissues are aligned parallel to that field. Then, an external radio frequency (RF) pulse (wave) is applied to the 
unpaired magnetic spins (proton) aligned in the static magnetic field, making them spin in different directions [26]. The interaction between the RF and proton spins leads to periodic absorption and emission of energy. When the protons relax back to their lower energy (equilibrium) state, they release detectable signals (energy) that are spatially encoded and are used to construct the MR image. Different types of tissues (muscle, fat, cerebral spinal fluid, etc.) send back measurably different types of tissue-specific signals following the application of the same RF pulse. The contrast of an MR image is strongly dependent on the way the image is acquired. Different components of the scanned area can be highlighted using different preselected pulse sequences: strength, shape, and timing of the RF and gradient pulses (external fields). Generally, MRI can be used to acquire planar 2D images (Figure 4), 3D volumes (Figure 5), or sequences of 3D volumes (i.e., 4D images see, Figure 6). Most commonly-known specialized MRI techniques are shown in Figure 7. Below, an overview of the main MRI types is given.

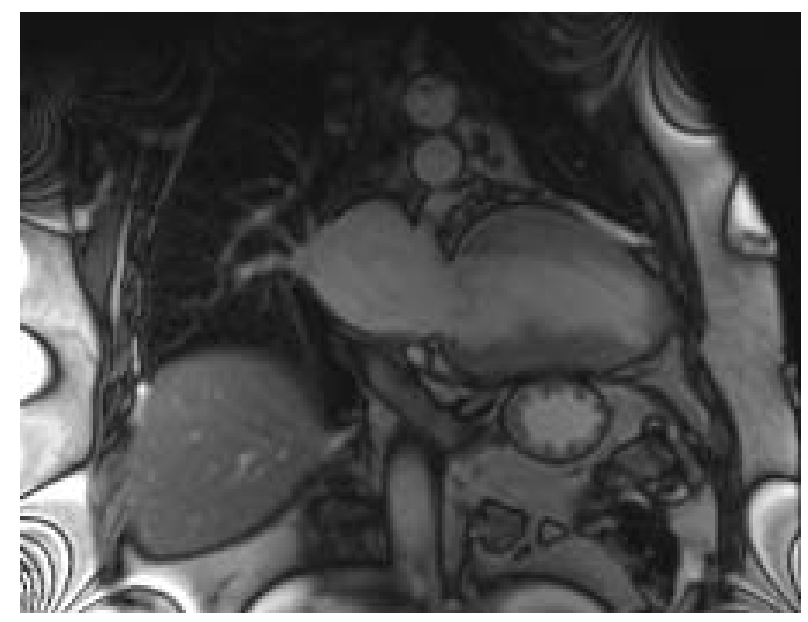

FIGURE 4: 2D cine MR image of the heart.

\section{Structural MRI}

The amount of energy (or signal strength) of the MRI primarily depends on the magnetic relaxation properties of body atomic nuclei. The time of the relaxation process (i.e, the time taken by nuclei to return to their baseline states after applying the RF pulse) is known as longitudinal relaxation time $\left(T_{1}\right)$ or transverse relaxation time $\left(T_{2}\right)$, based on the 


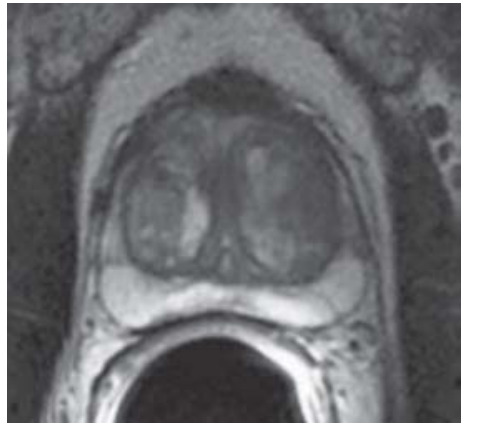

(a)

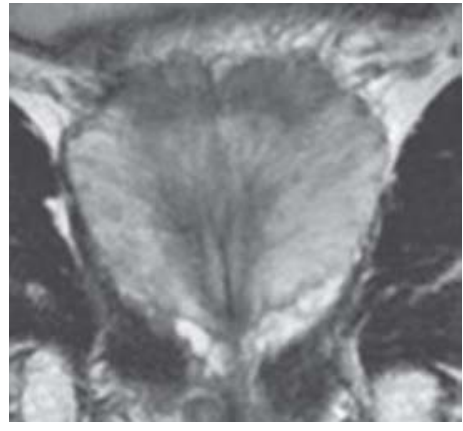

(b)

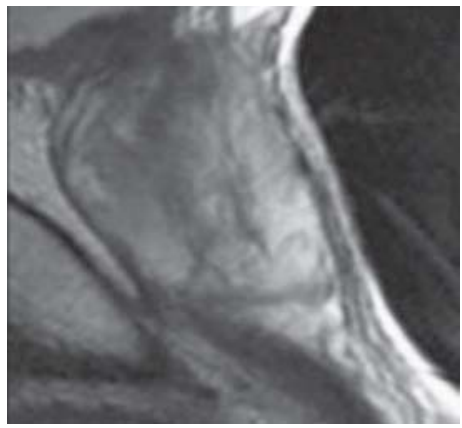

(c)

FIGURE 5: 3D $T_{2}$-weighted MRI of the prostate, captured in the axial $(a)$, coronal $(b)$, and sagittal (c) planes. Courtesy of [9]

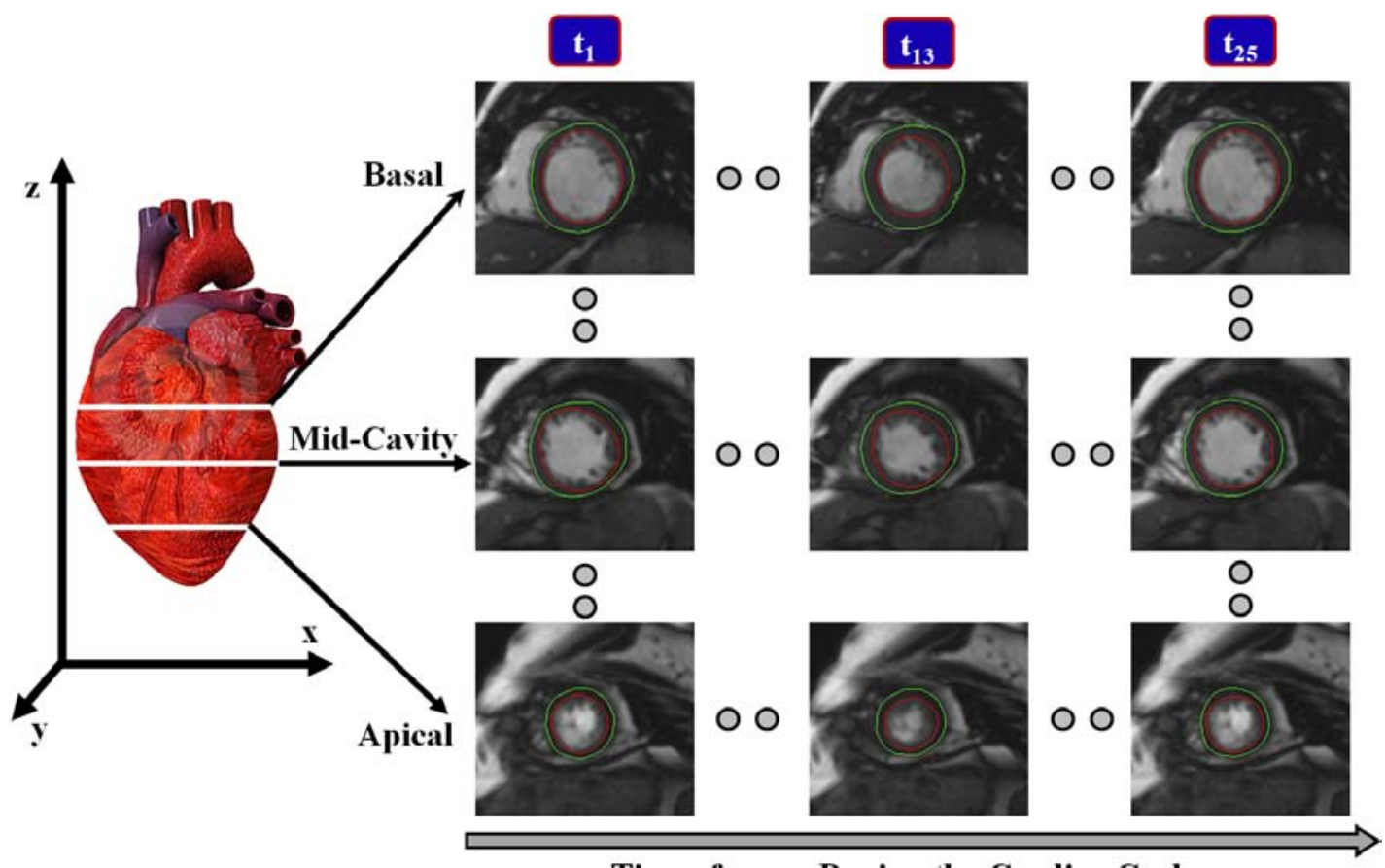

Times frames During the Cardiac Cycle

FIGURE 6: 4D (3D + time) cardiac MRI data. Images are acquired at different sections covering the heart (from basal to apical) and each section consists of a time series of 25 image frames over the cardiac cycle $\left(t_{1} \longrightarrow t_{25}\right)$. The inner and the outer borders of the heart wall are shown in red and green, respectively.

orientation of the component with respect to the magnetic field. Every tissue in the human body has its own $T_{1}$ and $T_{2}$ values, which depend on the concentration of protons in the tissue in the form of water and macromolecules. $T_{1}$-weighted MRI is the commonly-run clinical MRI scan that emphasizes $T_{1}$-contrast, i.e., most of the contrast between tissues is 


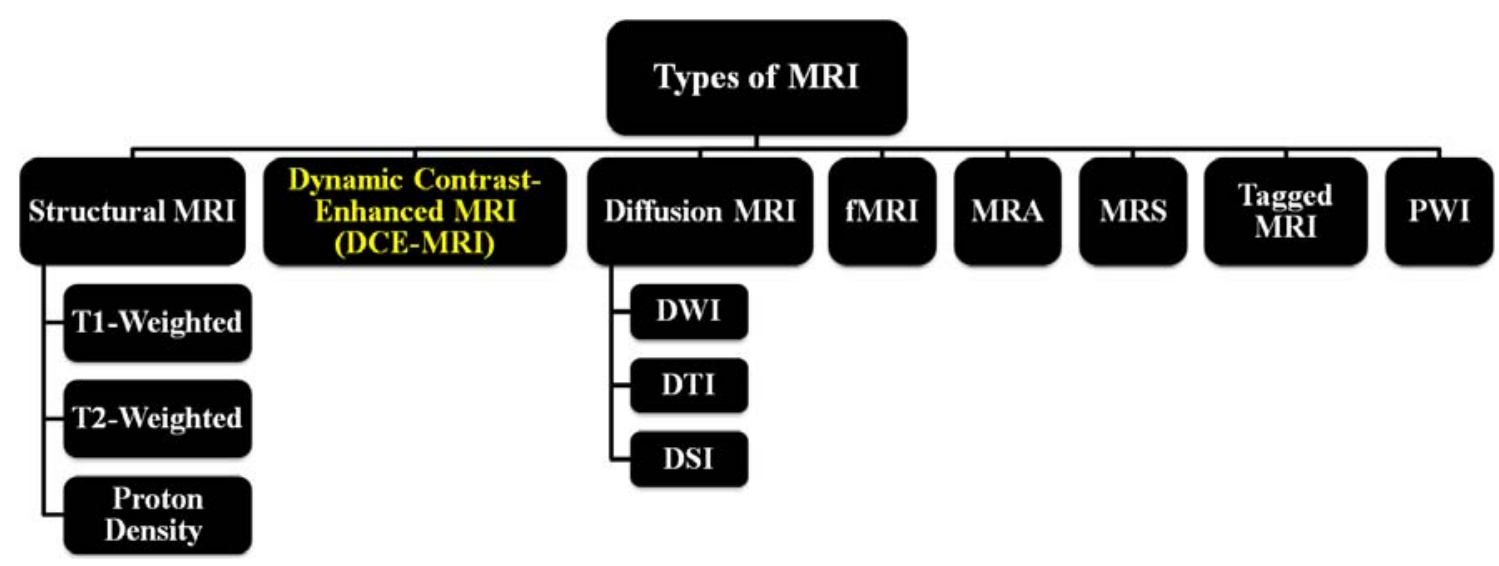

FIGURE 7: Different specialized MRI acquisition techniques.

due to differences in tissue $T_{1}$ values. Thus, it is the best MRI method for demonstrating anatomical details. $T_{2}$-weighted is a basic type of MRI in which most of the contrast between tissues is due to differences in tissue $T_{2}$ values. Usually, $T_{2}$-weighted scans are used when contrast between fluid, abnormalities (e.g., tumors, inflammation, trauma), and the surrounding tissues is required. Therefore, it is the best MRI method for pathological details. In practice, $T_{1}$ - and $T_{2}$-weighted images provide complementary information, so both are important for characterizing abnormalities. Finally, the spin or proton densityweighted scans try to have no contrast from either $T_{1}$ or $T_{2}$. The only signal change is due to differences in the amount of available spins (hydrogen nuclei in water). The main advantage of the proton density-weighted images is the increase in contrast between fluid and non-fluid tissues. However, proton density-weighted images usually show less contrast resolution than $T_{1}$ - and $T_{2}$-weighted images. This is due to the fact that the difference in hydrogen concentration (proton density) of soft tissues is relatively small.

The main strength of MRI is that it offers the best soft tissue contrast among all image modalities. Moreover, it is a dynamic technology that can be optimized to tailor the imaging study to the anatomical part of interest and to the disease process being studied. In this regard, MRI offers different degrees of dynamic optimization. For example, the imaging plane can be optimized to the anatomical area being studied (axial, coronal, sagittal, see Figure 5, and multiple oblique planes can be captured with equal ease). In addition, as previously described, the signal intensities of the imaged tissues can be controlled by 
selecting the type of the scan (see, Figure 8): either proton density-weighted, $T_{1}$-weighted, or $T_{2}$-weighted [25].

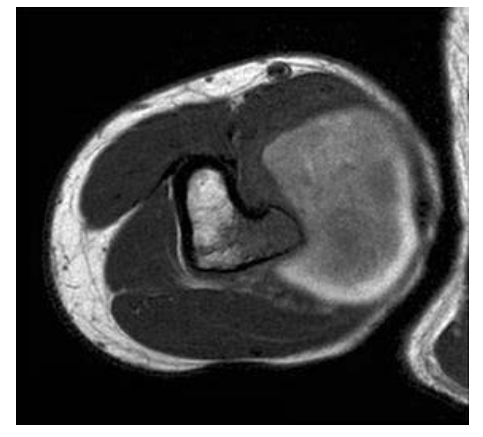

(a)

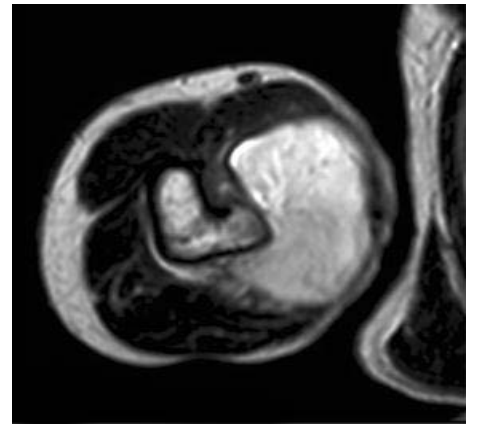

$(b)$

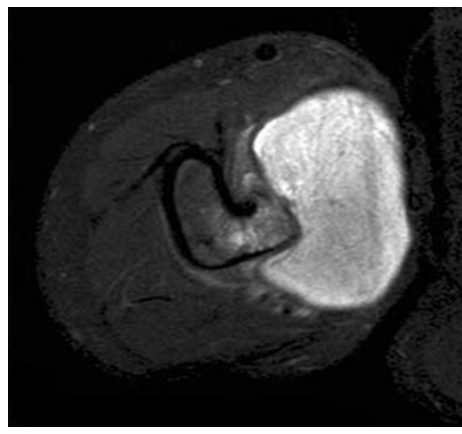

(c)

FIGURE 8: Examples of axial MRI scans of the osteochondroma underlying the pseudoaneurysm: (a) $T_{1}$-weighted, (b) proton density-weighted, and (c) $T_{2}$-weighted images. The images have different contrast that reveal specific information about the structures in the knee. Courtesy of [10].

\section{Dynamic Contrast-Enhanced MRI (DCE-MRI)}

Although structural MRI provides excellent soft tissue contrast, it lacks functional information. Dynamic contrast-enhanced MRI (DCE-MRI) is a special MR technique that has the ability to provide superior information of the anatomy, function, and metabolism of target tissues [27]. The technique involves the acquisition of serial MR images with high temporal resolution before, during, and at several times after the administration of a contrast agent (CA) into the blood stream. In DCE-MRI, the signal intensity in the target tissue changes in proportion to the CA concentration in the volume element of measurement, or voxel. DCE-MRI is commonly used to enhance the contrast between different tissues, particularly normal and pathological. Dynamic MRI has gained considerable attention due to the lack of ionizing radiation, increased spatial resolution, ability to yield information about the hemodynamic (i.e., perfusion) properties of tissues, micro-vascular permeability, and extracellular leakage space [28]. Typical examples of dynamic MRI time series data of the kidney, heart, and prostate are shown in Figure 9. 


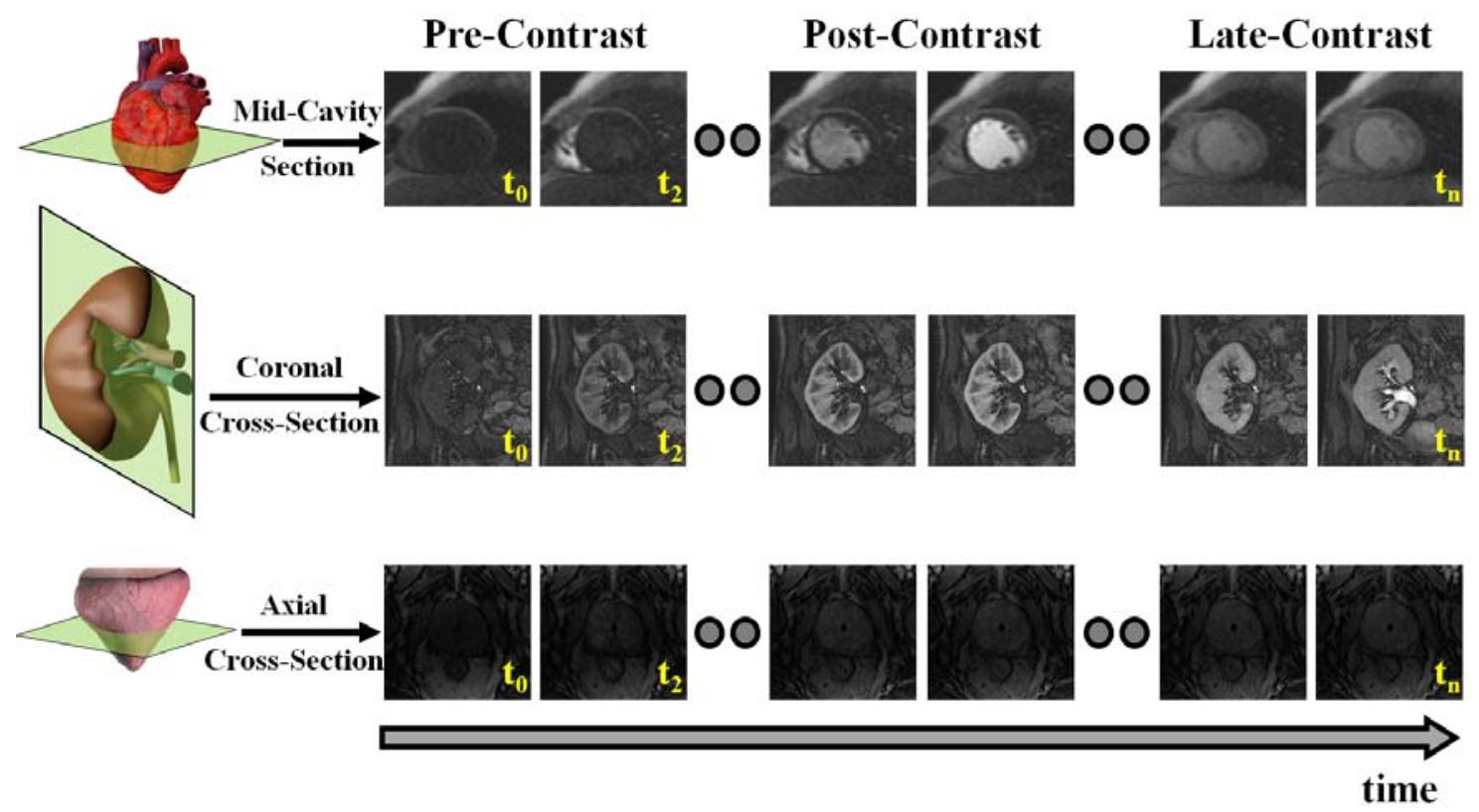

FIGURE 9: Dynamic MR images taken at different time points post the adminstration of the contrast agent (CA) showing the change of the contrast as the CA perfuse into the tissue for the heart, kidney, and prostate.

Unlike structural MRI where the contrast mainly depends on the intrinsic magnetic relaxation times $T_{1}$ and $T_{2}$, the DCE-MRI technique employs the administration of a CA prior to the medical scan. The main role of the CA is to increase the image contrast of anatomical structures (e.g., blood vessels) that are not easily visualized by the alteration of the magnetic properties of water molecules in their vicinity. This in turns improves the visualization of tissues, organs, and physiological processes. In clinical practice, several types of CAs are in use and their choice is based on the imaging modality. In particular, for MRI there are several types of CAs such as paramagnetic agents, superparamagnetic agents, extracellular fluid space (ECF) agents, and tissue (organ)-specific agents, see Figure 10.

Superparamagnetic CAs are based on water insoluble iron oxide crystals, usually magnetite (Fe3O4) or maghemite $(\gamma$-Fe2O3). These CAs are suitable for MRI scans of the gastrointestinal tract, including the liver, spleen, esophagus, the stomach, etc. The superparamagnetic can be classified into superparamagnetic iron oxide particles and ultrasmall superparamagnetic iron oxide particles [29]. The most successful MRI contrast agents that have been widely investigated are gadolinium-based. Gadolinium, a rare metal, is a non- 


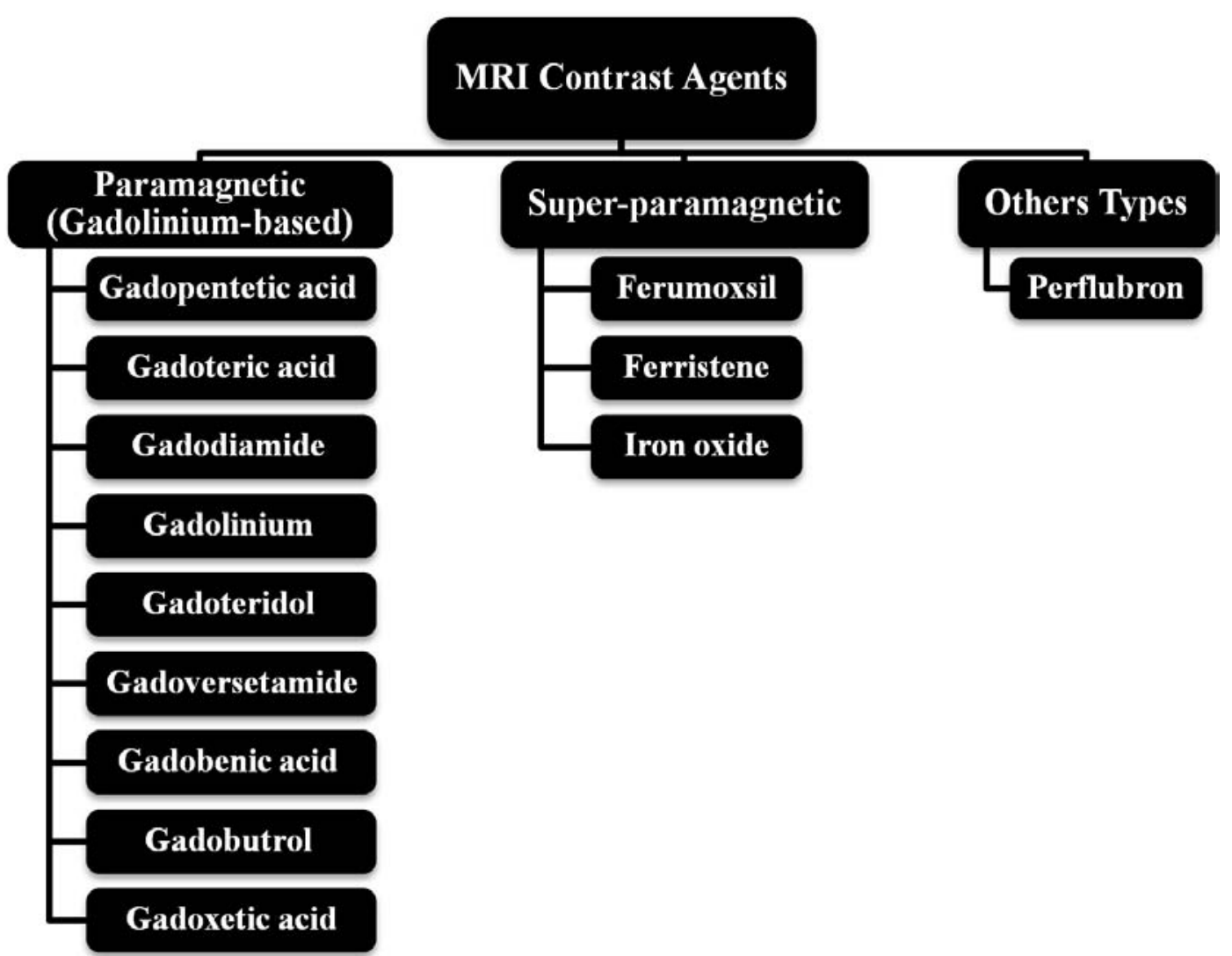

FIGURE 10: Different types of contrast agents used in MRI medical scans.

toxic paramagnetic CA that enhances the detected MR signal and produces high contrast images of soft tissues by decreasing $T_{1}$ relaxation times of water protons in living tissue in the vicinity of the paramagnetic CA. Used for cardiovascular, oncological, and neurological imaging, the MRI does not utilize radioactive materials such as X-ray.

\section{Diffusion MRI}

Sometimes MRI techniques that depend on using CA (e.g., gadolinium-based) may be harmful to some patients (e.g., patients with kidney problems). In order to avoid the use of CAs, in recent years researchers have started to investigate an MRI technique called diffusion imaging. Diffusion MRI is a functional technique that is based on the measurement of the micromovements (random, Brownian) of extracellular water molecules inside the body. These movements provide indirect information about the structures surrounding these water molecules. Diffusion MRI is a noninvasive technique that has the advantage of 
being acquired very rapidly, without the use of any CA or specialized hardware. Basically, DWI focuses on the movements of the water molecules inside the body. Diffusion MRI can be classified into three main types, namely, diffusion-weighted imaging (DWI), diffusion tensor imaging (DTI) and diffusion spectrum imaging (DSI).

a. Diffusion-weighted imaging (DWI) is designed to obtain images whose contrast depends on the differences in water molecule mobility. This is achieved during data acquisition by adding diffusion magnetic field gradients. The degree of diffusion weighting of the sequence, expressed as the b-factor (in s/mm2), depends on the amplitude of the field gradient, time of application, and time interval between the magnetic field gradients. A typical DWI-MRI for the prostate is shown in Figure 11. DWI is a well-established MRI method that has been successfully used for tumor localization and diagnosis [30], investigation of brain disorders, such as epilepsy, multiple sclerosis, brain abscesses and tumors, and hypertensive encephalopathy [31], and in-vivo study of tissue microstructure [32].

(a)

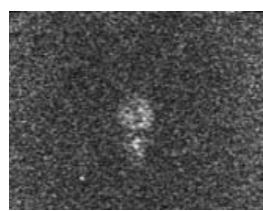

(b)

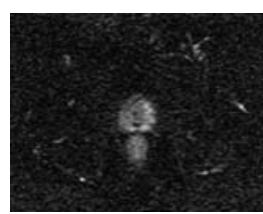

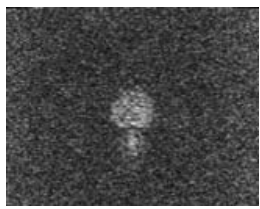

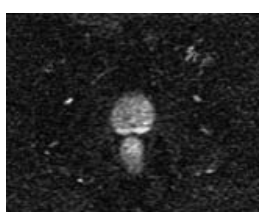

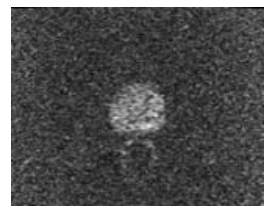

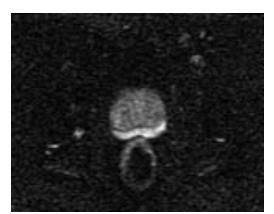

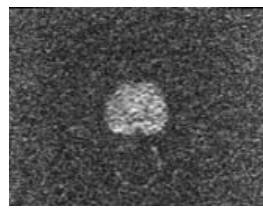
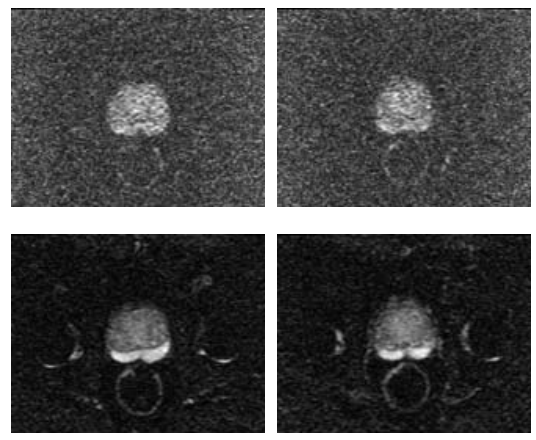

FIGURE 11: Diffusion MR images for the prostate at $(a)$ b-value of $0 \mathrm{~s} / \mathrm{mm}^{2}$ and $(b) \mathrm{b}$ value of $800 \mathrm{~s} / \mathrm{mm}^{2}$.

b. Diffusion tensor imaging (DTI) is another type of diffusion MRI that is based on the measurement of the Brownian motion of water molecules in tissue. DTI is a newlydeveloped MRI technique to study in vivo tissue microstructure, e.g., the connectivity between different brain areas. This MRI modality allows the scientist to be able to look at the network of nerve fibers. Nowadays, DTI has been used by neuroscientists to study a number of disorders (e.g., addiction, epilepsy, traumatic brain injury, and various neurodegenerative diseases) and to identify subtle abnormalities in a variety of diseases, (e.g., 
stroke, multiple sclerosis, dyslexia, and schizophrenia) [32]. An example of a brain nerve's connectivity bundle obtained from a 3D DTI data set is shown in Figure 12.

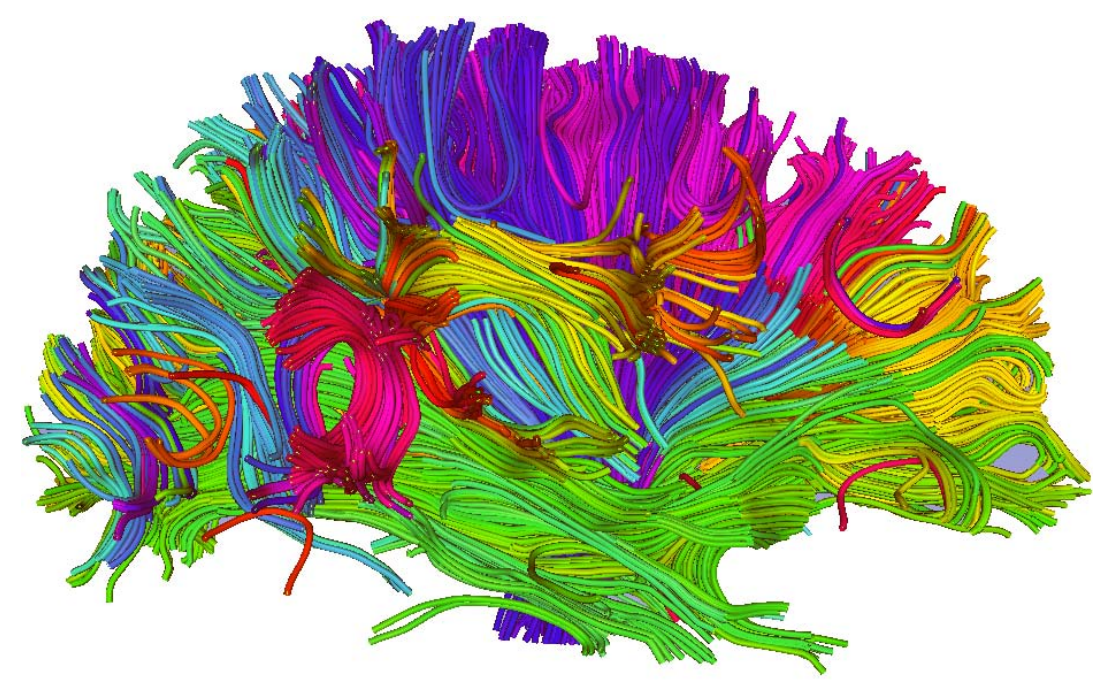

FIGURE 12: A 2D projection of the tractographic color map estimated from a DTI imaging data set. Color codes have been assigned based on the mean orientation of the fibers. Note that the data set may be rotated continuously into various planes to provide better tractographic assessments of the brain.

c. Diffusion spectrum imaging (DSI) is the third type of diffusion MRI. Although DTI has been proven as a useful MR technique in studying in vivo fibrous connectivity, it cannot directly image fiber crossings within a single voxel [33]. To overcome this limitation, DSI has been developed as a generalization of DTI to map complex structures such as crossing fibers. Thus, DSI allows more accurate mapping of axonal trajectories than other diffusion imaging approaches [33]. The disadvantages of DSI are that it requires several hundred images and long acquisition times compared with DTI and DWI [34].

\section{Functional Magnetic Resonance Imaging (fMRI)}

Functional magnetic resonance imaging (fMRI) is a noninvasive MRI technique that is used to study the activated area of the brain after certain stimuli and to map changes of brain hemodynamics that correspond to mental operations. The technique has the ability to observe brain function as well as to determine which structures participate in specific func- 
tions [35]. fMRI acquires consecutive images, one while the brain is in rest state followed by another one after the brain is stimulated in some way. The areas of brain activation are determined as any regions that are different between the two scans. Functional MRI allows radiologists to better understand brain organization and to assess neurological status and neurosurgical risk. Unlike Electroencephalography that provides surface information (brain waves) through electrodes mounted on the patients' scalp, fMRI has the advantage of providing in-depth details of what is inside the brain. Figure 13 shows a typical fMRI for the brain of a normal person reading. The arrows point to parts of the brain that are activated. As shown in the figure, the fMRI can determine the changes in particular regions of the brain in response to a certain stimuli.

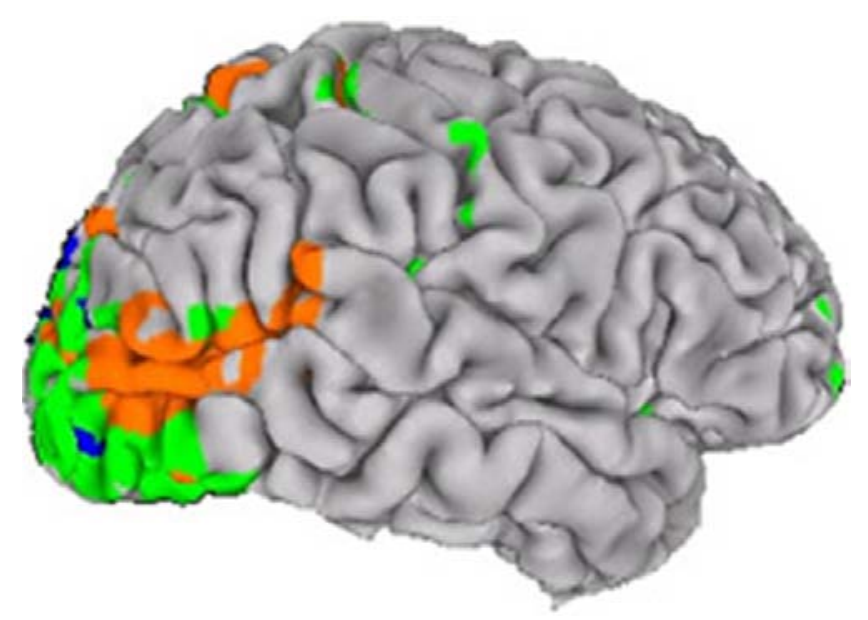

FIGURE 13: A lateral view showing the active human brain areas of a subject viewed moving and static figures. Orange regions are the activated brain areas when the subject viewed moving figures, while green and blue regions responded similarly to both moving and static figures. Courtesy of [11].

5. Tagged Magnetic Resonance Imaging

Tagged MRI is a well-known MRI technique for detailed and noninvasive visualization of cardiac motions [36]. This imaging modality can help in the localization of heart diseases (e.g., coronary atherosclerosis) and global conditions (e.g., heart failure and diabetes) that result in heart wall dysfunction. Cardiac MRI tagging places a pre-specified 
pattern of temporary markers (called tags) inside soft body tissues, e.g. tag lines created by patterns of magnetic spin in the examined tissue so that the motion in the tagged tissue can be measured from the images [37]. This technique complements traditional anatomical images and can capture detailed information about the heart over time. The tag lines allow for computing displacement, velocity, rotation, elongation, strain, and twist of the heart. While traditional MRI techniques carry only information about the motion at the boundaries of an object, the tag lines allow us to examine the strain and displacement of the interior of the tissue in close detail [38]. Figure 14 shows a typical tagged MRI time-series of the heart.

\section{Time Frames During the Cardiac Cycle}

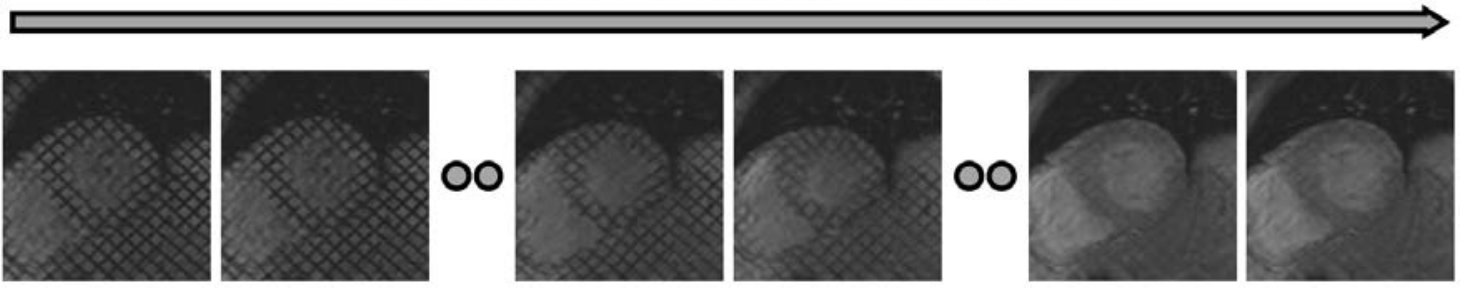

FIGURE 14: A example of the tagged MRI time-series for the heart.

\section{Perfusion-Weighted Imaging (PWI)}

Perfusion-weighted imaging (PWI) is a serial MRI technique designed to image blood flow into brain vasculature. PWI uses an MR contrast to provide information about the location and extent of cell death within a few hours of a stroke, and it has been shown to be superior to conventional MRI [39]. The most common technique is known as dynamic susceptibility contrast imaging, which has been thoroughly studied to measure the cerebral blood flow of the brain for patients with vascular stenosis, stroke, and brain tumors [40]. This MR technique helps the neuroradiologist to more accurately understand brain perfusion by providing other important parameters, such as blood volume and perfusion enhancement time.

In total, potential advantages of MRI include ( $i$ ) MRI does not involve exposure to any harmful radiation, can be repeated sequentially over time, and has the ability to generate cross-sectional images in any plane (including oblique planes); (ii) MRI provides 
superior resolution with far better contrast (the ability to distinguish the differences between two arbitrarily similar but not identical tissues) compared with other medical image modalities [25]; and (iii) MRI plays an important role in assessing the extent and location of tumors, directing biopsies, planning proper therapy, and evaluating therapeutic results [41]. Nonetheless, MRI imaging modality has its own disadvantages: (i) MRI data acquisition is relatively long and complex-for each scan the imaging parameters and pulse sequence need to be fixed; (ii) MRI is not suitable for patients with metal implants due to its magnetic nature; (iii) MRI suffers from sensitivity to noise and image artifacts; (iv) MRI signals are dependent on the imaging sequence used and can become non-linear beyond certain concentrations leading to errors in extracted physiology; (v) MRI scanning processes may be uncomfortable for some people because it can produce claustrophobia. Recent improvements in MRI design aim to aid claustrophobic patients by using more open magnet designs and shorter exam times.

\section{B. QUANTIFICATION OF ORGAN FUNCTIONALITY USING CE IMAGES}

The use of contrast-enhanced (CE) medical images for the quantification of the human organ functionality has been increased in recent years due to its ability to provide superior anatomical and functional information about the tissue being imaged. For example, the potential of CE images has shown an important role in many clinical applications, such as localizing the locations and the extent of pathological tissues [42-44], differentiation between various brain tumors [45-48], early detection of breast cancer [49-55], planning proper therapy [56], and evaluating therapeutic results [57-60].

The goal of this work is to develop a noninvasive image-based framework for the analysis of the complex data from CE medical images. The dissertation focuses on building new mathematical models and learning techniques in order to facilitate the accurate analysis of the perfusion of the CA in living organs through imaging. The ultimate goal of the proposed work is to be applicable to medical imaging of different organs in living subjects. In this dissertation, the proposed models have been applied to three case studies. The first 
case study defines a CAD system for the early detection of acute renal transplant rejection using DCE-MRI. In the second case study, a comprehensive framework is proposed for the analysis of the transit of the CA at cardiac first-pass MRI (FP-MRI) to depict regional and transmural effects across the myocardial wall. Finally, a CAD system for early detection of prostate cancer using DCE-MRI is proposed. In the following sections, a brief overview is given for each of the proposed analysis systems.

\section{Early Detection of Renal Transplanted Rejection}

Early detection of acute renal rejection-the immunological response of the human immune system to a foreign kidney-is important to institute salvage medical and immune therapy in patients with transplanted kidneys [61]. In the United States, approximately 17,736 renal transplants are performed annually [62], and given the limited number of donors, transplanted kidney salvage is an important medical concern. Functional assessment of renal transplant is initially evaluated using blood tests and urine sampling, e.g., plasma creatinine and creatinine clearance. However, these indexes have low sensitivity, since a significant change in creatinine levels is only detectable after the loss of $60 \%$ of kidney function. Therefore, more sophisticated techniques, such as radionuclide approaches and Ultrasound, are used for noninvasive evaluation of kidney dysfunction. However, radionuclide-based approaches involve radiation exposure, thereby limiting the applicability of these techniques. Ultrasound imaging, while noninvasive, suffers from low signal-to-noise ratios, shadowing artifacts, and speckles that greatly decrease image quality and diagnostic confidence. These shortcomings make these techniques impractical for clinical use. Thus far, biopsy (see Figure 15) remains the gold standard for the assessment of renal transplant dysfunction, but only as the last resort because of its invasive nature, high cost, and potential morbidity rates. Also, the relatively small needle biopsy sample may lead to over- or under-estimation of the extent of inflammation in the entire graft. Therefore, there is an urgent need for new technology based on noninvasive techniques that possesses the ability of early and accurate diagnosis of the transplanted kidney status. 


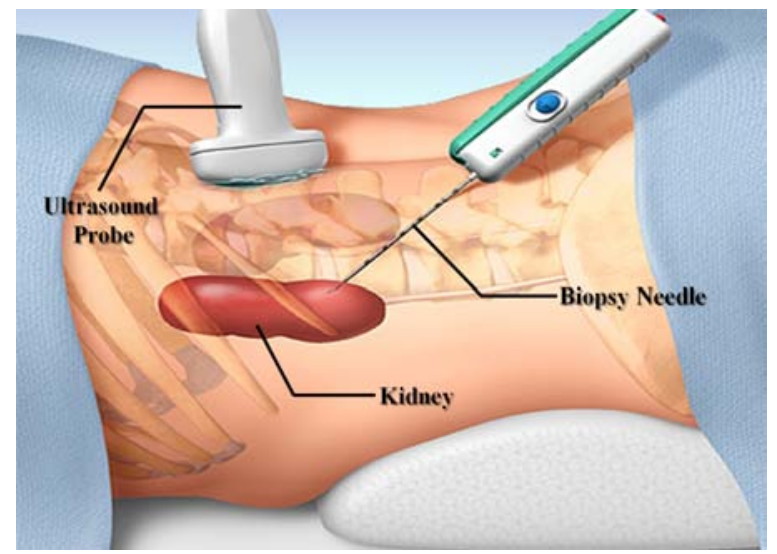

FIGURE 15: An illustration of taking biopsy samples from the kidney. A thin needle is inserted through the skin to perform the operation, and the procedure is usually directed by an imaging device (e.g., ultrasound) to help in placing the needle into the right location [12].

The proposed work in this dissertation seeks to provide a new noninvasive CAD system that possesses the ability to facilitate the accurate analysis of the perfusion of a CA in the kidney tissue in an effort to extract perfusion parameters that distinguish non-rejection and acute rejection transplants. In DCE-MRI of the kidney, the widely used clinical agent gadolinium behaves as a leakage agent, namely it distributes in the extracellular extravascular space, and at short times (up to about 2 minutes ( $\mathrm{min}$ ) after administration) perfusionrelated parameters that reflect the CA delivery to the tissue can be derived from the tissue kinetic curves or time-intensity curves, see Figure 17. To characterize the transplanted kidney, the proposed framework segments the kidney and cortex (as it is the functional unit that is most affected by rejection) after handling the global and local motions. Then, agent kinetic curves of the segmented cortex are constructed and perfusion-related parameters are estimated and are used for the evaluation of kidney status, see Figure 16. In addition to the global information about the transplanted kidney condition, pixel-by-pixel displays of perfusion for local assessment was developed (see Figure 18). These pixel-wise perfusion maps are important for the radiologist to investigate which kidney regions need attention during follow-up of the treatment and thus to determine the appropriate therapy. 


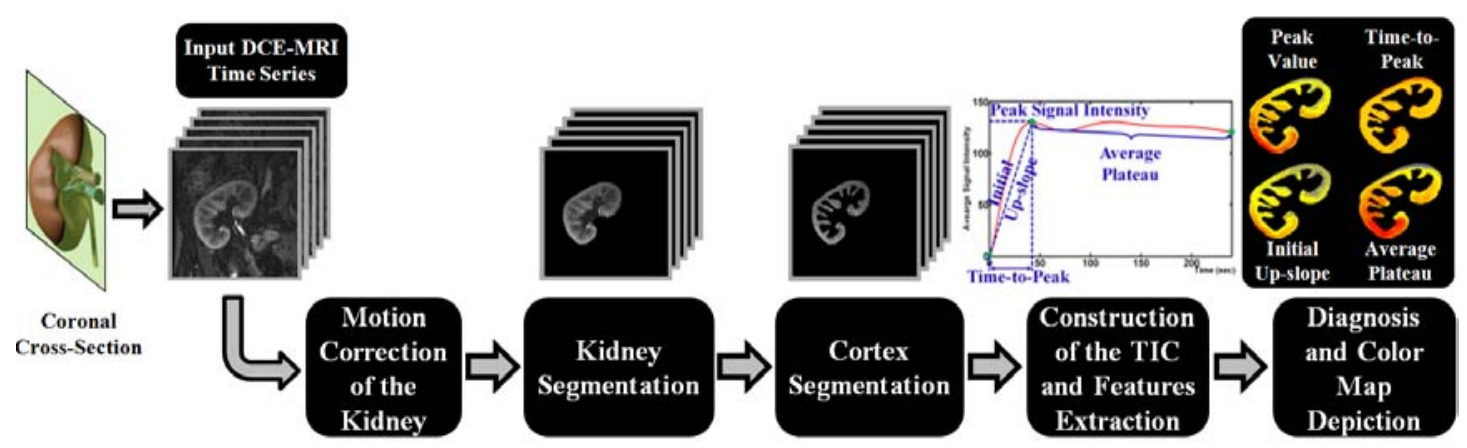

FIGURE 16: A typical CAD system for the detection of acute renal transplant rejection. The motion correction step is used to handle global and/or local motions of the input DCEMRI data. Then, the kidney and its functional unit (cortex) are extracted from the surrounding abdomen tissue. Next, agent kinetic curves are constructed and perfusion parameters are extracted and are used for diagnosis.

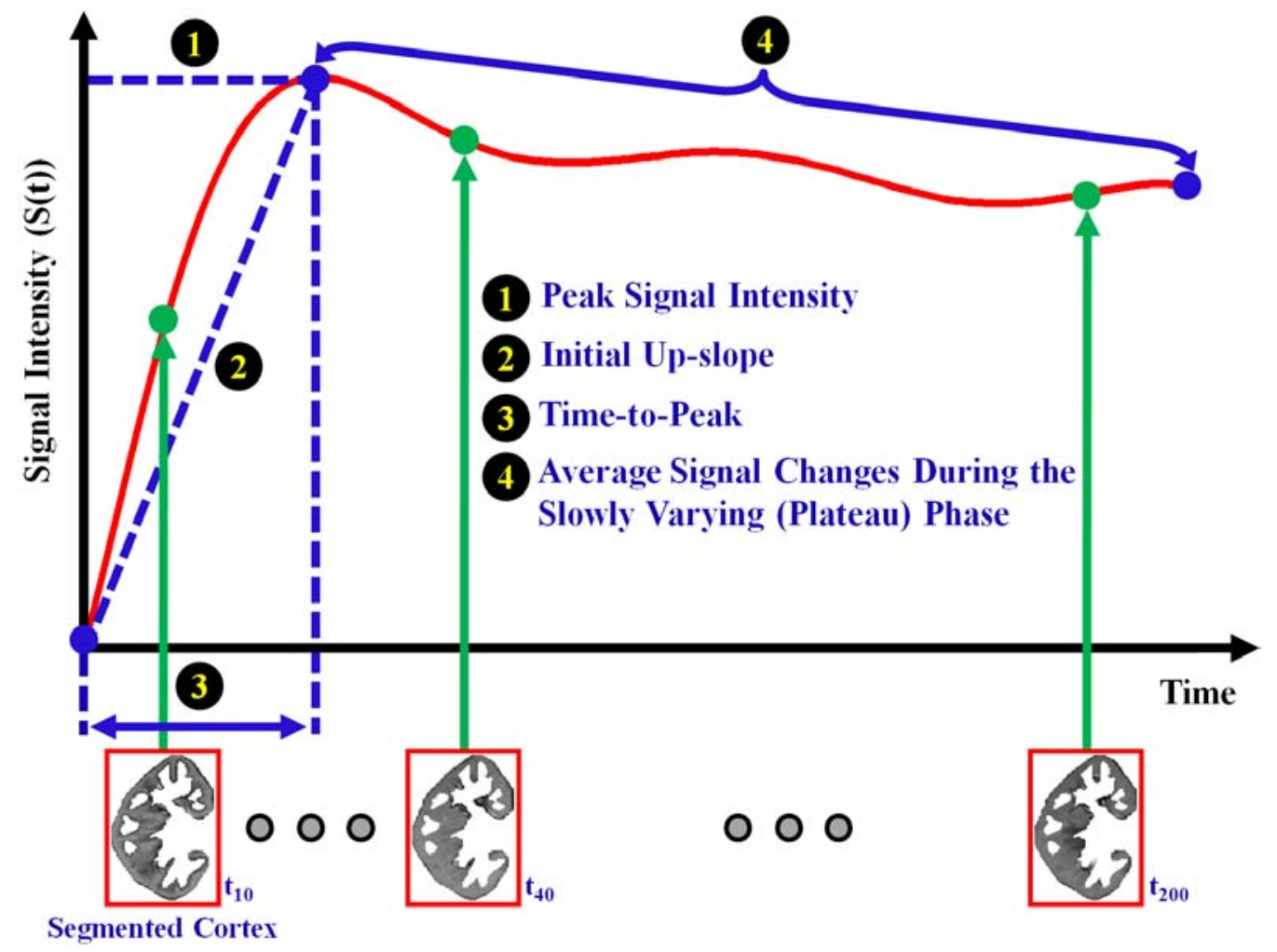

FIGURE 17: A typical time-intensity curve of the average intensity of the kidney measured before and after contrast agent adminstration. The curve illustrates typical perfusion-related indexes that can be estimated and used for diagnosis of the kidney status. 

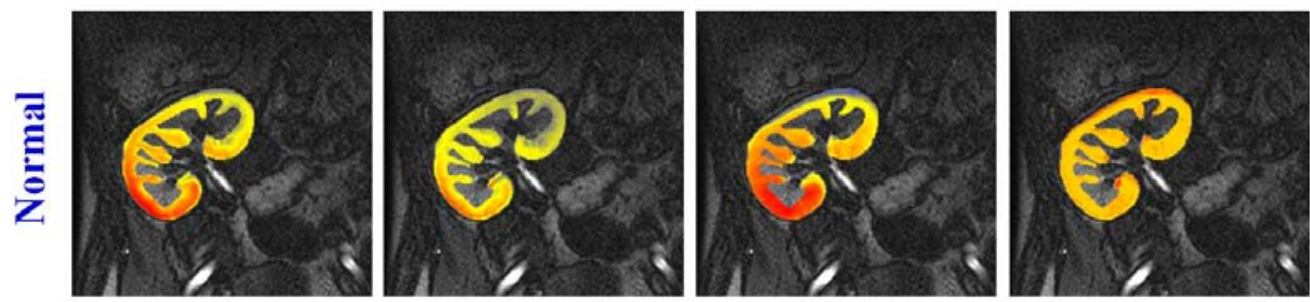

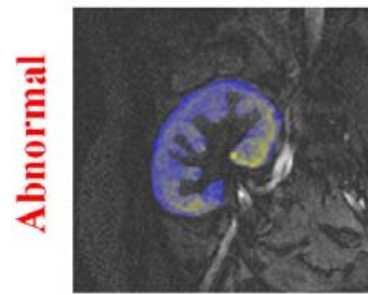

(a)

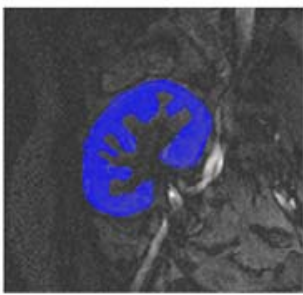

(b)

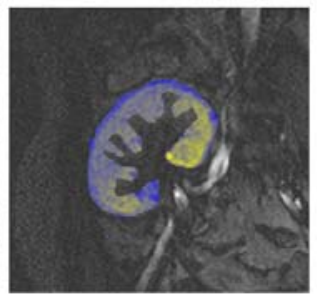

(c)

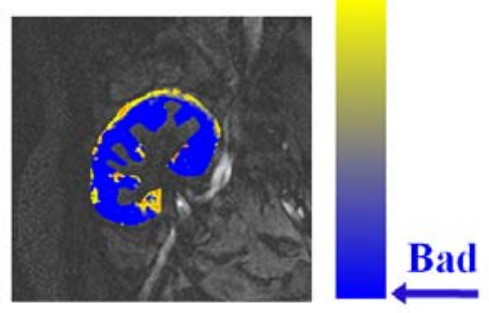

(d)

FIGURE 18: Pixel-wise perfusion maps of the perfusion indexes shown in Figure 17: $(a)$ peak signal intensity, $(b)$ initial up-slope, $(c)$ average plateau, and $(d)$ time-to-peak; for one normal (upper row) and one abnormal (lower row) kidney. The red and blue hues of each color scale correspond to the highest and lowest values, respectively. Note all indexes show worsening of perfusion with pathology.

\section{Functional Assessment of Myocardial Perfusion}

Heart failure is considered the most important cause of morbidity and mortality in cardiovascular disease, which affects approximately 6 million US patients annually. Thus it is critical in these patients to detect precursors in order to prevent progression to end-stage disease. Ischemic heart disease is considered to be the most common underlying cause of heart failure [63]. Therefore, the detection of the heart failure in its earliest forms is essential to prevent its relentless progression to premature death. Recent clinical research has explored a novel therapeutic strategy for restoring damaged heart wall tissue due to heart attack based on harnessing normal cells from the patient's own heart $[64,65]$. To reliably detect clinically meaningful responses to therapy, cardiac magnetic resonance images are usually used as the "gold standard" for evaluating heart function, e.g., quantifying the LV function and measuring the infarct size [66]. While several MRI studies have focused on viability [42-44] and contractile function [67-69] in areas with myoregeneration, there is 
a dearth of in-vivo studies designed to address the key issue of the effects of the myoregeneration therapy on the associated blood nutrient supply.

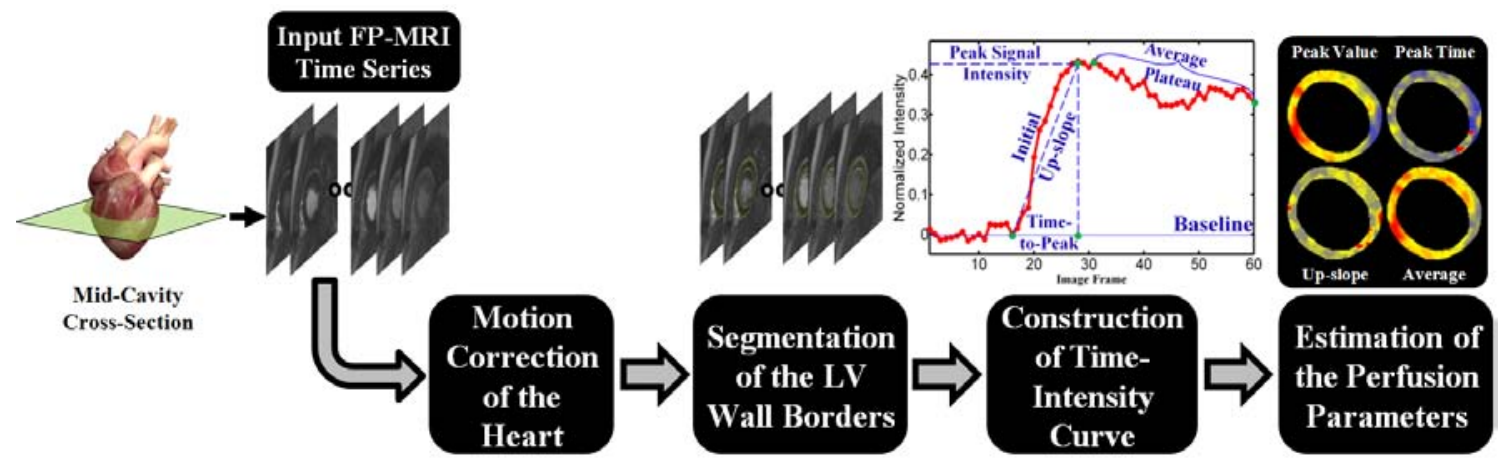

FIGURE 19: A schematic diagram of the proposed CAD system for the analysis of myocardial perfusion. The input to the system is the cardiac FP-MRI data. The motion correction step is used to handle the global and/or the local motions of the heart. Then, the left ventricle (LV) wall is segmented, the agent kinetic curve is constructed, and perfusion parameters are estimated and used to depict regional and transmural effects across the myocardial wall.

In this dissertation, a comprehensive framework for functional assessment of myocardial perfusion is proposed based on the study of first-pass dynamics of gadolinium CA, see Figure 19. First-pass MRI was employed because of its strong relation to perfusion kinetic parameters, such as permeability-surface area product, blood flow, and proportional blood volume [70]. The proposed system is evaluated in patients with ischemic heart damage who are undergoing a novel myoregeneration therapy [65]. The innovation in this case study is a comprehensive analysis framework, with robust automated steps, which optimally characterizes myocardial perfusion and clinical status in heart failure and novel myoregeneration therapies. The proposed framework possesses the ability $(i)$ to followup on treatment, and (ii) to determine whether regions showing improvement with other functional indexes (e.g., derived from viability or cine MRI) also manifest improvement in vascularity as indexed at first-pass myocardial transit of the CA (see Figure 20). 


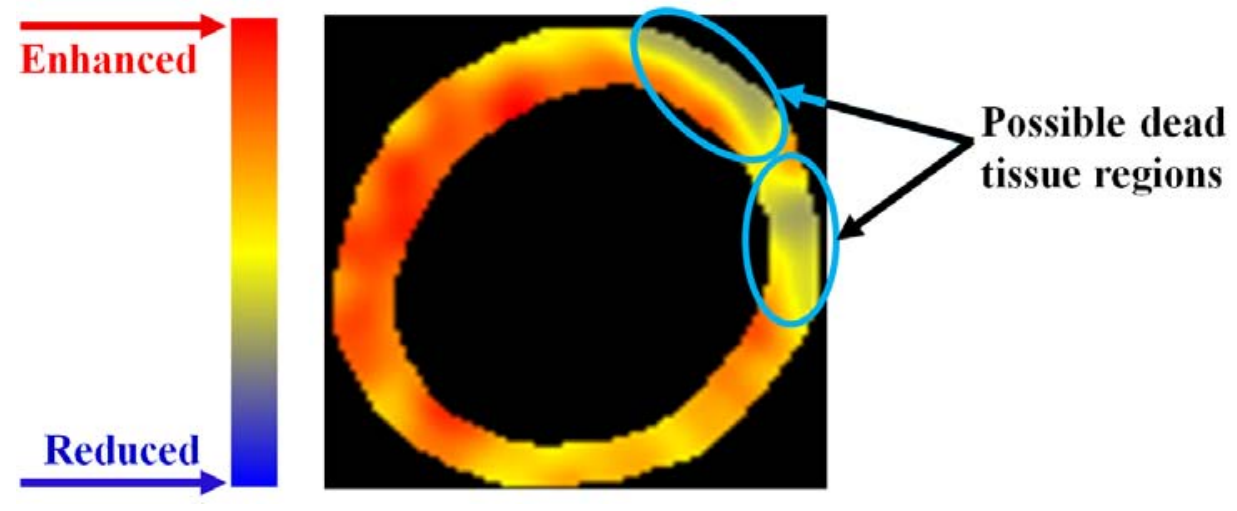

FIGURE 20: Pixel-wise parametric map display of the peak signal intensity demonstrating local LV areas that may be damaged due to the heart attack. The red and blue hues of each color scale correspond to enhanced and reduced perfusion, respectively.

\section{Early Diagnosis of Prostate Cancer}

Prostate cancer is the most frequently diagnosed malignancy among men and remains the second leading cause of cancer-related deaths in the U.S. In 2013, prostate cancer studies reported an estimate of 238,590 new cases and a mortality rate of close to 29,720 [71]. Therefore, early diagnosis of prostate cancer can improve the effectiveness of treatment and increase the patient's chance of survival. Currently, there are different techniques that are used for early diagnosis of prostate cancer. However, the accuracy of these techniques are clearly unsatisfactory. For example, prostate specific antigen (PSA) has a high chance of false positives, ranging from 28\%-58\% [72] and doesn't offer accurate information about the location and extent of the lesion [73]. $T_{2}$-weighted MRI modality, while possessing the ability to provide reliable information about the size and shape of the prostate gland, is limited by unsatisfactory sensitivity and specificity for cancer detection and localization [74]. More recent MRI modalities, such as DCE-MRI [75-77] and DWI [78-80], have demonstrated the promise for early detection of prostate cancer.

The innovation in this case study is a CAD system that integrates stochastic approaches using new Markov-Gibbs random field (MGRF) energy models and the geometric approaches to accurately separate the prostate from the surrounding tissues, after handling 
the global and local motion, in order to optimally distinguish between malignant and benign prostate cancers. The proposed CAD system is based on DCE-MRI and possesses the ability to be used as a supplement for the current technology for early diagnosis of the prostate cancer.

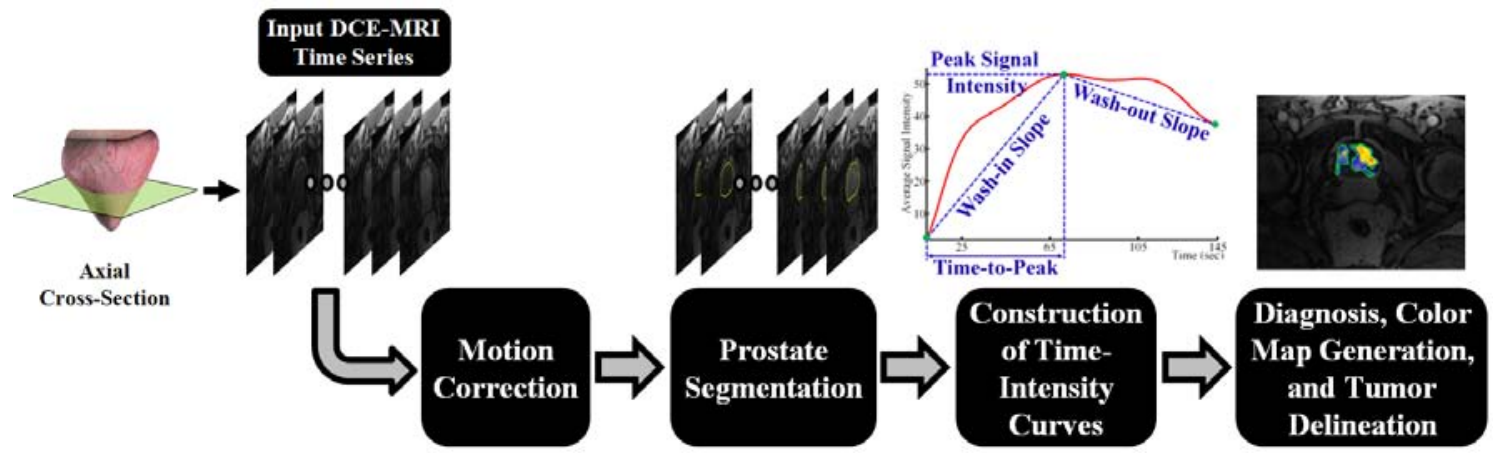

FIGURE 21: A block diagram of the proposed CAD system for the early diagnosis of prostate cancer using DCE-MRI. The time series data is first corrected for motion artifacts. Then, the prostatic tissue is segmented and the agent kinetic curves are constructed. Finally, perfusion-related parameters are extracted from the kinetic curves and are used for the classification of both malignant and benign cases.

\section{LIMITATION OF EXISTING WORK AND THE INNOVATION OF THIS WORK}

The ultimate goal of this work is to develop an image-based, noninvasive framework for the analysis of CE medical images. A generic framework for the analysis of CE images can include one or more of the following steps: (i) motion correction models, (ii) segmentation approaches to extract the object of interest, (iii) construction of enhancement (kinetic or perfusion) curves, and (iii) estimation of perfusion-related features to understand the functionality of the object being diagnosed. Motion correction, object segmentation, and features extraction are the main processing steps in any pipeline for the analysis of $\mathrm{CE}$ images. In the literature, a tremendous number of image segmentation techniques have been proposed for the extraction of medical structures from CE medical images. However, 
existing techniques for image segmentation have their own shortcomings:

- Threshold-based methods are too inaccurate, due to similar intensities (gray levels) of the object of interest and the surrounding tissues. In addition, these methods work fine for post- and late-contrast images, but fail in segmenting pre-contrast images.

- Region growing and split-and-merge methods do not guarantee connectedness of the found objects, which is a basic requirement in many medical imaging and CAD applications. Additionally, region growing-based approaches are sensitive to initialization and often need user assistance to select proper seed points.

- Deformable model-based methods without adequate shape and appearance models fail in the case of excessive image noise, poor image resolution, and diffused boundaries, and they are quite sensitive to imperfect organ contours.

- Most of the existing shape-based methods do not impose temporal constrains on organ segmentation.

- Most of the existing segmentation approaches do not take into account the spatial dependencies between the pixels and therefore are quite sensitive and may perform poorly on some image frames due to the lack of well-defined organ contours.

Moreover, motion correction models are essential for the accurate estimation of perfusion-related parameters to insure that the same pixels be evaluated between the image frames of a given CE time series data. In the literature, several motion correction approaches have been proposed for the alignment of CE images. However, existing motion correction models show the following limitations:

- Global motion correction methods do not take into account the local motion of the objects due to transmitted respiratory and peristaltic effects.

- Local motion correction methods and pharmacokinetic (PK)-based methods register the original grey level data without any prior segmentation, thus they do not guarantee pixel-on-pixel matches of the registered perfusion data.

- PK-based methods increase the computational cost of registration, since a full model fitting has to be performed on a per-pixel basis. 
- Intensity-based registration methods are prone to nonlinear intensity variations over the time series and perform poorly in pre-contrast images.

After motion correction and object segmentation, the next step in a typical CAD system for the analysis of CE images is to extract perfusion-related parameters (indices or features), which are numerical values that correspond to attributes of the segmented region (e.g. mean pixel/voxel intensity). In the literature, established dynamic perfusion analyses derive empirical parameters from the CA kinetic (or signal intensity versus time, see Figure 17), which consists of two time sections or phases: the wash-in (transient or first-pass) and wash-out (tissue distribution or plateau) phases. Typical parameters that can be estimated from these curves include: initial up-slope, peak signal intensity, and time-topeak, etc. The main challenge for CE analysis is the determination of the best parameter(s) that can be used to evaluate the object functionality. For example, while transient phase parameters gives an indication about how the tissue of interest responds to rapid changes in CA kinetics, the resulting limited temporal sampling of the transient phase leads to noisy estimates of those parameters. Therefore, it is crucial to extimae new indices using a large number of data points in order to better characterize tissue perfusion. This can be achieved by calculating the total area under the curve and the average of signal change during the more slowly varying phase (plateau or tissue distribution phase, see Figure 17). For accurate analysis of $\mathrm{CE}$ images it is therefore important to estimate parameters from both wash-in and wash-out phases to completely describe the whole agent kinetic curve. In addition, the fusion between the estimated parameters, using appropriate weights, is of great importance for a more robust characterization of tissue perfusion. The augmentation of multiple parameters is expected to increase the accuracy of CAD decision.

In this dissertation the aim is to address the above-mentioned shortcomings and discrepancies of motion correction and organ segmentation for accurate analysis of CE medical images. In order to achieve this, comprehensive mathematical models for the segmentation and motion correction techniques are proposed. In particular, two novel segmentation models are developed for precise segmentation of the organ from CE data. First, 
a geometric (level set-based) deformable model is proposed for the segmentation of the medical structures from the CE images, in which the evolution is constrained by a novel stochastic force that counts for an adaptive probabilistic shape model and appearance features of the organ (e.g., kidney) in terms of pixel-wise image intensities and their spatial interactions. Experiments, presented in Chapters III and IV, confirm that the proposed segmentation approach has superior results in segmenting the kidney tissues as well as the LV boundaries of the heart. The second segmentation model is a maximum A posteriori (MAP)-based approach aimed at accurate segmentation of different organs from CE medical images. The proposed segmentation approach is based on the integration of statistical approaches (the first- and second-order appearance models and a probabilistic shape of the object of interest) into a log-likelihood function for the purpose of segmentation. The experiments presented in Chapter $\mathrm{V}$ demonstrate promising results of the proposed approach in segmenting the prostate tissue from DCE-MRI. The preliminary results of the proposed segmentation approaches confirm the benefits of these models and encourage applying them in other applications.

In addition to the proposed segmentation models, this work proposes a novel nonrigid registration approach for the local motion correction of medical structures. The proposed approach exploits geometric features, rather than image intensities in order to avoid problems associated with intensity variations over the temporal dynamic contrast agent data set. Namely, it is based on deforming the medical objects over a set of nested, equispaced contours (i.e., iso-contours) to closely match a reference object. The solution of the Laplace equation is used to co-allocate the pixel-wise correspondences between the isocontours. Both in-vivo and phantom experiments in Chapter III confirm that the proposed Laplace-based method outperforms well-known literature methods in co-allocation of the corresponding pixel pairs between the target and reference objects. It is worth mentioning that the proposed local alignment possesses the ability to provide accurate pixel-on-pixel matches of the registered data for generating and displaying pixel-wise parametric maps.

Moreover, a new similarity function for nonrigid registration of medical images is presented, which accounts for both the first- and second-order normalized mutual infor- 
mation (NMI). Unlike the traditional NMI metric that depends solely on the first-order term (i.e., intensity or grey levels), the proposed similarity metric incorporates a second-order NMI term (i.e., spatial information). The incorporation of the second-order term increases the ability of the motion correction approach to capture more features from the CE images, and therefore can improve the registration accuracy for image frames with significant intensity differences. The proposed similarity metric has been applied for the registration of the cardiac FP-MRI data, see Chapter IV. Validation on realistic synthetic phantoms of the heart with different types deformations document the high registration accuracy of the proposed similarity function compared with the first-order NMI alone.

Finally, in the present work both the transient (time-to-peak, peak signal intensity) and slowly varying (the signal change index) phase indexes were employed for the analysis of CE images. Experiments, presented in Chapter III demonstrated that the weightedaugmentation of perfusion parameters leads to more accurate diagnosis of the transplanted kidney status when compared with individual indexes.

\section{BROADER IMPACT OF THE PROPOSED RESEARCH IN THE U.S. AND WORLDWIDE}

The goal of this work is to develop computationally flexible, and feasible mathematical models for efficient analysis of contrast-enhanced medical images. The proposed models in this dissertation are based on higher-order Markov-Gibbs random field models, shape models, motion correction models, and perfusion analysis models, that are essential in developing CAD systems for early detection of a variety of diseases and medical conditions. The developed models and learning techniques has been successfully tested on, but not limited to, three case studies of the kidney, heart, prostate. The work presented in this dissertation and the obtained results is expected to notably amplify the accuracy of CAD decisions based on the automated analysis of CE images, which is expected to substantially benefit research and healthcare in the U.S. and worldwide by providing noninvasive technology. 


\section{E. DISSERTATION ORGANIZATION}

This dissertation consists of six chapters. The following remarks summarize the scope of each chapter:

- Chapter I presents some basic concepts about medical images and CE images. A brief summary of the basic contributions of the proposed research for analyzing CEimages and the clinical applications are also outlined.

- Chapter II overviews the existing methods for the analysis of dynamic contrastenhanced magnetic resonance imaging (DCE-MRI) and outlines their strengths and weaknesses. Additionally, the state-of-the-art clinical applications and findings using these computational methods are covered.

- Chapter III presents a novel image-based framework in renal application for accurate and early detection of acute renal transplant rejection after kidney transplantation using DCE-MRI.

- Chapter IV presents a comprehensive, noninvasive framework in cardiac application for the improved visualization of myocardial transit of a CA using first-pass perfusion MRI (FP-MRI), including the ability to depict regional and transmural effects.

- Chapter V presents a novel, noninvasive framework in prostate application for early detection of prostate cancer using DCE-MRI.

- Chapter VI concludes the work and outlines the future directions. 


\section{CHAPTER II}

\section{MODELS AND METHODS FOR ANALYZING DCE-MRI: A SURVEY}

Dynamic contrast-enhanced magnetic resonance imaging (DCE-MRI) allows for noninvasive quantitative analysis of contrast agent (CA) transient in soft tissues. Thus it is an important and well-established tool to reveal microvasculature and perfusion in various clinical applications. For the last three decades, a host of nonparametric and parametric models and methods have been developed in order to quantify the CA's perfusion into the tissue and estimate perfusion-related parameters (indexes) from signal enhancement or concentration-time curves. These indexes are widely used in various clinical applications for the detection, characterization, and therapy monitoring of different diseases, such as breast and prostate cancer, renal rejection, liver tumors, etc. This chapter reviews most popular nonparametric and parametric approaches to analyze DCE-MRI data, discusses their strengths and weaknesses, and outlines state-of-the-art clinical applications of and findings from these approaches.

\section{A. INTRODUCTION}

Dynamic contrast-enhanced magnetic resonance imaging (DCE-MRI) is a specialized MR technique that has the ability to provide superior information about the anatomy, function, and metabolism of the tissues [27]. Dynamic MRI is widely explored in many clinical studies for noninvasive detection, characterization, and therapy monitoring of different diseases, heart failure, breast and prostate cancer, renal rejection, liver tumors, etc. In, DCE-MRI a tracer, or a CA is injected into the blood stream and then a time series of MR images of the same organ is acquired with fast imaging techniques. MR signal intensities in volume elements (voxels) of a target tissue under the measurement change in proportion to a CA's concentrations. Examples of cross-sectional DCE-MRI time series of 
the heart, kidney, and prostate are shown in Figure 9.

Due to its ability to describe organ functionality in addition to the anatomy, DCEMRI has been widely investigated in perfusion-related studies in many clinical applications, e.g., in evaluation of the kidney, heart, breast, and prostate. Parameters of the CA's delivery to a tissue of interest can be derived from DCE-MRI shortly (up to about two min) after the administration of the CA, e.g., gadolinium [81]. Kinetics (spatial and temporal distributions) of the CA transit depend heavily on the tissue perfusion, vessel permeability, and volume of the extracellular and extravascular space (EES). Following the CA administration, the perfusion can be depicted using changes over time in the recorded MR signal intensity, $S(t)$. A typical time varying signal $S(t)$ shown in Figure 22 demonstrates that the tissue intensity rises at the bolus arrival (wash-in), reaches its maximum, and then decreases slowly afterwards (wash-out). Such an $S(t)$-curve allows for deriving or estimating perfusion-related indexes (parameters) of the tissue's vascularization. The next sections detail and discuss most popular approaches for analyzing $S(t)$.

\section{B. TECHNIQUES FOR ANALYZING DYNAMIC MRI}

Traditional DCE-MRI analysis is based on subjective evaluation by an experienced observer of signal enhancement curves in voxels or a region-of-interest (ROI) and associates each curve with one out of a small number of predefined shape categories (see e.g., Figure 23). Although this approach is most intuitive [82], it is prone to errors due to expert's experience and bias, and provides no quantifiable indexes (like a rate of tracer uptake or wash-out) and measurements of tissue perfusion and permeability. Therefore, other quantitative methods have been proposed for the analysis of DCE-MRI.

This chapter focuses on the two most well-known groups of approaches to quantitatively analyze DCE-MRI, namely, nonparametric (model-free) and parametric (analytical) ones. The nonparametric approaches derive empirical parameters (indexes) that characterize the shape and structure of the $S(t)$. Typical examples of these parameters are shown in Figure 22. Straightforward and simple definitions and computations of these empirical 


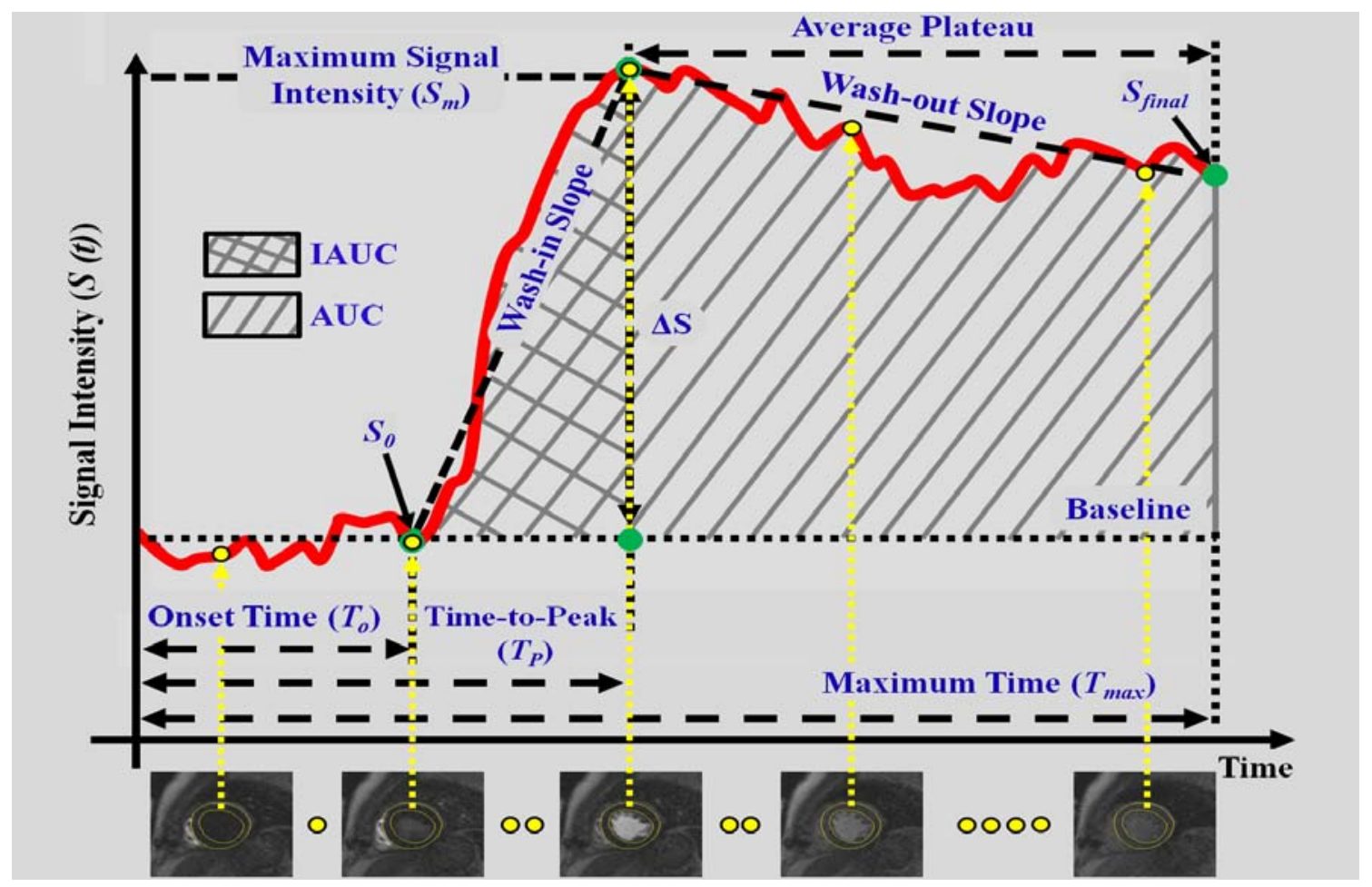

FIGURE 22: Typical $S(t)$ curve showing the time points that quantifies the CA's dynamics and results in different metrics that qualitatively characterize the agent's perfusion: the onset time $\left(T_{o}\right)$, time-to-peak $\left(T_{P}\right)$, peak signal intensity, wash-in slope (initial up-slope), wash-out slope (down-slope), area under the curve (AUC), and initial area under the curve (IAUC). Note that $S_{0}$ is the intensity before the adminstration of CA and $T_{\max }$ is the time period of the MR experiment.

parameters are their main advantages. The empirical indexes correlate with the physiology of the organ as evidenced by their change with diseases (e.g., cancer, renal rejection); however, it is difficult to estimate the physiological quantities of the tissue, such as vascular permeability and blood flow, directly from these empirical indexes. The parametric approaches, on the other hand, aim to estimate kinetic parameters directly by fitting one out of several well-known pharmacokinetic (PK) models to the concentration curves. The PK models are potentially able to extract a set of kinetic parameters, which are physiologically interpretable, e.g., the EES volume and capillary permeability [83]. However, the underlying assumptions of each PK model may not be applicable to all types of tissues or 
tumors. Therefore, the choice of a PK model depends on the clinical application [84]. The key point of the PK modeling is the accuracy of estimating the CA concentration in the blood plasma, which is known as the arterial input function (AIF).

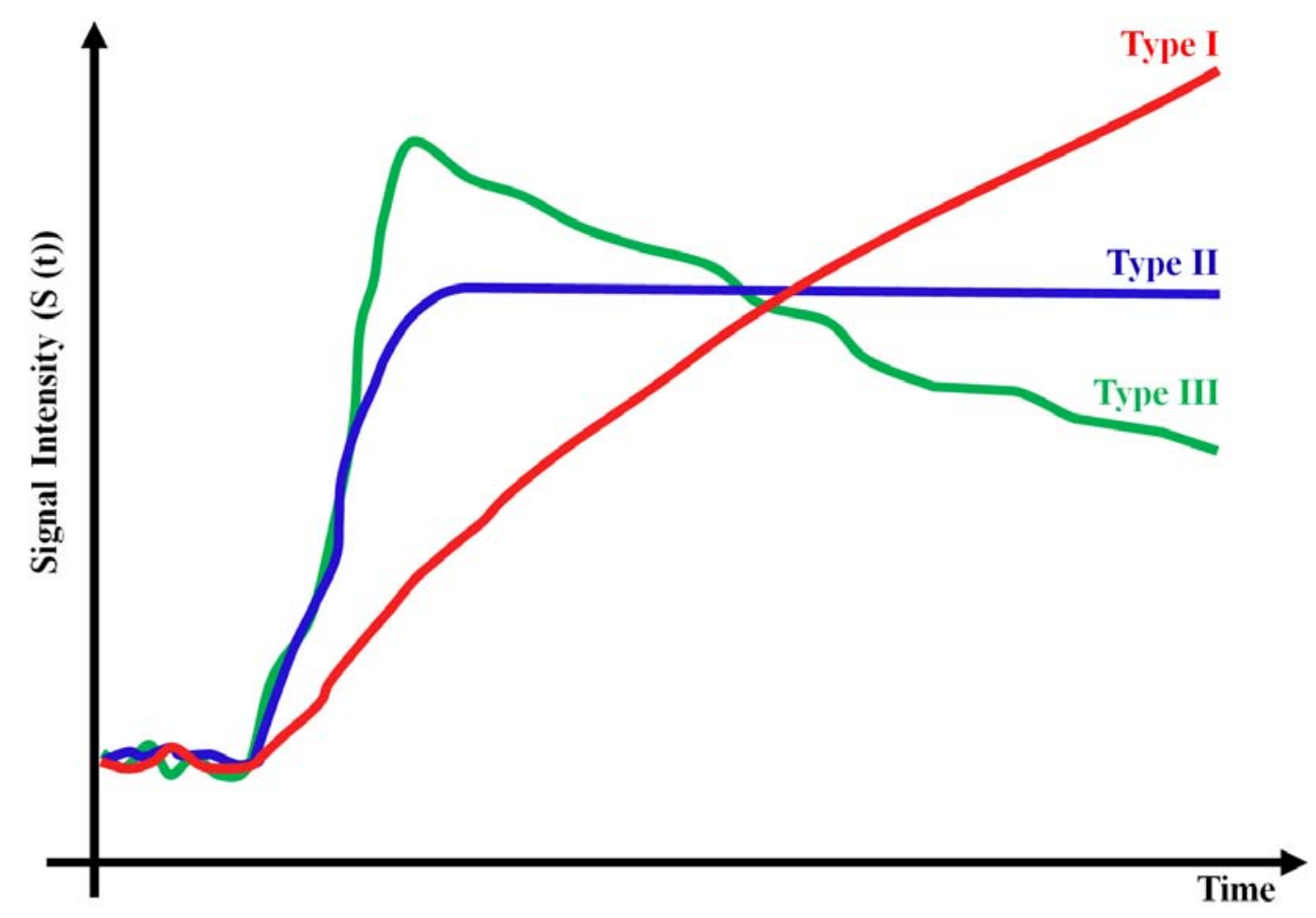

FIGURE 23: Different enhancement patterns: Type I - a progressive signal intensity $(S(t))$ increase during the experiment; Type II - an initial peak followed by a relatively constant enhancement (no wash-out), and Type III - a sharp uptake followed by an enhancement decrease over time (wash-out).

The nonparametric approaches are suited more to a fast and simple noninvasive image-based diagnostics, whereas the parametric ones support studying the CA kinetics in a certain tissue or organ when physiological parameters of the underlying tissue are required. Strengths and weaknesses of the popular nonparametric and parametric approaches for analyzing the dynamic MRI, together with various clinical applications and findings using these methods, are discussed below. 


\section{NONPARAMETRIC DCE-MRI ANALYSIS}

The first category for the analysis of CA perfusion is based on nonparametric or model-free techniques. The established nonparametric dynamic perfusion analysis of extracellular extravascular CA, such as gadolinium, measures empirical indexes directly from $S(t)$. The perfusion-related measurements, shown in Figure 22, include the onset (lag, or arrival) time, peak signal intensity, wash-in slope (maximum or initial up-slope) [81], washout slope (down-slope) [85], maximum intensity time ratio (MITR) [86], time-to-peak, and others, which are defined as follows:

$T_{o}$ - the onset (lag or bolus arrival) time of an enhancement curve, i.e., the time from the CA injection to the appearance of contrast in the tissues.

$S_{m}=\max _{t} S(t)$ - the maximum signal intensity (peak enhancement) of a given timevarying signal $S(t)$.

$\Delta S=S_{m}-S_{o}$ - the peak (maximal absolute) enhancement of a given signal $S(t)$, i.e., the difference between the maximum $\left(S_{m}\right)$ and baseline $\left(S_{o}\right.$ intensities.

$\frac{\Delta S}{S_{o}}-$ the relative signal intensity (RSI) or peak enhancement ratio (PER), i.e., the relative peak enhancement.

$T_{P}$ - the time-to-peak, i.e., the time before the CA circulating in the blood reaches its highest value during the first-pass cycle.

$T_{90}$ - the time before the CA circulating in the blood reaches $90 \%$ of the maximal signal intensity.

$\frac{\Delta S}{T_{P}}$ - the maximum intensity time ratio (MITR), i.e., the ratio between the peak enhancement $\Delta S$ and the time-to-peak $T_{P}$. Also, the normalized MITR (nMITR), $\frac{\triangle S}{S_{o} T_{P}}$, is used.

- the wash-in slope (the maximal or initial up-slope), $\frac{\triangle S}{\left(T_{P}-T_{o}\right)}$, i.e., the slope of the line connecting the baseline $\left(S_{o}\right)$ and the peak intensity $\left(S_{m}\right)$ points: 
- the wash-out slope (down-slope), i.e., the slope of the line connecting the peak intensity $S_{m}$ and the last point of the signal curve.

- the average plateau, i.e., the average signal change during the tissue distribution (washout) phase, starting at approximately $30 \mathrm{sec}$ after the CA injection and extending to approximately two minutes ( $\mathrm{min}$ ) for peripheral injections [87].

- the area under the curve (AUC), i.e., the area under the $S(t)$ curve (relative or absolute). Some methods use the area under the $S(t)$ curve for a time point $t$ (e.g., $\mathrm{AUC}_{60}, \mathrm{AUC}_{90}$, etc.) and the area under the initial uptake portion of the curve, called the initial AUC (IAUC).

However, the CA kinetics change rapidly during the transient phase of the CA transit, so that the limited temporal sampling results in the noisy measurements. To overcome this problem, the perfusion can be characterized using a large number of data points over the signal intensity time series. This can be achieved by calculating the total AUC and the average signal change during the more slowly varying phase (plateau or tissue distribution), as shown in Figure 22. In the case of peripheral injections, the plateau phase starts at approximately 30 seconds ( $\mathrm{sec}$ ) and effectively extends to approximately two $\min$ [87].

Obvious advantages of the nonparametric DCE-MRI analysis include $(i)$ less complicated and time-consuming acquisition requirements (e.g., no AIF measurement is required), (ii) parameter estimation is performed directly from the $S(t)$-curves without converting them into CA concentrations, and (iii) possibilities to completely describe the $S(t)$ curve with a large set of measurements (see Figure 22). However, since the analysis is based on the signal intensity, MR acquisition parameters and scanner type and settings can influence the measurements. Comparisons of results obtained at different times and/or at different sites are also difficult unless totally identical settings are used [88]. Although the nonparametric analysis cannot derive physiological information (e.g., vascular permeability and blood flow) directly from $S(t)$, there exists a correlation between the curve-related measurements and the underlying physiology. For example, the increased wash-in slope, 
AUC, and peak enhancement and the decreased time-to-peak are likely related to an improved response to therapy or increased vascular density and/or vascular permeability.

\section{Clinical Applications of Nonparametric Approaches}

The promise of DCE-MRI as a new diagnostic modality and the feasibility of the nonparametric analysis of perfusion MRI for developing noninvasive CAD systems were investigated in various clinical studies. These studies try to correlate DCE-MRI measurements with diseases. The early DCE-MRI-based diagnosis was explored in different clinical studies, including head and neck [89], cardiac [90-98], pelvic [99], rectal [100], pancreatic cancer [101], liver [102], lung [103], colon [104], breast [52, 56, 105-117], renal $[3,118-129]$, and prostate $[75,77,130-140]$, applications. Recent applications of the nonparametric DCE-MRI analysis and their findings for the assessment of heart disease, kidney function, and prostate and breast cancers are overviewed below.

Ischemic heart disease is the most common cause of heart failure, which affects approximately 6 million US patients annually [63]. Therefore, detecting precursors to prevent progression to end-stage disease is of important clinical concern. The nonparametric DCE-MRI analysis has been used for the assessment of myocardial perfusion in patients with heart diseases in [90-98]. Schwitter et al. [90] detected and sized the compromised myocardium by using MRI, comparing with quantitative measures of coronary angiography and positron emission tomography (PET). The up-slope index was used to measure the myocardial perfusion. According to their results, the MRI measurements could reliably detect and quantify perfusion deficits in patients with the coronary artery disease, even when perfusion abnormalities were confined to the subendocardial layer. Ibrahim et al. [91] measured in a similar way the coronary flow reserve (CFR) defined as the stress-to-rest ratio of the maximal up-slope and myocardial peak signal intensity indexes. The MRI-based CFR was underestimated with respect to the PET-based one. A semi-automated approach by Positano et al. [92] characterized the myocardial perfusion in patients with suspected coronary artery diseases using the wash-in slope, the time-to-peak, and the peak signal intensity 
for a number of user-defined equiangular sectors. Semi-automated evaluation of regional myocardial perfusion by Tarroni et al. [98] quantified the perfusion regionally with the peak signal intensity, the initial up-slope, and the product of the amplitude and the slope. The up-slope index had the highest diagnostic accuracy compared to a coronary angiography reference for the presence of obstructive coronary artery disease. An automated assessment of cardiac perfusion in patients with acute myocardial infarction by Ólafsdóttir et al. [93] used parametric maps of three perfusion-related indexes (the maximum up-slope, the time-to-peak, and the peak value) to reveal severe perfusion deficit across the myocardial wall. A similar approach by Xue et al. [94] employed the scale-space theory and the non-maximal suppression [141]. A framework for evaluating the perfusion indexes for normal and ischemic myocardium was proposed by Su et al. [95]. The segment-wise ratio of the maximum up-slope (i.e., the up-slope at stress) to the up-slope at rest using the 17-segment model [6] differentiated between ischemic and non-ischemic myocardium. In addition to studying ischemic heart diseases, the dynamic MRI was also employed for evaluating the follow-up on therapy. Khalifa et al. [96,97] analyzed the myocardial first-pass MRI of patients with ischemic damage from heart attacks who were undergoing a stem cell myoregenrataion therapy. The perfusion was quantified using pixel-wise perfusion-related maps of the peak signal intensity, time-to-peak, initial up-slope, and average plateau indexes. The derived perfusion maps demonstrated the ability to show regional perfusion differences and improvements with treatment, including transmural effects.

Breast cancer is one of the most common female malignancies worldwide that accounts in total for more than $20 \%$ of new cancer cases and about $15 \%$ of cancer deaths [71]. Therefore, its early detection, diagnosis, and treatment are of prime importance. The accuracy of early detection and/or diagnosis using the nonparametric DCE-MRI measurements has been tested and improved in a number of CAD systems [52, 56, 105-117]. An approach for the extraction and visualization of perfusion parameters of breast DCE-MRI was proposed by Glaßer et al. [105]. To reveal the most suspicious region and the heterogeneity of the tumor, their study employed voxel-wise parametric maps of relative enhancement of breast tumors. Karahaliou et al. [106] investigated the feasibility of discriminating between 
the malignant and benign breast tumors by texture analysis. Discriminatory features quantifying the heterogeneity of the lesion enhancement kinetics were obtained from the maps of three indexes $\left(S_{m}\right.$, measured for the first three $\min$ after the CA injection; $\left(S_{m}-S_{\text {final }}\right) / S_{m}$, and the signal enhancement ratio, $\left(S_{m}-S_{0}\right) /\left(S_{\text {final }}-S_{0}\right)$, see Figure 22). Discriminating abilities of the texture features were investigated using the minimum least-squares distance classifier. Abramson et al. [107] characterized the breast lesions as benign or malignant with three metrics: the lesion volume, the percentage of the lesion volume demonstrating enhancement (assessed by a radiologist), and an enhancement kinetic index calculated from the voxels with either $\geq 50 \%$ - or $\geq 100 \%$-increase of intensity over the baseline following the CA injection. The ability to discriminate between the types of tumors was obtained after one cycle of neoadjuvant chemotherapy (NAC). The NAC reduces cancer burdens for patients before surgery and allows for an earlier treatment of cancer cells [142]. To improve the diagnostic performance of the Type II enhancement curves of the breast, Fusco et al. [108] used the difference between the percentage enhancement at the last time point and the peak percentage enhancement as a discriminatory feature. In addition to the breast cancer detection and diagnosis, the nonparametric DCE-MRI based evaluation and followup on treatment have been also explored [56, 109-111]. Martincich et al. [109] predicted histological responses in patients undergoing primary chemotherapy for breast cancer using the total lesion volume, the pre-contrast uptake (the signal intensity over the baseline normalized by the baseline intensity), and the enhancement pattern categorized into Types I, II and III, shown in Figure 23. A similar study by El Khoury et al. [110] for patients with breast cancer under preoperative chemotherapy quantified the tumors using the washout variation maps (i.e., the maps of the difference between $S_{\text {final }}$ and $S_{m}$ of the dynamic series in each voxel, see Figure 22). The nonparametric MRI-based indexes were used by Johansen et al [56] for the early prediction of the response to the NAC and the five-year survival for patients with locally advanced breast cancer. In the baseline DCE-MRI study, which was performed prior to the start of therapy, the patients surviving for more than five years had significantly less heterogeneous RSI distribution than the non-survivors. The usefulness of DCE-MRI in analyzing and predicting the survival of the breast cancer patients 
has been demonstrated also by Tuncbilek et al. [111].

Renal diseases, including cancer, artery stenosis, and transplant rejection, can also be diagnosed with the nonparametric DCE-MRI techniques [3, 118-129]. A semi-automated framework by Ho et al. [118] evaluated renal lesions, which were identified manually by observers, with a percentage of the enhancement ratio between the pre- and post-contrast signals in each set of images. A $15 \%$ threshold was used to distinguish between cysts and solid renal lesions. All malignancies were accurately diagnosed between two min and four min after administering the CA (100\% sensitivity for true tumors and $6 \%$ or fewer falsepositive tumor diagnoses). Michaely et al. [119] assessed the feasibility of the renal MR perfusion for grading renal artery stenosis effects on parenchymal perfusion. The gamma variate function [143] was used to describe the transient (first pass, or wash-in) phase of the time varying signal $S(t)$. Then perfusion-related indexes after agent bolus, such as the mean transit time (MTT), the maximal up-slope, the maximum signal intensity, and the time-to-peak, were calculated from the fitted $S(t)$. The evaluated perfusion reflected the renal function measured with serum creatinine in a cohort of 73 patients. The peak signal intensity, the MTT, the initial up-slope, and the time to peak were also used to analyze the perfusion by Positano et al. [120]. Other research groups have exploited DCE-MRI for early detection of the renal rejection following the kidney transplantation [3,121-129]. A DCE-MRI based CAD system for early diagnosis of the acute renal transplant rejection proposed by Farag et al. [122] and El-Baz et al. [123, 125] classified the kidney status of each patient using four indexes: the peak signal intensity, the time-to-peak, the wash-in slope, and the wash-out slope, calculated from the MRI signal for the kidney cortex. Similar approaches, but with the perfusion curves for the whole kidney, rather than only the cortex, were proposed in $[126,127]$. The latter CAD system was tested on 100 patients. A novel nonparametric MRI-based technique by Khalifa et al. [128] for analyzing the kidney perfusion accounts for kidney deformations in order to accurately calculate indexes for classifying the transplanted kidney status and evaluating the acute renal transplant rejection. The kidney status is characterized by both the transient phase indexes (the peak signal intensity, the initial up-slope, and the time-to-peak) and the tissue phase signal change in- 
dex (the average plateau). This technique was extended in [129] by applying a simplified gamma variate fit [144] to the $S(t)$-curves.

Prostate cancer is the most frequently diagnosed male malignancy and the second leading cause (after lung cancer) of cancer-related death in the USA with more than 238,000 new cases and a mortality rate of about 30,000 in 2013 [71]. Early diagnosis improves the effectiveness of the treatment and increases the patient's chances of survival. The nonparametric DCE-MRI analyses have been widely used to identify and classify prostate cancer $[75,77,130-140]$. Engelbrecht et al. [130] separated cancerous and normal prostate tissues in the peripheral zone $(\mathrm{PZ})$ and central zone $(\mathrm{CZ})$ by combining the $T_{2}$-relaxation rate with DCE-MRI indexes, calculated from the concentration curves, rather than the $S(t)$-curves. According to receiver operating characteristics (ROC), the relative peak enhancement index was the best for discriminating the prostate carcinoma in the PZ and CZ. Noworolski et al. [131] used DCE-MRI data to classify the prostate tissues into cancerous or normal PZ and stromal benign or glandular hyperplasia. Capabilities of the DCE-MRI indexes in diagnosing the benign and malignant prostate tissues were evaluated by Ren et al. [132], who also investigated relationships between characteristics of the $S(t)$ curves and angiogenesis. Their studies confirmed that DCE-MRI and histological findings are correlated. DCE-MRI based CAD systems introduced by Puech et al. $[133,134]$ and Firjani et al. $[75,77]$ classified the prostatic tissue using the wash-in and wash-out slopes derived from the $S(t)$-curves. Isebaert et al. [138] evaluated the correlation to histopathology of nonparametric DCE-MRI characteristics, such as the time-to-peak, the maximal signal enhancement, the wash-in slope, and the clearance rate of the CA (the wash-out), for detecting prostate carcinoma and separating malignant and benign prostate tissue regions. According to this study, the wash-in slope is the most accurate separator of the malignant and benign tissues. Niaf et al. [139] developed a CAD system using multiple MRI data (namely, the $T_{2}$-weighted MRI, DCE-MRI and DWI-MRI) to diagnose prostate cancer in the PZ. Four supervised classifiers of malignant and benign tissues were compared: a nonlinear support vector machine (SVM), the linear discriminant analysis, a $k$-nearest neighbor, and a naïve Bayes one, combining image intensity, texture, and gradient with 
functional features (e.g., PK features, peak intensity, and the wash-in and wash-out slopes). In addition to cancer detection and classification, DCE-MRI facilitated prostate cancer therapy evaluations. Haider et al. [135] compared the DCE-MRI diagnostic accuracy with the conventional $T_{2}$-weighted MRI for detecting and localizing the recurrent prostate cancer in the PZ for patients with biochemical failures after the external beam radiotherapy. A DCEMRI based voxel-enhancement criterion at $46 \mathrm{sec}$ after the CA injection outperformed the $T_{2}$-weighted MRI. A similar study was conducted by Casciani et al. [136] to detect the local cancer recurrence after radical prostatectomy using the combined endorectal MRI and DCE-MRI. Multivariate analysis of magnetic resonance spectroscopy (MRS) imaging and DCE-MRI was used by Valerio et al. [137] to differentiate between various prostate diseases, such as chronic inflammation, fibrosis and adenocarcinoma. Capabilities of DCEMRI in staging the prostate cancer were investigated in [140]. To separate the stage 2 and stage 3 prostate carcinoma, four perfusion-related indexes have been calculated from the concentration curves of the dynamic MRI data instead of the $S(t)$-ones, just as in [130]. A summary of the studies covered in this section and their findings is given in Table 1.

TABLE 1: Recent studies for nonparametric analysis of DCE-MRI. Note that DIM and AL stand for data dimension (i.e., 2D, 3D, or 4D) and the automation level (i.e., automated or semi-automated), respectively

\begin{tabular}{|c|c|c|c|}
\hline Study & DIM, AL, and Data & Tissue of Interest & MRI Parameters \\
\hline $\begin{array}{l}\text { Schwitter et } \\
\text { al. [90] }\end{array}$ & $\begin{array}{l}\text { - } 2 \mathrm{D} \\
\text { - Semi-automated } \\
\text { - } 66 \text { subjects (18 } \\
\text { volunteers and } 48 \\
\text { patients) }\end{array}$ & - Heart & - Up-slope \\
\hline $\begin{array}{ll}\text { Ibrahim } & \text { et } \\
\text { al. [91] } & \end{array}$ & $\begin{array}{l}\text { - } 2 \mathrm{D} \\
\text { - Semi-automated } \\
\text { - } 45 \text { subjects (20 } \\
\text { volunteers and } 25 \\
\text { patients) } \\
\end{array}$ & - Heart & $\begin{array}{l}\text { - Peak signal intensity (over the base- } \\
\text { line) } \\
\text { - Up-slope }\end{array}$ \\
\hline $\begin{array}{l}\text { Postiano et } \\
\text { al. [92] }\end{array}$ & $\begin{array}{l}\text { - } 2 \mathrm{D} \\
\text { - Automated } \\
\text { - } 10 \text { patients }(70 \\
\text { image sequences })\end{array}$ & - Heart & $\begin{array}{l}\text { - Peak signal intensity } \\
\text { - Time-to-peak } \\
\text { - Wash-in slope }\end{array}$ \\
\hline $\begin{array}{l}\text { Ólafsdóttir } \\
\text { et al. [93] }\end{array}$ & $\begin{array}{l}\text { - } 2 \mathrm{D} \\
\text { - Automated } \\
\text { - } 10 \text { subjects }\end{array}$ & - Heart & $\begin{array}{l}\text { - Peak signal intensity } \\
\text { - Time-to-peak } \\
\text { - Maximum up-slope }\end{array}$ \\
\hline & & & Continued on next page ... \\
\hline
\end{tabular}


TABLE 1 - continued from previous page

\begin{tabular}{|c|c|c|c|}
\hline Study & DIM, AL, and Data & Tissue of Interest & MRI Parameters \\
\hline Su et al. [95] & $\begin{array}{l}\text { - } 2 \mathrm{D} \\
\text { - Automated } \\
\text { - } 42 \text { subjects }\end{array}$ & - Heart & $\begin{array}{l}\text { - Maximum up-slope } \\
\text { - Up-slope ratios }\end{array}$ \\
\hline $\begin{array}{l}\text { Xue et } \\
\text { al. [94] }\end{array}$ & $\begin{array}{l}\text { - } 2 \mathrm{D} \\
\text { - Automated } \\
\text { - } 260 \text { perfusion } \\
\text { time series }\end{array}$ & - Heart & $\begin{array}{l}\text { - Peak signal intensity } \\
\text { - Time-to-peak } \\
\text { - Maximum up-slope }\end{array}$ \\
\hline $\begin{array}{l}\text { Tarroni et } \\
\text { al. [98] }\end{array}$ & $\begin{array}{l}\text { - N/A } \\
\text { - Semi-automated } \\
\text { - } 42 \text { subjects }\end{array}$ & - Heart & $\begin{array}{l}\text { - Peak signal intensity } \\
\text { - Initial up-slope } \\
\text { - Product of the amplitude and slope }\end{array}$ \\
\hline $\begin{array}{l}\text { Khalifa et } \\
\text { al. }[96,97]\end{array}$ & $\begin{array}{l}\text { - } 2 \mathrm{D} \\
\text { - Automated } \\
\text { - } 24 \text { data sets }\end{array}$ & - Heart & $\begin{array}{l}\text { - Peak signal intensity } \\
\text { - Time-to-peak } \\
\text { - Initial up-slope } \\
\text { - Average plateau. }\end{array}$ \\
\hline $\begin{array}{l}\text { Martincich } \\
\text { et al. [109] }\end{array}$ & $\begin{array}{l}\text { - } 3 \mathrm{D} \\
\text { - } \text { Automated } \\
\text { - } 30 \text { patients }\end{array}$ & - Breast & $\begin{array}{l}\text { - Total lesion volume } \\
\text { - Early contrast uptake } \\
\text { - } S(t) \text {-curve pattern type }\end{array}$ \\
\hline $\begin{array}{l}\text { El Khoury } \\
\text { et al. }[110]\end{array}$ & $\begin{array}{l}\text { - } 3 \mathrm{D} \\
\text { - Semi-automated } \\
\text { - } 33 \text { patients }\end{array}$ & - Breast & - Wash-out variation $\left(S_{\text {final }}-S_{P}\right)$ \\
\hline $\begin{array}{l}\text { Johansen et } \\
\text { al. [56] }\end{array}$ & $\begin{array}{l}\text { - } 3 \mathrm{D} \\
\text { - Semi-automated } \\
\text { - } 24 \text { patients }\end{array}$ & - Breast & $\begin{array}{l}\text { - RSI in each voxel, } \\
\text { - } S(t) \text {-curve pattern type }\end{array}$ \\
\hline $\begin{array}{l}\text { Karahaliou } \\
\text { et al. [106] }\end{array}$ & $\begin{array}{l}\text { - } 3 \mathrm{D} \\
\text { - Semi-automated } \\
\text { - } 74 \text { subjects }\end{array}$ & - Breast & $\begin{array}{l}\text { - Initial enhancement } \\
\text { - Post-initial enhancement } \\
\text { - Signal enhancement ratio }\end{array}$ \\
\hline $\begin{array}{l}\text { Glaßer } \\
\text { al. }[105]\end{array}$ & $\begin{array}{l}\text { - } 3 \mathrm{D} \\
\text { - Semi-automated } \\
\text { - } 5 \text { data sets }\end{array}$ & - Breast & $\begin{array}{l}\text { - Peak enhancement } \\
\text { - Time-to-peak } \\
\text { - Up-slop } \\
\text { - Wash-out slope } \\
\text { - AUC }\end{array}$ \\
\hline $\begin{array}{l}\text { Tuncbilek et } \\
\text { al. [111] }\end{array}$ & $\begin{array}{l}\text { - } 2 \mathrm{D} \\
\text { - Semi-automated } \\
\text { - } 49 \text { subjects }\end{array}$ & - Breast & $\begin{array}{l}\text { - Maximal relative enhancement within } \\
\text { the first minute } \\
\text { - Maximal relative enhancement of the } \\
\text { entire study } \\
\text { - Steepest slope } \\
\text { - Time-to-peak }\end{array}$ \\
\hline $\begin{array}{l}\text { Abramson } \\
\text { et al. [107] }\end{array}$ & $\begin{array}{l}\text { - } 3 \mathrm{D} \\
\text { - Automated } \\
\text { - } 21 \text { patients }\end{array}$ & - Breast & $\begin{array}{l}\text { - Total lesion volume } \\
\text { - Early contrast uptake } \\
\text { - } S(t) \text {-curve pattern type }\end{array}$ \\
\hline $\begin{array}{l}\text { Fusco et } \\
\text { al. }[108]\end{array}$ & $\begin{array}{l}\text { - } 2 \mathrm{D} \\
\text { - Semi-automated } \\
\text { - } 44 \text { patients }\end{array}$ & - Breast & $\begin{array}{l}\text { - Difference between the percentage- } \\
\text { enhancement at the last time point and } \\
\text { the peak percentage-enhancement }\end{array}$ \\
\hline $\begin{array}{l}\text { Ho } \\
\text { al. }[118]\end{array}$ & $\begin{array}{l}\text { - } 2 \mathrm{D} \\
\text { - Semi-automated } \\
\text { - } 74 \text { patients }\end{array}$ & - Kidney & $\begin{array}{l}\text { - Percentage enhancement ratio: } \\
\frac{S I_{\text {post }}-S I_{\text {pre }}}{S I_{\text {pre }}} \%\end{array}$ \\
\hline $\begin{array}{l}\text { Farag et } \\
\text { al. }[122] \text { and } \\
\text { El-Baz et } \\
\text { al. }[123,125] \\
\end{array}$ & $\begin{array}{l}\text { - } 2 \mathrm{D} \\
\text { - Automated } \\
\text { - } 30 \text { data sets }\end{array}$ & - Kidney & $\begin{array}{l}\text { - Peak signal intensity } \\
\text { - Time-to-peak } \\
\text { - Wash-in slope } \\
\text { - Wash-out slope } \\
\end{array}$ \\
\hline & & & Continued on next page ... \\
\hline
\end{tabular}


TABLE 1 - continued from previous page

\begin{tabular}{|c|c|c|c|}
\hline Study & DIM, AL, and Data & Tissue of Interest & MRI Parameters \\
\hline $\begin{array}{l}\text { El-Baz et } \\
\text { al. }[126,127]\end{array}$ & $\begin{array}{l}\text { - } 2 \mathrm{D} \\
\text { - Automated } \\
\text { - } 100 \text { data sets }\end{array}$ & - Kidney & $\begin{array}{l}\text { - Peak signal intensity } \\
\text { - Time-to-peak } \\
\text { - Wash-in slope } \\
\text { - Wash-out slope }\end{array}$ \\
\hline $\begin{array}{l}\text { Michaely et } \\
\text { al. [119] }\end{array}$ & $\begin{array}{l}\text { - } 2 \mathrm{D} \\
\text { - Automated } \\
\text { - } 73 \text { patients }\end{array}$ & - Kidney & $\begin{array}{l}\text { - Mean Transit time (MMT) } \\
\text { - Maximum signal intensity } \\
\text { - Time-to-peak } \\
\text { - Maximum up-slope }\end{array}$ \\
\hline $\begin{array}{l}\text { Positano et } \\
\text { al. [120] }\end{array}$ & $\begin{array}{l}\text { - } 2 \mathrm{D} \\
\text { - Automated } \\
\text { - } 20 \text { subjects }\end{array}$ & - Kidney & $\begin{array}{l}\text { - Peak signal intensity } \\
\text { - Time-to-peak } \\
\text { - Mean transit time (MTT) } \\
\text { - Initial up-slope }\end{array}$ \\
\hline $\begin{array}{l}\text { Khalifa et } \\
\text { al. [128] }\end{array}$ & $\begin{array}{l}\text { - } 2 \mathrm{D} \\
\text { - Automated } \\
\text { - } 50 \text { data sets }\end{array}$ & - Kidney & $\begin{array}{l}\text { - Peak signal intensity } \\
\text { - Time-to-peak } \\
\text { - Initial up-slope } \\
\text { - Average plateau }\end{array}$ \\
\hline $\begin{array}{l}\text { Khalifa et } \\
\text { al. [129] }\end{array}$ & $\begin{array}{l}\text { - } 2 \mathrm{D} \\
\text { - Automated } \\
\text { - } 50 \text { data sets }\end{array}$ & - Kidney & $\begin{array}{l}\text { - Time-to-peak } \\
\text { - Average plateau } \\
\text { - Gamma-variate parameters }\end{array}$ \\
\hline $\begin{array}{l}\text { Engelbrecht } \\
\text { et al. [130] }\end{array}$ & $\begin{array}{l}\text { - } 3 \mathrm{D} \\
\text { - Automated, } \\
\text { - } 58 \text { image se- } \\
\text { quences }\end{array}$ & - Prostate & $\begin{array}{l}\text { - Onset time } \\
\text { - Time-to-peak } \\
\text { - Peak enhancement } \\
\text { - Relative peak enhancement } \\
\text { - Wash-out, } \\
\text { - T2 relaxation rates. }\end{array}$ \\
\hline $\begin{array}{l}\text { Noworolski } \\
\text { et al. [131] }\end{array}$ & $\begin{array}{l}\text { - } 3 \mathrm{D} \\
\text { - Semi-automated } \\
\text { - } 25 \text { patients }\end{array}$ & - Prostate & $\begin{array}{l}\text { - Peak signal intensity } \\
\text { - Time to } 90 \% \text { peak } \\
\text { - Initial up-slope } \\
\text { - Wash-out slope }\end{array}$ \\
\hline $\begin{array}{l}\text { Fütterer et } \\
\text { al. [140] }\end{array}$ & $\begin{array}{l}\text { - } 3 \mathrm{D} \\
\text { - } \mathrm{N} / \mathrm{A} \\
\text { - } 124 \text { patients }\end{array}$ & - Prostate & $\begin{array}{l}\text { - Onset time } \\
\text { - Time to peak } \\
\text { - Peak enhancement } \\
\text { - Wash-out slope }\end{array}$ \\
\hline $\begin{array}{ll}\text { Ren } & \text { et } \\
\text { al. [132] } & \end{array}$ & $\begin{array}{l}\text { - N/A } \\
\text { - Automated } \\
\text { - } 50 \text { patients }\end{array}$ & - Prostate & $\begin{array}{l}\text { - Peak enhancement } \\
\text { - Time-to-peak } \\
\text { - Enhancement rate (up-slope) }\end{array}$ \\
\hline $\begin{array}{l}\text { Haider } \\
\text { al. [135] }\end{array}$ & $\begin{array}{l}\text { - } 3 \mathrm{D} \\
\text { - } \mathrm{N} / \mathrm{A} \\
\text { - } 33 \text { patients }\end{array}$ & - Prostate & $\begin{array}{l}\text { - Voxel-enhancement at } 46 \mathrm{sec} \text { after CA } \\
\text { injection to a greater degree than the } \\
\text { mean signal intensity of the prostate at } \\
618 \mathrm{sec}\end{array}$ \\
\hline $\begin{array}{l}\text { Casciani et } \\
\text { al. }[136]\end{array}$ & $\begin{array}{l}-2 \mathrm{D} \\
-\mathrm{N} / \mathrm{A} \\
-51 \text { patients }\end{array}$ & - Prostate & $\begin{array}{l}\text { - Initial signal increase over the baseline } \\
\text { (90 sec after CA injection) } \\
\text { - Post-initial signal increase over the } \\
\text { baseline (90-325 sec after CA injec- } \\
\text { tion) }\end{array}$ \\
\hline $\begin{array}{ll}\text { Valerio } & \text { et } \\
\text { al. [137] } & \end{array}$ & $\begin{array}{l}\text { - } 2 \mathrm{D} \\
\text { - Semi-automated } \\
\text { - } 51 \text { patients } \\
\end{array}$ & - Prostate & $\begin{array}{l}\text { - Onset time } \\
\text { - Time-to-peak } \\
\text { - Peak enhancement }\end{array}$ \\
\hline $\begin{array}{l}\text { Puech et } \\
\text { al. [134] }\end{array}$ & $\begin{array}{l}\text { - } 3 \mathrm{D} \\
\text { - Semi-automated } \\
\text { - } 100 \text { patients }\end{array}$ & - Prostate & $\begin{array}{l}\text { - Wash-in slope } \\
\text { - Wash-out slope }\end{array}$ \\
\hline
\end{tabular}


TABLE 1 - continued from previous page

\begin{tabular}{|c|c|c|c|}
\hline Study & DIM, AL, and Data & Tissue of Interest & MRI Parameters \\
\hline $\begin{array}{l}\text { Firjani et } \\
\text { al. }[75,77]\end{array}$ & $\begin{array}{l}-3 \mathrm{D} \\
\text { - Automated } \\
\text { - } 21 \text { data sets }\end{array}$ & - Prostate & $\begin{array}{l}\text { - Wash-in slope } \\
\text { - Wash-out slope }\end{array}$ \\
\hline $\begin{array}{l}\text { Isebaert et } \\
\text { al. [138] }\end{array}$ & $\begin{array}{l}\text { - } 3 \mathrm{D} \\
\text { - Semi-automated } \\
\text { - } 53 \text { subjects }\end{array}$ & - Prostate & $\begin{array}{l}\text { - Time-to-peak } \\
\text { - Maximal contrast enhancement } \\
\text { - Wash-in slope } \\
\text { - Clearance rate of the CA (Wash-out) }\end{array}$ \\
\hline $\begin{array}{ll}\text { Niaf } & \text { et } \\
\text { al. [139] } & \end{array}$ & $\begin{array}{l}\text { - } 3 \mathrm{D} \\
\text { - Semi-automated, } \\
\text { - } 30 \text { subjects }\end{array}$ & - Prostate & $\begin{array}{l}\text { - Image features (grey-level, texture, } \\
\text { and gradient features) } \\
\text { - Functional features (onset time, time- } \\
\text { to-peak, peak value, } 95 \% \text { of peak } \\
\text { value, time to max, AUC, and wash-in } \\
\text { and wash-out slopes) } \\
\text { - parametric features ( } K^{\text {trans }, k_{\text {ep }} \text {, and }} \\
\left.v_{\mathrm{p}}\right)\end{array}$ \\
\hline
\end{tabular}

\section{PARAMETRIC DCE-MRI ANALYSIS}

Parametric approaches fit mathematical pharmacokinetic (PK) models to the dynamically acquired tissue concentration curves, so that quantitative tissue parameters (e.g., permeability and volume fractions) that are related to vascularity can be estimated. The pioneering works by Larsson [145], Brix [146], and Tofts [13] for the study of multiple sclerosis $[13,145]$ and brain tumors $[145,146]$ showed the potential promise of the PK models to better understand the CA perfusion kinetics in human tissue. Later on, these initial models enabled modeling of the CA kinetics with DCE-MRI and were used to estimate perfusion and permeability in several clinical studies. Recent PK analyses reveal physiological tissue characteristics by relating the perfusion to the tissue vascular functionality, which enables measurement of the blood volume and capillary permeability [70].

The literature's PK models proposed for quantitative analysis of DCE-MRI data are based on different assumptions and simplifications. The choice of a particular model for solving a certain clinical problem depends on many factors, including $(i)$ the unique physiology of the tissue of interest (e.g., brain, breast, or prostate) that governs the CA behavior; (ii) dominant conditions identifying the MR signal (e.g., fast or limited water exchange); (iii) whether the depicted anatomy allows for determining an AIF; and (iv) the temporal MR data resolution needed to accurately capture CA uptake, etc. [147]. The 
dynamic perfusion data analysis involves two main PK models, namely, compartment and distributed models. The former category includes the Larsson (LM), Brix (BM), Tofts and Kermode (TK), extended TK (ETK), two-compartment exchange (2CXM), and Patlak (PM) models, whereas the latter category comprises the distributed-parameter (DP) and tissue homogeneity (TH) models and its adiabatic approximation or the AATH model.

The compartment PK model proposed originally by Kety [148] describes complex blood-tissue exchanges of an administered CA with a collection of interacting homogeneous components, called compartments. Two assumptions are sufficient to completely define the CA kinetics: (i) compartments are well-mixed, i.e., the CA concentration is spatially uniform at any given time within the volume, and (ii) an output CA flux of any compartment is directly proportional to its concentration. Generally, the larger the number of compartments, the higher the accuracy of the PK model, but the higher the analysis complexity [149]. Due to simplicity and small numbers of parameters to be estimated, the compartment models have gained considerable attention in many clinical investigations over the past two decades.

The distributed kinetic model $[150,151]$ is based on a plug-flow model, carrying an administered CA through a tube by a flow, where all particles travel with the same velocity. Unlike the compartment models, the distributed ones account for both spatial and temporal variations of an administered CA. Therefore, these models correspond more closely to reality, are expected to reflect the underlying physiology more accurately than the compartment models, and potentially increase modeling accuracy and provide additional information. However, their higher complexity (in terms of larger numbers of parameters) requires higher data quality to maintain the accuracy and precision of the estimated model parameters and thus limits their widespread popularity [152].

Since most of the PK analysis requires the CA concentration in the blood supply, i.e., measuring or determining a so-called arterial input function (AIF), most popular AIF determination methods are outlined in the next section. 


\section{Arterial Input Function (AIF)}

The AIF describing the changes of the CA concentration over time in a blood vessel feeding the tissue of interest has to be determined or measured for almost all the parametric DCE-MRI models [153]. However, the true AIF is difficult to be accurately determined or estimated due to problems including flow artefacts, inflow and non-linear effects of high CA concentrations, and partial volume effects [14]. The AIF kinetics differ from the tissue concentration as it is characterized by a sharp uptake, followed by a short-lived peak value, and subsequently a longer wash-out period. Current techniques to measure or determine the AIF can be stratified into five groups: the gold standard, population-based, subject-specific, reference tissue-based, and jointly-estimated AIFs, which are briefly reviewed below.

The gold standard AIF is determined by analyzing blood samples collected during DCE-MRI acquisition from an arterial catheter inserted into the subject. Larsson et al. [145] measured the CA amount in a series of blood samples taken at intervals of $15 \mathrm{sec}$ after a CA bolus injection. The main advantage of this method for AIF determination is the precise measurement of $C_{\mathrm{p}}$ in each sample over time, i.e., the accurate characterization of the AIF as a function of time. However, this invasive approach is inconvenient for patients and its accuracy depends on temporal resolution (the number of samples that can be collected), especially for depicted small lesions. Additionally, it is unsuitable for some clinical applications, such as breast DCE-MRI, due to the lack of big vessels in the field of view.

The population-based AIF is determined by measuring blood samples from a small group of subjects and using their average measurement for subsequent studies [154]. Tofts and Kermode [13] used a population-based AIF and described it by a sum of two decreasing exponentials (see Figure 24 (a)) with parameters estimated by fitting plasma concentration measurements, taken from control subjects, in the earlier work by Weinmann [154]:

$$
C_{\mathrm{p}}(t)=D\left(a_{1} e^{-m_{1} t}+a_{2} e^{-m_{2} t}\right)
$$

where $D$ is the CA dose $\left(\mathrm{mM} \mathrm{kg}^{-1}\right) ; a_{1}=3.99 \mathrm{~kg}$ and $a_{2}=4.78 \mathrm{~kg}$ are amplitudes of the exponentials, and $m_{1}=0.144 \mathrm{~min}^{-1}$ and $m_{2}=0.011 \mathrm{~min}^{-1}$ are their rate constants [13]. 


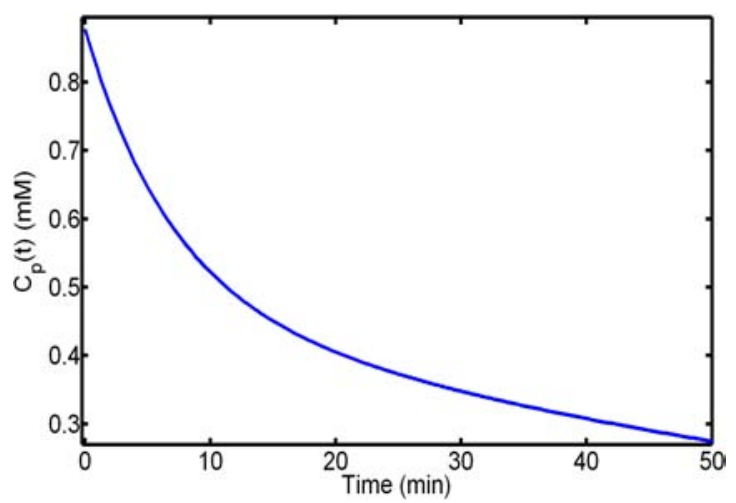

(a)

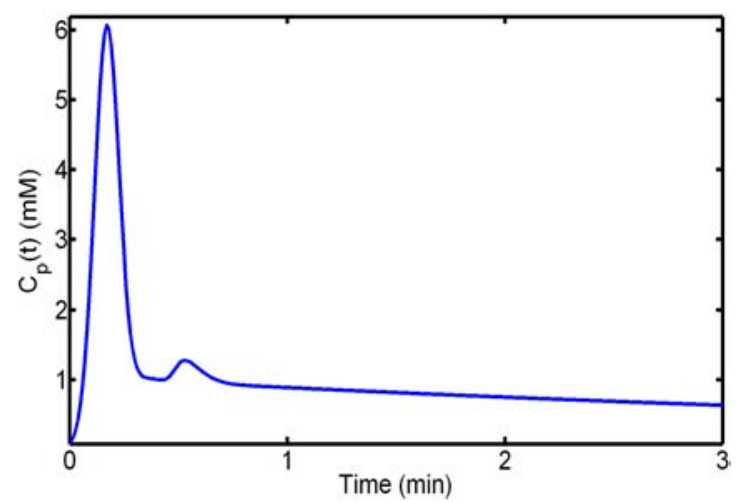

(b)

FIGURE 24: The population-based arterial input functions (AIF) proposed by (a) Tofts and Kermode [13] with $D=0.1 \mathrm{mM} \mathrm{kg}^{-1}$; and (b) Parker et al. [14]. Different time scales are used to visualize better the CA uptake and wash-out phases in each AIF.

Parker et al. [14] proposed another population-based AIF where a mixture of two Gaussian kernels plus an exponential modulated with a sigmoid function (see Figure 24(b)) fits the average of plasma concentration measurements for 23 cancer patients:

$$
C_{\mathrm{p}}(t)=\frac{B e^{-m_{1} t}}{1+e^{-m_{2}\left(t-t_{c}\right)}}+\sum_{i=1}^{2} \frac{a_{i}}{\sigma_{i} \sqrt{2 \pi}} e^{-\left(\frac{t-\mu_{i}}{\sigma_{i} \sqrt{2}}\right)^{2}}
$$

where $B=1.050 \mathrm{mM}$ and $m_{1}=0.1682 \mathrm{~min}^{-1}$ are the amplitude and the decay constant of the exponential; $m_{2}=38.078 \mathrm{~min}^{-1}$ and $t_{c}=0.1483 \mathrm{~min}$ are the sigmoid width and center, and $a_{1}=0.809 \mathrm{mM} \min , a_{2}=0.330 \mathrm{mM} \min , \sigma_{1}=0.0563 \min , \sigma_{2}=0.132 \mathrm{~min}$, $\mu_{1}=0.170 \mathrm{~min}$, and $\mu_{2}=0.365 \min$ are the scales, widths, and centers of the Gaussian kernels, respectively.

As demonstrated in Figure 24, the AIF by Parker et al. [14] is closer by kinetics to the true AIF and therefore is more realistic than the AIF proposed by Tofts and Kermode [13]. The population-based AIFs are widely used in quantitative DCE-MRI studies due to their simplicity and the fact that no additional MR measurements in other regions of interest are required [155]. However, ignoring variations of the CA injection rates and presuming small inter-subject variabilities are their main limitations, which can result in large errors in both the AIF characterization and subsequent PK analysis. 
The subject-specific, or individual-based AIF is determined from the patient's DCEMRI data $[156,157]$. Typically, the MRI signal from a region, which contains a large feeding artery located near the tissue of interest, is monitored and converted to the CA concentration (such a conversion is detailed in Section II.D.2) to directly characterize the AIF. This approach is a completely noninvasive technique and is expected to closely approximate the true AIF. However, the required large arterial vessel within the depicted field of view may not exist if small lesion areas, e.g., breast cancer, are imaged. Also, the accuracy of the measured AIF depends on the chosen MRI pulse sequence parameters [158], namely, the optimization of temporal resolution for determining the AIF accurately could result in undesirable spatial resolution and signal-to-noise ratio (SNR) [155]. It is worth mentioning that the AIF obtained from a feeding artery characterizes the whole arterial blood and must be corrected to account for the hematocrit factor in order to represent the plasma concentration.

The reference tissue-based AIF [159-162] overcomes limitations of the subjectspecific AIF and inaccuracies of the population-based AIF. Instead of measuring the MRI signal in a nearby feeding artery or assuming a particular form of the AIF, the CA concentration in a well-characterized, healthy reference tissue (e.g., a muscle) is measured to calibrate signal intensity changes in the tissue of interest. These techniques are stratified into two groups called the single and multiple reference tissue-based methods, respectively. The former presume the known PK parameter values for a single healthy reference tissue and use its CA uptake curve to inversely derive the AIF [159]. However, this assumption does not necessarily hold due to inter-individual variability of the kinetic parameters [163], which affects the accuracy and reduces the reproducibility of the results [161]. Additionally, the single reference tissue-based AIF is assumed to be the same for both the reference tissue (e.g., a muscle) and the tissue of interest (e.g., a tumor). Moreover, single reference tissue-based AIF is applicable only for simple DCE-MRI modeling, which does not include the fractional volume of plasma per unit volume of tissue, or $v_{\mathrm{p}}[164,165]$. The double $[164,165]$ and multiple $[166,167]$ reference tissue-based techniques involve no assumptions about the kinetic parameters in the reference tissue. The multiple reference 
tissue-based AIFs have demonstrated better PK modeling compared with the populationbased ones [166].

The jointly estimated AIF is specified as an acceptable function with adjustable parameters of blood plasma concentration, which can be jointly estimated with the PK parameters. No measurements of or assumptions about an AIF are involved. Both the PK and AIF parameters are jointly adjusted for fitting the tissue CA concentration curve, while the AIF parameters are tuned for obtaining the best fit [168]. The main advantage is that no special DCE-MRI protocol for measuring the AIF is required. As shown in [169], the joint estimation of both the AIF and PK model parameters decreases biases and uncertainties in the PK estimates compared to a population-based technique. Mono-exponential functions [170] are the simplest AIF forms; however, these simplifications may give significant errors in the estimated PK parameters [165]. More realistic AIF forms with a large number of free parameters, like, e.g., Eq. (1), increases the computational cost of DCE-MRI analysis. For example, the Tofts model [171] and the AIF by Parker et al. [14] require in total 12 free parameters ( 2 for the Tofts model and 10 for the AIF), which makes the search space for the optimum parameters of the tissue concentration curve in each voxel very big, and therefore the probability of being trapped in local minima is high. This most recent AIF determination technique requires additional investigation, similar to the multiple reference tissue-based approach.

An essential requirement for building a PK model for analyzing DCE-MRI in clinical applications is to convert the measured MR signal intensity $(S(t))$ into the CA concentration curve. The estimation of the CA concentration from the signal data follows.

\section{Estimation of Contrast Agent Concentration}

Almost all parametric DCE-MRI analysis calls for determining both the tissue, $C_{\mathrm{t}}(t)$, and blood plasma, $C_{\mathrm{p}}(t)$, CA concentration curves from the signal intensity or strength $(S(t))$. Determination of CA concentrations may not be necessary as long as the relationship between $S(t)$ and CA concentration is linear and is the same for blood and tis- 
sue. In other words, one could change the scale on which CA concentrations are measured and it would not change the results for blood flow, permeability surface area products, etc. However, in most cases, the relationship between $S(t)$ and CA concentration is non-linear due to the effects of signal saturation at higher CA concentrations [172]. DCE-MRI measures the CA presence effect on proton relaxation times, $T_{1}$ and $T_{2}$, rather than the signal change from the CA uptake. But since changes in the relaxation times affect the recorded MRI signal, the $S(t)$-curve can be transformed into CA concentration curves using the Bloch's equations [173] for any MRI sequence.

Typical MRI acquisition techniques include saturation recovery, inversion recovery, spin echo, and gradient echo sequences. A spoiled gradient echo (SPGRE) sequence is frequently used in gadolinium-diethylene triamine pentacetate (Gd-DTAP) acid-based studies of the $T_{1}$-weighted DCE-MRI [155]. This sequence provides high temporal sampling, being adequate to characterize the CA transit, while maintaining an acceptable SNR and spatial resolution for visualizing the anatomy. The expected signal intensity using an SPGRE sequence is [174]:

$$
S(t)=M_{0} \frac{\exp \left(-\frac{T_{E}}{T_{2}^{*}}\right)\left(1-\exp \left(-\frac{T_{R}}{T_{1}(t)}\right)\right) \sin (\alpha)}{1-\cos (\alpha) \exp \left(-\frac{T_{R}}{T_{1}(t)}\right)}
$$

where $M_{0}$ is a scaling factor that depends on the scanner gain and proton density; $\alpha$ is the flip angle; $T_{R}$ is the repetition time ( $\left.m s e c\right), T_{E}$ is the echo time (msec), and $T_{1}$ and $T_{2}$ are the spin-lattice (longitudinal) and spin-spin (transverse) relaxation times, respectively. In heavily $T_{1}$-weighted scenarios $\left(T_{E} \ll T_{2}^{*}\right)$ the $T_{2}^{*}$ effect on signal degradation can be ignored and Equation (3) can be simplified by incorporating the effects of $T_{2}, T_{E}$ and other scanner parameters into the $M_{0}$ term, denoted below $S_{0}$ :

$$
S(t)=S_{0} \frac{\left(1-\exp \left(-\frac{T_{R}}{T_{1}(t)}\right)\right) \sin (\alpha)}{1-\cos (\alpha) \exp \left(-\frac{T_{R}}{T_{1}(t)}\right)}
$$

In theory, the tissue CA concentration, $C_{\mathrm{t}}$, relates linearly to the time-variant relaxation rate [171]:

$$
\frac{1}{T_{1}(t)}=\frac{1}{T_{10}}+r_{1} C_{\mathrm{t}}
$$


where the longitudinal CA relaxation coefficient $r_{1}\left(\mathrm{mM}^{-1} \mathrm{sec}^{-1}\right)$ depends on temperature, field strength, and chemical structure of the CA [175] and $T_{10}$ is a so-called native relaxation time, i.e., the value of $T_{1}$ before injecting any $\mathrm{CA}$. To determine the CA concentration, both $T_{1}(t)$ and $T_{10}$ essentially have to be calculated. In MR literature, Equation (5) applies for tissue in the fast exchange limit (FXL) scenarios.

Both $S_{0}$ and $T_{10}$ are estimated most frequently by a multiple-angle acquisition, from images taken before administering the CA at two or more different flip angles [176]. In the case of two flip angles, $\alpha_{1}$ and $\alpha_{2}$, the signal intensities are calculated from the two SPGRE pulse sequences acquired using these angles. Then, using Equation (3), the ratio, $R_{\alpha}=\frac{S_{\alpha_{2}}}{S_{\alpha_{1}}}$, of the two measurements allows for finding the $T_{10}$ value [177]. The $T_{1}(t)$-curve can be calculated analytically:

$$
T_{1}(t)=T_{R}\left[\ln \left(\frac{S_{1}(t) \cos \left(\alpha_{1}\right) \sin \left(\alpha_{2}\right)-S_{2}(t) \sin \left(\alpha_{1}\right) \cos \left(\alpha_{2}\right)}{S_{1}(t) \sin \left(\alpha_{2}\right)-S_{2}(t) \sin \left(\alpha_{1}\right)}\right)\right]^{-1}
$$

The multiple flip angle method [178] requires three or more SPGRE signal intensities $S_{\alpha_{i}}$ at different angles $\alpha_{i} ; i=3,4, \ldots$ Rearranging Equation (4) yields a straight line equation:

$$
Y=m X+S_{0}(1-m)
$$

where $Y=\frac{S_{\alpha_{i}}}{\sin \left(\alpha_{i}\right)}, X=\frac{S_{\alpha_{i}}}{\tan \left(\alpha_{i}\right)}$, and $m=\exp \left(-\frac{T_{R}}{T_{1}}\right)$ represents the slope of the line. Hence from the $Y$-against- $X$ plot, the $T_{10}=-\frac{T_{R}}{\ln (m)}$ value can be calculated. For a more accurate DCE-MRI analysis and better evaluation of the PK parameters, the estimated $T_{1}(t)$ and $T_{10}$ values should be compared with the known ones for different tissues (e.g., muscles, grey matter, and white matter) to ensure that these estimates are in the acceptable ranges.

Once the AIF is measured or determined, the $S(t)$ is converted to the tissue CA concentration curve using Equation (5), and the PK model is then used to fit the latter. The final solution describes the CA concentration, $C_{\mathrm{t}}$, in terms of various rates and volume parameters of the PK model used. Strengths and weaknesses of the most common compartment and distributed tracer-kinetic models that are used for analyzing DCE-MRI, are outlined below. 


\section{Compartment Models}

For the last two decades, many compartment models of various complexities and under different assumptions have been proposed for quantifying the CA uptake in the tissue. The well-known compartment models are based on the original Kety's [148] model that captures exchanges of blood, which contains the CA, between different compartments. A two-compartment model, widely used for quantitative analysis of tissue perfusion, is sketched in Figure 25. Its compartments specify the CA concentrations in the EES and the blood plasma (intravascular space). An arterial input CA amount, $C_{\mathrm{a}}(t)$, administered to the system yields a dynamic concentration, $C_{\mathrm{p}}(t)$, in the first compartment, whereas the intercompartment exchange of the CA results in a dynamic concentration, $C_{\mathrm{e}}(t)$, in the second compartment. Inter-compartment exchange rates are governed by forward and backward volume transfer constants, $k_{12}$ and $k_{21}$, respectively, and CA losses from the system are described by an excretion rate, $k_{\mathrm{el}}$. Since, the parameters $k_{12}$ and $k_{21}$ control the CA transfer from the blood plasma to the tissue, they are related to capillary permeability [171]. The total tissue CA concentration is as follows: $C_{\mathrm{t}}(t)=v_{\mathrm{p}} C_{\mathrm{p}}(t)+v_{\mathrm{e}} C_{\mathrm{e}}(t)$ where $v_{\mathrm{p}}$ and $v_{\mathrm{e}}$ $\left(0<v_{\mathrm{p}}, v_{\mathrm{e}}<1\right)$ are the fractional plasma and EES volumes, respectively. The most popular compartment models for analyzing the CA perfusion in the tissue are detailed below.

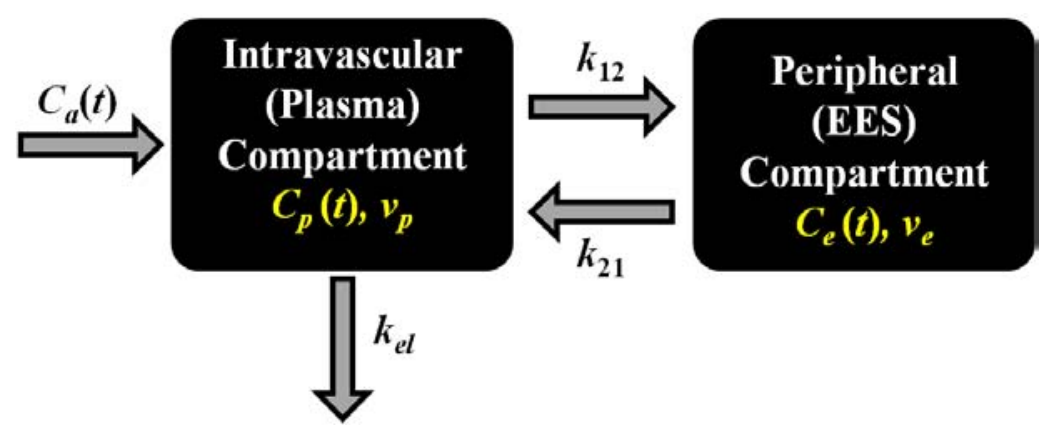

FIGURE 25: Two-compartment model: the function $C_{\mathrm{a}}(t)$ quantifies the arterial input into the plasma compartment; the peripheral compartment receives the contrast agent $(\mathrm{CA})$ from and returns it to the plasma compartment at the rates $k_{12}$ and $k_{21}$, respectively, and the rate $k_{\mathrm{el}}$ specifies the CA loss from the system. 
a. The Larsson model (LM) Developed by Larsson et al. [145], it is one of the earliest kinetic models for analyzing DCE-MRI. In this model, the CA flow between the blood plasma in the capillary and EES (also called the interstitial water space) is assumed to be controlled by a single transfer constant $k_{\mathrm{ep}}\left(\mathrm{min}^{-1}\right)$ combining three parameters: the capillary blood flow, $F_{\mathrm{p}}$; the extraction fraction, $E$, and the fractional EES volume, see Figure 26.
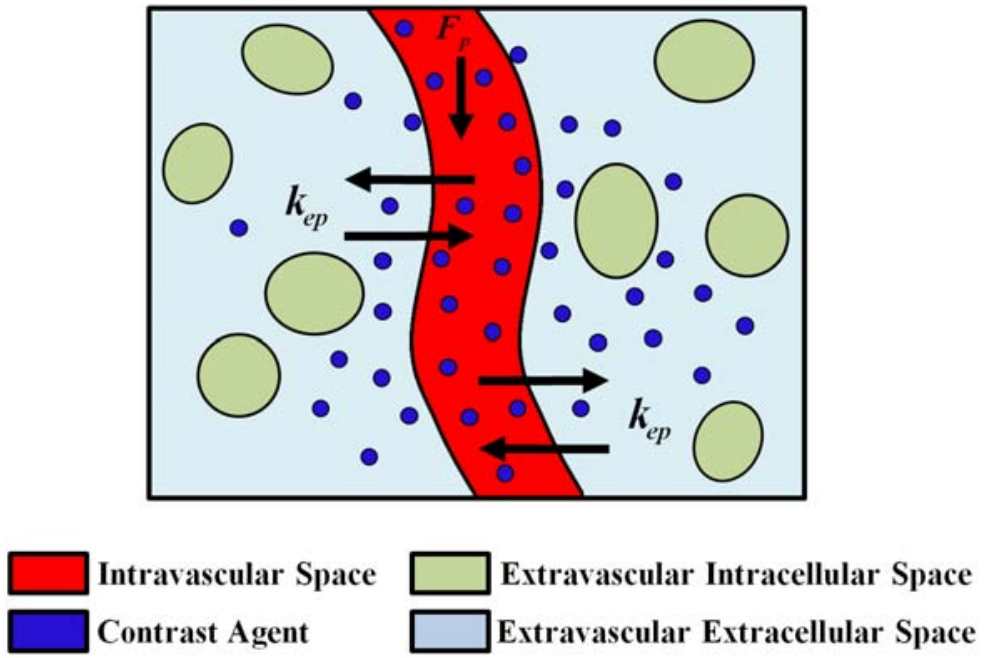

FIGURE 26: The Larsson model (LM) for a capillary-tissue system: the blood plasma flows in the capillary at a rate of $F_{\mathrm{p}}$ and exchanges the contrast agent (CA) with the extracellular and extravascular space (EES) at a rate of $k_{\mathrm{ep}}$.

To build the model, the CA concentration in the plasma compartment, $C_{\mathrm{p}}(t)$, is obtained using the gold standard AIF, namely, by measuring the CA amount in a series of blood samples taken in $15 \mathrm{sec}$ intervals after a bolus CA injection. The measurements are then fitted with a sum of three exponentials with the amplitudes $a_{i}$ and time constants $m_{i}$, respectively $(i=1,2,3)$ :

$$
C_{\mathrm{p}}(t)=\sum_{i=1}^{3} a_{i} e^{-m_{i} t}
$$

The temporal tissue CA concentration uptake change in the EES compartment is described using the transfer equation:

$$
\frac{d C_{t}}{d t}=k_{\mathrm{ep}}\left(C_{\mathrm{p}}(t)-C_{\mathrm{t}}\right)
$$


which can be solved for $C_{\mathrm{t}}$ using $C_{\mathrm{p}}(t)$ in Equation (8) as follows:

$$
C_{\mathrm{t}}(t)=k_{\mathrm{ep}} \sum_{i=1}^{3} \frac{a_{i}\left(e^{-k_{\mathrm{ep}} t}-e^{-m_{i} t}\right)}{m_{i}-k_{\mathrm{ep}}}
$$

The LM assumes that the MR signal $S(t)$ relates linearly to the CA concentration:

$$
S(t)=S_{0}+\left(\frac{k^{\prime}(t)}{k_{\mathrm{ep}}}\right) C_{\mathrm{t}}
$$

where $k^{\prime}(t)=\frac{S^{\prime}(t)}{\sum_{i=1}^{3} a_{i}} ; S_{0}$ is the baseline signal intensity before the CA injection, and $S^{\prime}(t)$ is the initial signal slope, or equivalently

$$
S(t)=S_{0}+k^{\prime}(t) \sum_{i=1}^{3} \frac{a_{i}\left(e^{-k_{\mathrm{ep}} t}-e^{-m_{i} t}\right)}{m_{i}-k_{\mathrm{ep}}}
$$

The LM is applicable under a very limited tissue permeability, i.e., when the permeability is considerably lower than the flow, and is fully described by the single transfer constant, $k_{\mathrm{ep}}$. The latter can be estimated via optimization, e.g., by the least-squares techniques. The main limitation of the LM is its assumed negligible contribution of the plasma (intravascular space) tracer. Additionally, the work presented in [145] provide only a combined estimation of both permeability and mean extravascular space. However, Larsson and coworkers provisioned a method that allows for separate estimation of permeability and $v_{\mathrm{e}}$, which was introduced in [179] using in vitro value of relaxivity, and a measurement of $T_{10}$.

b. The Brix model (BM) Proposed by Brix et al. [146], it is one of the most wellknown compartment models for analyzing DCE-MRI. In the Brix model, kinetics of the CA exchange between the blood plasma and the peripheral (interstitial) EES compartments are described with several rate and transfer constants, shown in Figure 27. The CA is administered at a constant rate of $k_{\text {in }}$ over a time-span $\tau$, exchanged between the plasma and EES compartments at $k_{\mathrm{pe}}$ (forward) and $k_{\mathrm{ep}}$ (reverse) transfer rates, and eliminated from the plasma at a rate of $k_{\mathrm{el}}$. Unlike the LM, which requires a predetermined AIF, for the $\mathrm{BM}$ a particular AIF is taken to be known from the infusion rate (the flux) entering the body. However, it is not correct to assume that the infusion is the same flux that enters the tissue compartment in focus. 


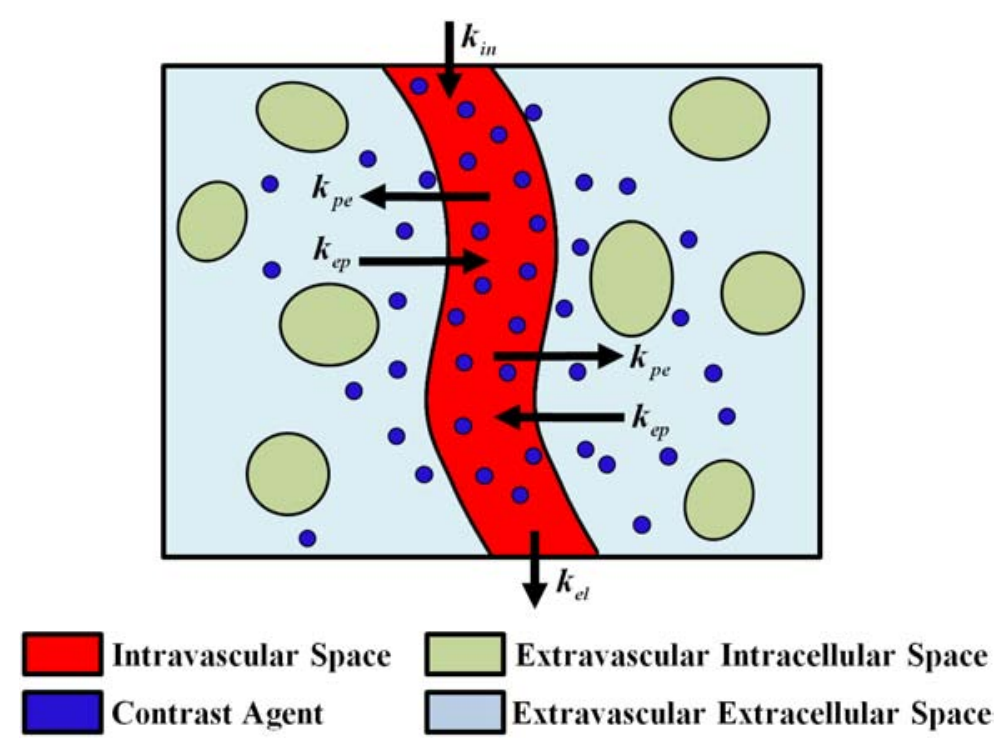

FIGURE 27: The Brix model (BM): the CA is administered at a constant rate of $k_{\text {in }}$ into the plasma compartment; exchanged between the two compartments at rates of $k_{\mathrm{ep}}$ and $k_{\mathrm{pe}}$, respectively, and eliminated (cleared) from the plasma at a rate of $k_{\mathrm{el}}$.

Relationships between the intravascular and peripheral compartments in the BM are described using the mass conservation principle [146]:

$$
\begin{gathered}
\frac{d C_{\mathrm{p}}}{d t}=\frac{k_{\text {in }}}{V_{\mathrm{p}}}(u(t)-u(t-\tau))-k_{\mathrm{el}} C_{\mathrm{p}}(t) \\
\frac{d C_{\mathrm{t}}}{d t}=k_{\mathrm{pe}} \frac{V_{\mathrm{p}}}{V_{\mathrm{e}}} C_{p}-k_{\mathrm{ep}} C_{\mathrm{p}}(t)
\end{gathered}
$$

where $u(t)$ is the Heaviside step function, and $V_{\mathrm{p}}$ and $V_{\mathrm{e}}$ are the intravascular plasma and the EES compartment volumes, respectively. Solving Equations (13) and (14) under the initial conditions $C_{\mathrm{p}}(t)=0$ and $C_{\mathrm{t}}(t)=0$ for $t=0$ gives the following CA concentrations in the blood plasma and tissue [180]:

$$
\begin{gathered}
C_{\mathrm{p}}(t)=\frac{k_{\mathrm{in}}}{V_{\mathrm{p}} k_{\mathrm{el}}}\left(e^{k_{\mathrm{el}} t^{\prime}}-1\right) e^{k_{\mathrm{el}} t} \\
C_{\mathrm{t}}=\frac{k_{\mathrm{in}} k_{\mathrm{pe}}}{V_{\mathrm{p}}\left(k_{\mathrm{ep}}-k_{\mathrm{el}}\right)}\left[\frac{e^{-k_{\mathrm{el}} t}}{k_{\mathrm{el}}}\left(e^{k_{\mathrm{el}} t^{\prime}}-1\right)-\frac{e^{-k_{\mathrm{ep}} t}}{k_{\mathrm{ep}}}\left(e^{k_{\mathrm{ep}} t^{\prime}}-1\right)\right]
\end{gathered}
$$

where $t^{\prime}=t$ if $0 \leq t \leq \tau$, and $t^{\prime}=\tau$ if $\tau \leq t$. To fit the measured signal $S(t)$, the BM uses three parameters, namely, the CA exchange rate, $k_{\mathrm{ep}}$; the elimination rate, $k_{\mathrm{el}}$, and an additional parameter, $A^{\text {Brix }}$, being an arbitrary constant that depends on the tissue properties 
and the MR sequence parameters. The relationship between the signal $S(t)$ and these free model parameters at any time is as follows [146]:

$$
\frac{S(t)}{S_{0}}=1+\frac{A^{\text {Brix }}}{k_{\mathrm{ep}}-k_{\mathrm{el}}}\left[\frac{e^{-k_{\mathrm{el}} t}}{k_{\mathrm{el}}}\left(e^{k_{\mathrm{el}} t^{\prime}}-1\right)-\frac{e^{-k_{\mathrm{ep}} t}}{k_{\mathrm{ep}}}\left(e^{k_{\mathrm{ep}} t^{\prime}}-1\right)\right]
$$

where $t^{\prime}=t$ if $0 \leq t \leq \tau$ and $t^{\prime}=\tau$ if $\tau \leq t$. After a CA bolus injection, Equation (17) is reduced to:

$$
\frac{S(t)}{S_{0}} \approx 1+\tau A^{\text {Brix }}\left(\frac{e^{-k_{\mathrm{el}} t}-e^{-k_{\mathrm{ep}} t}}{k_{\mathrm{ep}}-k_{\mathrm{el}}}\right)
$$

A modified version of the BM proposed by Hoffmann et al. [170] reduces the CA infusion length to one minute. The after-bolus signal $S(t)$ is fitted by using the following equation:

$$
\frac{S(t)}{S_{0}} \approx 1+k_{\mathrm{ep}} A^{\mathrm{H}}\left(\frac{e^{-k_{\mathrm{el}} t}-e^{-k_{\mathrm{ep}} t}}{k_{\mathrm{ep}}-k_{\mathrm{el}}}\right)
$$

where the amplitude parameter, $A^{\mathrm{H}}$ approximately corresponds to the EES size if the CA relaxation properties, the native $\mathrm{T} 1$, and the CA dose do not vary significantly [171].

Although the BM has been widely used due to its simplicity and proved ability to closely fit the tissue DCE-MRI data, its basic assumption of approximating $C_{\mathrm{p}}(t)$ with a single exponential function for up to $20 \mathrm{~min}$ after the CA injection is seldom supported by experimental observations $[13,154]$. Additionally, the BM provides no direct measure of capillary permeability and is applicable only under specific permeability-limiting conditions [181]. However, the vasculature permeability can be roughly estimated with the product of the amplitude parameter, $A^{\text {Brix }}$, and the rate constant, $k_{\mathrm{ep}}[146,182]$.

c. The Tofts and Kermode (TK) model The most popular PK model proposed by Tofts and Kermode (TK) [13] has unified many previous ones and introduced common characteristic parameters and naming conventions [183]. It assumes that the CA diffuses from and returns to the blood plasma at rates governed by the forward transfer constant, $K^{\text {trans }}\left(\min ^{-1}\right)$, and the reverse constant, $k_{\text {ep }}\left(\mathrm{min}^{-1}\right)$, respectively (see Figure 28 ).

The tissue CA concentration is derived in the TK from the EES components only, while the intravascular (plasma) compartment contribution is ignored, i.e., $C_{\mathrm{t}}(t)=v_{\mathrm{e}} C_{\mathrm{e}}(t)$. 

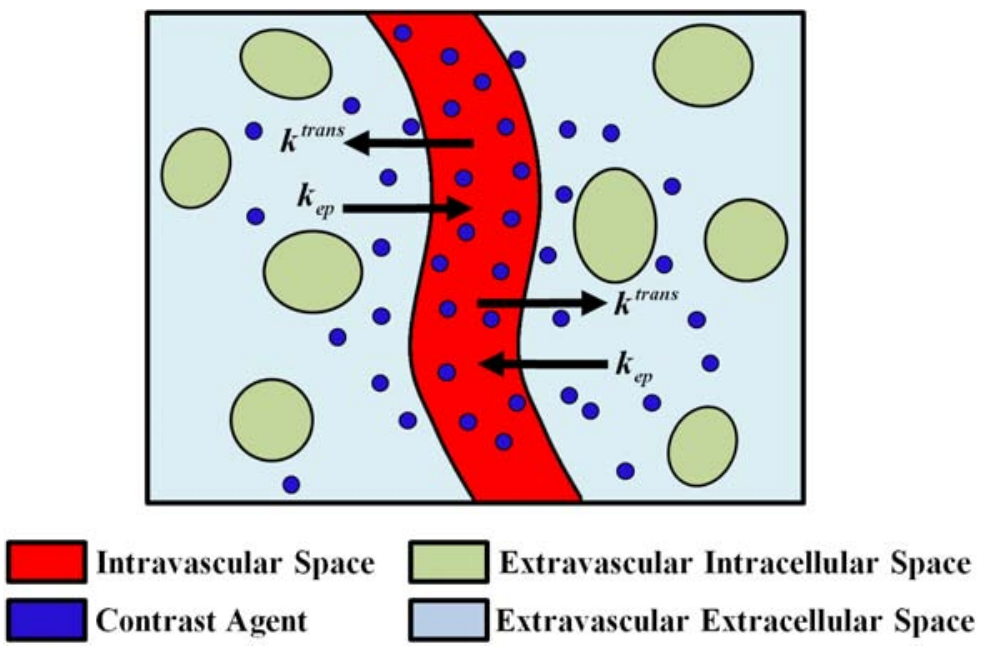

FIGURE 28: Schematic illustration of the CA transfer in the Tofts and Kermode (TK) model between the central (plasma) compartment and the EES space with the $K^{\text {trans }}$ and $k_{\text {ep }}$ rates, respectively.

The tissue concentration, $C_{\mathrm{t}}(t)$, is described by the transfer equation:

$$
\frac{d C_{\mathrm{t}}}{d t}=K^{\text {trans }} C_{\mathrm{p}}(t)-k_{\mathrm{ep}} C_{\mathrm{t}}(t)=K^{\mathrm{trans}}\left(C_{\mathrm{p}}(t)-\frac{C_{\mathrm{t}}(t)}{v_{\mathrm{e}}}\right)
$$

where $k_{\mathrm{ep}}=K^{\text {trans }} / v_{\mathrm{e}}$. The CA concentration in the plasma, $C_{\mathrm{p}}(t)$, after injection specifies the AIF and is used as the initial condition to estimate $C_{\mathrm{t}}(t)$. Under the initial conditions $C_{\mathrm{p}}(t)=C_{\mathrm{t}}(t)=0$ at $t=0$, Equation (20) has the following solution [183]:

$$
C_{\mathrm{t}}(t)=K^{\text {trans }} \int_{0}^{t} C_{\mathrm{p}}\left(t^{\prime}\right) \exp \left(-\frac{K^{\text {trans }}}{v_{\mathrm{e}}}\right)\left(t-t^{\prime}\right) d t^{\prime}
$$

Alternatively, the TK output, $C_{\mathrm{t}}(t)$, in Equation (21), can be found by using the convolution theory. Namely, $C_{\mathrm{t}}(t)$ is obtained by the convolution (denoted $\otimes$ ) of the input signal, $C_{\mathrm{p}}(t)$, with the tissue impulse response, $H_{\mathrm{TK}}(t)$, i.e., $C_{\mathrm{t}}(t)=C_{\mathrm{p}}(t) \otimes H_{\mathrm{TK}}(t)$ where

$$
H_{\mathrm{TK}}(t)=K^{\text {trans }} \exp \left(-\frac{K^{\text {trans }}}{v_{\mathrm{e}}} t\right)
$$

A population-based AIF in the original TK [13], described by a sum of two exponentials (see Equation (1) and Figure 24 (a)) results in the following output $C_{\mathrm{t}}(t)$ of 
Equation (21):

$$
\begin{aligned}
C_{\mathrm{t}}(t)= & D K_{\text {trans }} \frac{a_{1}}{m_{1}-k_{\mathrm{ep}}}\left(\exp \left(-\frac{K^{\text {trans }}}{v_{\mathrm{e}}} t\right)-\exp \left(-m_{1} t\right)\right) \\
& +\frac{a_{2}}{m_{2}-k_{\mathrm{ep}}}\left(\exp \left(-\frac{K^{\text {trans }}}{v_{\mathrm{e}}} t\right)-\exp \left(-m_{2} t\right)\right)
\end{aligned}
$$

where $D$ is the CA dose (mM kg-1 of body mass) and $K^{\text {trans }}$ and $v_{\mathrm{e}}$ are the TK model free parameters that determine the shape of the fitted data, and $k_{\mathrm{ep}}=K^{\text {trans }} / v_{\mathrm{e}}$.

Physiologically, $K^{\text {trans }}$ is the most important and significant tissue-dependent parameter in the TK model. It assesses either plasma flow $F_{\mathrm{p}}$ in flow-limited scenarios or tissue permeability (represented by the tissue permeability-surface area product, $P S$ ) in permeability-limited scenarios for the uptake. In mixed scenarios, it indicates a combination of flow and permeability properties of the tissue and acts as a lump measure of their joint effect.

d. Extended Tofts and Kermode (ETK) model The original TK depends on the two parameters, $K^{\text {trans }}$ and $v_{\mathrm{e}}$, and assumes that the tissue is weakly vascularized $\left(v_{\mathrm{p}}=0\right)$. However, this assumption is invalid for many tissues, especially tumors. The generalized TK [171], known commonly as the extended TK (ETK), includes the intravascular contribution $v_{\mathrm{p}} C_{\mathrm{p}}(t)$ to the tissue concentration by representing the $C_{\mathrm{t}}(t)$ as:

$$
\begin{aligned}
C_{\mathrm{t}}(t) & =v_{\mathrm{p}} C_{\mathrm{p}}(t)+C_{\mathrm{p}}(t) \otimes H_{\mathrm{TK}}(t) \\
& =v_{\mathrm{p}} C_{\mathrm{p}}(t)+K^{\text {trans }} \int_{0}^{t} C_{\mathrm{p}}\left(t^{\prime}\right) \exp \left(-\frac{K^{\text {trans }}}{v_{\mathrm{e}}}\left(t-t^{\prime}\right)\right) d t^{\prime}
\end{aligned}
$$

where $v_{\mathrm{p}}$ is the fractional plasma volume per unit tissue volume. Free ETK parameters, $K^{\text {trans }}, v_{\mathrm{e}}$, and $v_{\mathrm{p}}$, can be estimated by fitting an empirical tissue concentration estimated from the MRI data by the curve $C_{\mathrm{t}}(t)$ of Equation (24) with a measured or determined AIF (as described in Section II.D.1). For fitting, the signal intensity is converted to the CA concentration using Equation (5). Both the TK [13] and ETK [171] are considered the bestestablished models for analyzing the $T_{1}$-weighted DCE-MR images. However, because the volume transfer constant, $K^{\text {trans }}$, incorporates both the plasma flow and tissue permeability, these latter parameters cannot be estimated separately. The use of a population-based AIF, which was originally introduced by Weinmann et al. [154], differs significantly from the true AIF and is an additional disadvantage of both the models. 
e. Patlak model (PM) Unlike the above PK models, Patlak et al. [184] have proposed a graphical approach, called Patlak plot, for compartment analysis in order to estimate the CA transfer constant between the blood plasma and the EES space. The Patlak model (PM) assumes the reverse vascular transfer constant $\left(k_{\mathrm{ep}}\right)$ from the EES back to the plasma in Figure 28) and Equation (24) is negligibly small due to low permeability and short measuring time. This assumption results in the following tissue concentration:

$$
C_{\mathrm{t}}(t)=v_{\mathrm{p}} C_{\mathrm{p}}(t)+K^{\mathrm{trans}} \int_{0}^{t} C_{\mathrm{p}}\left(t^{\prime}\right) d t^{\prime}
$$

where $v_{\mathrm{p}}$ is the vascular fraction. The Patlak plot linearizes Equation (25) as:

$$
Y=K^{\text {trans }} X+v_{\mathrm{p}}
$$

where $Y=\frac{C_{\mathrm{t}}(t)}{C_{\mathrm{p}}(t)}$ and $X=\frac{\int_{0}^{t} C_{\mathrm{p}}\left(t^{\prime}\right) d t^{\prime}}{C_{\mathrm{p}}(t)}$. Estimation of the parameter $K^{\text {trans }}$ by constructing visual linear graphical plots and simple interpretation are the main advantages of the PM. This linearized graphical analysis has a widespread popularity in certain clinical studies, such as renal applications [185-188] where $K^{\text {trans }}$ is equal to the kidney's glomerular filtration rate (GFR). However, this model does not take into account the reverse flow $\left(k_{\mathrm{ep}}\right)$; therefore, its estimates can be highly inaccurate and the analysis results could have some limitations [189]. Moreover, if the model assumption is violated, the plotted points are not collinear and the estimation of the parameters is no longer correct [190].

Chen et al. [190] developed an extended graphical PM, which is an intermediate between the ETK and PM and yields more stable and unbiased estimates of $K^{\text {trans }}$ within short acquisition durations. It expands the ETK by correcting for reflux while retaining the central PM's advantages, such as linearity in the parameters estimated, simple graphical interpretation, and stable fitting procedures. Due to accommodating the CA efflux, the extended graphical PM became less susceptible to bias [191].

f. Two-compartment exchange model (2CXM) The earlier PK models [13, 145, $146,171]$ allowed, in principle, for estimating the volume transfer constant, $K^{\text {trans }}$, that combines both the blood flow and tissue permeability. A recent more general two-compartment exchange model (2CXM) [192-194] allows for separate estimation of the permeability, 
$P S$, and the plasma blood flow, $F_{\mathrm{p}}$. A block diagram of the 2CXM is schematized in Fig. 29 and consists of two compartments, namely the intravascular plasma and the EES compartments. The intravascular compartment experiences an external flow, $F_{\mathrm{p}}$, of the plasma and the CA exchanges between both compartments at a symmetric rate of PS.

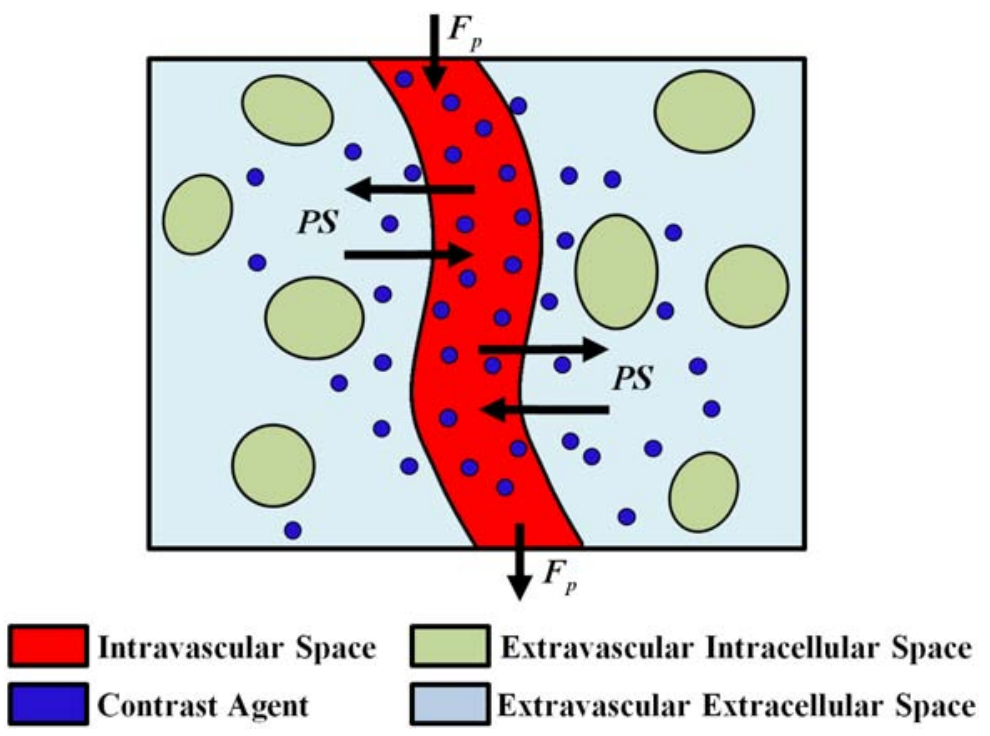

FIGURE 29: Schematic illustration of the two-compartment exchange model (2CXM). The CA delivered via arteries to the plasma compartment at an $F_{\mathrm{P}}$ rate, is exchanged between the intravascular plasma and EES compartments at a symmetric rate of $P S$, and is eliminated subsequently from the plasma compartment.

Using the mass conservation principle, the CA diffusion between the capillary plasma and the EES space is described by the coupled system of differential equations [192]:

$$
\begin{aligned}
\frac{d C_{\mathrm{p}}}{d t} & =\frac{P S}{v_{\mathrm{p}}}\left(C_{\mathrm{e}}(t)-C_{\mathrm{p}}(t)\right)+\frac{F_{\mathrm{p}}}{v_{\mathrm{p}}}\left(C_{\mathrm{a}}(t)-C_{\mathrm{p}}(t)\right) \\
\frac{d C_{\mathrm{e}}}{d t} & =\frac{P S}{v_{\mathrm{e}}}\left(C_{\mathrm{p}}(t)-C_{\mathrm{e}}(t)\right) \\
C_{\mathrm{t}}(t) & =v_{\mathrm{p}} C_{\mathrm{p}}(t)+v_{\mathrm{e}} C_{\mathrm{e}}(t)
\end{aligned}
$$

where $C_{\mathrm{p}}(t), C_{\mathrm{e}}(t)$, and $C_{\mathrm{a}}(t)$, are the intravascular plasma, EES space, and arterial plasma CA concentrations, respectively. Here $v_{\mathrm{p}}$, and $v_{\mathrm{e}}$ are the respective fractional capillary plasma and EES compartments' volumes. The $C_{\mathrm{t}}(t)$ is specified by convolving the AIF 
with the tissue impulse response function, multiplied by the blood plasma flow, $F_{\mathrm{p}}$ :

$$
C_{\mathrm{t}}(t)=F_{\mathrm{p}} H_{2 \mathrm{CXM}}(t) \otimes C_{\mathrm{a}}(t)
$$

where the tissue response, $H_{2 \mathrm{CXM}}(t)$, is found by solving Equations (27) and (28) for the input delta-function $C_{\mathrm{a}}(t)=\delta(t)$ under the initial conditions $C_{\mathrm{p}}(t)=C_{\mathrm{e}}(t)=0$ for $t=0$ and using Equation (29) [193]:

$$
H_{2 \mathrm{CXM}}(t)=B e^{-m_{1} t}+(1-B) e^{-m_{2} t}
$$

where $B, m_{1}$, and $m_{2}$ relate to the model parameters $\left(F_{\mathrm{p}}, P S, v_{\mathrm{p}}\right.$, and $\left.v_{\mathrm{e}}\right)$ as follows:

$$
\begin{gathered}
m_{1}=\frac{1}{2}\left(a+b+\sqrt{(a+b)^{2}-4 b c}\right) \\
m_{2}=\frac{1}{2}\left(a+b-\sqrt{(a+b)^{2}-4 b c}\right) \\
B=\frac{m_{2}-c}{m_{2}-m_{1}}
\end{gathered}
$$

where

$$
a=\frac{F_{\mathrm{p}}+P S}{v_{\mathrm{p}}} ; b=\frac{P S}{v_{\mathrm{e}}} ; c=\frac{F_{\mathrm{p}}}{v_{\mathrm{p}}}
$$

Generally, most of the well-known PK models can be derived from the 2CXM under specific assumptions. For example, the ETK model is derived from the 2CXM model by assuming that the plasma flow is so high such that the time taken for the CA to pass through the plasma compartment, i.e., the mean transit time (MTT), is negligible. Under this assumption, the intravascular plasma concentration cannot be distinguished from the AIF, i.e., $C_{\mathrm{p}}(t) \cong C_{\mathrm{a}}(t)$.

As the most general compartment model, the 2CXM is gradually becoming popular for fitting the MRI data in many clinical applications [193-195]. Its main advantage is the possibility to estimate both the regional blood flow and capillary permeability as well as the volume fractions of the intravascular (plasma) and interstitial (EES) space [192]. The main limitation of this and other compartment models is the assumed well-mixed tissue compartments so that spatial variations of the CA diffusion are not taken into account. In addition, all the compartment models assume a fast exchange limit regime, which is not always valid or true, especially for the high CA concentration in the voxel of interest, 
as was recently shown in $[196,197]$. The extravascular space is divided in these studies into two separate compartments, namely, the EES and the extravascular-intracellular space, and a special shutter speed model is introduced in order to account for a limited water exchange rate between the compartments [198]. More details about these models can be found in $[196,197,199,200]$.

\section{Distributed Models}

The main limitation of the compartment models-the assumed fast CA movement and even distribution throughout the compartment-makes the CA concentration a function of time only, but not space. Advanced distributed kinetic models, detailed below, that account for both temporal and spatial CA concentration distributions [150,151, 201, 202] have been introduced for a more precise perfusion data analysis.

a. Distributed-parameter (DP) model The DP model [203] is the first type of the distributed kinetic models that is based on a plug flow model, which assumes that the administered CA is carried through a tube by a flow where all particles are traveling with the same velocity. In contrast to the compartment models, the DP does not assume homogeneous (well-mixed) compartments, but accounts instead for a CA concentration gradient within the plasma and EES compartments making their CA concentrations functions of both the time and distance along the capillary length (see Figure 30). Within the DP, the EES is modeled as a series of infinitesimal compartments exchanging the CA with only nearby locations in the capillary bed [70]. Also, no axial CA transportation (along the $x$-direction in Figure 30) is allowed in the EES.

From the mass conservation, the DP can be represented with a system of differential equations for an elementary volume $d x$ along the axial length $L$ of a capillary tube [203]:

$$
\begin{aligned}
v_{\mathrm{p}} \frac{\partial C_{\mathrm{p}}(x, t)}{\partial t} & =-L F_{\mathrm{p}} \frac{\partial C_{\mathrm{p}}(x, t)}{\partial x}-P S\left[C_{\mathrm{p}}(x, t)-C_{\mathrm{e}}(x, t)\right] ; \\
v_{\mathrm{e}} \frac{\partial C_{\mathrm{e}}(x, t)}{\partial t} & =P S\left[C_{\mathrm{p}}(x, t)-C_{\mathrm{e}}(x, t)\right]
\end{aligned}
$$

Similarly to the 2CXM, the analytical DP solution is obtained by the convolution of 


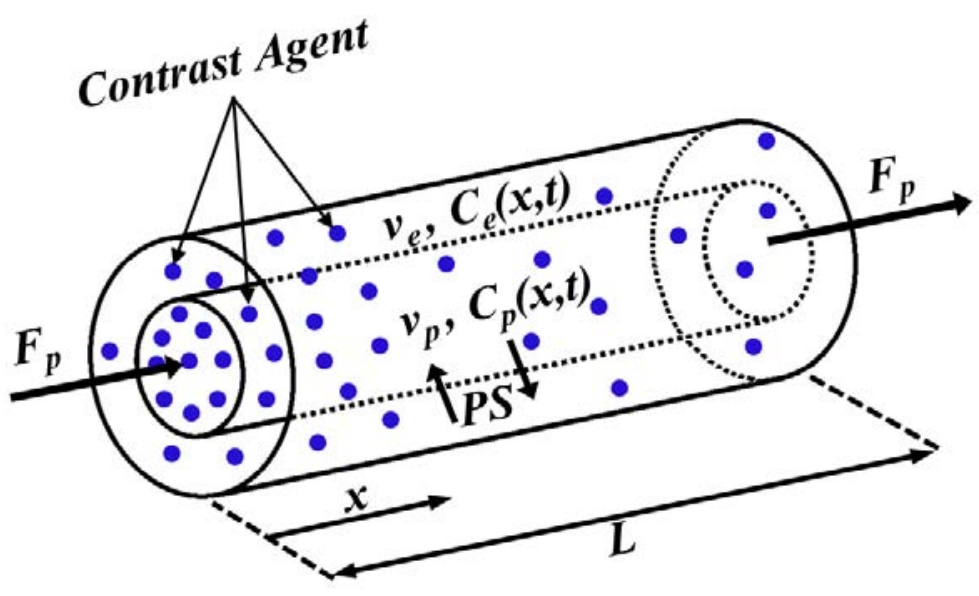

FIGURE 30: Schematic illustration of the Distributed-parameter (DP) model. The CA concentration within the capillary decreases with position $(x)$ along the capillary length $(L)$, producing concentration gradients between the arterial $(x=0)$ and venous $(x=L)$ capillary ends. During the CA passage, some amounts diffuse between the plasma and EES at a controlled $P S$ rate, so that the plasma, $C_{\mathrm{p}}(x, t)$, and EES, $C_{\mathrm{e}}(x, t)$, concentrations show both the spatial and temporal dependence.

$C_{\mathrm{a}}(t)$ (the AIF) with the tissue impulse response function, multiplied by the blood plasma flow, $F_{\mathrm{p}}$. The latter function is found again by solving Equations (34) and (35) for the delta-function input of CA $[70,147]$ as:

$$
H_{\mathrm{DP}}(t)=u(t)-u\left(t-T_{\mathrm{c}}\right) \exp \left(-\frac{P S}{F_{\mathrm{p}}}\right)\left(1+H_{\mathrm{t}^{\prime}}(t)\right)
$$

where and $u(t)$ is the Heaviside unit step function, $T_{\mathrm{c}}=\frac{v_{\mathrm{p}}}{F_{\mathrm{p}}}$ is the MMT of the capillary, and $H_{\mathrm{t}^{\prime}}(t)$ is defined as

$$
H_{\mathrm{t}^{\prime}}(t)=P S \int_{0}^{t-T_{\mathrm{c}}}\left(\frac{1}{t^{\prime} F_{\mathrm{p}} v_{\mathrm{e}}}\right)^{0.5} \exp \left(-\frac{P S}{v_{\mathrm{e}}} t^{\prime}\right) \times I_{1}\left(2 P S\left(\frac{t^{\prime}}{F_{\mathrm{p}} v_{\mathrm{e}}}\right)^{0.5}\right) d t^{\prime}
$$

where $I_{1}($.$) is the modified Bessel function [204]. Compared to the compartment models,$ the DP is more realistic and makes fewer assumptions about microcirculation. However, like all distributed kinetic models in general, the DP is computationally more intensive and requires data with higher temporal resolution in order to derive meaningful results [152]. 
b. Tissue homogeneity (TH) model Another distributed-parameter model is the tissue homogeneity (TH) model that was first described by Johnson and Wilson [150] and applied in nuclear medicine by Sawada et al. [202]. The TH model is a special case of the DP assuming the homogeneous (well-mixed) spatial distribution of the CA concentration within the EES, and therefore, only the time-dependent EES concentration. The TH and DP equations are identical apart from the $x$-position-independent EES concentration [150, 205]:

$$
\begin{aligned}
v_{\mathrm{p}} \frac{\partial C_{\mathrm{p}}(x, t)}{\partial t} & =-L F_{\mathrm{p}} \frac{\partial C_{\mathrm{p}}(x, t)}{\partial x}-P S\left[C_{\mathrm{p}}(x, t)-C_{\mathrm{e}}(t)\right] ; \\
v_{\mathrm{e}} \frac{\partial C_{\mathrm{e}}(x, t)}{\partial t} & =P S\left[C_{\mathrm{p}}(t)-C_{\mathrm{e}}(t)\right]
\end{aligned}
$$

where $C_{\mathrm{p}}(t)$ and $C_{\mathrm{p}}(x, t)$ denote the average CA concentration in plasma and the local CA concentration at $x$, respectively [70]. Unlike the DP, the $\mathrm{TH}$ has no analytical solution in the time domain, hindering its widespread applicability for DCE-MRI analysis [151]. The closed-form model solution exists only in the Laplace space [205]. According to Garpebring et al. [206], the TH solution could be found with the fast Fourier transform. This approach removes many practical obstacles to using the TH in DCE-MRI analysis [70].

St. Lawrence and Lee [151] found a time-domain TH solution by assuming adiabatic (slow) changes in the EES compartment with respect to the change rate in the capillaries. Unlike the $\mathrm{TH}$, their model is based on two basic assumptions: the capillary walls are impermeable to the $\mathrm{CA}$ and the EES receives influx with clearance $E F_{\mathrm{p}}$ from the venous capillary end; where the extraction fraction $E=1-\exp \left(-\frac{P S}{F_{\mathrm{p}}}\right)$ is the intravascular-toEES CA fraction extracted in the first CA pass through the capillary bed. The assumptions lead to the following coupled transfer equations [70]:

$$
\begin{aligned}
v_{\mathrm{p}} \frac{\partial C_{\mathrm{p}}(x, t)}{\partial t} & =-L F_{\mathrm{p}} \frac{\partial C_{\mathrm{p}}(x, t)}{\partial x} \\
v_{\mathrm{e}} \frac{\partial C_{\mathrm{e}}(x, t)}{\partial t} & =E F_{\mathrm{p}}\left[C_{\mathrm{p}}(L, t)-C_{\mathrm{e}}(t)\right]
\end{aligned}
$$

where $C_{\mathrm{p}}(L, t)$ is the CA concentration at the venous capillary end. The resulting compact closed-form solution, called the adiabatic approximation of the TH (AATH), determines 
$C_{\mathrm{t}}(t)$ by convolving the AIF with the tissue impulse response function $H_{\mathrm{AATH}}(t)$ [151]:

$$
C_{\mathrm{t}}(t)=F_{\mathrm{p}} H_{\mathrm{AATH}}(t) \otimes C_{\mathrm{a}}(t)
$$

where

$$
H_{\mathrm{AATH}}(t)=u(t)+E u\left(t-T_{\mathrm{c}}\right) \exp \left(-\frac{E F_{\mathrm{p}}}{v_{\mathrm{e}}}\left(t-T_{\mathrm{c}}\right)\right)
$$

The AATH has four free parameters: $F_{\mathrm{p}}, E, v_{\mathrm{e}}$, and $T_{\mathrm{c}}$. Also, other physiological parameters can also be calculated [183]: $K^{\text {trans }}=E F_{\mathrm{p}}, k_{\mathrm{ep}}=\frac{E F_{\mathrm{p}}}{v_{\mathrm{e}}}, v_{\mathrm{p}}=F_{\mathrm{p}} T_{\mathrm{c}}$, and $P S=$ $-\frac{F_{\mathrm{p}}}{\ln (1-E)}$. The main advantage of the AATH is a closed time-domain solution, which allows for estimating the TH parameters from DCE-MRI data. However, the TH estimation is somewhat difficult and slow even with the AATH, due to a large number of initial guesses in order to avoid too large parameter values [207]. Also, the increased computational cost if voxel-wise parametric maps are needed hinders the wider use of the model [206].

\section{Clinical Applications of Parametric Models}

Over the past two decades several PK models have been developed to capture the dynamics of the CA perfusing into the tissue. These models are able to extract microvascular characteristics and physiological parameters, such as the fractional blood volume and permeability, that describe the blood flow (perfusion) in a biological tissue. The PK analyses provide radiologists with additional functional information about the tissue perfusion, which can facilitate the diagnosis, prognosis, treatment choice, or follow-up on treatment. The dynamic perfusion data analysis can be performed by using two common types of the kinetic models, namely, the compartment and distributed ones. The choice of a particular PK type influences notably the accuracy and precision of the estimated PK parameters [84] and depends on many factors including the underlying application, and data quality and structure, e.g., injection protocol, temporal resolution, acquisition time and noise level.

The compartment models have gained a widespread popularity due to their simplicity and a small number of parameters to be estimated. While being introduced initially to study the blood-brain barrier, they have been used then to analyze DCE-MRI in a wide 
range of clinical applications. In particular, the LM has been applied to study multiple sclerosis [145], assess heart diseases [208-211], quantify regional myocardial perfusion in healthy humans [212,213], and diagnose breast cancer [214,215]. The BM, which is particularly attractive since the AIF need not be known a priori [216], has been used to study the brain $[146,170]$ and breast tumors [217-220]. It is frequently used to analyze the contrast uptake patterns in other applications, including intracranial meningiomas [221], malignant pleural mesothelioma [222], cervical cancer [223] and its chemoradiotherapy outcome [216], colorectal [224] and liver tumors [225], prostate diseases [180, 226-229], and recently, bone perfusion [182].

The most straightforward to interpret are the TK and ETK models, which have been extensively applied to characterize the brain [48, 230-233]), lung [234, 235], breast [51, 53-55, 197, 236-240], prostate [163, 241-254], liver [225], and colorectal [57, 255-257] tumors. These models have also shown promise in a variety of other clinical applications, such as renal carcinoma [258], rheumatoid arthritis [259,260], quantification of myocardial blood flow (MBF) [261, 262], nasopharyngeal carcinoma [263], arterial occlusive disease [264] and carotid atherosclerotic plaque [191, 265], hepatocellular carcinomas [266], and tumor heterogeneity analysis $[57,58,267]$. New fields, such as assessment of preoperative oral cancer therapy [60], pancreatic [59] and cervical cancer [167, 194, 216, 268, 269], head and neck cancers [270-274], and cardiac diseases [275-277] are also regularly explored. The Patlak plot is the simplest PK technique having been widely used in the dynamic MRI analysis. The slope of the Patlak plot is a useful quantitative index for characterizing CA kinetics in certain applications, such as quantifying the MBF [262, 278, 279], assessing kidney function [185-187, 280], predicting blood-brain barrier disruption after an embolic stroke in rats [281], measuring blood-brain barrier permeability [233], and studying abdominal aortic aneurysm [282] and carotid stenosis [191].

The more general 2CXM resolves the ambiguity in interpreting the $K^{\text {trans }}$-estimates from the TK and ETK models. This is a well-known model in classical pharmacokinetics [283] and has been applied to analyze nuclear medicine data by Larson et al. [201] and adopted for the perfusion analysis by Brix et al. [284, 285] and Cheong et al. [286]. Classifi- 
cation of breast tumors by Brix et al. [192] was its first DCE-MRI application. Recently, the 2CXM is gradually becoming popular in various applications, such as brain $[193,195,287]$ and lung cancer [288], myometrium [289], cervix [194] and bladder cancer [290], head and neck tumors [291], and carotid atherosclerotic plaques [191].

Compared to the compartment models, the distributed kinetic models, such as DP, $\mathrm{TH}$, and AATH, account much more accurately for the underlying physiology. The DP has been first described by Sangren and Sheppard [203] and applied to DCE-MRI by Bisdas et al. [292] for studying head and neck cancer and more recently by Koh et al. [293] for studying hepatic metastases. In contrast to the $\mathrm{DP}$, the $\mathrm{TH}$ has not been widely used for DCE-MRI analysis due to the lack of a closed-form solution in the time domain. Because the closed-form solution exists only in the Laplace space [205], the fast Fourier transform was used by Garpebring et al. [206] to apply the TH for studying brain tumors.

The AATH has been first applied to characterize animal brain tumors using DCEMRI by Henderson et al. [294]. Among all current models measuring $F_{\mathrm{p}}$ and $P S$ separately, the AATH has been most widely used in a number of DCE-MRI studies, such as detecting viable myocardium [295] and lung nodules [235], exploring breast tumors [220,296] and prostate cancer [207,297-299], cerebral perfusion mapping [287], and investigating hepatocellular carcinomas in animals [266].

In summary, the PK modeling capabilities to noninvasively characterize microvascular physiology have been explored and validated in a wide range of clinical applications. This section covered the clinical applications of the reviewed PK models. Table 2 summarizes a number of important findings in a wide range of non-tumor and tumor studies, including tumor detection, characterization, and staging, as well as therapy monitoring.

TABLE 2: Recent parametric DCE-MRI studies in different clinical applications.

\begin{tabular}{|c|c|c|c|}
\hline Study & PK model & Tissue of interest & Objectives (o) and conclusions (c) \\
\hline $\begin{array}{l}\text { Harrer et } \\
\text { al. [230] }\end{array}$ & $\begin{array}{l}\text { TK, ETK, } \\
\text { and the first- } \\
\text { pass leak- } \\
\text { age profile } \\
(\text { FPLP) [300] }\end{array}$ & $\begin{array}{l}\text { Brain; } 18 \text { pa- } \\
\text { tients. }\end{array}$ & $\begin{array}{l}\text { (o) Characterizing human gliomas with three PK } \\
\text { models } \\
\text { (c) Highly correlated ETK- and FPLP-based esti- } \\
\text { mates of } K^{\text {trans }} \text { and } v_{\mathrm{p}} \text { validate the use of these } \\
\text { models for the evaluation of perfusion and per- } \\
\text { meability in tumors }\end{array}$ \\
\hline & & & continued on the next page ... \\
\hline
\end{tabular}


TABLE 2 - continued from the previous page

\begin{tabular}{|c|c|c|c|}
\hline Study & PK model & Tissue of interest & Objectives (o) and conclusions (c) \\
\hline $\begin{array}{l}\text { Haris et } \\
\text { al. }[231]\end{array}$ & TK & $\begin{array}{ll}\text { Brain; } & 103 \\
\text { patients. } & \end{array}$ & $\begin{array}{l}\text { (o) Assessing capabilities of various perfusion in- } \\
\text { dexes to separate infective from neoplastic } \\
\text { brain lesions } \\
\text { (c) } K^{\text {trans }} \text { and } v_{\mathrm{e}} \text { are useful indexes for discriminat- } \\
\text { ing between infective brain lesions and gliomas }\end{array}$ \\
\hline $\begin{array}{l}\text { Bergamino } \\
\text { et al. }[233]\end{array}$ & $\begin{array}{l}\text { ETK and } \\
\text { PM }\end{array}$ & $\begin{array}{l}\text { Brain; } 25 \text { pa- } \\
\text { tients. }\end{array}$ & $\begin{array}{l}\text { (o) PK analysis of the T1-weighted DCE-MRI } \\
\text { to investigate blood-brain-barrier permeability } \\
\text { associated with different brain tumors } \\
\text { (c) Different permeability measurements based on } \\
K^{\text {trans }} \text { for different tumor grades: the higher the } \\
\text { histological grades, the higher the permeability } \\
\text { values } \\
\text { (c) Significantly different ETK- and PM-related } \\
K^{\text {trans }} \text {-values for the high grade tumors }\end{array}$ \\
\hline $\begin{array}{ll}\text { Lee } & \text { et } \\
\text { al. }[272] & \end{array}$ & ETK & $\begin{array}{lr}\text { Head } & \text { and } \\
\text { neck; } & 21 \\
\text { patients. } & \end{array}$ & $\begin{array}{l}\text { (o) Investigating the radiation exposure effect on } \\
\text { the DCE-MRI parameters and correlating the } \\
\text { radiation dose and the degree of parotid gland } \\
\text { atrophy } \\
\text { (c) The correlation between the greater glandular } \\
\text { atrophy and a lower baseline } v_{\mathrm{e}} \text { and } v_{\mathrm{p}} \text {, as } \\
\text { well as a higher post-treatment increase in } v_{\mathrm{e}} \\
\text { showed sound potentialities of DCE-MRI for } \\
\text { predicting and assessing the radiation injury in } \\
\text { the parotid glands }\end{array}$ \\
\hline $\begin{array}{ll}\text { Lee } & \text { et } \\
\text { al. }[274]\end{array}$ & ETK & $\begin{array}{lr}\text { Head } & \text { and } \\
\text { neck; } & 63 \\
\text { patients. } & \end{array}$ & $\begin{array}{l}\text { (o) Examining DCE-MRI capabilities in differen- } \\
\text { tial diagnostics of various head and neck can- } \\
\text { cers } \\
\text { (c) Significantly different } K^{\text {trans }} \text {-values obtained } \\
\text { by the PK analysis between the undifferenti- } \\
\text { ated carcinomas (UDC) and squamous cell car- } \\
\text { cinoma (SCC), as well as between the UDC } \\
\text { and lymphoma } \\
\text { (c) The obtained } K^{\text {trans }- \text { values correlate with the }} \\
\text { vascular endothelial growth factor (VEGF) ex- } \\
\text { pression }\end{array}$ \\
\hline $\begin{array}{l}\text { Donaldson } \\
\text { et al. [291] }\end{array}$ & 2CXM & $\begin{array}{lr}\text { Head } & \text { and } \\
\text { neck; } & 8 \\
\text { patients. } & \end{array}$ & $\begin{array}{l}\text { (o) Analyzing DCE-MRI data and correlating the } \\
\text { obtained PK parameters with measurements of } \\
\text { hypoxia and VEGF expression in patients with } \\
\text { squamous cell carcinoma } \\
\text { (c) Negative correlation between the perfusion and } \\
\text { both the VEGF expression and hypoxia }\end{array}$ \\
\hline $\begin{array}{l}\text { Bisdas et } \\
\text { al. [48] }\end{array}$ & TK & $\begin{array}{l}\text { Brain; } 18 \text { pa- } \\
\text { tients. }\end{array}$ & $\begin{array}{l}\text { (o) Investigating the feasibility of the PK model- } \\
\text { ing to distinguish recurrent high-grade gliomas } \\
\text { from radiation injury } \\
\text { (c) Significantly higher } K^{\text {trans }} \text {-values in recurrent } \\
\text { gliomas in comparison to radiation-induced } \\
\text { necrosis sites } \\
\text { (c) } 100 \% \text { sensitivity and } 83 \% \text { specificity of detect- } \\
\text { ing recurrent gliomas on the basis of a cut- } \\
\text { off } K^{\text {trans }} \text {-value, compared to } 71 \% \text { sensitivity } \\
\text { and } 71 \% \text { specificity of the like diagnostics with } \\
\text { nonparametric AUC }\end{array}$ \\
\hline
\end{tabular}

continued on the next page ... 
TABLE 2 - continued from the previous page

\begin{tabular}{|c|c|c|c|}
\hline Study & PK model & Tissue of interest & Objectives (o) and conclusions (c) \\
\hline $\begin{array}{l}\text { Chih-Feng } \\
\text { et al. }[301]\end{array}$ & ETK & $\begin{array}{l}\text { Brain; } 10 \text { pa- } \\
\text { tients. }\end{array}$ & $\begin{array}{l}\text { (o) Correlating the } K^{\text {trans }} \text {-values and the ini- } \\
\text { tial area under the concentration time curve } \\
\text { (IAUC) for parametric and nonparametric PK } \\
\text { modeling, respectively, in clinical patients with } \\
\text { brain tumors } \\
\text { (c) High correlation coefficient }(0.913) \text { between } \\
\text { the IAUC and } K^{\text {trans }} \text { suggests the IAUC as an } \\
\text { alternative for evaluating physiological condi- } \\
\text { tion in DCE-MRI }\end{array}$ \\
\hline $\begin{array}{l}\text { Adluru et } \\
\text { al. }[261]\end{array}$ & TK & $\begin{array}{l}\text { Heart; } 10 \text { pa- } \\
\text { tients. }\end{array}$ & $\begin{array}{l}\text { (o) Assessing the accuracy of a PK-based registra- } \\
\text { tion of the myocardial DCE-MRI for quantify- } \\
\text { ing the myocardial blood flow (MBF) } \\
\text { (c) Improved estimation of the regional perfusion } \\
\text { flow indexes for } 77 \% \text { out of all the data sets }\end{array}$ \\
\hline $\begin{array}{ll}\text { Pärkkä } & \text { et } \\
\text { al. [212] } & \end{array}$ & LM & $\begin{array}{l}\text { Heart; } 18 \text { pa- } \\
\text { tients. }\end{array}$ & $\begin{array}{l}\text { (o) Assessing the myocardial perfusion reserve } \\
\text { (MPR) in healthy humans by using DCE-MRI } \\
\text { in comparison with the positron emission to- } \\
\text { mography (PET) } \\
\text { (c) Significant correlation between the MRI- and } \\
\text { PET-based MPR } \\
\text { (c) Myocardial perfusion can be quantified by PK } \\
\text { modeling of DCE-MRI }\end{array}$ \\
\hline $\begin{array}{l}\text { Fritz- } \\
\text { Hansen et } \\
\text { al. }[213]\end{array}$ & LM & $\begin{array}{l}\text { Heart; } 10 \text { pa- } \\
\text { tients. }\end{array}$ & $\begin{array}{l}\text { (o) Evaluating the MPR in humans using DCE- } \\
\text { MRI and }{ }^{13} \mathrm{~N} \text {-ammonia PET as a reference } \\
\text { (c) High correlation coefficient }(0.96) \text { between the } \\
\text { mean perfusion values at rest and hyperemia } \\
\text { derived from both DCE-MRI and PET data }\end{array}$ \\
\hline $\begin{array}{l}\text { Kerwin } \\
\text { al. }[277]\end{array}$ & ETK & $\begin{array}{l}\text { Heart; } 45 \\
\text { patients } \quad(20 \\
\text { with carotid } \\
\text { atheroscle- } \\
\text { rosis and } 25 \\
\text { after carotid } \\
\text { endarterec- } \\
\text { tomy). }\end{array}$ & $\begin{array}{l}\text { (o) Characterizing vasa vasorum in the carotid } \\
\text { artery in patients with carotid atherosclerosis } \\
\text { disease } \\
\text { (c) } K^{\text {trans }} \text {-values estimated for the adventitial } \\
\text { carotid region are significantly correlated with } \\
\text { serum inflammation markers, such as C- } \\
\text { reactive protein levels }(r=0.57 ; p=0.01) \\
\text { (c) The adventitial } K^{\text {trans }} \text {-value may be a risk } \\
\text { marker }\end{array}$ \\
\hline $\begin{array}{ll}\text { Kurita } & \text { et } \\
\text { al. }[278] & \end{array}$ & PM & $\begin{array}{l}\text { Heart, } 20 \text { pa- } \\
\text { tients. }\end{array}$ & $\begin{array}{l}\text { (o) Comparing the regional MPR with the CFR } \\
\text { found, respectively, by DCE-MRI-based esti- } \\
\text { mation of myocardial perfusion and with the } \\
\text { intracoronary Doppler flow wire } \\
\text { (c) Significant direct correlations between DCE- } \\
\text { MRI-based MPR assessments and Doppler- } \\
\text { based CFR assessments (the correlation coef- } \\
\text { ficients of } 0.87 \text { and } 0.86 \text { for culprit and non- } \\
\text { culprit arteries, respectively) }\end{array}$ \\
\hline $\begin{array}{l}\text { Ichihara et } \\
\text { al. [279] }\end{array}$ & PM & $\begin{array}{l}\text { Heart; } 10 \text { pa- } \\
\text { tients. }\end{array}$ & $\begin{array}{l}\text { (o) Quantifying the MBF estimated from DCE- } \\
\text { MRI and comparing with the MBF estimate } \\
\text { obtained from the coronary sinus blood flow } \\
\text { (c) The means of } 86 \pm 25 \text { and } 89 \pm 30 \mathrm{ml} / \mathrm{min} / 100 \\
\mathrm{~g} \text {, for the MBFs from DCE-MRI-related per- } \\
\text { fusion estimates and the coronary sinus blood } \\
\text { flow, respectively }\end{array}$ \\
\hline
\end{tabular}


TABLE 2 - continued from the previous page

\begin{tabular}{|c|c|c|c|}
\hline Study & PK model & Tissue of interest & Objectives (o) and conclusions (c) \\
\hline $\begin{array}{l}\text { Pack } \\
\text { and Di- } \\
\text { Bella. }[262]\end{array}$ & TK and PM & $\begin{array}{l}\text { Heart, } \\
\text { subjects. }\end{array}$ & $\begin{array}{l}\text { (o) Comparing different quantitative techniques } \\
\text { for regional myocardial perfusion quantifica- } \\
\text { tion } \\
\text { (c) No significant differences between the aggre- } \\
\text { gate } K^{\text {trans }} \text {-values for both the TK and the Pat- } \\
\text { lak plot analysis }\end{array}$ \\
\hline $\begin{array}{l}\text { Furman- } \\
\text { Haran } \\
\text { al. [51] }\end{array}$ & TK & $\begin{array}{ll}\text { Breast; } & 121 \\
\text { patients. } & \end{array}$ & $\begin{array}{l}\text { (o) Quantifying microvascular perfusion parame- } \\
\text { ters in various breast lesions and determining } \\
\text { whether the parameters vary between benign } \\
\text { and malignant lesions. } \\
\text { (c) High specificity }(96 \%) \text { and sensitivity }(93 \%) \text { of } \\
\text { the } K^{\text {trans }-v a l u e s ~ f o r ~ b r e a s t ~ c a n c e r ~ d e t e c t i o n ~} \\
\text { (c) Significant improvements of breast cancer di- } \\
\text { agnosis using the PK models }\end{array}$ \\
\hline $\begin{array}{l}\text { Padhani et } \\
\text { al. [58] }\end{array}$ & TK & $\begin{array}{ll}\text { Breast; } & 25 \\
\text { patients. } & \end{array}$ & $\begin{array}{l}\text { (o) Correlating early changes in the PK parame- } \\
\text { ters' distribution with treatment response as- } \\
\text { sessments } \\
\text { (c) Changes in vascular heterogeneity quantified } \\
\text { by } K^{\text {trans }} \text { at the end of the second cycle of sys- } \\
\text { temic chemotherapy can predict both clinical } \\
\text { and histopathological responses after three to } \\
\text { six cycles of the therapy }\end{array}$ \\
\hline $\begin{array}{l}\text { Vincensini } \\
\text { et al. [53] }\end{array}$ & TK & $\begin{array}{l}\text { Breast; } \\
\text { patients. }\end{array}$ & $\begin{array}{l}\text { (o) Studying the effectiveness of quantitative } \\
\text { DCE-MRI parameters to characterize malig- } \\
\text { nant breast lesions } \\
\text { (c) Excellent classification sensitivity and speci- } \\
\text { ficity of the } k_{\mathrm{ep}} \text {-parameter } \\
\text { (c) Monitoring the } k_{\mathrm{ep}} \text {-value may be used to dis- } \\
\text { criminate between lesions that respond well or } \\
\text { poorly to therapy at the early treatment stage }\end{array}$ \\
\hline $\begin{array}{l}\text { Radjenovic } \\
\text { et al. [302] }\end{array}$ & BM & $\begin{array}{l}\text { Breast; } \\
\text { patients. }\end{array}$ & $\begin{array}{l}\text { (o) Studying the effectiveness of quantitative } \\
\text { DCE-MRI parameters for monitoring neoadju- } \\
\text { vant chemotherapy (NAC) } \\
\text { (c) The parameters } K^{\text {trans }} \text { and } k_{\text {ep }} \text { exhibit the high- } \\
\text { est correlation with the high-grade breast carci- } \\
\text { nomas, whereas differ significantly in the low- } \\
\text { grade ones }\end{array}$ \\
\hline $\begin{array}{ll}\text { Ah-See } & \text { et } \\
\text { al. [54] } & \end{array}$ & TK & $\begin{array}{l}\text { Breast; } \\
\text { patients. }\end{array}$ & $\begin{array}{l}\text { (o) Investigating whether the PK modeling param- } \\
\text { eters for pre- and post-NAC could predict final } \\
\text { clinical and pathological response } \\
\text { (c) According to the ROC analysis, the parame- } \\
\text { ter } K^{\text {trans }} \text { is the best pathological non-response } \\
\text { predictor (the AUC of } 0.93 \text {; sensitivity of } 94 \% \text {, } \\
\text { and specificity of } 82 \% \text { ) } \\
\text { (c) Correctly identified } 94 \% \text { of non-responders } \\
\text { and } 73 \% \text { of responders, being comparable to } \\
\text { the change of the MRI-derived tumor size that } \\
\text { failed to predict the pathological response }\end{array}$ \\
\hline
\end{tabular}


TABLE 2 - continued from the previous page

\begin{tabular}{|c|c|c|c|}
\hline Study & PK model & Tissue of interest & Objectives (o) and conclusions (c) \\
\hline $\begin{array}{ll}\text { Schmid et } \\
\text { al. [296] }\end{array}$ & AATH & $\begin{array}{ll}\text { Breast; } & 12 \\
\text { patients. } & \end{array}$ & $\begin{array}{l}\text { (o) Evaluating the accuracy of a Bayesian P- } \\
\text { spline-based semi-parametric quantification of } \\
\text { concentration curves obtained from DCE-MRI } \\
\text { (c) The P-spline model demonstrates a superior fit } \\
\text { to the observed concentration curves and cap- } \\
\text { tures accurately the time series up-slope }\end{array}$ \\
\hline $\begin{array}{l}\text { Schabel et } \\
\text { al. [55] }\end{array}$ & ETK & $\begin{array}{ll}\text { Breast; } & 74 \\
\text { patients. } & \end{array}$ & $\begin{array}{l}\text { (c) Prospective investigation of whether the PK } \\
\text { parameters could provide diagnostically useful } \\
\text { information to distinguish between benign and } \\
\text { malignant breast lesions } \\
\text { (c) The AUC of } 0.915 \text { and the excellent sensitivity } \\
(91 \%) \text { and specificity ( } 85 \%) \text { of the } K^{\text {trans }} \text { and } \\
k_{\text {ep based classification with respect to mam- }} \text { mography (the sensitivity of } 88 \% \text { and speci- } \\
\text { ficity of } 68 \%)\end{array}$ \\
\hline $\begin{array}{l}\text { El Khouli et } \\
\text { al. }[238]\end{array}$ & ETK & $\begin{array}{ll}\text { Breast; } & 95 \\
\text { patients. } & \end{array}$ & $\begin{array}{l}\text { (c) Comparing the ETK performance with the con- } \\
\text { ventional morphology plus kinetic curve type } \\
\text { analysis } \\
\text { (c) The kinetic curve type assessment or PK mod- } \\
\text { eling improve similarly the diagnostic perfor- } \\
\text { mance }\end{array}$ \\
\hline $\begin{array}{ll}\text { Fusco } & \text { et } \\
\text { al. }[220] & \end{array}$ & $\begin{array}{l}\text { ETK, BM, } \\
\text { and AATH }\end{array}$ & $\begin{array}{l}\text { Breast; } 4 \text { pa- } \\
\text { tients. }\end{array}$ & $\begin{array}{l}\text { (o) Analyzing the performance of different DP and } \\
\text { compartment PK models } \\
\text { (o) Comparing the TK and BM on the real breast } \\
\text { DCE-MRI data } \\
\text { (c) The AATH achieved better fit than the TK and } \\
\text { BM with respect to three goodness-of-fit met- } \\
\text { rics: the residual sum of squares, the Bayesian } \\
\text { information criterion, and the Akaike informa- } \\
\text { tion criterion (AIC) }\end{array}$ \\
\hline $\begin{array}{ll}\text { Naish } & \text { et } \\
\text { al. }[235] & \end{array}$ & $\begin{array}{l}\text { TK, ETK, } \\
\text { and AATH }\end{array}$ & $\begin{array}{l}\text { Lung; } 6 \text { pa- } \\
\text { tients. }\end{array}$ & $\begin{array}{l}\text { (o) Comparing the performance of three PK mod- } \\
\text { els and a model-free analysis in distinguishing } \\
\text { malignant from nonmalignant lung tissues } \\
\text { (c) The AATH gives the best description of the } \\
\text { lung tumor data with respect to the AIC }\end{array}$ \\
\hline $\begin{array}{l}\text { Michoux et } \\
\text { al. [266] }\end{array}$ & $\begin{array}{l}\text { TK, ETK, } \\
\text { and AATH }\end{array}$ & $\begin{array}{l}\text { Liver; } 12 \\
\text { Wistar rats. }\end{array}$ & $\begin{array}{l}\text { (o) Assessing which PK parameters reflect de- } \\
\text { creased transvascular and interstitial transport } \\
\text { if low- and high-molecular-weight CA are used } \\
\text { in rat hepatocellular carcinomas } \\
\text { (c) Both the kinetic parameters } v_{\mathrm{e}} \text { and } E \text { have } \\
\text { better potential to show differences of the } \\
\text { transvascular and interstitial transport than the } \\
\text { parameter } K^{\text {trans }} \text {, and therefore may be of in- } \\
\text { terest for observing the effect of antiangiogenic } \\
\text { agents }\end{array}$ \\
\hline $\begin{array}{l}\text { De Sen- } \\
\text { neville }[280]\end{array}$ & PM & $\begin{array}{l}\text { Kidney; } \\
\text { patients. }\end{array}$ & $\begin{array}{l}\text { (o) Evaluating the effect of DCE-MRI motion cor- } \\
\text { rection on the estimated glomerular filtration } \\
\text { rate (GFR) } \\
\text { (c) Significant uncertainty reduction on the com- } \\
\text { puted GFR for native, but not the transplanted } \\
\text { kidneys }\end{array}$ \\
\hline & & & continued on the next page ... \\
\hline
\end{tabular}


TABLE 2 - continued from the previous page

\begin{tabular}{|c|c|c|c|}
\hline Study & PK model & Tissue of interest & Objectives (o) and conclusions (c) \\
\hline $\begin{array}{l}\text { Sourbron et } \\
\text { al. [188] }\end{array}$ & $\begin{array}{l}\text { Sourbron } \\
\text { model [188] }\end{array}$ & $\begin{array}{l}\text { Kidney; } 15 \\
\text { volunteers. }\end{array}$ & $\begin{array}{l}\text { (o) Measuring the GFR with the gadobenate } \\
\text { dimeglumine (Gd-BOPTA) CA using a Sour- } \\
\text { bron mode-the 2-compartment PK model with } \\
\text { bolus dispersion and tubular outflow } \\
\text { (c) About } 40 \% \text {-underestimation of the GFR (ar- } \\
\text { guably, due to low albumin binding of the Gd- } \\
\text { BOPTA, which leads to reduced relaxivity in } \\
\text { the tubular system and incomplete glomerular } \\
\text { filtration) }\end{array}$ \\
\hline $\begin{array}{l}\text { Anderlik et } \\
\text { al. [303] }\end{array}$ & $\begin{array}{l}\text { Sourbron } \\
\text { model [188] }\end{array}$ & $\begin{array}{l}\text { Kidney; } 11 \\
\text { volunteers. }\end{array}$ & $\begin{array}{l}\text { (o) Quantitative assessment of kidney function us- } \\
\text { ing DCE-MRI } \\
\text { (c) Promising estimates of the GFR }\end{array}$ \\
\hline $\begin{array}{ll}\text { Hahn } & \text { et } \\
\text { al. }[258] & \end{array}$ & ETK & $\begin{array}{l}\text { Kidney; } \\
\text { patients. }\end{array}$ & $\begin{array}{l}\text { (o) Investigating DCE-MRI as a pharmacody- } \\
\text { namic biomarker of a Sorafenib antiangiogenic } \\
\text { agent with renal cancer activity } \\
\text { (c) The derived } K^{\text {trans }} \text { and IAUC } 90 \text { are pharmaco- } \\
\text { dynamic biomarkers of Sorafenib in metastatic } \\
\text { renal cancer } \\
\text { (c) The high baseline } K^{\text {trans }} \text { and } v_{\mathrm{p}} \text { may act as a } \\
\text { prognostic or predictive biomarker, which is } \\
\text { beneficial to Sorafenib }\end{array}$ \\
\hline $\begin{array}{ll}\text { Buckley } & \text { et } \\
\text { al. }[298] & \end{array}$ & AATH & $\begin{array}{ll}\text { Prostate; } & 22 \\
\text { patients. } & \end{array}$ & $\begin{array}{l}\text { (o) Prospective evaluation of vascular characteris- } \\
\text { tics of prostate cancer using DCE-MRI and the } \\
\text { kinetic DP model } \\
\text { (c) Increased plasma flow and EES-space fraction } \\
\text { within prostate cancer tissue, when compared } \\
\text { to its peripheral zone (PZ) } \\
\text { (c) Similar permeability and plasma volume frac- } \\
\text { tion estimates in both regions of interest }\end{array}$ \\
\hline $\begin{array}{ll}\text { Kelm } & \text { et } \\
\text { al. }[180] & \end{array}$ & BM & $\begin{array}{ll}\text { Prostate; } & 37 \\
\text { patients. } & \end{array}$ & $\begin{array}{l}\text { (o) Assessing the efficacy of spatial prior knowl- } \\
\text { edge for estimating PK parameter maps from } \\
\text { DCE-MRI } \\
\text { (c) The spatial prior knowledge reduces both } \\
\text { the bias and variance of the estimated } k_{\text {ep- }} \\
\text { parameter maps }\end{array}$ \\
\hline $\begin{array}{l}\text { Kiessling et } \\
\text { al. [226] }\end{array}$ & BM & $\begin{array}{ll}\text { Prostate; } & 27 \\
\text { patients. } & \end{array}$ & $\begin{array}{l}\text { (o) Evaluating the accuracy of discrimination of } \\
\text { prostate cancers from the peripheral gland with } \\
\text { descriptive and PK parameters } \\
\text { (c) Potentialities of the PK parameters ( } A_{\text {Brix }} \text { and } \\
k_{\text {ep }} \text { ) in discriminating the prostate cancers from } \\
\text { the peripheral prostate tissue } \\
\text { (c) The nonparametric indexes of the early signal } \\
\text { enhancement after the CA injection, such as the } \\
\text { AUC and up-slopes at } 26,39,52 \text {, and } 65 \text { sec, } \\
\text { have higher sensitivity and specificity, although } \\
\text { at the start of the signal intensity increase, } T_{\mathrm{o}} \text {, } \\
\text { these indexes do not discriminate the carcino- } \\
\text { mas from the glandular tissue }\end{array}$ \\
\hline
\end{tabular}


TABLE 2 - continued from the previous page

\begin{tabular}{|c|c|c|c|}
\hline Study & PK model & Tissue of interest & Objectives (o) and conclusions (c) \\
\hline $\begin{array}{l}\text { van Dorsten } \\
\text { et al. [304] }\end{array}$ & LM & $\begin{array}{ll}\text { Prostate; } & 50 \\
\text { patients. } & \end{array}$ & $\begin{array}{l}\text { (o) Differentiating prostate carcinoma from } \\
\text { healthy PZ and CZ using DCE-MRI and } \\
\text { two-dimensional } 1 \mathrm{H} \text { MR MRS imaging. } \\
\text { (c) Excellent potentialities of the combined spatio- } \\
\text { vascular information from DCE-MRI and } \\
\text { metabolic information from MRS for improv- } \\
\text { ing the localization and characterization of the } \\
\text { prostate cancer in a clinical setting. }\end{array}$ \\
\hline $\begin{array}{ll}\text { Ocak } & \text { et } \\
\text { al. }[241] & \end{array}$ & TK & $\begin{array}{ll}\text { Prostate; } & 50 \\
\text { patients. } & \end{array}$ & $\begin{array}{l}\text { (o) Determining PK parameters, being useful for } \\
\text { prostate cancer diagnostics } \\
\text { (c) Four PK parameters: ( } K^{\text {trans }}, k_{\mathrm{ep}}, v_{\mathrm{e}} \text {, and the } \\
\text { AUC of the gadolinium concentration curve } \\
\text { were determined and compared for cancer, in- } \\
\text { flammation, and healthy peripheral } \\
\text { (c) Improved prostate cancer specificity of the } \\
\text { conventional } T_{2} \text {-weighted MRI for the } K^{\text {trans }} \\
\text { and } k_{\text {ep }} \text { parameters }\end{array}$ \\
\hline $\begin{array}{l}\text { Kershaw et } \\
\text { al. [297] }\end{array}$ & AATH & $\begin{array}{ll}\text { Prostate; } & 13 \\
\text { patients. } & \end{array}$ & $\begin{array}{l}\text { (o) Evaluating microvascular and relaxation pa- } \\
\text { rameters of prostate and nearby muscle in pa- } \\
\text { tients with benign prostatic hyperplasia and ex- } \\
\text { amining measurement reproducibility } \\
\text { (c) Significantly different bootstrap analysis of the } \\
\text { PK parameters }\left(F_{\mathrm{p}}, T_{\mathrm{c}}, K^{\text {trans }} \text {, and } P S\right) \text { in the } \\
\text { prostate's } \mathrm{CZ} \text {, comparing with the PZ }\end{array}$ \\
\hline $\begin{array}{ll}\text { Jackson } & \text { et } \\
\text { al. }[242] & \end{array}$ & TK & $\begin{array}{ll}\text { Prostate; } & 19 \\
\text { patients. } & \end{array}$ & $\begin{array}{l}\text { (o) Assessing the efficacy of spatial prior knowl- } \\
\text { edge for estimating PK parameter maps } \\
\text { (c) Pixel-wise parametric maps for the } K^{\text {trans }}, v_{\mathrm{e}} \text {, } \\
\text { and } k_{\text {ep parameters reveal significant differ- }} \text { ences between the benign and malignant tu- } \\
\text { mors in the PZ, while the ROC analysis shows } \\
\text { that the PK parameters are only "fair discrimi- } \\
\text { nators between the cancer and benign gland } \\
\text { (c) The radiologist interpretation shows similar } \\
\text { specificity ( } 85 \% \text { vs } 81 \% \text {; }=0.593 \text { ) and higher } \\
\text { sensitivity ( } 50 \% \text { vs } 21 \% \text {; }=0.006 \text { ) of DCE- } \\
\text { MRI with respect to } T_{2} \text {-weighted MR images } \\
\text { for cancer localization } \\
\text { (c) Guiding the radiotherapy beam with DCE-MRI } \\
\text { and PK modeling can improve the outcome of } \\
\text { radiotherapy }\end{array}$ \\
\hline $\begin{array}{l}\text { Langer et } \\
\text { al. }[244,247]\end{array}$ & TK & $\begin{array}{l}\text { Prostate; } 25[244] \\
\text { and } 24[247] \\
\text { patients. }\end{array}$ & $\begin{array}{l}\text { (o) Investigating relationships between the multi- } \\
\text { modal MRI (DTI, } T_{2} \text {-weighted, DCE-MRI) } \\
\text { measurements and the underlying composition } \\
\text { of normal and malignant prostate tissues } \\
\text { (c) Significant differences between the cancer and } \\
\text { normal PZ tissues for the MRI-derived appar- } \\
\text { ent diffusion coefficient (ADC), } T_{2}, K^{\text {trans }} \text {, and } \\
v_{\mathrm{e}} \text { ) parameters and the percentage areas of all } \\
\text { tissue components except stroma }\end{array}$ \\
\hline
\end{tabular}


TABLE 2 - continued from the previous page

\begin{tabular}{|c|c|c|c|}
\hline Study & PK model & Tissue of interest & Objectives (o) and conclusions (c) \\
\hline $\begin{array}{ll}\text { Vos } & \text { et } \\
\text { al. }[246] & \end{array}$ & TK & $\begin{array}{ll}\text { Prostate; } 34 \\
\text { patients. }\end{array}$ & $\begin{array}{l}\text { (o) Developing a multi-modality }\left(T_{2} \text {-weighted }\right. \\
\text { MRI and DCE-MRI) CAD system with an } \\
\text { SVM classifier to diagnose prostate cancer in } \\
\text { the PZ } \\
\text { (c) Using the } T_{2} \text {-weighted sequence significantly } \\
\text { improves the diagnosing performance obtained } \\
\text { with only DCE-MRI PK parameters, namely, } \\
\text { the ROC's AUC increases from } 0.83(0.75- \\
0.92) \text { to } 0.89(0.81-0.95)\end{array}$ \\
\hline $\begin{array}{ll}\text { Vos } & \text { et } \\
\text { al. }[251] & \end{array}$ & TK & $\begin{array}{l}\text { Prostate; } 177 \\
\text { patients. }\end{array}$ & $\begin{array}{l}\text { (o) Investigating the feasibility of an automated } \\
\text { CAD system with a linear discriminant analy- } \\
\text { sis (LDA) classifier to detect prostate's cancer- } \\
\text { suspicious regions } \\
\text { (c) The CAD system detects } 74 \% \text { of all tumors at } \\
\text { a false positive (FP) level of } 5 \\
\text { (c) The system's sensitivity of } 88 \% \text { for the high- } \\
\text { grade tumors at the FP level of } 5\end{array}$ \\
\hline $\begin{array}{ll}\text { Vos } & \text { et } \\
\text { al. [252] }\end{array}$ & TK & $\begin{array}{l}\text { Prostate; } 45 \\
\text { patients. }\end{array}$ & 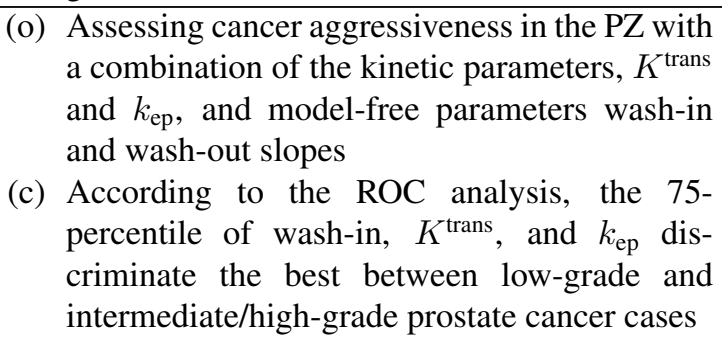 \\
\hline $\begin{array}{ll}\text { Li } & \text { et } \\
\text { al. [253] }\end{array}$ & TK & $\begin{array}{l}\text { Prostate; } 33 \\
\text { patients. }\end{array}$ & $\begin{array}{l}\text { (o) Investigating and comparing the diagnostic } \\
\text { performance of DTI, DCE-MRI, or their com- } \\
\text { bination in detecting the prostate's cancerous } \\
\text { areas in the PZ } \\
\text { (c) Good agreement between the PK parameters } \\
\text { estimated from the DCE-CT and DCE-MRI } \\
\text { (only } v_{\mathrm{e}} \text {-values differ significantly when esti- } \\
\text { mated from the MRI and CT) }\end{array}$ \\
\hline $\begin{array}{l}\text { Chikui et } \\
\text { al. [60] }\end{array}$ & TK & $\begin{array}{l}\text { Oral cancer, } \\
29 \text { patients. }\end{array}$ & $\begin{array}{l}\text { (o) Evaluating usefulness of a PK analysis to mon- } \\
\text { itor the oral cancer response to chemoradio- } \\
\text { therapy (CRT) } \\
\text { (c) Statistical analysis reveals that the } v_{\mathrm{e}} \text {-increase } \\
\text { strongly suggests a good tumor response to the } \\
\text { CRT } \\
\text { (c) Significantly larger } K^{\text {trans }} \text {-changes for respon- } \\
\text { ders, than for non-responders }\end{array}$ \\
\hline $\begin{array}{l}\text { De Lussanet } \\
\text { et al. [57] }\end{array}$ & ETK & $\begin{array}{l}\text { Rectal cancer; } \\
17 \text { patients. }\end{array}$ & $\begin{array}{l}\text { (o) Evaluating radiation therapy-related microvas- } \\
\text { cular changes in locally advanced rectal cancer } \\
\text { using DCE-MRI and histology } \\
\text { (c) Lower intratumoral heterogeneity in } K^{\text {trans }} \text { and } \\
v_{\mathrm{p}} \text { for primary rectal cancer patients receiving } \\
\text { the radiation therapy than for those without the } \\
\text { radiation therapy }\end{array}$ \\
\hline
\end{tabular}

continued on the next page ... 
TABLE 2 - continued from the previous page

\begin{tabular}{|c|c|c|c|}
\hline Study & PK model & Tissue of interest & Objectives (o) and conclusions (c) \\
\hline $\begin{array}{l}\text { deLussanet } \\
\text { et al. [264] }\end{array}$ & TK & $\begin{array}{l}\text { Thigh; } 15 \\
\text { male rabbits. }\end{array}$ & $\begin{array}{l}\text { (o) Assessing the use of the MRI (DCE-MRI and } \\
\text { MRA) to evaluate the muscle perfusion recov- } \\
\text { ery and the growth of collateral arteries in a } \\
\text { rabbit femoral artery ligation model } \\
\text { (c) The combined DCE-MRI and MRA data allow } \\
\text { for noninvasive serial monitoring of changes } \\
\text { in the muscle blood flow and growth of sub- } \\
\text { millimeter sized collateral arteries }\end{array}$ \\
\hline $\begin{array}{ll}\text { Akisik } & \text { et } \\
\text { al. [59] } & \end{array}$ & TK & $\begin{array}{l}\text { Pancreas; } 11 \\
\text { patients. }\end{array}$ & $\begin{array}{l}\text { (o) Evaluating capabilities of the PK modeling of } \\
\text { DCE-MRI to predict a pancreatic cancer re- } \\
\text { sponse to combined chemotherapy and antian- } \\
\text { giogenic therapy } \\
\text { (c) Significant reduction of all perfusion parame- } \\
\text { ters, such as } K^{\text {trans }}, v_{\mathrm{e}} \text {, the peak concentration, } \\
\text { up-slope, and AUC at } 60 \mathrm{sec} \text {, after the com- } \\
\text { bined therapy } \\
\text { (c) The pre-treatment } K^{\text {trans }} \text {-measurement in pan- } \\
\text { creatic tumors can predict response to antian- } \\
\text { giogenic therapy }\end{array}$ \\
\hline $\begin{array}{ll}\text { Zahra } & \text { et } \\
\text { al. }[268] & \end{array}$ & TK & $\begin{array}{lr}\text { Cervix; } & 13 \\
\text { patients (each } \\
\text { with three } \\
\text { scans). }\end{array}$ & $\begin{array}{l}\text { (o) Evaluate DCE-MRI-based prediction of the re- } \\
\text { sponse to cervix cancer radiotherapy } \\
\text { (c) In spite of statistically significant correlation } \\
\text { between the percentage tumor regression and } \\
\text { both nonparametric indexes (peak time, slope, } \\
\text { maximum slope, and contrast enhancement ra- } \\
\text { tio) and PK parameters ( } K^{\text {trans }}(\mathrm{p}=0.043) \text { and } \\
k_{\mathrm{ep}}(\mathrm{p}=0.022) \text { ) for the pre-treatment DCE- } \\
\text { MRI, the same characteristics for the second } \\
\text { and third scans show no correlation }\end{array}$ \\
\hline $\begin{array}{l}\text { Donaldson } \\
\text { et al. [194] }\end{array}$ & $\begin{array}{l}\text { 2CXM and } \\
\text { TK }\end{array}$ & $\begin{array}{l}\text { Cervix; } \\
\text { patients. }\end{array}$ & $\begin{array}{l}\text { (o) Comparisons of the 2CXM and TK on report- } \\
\text { ing microvascular parameters in patients with } \\
\text { cervical cancer } \\
\text { (c) The } 2 \mathrm{CXM} \text { model demonstrated better fit to the } \\
\text { data for all patients } \\
\text { (c) Inaccurate PK parameter estimates for the TK } \\
\text { due to its inherent assumption of the negligible } \\
\text { plasma MTT }\end{array}$ \\
\hline $\begin{array}{l}\text { Andersen et } \\
\text { al. [216] }\end{array}$ & $\mathrm{BM}$ and $\mathrm{TK}$ & $\begin{array}{l}\text { Cervix; } \\
\text { patients. }\end{array}$ & $\begin{array}{l}\text { (o) Assessing the prognostic value of the PK pa- } \\
\text { rameters derived from pre-chemoradiotherapy } \\
\text { DCE-MRI of cervical cancer patients } \\
\text { (c) According to the prognostic significance of } \\
\text { the PK parameters (FIGO stage and tumor } \\
\text { volume), being assessed with the multivari- } \\
\text { ate analysis, the estimated imaging parame- } \\
\text { ters prior to chemoradiotherapy may be used } \\
\text { to identify patients at risk of treatment failure }\end{array}$ \\
\hline
\end{tabular}


TABLE 2 - continued from the previous page

\begin{tabular}{|c|c|c|c|}
\hline Study & PK model & Tissue of interest & Objectives (o) and conclusions (c) \\
\hline $\begin{array}{ll}\text { Ma } & \text { et } \\
\text { al. }[182] & \end{array}$ & $\mathrm{BM}$ & $\begin{array}{ll}\text { Bone } & \text { per- } \\
\text { fusion; } & 165 \\
\text { subjects. } & \end{array}$ & $\begin{array}{l}\text { (o) Investigating PK modeling of bone perfusion } \\
\text { in subjects with varying bone mineral density } \\
\text { (c) Notable reduction of the PK parameter } A^{\text {Brix }} \text { in } \\
\text { osteoporotic subjects compared to the normal } \\
\text { ones } \\
\text { (c) Less pronounced reductions in the permeabil- } \\
\text { ity constant, } A^{\text {Brix }} k_{\mathrm{ep}} \text {, and the elimination one, } \\
k_{\mathrm{el}}\end{array}$ \\
\hline
\end{tabular}

\section{E. SUMMARY AND DISCUSSION}

In this chapter, an overview of the explored DCE-MRI analysis and modeling techniques as well as their applications in a wide range of clinical studies in the last two decades has been presented. Promising theoretical findings and experimental results for the reviwed models and techniques in a variety of clinical applications suggest that DCE-MRI is a clinically relevant imaging modality. For example, the classical mammography has a significantly lower sensitivity (33\%-59\%) in early detection of breast cancer than DCE-MRI analysis (71\%-96\%) [51-55]. Among all the MRI modalities, DCE-MRI offers also the highest diagnostic accuracy of small (less than $1 \mathrm{~cm}$ ) breast lesions [86, 237,305] and the most accurate localization and staging of prostate cancer $[75,77,130,140,226,241,251,252]$. The DCE-MRI allows for differentiating very accurately between various brain tumors, such as glioma, meningioma, acoustic neuroma, or metastases $[45,47,48]$ and is a promising noninvasive tool for detecting acute renal rejection at its earliest stage [3, 124-129], even one day after transplantation [128].

As discussed in this chapter, both nonparametric and parametric approaches for DCE-MRI analysis possess the ability to quantify tissue perfusion. The main advantage of these analysis methods is that they reduce the original DCE-MRI data dimensionality to a small set of parameters that describe the tissue perfusion. The straightforward nonparametric techniques characterize shapes and structures of signal enhancement curves by computing descriptive indexes (e.g., the maximum enhancement, time-to-peak, up-slope, etc.) directly from signals of the curves. The advantages of these techniques are two-fold: $(i)$ no 
need to convert the recorded MR signals into CA concentrations and (ii) the complete analysis of the whole enhancement curve cycle by computing perfusion-related indexes from both wash-in and wash-out phases. However, the relation of the indexes to the underlying physiology is often unclear, although some correlation with quantitative or at least qualitative physiological measurements can be established. For example, an increased wash-in slope, AUC, peak enhancement, and decreased time-to-peak indicate likely an improved response to therapy or increased vascular density and/or vascular permeability. Nevertheless, the indexes can be reproducible at different sites only if an identical data acquisition protocol is used [306].

The parametric techniques fit one of the well-known mathematical PK models to the concentration curves in order to estimate physiologically-meaningful parameters, e.g., the EES volume and capillary permeability of a tissue of interest. The parametric PK modeling has the following advantages: $(i)$ estimation of a set of kinetic parameters that have physiological interpretations; (ii) they provide a way for better understanding of the interaction between drugs (i.e., contrast agent) and the human tissue, and (iii) the lesser sensitivity of parameter estimates to noise, image settings and data acquisition protocols [83]. However, both the data acquisition and analysis become more complicated in comparison with the nonparametric techniques. The complexity arises from the required conversion of MR signal intensities into CA concentrations involving the measurements of tissue relaxation time $\left(T_{1}\right)$, native relaxation time $\left(T_{10}\right)$, and the tissue AIF. The PK models are typically refined to more closely reflect physiological processes by including additional parameters, e.g., water exchange and CA diffusion. However, the refinement mostly complicates DCE-MRI analysis, and its usefulness requires additional investigation [197, 290, 307-309].

One of the challenges in gadolinium-based DCE-MRI analysis is the risk of nephrogenic systemic fibrosis, which is increased in patients with renal dysfunction [310]. Also, the choice of an appropriate CA dose for the quantitative DCE-MRI analysis is a challenging problem: the high dose increases the signal enhancement in the tissue, but may cause overestimation of the PK parameters due to saturation or nonlinearity of the MRI signal [188] or their underestimation due to transendothelial water exchange effects [311]. 
Investigations of alternative CA types, such as superparamagnetic iron oxide (SPIO) and ultra SPIO (USPIO) particles remain an open research area.

Comparison of different published results is still a challenging problem, in part, due to the lack of standardized DCE-MRI acquisition protocols; difficulties of achieving adequate spatial and temporal DCE-MRI resolutions simultaneously; various analytical post-processing, which sometimes may not describe adequately the relevant physiology, and different types of diseases, number of subjects, and treatment scenarios.

Recent trends to increase the reliability and accuracy of the DCE-MRI-derived perfusion parameters include the searches for $(i)$ objective criteria for choosing a suitable PK model among the existing ones to fit a given application; (ii) a standard consensus for a DCE-MRI data acquisition protocol in each specific application; (iii) better data normalization techniques that account for different physiological and scanning factors, e.g., patient weight/size and scanner type/acquisition parameters, respectively; (iv) since the analysis techniques describe a direct relation between the MRI signal and the underlying model, a new trend is to apply motion correction techniques before analyzing DCE-MRI data [128] to remove noise and motion effects in order to assure that the change in the signal is related to the CA transit in the tissue; $(v)$ since nonparametric approaches are simple and fast, a new trend is to develop complete noninvasive image-based diagnostic systems for early diagnosis of different diseases, e.g., lung and prostate cancer; (vi) fusion of parametric and nonparametric approaches is one more new trend towards more robust diagnostic decisions in each application [48, 129, 139, 229, 239, 249, 254, 263, 268, 301]. The high correlation between the estimated physiological (parametric) and nonparametric indexes [129, 239, 254,301] suggests the nonparametric DCE-MRI analysis can help in avoiding the complexity and limitations of the parametric methods for evaluation of physiological conditions; and (vii) distributed-parameter models are more realistic and makes fewer assumptions about microcirculation, therefor they are the main focus of research in recent years and their usefulness requires additional investigation. 


\section{CHAPTER III \\ EARLY DETECTION OF ACUTE RENAL TRANSPLANT REJECTION}

In this chapter a novel framework for the classification of acute rejection versus non-rejection status of renal transplants from 2D dynamic contrast-enhanced magnetic resonance imaging (DCE-MRI) is proposed. The proposed framework consists of four steps. In the first step, kidney objects are segmented from adjacent structures with a level setbased deformable model guided by a novel stochastic speed function. The latter accounts for a fourth-order Markov-Gibbs random field (MGRF) model of the kidney/background shape and appearance. Second, a Laplace-based nonrigid registration approach is employed to account for local kidney deformations caused by physiological effects. Namely, the target kidney object is deformed over closed, equi-spaced contours (i.e., iso-contours) to closely match the reference object. In the third step, the renal cortex is segmented as it is the functional kidney unit that is primarily affected by by the perfusion deficits that underlie the pathophysiology of acute rejection. To characterize rejection, perfusion is estimated from contrast agent kinetics using empirical indexes, namely, the transient phase indexes (peak signal intensity, time-to-peak, and initial up-slope), and a steady-phase index defined as the average signal change during the slowly varying tissue phase of agent transit. Finally, a $k_{n}$-nearest neighbor classifier is used to distinguish between acute rejection and non-rejection status. Performance of the proposed framework was evaluated using the receiver operating characteristics (ROC). Experimental results in 50 subjects, using a combinatoric $k_{n}$-classifier, correctly classified $92 \%$ of training subjects, $100 \%$ of the test subjects, and yielded an area under the ROC curve that approached the ideal value. The proposed framework thus holds promise as a reliable noninvasive diagnostic tool. 


\section{A. KIDNEY: ANATOMY, FUNCTION, AND RENAL TRANSPLANT DISEASES}

The kidneys are bean-shaped organs, each approximately has the size of the fist and a length of about four or five inches. They are vital body organs as they filter the blood before flowing to the rest of the bodys tissues. As the kidneys filter blood, the waste and extra water become urine that flows to the bladder and through the urethra to the outside environment [312]. In this section, a brief description of the kidney anatomy and function, as well as the diseases that affect the transplanted kidneys are described.

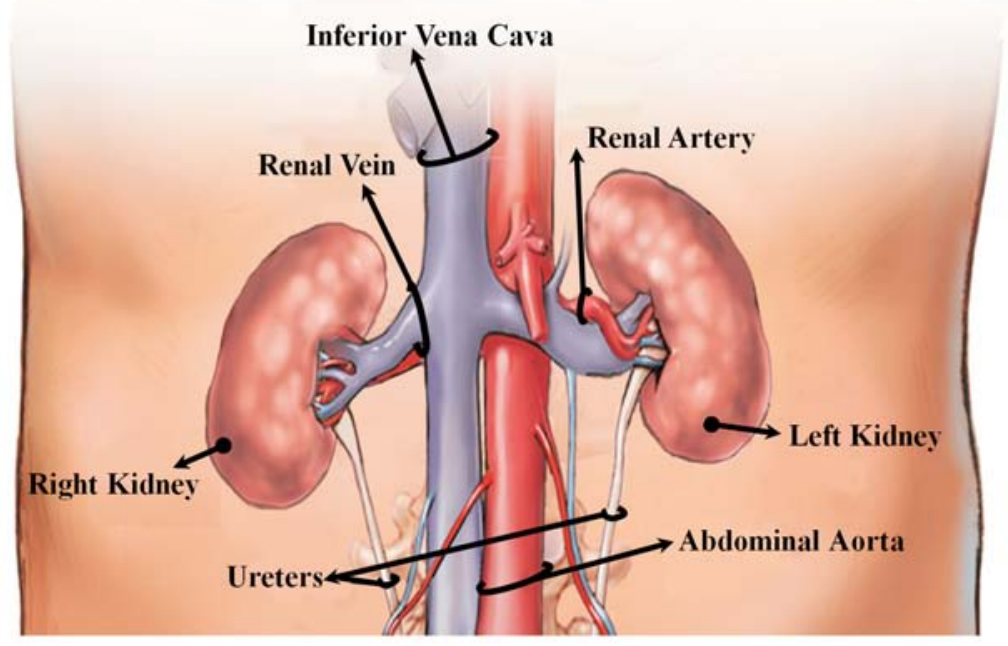

FIGURE 31: The abdominal area of the human body showing the kidney location [15].

Kidneys are bean-shaped organs, located at the back of the abdominal cavity, one on each side of the spinal column, just below the rib cage [312] as shown in Figure 31. Every day, they process about 200 quarts of blood to make the two quarts of waste products and extra water which becomes urine [313]. It is the urine production that keeps the blood clean and chemically balanced, making the kidneys vital organs for the body. A schematic illustration of a cross-section of the right kidney showing its internal structures is shown in Figure 32. The main three regions of the kidney are the pelvis, cortex, and medulla. The pelvis region is only an extension of the ureter into the kidney, while the cortex (outer portion) and the medulla (inner portion) are the main two structural regions. The cortex and medulla consist of nearly 1 million functional units called nephrons, which are 45 to 
$65 \mathrm{~mm}$ in length and $0.05 \mathrm{~mm}$ in width [313]. Nephrons in the cortex and the medulla process the blood that enters the kidney in several steps to form the urine. Once the urine is formed, it escapes into the pelvis to be transported via ureter tubes to the urinary bladder and through the urethra to the outside environment [312].

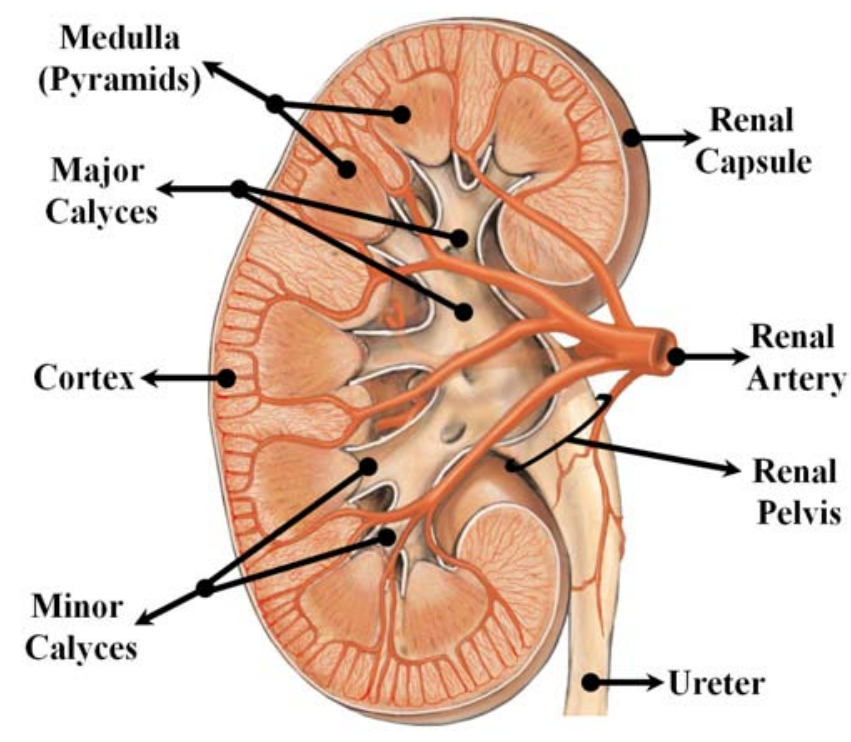

FIGURE 32: A schematic illustration of a corronal cross-section of the human right kidney showing its internal structures [15].

The actual process of creating the urine from the blood takes place in the nephrons. Each nephron consists of a glomerulus, its tubule, and its blood supply as shown in Figure 33. The tubule is divided into four parts: Bowman capsule, proximal tubule, loop of Henle, and distal tubule (see Figure 33). The blood meets the glomerulus structure and the urine starts to formulate through three consecutive processes, namely filtration by the glomerulus, reabsorption, and secretion by the tubular cells (see Figure 34). By means of these processes, the important products, such as the amino acids and water in the body are conserved, while the metabolic wastes (urea, uric acid, creatinine, ammonia) are excreted out of the body. The first process of urine formation is the filtration, which occurs in the glomerulus. The differences in the blood pressure and the protein osmotic (oncotic) pressure allows the glomerulus to act as an ultra-filter that allows only small particles to enter the fluid that goes into the Bowman's capsule. Thus, the fluid that enters the Bowman's 


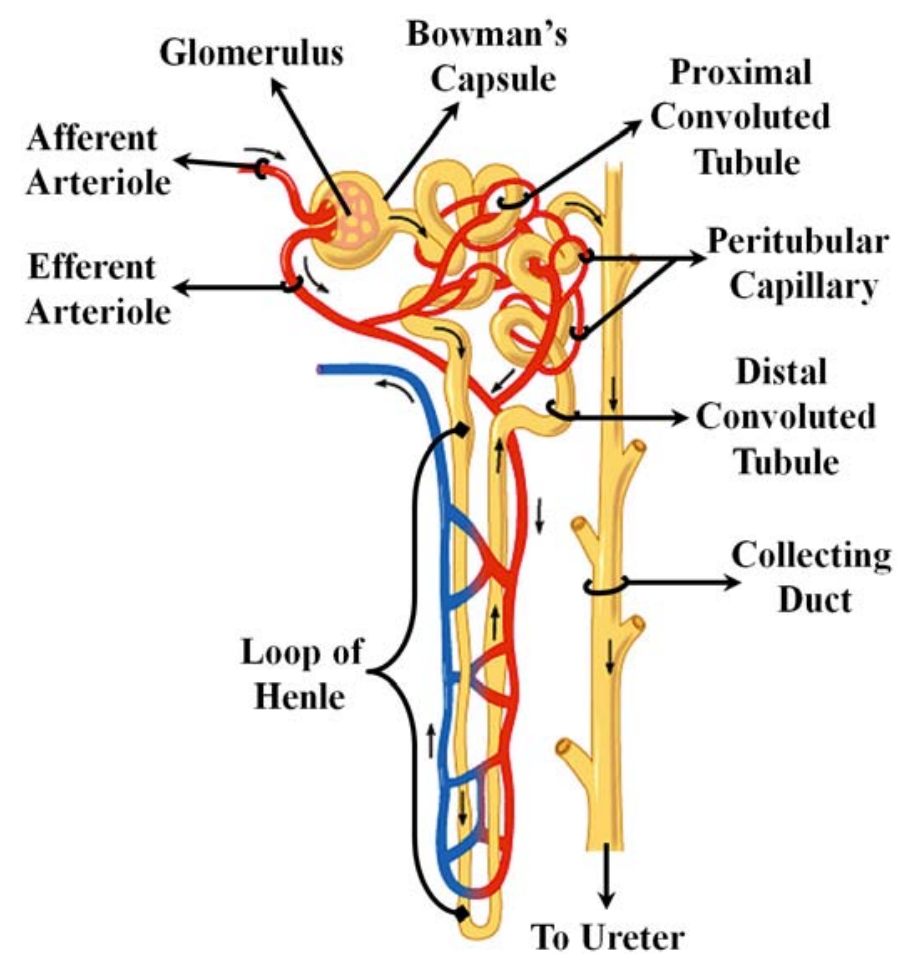

FIGURE 33: A schematic illustration of the detailed structure of a nephron [16].

capsule lacks the blood cells and the proteins. In the second process, the filtrated fluid in the Bowman's capsule goes into the tubular cells, which actively transport the necessary materials (e.g., glucose, amino acids) back into the body via a process called reabsorption. This process helps to retain normal levels of necessary materials of the blood. The final process of urine creation is the secretion, which is responsible for removing some substances from the blood and adding them to the tubular [313]. In this respect, secretion is reabsorption in reverse (see Figure 34). By the end of these three steps, the urine of a healthy kidney should be free of protein, glucose and any blood cells.

The function of the transplanted kidney can suffer from different types of renal complications, which, as shown in Figure 35, can be divided into six classes: (i) urologic complications, (ii) fluid collections, (iii) vascular complications, (iv) neoplasms, (v) recurrent native renal disease, and (vi) graft dysfunction [314]. Urologic complications include urine leaks associated with discharged urinomas, which have different sizes and occur within two weeks from transplantation. In addition, transplant patients face the high risk of developing calculous disease and urinary obstruction. Transplant fluid collections (e.g., urinomas, 


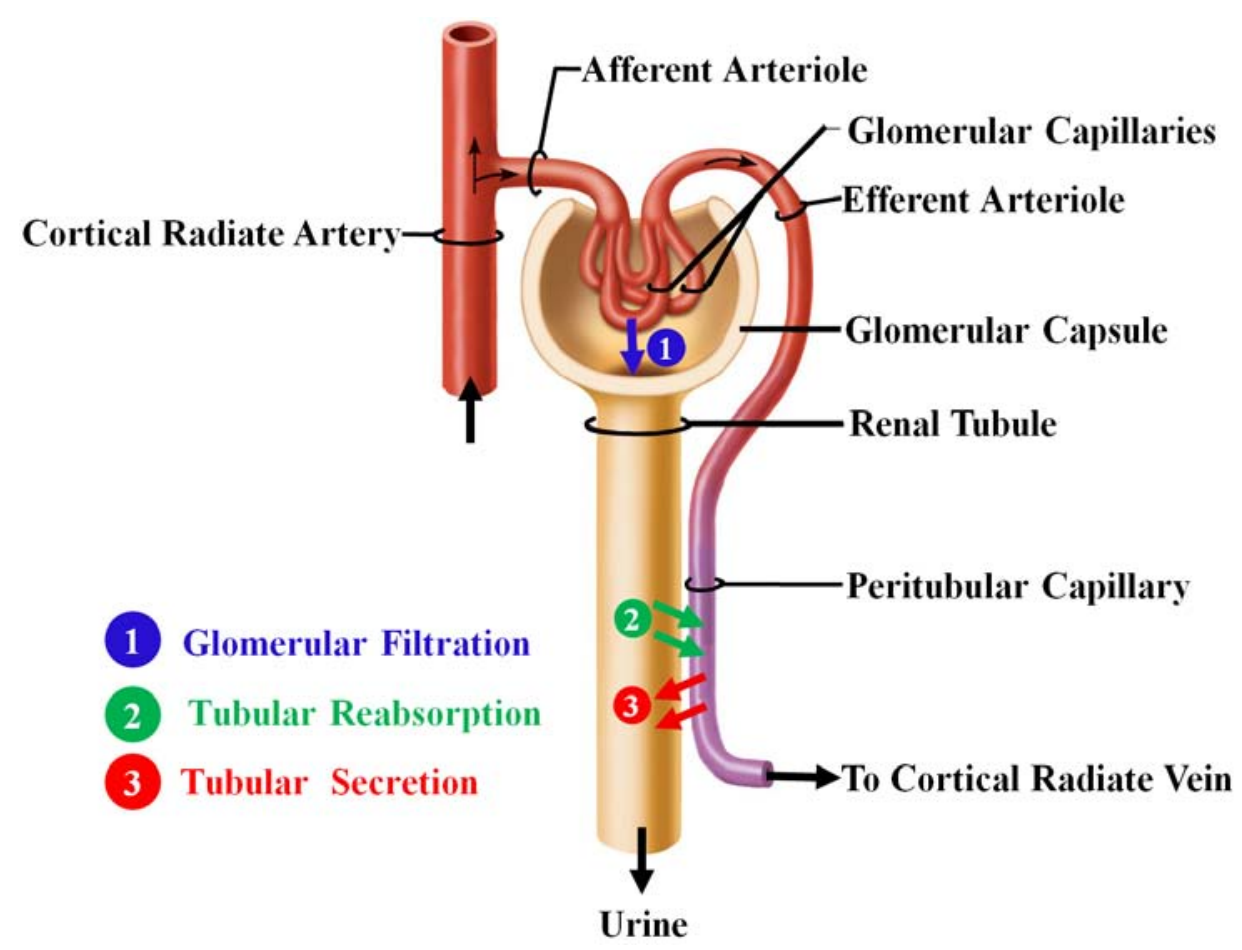

FIGURE 34: Illustration of the basic processes of urine formulation [17].

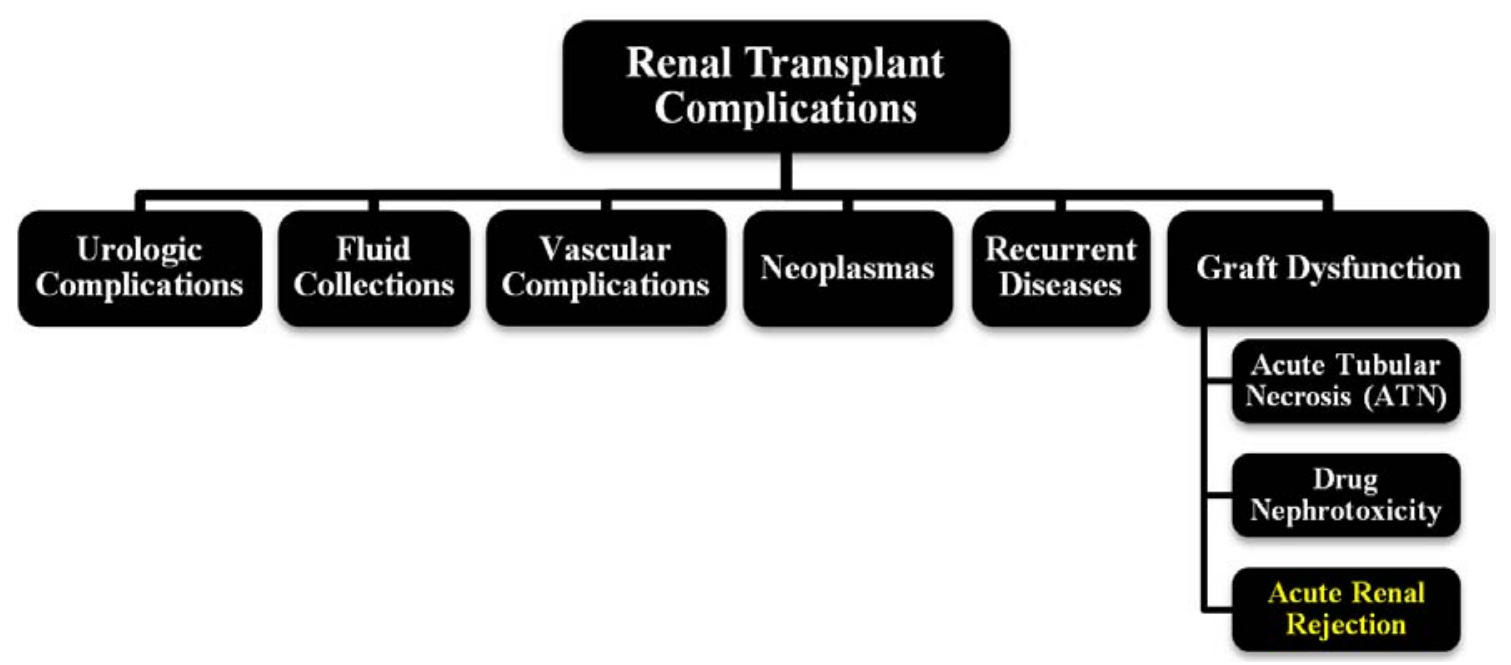

FIGURE 35: Different types of renal transplant complications.

hematomas, lymphoceles, and abscesses) are another type of renal transplant complications that have been recorded in up to $50 \%$ of renal transplantations. The size, location, and growth possibility of these collections greatly influence their clinical relevance [315]. Vascular complications include transplanted artery stenosis, infarction, arteriovenous fis- 
tulas and pseudoaneurysms, and renal vein thrombosis. Although these complications are found in only $10 \%$ of transplantation cases, they represent significant causes for serious graft dysfunction with high mortality rates [316]. Kidney transplantation increases cancer development, especially when the immunosuppression period is extended. Neoplasm risks include renal cell carcinomas and lymphomas [317]. Recurrent disease is rare in the early stage post-transplantation, but it is usually detected in long-term renal transplant recipients who suffer from diabetes, amyloidosis, and cystinosis [318]. Another type of renal transplant complications is the graft dysfunction, which is one of the major causes of renal transplant loss [319]. Renal graft dysfunction causes are acute tubular necrosis (ATN), drug nephrotoxicity, and rejection [320]. ATN is found initially in most cadaveric grafts, and usually diminishes within two weeks depending on ischemic insult. ATN is usually related to the donor kidney and is commonly observed in patients whose transplants are from living relatives [321]. Drug toxicity also contributes in degrading the grafted kidney functions. Cyclosporine imposes a high nephrotoxic potential as it can affect glomerular arterioles [322]. Acute renal rejection-the immunological response of the human immune system to the foreign kidney-is a major cause of allograft dysfunction, which can lead to the loss of the transplanted kidney. In the next section, more details about acute renal rejection, which is a case study in this dissertation, and current approaches for early detection of this kidney condition is provided.

\section{B. ACUTE RENAL TRANSPLANT REJECTION}

Acute renal transplant rejection is one of the most critical problems in urology. In the United States, approximately 17,736 renal transplants are performed annually [62], and given the limited number of donors, the salvage of transplanted kidney is an important medical concern. Acute renal rejection is found in up to $40 \%$ of patients within three weeks after transplantation and it is typically reversible through high-dose steroids or antibody therapy [323]. A gradual deterioration in graft function results in chronic rejection, which starts approximately at three months post-transplantation [322]. Since finding an effective 
treatment of chronic rejection is still an ongoing area of research, avoiding acute rejection episodes is the ideal way of preventing chronic rejection [321]. Therefore, early detection of rejection is important to institute appropriate medical and immune therapy in patients with transplanted kidneys [61].

At present, initial evaluation of renal transplant dysfunction is based on multiple blood tests and urine sampling (e.g., plasma creatinine, creatinine clearance). Creatinine clearance is a laboratory measurement used to estimate renal function, such as glomerular filtration rate (GFR). The creatinine level is determined by measuring the concentration of endogenous creatinine, which body produces in both plasma and urine. Creatinine clearance is still rendered as the more practical clinical measurement to assess renal function. However, the efficiency of such index in detecting renal rejection is limited due to the fact that creatinine clearance provides information on both kidneys together not unilateral information [324], as well as a significant change in creatinine level is only detectable after the loss of $60 \%$ of the kidney function [325]. Nonetheless, biopsy remains the gold standard, but only as the last resort because of its high costs and potential morbidity rates. Also, biopsy is an invasive procedure that imposes the risk of bleeding and infection to patients. Additionally, the relatively small needle biopsy sample may lead to over- or underestimation of the extent of inflammation in the entire graft [326]. Therefore, there is an urgent need for new technology based on noninvasive techniques that possess the ability of early and accurate diagnosis of the transplanted kidney status.

Several noninvasive imaging modalities have been used clinically to assess transplanted kidneys. Radionuclide imaging (also called scintigraphy), the traditional method in renal imaging, is an excellent modality for evaluating graft function, both qualitatively and quantitatively, while screening for common complications [320]. However, this technique fails in showing accurate anatomical details due to its limited spatial resolution, so functional abnormalities inside different parts of the kidney (e.g., the cortex and medulla) cannot be discriminated precisely [327]. Furthermore, radionuclide imaging includes radiation exposure [328], thus limiting the range of its applications, especially in monitoring such diseases as ATN or cyclosporin [329]. Ultrasound imaging is usually used to 
evaluate the transplanted kidney early in the postoperative period, and it can also be used for long-term follow-up assessment of the transplanted kidney. Ultrasound is a relatively cheap and non-nephrotoxic modality. However, sensitivity and reliability of this method mainly depend on the investigator's experience and suffers from low signal-to-noise ratios, shadowing artifacts, and speckles that greatly decrease image quality and diagnostic confidence [330]. Computed tomography (CT) is a commonly available imaging technique that allows accurate evaluation of various diseases in renal transplantation with lower costs than magnetic resonance imaging [331]. However, CT has a limited role in diagnosing acute renal rejection because the information gathered by CT is unspecific and the contrast agent (CA) used are still nephrotoxic [332]. More recently, dynamic contrast-enhanced MRI (DCE-MRI) is a non-invasive imaging technique that has been explored in perfusionrelated concerns in many clinical applications (e.g., in evaluation of the kidney, brain, and heart) due its ability to provide both functional and anatomical information. The potential advantages of DCE-MRI over other noninvasive techniques include (i) the lack of ionizing radiation, (ii) increased spatial resolution, (iii) the ability to provide both anatomical and functional information, and (iv) the feasibility to be used as early as possible (even one day post-transplantation) for the assessment and follow-up of the transplanted kidney

Developing a CAD system for early and noninvasive diagnosis of the kidney using DCE-MRI is an ongoing area of research. However, DCE-MRI exhibits multiple challenges stemming from $(i)$ the need to image very quickly, to capture the transient first-pass transit effects, while maintaining adequate spatial resolution; (ii) varying signal intensities over the time course of agent transit; and (iii) nonrigid deformations, or shape changes, may occur related to pulsatile or transmitted effects from adjacent structures, such as the bowel. A typical CAD system for the detection of acute rejection using DCE-MRI mainly involves motion correction to compensate for the global and/or local kidney motion, and segmentation of kidney and/or its functional unit (i.e., cortex). The related work on image segmentation and motion correction is provided below, and an overview of today's CAD systems for kidney diagnosis is detailed in Chapter II. 


\section{Related Work on Image Analysis for Acute Renal Rejection}

Dynamic MR images are subject to relatively low signal-to-noise, nonuniform intensity distribution over the time series images, and geometric kidney deformations caused by gross patient motion, transmitted respiratory effects, and intrinsic and transmitted pulsatile effects. Therefore, accurate segmentation and registration of dynamic MR renal images is a challenge. These two basic steps are commanding the major attention in this research area for automated analysis of dynamic perfusion MRI. Particularly, motion effects can be compensated for by specific use of global and local registration techniques. On the other hand, kidney segmentation techniques can be classified into three main categories: threshold-based, deformable boundary-based, and probabilistic or energy minimizationbased methods. The related work on kidney segmentation and registration techniques addressing the above-mentioned challenges are described below.

Signal thresholding segments the kidney and its internal structures (i.e., cortex and medulla) by analyzing an empirical probability distribution, or histogram of pixel intensities in a region-of-interest (ROI). Earlier computerized renal image analysis (e.g., [333336]) was usually carried out either manually or semi-automatically. Typically, the user defines an ROI in one image and for the rest of the images, image edges were detected and the model curve was matched to these edges. However, manual ROI placements are based on the users' knowledge of anatomy and thus are subject to inter- and intra-observer variability. Additionally, these approaches are very slow, even though semi-automated techniques (e.g., $[333,336])$ do reduce the processing time. Giele et al. [337] introduced an approach for the segmentation and registration of the kidney on DCE-MRI. First, the kidney contour is drawn manually by the user in a single high-contrast image. Then, the phase difference movement detection (PDMD) method is employed to correct kidney displacements. Their registration technique demonstrated better performance than direct image intensity matching and cross-correlation (CC) methods. However, when compared with the radiologist results, the PDMD method accuracy was about $68 \%$ and a manual mask to register the time frames was still required. Additionally, only translational motion was handled, while 
rotational motion was not mentioned. De Priester et al. [336] subtracted the average of pre-contrasted images (10 frames) from the average of early-enhanced images (30 frames) and thresholded the resulting difference image to obtain a kidney mask. Objects smaller than a certain size (700 pixels) were removed, and the remaining kidney object was closed using morphological erosion and manual processing. This approach was further expanded by Giele [327] by applying an erosion filter to the mask image in order to obtain a contour at a second subtraction stage. Koh et al. [338] segmented kidneys with the morphological 3D H-maxima transform. Rectangular masks and edge information were used to exclude training data or prior knowledge. In general, simple thresholding is too inaccurate to segment human organs in DCE-MRI, because these specific regions have similar gray level (intensity) distributions. Also, these methods work fine for post- and late-contrast phases, but fail in segmenting pre-contrast images.

Evolving deformable boundary methods have been explored as a more accurate means of kidney segmentation. A series of studies on both rats and human subjects [339343] has been conducted for the registration and segmentation of kidneys from DCE-MRI. A multi-step segmentation and registration in the study on humans by Sun et al. [341,343] initially corrected the large-scale motion by using a gradient-based rigid registration (only translational). Once roughly aligned, a high-contrast image is subtracted from a precontrast image and a level set approach was used to extract the kidney border from the difference image. Then, the segmented contour is propagated over other frames to search for the rigid (rotation and translation) registration parameters. For rat studies [339, 340,342] a variational level set approach that integrates a subpixel motion model and temporal smoothness constraints was used to find kidney borders. For segmenting the cortex and medulla, the level set approach by Chan and Vese [344] was used. Abdelmunim et al. [345] incorporated both image and shape prior information into a variational level set framework for kidney segmentation. However, their model did not adequately account for spatial dependencies between the pixels and therefore is quite sensitive to imperfect kidney contours and image noise. Yuksel et al. [346, 347] proposed a parametric deformable model approach for the segmentation of the kidney where the contour evolution was constrained using two 
density functions. The first described the kidney shape prior and was constructed using the average signed distance maps of the training samples. The second functional described the grey level distribution of the kidney and its background, estimated using adaptive linear combinations of discrete Gaussians (LCDG) [348-350]. A similar approach that incorporated shape and visual appearance priors was proposed by El-Baz et al. [351,352]. Their shape model is constructed from a linear combination of vectors of distances between the training boundaries and their common centroid. The appearance prior is modeled with a spatially homogeneous second-order Markov-Gibbs random field (MGRF) of gray levels with analytically estimated pairwise potentials. The current appearance model is described with the LCDG [348-350]. Khalifa et al [3,128] proposed an automated level set-based framework for the segmentation of kidney from dynamic MRI. They proposed a stochastic force that accounts for a shape prior and features of image intensity and pairwise MGRF spatial interactions. These features are integrated in to a joint MGRF image model of the kidney and its background to constrain the evolution of the deformable contour. They employed a two-stage registration methodology using first an affine transformation to account for the global motion, followed by a partial differential equation (PDE)-based approach for local motion correction. Gloger et al. [353] presented a level set-based approach using the shape prior information and Bayesian statistical concepts for generating the shape probability maps. However, the shape prior models in [346, 347, 351-353] did not impose temporal constraints on kidney segmentation.

The graph cut-based segmentation algorithm by Boykov et al. [354] minimizes the energy of a temporal MGRF model of intensity curves. Each voxel is described with a vector of intensity values over time. Initially, several seed points are placed on the objects and on the background to give user-defined constraints as well as expert samples of intensity curves. These samples are used to compute a two-dimensional histogram further acting as a data penalty function in minimizing the energy. Although the results looked promising, manual interaction was still required. Rusinek et al. [355] proposed a graph cut-based segmentation framework to assess cortical and medullary functional parameters. Their method employed a rigid registration step to account for the kidney displacements 
and the approach has been tested on simulated and in-vivo data. Ali et al. [356] used the graph cut-based minimization of an energy functional that combines a shape constraint with boundary properties. The constraint was built using a Poisson probability distribution and distance maps. Chevaillier et al. [357, 358] proposed a semi-automated method to segment internal structures (i.e., cortex, medulla) from DCE-MRI using $k$-means-based partitioning to classify pixels according to contrast evolution using a vector quantization algorithm. However, it was only tested on eight data sets for normal kidneys, and user interaction was still required. A similar segmentation by Song et al. [359] has only been tested on two MRI data sets, with simulated (rotation and translation) rigid motion, for one normal and one abnormal kidney. An automated framework proposed by Zöllner et al. [360] assesses renal function by deriving voxel-based functional information from DCE-MRI. They employed a B-splines based nonrigid image registration to compensate for the motion and deformation of the kidney. The $k$-means clustering method [361] was used for extracting functional information about different regions of the kidney according to their dynamic contrast enhancement patterns. An automated wavelet-based $k$-means clustering framework for segmenting the kidneys was proposed by Li et al. [362]. The images were co-aligned using B-splines registration and a $\mathrm{CC}$ cost function. Their framework was tested on seven subjects (four volunteers and three patients). Yang et al. [363] proposed a framework for the classification of kidney tissue using fuzzy $c$-means clustering. In order to reduce the motion artifacts, their framework employed a nonrigid registration step using the demons algorithm [364] and the squared pixel distance as a similarity metric and the squared gradient of the transformation field as the smoothness regularization term. In total, Table 3 summarize the reviewed methodologies for kidney segmentation and registration.

In summary, the segmentation and registration of DCE-MRI kidney data has been an area of increased research. As previously discussed, however, the existing DCE-MRI analysis techniques have their own limitations. To overcome these limitations, in this chapter novel mathematical models and learning techniques for motion correction and kidney segmentation are proposed. The developed techniques are combined in a comprehensive framework (shown in Figure 36) for noninvasive classification of non-rejection and acute 
renal transplant rejection from 2D DCE-MRI. In particular, a geometric deformable model approach is proposed for the segmentation of the kidney and its cortex. The proposed approach possess the ability to overcome shape variabilities, signal intensity variations, and image inhomogeneity by integrating appearance and shape features of the kidney into a joint probabilistic Markov-Gibbs random field (MGRF) image model to constrain the deformable model evolution. Additionally, a novel nonrigid registration approach for local kidney motion correction based on the solution of the Laplace equation is proposed. The proposed approach exploits geometric kidney features rather than image intensities, therefore it is not affected by intensity variations over the the time series. Details of the proposed framework are given in the following sections.

TABLE 3: Summary of kidney motion correction and segmentation techniques using magnetic resonance imaging (MRI).

\begin{tabular}{|c|c|c|}
\hline Study & Methodology & Data and Performance \\
\hline $\begin{array}{l}\text { De Priester et } \\
\text { al. }[336]\end{array}$ & $\begin{array}{l}\text { - } 2 \mathrm{D} \text {, semi-automated } \\
\text { - Image thresholding } \\
\text { - Morphological erosion and man- } \\
\text { ual processing. }\end{array}$ & $\begin{array}{l}\text { - } 18 \text { data sets (9 subjects) } \\
\text { - N/A }\end{array}$ \\
\hline $\begin{array}{l}\text { Giele et al. [327, } \\
\text { 337] }\end{array}$ & $\begin{array}{l}\text { - 2D, semi-automated } \\
\text { - Manual segmentation of the kid- } \\
\text { ney } \\
\text { - PDMD registration }\end{array}$ & $\begin{array}{l}\text { - } 5 \text { data sets } \\
\text { - ACC: } 68 \%\end{array}$ \\
\hline $\begin{array}{l}\text { Sun et al. [339, } \\
\text { 340, 342] }\end{array}$ & $\begin{array}{l}\text { - 2D, automated } \\
\text { - Variational level set }\end{array}$ & $\begin{array}{l}\text { - } 20 \text { data sets } \\
\text { - Visual evaluation by an } \\
\text { expert }\end{array}$ \\
\hline Sun et al. [341] & $\begin{array}{l}\text { - } 2 \mathrm{D} \text {, semi-automated } \\
\text { - Multi-step rigid registration } \\
\text { - level set segmentation for the kid- } \\
\text { ney and the cortex }\end{array}$ & $\begin{array}{l}\text { - } 5 \text { subjects } \\
\text { - Error is at most one } \\
\text { pixel size (for one se- } \\
\text { quence of } 150 \text { image } \\
\text { frames) }\end{array}$ \\
\hline $\begin{array}{l}\text { Boykov } \\
\text { al. [354] }\end{array}$ & $\begin{array}{l}\text { - 3D, semi-automated } \\
\text { - Graph cut-based segmentation } \\
\text { - Temporal MGRF }\end{array}$ & $\begin{array}{l}\text { - } 1 \text { data set } \\
\text { - N/A }\end{array}$ \\
\hline Song et al. [359] & $\begin{array}{l}\text { - 3D+time, semi-automated } \\
\text { - Fourier-based registration regis- } \\
\text { tration } \\
\text { - Template-based segmentation }\end{array}$ & $\begin{array}{l}\text { - } 4 \text { Subjects } \\
\text { - N/A }\end{array}$ \\
\hline
\end{tabular}


TABLE 3 - continued from the previous page

\begin{tabular}{|c|c|c|}
\hline Study & Methodology & Data and Performance \\
\hline $\begin{array}{l}\text { Rusinek et } \\
\text { al. [355] }\end{array}$ & $\begin{array}{l}\text { - } 3 \mathrm{D}+\text { time, semi-automated } \\
\text { - Mutual information (MI)-based } \\
\text { rigid registration (translation only) } \\
\text { - Graph cut-based segmentation }\end{array}$ & $\begin{array}{l}\text { - } 40 \text { data sets (18 sim- } \\
\text { ulated and } 22 \text { clinical } \\
\text { data sets) } \\
\text { - AD cortex: } 7.2 \pm 6.1 \text {, } \\
\text { AD medulla: } 6.5 \pm 4.6\end{array}$ \\
\hline Ali et al. [356] & $\begin{array}{l}\text { - } 2 \mathrm{D}, \text { semi-automated } \\
\text { - MI-based affine registration } \\
\text { - Graph cut shape-based segmenta- } \\
\text { tion }\end{array}$ & $\begin{array}{l}\text { - } \mathrm{N} / \mathrm{A} \\
\text { - } \mathrm{OAE} 5.7 \pm 0.9 \% \text { (tested } \\
\text { on } 33 \text { slices) }\end{array}$ \\
\hline $\begin{array}{l}\text { Yuksel et al. [346, } \\
\text { 347] }\end{array}$ & $\begin{array}{l}\text { - } 2 \text { D semi-automated, } \\
\text { - Shape-based segmentation using } \\
\text { parametric deformable model. }\end{array}$ & $\begin{array}{l}\text { - N/A, } \\
\text { - OAE } 0.382 \% \text { (for one } \\
\text { image only). }\end{array}$ \\
\hline $\begin{array}{l}\text { Abdelmunim et } \\
\text { al. [345] }\end{array}$ & $\begin{array}{l}\text { - } 2 \mathrm{D} \text {, automated } \\
\text { - Shape-based segmentation using } \\
\text { level set }\end{array}$ & $\begin{array}{l}\text { - } 39 \text { data sets to build } \\
\text { shape prior (Testing } \\
\text { data N/A) } \\
\text { - N/A }\end{array}$ \\
\hline $\begin{array}{l}\text { El-Baz et al. [351, } \\
\text { 352] }\end{array}$ & $\begin{array}{l}\text { - } 2 \text { D+time, semi-automated } \\
\text { - Scale invariant feature transform- } \\
\text { based alignment } \\
\text { - Shape-based segmentation using } \\
\text { parametric deformable model } \\
\text { - Second-order MGRF spatial inter- } \\
\text { action model of grey scale images }\end{array}$ & $\begin{array}{l}\text { - } 2700 \text { images } \\
\text { - } \mathrm{OAE} 0.83 \pm 0.45 \%\end{array}$ \\
\hline $\begin{array}{l}\text { Chevaillier } \\
\text { al. }[357,358]\end{array}$ & $\begin{array}{l}\text { - } 2 \mathrm{D} \text {, semi-automated } \\
\text { - } k \text {-means based clustering based } \\
\text { on contrast evolution using a vec- } \\
\text { tor quantization algorithm }\end{array}$ & $\begin{array}{l}\text { - } 8 \text { Data sets } \\
\text { - DSC : } 0.79,0.70 \text {, and } \\
0.77 \text { (cortex, medulla, } \\
\text { and cavities) }\end{array}$ \\
\hline $\begin{array}{l}\text { Zöllner } \\
\text { al. [360] }\end{array}$ & $\begin{array}{l}\text { - 3D+time, automated } \\
\text { - B-splines nonrigid registration } \\
\text { - k-means clustering }\end{array}$ & $\begin{array}{l}\text { - } 4 \text { Data sets } \\
\text { - Average similarity } \\
\text { score of } 0.96 \\
\end{array}$ \\
\hline Khalifa et al. [3] & $\begin{array}{l}\text { - } 2 \text { D+time, automated } \\
\text { - Affine registration } \\
\text { - Shape-based segmentation using } \\
\text { level set } \\
\text { - Second-order MGRF spatial inter- } \\
\text { action model }\end{array}$ & $\begin{array}{l}\text { - } 26 \text { Data sets } \\
\text { - OAE } 1.29 \pm 0.60\end{array}$ \\
\hline Li et al. [362] & $\begin{array}{l}\text { - } 3 \mathrm{D}+\text { time, automated } \\
\text { - } \mathrm{B} \text {-splines nonrigid registration } \\
\text { - wavelet-based k-means clustering }\end{array}$ & $\begin{array}{l}\text { - } 7 \text { Data sets } \\
\text { - ACC : } 88 \%, 91 \% \text {, and } \\
98 \% \text { (cortex, medulla, } \\
\text { and pelvis) }\end{array}$ \\
\hline
\end{tabular}


TABLE 3 - continued from the previous page

\begin{tabular}{|c|c|c|}
\hline Study & Methodology & Data and Performance \\
\hline Yang et al. [363] & $\begin{array}{l}\text { - } 3 \text { D+time, semi-automated } \\
\text { - Demons algorithm nonrigid regis- } \\
\text { tration } \\
\text { - Fuzzy c-mean clustering }\end{array}$ & $\begin{array}{l}-\mathrm{N} / \mathrm{A} \\
-\mathrm{N} / \mathrm{A}\end{array}$ \\
\hline $\begin{array}{l}\text { Khalifa } \\
\text { al. [128] }\end{array}$ & $\begin{array}{l}\text { - } 2 \text { D+time, automated } \\
\text { - Affine registration } \\
\text { - Shape-based segmentation using } \\
\text { level set } \\
\text { - Higher-order MGRF spatial inter- } \\
\text { action model }\end{array}$ & $\begin{array}{l}\text { - } 50 \text { Data sets } \\
\text { - DSC: } 0.982 \pm 0.016\end{array}$ \\
\hline $\begin{array}{l}\text { Khalifa } \\
\text { al. [129] }\end{array}$ & $\begin{array}{l}\text { - } 2 \text { D+time, automated } \\
\text { - Affine registration } \\
\text { - Shape-based segmentation using } \\
\text { level set } \\
\text { - Second-order MGRF spatial inter- } \\
\text { action model }\end{array}$ & $\begin{array}{l}\text { - } 50 \text { Data sets } \\
\text { - DSC: } 0.970 \pm 0.02\end{array}$ \\
\hline ACC: & \multicolumn{2}{|c|}{$\begin{array}{l}\text { Accuracy; } \mathrm{ACC}=\frac{\mathrm{TP}+\mathrm{TN}}{\mathrm{TP}+\mathrm{FP}+\mathrm{FN}+\mathrm{TN}} ; \text { where, } \mathrm{TP} \text { : true positive, } \\
\text { FP: false positive, } \mathrm{FN} \text { : false negative, TN: true negative. }\end{array}$} \\
\hline AD: & \multicolumn{2}{|l|}{ Absolute disparity. } \\
\hline DSC: & \multicolumn{2}{|c|}{ Dice similarity coefficient: $\mathrm{DSC}=\frac{2 \cdot \mathrm{TP}}{2 \cdot \mathrm{TP}+\mathrm{FP}+\mathrm{FN}}$} \\
\hline N/A: & \multicolumn{2}{|c|}{ Not applicable. } \\
\hline OAE: & \multicolumn{2}{|l|}{ Overlapping area error; $\mathrm{OAE}=\frac{\mathrm{FP}+\mathrm{FN}}{\mathrm{TP}+\mathrm{FN}} \%$} \\
\hline
\end{tabular}

\section{METHODS}

In this chapter, a noninvasive image-based framework (see Figure 36) aimed at classifying acute rejection versus non-rejection transplants by analyzing 2D DCE-MRI time series perfusion data is proposed. In the proposed DCE-MRI based framework, the classification of kidney status is performed using the following five steps: $(i)$ affine-based registration of the DCE-MRI data with the shape prior images, (ii) segmenting kidney objects from the surrounding structures, (iii) nonrigid registration for local motion correction, $(i v)$ segmenting the cortex and generating the physiological perfusion data and parameters; $(v)$ classifying the acute rejection versus non-rejection kidney status, evaluating the method as a diagnostic test, and depicting the parametric maps of the estimated perfusion indexes. The overall framework has been tested on a total of 50 dynamic MRI perfusion data sets to permit us to draw statistically meaningful inferences. 


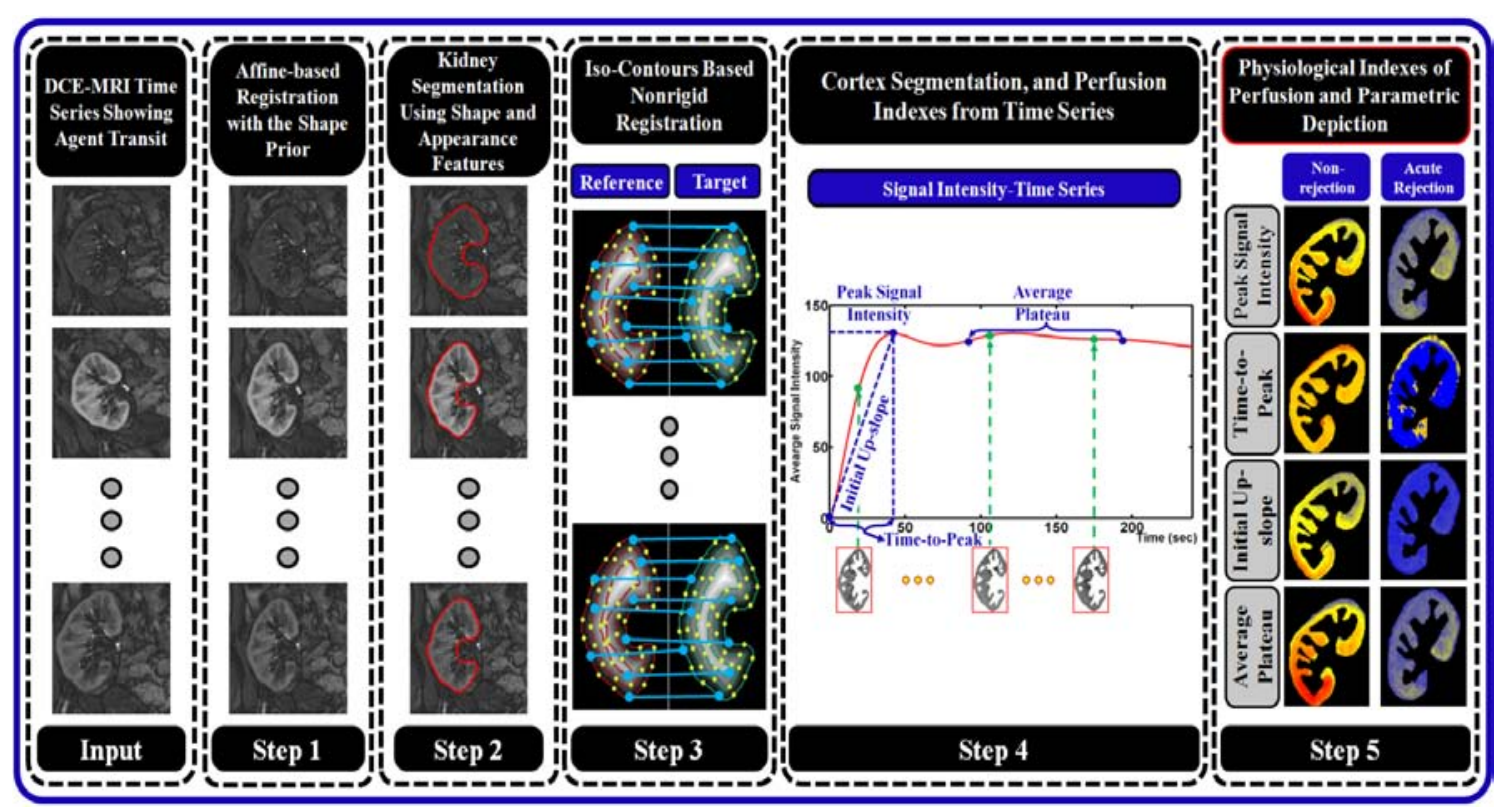

FIGURE 36: The proposed computer-aided diagnostic (CAD) system for noninvasive evaluation of acute renal transplant rejection.

\section{Kidney Segmentation Using a Joint Bi-Level MGRF Model}

Dynamic MRI time series are subject to relatively low signal-to-noise, non-uniform intensity distribution over the time series images, and respiratory and physiological motion. Therefore, accurate kidney segmentation from DCE-MRI is a challenging problem. In this chapter, the goal is to address these variations and discrepancies by integrating shape and appearance features (in terms of pixel-wise image intensities and their spatial interactions) of the kidney into a deformable model framework for precise segmentation of the kidney from DCE-MRI time series data. In the proposed approach, deformable prototypes using level sets are used due to its flexible evolution on the $x y$-plane and need no parametrization. The level set function $\phi$ is a distance map of the signed minimal Euclidean distances from every point $(x, y)$ of the plane to the boundary (negative for interior and positive for exterior points). It is evolving in the discrete time-space domain as follows [365]:

$$
\phi_{n+1}(x, y)=\phi_{n}(x, y)-\tau F_{n}(x, y)\left|\nabla \phi_{n}(x, y)\right|
$$


where $n$ is a discrete instant of time $t=n \tau$ taken with a step $\tau>0, F_{n}(x, y)$ is a speed function controlling the evolution, and $\nabla \phi_{n}(x, y)=\left[\frac{\partial \phi_{n}}{\partial x}, \frac{\partial \phi_{n}}{\partial y}\right]$ is the gradient of $\phi_{n}(x, y)$. For more accurate segmentation, a stochastic speed function that depends on three featuresa weighted probabilistic shape prior, pixel-wise image intensities, and high-order spatial interactions-is proposed. The features are integrated into a joint, bi-level, probabilistic MGRF model of the kidney and its background.

Let $\mathbf{R}=\{(x, y): 0 \leq x \leq X-1,0 \leq y \leq Y-1\} ; \mathbf{Q}=\{0,1, \ldots, Q-1\}$; and $\mathbf{L}=\{0,1\}$ denote a finite arithmetic lattice of the size of $X Y$ supporting grayscale images and their region (segmentation) maps, a finite set of $Q$ integer gray values, and a binary set of object ("1") and background ("0") labels, respectively. Let $\mathbf{g}=\left\{g_{x, y}:(x, y) \in\right.$ $\left.\mathbf{R} ; g_{x, y} \in \mathbf{Q}\right\}$ and $\mathbf{m}=\left\{m_{x, y}:(x, y) \in \mathbf{R} ; m_{x, y} \in \mathbf{L}\right\}$ be a gray scale image taking values from $\mathbf{Q}$, i.e., $\mathbf{g}: \mathbf{R} \rightarrow \mathbf{Q}$, and a region map taking values from $\mathbf{L}$, i.e., $\mathbf{m}: \mathbf{R} \rightarrow \mathbf{L}$, respectively. An input image $\mathbf{g}$, co-aligned to a shape prior, and its region map $\mathbf{m}$ are described with a joint probability model:

$$
P(\mathbf{g}, \mathbf{m})=P(\mathbf{g} \mid \mathbf{m}) P(\mathbf{m})
$$

where $P(\mathbf{g} \mid \mathbf{m})$ is a conditional distribution of the images given the map and $P(\mathbf{m})=$ $P_{\mathrm{sp}}(\mathbf{m}) P_{\mathbf{V}}(\mathbf{m})$ is an unconditional probability distribution of maps. Here, $P_{\mathrm{sp}}(\mathbf{m})$ denotes a weighted shape prior, and $P_{\mathbf{V}}(\mathbf{m})$ is a Gibbs probability distribution with potentials $\mathbf{V}$, which specifies a MGRF model of spatially homogeneous maps m. Details of the model's components are outlined below.

a. Weighted shape prior The level set evolution is constrained using an adaptive shape prior of the kidney that is built for a set of training images (Figure $38(a)$ ). To reduce the variability of kidney shapes in the training data and to maximize the overlap area of the estimated probabilistic shape prior, the training images are mutually coaligned by an affine 2D transformation, maximizing their MI [366]. To construct the shape prior, an expert in medical imaging delineated the kidney borders, which specify the region maps for the co-aligned training images (see Figure $37(c)$ ). Then, the shape prior was built as a spatially variant independent random field of region labels in these maps: 
$P_{\mathrm{sp}}(\mathbf{m})=\prod_{(x, y) \in \mathrm{R}} p_{\mathrm{sp}: x, y}\left(m_{x, y}\right)$ where $p_{\mathrm{sp}: x, y}(1)$ and $p_{\mathrm{sp}: x, y}(0)=1-p_{\mathrm{sp}: x, y}(1)$ are the empirical pixel-wise probabilities of kidney and background, respectively. Each input image to be segmented first is co-aligned to one of the training images, and the normalized cross-correlation (NCC) values, $\eta_{j} ; j=1, \ldots, N$, between the co-aligned input image and each of the $N$ training images are computed. Then, the pixel-wise kidney probability $p_{\mathrm{sp}: x, y}(1)$ (Figure $38(b)$ ) is estimated by a weighted sum of occurrences of the pixel $(x, y)$ in the kidney region in all training maps: $p_{\mathrm{sp}: x, y}(1)=\frac{1}{\alpha} \sum_{j=1}^{N} \eta_{j} m_{j: x, y}$ where $\alpha=\sum_{j=1}^{N} \eta_{j}$.

(a)
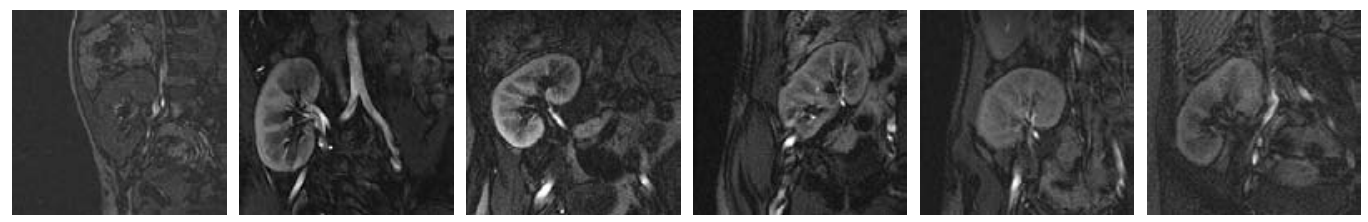

(b)
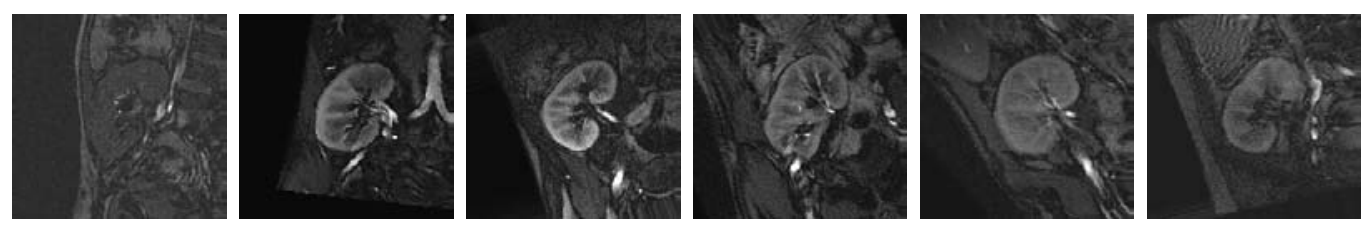

(c)
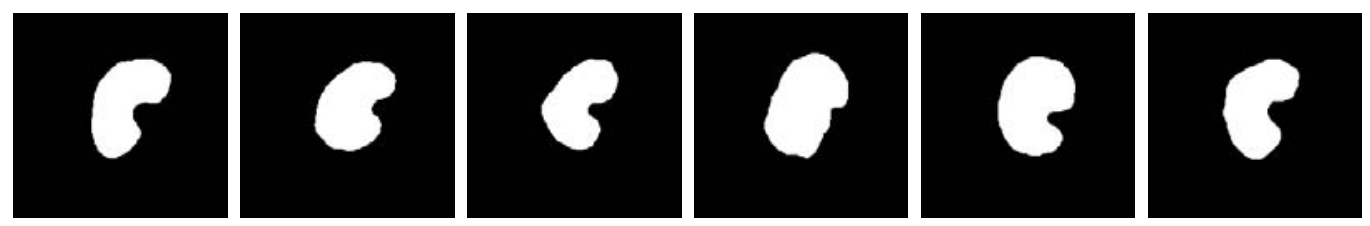

FIGURE 37: Forming kidney shape prior: (a) training samples, $(b)$ their affine alignment, and (c) manually segmented kidney objects . Note that the registration enhances the overlap between the kidney objects and thus reduce the variability of the final estimated shape.

b. MGRF model with second and higher-order spatial interaction To better account for the large inhomogeneity of the kidney (e.g., cortex, and medulla), the higherorder spatial interactions (i.e., the families of the triple and quad cliques, Figure $39(b, c)$ ) between region labels of a map $\mathbf{m}$ are added to the pairwise ones $[348,367]$. Let $\mathbf{C}_{a}$ denote a family of $s$-order cliques of an interaction graph with nodes in the lattice sites $(x, y)$ and edges connecting the interacting, or interdependent, sites (see Figure 39). To account for large variations of the DCE-MRI time series data related to the transit of the contrast agent, the label interactions are modeled by a spatially homogeneous MGRF with up to 

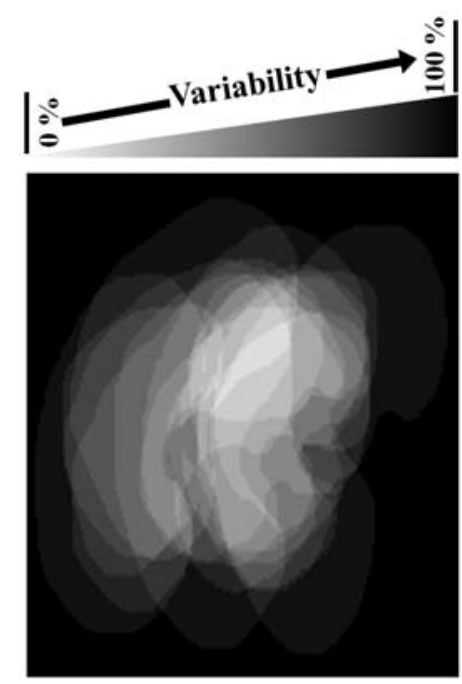

(a)
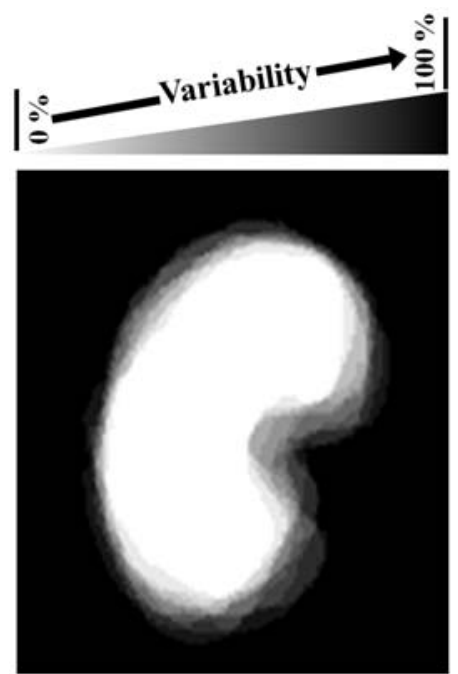

(b)
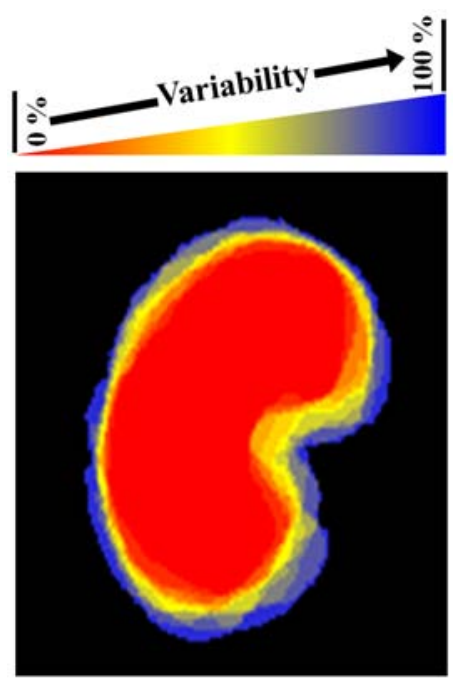

(c)

FIGURE 38: Gray-coded shape prior of the kidney before $(a)$ and after $(b)$ the affine mutual information (MI) based registration and the color-coded visualization of the shape prior $(c)$.

fourth-order interactions over the nearest 8-neighborhoods of pixels:

$$
P_{\mathbf{V}}(\mathbf{m})=\frac{1}{Z} \exp \left(\sum_{a=1}^{A} \sum_{\mathbf{c} \in \mathbf{C}_{a}} V_{a}(\mathbf{m}(x, y):(x, y) \in \mathbf{c})\right)
$$

where $A$ clique families describe the geometry of interactions, $\mathbf{V}=\left[V_{a}:\{0,1\} \rightarrow\right.$ $(-\infty, \infty): a=1, \ldots, A]$ is a collection of Gibbs potential functions $V_{a}$ for the families $\mathrm{C}_{a}$, and the partition function $Z$ normalizes the probabilities over the parent population $\mathbb{M}=\{0,1\}^{X Y}$ of all the maps.

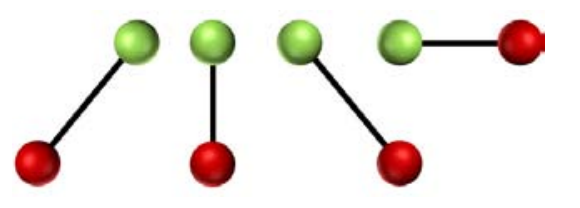

(a)

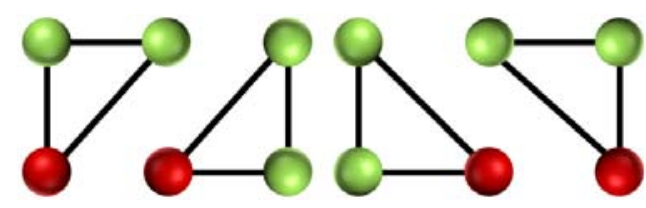

$(b)$

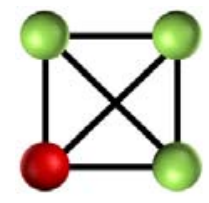

(c)

FIGURE 39: Second- $(a)$, third- $(b)$, and fourth-order $(c)$ cliques for the 8-neighborhood.

An initial map $\mathbf{m}$, obtained by the pixel-wise classification, allows for analyti- 
cally approximating the maximum likelihood estimates of the potentials and computing the pixel-wise probabilities of the region labels at each step of the contour evolution. For symmetry sake, only the equality or inequality of the labels in a clique $\mathbf{c}$ is taken into account. The second- and third-order potentials are given by Equations (47) and (48), respectively:

$$
\begin{gathered}
V_{a}\left(m_{x_{1}, y_{1}}, m_{x_{2}, y_{2}}\right)=\left\{\begin{array}{cl}
V_{2: a: e q} & \text { if } m_{x_{1}, y_{1}}=m_{x_{2}, y_{2}} \\
-V_{2: a: e q} & \text { otherwise }
\end{array}\right. \\
V_{a}\left(m_{x_{1}, y_{1}}, m_{x_{2}, y_{2}}, m_{x_{3}, y_{3}}\right)=\left\{\begin{array}{cl}
V_{3: a: \mathrm{eq}_{3}} & \text { if } m_{x_{1}, y_{1}}=m_{x_{2}, y_{2}}=m_{x_{3}, y_{3}} \\
-V_{3: a: \mathrm{eq}_{3}} & \text { otherwise }
\end{array}\right.
\end{gathered}
$$

and for the fourth-order potentials:

$$
V_{a}\left(m_{x_{1}, y_{1}}, m_{x_{2}, y_{2}}, m_{x_{3}, y_{3}}, m_{x_{4}, y_{4}}\right)= \begin{cases}V_{4: a: \mathrm{eq}_{4}} & \text { if there are } 4 \text { equal labels } \\ V_{4: a: \mathrm{eq}_{3}} & \text { if there are } 3 \text { equal labels } \\ -\left(V_{4: a: \mathrm{eq}_{3}}+V_{4: a: \mathrm{eq}_{4}}\right) & \text { otherwise }\end{cases}
$$

The proposed analytical approximation of the Gibbs potentials from a given map $\mathbf{m}$, i.e., the computation of the above values $V_{2: a: e q}, V_{3: a: \mathrm{eq}_{3}}, V_{4: a: \mathrm{eq}_{3}}$, and $V_{4: a: \mathrm{eq}_{4}}$, extends earlier second-order MGRFs [348, 367] to the higher-order models. To the best of our knowledge, the analytical estimation of the Gibbs potential for higher-order MGRF model is the first of its kind in the literature. For completeness, it is detailed in Appendix I.

c. Intensity model The visual appearance of both kidney region and surrounding tissues is modeled by separating a mixed empirical 1D distribution of pixel intensities into two individual components, associated with the dominant kidney and background modes, respectively. To model the current kidney appearance, the empirical distribution is precisely approximated with the LCDG model and automatically separated into the distinct kidney and background LCDG components ${ }^{1}$ [348-350]. The main advantage of the LCDG probabilistic model is that using both positive an negative Gaussian components can help to

${ }^{1}$ LCDG model is also applicable for images with more than two classes, which can be automatically estimated from the image using the modified Akiake information criterion $[69,368]$ 
fit any shape of the empirical density. In many cases of empirical densities, it is difficult (if not impossible) to accurately approximate the shape using only single symmetric kernels.

Let $\boldsymbol{\Psi}_{\theta}=(\psi(q \mid \theta): q \in \mathbf{Q})$ denote a discrete Gaussian (DG) ${ }^{2}$ with parameters $\theta=(\mu, \sigma)$, integrating a continuous 1D Gaussian density with mean $\mu$ and variance $\sigma^{2}$ over successive gray level intervals [348-350]. The LCDG with two dominant positive DGs and $M_{\mathrm{p}} \geq 2$ positive and $M_{\mathrm{n}} \geq 0$ negative subordinate DGs is defined as [348-350]:

$$
P_{\mathbf{w}, \boldsymbol{\Theta}}(q)=\sum_{i=1}^{M_{\mathrm{p}}} w_{\mathrm{p}: i} \psi\left(q \mid \theta_{\mathrm{p}: i}\right)-\sum_{j=1}^{M_{\mathrm{n}}} w_{\mathrm{n}: j} \psi\left(q \mid \theta_{\mathrm{n}: j}\right)
$$

where all the weights $\mathbf{w}=\left[w_{\mathrm{p}: i}, w_{\mathrm{n}: j}\right]$ are non-negative and meet an obvious constraint $\sum_{i=1}^{M_{\mathrm{p}}} w_{\mathrm{p}: i}-\sum_{j=1}^{M_{\mathrm{n}}} w_{\mathrm{n}: j}=1$. All the LCDG parameters, including the numbers of DGs, are estimated from the mixed empirical distribution to be modeled. Both the latter distribution and its two components are modeled with the LCDGs more accurately than with a conventional mixture [369] of only positive DGs or other unimodal distributions, thus yielding a better initial region map after the pixel-wise classification of intensities. The LCDG of Equation (50), including the numbers $M_{p}$ and $M_{n}$ of its components, is identified using the expectation-maximization (EM)-based algorithm introduced in [367, 370-375].

d. Deformable model evolution In overall summary, the weighted probabilistic shape prior, pixel-wise image intensities, and higher-order spatial interaction terms contribute to the pixel-wise guidance of the level set. Let $p(q \mid l)$ denote the pixel-wise probability of the intensity $q \in \mathbf{Q}$ for the LCDG model of current kidney $(l=1)$ or background $(l=0)$ appearance. Let $p_{\mathbf{V}: x, y}(1)$ be the probability of the kidney label for the pixel $(x, y)$ of the region map $\mathbf{m}$ in the MGRF model $P_{\mathbf{V}}(\mathbf{m})$ at the current evolution step. Let $P_{1: x, y}=\frac{\Omega_{1: x, y}}{\Omega_{1: x, y}+\Omega_{0: x, y}}$ and $P_{0: x, y}=\frac{\Omega_{0: x, y}}{\Omega_{1: x, y}+\Omega_{0: x, y}}=1-P_{1: x, y}$, where $\Omega_{1: x, y}=p(q \mid 1) p_{\mathbf{V}: x, y}(1) p_{\mathrm{s}: x, y}(1)$ and $\Omega_{0: x, y}=p(q \mid 0)\left(1-p_{\mathbf{V}: x, y}(1)\right)\left(1-p_{\mathrm{s}: x, y}(1)\right)$. Then, the speed function of Equation (44) is defined as: $F(x, y)=\kappa \vartheta(x, y)$ where $\kappa$ is the mean

\footnotetext{
${ }^{2}$ A Discrete Gaussian (DG) $\Psi_{\theta}=(\psi(q \mid \theta): q \in \mathbf{Q})$ with $\theta=\left(\mu, \sigma^{2}\right)$ is defined as $\psi(q \mid \theta)=\Phi_{\theta}(q+$ $0.5)-\Phi_{\theta}(q-0.5)$ for $q=1, \ldots, Q-2, \psi(0 \mid \theta)=\Phi_{\theta}(0.5)$, and $\psi(Q-1 \mid \theta)=1-\Phi_{\theta}(Q-1.5)$ where $\Phi_{\theta}(q)$ is the cumulative Gaussian function with the mean $\mu$ and the variance $\sigma^{2}$.
} 
contour curvature and $\vartheta(x, y)$ specifies the magnitude and direction of contour evolution:

$$
\vartheta(x, y)=\left\{\begin{array}{cl}
-P_{1: x, y} & \text { if } P_{1: x, y}>P_{0: x, y} \\
P_{0: x, y} & \text { otherwise }
\end{array}\right.
$$

In total, the key kidney segmentation steps are summarized in Algorithm 1.

\section{Iso-contours Based Nonrigid Registration}

After affine registration and kidney segmentation, a new nonrigid registration approach is proposed $[3,128]$ to compensate for local kidney motion and deformations over the time frame of image acquisition. In order to avoid problems associated with intensity variations over the temporal dynamic contrast agent data set, the proposed approach exploits geometric features, rather than image intensities. These geometric features are estimated from the electric field vectors that are calculated by solving the Laplace secondorder PDE between the segmented kidney borders. Estimating these field vectors allow for co-allocation of point-to-point correspondences between the segmented kidney objects. Mathematically, the second-order Laplace PDE defines a scalar field $\gamma$ is defined as:

$$
\nabla^{2} \gamma=\frac{\partial^{2} \gamma}{\partial x^{2}}+\frac{\partial^{2} \gamma}{\partial y^{2}}=0
$$

The solution $\gamma(x, y)$ of Equation (52) within the boundaries results in intermediate equipotential surfaces and streamlines (field lines), being everywhere orthogonal to all equipotential surfaces and establish natural point-to-point correspondences between the boundaries, e.g., the line connecting the points $\mathbf{B}_{a i}$ and $\mathbf{B}_{b j}$ in Figure 40. In medical imaging, the Laplace-equation based approaches have been previously used for colon surface flattening and centerline extraction [376], and thickness measurements [67,377]. To the best of our knowledge, the Laplace-based registration is the first of its kind in application to kidney motion correction in DCE-MRI for further improving the registration accuracy.

The proposed Laplace-based nonrigid registration approach is based on deforming each pixel of the segmented kidney objects over a set of nested, equi-spaced contours (i.e., iso-contours), which is generated for both the target and reference kidney objects as shown 
Algorithm 1 Key Steps for Level Set Segmentation of the Kidney

Input: greyscale image $\mathrm{g}$ to be segmented.

Output: segmented kidney border.

1. Build the kidney shape prior from the training grayscale images and their binary region maps.

2. For each input image $\mathrm{g}$ to be segmented:

(a) Co-align image $\mathrm{g}$ to one of the images used for building the shape prior using the affine transformation .

(b) Update the weighted shape prior:

- Calculate the NCCs between the co-aligned image and all training images.

- Compute the weighted sum of the training region maps in order to update the shape prior.

(c) Estimate the LCDG models of marginal intensity distributions for the kidney and its background.

(d) Form an initial kidney/background region map $\mathbf{m}$ by the pixel-wise classification with these LCDG models.

(e) Estimate analytically the Gibbs potentials for the fourth-order MGRF model of the map $\mathbf{m}$.

3. Find the speed function $\vartheta(x, y)$ of Equation (51) using Steps 2(b)-2(e).

4. Segment $\mathrm{g}$ by evolving the level set function $\phi$ guided by the speed function calculated in Step 3.

in Figure 41. To find the iso-contours, a distance map is generated inside the binary object area by finding the minimum Euclidean distance for every inner point to the object boundary. The external points are excluded from consideration. Then, the Laplace equation is 


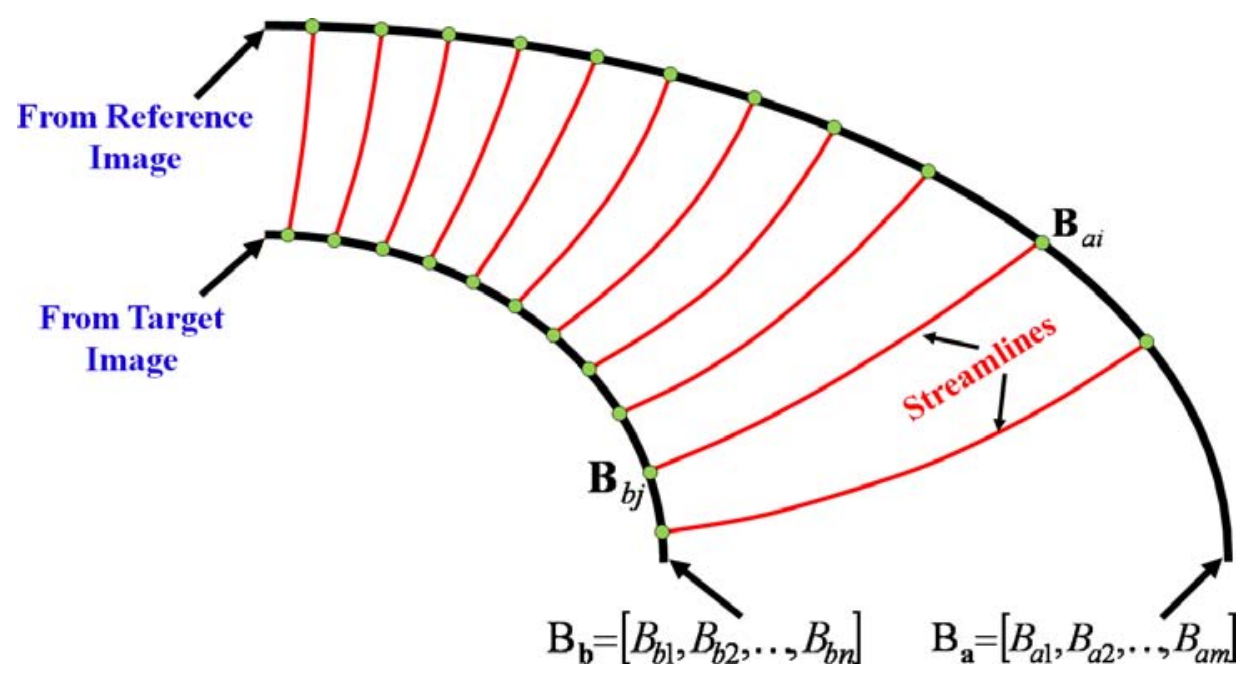

FIGURE 40: Schematic illustration of establishing point-to-point correspondences between two boundaries by a potential field.

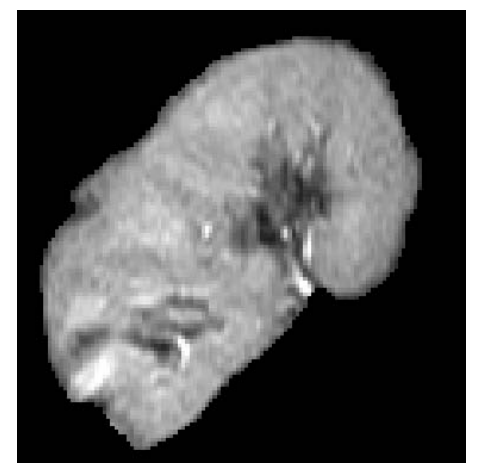

(a)

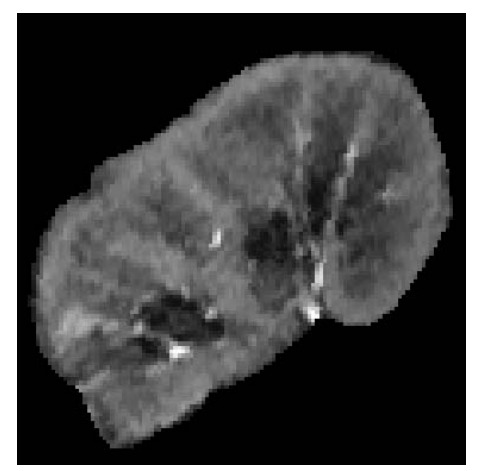

$(d)$

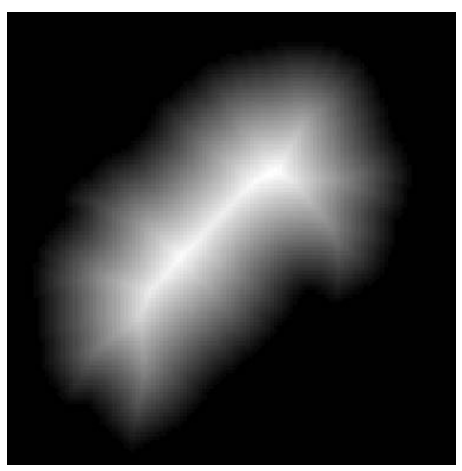

(b)

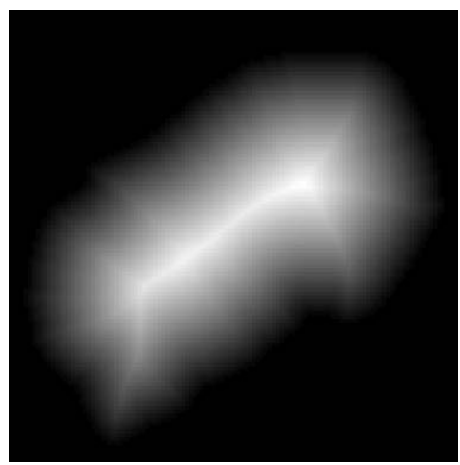

(e)

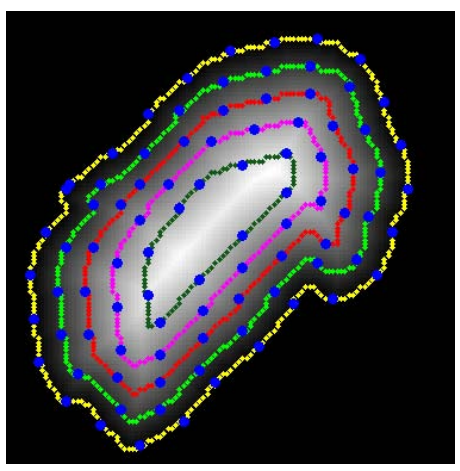

(c)

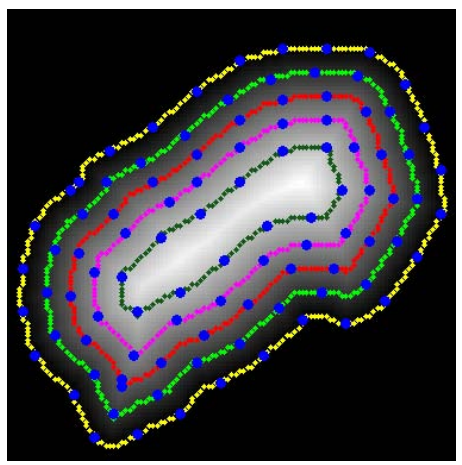

$(f)$

FIGURE 41: Generation of iso-contours: the reference and target images $(a, d)$, their distance maps $(b, e)$, and the generated iso-contours $(c, f)$. 
applied to the respective reference and target iso-contours to co-locate their corresponding points. Figure 42 illustrates the ability of the proposed nonrigid registration to find point-topoint correspondences in both possible misregistration scenarios; either when the surfaces overlap (bottom row) or they do not (top row). These scenarios are likely to occur due to unexpected large patient movements during MRI scanning. Note that the Laplace-based nonrigid registration is employed after affine alignment of the images (Figure 42 (b,e)). Basic steps of the Laplace-based nonrigid registration are summarized in Algorithm 2.

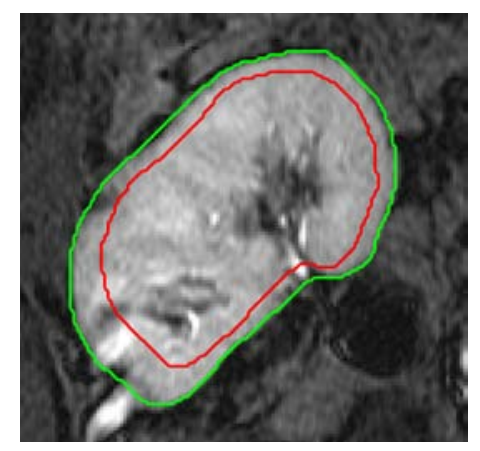

(a)

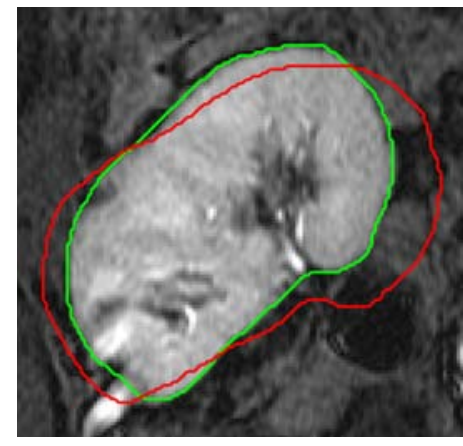

$(d)$

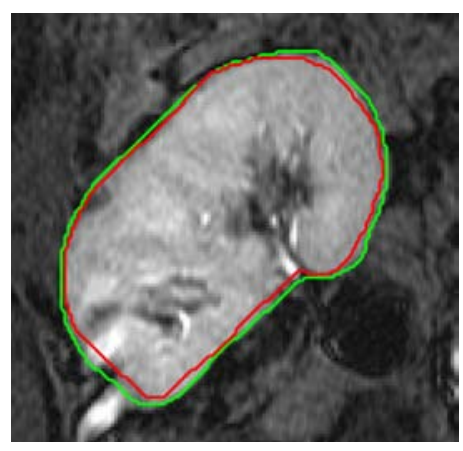

(b)

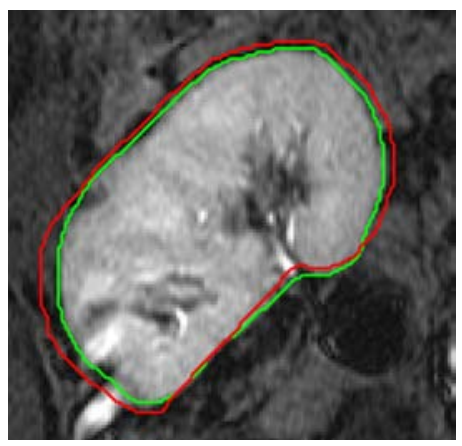

(e)

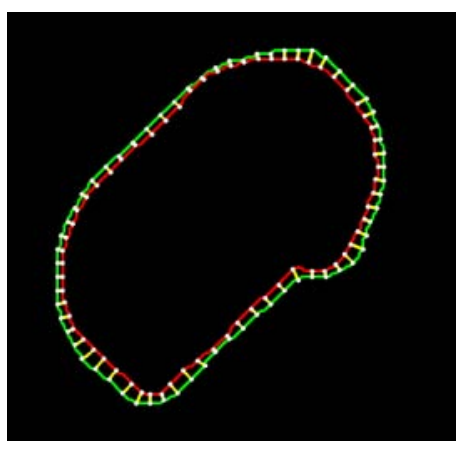

(c)

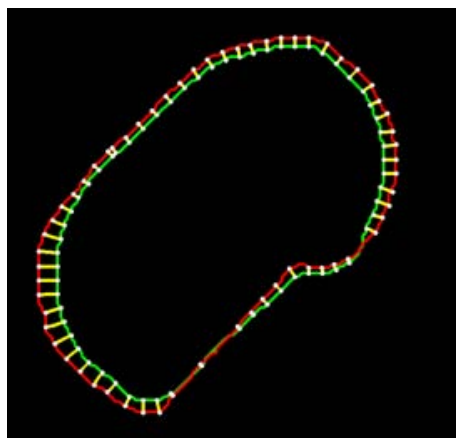

$(f)$

FIGURE 42: Co-allocation of point-to-point correspondences for two possible scenarios of kidney misregistration: the reference (green) and target (red) contours before $(a, d)$, and after $(b, e)$ affine alignment, and streamlines (yellow) linking the corresponding points found by solving the Laplace equation $(c, f)$. 
Algorithm 2 Laplace-Based Iso-Contours Nonrigid Registration

Input: segmented kidney objects.

Output: point-to-point correspondences.

1. Generate the distance maps inside the segmented kidney (Figure $41(b, e)$ ).

2. Generate the nested iso-contours for the target and reference maps (Figure $41(c, f)$ ).

3. Initial condition: Set the maximum and minimum (zero) potential $\gamma$ at the target iso-contour and the corresponding reference iso-contour, respectively.

4. Solve Equation (52) between the corresponding iso-contours using the above condition at Step 3.

5. Compute components of the gradient vectors in both $\mathrm{X}$ - and Y-directions for the estimated potential in Step 4.

6. Form the streamlines using the gradient vectors in Step 5, then find the corresponding points between the iso-contours that are matched by forming the streamlines.

7. Repeat Steps $3-6$ for the next set of the corresponding iso-contours.

3. Cortex Segmentation and Generation of Physiological Data

Vascular insults directly affect the kidney cortex [378]. Therefore, the cortex of the co-aligned kidneys is finally segmented after the nonrigid registration. To achieve this, the proposed level set approach is applied again, using only intensity and spatial features to guide the evolution. Since all the images are already co-registered, the deformable boundary evolves with respect to a circular contour initialized at the center of the registered kidney (see Section III.F.3, Figure 56). After the cortex is segmented, it serves as a mask that is propagated over the remaining co-registered image frames of a given perfusion time series data. 
Following the cortex segmentation, agent kinetic curves (signal intensity versus time curves) are constructed by estimating the average intensities over the entire cortex for each image frame of the time series (see Figure 57). To control for different physiological factors at different patient imaging exams, perfusion values obtained for the cortex were normalized by the perfusion of an adjacent segment of body wall muscle that was obtainable for each patient. Established dynamic perfusion analyses of extracellular extravascular agents, such as gadolinium agents, have previously used empirical parameters, including-initial up-slope, peak signal intensity, and time-to-peak [81]. However, due to rapidly changing contrast agent kinetics during the transient phase, the resulting limited temporal sampling leads to noisy estimates. Therefore, agent delivery during the more slowly varying phase (plateau, or tissue distribution phase, starting at approximately $30 \mathrm{sec}$ and effectively extending to approximately two $\min$ for peripheral injections) is characterized. This also serves to incorporate a large number of data points over the signal intensity time series to characterize perfusion [87]. Thus, both the transient phase indexes and a tissue phase signal change index are used. Please see Figure 36, Step \# 4.

\section{Classification, Performance Analysis, and Visualization of Perfusion Indexes}

To distinguish between the non-rejection and acute rejection cases, a $k_{n}$-nearest neighbor classifier learning statistical characteristics of the perfusion curves averaged over the entire cortex was used. The characteristics are obtained from the training sets containing both non-rejection and acute rejection cohorts. After training, the four perfusion indexes were chosen one by one to classify the test cases. For a more robust characterization, the $k_{n}$ classifier was augmented by combining all four indexes with appropriate weights, estimated by genetic optimization [379] using the training data sets. The weights were estimated by maximizing the Euclidean distance between the weighted-combined indexes of the nonrejection and acute rejection groups in order to better classify the training data, based on the biopsy ground truth. The dependence of the proposed framework on the selection of the training data set was evaluated using a cross-validation approach. All available data 
sets were divided into four groups and a four-fold cross-validation using the combined $k_{n}$-nearest classifier with new weights computed for each current training set.

The receiver operating characteristics (ROC) [380] is an alternate metric to test the performance of diagnostic systems. The ROC curve evaluates the sensitivity of a CAD system relative to the choice of its operating point (e.g., a classification threshold) by plotting the relationship between the true positive and false positive rates for different operating points. The ROCs for each of the individual indexes as well as the weighted combined indexes were computed. Also, a bootstrapping method [381] to compute the 95\% confidence interval $(95 \% \mathrm{CI})$ for each area under the ROC $\left(A_{z}\right)$ was employed. To carry out the bootstrapping method, a sample $(\mathrm{n}=50)$ was randomly drawn, with replacement, from the original data sets. Then, the $k_{n}$-classification was conducted based on individual perfusion indexes as well as the augmented indexes using this bootstrapping sample. The procedure was repeated 1,000 times and the $A_{z}$ was computed each time.

Whereas in many indications it is adequate to consider perfusion over the entire cortex, it is conceivable that a vascular insult can be confined to a local territory. Thus, for visual local assessment, the perfusion indexes can be displayed as parametric maps, on a pixel-by-pixel basis, and overlayed on an anatomic image. Briefly, for the registered images each perfusion index is computed over the entire time series for a given pixel, and then mapped to the corresponding pixel of the anatomic image.

\section{PERFORMANCE EVALUATION AND VALIDATION}

The proposed framework consists of the two main image processing steps: segmentation and nonrigid registration. Performance evaluation at each stage is outlined below.

\section{Evaluating Segmentation Accuracy Using the Dice Metric}

Segmentation accuracy is evaluated using the Dice similarity coefficient (DSC), characterizing the agreement between the segmented and ground truth regions. Mathemat- 
ically, the DSC is defined as [382]:

$$
\mathrm{DSC}=\frac{2 \cdot \mathrm{TP}}{2 \cdot \mathrm{TP}+\mathrm{FP}+\mathrm{FN}}
$$

where TP, FP, and FN denote the true positive, false positive, and false negative segmentation results, respectively (see Figure 43). For a segmented region, S, and its ground truth, $\mathbf{G}, \mathrm{TP}=|\mathbf{S} \cap \mathbf{G}|$ is the area of their overlap, i.e., the number of the common points in $\mathbf{S}$ and $\mathbf{G} ; \mathrm{FP}=|\mathbf{S}-\mathbf{S} \cap \mathbf{G}|$ is the number of points in the difference between $\mathbf{S}$ and TP, and $\mathrm{FN}=|\mathbf{G}-\mathbf{S} \cap \mathbf{G}|$ is the number of points in the difference between $\mathbf{G}$ and TP (see Figure 43). The closer the DSC to "1", the better the segmentation. To obtain the ground truth of the segmentation, an MRI expert delineated the kidney borders.

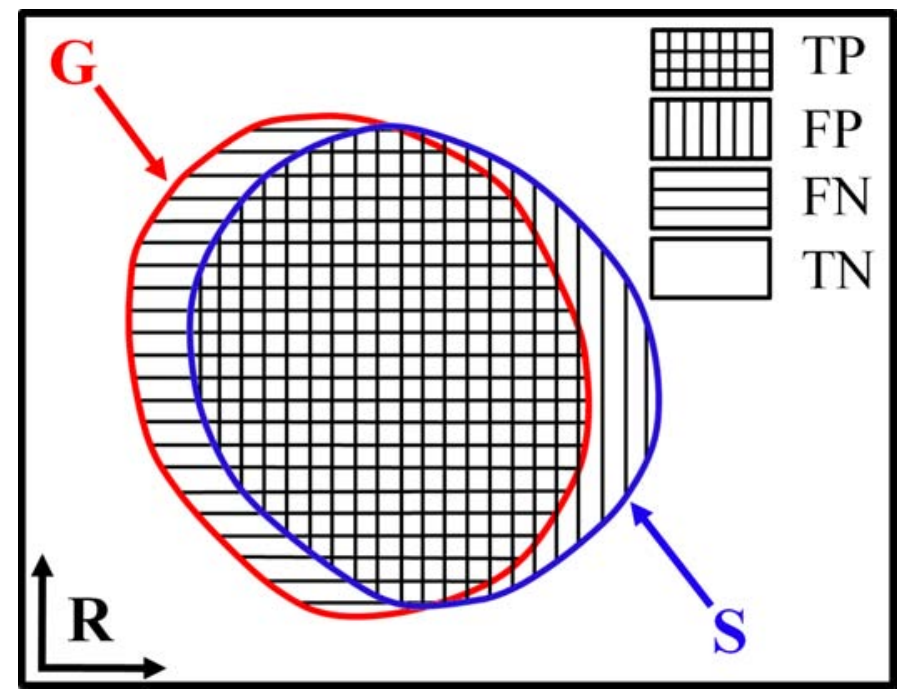

FIGURE 43: Measuring segmentation errors between the ground truth $\mathrm{G}$ and automatic segmentation $\mathbf{S}$.

2. Evaluating and Validating Accuracy of Nonrigid Registration

The accuracy of the proposed nonrigid registration is evaluated using both synthetic phantoms and in-vivo grayscale DCE-MRI data. For synthetic phantoms with inner and outer borders (see Figure 53), the registration accuracy is assessed for each inner border point of the phantom by a relative error metric, $e$. It is measured by the Euclidean distance 
between the known "ground-truth" outer point and the correspondences found by solving the Laplace equation ( $d_{1}$ in Figure 44). This relative error $e$ is defined with respect to the local distance, i.e., the Euclidian distance between this inner point and its corresponding "ground-truth" outer point ( $d_{2}$ in Figure 44$): e=\left(\frac{d_{1}}{d_{2}}\right) \%$. For real MRI data, the registration accuracy was evaluated using expert-identified anatomical landmarks in both the reference and target grayscale images (see Figure 50). After the correspondences were identified, the proposed nonrigid registration aligned the target to the reference, and the registration accuracy was assessed for each expert-identified target point. The accuracy was evaluated by the Euclidian distance between the registered position for each target point and its corresponding ground truth point on the reference image (see Figure 50)

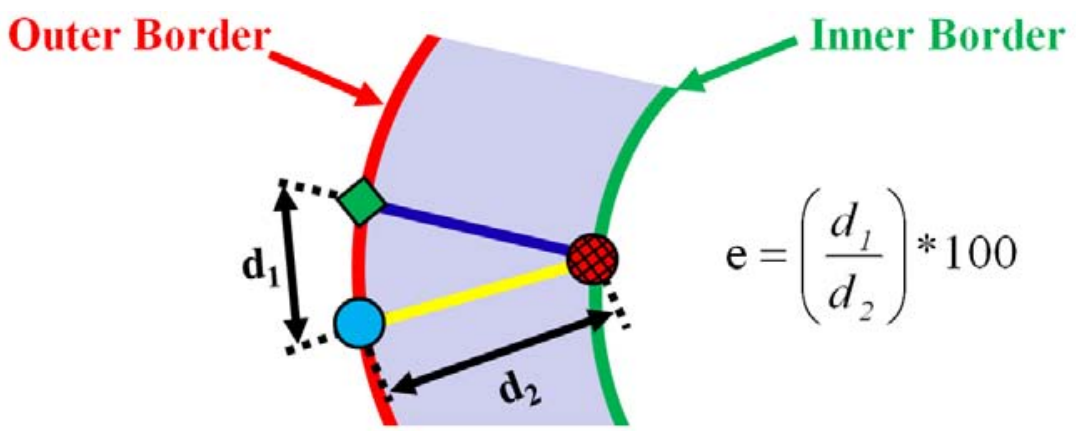

Inner Point

FIGURE 44: Illustration of the relative error $e=100 \cdot\left(d_{1} / d_{2}\right) \%$ measurement for the evaluation of nonrigid registration.

\section{E. PATIENT DATA AND MRI ACQUISITION PROTOCOL}

The proposed framework has been tested on 2D DCE-MRI data sets collected from 50 subjects ( 35 men and 15 women from 10 to 56 years old and with a mean age of $31 \pm 11$ years). Both the rejection (23 subjects) and non-rejection (27 subjects) groups, as part of the usual care after transplant, were routinely assessed with serum creatinine laboratory values. All non-rejection cases had a GFR above 30 milliliters/minute $(\mathrm{ml} / \mathrm{min})$. Patients 
manifesting renal dysfunction, as evidenced by values greater than 1.3 millilgrams/liter $(m g / L)$, were subsequently evaluated clinically using ultrasound to assess the kidney size and to exclude hydronephrosis. A Doppler study was also done for vascular patency. All patients were subsequently underwent ultrasound-guided biopsy, based on their clinical indication, as the gold standard. DCE-MRI was done before any biopsy procedure.

DCE-MRI was performed using temporal sampling adequate to characterize the transit of the CA during the first-pass, while maintaining adequate spatial resolution for visualizing the anatomy. A gradient-echo T1 imaging was employed using a $1.5 \mathrm{~T}$ MRI scanner (Signa Horizon LX Echo speed; General Electric Medical Systems, Milwaukee, WI, USA) with a phased-array torso surface coil. Gadoteric acid (Dotarem $0.5 \mathrm{mmol} / \mathrm{mL}$; Guerbet, France) CA was injected at the rate of 3-4 $\mathrm{ml} / \mathrm{sec}$, at the dose of $0.2 \mathrm{ml} / \mathrm{kilogram}$ body weight $(\mathrm{kgBW})$. Imaging parameters were: slice thickness 5 millimeter $(\mathrm{mm})$; TR = 30-40 millisecond $(\mathrm{msec}) ; \mathrm{TE}=2-3 \mathrm{msec}$; flip angle $70^{\circ} ; \mathrm{FOV}=38 \times 38$ centimeter $^{2}\left(\mathrm{~cm}^{2}\right)$, and matrix size $=256 \times 160$. For each patient, a single coronal section at the renal hilum level of the transplanted kidney was used and approximately 80 repeated temporal frames were obtained at $3 \mathrm{sec}$ intervals to obtain representative sampling to characterize perfusion.

\section{F. EXPERIMENTAL RESULTS}

\section{Kidney Segmentation}

The proposed CAD system was tested on the above DCE-MRI data from 50 subjects. The marginal intensity distributions of the MR images have two dominant modes: one mode for the kidney, and another mode for the abdomen and darker tissues. Figure 45 illustrates basic steps of building the LCDG models of both modes. Sample results of kidney segmentation for five independent subjects are shown in the first row of Figure 46. To highlight the advantages of using all three image features for level set segmentation, the test images were also segmented using (i) the shape prior only and (ii) the combined intensity and shape information. One of these results is shown in Figure 47, and the overall 


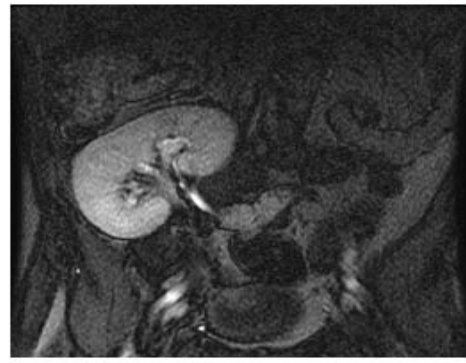

(a)

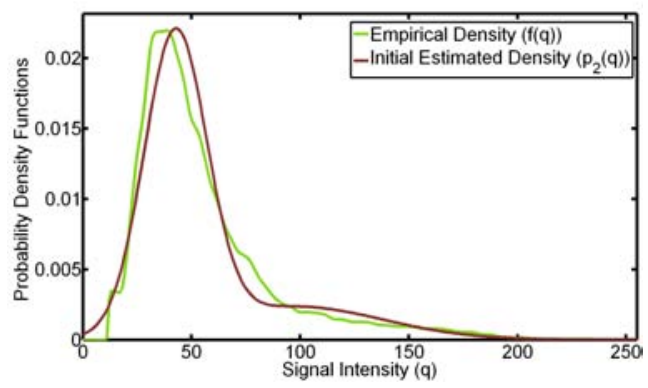

(b)

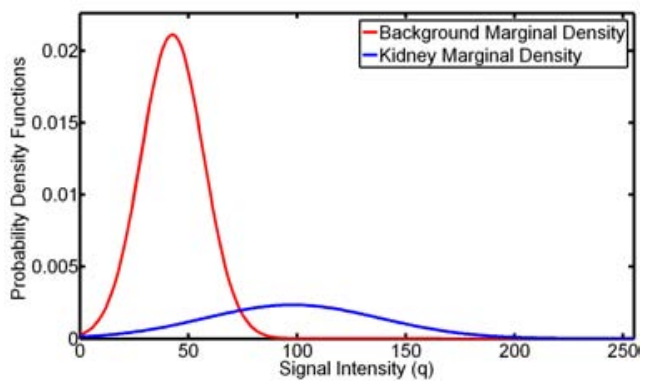

(c)
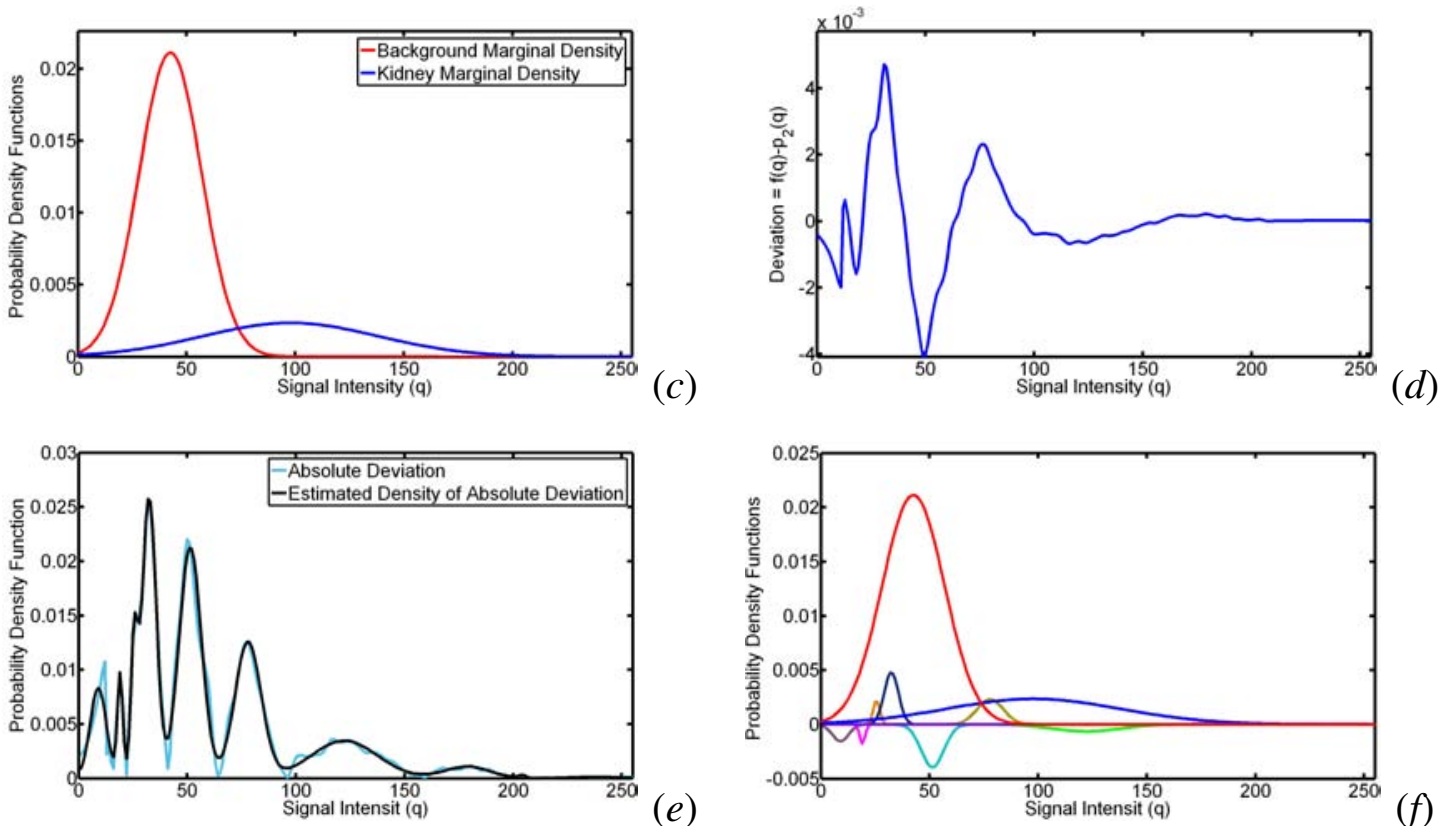

(e)
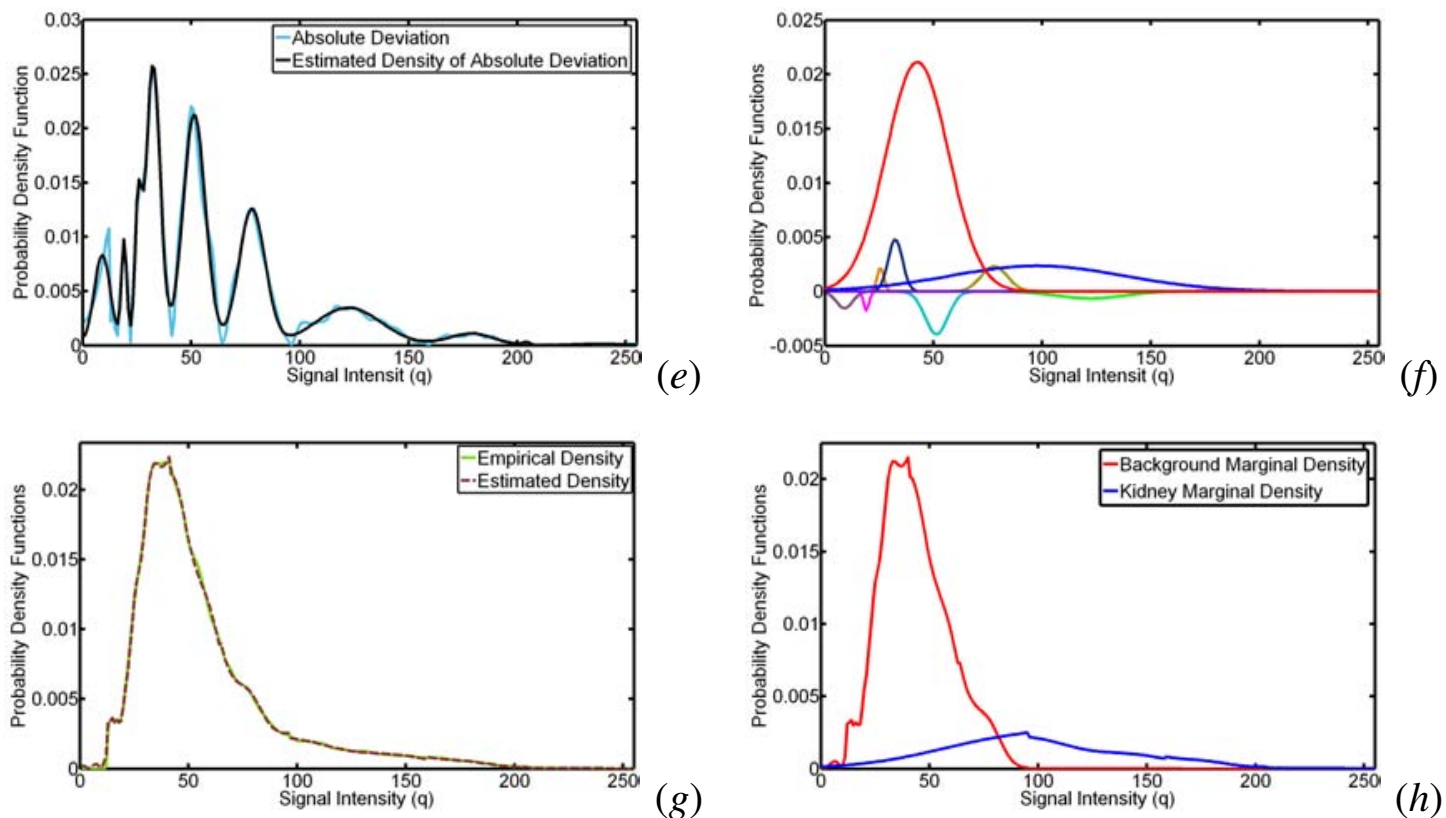

$(g)$

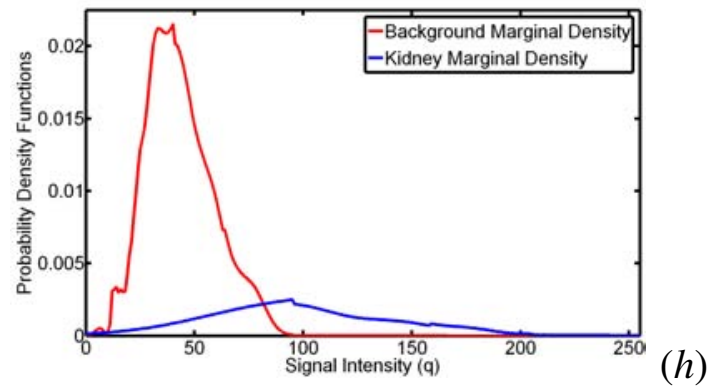

FIGURE 45: A typical DCE-MRI kidney image (a); and estimated density $(b)$ using only two dominant Gaussian components $(c)$, deviation between empirical and estimated densities $(d)$, estimated density of absolute deviation $(e)$ LCDG components $(f)$, final estimated density $(g)$, and the final estimated marginal density for each class $(h)$.

segmentation accuracy is summarized in Table 4 using the DSC with respect to ground truth (manual expert contouring). Figure $47(b)$ clearly demonstrates that the use of the shape information only as a guidance for the deformable model leads to high segmen- 
tation errors due to the variabilities of the learned prior with respect to the mean shape. Adding intensity information to shape prior allows the evolving contour to address the shape variabilities, thus enhancing the results as shown in Figure 47 (c). Finally, adding the higher-order spatial interactions to the intensity and shape features minimizes, to a large extent, the segmentation errors around the kidney edges. The results in Figure $47(d)$ thus highlight the advantages of the proposed integration of the weighted adaptive shape prior, kidney/background marginal intensity distributions, and up to the fourth-order spatial interaction characteristics into the level-set based segmentation.

(a)
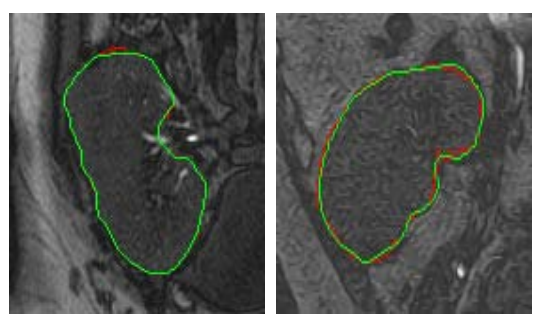

(b)
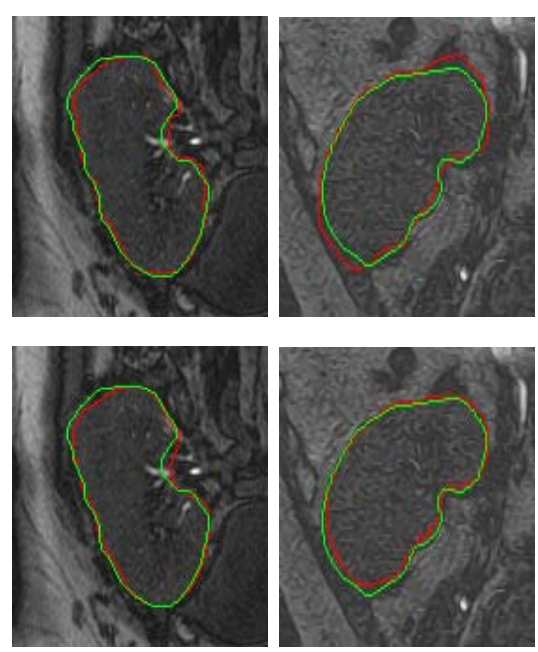
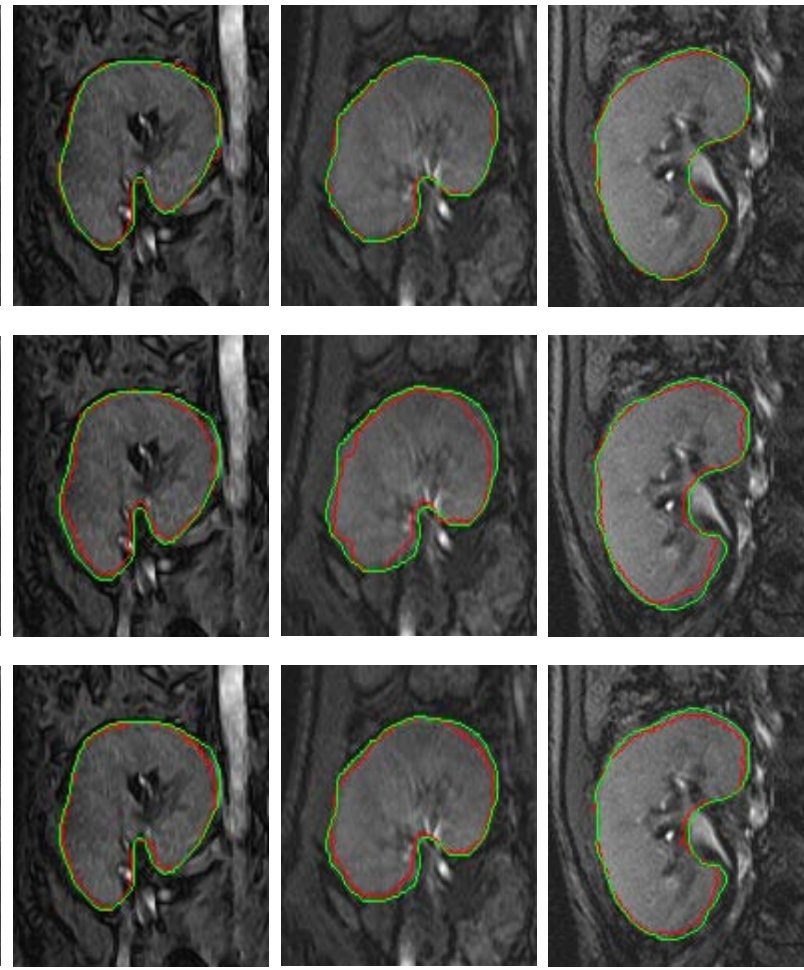

FIGURE 46: Segmentation results of the proposed level set-based approach $(a)$ in comparison with the shape-based (SB) approach (b) by Tsai et al. [1], and the vector level set (VLS) approach $(c)$ by Abdelmunim and Farag [2]. The segmented kidney boundary is in red, and the manual expert's segmentation is in green.

To show the advantage of the proposed level set-based segmentation, the proposed method is compared with the shape-based (SB) approach [1] and the vector level set (VLS) approach [2]. Figure 46 compares qualitatively the accuracy of these segmentation meth- 


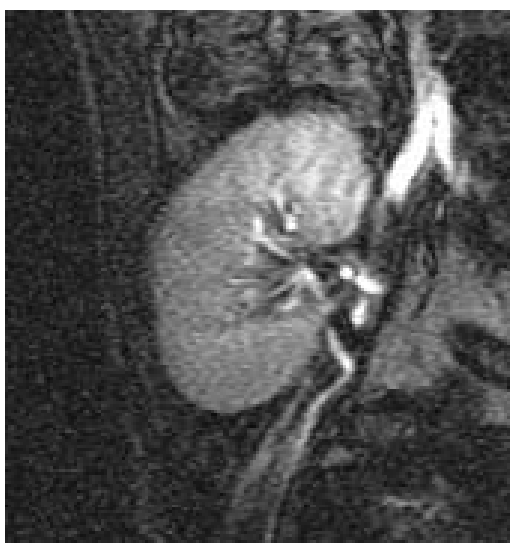

(a)

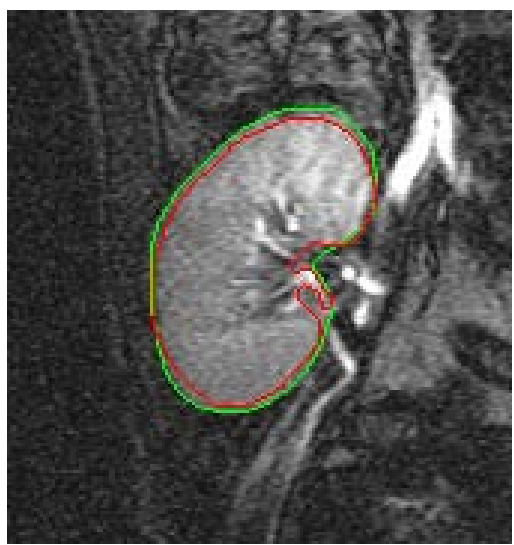

(c)

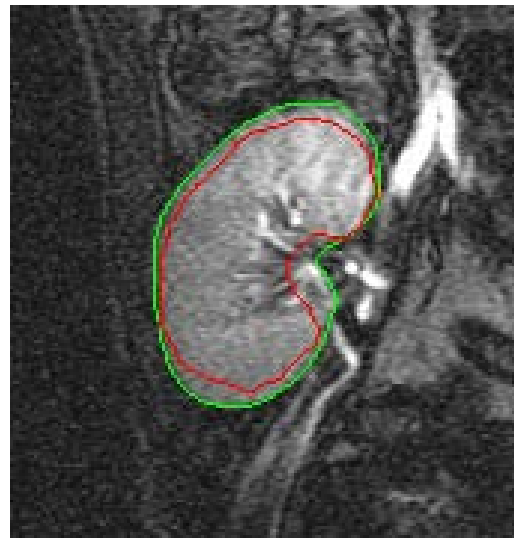

(b)

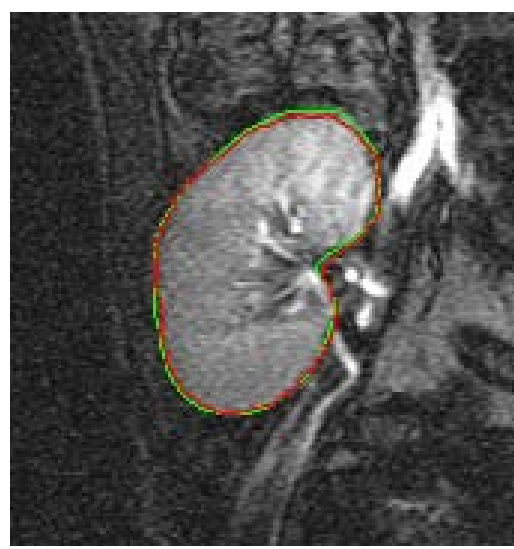

$(d)$

FIGURE 47: (a) Typical MRI slice to be segmented; and the segmentation results using $(b)$ the shape prior information only, $(c)$ the combined intensity and shape prior information, and $(d)$ the proposed approach. The segmented kidney boundary is in red, and the manual expert's segmentation is in green.

ods for sample data from five independent subjects, with respect to the ground truth. The ground truth is obtained by manually contouring the kidney borders by an imaging expert. Differences between the mean DSC for the proposed level set segmentation and other approaches in Table 5 are statistically significant using the paired $t$-test. Moreover, Table 5 demonstrates the comparison to the previous work in [3] and shows clear preference to use the adaptive shape in addition to the high-order MGRF model of the kidney region. 
TABLE 4: Kidney segmentation accuracy for the proposed level set segmentation (combined shape prior, higher-order MGRF, and intensity features) versus the level set segmentation based on the shape prior only or the combined shape prior and intensity information (“SD" stands for standard deviation).

\begin{tabular}{|l|c|c|}
\cline { 2 - 3 } \multicolumn{1}{c|}{} & DSC (Mean \pm SD) & $P$-value \\
\hline Level set with shape prior only & $0.884 \pm 0.023$ & $\leq 10^{-4}$ \\
Level set with combined shape and intensity & $0.928 \pm 0.021$ & $\leq 10^{-4}$ \\
The proposed level set approach & $0.982 \pm 0.016$ & \\
\hline
\end{tabular}

TABLE 5: Accuracy of the proposed level-set segmentation of the kidney versus the shapebased [1], the vector level set [2], and the second-order MGRF [3] approaches in comparison to the expert's ground truth using the Dice metric ("SD" - standard deviation).

\begin{tabular}{|l|c|l|}
\cline { 2 - 3 } \multicolumn{1}{c|}{} & DSC (Mean \pm SD) & $P$-value \\
\hline The proposed levelset approach & $0.982 \pm 0.016$ & \\
Previous approach [3] & $0.943 \pm 0.028$ & $\leq 10^{-4}$ \\
Shape-based (SB) approach [1] & $0.914 \pm 0.034$ & $\leq 10^{-4}$ \\
Vector level set (VLS) approach [2] & $0.902 \pm 0.083$ & $\leq 10^{-4}$ \\
\hline
\end{tabular}

\section{Nonrigid Registration}

After segmenting the DCE-MRI time series, the proposed Laplace-based nonrigid registration is applied to correct for the geometric kidney deformations by establishing point-to-point correspondences between the segmented kidney objects. As an intuitive visualization tool, Figure 48 presents checkerboard displays for four kidney samples before and after applying nonrigid registration (the reference and target objects are in a light and dark hues, respectively). This display uses the net contour as a metric of the agreement between the superimposed objects. The coinciding edges after registration demonstrate that the proposed Laplace-based approach effectively reduces the misregistration effects. 
The effect of registration on the physiological data of a typical time-intensity curve for one subject is also shown in Figure 49, illustrating the reduction of frame-to-frame signal intensity variability related primarily to motion effects.

A
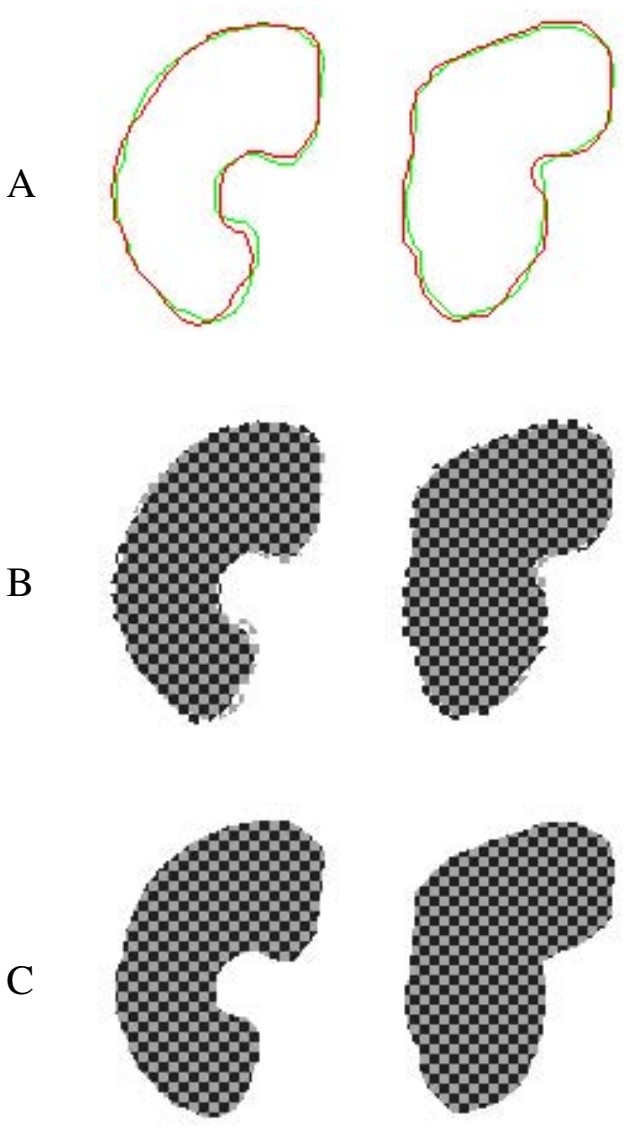

(a)

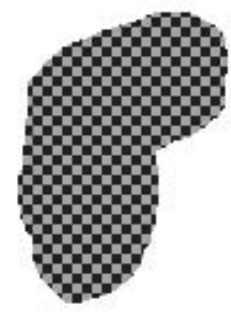

(b)
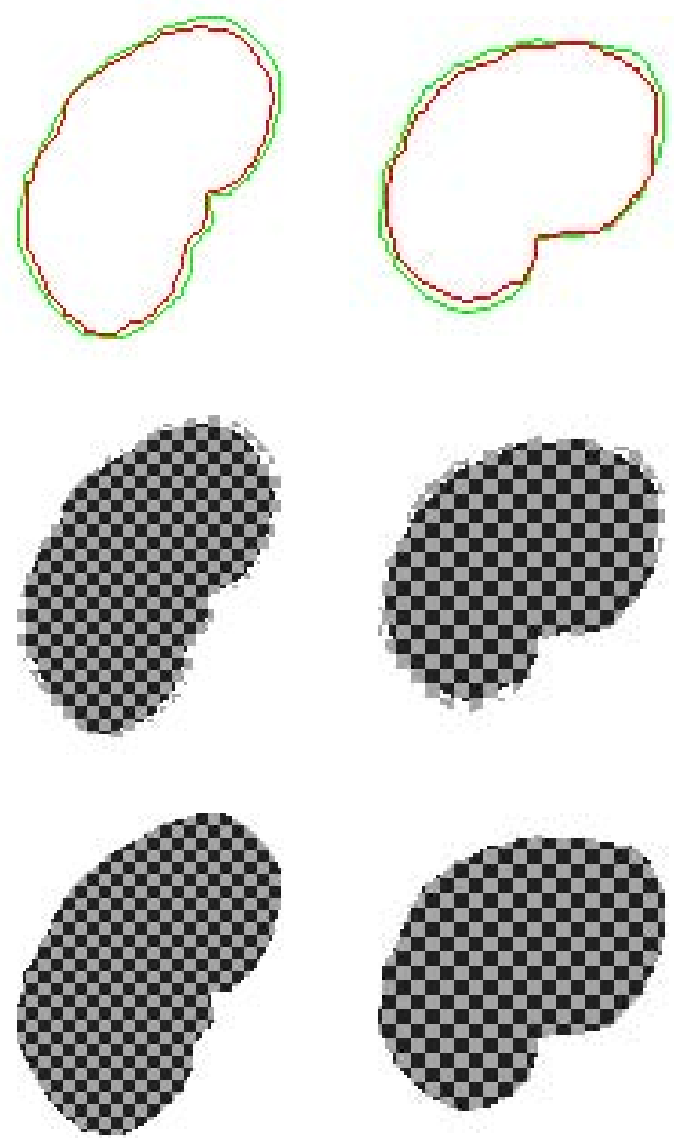

$(c)$

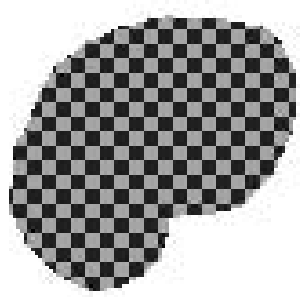

(d)

FIGURE 48: Superimposed reference (green) and target (red) kidney borders (A); and checkerboard displays before (B) and after (C) nonrigid registration for four independent subjects with $(a, b)$ and without $(c, d)$ overlapping edges.

In addition to checkerboard visual assessment and frame-to-frame signal intensity variability reduction, the accuracy of the proposed nonrigid registration was evaluated using anatomical landmark correspondences selected by a MRI expert using grayscale images (see Figure 50). The accuracy was assessed using the Euclidean distance metric defined in Section III.D.2. To account for inter-observer variability for landmark selection, the anatomical correspondences on both reference and target images were identified by three 


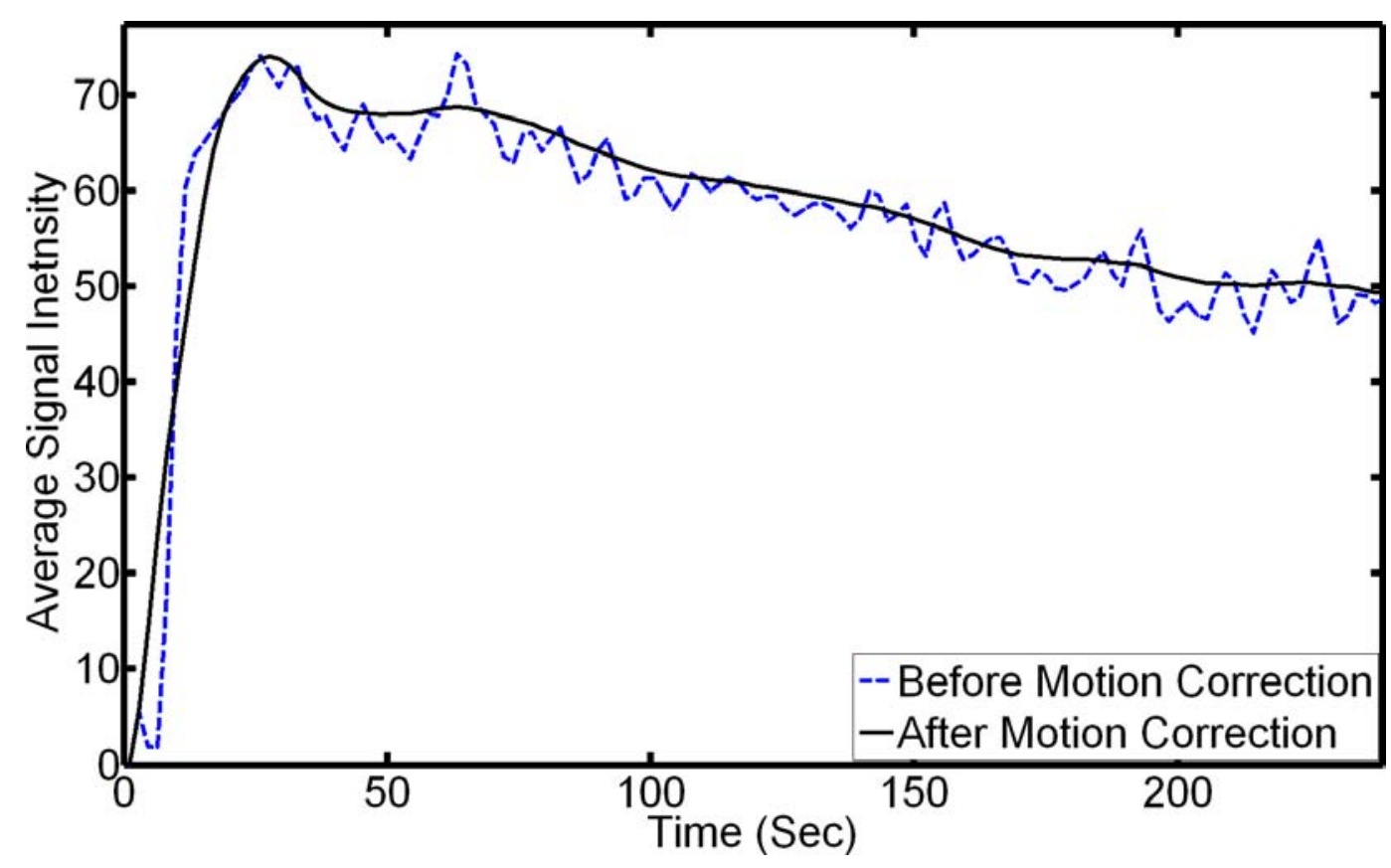

FIGURE 49: Typical time-intensity curve for one data set before and after registration.

independent experts (denoted below OB1, OB2, and OB3). The statistical paired $t$-test confirmed that differences between the alignment accuracy for the experts' annotations are insignificant (see $P$-values in Table 6). The advantages of the proposed approach are also highlighted by the comparison of the above summarized error statistics to the B-splines (BS) approach [4] using the paired $t$-test. The results in Table 7 show that improvements of the registration accuracy for all three observers in this experiment were statistically significant.

In addition, the alignment accuracy of the three observers' annotations was assessed using the Bland-Altman analysis [383]. For evaluation, the bias (mean difference) and the 95\% limits of agreement ( \pm 1.96 standard deviation around the bias) between the results of each two observers were calculated. The results are summarized in Figure 51 and show that the observers' accuracies are in close agreement for the proposed nonrigid registration and the BS approach [4] individually. It is evidenced by the near-zero biases of the observation $(-0.489,-0.134$, and -0.623 for the proposed approach; and $-0.137,-0.286$, and -0.423 for the BS approach), most data points fall within the $95 \%$ limits of agreement, and the small 


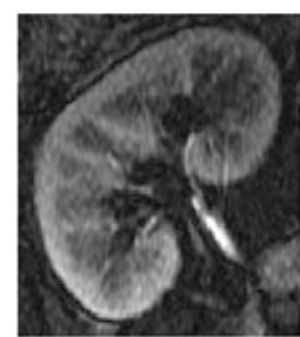

Reference

MRI of the kidney

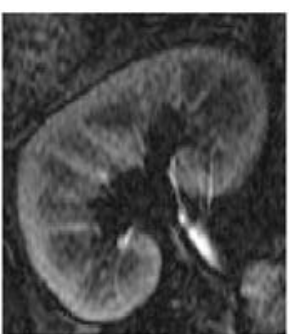

Target

MRI of the kidney

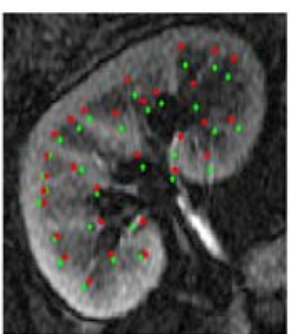

Superimposed Landmarks

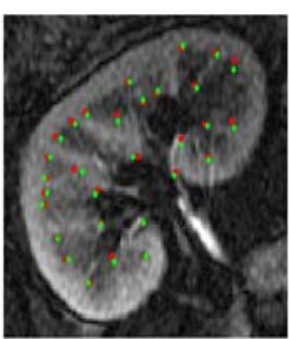

Proposed Approach Registration

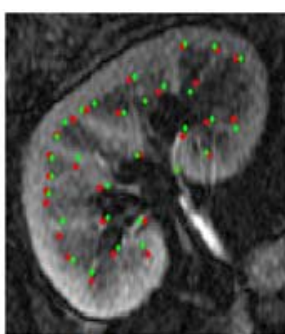

BS Approach Registration

FIGURE 50: Validating the proposed nonrigid registration and the B-Splines (BS) approach [4] using the corresponding anatomical landmarks identified by an expert in both the reference (red; $\bullet$ ) and target (green; $\diamond)$ images.

TABLE 6: Comparison of inter-observer variability in annotating corresponding anatomical landmarks for the proposed nonrigid registration and the B-splines approach [4], for real data using 30 data points ("SD" - standard deviation and "OB" - observer).

\begin{tabular}{|l|c|c|c|c|}
\cline { 2 - 5 } \multicolumn{1}{c|}{} & \multicolumn{4}{c|}{ Alignment error (in mm) } \\
\cline { 2 - 5 } \multicolumn{1}{c|}{} & \multicolumn{2}{c|}{ Proposed Approach } & \multicolumn{2}{c|}{ B-Splines Approach } \\
\hline Observer & Mean \pm SD & $P$-value & Mean \pm SD & $P$-value \\
\hline OB1 & $2.67 \pm 1.95$ & $(\mathrm{OB} 1, \mathrm{OB} 2): 0.23$ & $4.43 \pm 2.88$ & $(\mathrm{OB} 1, \mathrm{OB} 2): 0.76$ \\
\hline OB2 & $3.16 \pm 1.69$ & $(\mathrm{OB} 2, \mathrm{OB} 3): 0.73$ & $4.57 \pm 2.57$ & $(\mathrm{OB} 2, \mathrm{OB} 3): 0.42$ \\
\hline OB3 & $3.30 \pm 1.53$ & $(\mathrm{OB} 1, \mathrm{OB} 3): 0.09$ & $4.86 \pm 2.44$ & $(\mathrm{OB} 1, \mathrm{OB} 3): 0.10$ \\
\hline
\end{tabular}

TABLE 7: Comparison of the proposed nonrigid approach versus B-splines approach [4] for landmarks identified by three independent observers ("SD" - standard deviation).

\begin{tabular}{|l|c|c|c|}
\cline { 2 - 4 } \multicolumn{1}{c|}{} & \multicolumn{3}{c|}{ Alignment Error in mm (Mean \pm SD) } \\
\hline Approach & OB1 & OB2 & OB3 \\
\hline Proposed Approach & $2.67 \pm 1.95$ & $3.16 \pm 1.69$ & $3.30 \pm 1.53$ \\
\hline B-Splines Approach & $4.43 \pm 2.88$ & $4.57 \pm 2.57$ & $4.86 \pm 2.44$ \\
\hline$P$-value & 0.0005 & 0.0143 & 0.0019 \\
\hline
\end{tabular}




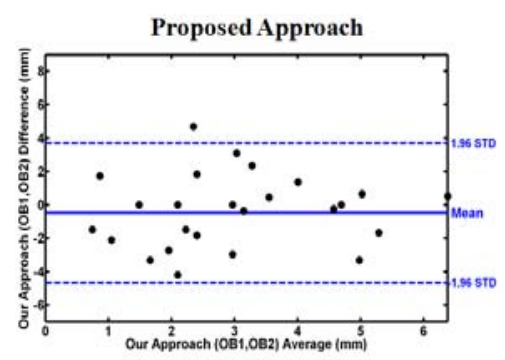

(a)

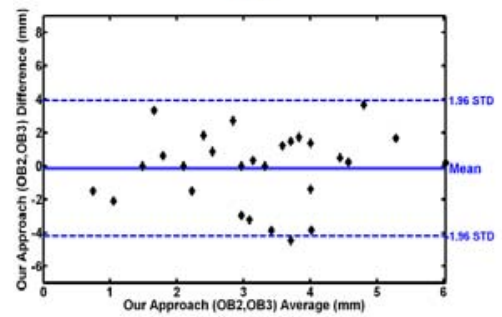

(d)

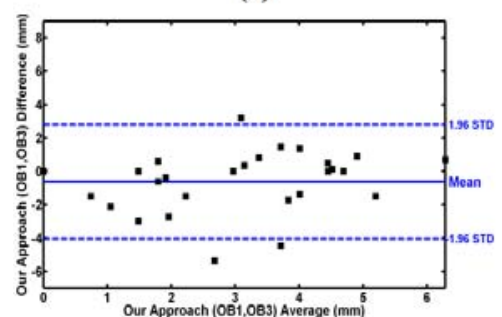

(g)

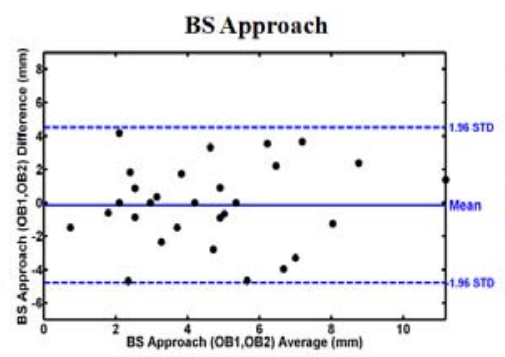

(b)

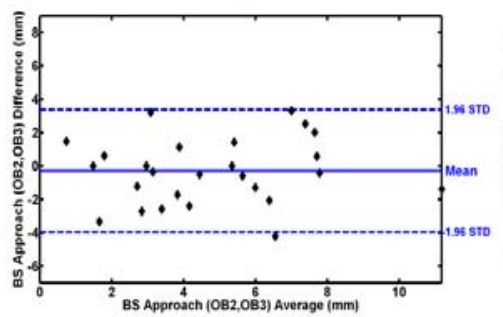

(e)

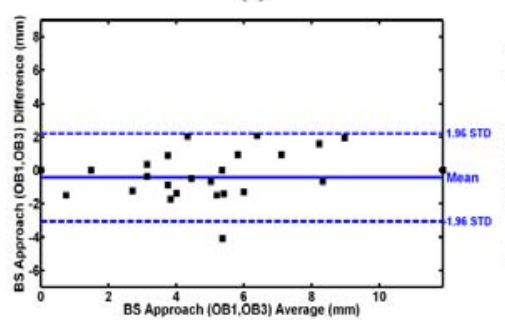

(h)

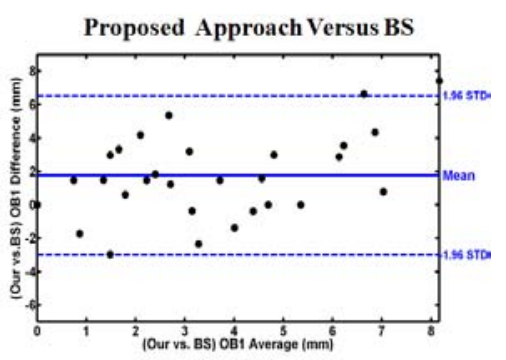

(c)

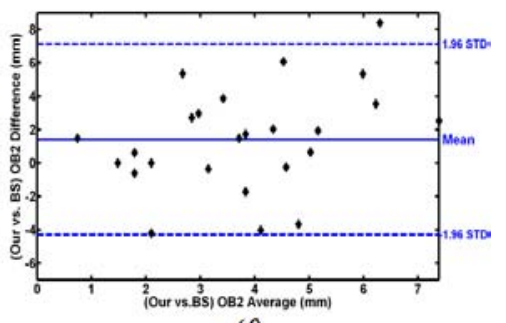

(f)

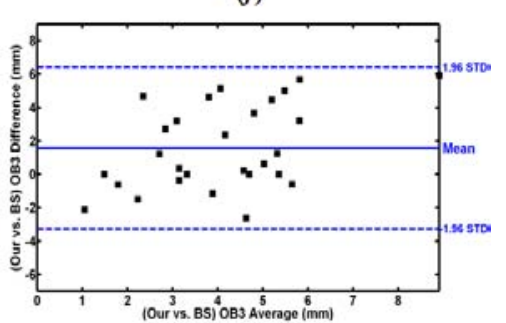

(i)

FIGURE 51: Bland-Altman plots for alignment errors with respect to annotations of the three observers (OB1, OB2, and OB3). Each plot shows the difference errors ( $y$-axis) versus their average ( $x$-axis) and the $95 \%$ confidence interval $(95 \% \mathrm{CI} ; \pm 1.96 \mathrm{SD}$, dotted lines) for inter-observer comparison for $\mathrm{OB} 1$ and $\mathrm{OB} 2(a, b), \mathrm{OB} 2$ and $\mathrm{OB} 3(d, e)$, and OB 1 and $\mathrm{OB} 3(g, h)$. The comparison between the proposed nonrigid registration and the B-splines (BS) approach [4], for OB1, OB2, and OB3 annotations are shown in $(c),(f)$, and $(i)$, respectively. For close agreement the bias (mean, solid lines) should be near zero, while the data points should fall within the $95 \%$ CI. These plots document that while there is good inter-observer agreement for both the proposed approach and the BS approach, it also demonstrates the significant differences in performance between both methods.

range of the $95 \%$ agreement interval. Contrastingly, the large biases $1.763,1.411$, and 1.562 in Figure $51(c),(f)$, and $(i)$, respectively, demonstrate the significant differences between the observers' results of the proposed approach registration versus the BS approach. 
To demonstrate that the proposed nonrigid registration is not affected by nonlinear intensity variations due to agent transit, an experiment is conducted using MR images at different stages of contrast enhancement phases (baseline, peak-intensity, and tissue distribution) of the DCE-MRI time series (see Figure 52). Anatomical landmark correspondences in both the reference image (selected from the peak-intensity phase) and all target images were chosen once again by an imaging expert. Then, the proposed nonrigid registration aligned all target images to the reference, and the registration accuracy was assessed just as described in Section III.D.2. The results in Figure 52 and error statistics summarized in Table 8 justify that the proposed nonrigid registration is independent of intensity variations over the given temporal image.
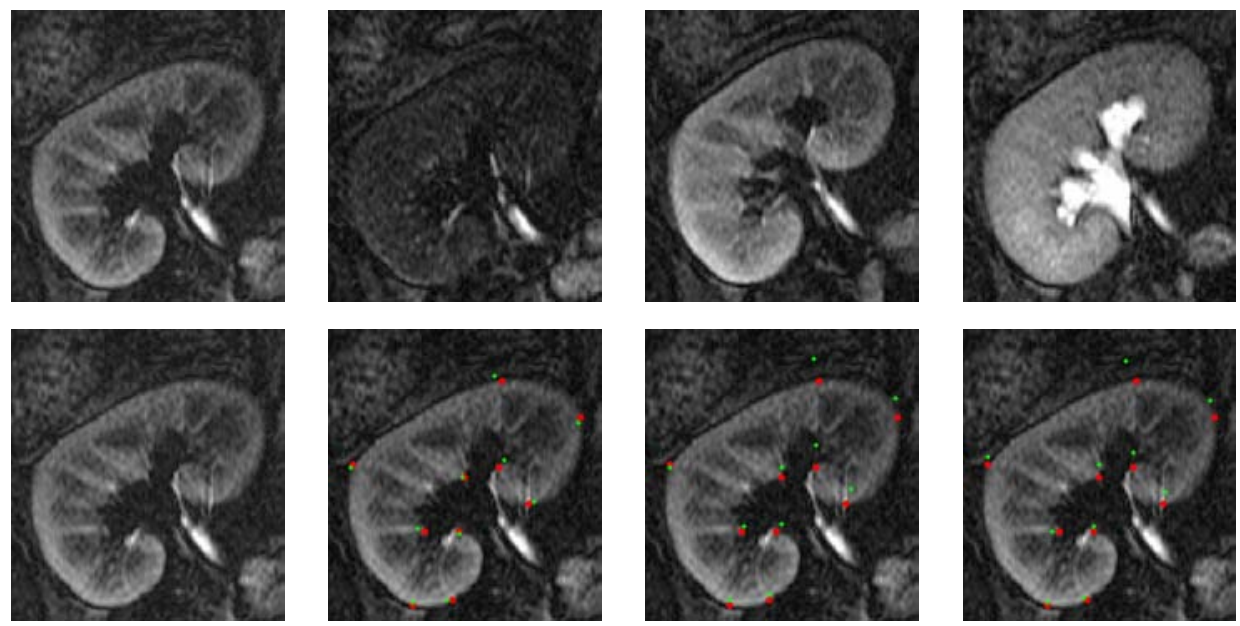

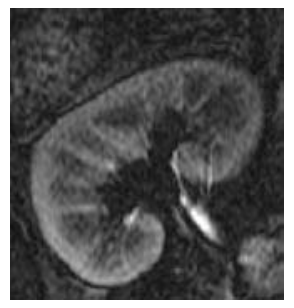

(a)

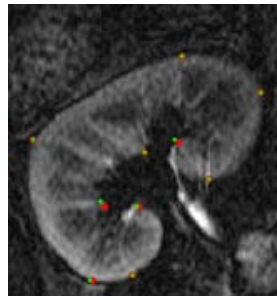

(b)

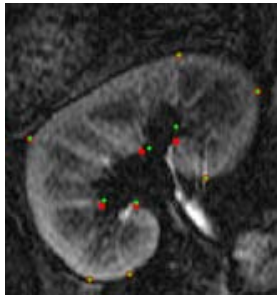

(c)

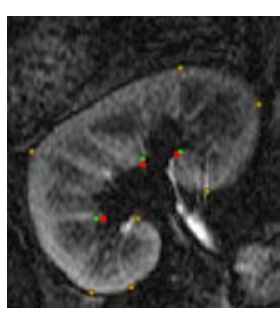

(d)

FIGURE 52: Validating the proposed nonrigid registration with the anatomical landmarks identified by an expert for different phases of contrast-enhancement. Top row: the reference $(a)$, baseline $(b)$, peak-intensity $(c)$, and tissue distribution $(d)$ images. Middle row: superimposed expert-identified correspondences for both the reference (red; •) and target (green; $\checkmark$ ) images. Bottom row: the registration results obtained by the proposed approach. 
TABLE 8: Alignment errors (in $\mathrm{mm}$ ) of the proposed nonrigid registration for the expertidentified landmarks at different phases of contrast enhancement.

\begin{tabular}{|l|c|c|c|c|c|c|}
\cline { 2 - 7 } \multicolumn{1}{c|}{} & \multicolumn{6}{c|}{ Alignment errors (in mm) } \\
\cline { 2 - 7 } \multicolumn{1}{c|}{} & \multicolumn{2}{c|}{ Baseline } & \multicolumn{2}{c|}{ Peak-Intensity } & \multicolumn{2}{c|}{ Tissue Distribution } \\
\cline { 2 - 7 } & Before & After & Before & After & Before & After \\
\hline Maximum & 5.35 & 4.20 & 13.69 & 8.91 & 13.27 & 4.45 \\
Mean & 3.16 & 1.05 & 7.04 & 1.84 & 6.34 & 0.95 \\
SD & 1.55 & 1.57 & 4.66 & 2.30 & 3.91 & 1.63 \\
\hline$P$-Value & \multicolumn{2}{|c|}{0.0035} & 0.0072 & \multicolumn{2}{c|}{0.0030} \\
\hline
\end{tabular}

Because the manual landmark selection may cause inaccurate identification of the corresponding points, the accuracy of the proposed approach was also assessed using synthetic phantoms with known (ground truth) point-to-point correspondences and also by applying simulated deformation to both synthetic and real MRI data.

A synthetic 2D phantom that mimics kidney shape with known (ground-truth) point-to-point correspondences was generated (see Figure 53). The phantom consists of concentric shapes with sharp edges. The registration accuracy was evaluated using the relative error $e$ defined in Section III.D.2. The average relative error over all the ground truth points of the phantom was $0.0 \%$.

Secondly, more realistic phantoms with curved and overlapping edges (see Figure 54) were generated by deforming the border of a manually segmented kidney using the $2 \mathrm{D}$ cubic B-spline model of local transformation [18]. The phantom images were generated by inverse mapping in accord with the 1D marginal probability distribution of the kidney signals in DCE-MRI. Visual appearances of the phantom images in Figure 54 were generated to mimic the varying contrast-enhanced intensity during the agent transit. To assess the accuracy of localizing the point-to-point correspondences of the proposed approach, three types of the deformations (small, moderate, and large) were generated, and the ground truth correspondences were established. Similarly, the error for each point on 


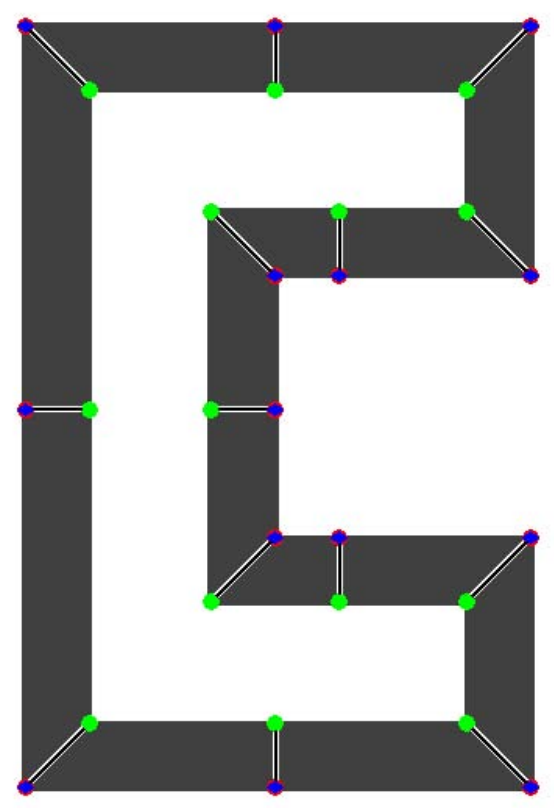

FIGURE 53: Validation of the proposed nonrigid registration on synthetic kidney-shaped phantom with sharp edges: ground truth (white lines) versus estimated (black lines) correspondences.

the un-deformed kidney border was defined by the Euclidian distance between its matching "simulated point" after deformation and the point found by solving the Laplace equation. To highlight the advantages of the proposed nonrigid registration, the deformed images were registered using an affine transformation with six degrees of freedom (two coordinatewise scales and shifts, one rotation angle, and one shear parameter). Results for the three types of deformations, at different contrast-enhancement phases, for the proposed nonrigid approach and the affine registration are shown in Figure 54. The figure clearly demonstrates the advantages of the proposed approach, as evidenced by small errors in co-allocating corresponding points, as well as by the checkerboard overlay displays in Figure 55.

Nonrigid deformations on in-vivo MRI data were obtained by simulating local deformations on a real grayscale MRI using the transformation model proposed in [18]. The registration accuracy was assessed for three types of generated deformation fields (with small, moderate, and large deformations, respectively), as indicated in Table 9. The proposed nonrigid registration was applied to each type of deformation field, and the performance was 


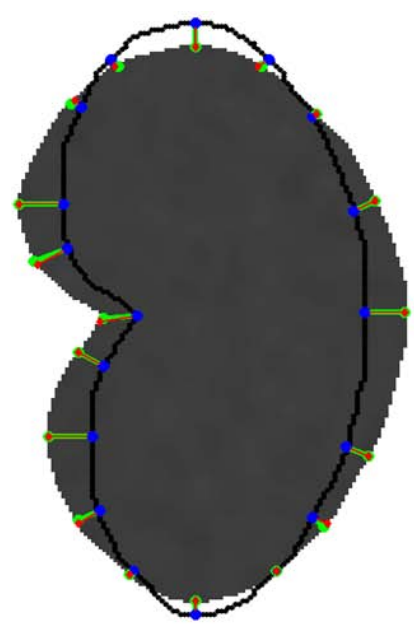

Error: $3.00 \%$

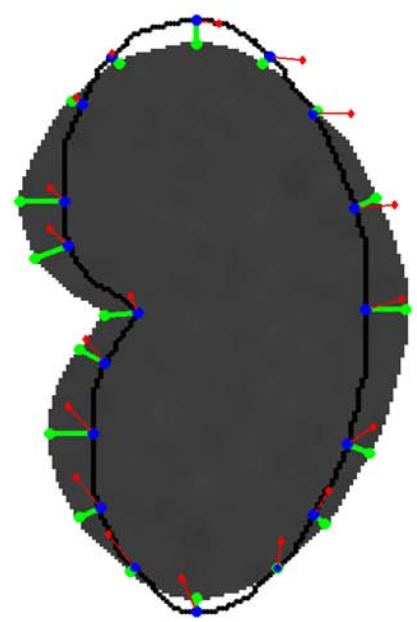

Error: $49.01 \%$

(a)

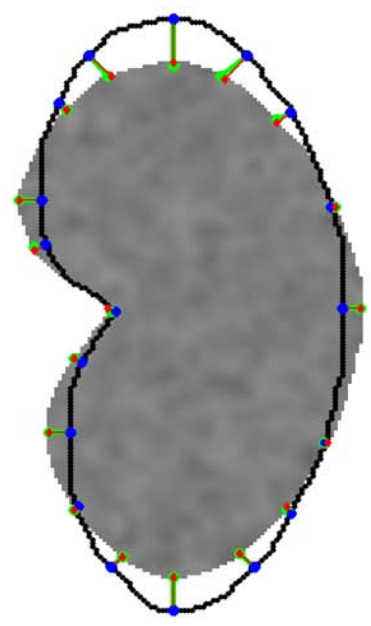

Error: $3.06 \%$

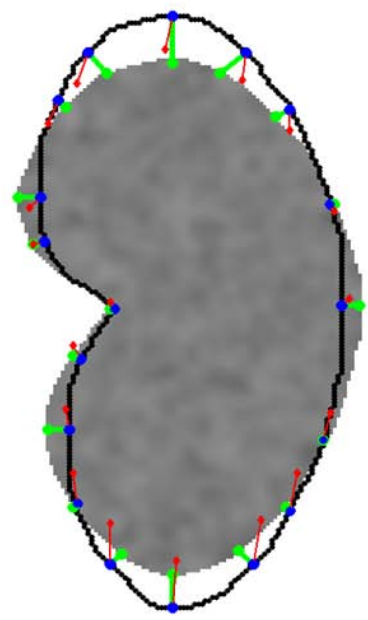

Error: $37.44 \%$

(b)

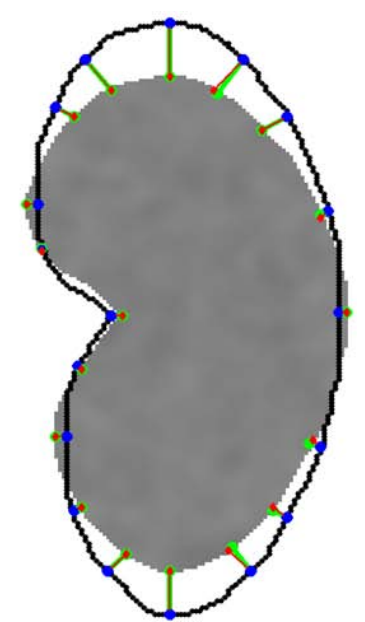

Error: $2.51 \%$

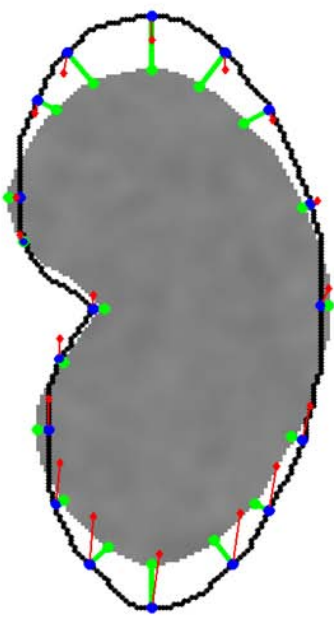

Error: $46.36 \%$

(c)

FIGURE 54: Validation of the proposed nonrigid alignment (top row) versus affine alignment (bottom row) on three realistic simulated kidney-shaped phantoms at different contrast-enhancement stages (from left to right: baseline, peak intensity, and tissue distribution phase). Blue and green circles represent ground truth correspondences on the reference and target objects, respectively. Laplace and affine-determined correspondences are represented by red diamonds. The ground truth and correspondences estimated on the target object are connected to the reference ground truth points by green and red lines, respectively. Small (a), moderate $(b)$, and large ( $c$ ) deformations of a manually segmented kidney (black) were generated by using the 2D cubic B-spline transformation model [18]. 


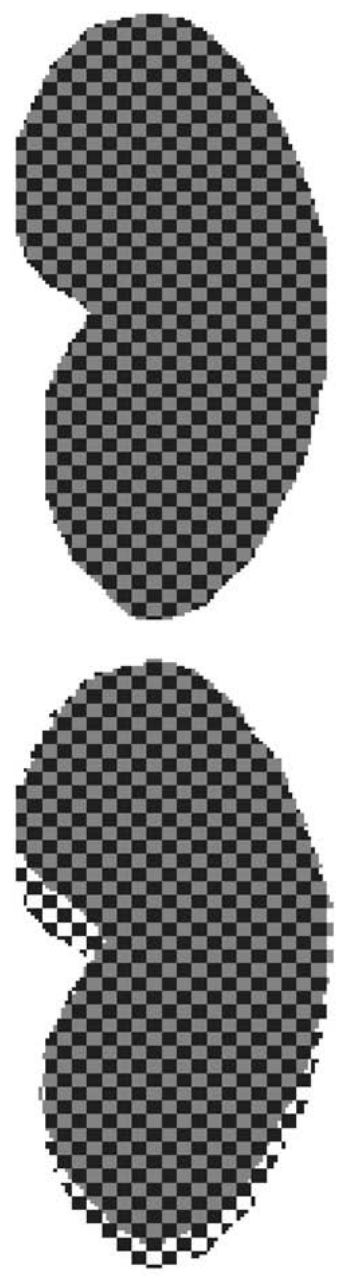

(a)
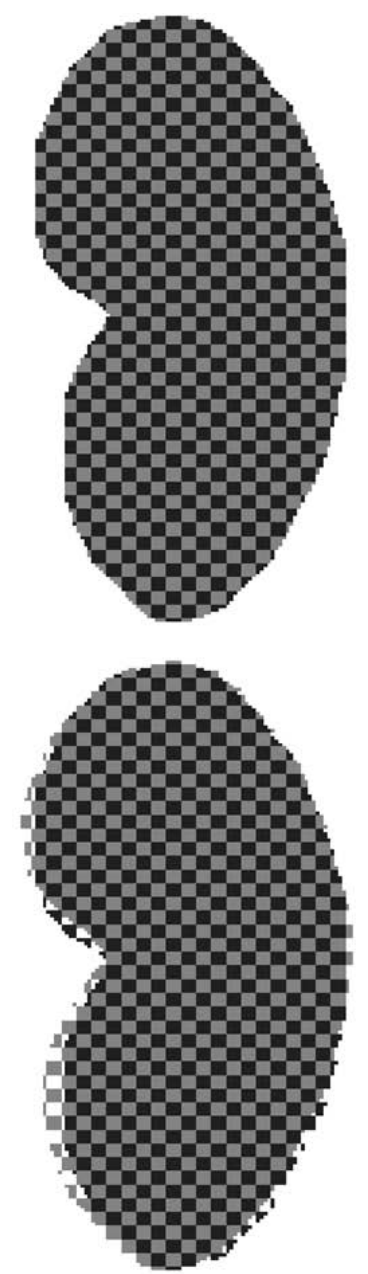

(b)
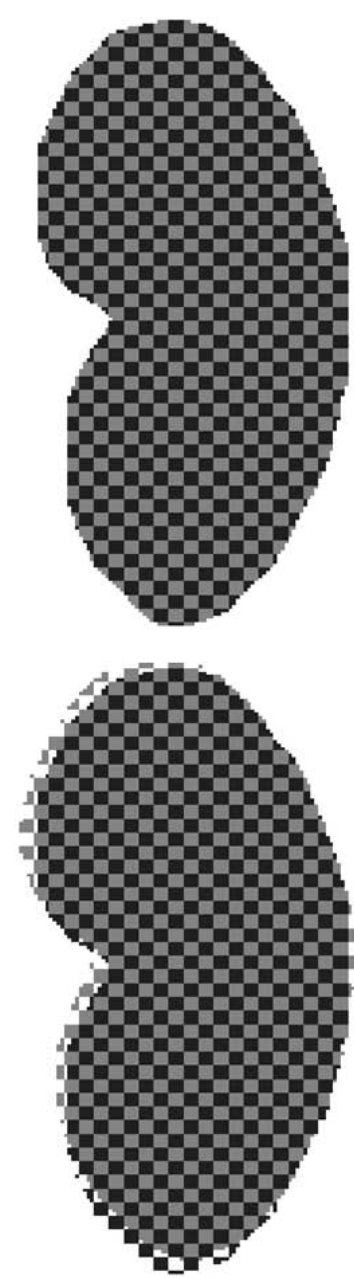

(c)

FIGURE 55: Checkerboard display for the proposed (top row) and the affine registration (bottom row) for small $(a)$, moderate $(b)$, and large $(c)$ deformations of the phantom shown in Figure 54. The reference object is in light gray, and target objects are in dark gray.

quantitatively assessed by comparing the simulated and recovered pixel displacements. The deformed image had also been registered using the multiresolution BS approach by Rueckert et al. [4]. Table 9 summarizes the registration errors in these experiments along with the $P$-values of the paired $t$-test comparing the proposed and the BS approaches. 
TABLE 9: Accuracy of the proposed nonrigid registration approach (PA) versus the Bsplines (BS) approach for displacements simulated with cubic B-splines, for real data using 30 data points ("SD" - standard deviation).

\begin{tabular}{|c|c|c|c|c|c|c|}
\hline & \multicolumn{6}{|c|}{ Simulated displacements (in mm) } \\
\hline & \multicolumn{2}{|c|}{ Small } & \multicolumn{2}{|c|}{ Moderate } & \multicolumn{2}{|c|}{ Large } \\
\hline Maximum & \multicolumn{2}{|c|}{3.33} & \multicolumn{2}{|c|}{9.05} & \multicolumn{2}{|c|}{11.86} \\
\hline Mean & \multicolumn{2}{|c|}{1.68} & \multicolumn{2}{|c|}{6.56} & \multicolumn{2}{|c|}{8.37} \\
\hline $\mathrm{SD}$ & \multicolumn{2}{|c|}{0.95} & \multicolumn{2}{|c|}{1.38} & \multicolumn{2}{|c|}{2.32} \\
\hline & \multicolumn{6}{|c|}{ Alignment errors (in mm) } \\
\hline & PA & BS & PA & $\mathrm{BS}$ & PA & $\mathrm{BS}$ \\
\hline Maximum & 2.97 & 3.33 & 3.31 & 4.69 & 4.69 & 5.94 \\
\hline Mean & 1.25 & 1.75 & 1.63 & 2.52 & 1.87 & 2.95 \\
\hline $\mathrm{SD}$ & 0.92 & 0.77 & 0.94 & 1.38 & 1.13 & 1.50 \\
\hline$P$-Value & \multicolumn{2}{|c|}{0.025} & \multicolumn{2}{|c|}{0.005} & \multicolumn{2}{|c|}{0.003} \\
\hline
\end{tabular}

\section{Cortex Segmentation}

After segmenting the whole kidney from the background and correcting for the local motion, the cortex of the aligned kidney is segmented. Figure 56 shows basic steps of the cortex segmentation for one subject. Since the cortex does not have specific shape, the proposed deformable model is automatically initialized at the center of the segmented kidney region and evolves on the basis of the combined spatial interaction and intensity information. The extracted cortex area (Figure $56(b)$ ) is used as a mask (Figure $56(c)$ ) and is applied to the rest of the time series as shown in Figure $56(f-j)$. To demonstrate the advantages of the proposed cortex segmentation method, a comparison to the most popular level set approach of Chan and Vese [344] is conducted. The DSC values for this comparison, with respect to the ground truth, were $0.937 \pm 0.045$ and $0.814 \pm 0.036$ for the proposed approach and the Chan and Vese approach [344], respectively. 


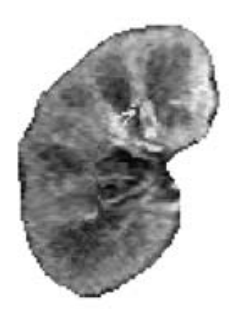

(a)

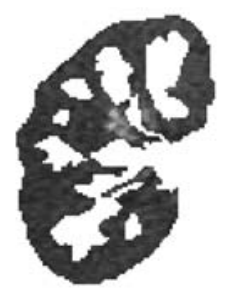

$(f)$

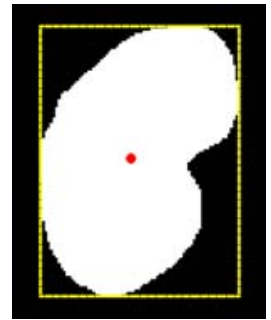

(b)

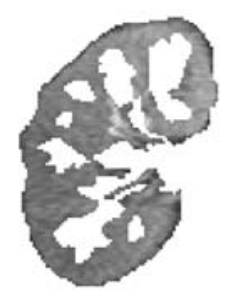

$(g)$

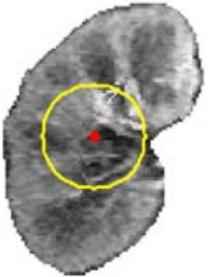

(c)

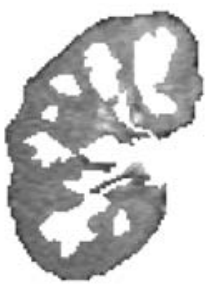

$(h)$

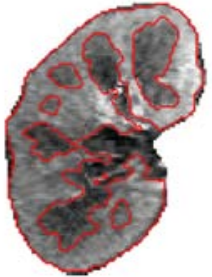

(d)

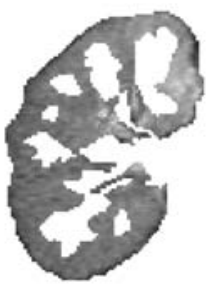

(i)

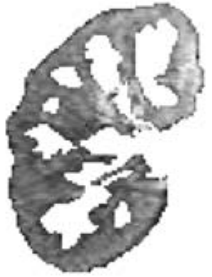

(e)

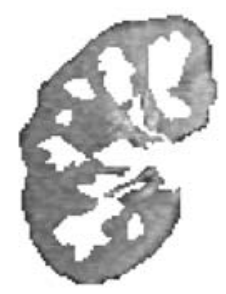

(j)

FIGURE 56: Step-by-step cortex segmentation for one subject: (a) kidney object after nonrigid alignment; (b) automatically-determined bounding box (yellow) that yields the seed point (red) for level set initialization; $(c)$ initial contour (yellow); (d) final contour (red); $(e)$ the extracted cortex mask; and $(f)-(j)$ the propagation of the mask over the registered time series images at representative time instants $6,42,120,180$, and 234 sec, respectively.

4. Perfusion Indexing and Diagnostic Results

The ultimate goal of the proposed CAD system is to provide a reproducible, noninvasive diagnostic tool for reliable detection of renal transplant rejection physiology. In a pathophysiological sense, transplant rejection is believed to have a microvasculature basis. Therefore, the focus is on vascular parameters (or indexes) obtained from the dynamic contrast agent transit, characterized by the signal intensity-time plots of contrast agent kinetics. Plots grouping all rejection versus non-rejection patients (see Figure 57) reveal obvious separation between the two groups both in the transient phase and the more slowly varying phase of bolus contrast agent transit. Due to the few number of data points that characterize the transit first-pass effect, the plateau perfusion parameter was also used because it permits characterization using a large number of averaged data points. This parameter indexes the amount of contrast agent delivered to the tissue and approximates the area under the signal 
intensity-time curve, namely a parameter related to intra-vascular blood volume [87].

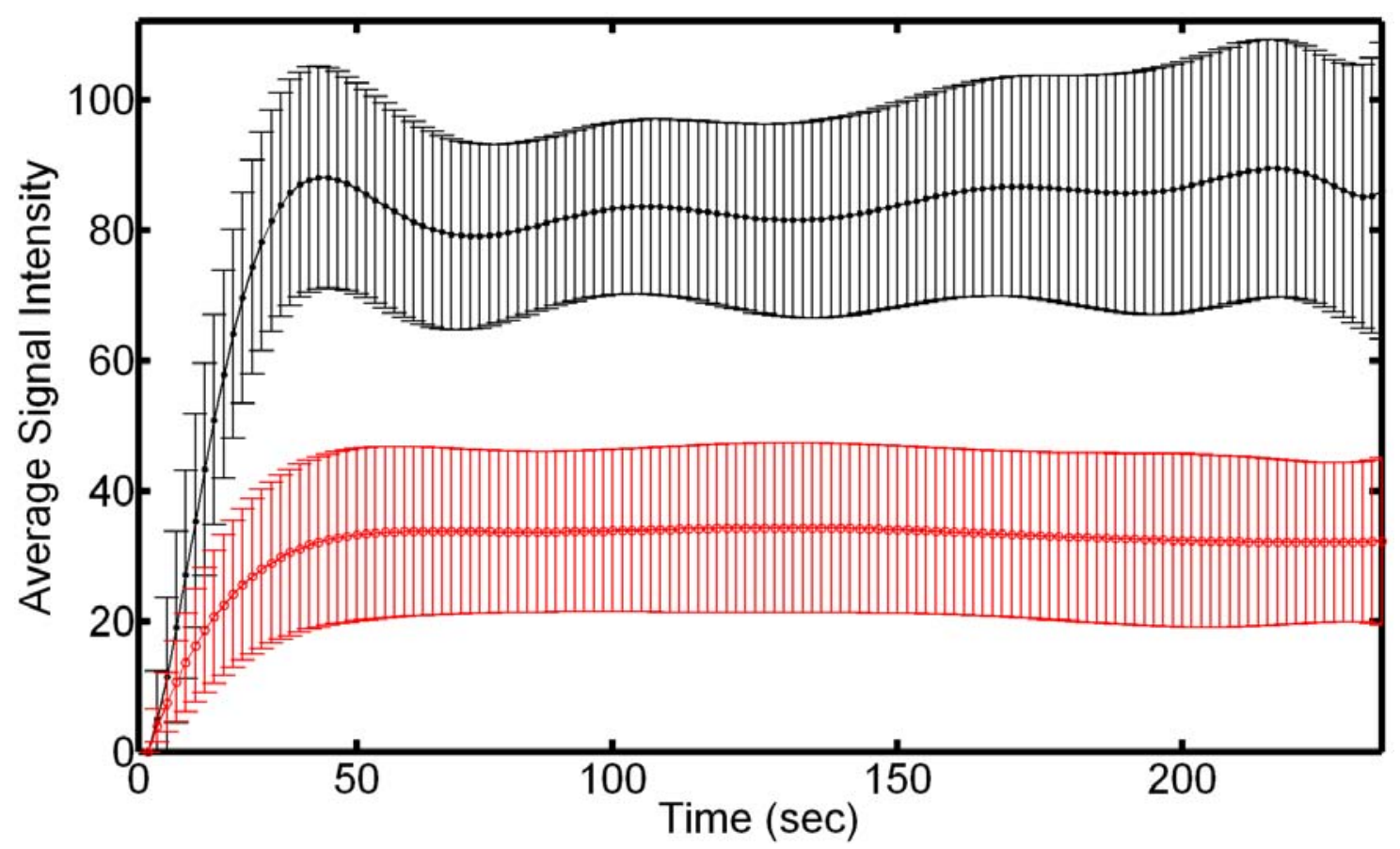

FIGURE 57: Normalized signal intensity, averaged over the entire cortex, with respect to timing of agent delivery. Standard deviations (error bars) for the non-rejection (black; •) and acute rejection (red; ○) subjects indicate the clear separation between the two groups by the transient phase kinetic parameters (peak signal, time-to-peak, and initial up-slope), and the average signal magnitude at the more slowly varying tissue distribution plateau phase. Note that the time-to-peak index has the least selectivity, and the area under the signal intensity time curve is related to the kinetic parameter blood volume.

To build the $k_{n}$-nearest neighbor classifier that characterizes the kidney status, $50 \%$ (13 non-rejection and 12 rejection subjects) was used for training, and the other 50\% (14 non-rejection and 11 rejection subjects) for testing. The diagnostic accuracy for each of the perfusion indexes is shown in Table 10. Note that the time-to-peak has the worst performance. When a weighted combination of the indexes is used, given by the genetic algorithm [379], all the 25 test data sets (100\%) were correctly classified. Building an accurate classifier requires a large number of data points, ideally the entire time-intensity curve. The proposed augmented weighted index results in virtually the same diagnostic accuracy as all 
the data points of the entire time-intensity curve (about 48 , or $96 \%$ out of the 50 training and test data sets), whereas it notably accelerates the classification process.

TABLE 10: Diagnostic accuracy of the $k_{n}$-nearest neighbor classification using individual perfusion-related renal indexes.

\begin{tabular}{|c|c|c|c|c|}
\cline { 2 - 5 } \multicolumn{1}{c|}{} & \multicolumn{4}{c|}{ The index used for classification: } \\
\cline { 2 - 5 } & Peak value & Time-to-peak & Initial up-slope & Average plateau \\
\hline Accuracy & $23 / 25$ & $19 / 25$ & $24 / 25$ & $23 / 25$ \\
\hline
\end{tabular}

As a demonstration of the importance of using perfusion of the kidney cortex, when perfusion over the entire kidney [3] was used, the diagnostic performance for the test data sets was reduced to $80 \%$ (20 out of 25). Moreover, the diagnostic accuracy to the current clinical approach used by radiologists is compared with the proposed perfusion analysis. Using clinical software, radiologists manually define multiple ROIs inside the kidney cortex. Then, these ROIs are used as a mask applied to all images (see Figure 58), notably, without any segmentation or motion correction. The perfusion curve is then obtained from the average intensity of these ROIs over all the time series images and three features, namely wash-in slope, time-to-peak, and wash-out slope, are extracted for the classification of kidney status (Figure 58). The diagnostic accuracy of this comparative method is reduced to $92 \%$ (23 out of 25 ) and $76 \%$ (19 out of 25 ) for the training and testing data sets, respectively. This reduced classification accuracy is due to the high frame-to-frame signal intensity variability, which is related primarily to uncorrected motion effects (Figure 58). This eventually leads a noisy estimation of the perfusion-related parameters. These results highlight the advantage of the proposed CAD system over currently available clinical software for perfusion analysis using the automatically segmented cortical area on images that has been corrected for global and local motions. Finally, a 4-fold cross-validation was performed and have documented the independence of the diagnostic accuracy of the combined $k_{n}$ classification system on the choice of the training data sets, see Table 11. 


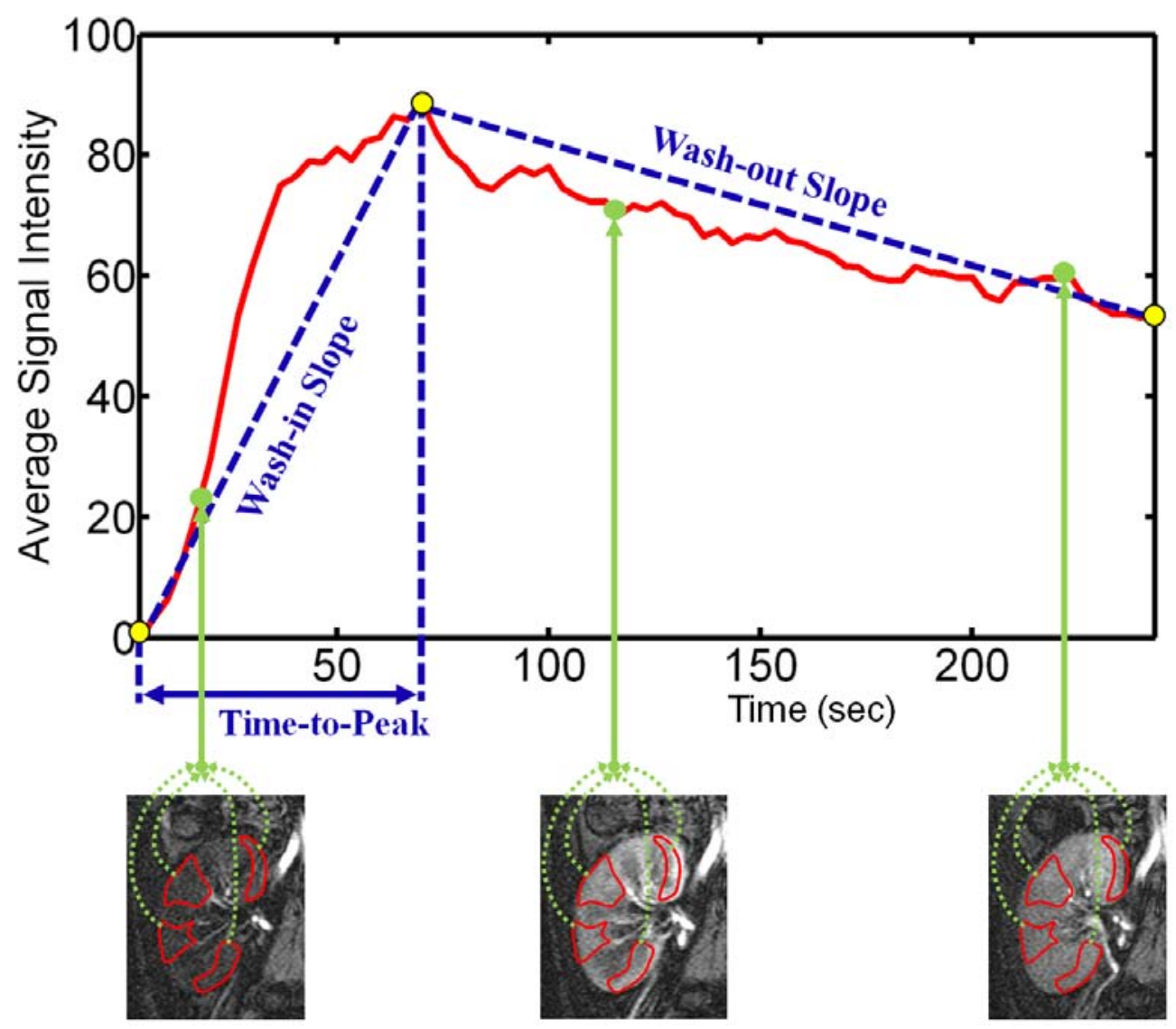

FIGURE 58: Radiologist clinical method for the estimation of the perfusion parameters for the classification of the transplanted kidney status.

TABLE 11: Sensitivity to a training set using a 4-fold cross-validation.

\begin{tabular}{|l|c|c|c|c|}
\cline { 2 - 5 } \multicolumn{1}{c|}{} & \multicolumn{4}{c|}{ Cross-validating the classifier } \\
\hline Selection & 1 & 2 & 3 & 4 \\
\hline Accuracy & $25 / 25$ & $24 / 25$ & $23 / 25$ & $23 / 25$ \\
\hline
\end{tabular}

5. ROC Analysis and Regional Perfusion Displays

To further test the robustness of the proposed CAD system, the ROCs for the $k_{n}$ classifiers were computed. Figure 59 shows the ROC curves for each of the single $k_{n}$ - classifiers, and the weighted-combined classifier. Based on the ROC analysis, the augmented $k_{n}$-classification attained essentially the ideal performance; the area under the ROC curve approached the ideal value of " 1 " (i.e., $A_{z}=1.0$ ). Note that based on the visual inspection 
of the ROC curves, characterization using time-to-peak has the worst performance. As a more refined statistical comparison uing the bootstrapping method, the 95\% CI, defined as the 2.5 percentile to the 97.5 percentile, was computed for the 1,000 bootstrapped values for parameter area under the curve $\left(A_{z}\right)$. The 95\% CIs were [0.951, 0.979], [0.791, 0.975], [0.951, 0.979], [0.906, 0.978], and [0.974, 1.000] for individual classifiers corresponding to the peak signal intensity, time-to-peak, initial up-slope, average plateau, and for the combined classifier, respectively. Based on the objective 95\% CIs, it does appear that the time-to-peak has the worst observable performance.

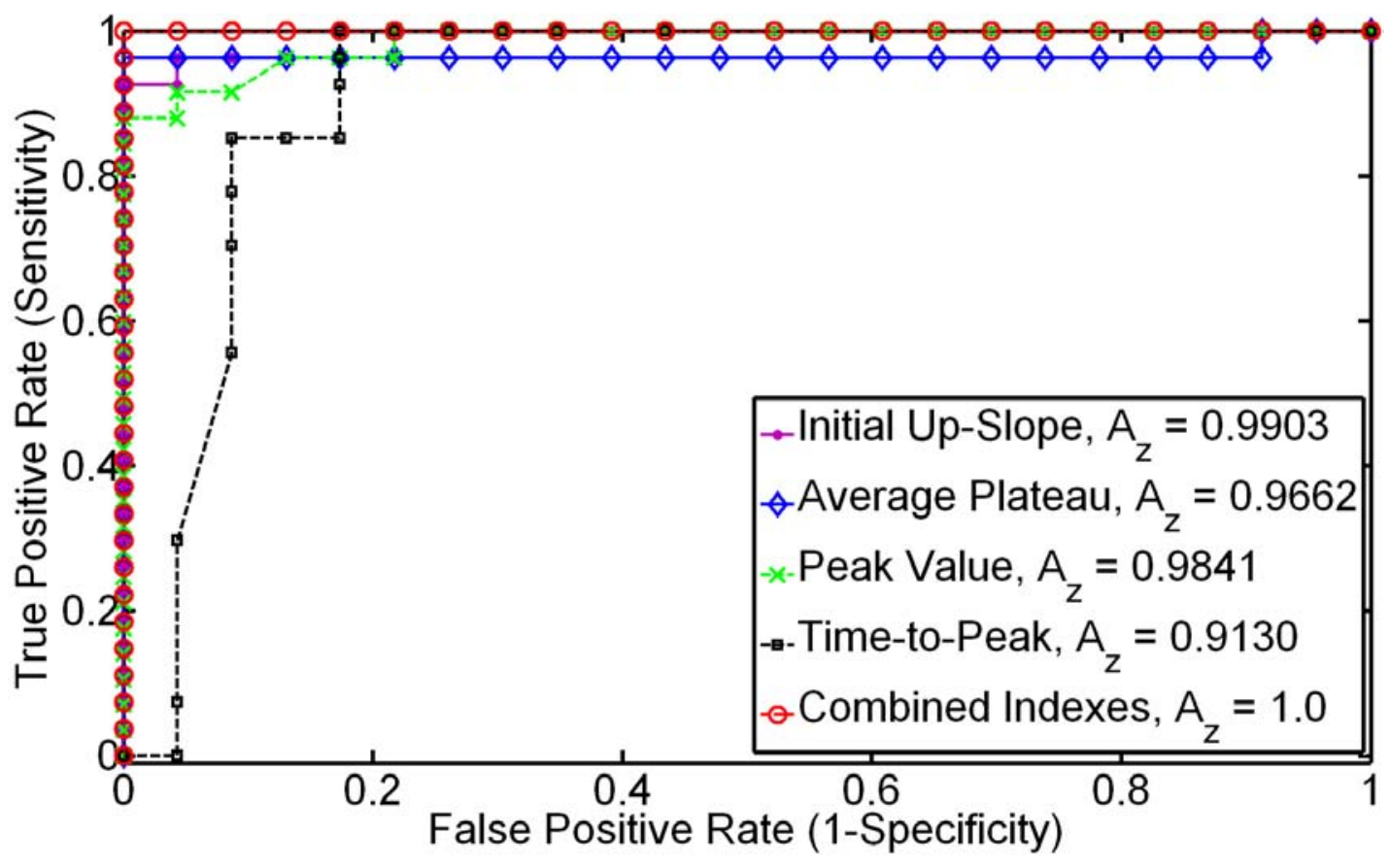

FIGURE 59: ROC curves for the $k_{n}$ diagnostic classification based on the individual perfusion indexes extracted from the cortex - the peak signal intensity (green; $\times$ ); time-to-peak (black; $\mathbf{\square})$; initial up-slope (pink; •), the average plateau (blue; $\diamond)$, and the combined classifier (red; ○). Note that the weighted combination of the four indexes has almost the ideal performance, as evidence by the area under the ROC curve approaching unity $\left(A_{z} \approx 1.0\right)$.

Finally, as a method of regional display pixel-by-pixel mapping of the indexes was explored using the registered perfusion data. Figure 60 demonstrates parametric maps corresponding to the four indexes-peak signal intensity, time-to-peak, initial up-slope,and av- 
erage plateau-for a non-rejection case and an acute rejection case. These data reveal the expected relation of the perfusion indexes for non-rejection versus rejection status.

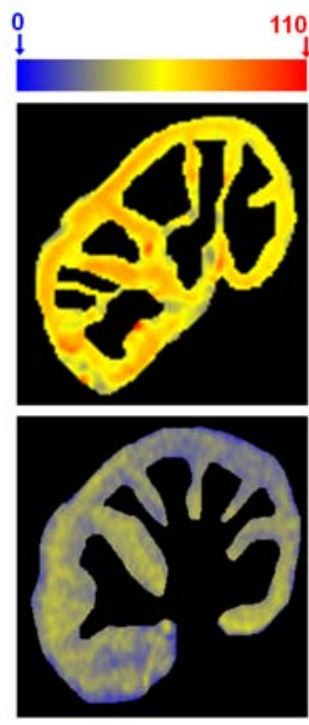

(a)
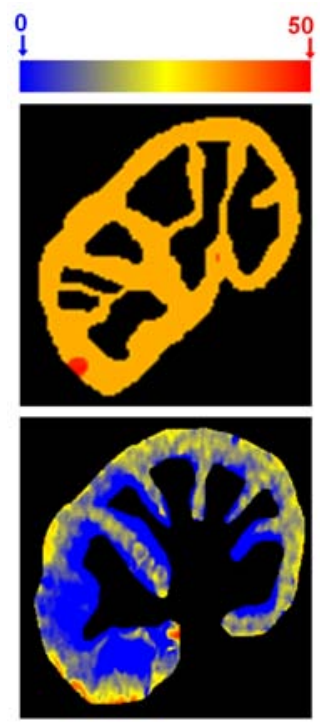

(b)
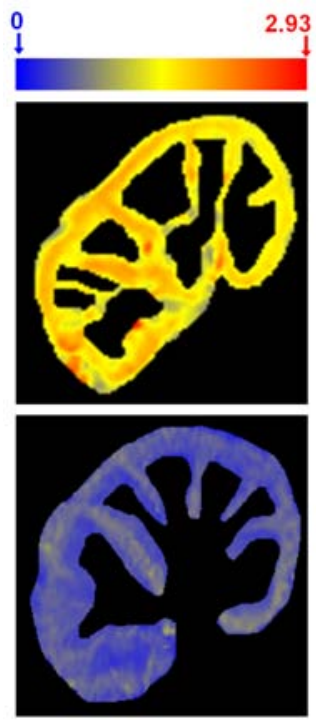

$(c)$
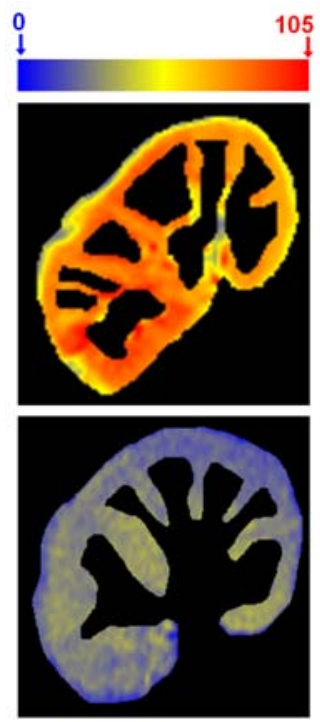

(d)

FIGURE 60: Perfusion maps for the four perfusion indexes, using the normalized signal intensity: peak signal intensity $(a)$; time-to-peak $(b)$; initial up-slope $(c)$, and average plateau ( $d$ ) for a representative non-rejection subject (upper row) and acute rejection subject (lower row). The red and blue hues of each color scale correspond to highest and lowest values, respectively. Note all indexes show worsening of perfusion with pathology.

\section{G. DISCUSSIONS}

In this chapter, a method based on the pathophysiology of renal transplant rejection, specifically perfusion derangements, was investigated to develop a novel image-based CAD system for the classification of transplant physiological status. Experiments documented that the estimated empirical perfusion indexes (peak signal intensity, time-to-peak, initial up-slope, and average plateau) are able to separate acute rejection versus non-rejection, when referenced against a biopsy gold standard in a cohort of 50 participants, using a $k_{n^{-}}$ nearest classifier to identify each subject as rejection or non-rejection. This resulted in an overall diagnostic accuracy of $92 \%$ and $100 \%$ for the training and testing data sets, respec- 
tively. The ROC analysis was used to test the diagnostic performance of the individual $k_{n}$ classifiers and their use in a combinatorial fashion. The combined classifier has near ideal ROC performance, while the time-to-peak index consistently has the worst performance. Whereas rejection would be expected to result in a global reduction of perfusion over the entire cortex, it is conceivable that a vascular insult can be confined to a local region of the cortex. Thus, for local visualization of perfusion deficits, pixel-by-pixel displays were developed. In a sample exploration of non-rejection versus acute rejection, all four perfusion indexes revealed the expected behavior for perfusion reduction.

Unlike approaches that involve free parameters, the proposed CAD system parameters are based on the mathematical definitions and are computed directly from the image data. Specifically, the mean, variances, and weights of the components of the LCDG visual appearance model; Gibbs potentials for the MGRF region map model; and mixing weights of the probabilistic weighted shape prior are automatically estimated first from the image data. Then, these parameters are integrated in a joint MGRF probabilistic model to derive a speed function that controls the deformable model evolution. Further, unlike other methods that are prone to image intensity variations over the time series, the proposed nonrigid registration involves a Laplace-based search for point-to-point correspondences between the boundaries that explicitly depends only on the geometric features of the segmented kidney. It is worth mentioning that the proposed local alignment is not generic and was designed specifically for the current application to provide accurate pixel-on-pixel matches for generating and displaying parametric maps. These capabilities are of great importance for the radiologists to help investigate, in the case of renal dysfunction, which region of the kidney needs attention and follow-up with appropriate therapeutic treatment after transplantation.

If detected early, renal transplant rejection can be potentially mitigated by the adminstration of anti-rejection medications. Reliable, early detection of the kidney rejection is thus important for the management of these patients. Renal biopsy with histopathology assessment is the gold standard for diagnosing acute graft rejection. However, this procedure is invasive and has the risk of serious complications. Additionally, radionuclide techniques and biopsy procedures cannot be used early after transplantation, and therefore 
will not help in the early detection of kidney failure. On the other hand, dynamic MRI can be used at anytime, even at day one post-transplantation, for the assessment and follow up of the functionality of the transplanted kidney. In this study, dynamic MRI combined with computer-aided segmentation and motion correction was used to derive perfusion indexes that characterize the renal cortex. The proposed CAD system documented the ability to differentiate between normal kidneys and kidneys with altered function without the need for invasive procedures, thus it can aid in early diagnosis and the institution of appropriate therapy for detected rejection cases.

\section{H. SUMMARY}

Determination of rejection status for an individual patient, in the earliest stages when there is an optimal opportunity to intervene using existing clinical strategies to slow or reverse rejection, may represent an important advance in the personalized management of this condition. In this chapter, a noninvasive MRI-based CAD system for early detection of acute renal transplant rejection has been introduced. The proposed framework demonstrated the documented ability to reliably distinguish rejection from non-rejection, in a biopsy-proven preliminary cohort of 50 participants. Essential contributions of this chapter are $(i)$ an accurate level set segmentation approach to extract kidney objects from surrounding abdomen structures; (ii) an unsupervised probabilistic model to learn the second- and higher-order spatial interactions between the kidney pixels from DCE-MRI data; (iii) a two-stage registration methodology that separately accounts for global (based on affine transformation) and local (based on the solution of the Laplace equation) motions of the kidney to reduce the effect of frame-to-frame variability due to transmitted respiratory, peristaltic effects, or intrinsic pulsatile effects, and (iv) fusion of four physiologically-motivated indexes that characterize both transient and slowly varying phase perfusion effects for the classification of kidney status. The work presented in this chapter has been published in the IEEE Transactions on Medical Imaging [128], NMR in Biomedicine [129], and the Medical Image Computing and Computer-Assisted Intervention (MICCAI) conference [3]. 


\section{CHAPTER IV \\ FUNCTIONAL ASSESSMENT OF MYOCARDIAL PERFUSION}

This chapter presents an automated framework for accurate analysis of myocardial perfusion using first-pass magnetic resonance imaging (FP-MRI). The main processing steps of the proposed framework include, motion correction of the heart, level set segmentation of the heart wall, and myocardial perfusion analysis. First, in order to account for heart deformations due to respiratory motion and heart contraction, a two-step registration methodology is proposed. The proposed method involves an affine-based registration followed by a local B-splines alignment to maximize a new similarity function that accounts for the first- and second-order normalized mutual information (NMI). Then the myocardium is extracted using a level set deformable model, its evolution being constrained by three features, namely, a weighted shape prior, a pixel-wise mixed object/background image intensity distribution, and a second-order binary Markov-Gibbs random field (MGRF) spatial model. At the third stage, residual segmentation errors and imperfection of image alignment are reduced by employing a Laplace-based registration refinement step that provides accurate pixel-on-pixel matches on all segmented frames to generate accurate parametric perfusion maps. Finally, physiology is characterized by pixel-wise mapping of empirical indexes-peak signal intensity, time-to-peak, initial up-slope, and the average signal change of the slowly varying agent delivery phase-that are extracted from contrast agent kinetics. The proposed framework has been tested on 24 perfusion data sets from eight patients with ischemic damage who are undergoing a novel myoregeneration therapy. The performance of the processing steps of the proposed framework is evaluated using both synthetic and in-vivo data. By quantitative and visual assessment, the proposed framework documented the ability to visualize regional and transmural perfusion; thus, it can aid in follow-up on treatment for patients undergoing myoregeneration therapy. 


\section{A. HEART: ANATOMY, FUNCTION, AND DISEASES}

The human heart is a hollow muscular organ that is approximately the size of a fist and approximately weighs $250-300$ grams in females and 300-350 grams in males [384]. It is the most important organ of the human body as it pumps blood and supplies all body parts and organs with oxygen. In this section, brief overviews of heart anatomy and function, as well as the diseases that affect the heart, are given.

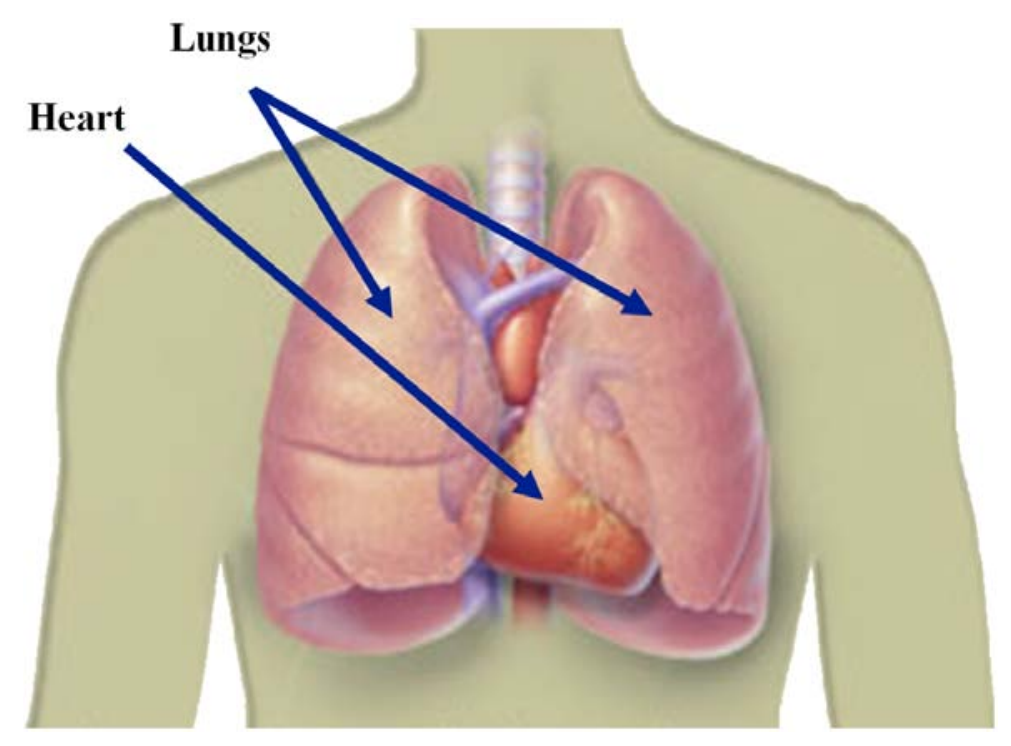

FIGURE 61: Illustration of the location of the heart in the body [19].

The human heart is located under the ribcage in the center of the chest in the direct neighborhood of the lungs (see Figure 61) slightly to the left of the breastbone. The heart is divided into left and right parts separated by a muscle called the interventricular septum (see Figure 62). Each part (left or right) of the heart has two chambers, the atrium and the ventricle (see Figure 62). The four heart chambers are right atrium, right ventricle, left atrium, and left ventricle (LV). The heart's wall consists of three layers as shown in Figure 63: the outermost wall layer (epicardium); the contracting muscle (myocardium); and the innermost wall layer (endocardium), which is in direct contact with the heart blood pool. The ventricles walls are lined with an irregular muscle called trabeculae carneae [385].

The function of the heart is to pump blood to both the lungs and all other body systems. To control blood flow, the pumped blood passes through certain types of valves before leaving each chamber (atrium or ventricle) of the heart, which are separated by 


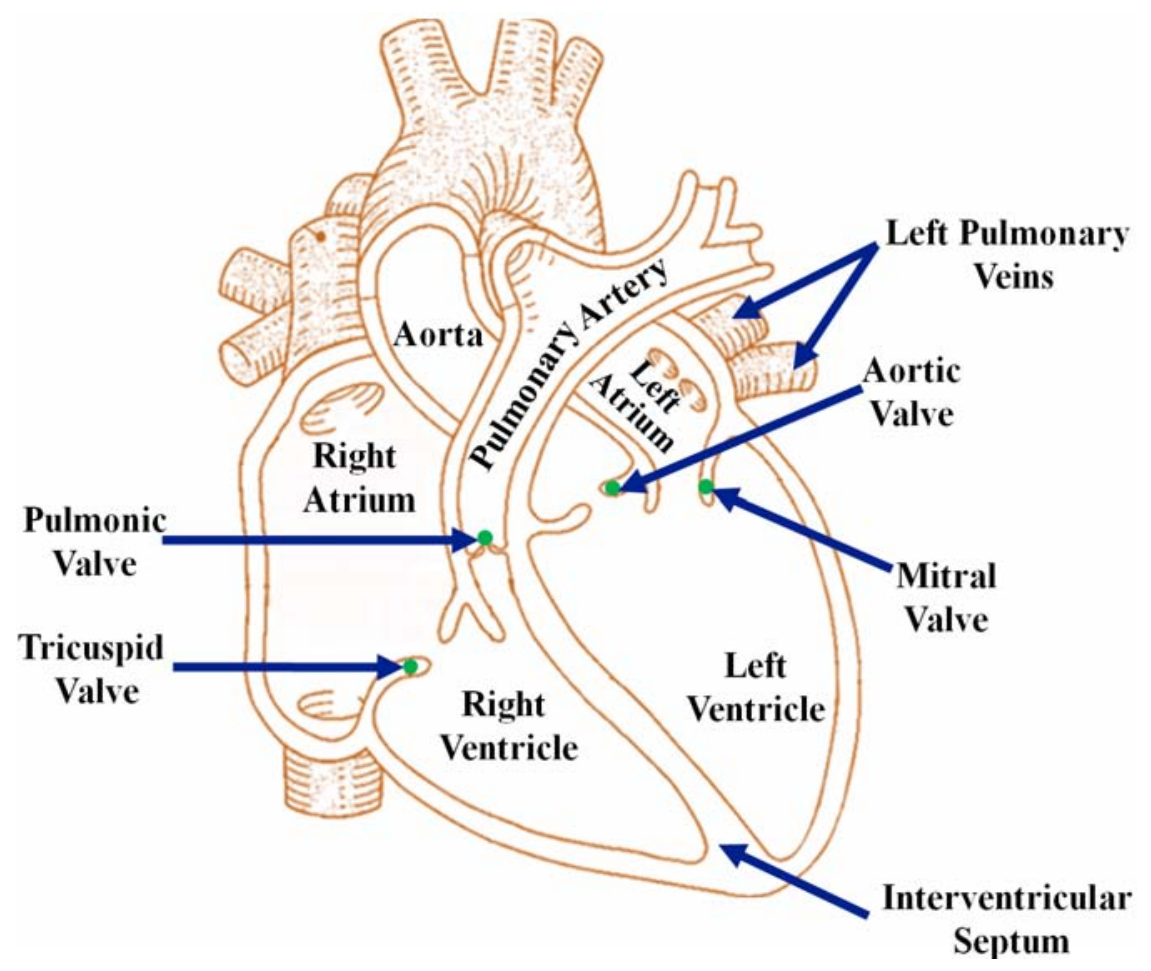

FIGURE 62: A schematic illustration of a long-axis cross-section of the heart showing the four chambers of the heart and the atrioventricular valves that controls blood flow [20].

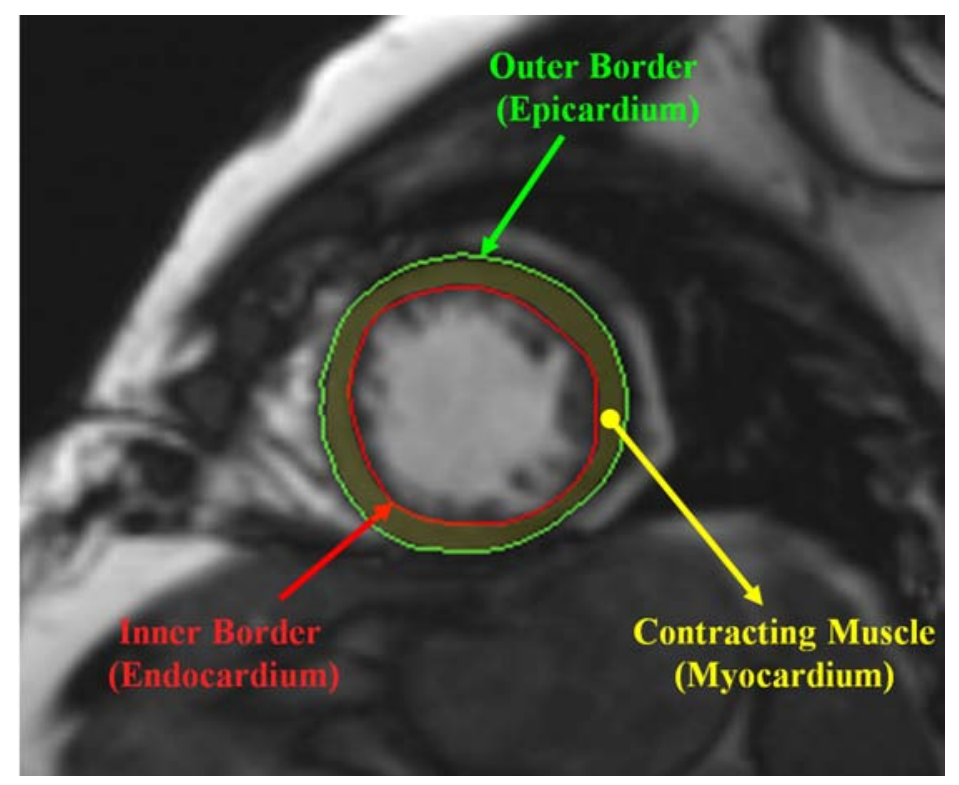

FIGURE 63: A short-axis MR image taken at the mid-cavity level of the heart showing different layers of the cardiac wall.

atrioventricular valves (tricuspid and mitral valves). Additionally, both ventricles are closed by valves (pulmonary and aortic valves) that define the flow direction of the blood and 
prevent the blood from flowing backwards or "regurgitating" back into the heart [385]. All heart valves are actually flaps or cusps that act as one-way inlets (or outlets) for blood coming into (or leaving) a ventricle. The cardiac cycle is defined as the occurring events during one heartbeat (an adult heart beats about 60-80 beats per minute). During this cycle, any of the heart chambers can be found in one of the two phases: systolic or diastolic. During systole, the heart contracts to push blood out of the chamber. Whereas, during diastole the heart muscle relaxes to allow the chamber to be filled with blood [385]. During these ventricular systole and diastole phases, the arterial blood pressure changes between a maximum (systolic) and a minimum (diastolic) value. Typical blood pressure value ranges of healthy adults are 90-120 and 60-80 for systolic and diastolic pressure, respectively.

The function and structures of the heart are affected by a broad range of diseases or conditions, referred to as cardiovascular diseases (CVDs). CVDs affect approximately 6 million US patients annually and are considered to be the leading cause of death for both men and women in the USA. CVDs include a group of disorders of the heart and blood vessels, such as coronary artery disease (atherosclerosis), abnormal heart rhythms (arrythmia), ischemic heart disease (myocardial infarction), congenital heart disease, heart muscle disease (cardiomyopathy), pericardial disease, vascular disease (blood vessel disease), and heart failure [386]. Coronary artery disease is a condition where the inner walls of the arteries that provide vital oxygen and nutrients to the heart become narrow due to buildup of plaque or blood clots. This is known as atherosclerosis, or hardening of the arteries. Arrhythmia is an irregular or abnormal condition with the rhythm of the heartbeat, which can involve too fast, too slow, or uneven (irregular) heartbeats. Ischemic heart damage is a severe form of heart disease that occurs due to the blocking of blood flow to part of the heart and causes myocardial infarction. This leads to the damage or death of the heart muscle if untreated. Congenital heart disease is a problem with the structure of the heart or blood vessels that is present at birth and disrupts the normal blood flow through the heart. This cardiac disorder can allow the blood to flow in the wrong direction, slow down, or be blocked completely [387]. Cardiomyopathies are heart diseases that weaken and enlarge the heart muscle, thereby weakening the heart's ability to pump blood to the rest of the 
body. Most patients with untreated cardiomyopathy can have heart failure and abnormal heart rhythms. Pericardial disease is inflammation that affects the membrane that surrounds and protects the heart, which is known as the pericardium. Vascular diseases include any condition that affects the blood vessels (circulatory system) that carry the blood pumped by the heart to every part of the body. Another major heart condition is heart failure, which occurs when the heart muscle does not pump the blood to meet the body's needs. Heart failure can be caused by many factors, such as coronary artery disease, heart defects present at birth, diabetes, lung diseases, etc., and is considered as the most salient cause of morbidity and mortality in cardiovascular disease. The only therapy once end-stage symptoms set in is heart transplant. Due to the paucity of transplant organs, early detection of heart failure is essential to prevent its relentless progression to premature death.

In this chapter, the main focus is on the assessment of heart functionality for patients with ischemic heart damage due to heart attack who are undergoing a novel myoregeneration therapy $[64,65]$. The evaluation of heart function is based on using cardiac perfusion MRI. The next section overviews different MRI modalities for the evaluation of heart function and the related work on cardiac perfusion MR image analysis.

\section{B. ANALYSIS OF MYOCARDIAL PERFUSION}

Heart failure is considered the most important cause of morbidity and mortality in cardiovascular disease, which affects approximately 6 million US patients annually. Thus it is critical in these patients to detect precursors in order to prevent progression to end-stage disease. The most common underlying cause of heart failure is ischemic heart disease [63]. Therefore, early detection of heart failure is essential to prevent its relentless progression to premature death. In order to characterize the workings of the heart status different cardiac MRI techniques, such as cine MRI, tagged MRI, or late-gadolinium MRI, have been exploited. The status of cardiac physiology in health and disease is quantified with multiple important indicators of cardiac function, including global performance indexes (e.g.,wall mass, end-systolic and end-diastolic volumes, and ejection fraction), and local functional 
indexes (e.g., functional strain and wall thickening). These characteristics assist cardiologists in detecting myocardial dysfunction, specifically major heart conditions leading to heart attack or failure, and in optimizing therapies for individual patients [67]. While several MRI studies have focused on viability [42-44] and contractile function [67-69] in areas with myoregeneration, there is a dearth of in-vivo studies designed to address the key issue of the effects of the myoregeneration therapy on the associated blood nutrient supply.

First-pass magnetic resonance imaging (FP-MRI) is another MRI technique that has also been used for the assessment of heart status in ischemic heart disease. Perfusion MRI has long been investigated for non-invasive analysis of myocardial perfusion [388-393]. As opposed to late-gadolinium analysis, FP-MRI characterizes the dynamic phase of contrast agent (CA) transient, therefore yield parameters that reflects tissue vascularity (perfusion). Visual myocardial perfusion assessment is usually adequate for clinical purposes [394]. However, the cardiac motion, large data dimensionality (3D+time) the requirement of rapid temporal sampling of first-pass images (with compromises in spatial resolution), and the relatively low-contrast resolution images, sometimes limit visual assessment and reliability. Thus, the goal of this chapter is to to develop a processing framework to aid the analysis of myocardial perfusion images and to improve the visualization of perfusion effects across the myocardial wall. The proposed framework accounts for rigid and nonrigid motion of the heart, and characterizes and displays perfusion indexes using color- coded regional maps to aid visual assessment of myocardial perfusion.

Two basic steps of automated analysis of myocardial perfusion MRI-image segmentation and motion correction models-are commanding major attention in this area. In addition, the incorporation of these methods into a comprehensive work flow has been an area of research interest. Next, an overview of the state-of-the-art computational techniques for the analysis of myocardial perfusion using MRI is presented. The state-of-the-art CAD systems for the analysis of myocardial perfusion are discussed in Chapter II. 


\section{Related Work on the Segmentation of Cardiac Perfusion MRI}

Accurate analysis of cardiac perfusion MRI requires precise delineation of the LV wall borders. However, this is subject to multiple challenges due to the need to maintain adequate spatial resolution while imaging very quickly to capture the transient first-pass transit event, nonlinear intensity variations due to agent transit, relatively low signal-tonoise ratio (SNR), and partial influence of adjacent structures. Several studies have been proposed to address these challenges by integrating both temporal and spatial information of the perfusion images into their segmentation methods. Spreeuwers and Breeuwer [395] proposed to segment the myocardium using active contours. In their approach, temporal maximum intensity projection for pre- and post-contrast time periods of the dynamic acquisition are calculated to serve as feature images for the active contour evolution. Postiano et al. [92] proposed a semi-automated approach for the segmentation of perfusion images using a gradient vector flow snake. The LV wall borders were segmented only on one user-defined frame (the one with the best SNR), and were then used as a mask that was applied to all co-registered frames of the time series. A similar approach was proposed by Santarelli et al. [396]. They employed non-linear anisotropic diffusion filtering to reduce gray scale inhomogeneity and to selectively preserve the myocardium edges. To segment the myocardium, the active contour is initialized by a manually traced polygon in the LV. Pluempitiwiriyawej and Sotthivirat [397] proposed an automated approach for myocardial border detection using active contours. To find the initial contours, thresholding and morphological operations were employed. Then, an energy minimization approach that incorporated an elliptical shape prior of the heart and probabilistic region- and edgebased energy terms were used to guide the contour evolution. A level set-based approach to extract the LV borders on perfusion MRI was proposed by Adluru et al. [398]. However, deformable contour-based methods fail in the case of excessive image noise, poor image resolution, or diffused boundaries, if they do not take advantage of a priori models and spatial relationship between image pixels. Active appearance model (AAM) based techniques were also proposed by Stegmann et al. [399] and Gupta et al. [400] to segment the LV 
borders of dynamic MRI time series. A multi-band AAM framework for the segmentation of cardiac perfusion MRI that integrated both spatial priors about myocardial shape and temporal priors about characteristic perfusion patterns was proposed by Baka et al. [401]. However, parametric shape-based approaches depend on the existence of good texture features in perfusion images and may perform poorly on some frames due to noise and the lack of well-defined features. Mahapatra and Sun [402] proposed a segmentation framework of cardiac perfusion MRI using Markov random field (MRF). They used a graph cut-based approach to optimize a cost function that combines the mutual dependence of registration and segmentation information. This framework was later extended by using a groupwise registration that incorporated temporal information of the time series data [403].

The above overview shows the following limitations of existing segmentation approaches: (i) some of them require manual user input, and are prone to variability (ii) most of them did not account for spatial interaction between image pixels; (iii) deformable model-based methods without appearance and shape priors result in errors related to diffused or discontinuous object boundaries, excessive image noise, and poor image resolution; and (iv) parametric shape-based approaches depend on the existence of good texture features in perfusion images and may perform poorly on some frames due to noise and the lack of well-defined features.

\section{Related Work on Motion Correction of Cardiac Perfusion MRI}

Patient and respiratory motions and heart contraction result in geometric deformations in the LV wall. These rigid and nonrigid deformations limit accurate quantification of perfusion parameters on time series data. Therefore, for accurate evaluation of perfusion MRI, the first-pass temporal data must be registered to compensate for these artifacts. In literature, several motion correction techniques have been introduced to correct the motion of the heart on perfusion MRI time series. Breeuwer et al. [404] proposed a translation/rotation-based rigid-body registration approach using the normalized crosscorrelation (NCC) as a similarity measure. A similar approach was proposed by Wong et 
al. [405] using normalized mutual information (NMI). Bidaut and Vallee [406] introduced a multiresolution registration approach employing cardiac masks to restrict the registration to the area of the nearly rigid motion of the heart. Their approach is based on minimizing the mean squared differences between perfusion time series images and the reference image. A similar approach was introduced by Dornier et al. [407] using tightly cropped masks with no signal intensity changes throughout the time series. S. N. Gupta et al. [408] have also reported a technique for rigid-body registration that considered translation effects of the center of mass, but did not explicitly consider rotation or shape changes. However, all of these registration methods [404-408] account for the global motion, and do not account for the nonrigid deformations of the heart. Gallippi et al. [409] corrected cardiac motion using a statistics-based registration approach. All the images are registered to the central image of the time series using intensity variations and edge directions as similarity measures. However, this method depends only on first-order image intensity for registration criteria, which performs poorly for frame with significant intensity changes. An iterative model-based nonrigid registration approach was proposed by Adluru et al. [261]. Each image frame was registered to its corresponding model image, which was obtained in a preliminary PK model registration step by minimizing the mean square difference between a user-selected reference image and all other perfusion images. However, PKbased techniques increase the computational cost of the registration approach, since a full model fitting has to be performed on a pixel basis. Milles et al. [410] proposed a two-pass coarse-to-fine registration approach using independent component analysis (ICA) for dealing with local intensity change of the perfusion images. In their framework, each image frame is registered to a time-varying reference image that is constructed from three identified images of the left and the right ventricle intensity curves using ICA. The registration is achieved based on minimizing the sum of squared differences. However, only translationalmotion was corrected. A similar ICA-based nonrigid registration approach was proposed by Wollny et al. [411] using an improved independent component labeling approach that is based on time-frequency analysis of the perfusion images. Wollny et al. [412] proposed a multiresolution nonrigid registration approach based on the quasi-periodicity of respi- 
ratory motion. Their registration framework uses a semilocal B-spline parametric transformation to optimize the normalized gradient field similarity metric. To compensate for cardiac translation and deformation, Tarroni et al. [413] proposed a nonrigid registration algorithm using a 2D multi-scale extension of NCC. In their approach, each image frame is registered to a template frame and five additional frames created by resizing the reference frame. The transformation parameters are determined according to the template with peak cross-correlation value. Then, contour adaptation is achieved using an edge-based level set method. To overcome the intensity change on myocardial perfusion images, Li et al. [414] proposed a registration framework that is based on an initial semi-automatic segmentation of the heart ventricles. In their framework, an approximation of ground truth of the image sequence is obtained using spatio-temporal smoothness constraints derived from the initial segmentation. This idealized ground truth is then used as reference for non-linear registration to reduce motion within the perfusion time series images. Tautz et al. [415] proposed a multi-scale nonrigid registration approach that utilized local phase (rather than intensity or gradient information) and the Demons algorithm [416]. The central image frame of the time series was selected as the reference image.

In total, the aforementioned motion correction models have their own limitations: (i) most of the methods depend on using only rigid alignment and do not account for the nonrigid deformations of the heart; (ii) most of them heavily depend on first-order image intensity characteristics for registration criteria; (iii) PK- based techniques increase the computational cost of the registration approach, since a full model fitting has to be performed on a pixel basis; and (iv) none of the existing techniques provide accurate pixel-on-pixel matches of the co-aligned image frames of a given time series.

To overcome the shortcomings and discrepancies of the existing segmentation and motion correction techniques, a comprehensive image-based framework, shown in Figure 64, is proposed for the analysis of myocardial perfusion based on the study of first-pass dynamics of gadolinium CA. For the myocardium segmentation, a level set-based approach that has the ability to overcome LV shape variabilities, signal intensity variations over the time series, and image inhomogeneity is proposed. This is achieved by integrating shape 
and appearance features of the heart into a joint probabilistic Markov-Gibbs random field (MGRF) image model to constrain the deformable model evolution. To reduce the variabilities of the LV wall in FP-MRI, it is first imperative to co-align the time series images to account for the heart deformations. Therefore, a novel two-step registration methodology is proposed that has the ability to handle both the global (using affine alignment) and local (using B-splines alignment) motions of the heart. The proposed methodology is based on using a new similarity metric that accounts for the first-and second-order NMI. The second-order NMI similarity term is proposed to increase the ability of the NMI to capture more features of the perfusion images, and therefore can improve the registration accuracy for frames with significant intensity differences. Details of the proposed framework are described in the following sections.

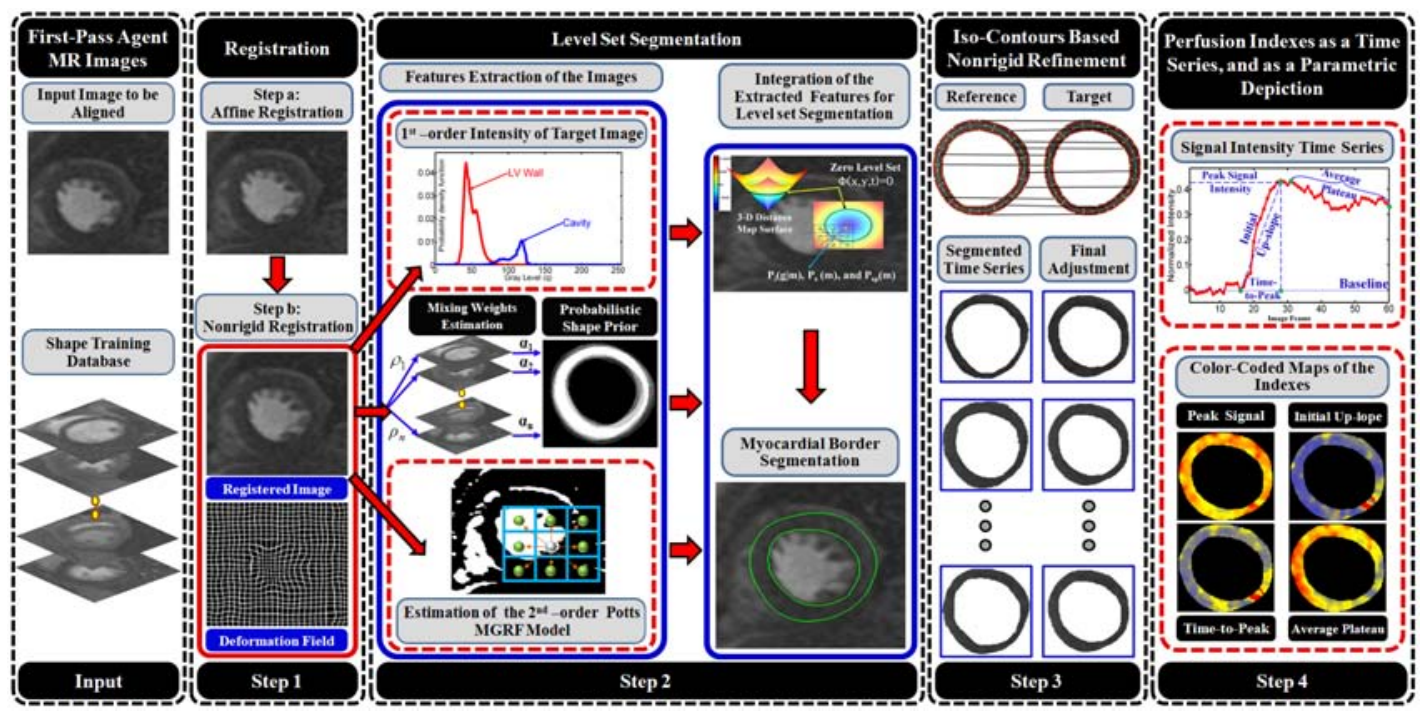

FIGURE 64: The proposed framework for the analysis of cardiac first-pass perfusion MRI.

\section{MYOCARDIAL BORDERS SEGMENTATION}

A novel geometric (level set-based) deformable model approach is proposed for the segmentation of the LV wall borders from FP-MRI time series. The evolution of the level set is controlled by three features: a weighted probabilistic shape prior, the first-order pixel-wise image intensities, and a binary pair-wise Potts MGRF spatial interaction model. These features are integrated into a joint MGRF model to guide the evolution of the level set deformable model as described in Section III.C.1. 
In order to constrain the level set evolution, a weighted probabilistic shape prior of the myocardium that is built for a set of training images is employed (Figure $65(a)$ ). In order to reduce the shape variability of the LV wall and get more accurate segmentation, the training images are mutually co-aligned using a two-step registration approach that accounts for both global and local motions related to heart contraction and respiratory motion. First, the training image frames are co-aligned by an affine transformation to account for the global motion of the heart (Figure $65(b)$ ). Second, the B-splines transformation model [18] is used to locally register the globally aligned frames due to its flexibility and effectiveness for modeling large deformations (Figure $65(c)$ ). The basic idea of using $B-$ splines is to deform an object by manipulating an underlying lattice, $\Pi$, of control points, $\Pi_{u, v}: u=0, \ldots, U-1 ; v=0, \ldots, V-1$, to maximize a new similarity function that accounts for first-and second-order NMI. The B-splines deformation model is defined as [18]:

$$
f(x, y)=\sum_{i=-1}^{2} \sum_{j=-1}^{2} \beta_{i}(s) \beta_{j}(h) \psi_{u+i, v+j}
$$

where $u=\lfloor x\rfloor, v=\lfloor y\rfloor,\lfloor$.$\rfloor denotes the integer part of a real-valued number; (s, h): s=$ $x-u \in[0,1)$ and $h=y-v \in[0,1)$, is the relative position of the point $(x, y)$ with respect to the four nearest lattice points $(u, v),(u+1, v),(u, v+1)$ and $(u+1, v+1)$; and $\beta_{j}(h)$ is the $j^{\text {th }}$ basis function of the uniform cubic B-spline [18]:

$$
\begin{array}{ll}
\beta_{-1}(h)=\frac{-h^{3}+3 h^{2}-3 h+1}{6} ; & \beta_{0}(h)=\frac{3 h^{3}-6 h^{2}+4}{6} ; \\
\beta_{1}(h)=\frac{-3 h^{3}+3 h^{2}+3 h+1}{6} ; & \beta_{2}(h)=\frac{h^{3}}{6}
\end{array}
$$

\section{Similarity Function}

A new similarity metric $\Upsilon$ that accounts for both first $(I)$ - and second $(S)$-order NMI between the reference $\left(\mathrm{g}_{\mathrm{r}}\right)$ and the target $\left(\mathrm{g}_{\mathrm{t}}\right)$ images is proposed:

$$
\Upsilon\left(\mathbf{g}_{\mathbf{r}}, \mathbf{g}_{\mathbf{t}}\right)=\frac{H_{I}\left(\mathbf{g}_{\mathbf{r}}\right)+H_{I}\left(\mathbf{g}_{\mathbf{t}}\right)}{H_{I}\left(\mathbf{g}_{\mathbf{r}}, \mathbf{g}_{\mathbf{t}}\right)}+\frac{H_{S}\left(\mathbf{g}_{\mathbf{r}}\right)+H_{S}\left(\mathbf{g}_{\mathbf{t}}\right)}{H_{S}\left(\mathbf{g}_{\mathbf{r}}, \mathbf{g}_{\mathbf{t}}\right)}
$$

where $I$ stands for intensity and $S$ stands for spatial, $H($.$) (referring to either H_{I}$ or $H_{S}$ ) is the Shannon's entropy $\left(H()=.-\sum_{(x, y) \in \mathbf{R}} p_{x, y}(.) \log p_{x, y}().\right)$ of the image signals, $H(.,$. 
(a)
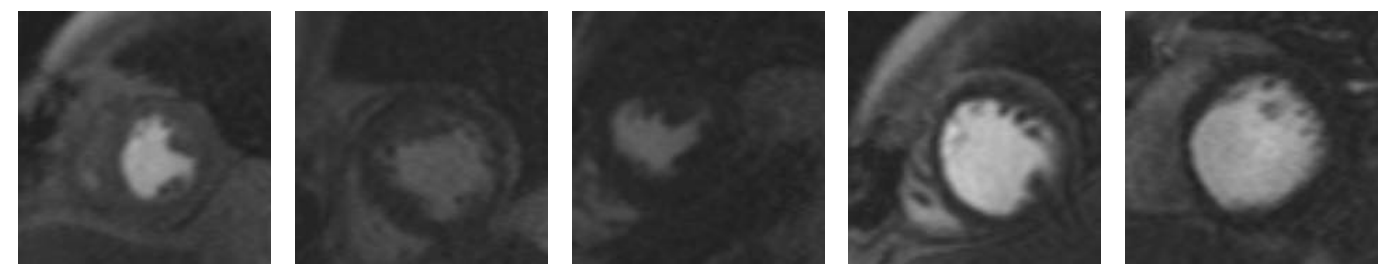

(b)
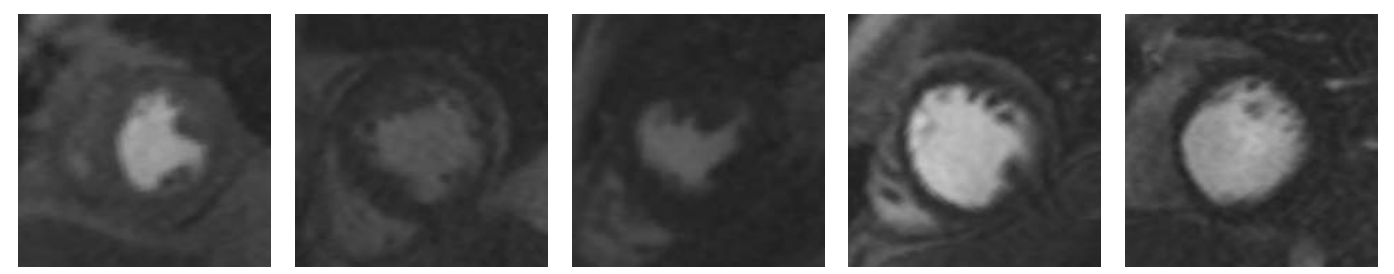

(c)
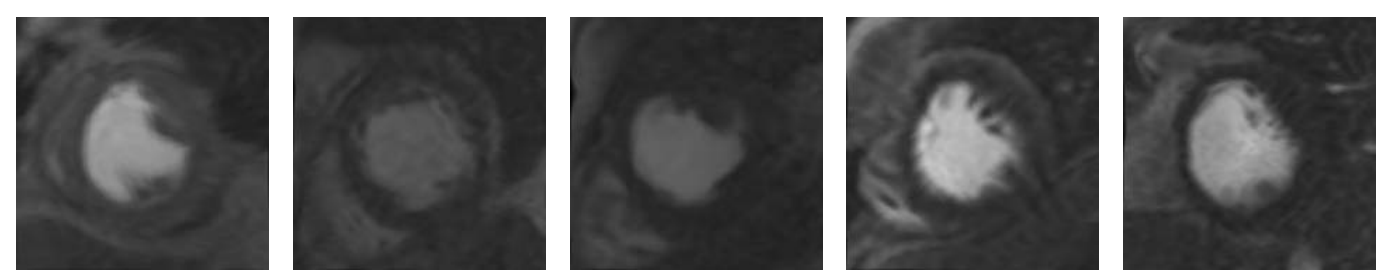

(d)
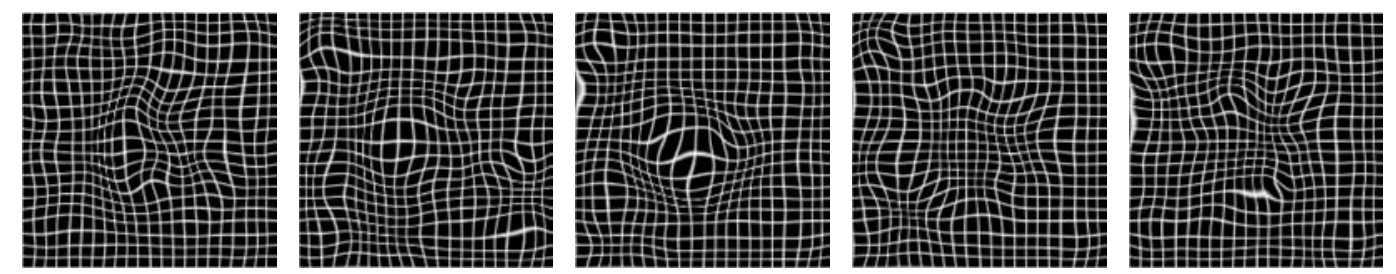

(e)
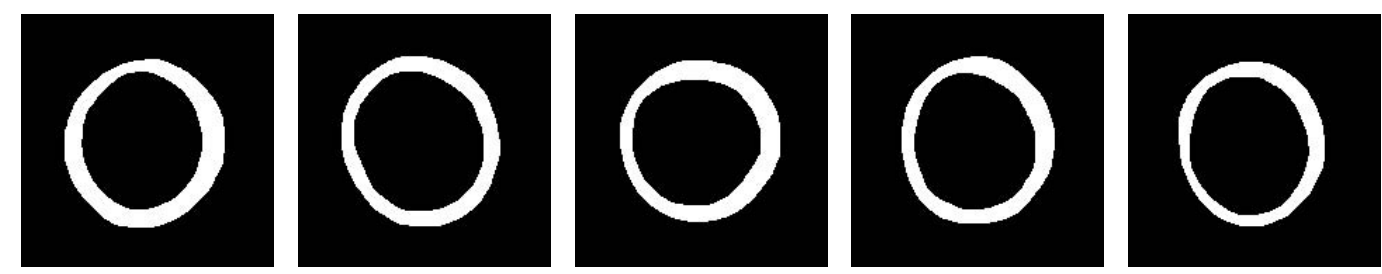

FIGURE 65: Building the LV wall probabilistic shape prior: $(a)$ training samples, $(b)$ their affine-based alignment, $(c)$ their B-splines based nonrigid registration, $(d)$ the deformation fields used for the B-splines registration, and $(e)$ the manually segmented myocardium.

is their joint entropy $\left(H(.,)=.-\sum_{(x, y) \in \mathbf{R}} p_{x, y}(.,.) \log p_{x, y}(.,\right.$.$) . Here \mathbf{R}=\{(x, y): 0 \leq$ $x \leq X-1,0 \leq y \leq Y-1\}$ denotes a finite 2D arithmetic lattice of the size of $X Y$ supporting the gray scale images $g_{r}$ and $g_{t}$.

a. Estimation of the first-order NMI is performed using the linear combination of discrete Gaussians (LCDG) as described in Section III.C.1.c. The LCDG was employed for more accurate approximation of both the marginal $\left(P_{I}\left(\mathbf{g}_{\mathbf{r}}\right)\right.$ or $\left.P_{I}\left(\mathbf{g}_{\mathbf{t}}\right)\right)$ and joint $\left(P_{I}\left(\mathbf{g}_{\mathbf{r}}, \mathbf{g}_{\mathbf{t}}\right)\right)$ graylevels probability distributions, 
b. Estimation of the second-order NMI is performed using a generic secondorder MGRF model of greyscale images. Generally, the interaction structure and Gibbs potentials of an MGRF model can be arbitrary and are identified from training data. For simplicity and by symmetry considerations, the interaction structure is limited to the pixel's nearest 8-neighbors shown in Figure $66(a)$ for the estimation of second-order marginal MGRF probability distributions $P_{S}\left(\mathbf{g}_{\mathbf{r}}\right)$ and $P_{S}\left(\mathbf{g}_{\mathbf{t}}\right)$. On the other hand, for the estimation of joint MGRF probability distribution $P_{S}\left(\mathbf{g}_{\mathbf{r}}, \mathbf{g}_{\mathbf{t}}\right)$ the structure includes 17-neighbors as shown in Figure $66(b)-8$-neighbors within the target frame and 9-neighbors within the reference frame.

(a)

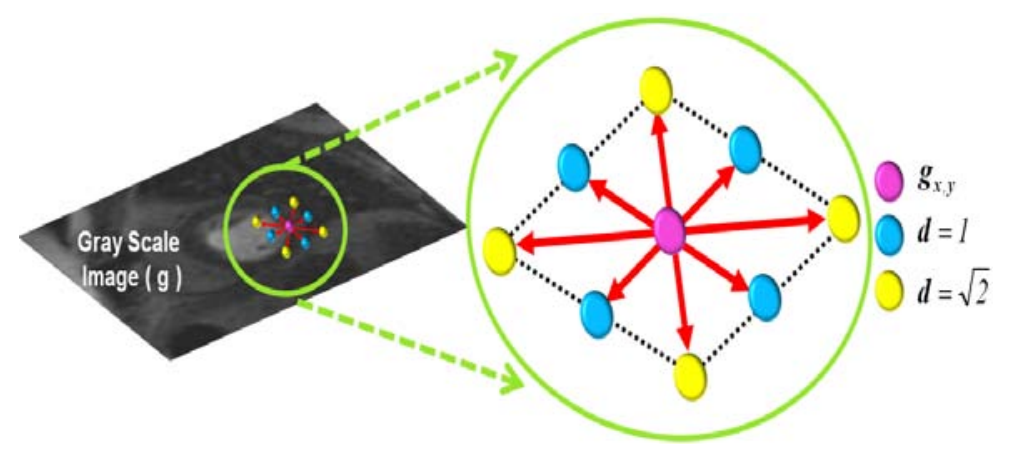

(b)

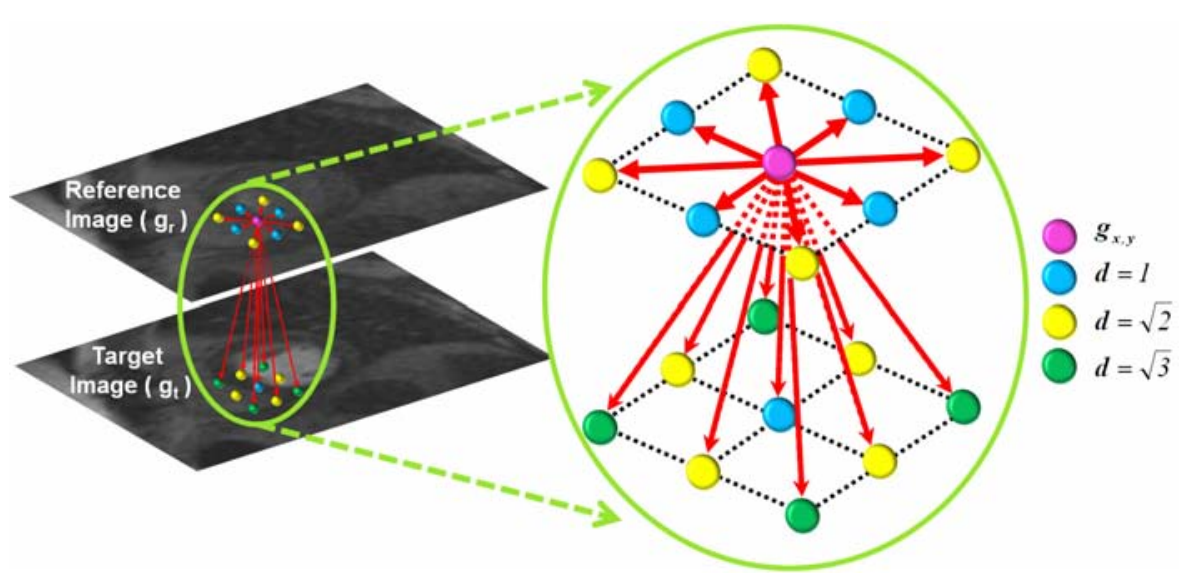

FIGURE 66: Neighborhood systems for the estimation of the second-order marginal $(a)$, and joint (b) MGRF probabilities.

For both structures, the Gibbs potentials are bi-valued and depend only on whether each pair of gray levels are equal or not. Under these assumptions, it is similar to the Potts model [417] and differs only in that the potentials are estimated analytically. The 8-neighborhood (Figure $66(a)$ ) has two types of symmetric pairwise interactions that are 
specified by the distance $d$ between two pixels in a given image: $(i)$ horizontal and vertical pairs with $d=1$, and (ii) the diagonal pairs with $d=\sqrt{2}$. In addition to $d \in \mathbf{D}=\{1, \sqrt{2}\}$, the 17-neighborhood has an additional type of pairwise interactions $(d=\sqrt{3})$ between a given pixel location on the target image and the reference image (Figure $66(b)$ ).

In order to use the Potts model of the spatial interactions in grayscale images and reduce the noise effect on estimating the Gibbs potentials, the 256 gray levels were reduced to 64 levels by representing every four adjacent gray levels by one label. Note that such a grey level compression is performed only for obtaining the transformation parameters that optimize the registration. Once obtained, the transformation is applied to the original uncompressed images. Therefore, the analysis of the contrast agent's perfusion is done on the original recorded images and is not affected by the compression. Let $\mathbf{N}=\left\{\mathbf{N}_{d}, d \in\right.$ $\mathbf{D}=\{1, \sqrt{2}, \sqrt{3}\}\}$ denote the family of the neighboring pixel pairs supporting the Gibbs potentials and $\left|\mathbf{C}_{\mathbf{N}}\right|$ denote its cardinality. Let $\mathbf{V}_{a}=\left\{V_{a}\left(q, q^{\prime}\right)=V_{a, \text { eq }}\right.$ if $q=q^{\prime}$ and $V_{a}\left(q, q^{\prime}\right)=V_{a, \text { ne }}$ if $\left.q \neq q^{\prime} ; q, q^{\prime} \in \mathbf{Q}=\{0,1, \ldots, Q-1\}\right\}$ denote bi-valued Gibbs potentials describing symmetric pairwise interactions. Then, the MGRF probability of an individual greyscale image $\mathbf{g}$ (standing for $\mathbf{g}_{\mathbf{r}}$ or $\mathbf{g}_{\mathbf{t}}$ ) is as follows:

$$
P_{S}(\mathbf{g})=\frac{1}{Z} \exp \sum_{(x, y) \in \mathbf{R}} \sum_{(\xi, \zeta) \in \mathbf{N}} V_{a}\left(g_{x, y}, g_{x+\xi, y+\zeta}\right)
$$

where $Z$ is the approximate partition function [348, 367]: $Z \approx e^{\left(V_{a}\left|\mathbf{C}_{\mathbf{N}}\right|\right)}$. The MGRF model is identified analytically using the maximum likelihood estimates of the potentials [367]:

$$
V_{a, \mathrm{eq}}=-V_{a, \mathrm{ne}}=2 \times f_{a, \mathrm{eq}}(\mathbf{g})-1
$$

where $f_{a, \text { eq }}(\mathbf{g})$ denotes the relative frequency of the label pairs in the neighboring pixel pairs $\{(x, y),(x+\xi, y+\zeta)\} \in \mathbf{R}^{2} ;(\xi, \zeta) \in\{( \pm 1,0),(0, \pm 1),( \pm 1, \pm 1)\}$. Similarly, the joint MGRF probability, $P_{S}\left(\mathbf{g}_{\mathbf{r}}, \mathbf{g}_{\mathbf{t}}\right)$, can be estimated using Equation (57) and the neighborhood system of Figure $66(b)$ with $d \in \mathbf{D}=\{1, \sqrt{2}, \sqrt{3}\}$. A gradient descent method to find the best lattice resolution (i.e., control points) to maximize the proposed similarity metric in Equation (56) is used. 


\section{Level Set Evolution}

After the co-alignment of the training images as described above, their region maps are obtained by a medical imaging expert delineation of the LV wall borders. Then, the shape prior, shown Figure 67, is built using these training maps and is updated for each input image to be segmented as described in Section III.C.1.a. Then, the weighted probabilistic shape prior, the pixel-wise image intensities (Section III.C.1.c), and the binary pairwise spatial interaction (Section III.C.1.b) are integrated into a joint MGRF model to provide the pixel-wise guidance of the level set deformable model as described in Section III.C.1.d. In total, the key steps of the LV wall borders segmentation are summarized in Algorithm 3.

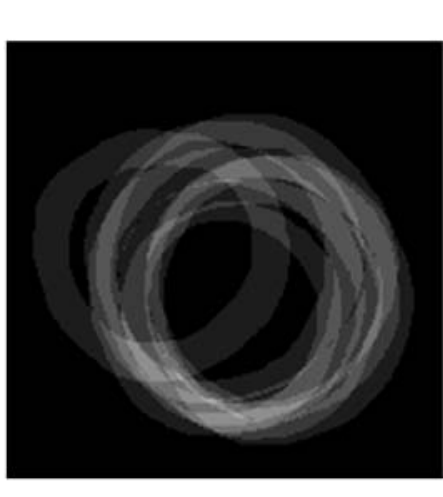

(a)

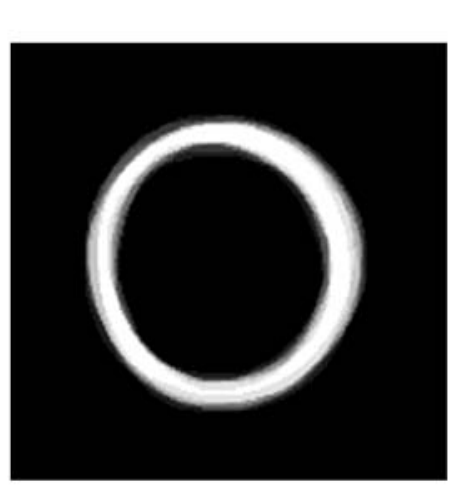

(c)
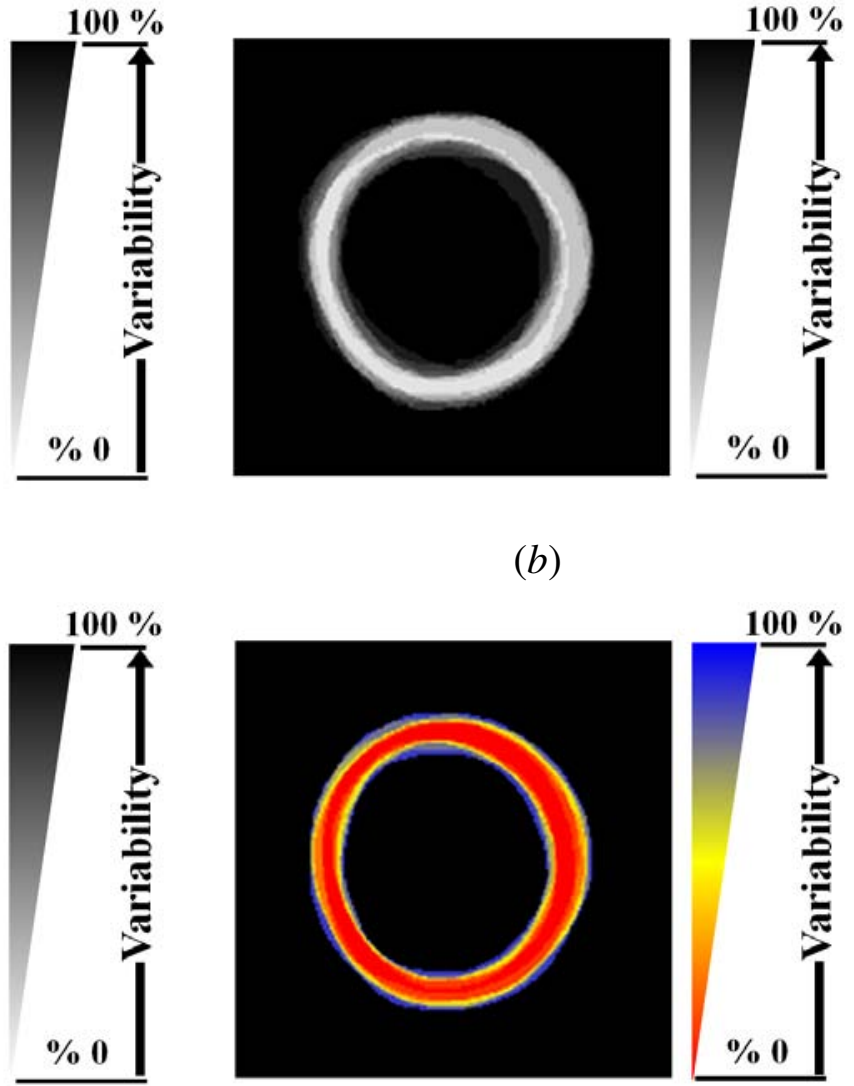

$(d)$

FIGURE 67: Overlaps of the training maps before $(a)$ and after $(b)$ affine registration; $(c)$ after the two-step registration; and $(d)$ color-coded visualization of shape prior. 
Input: grayscale image $\mathrm{g}$ to be segmented.

Output: segmented LV borders.

1. Construct the shape prior using the training images and their binary maps.

2. For each input image $\mathrm{g}$ to be segmented:

(a) Co-align g to one of the training images using the proposed two-steps registration methodology.

(b) Update the weighted shape prior.

- Calculate the NCCs between the co-aligned image and all training images.

- Compute the weighted sum of all training maps to update the shape prior.

(c) Estimate the LCDG models of marginal intensity distributions for the LV wall and its background.

(d) Use the estimated LCDG to form an initial LV wall/background map $\mathbf{m}$.

(e) Estimate the Gibbs potentials for the binary MGRF model of $\mathbf{m}$.

3. Find the speed function defined in Equation (51) using Steps $2(b)-2(e)$.

4. Evolve the level set using the determined speed function.

\section{ISO-CONTOURS REFINEMENT}

To generate accurate pixel-by-pixel parametric maps that represent the transit of the $\mathrm{CA}$ in the LV wall of a given patient, one-on-one pixel matches in all segmented walls of the time series images are required. To account for the segmentation errors and imperfection of image alignment, an additional registration refinement step is proposed, which is based on deforming each pixel of a target wall over evolving iso-contours to closely match the reference wall. To generate these iso-contours, the first step is to extract the centerline of both reference and target walls. A description of this follows. 


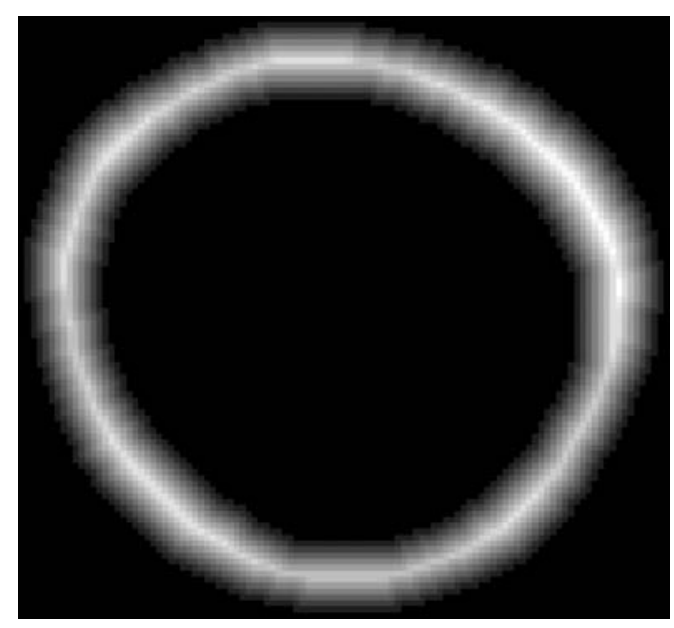

(a)

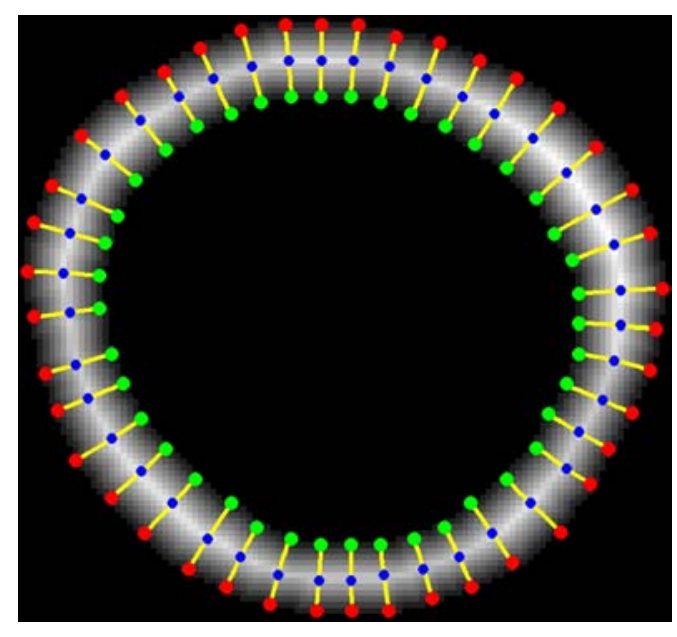

(c)

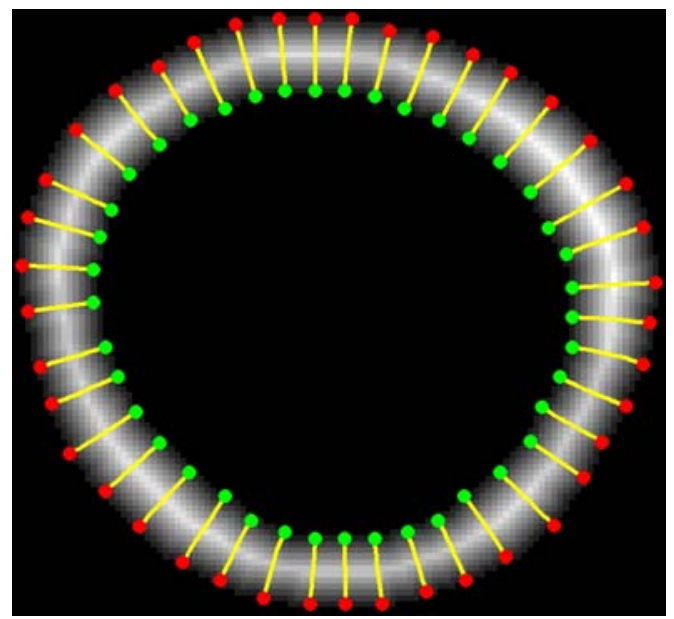

(b)

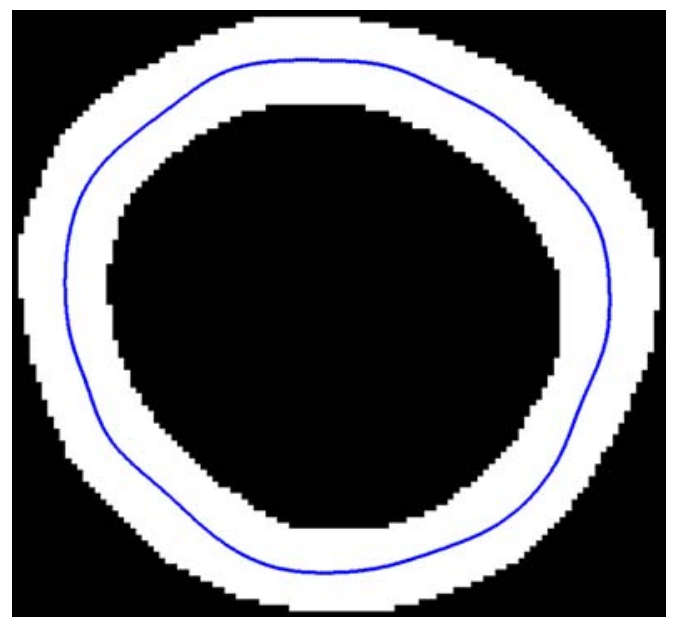

$(d)$

FIGURE 68: Illustration of the centerline extraction: $(a)$ the distance map of a typical LV wall, $(b)$ the streamlines found by solving Laplace equation between the inner and the outer borders of the LV wall, $(c)$ the identified centerline points (blue), and $(d)$ the extracted centerline overlaid on the binary mask of the LV wall.

\section{Centerline Extraction of the LV Wall}

The main idea of the proposed centerline approach is to find point-to-point correspondences between the inner and outer borders of the segmented LV wall. Then, the centerline is extracted by picking the points that are located at equidistance from each two correspondence points (see e.g., blue points in Figure 68 (c)). The point-to-point correspon- 
dences between the wall borders are estimated based on solving the linear second-order Laplace equation as described in Section III.C.2, Equation (52). Basic steps of centerline extraction using the Laplace method are summarized in Algorithm 4 and Figure 68. A distance map is generated inside the LV wall by finding for every inner point the minimum Euclidean distance to the wall boundaries, as shown in Figure $68(a)$. The Laplace equation is then applied to wall borders to co-locate the corresponding border points (see Figure 68 (b)). Then, for each streamline linking corresponding border points, the streamline point located at equidistance from both borders is selected as a candidate location on the centerline (see Figure $68(c)$ ). Finally, the centerline is generated using a closed spline fit for the selected points (see Figure $68(d)$ ).

\section{Algorithm 4 Centerline Determination of the Left Ventricle Wall}

Input: segmented LV wall.

Output: centerline of the LV wall.

1. Generate a distance map inside the segmented LV wall (see Figure $68(a)$ ).

2. Apply the Laplace equation to the inner and outer borders of the segmented myocardium to c-allocate the point-to-point correspondences (see Figure $68(b)$ ).

3. Locate the points located equidistant from the boundary point-pairs using distance map obtained in Step (1) (see Figure $68(c)$ ).

4. Determine the centerline using a closed spline fit for the identified points in Step 3 (see Figure $68(d)$ ).

\section{Evolution of the Generated Iso-contours}

Following the extraction of the centerline, a collection of equi-spaced contours (isocontours) within the LV wall, generated at equal distances from the centerline, is formed 
(see Figure 69). Subsequently, the Laplace equation was applied to the corresponding isocontours of the reference and target LV walls to co-locate the corresponding contour points.

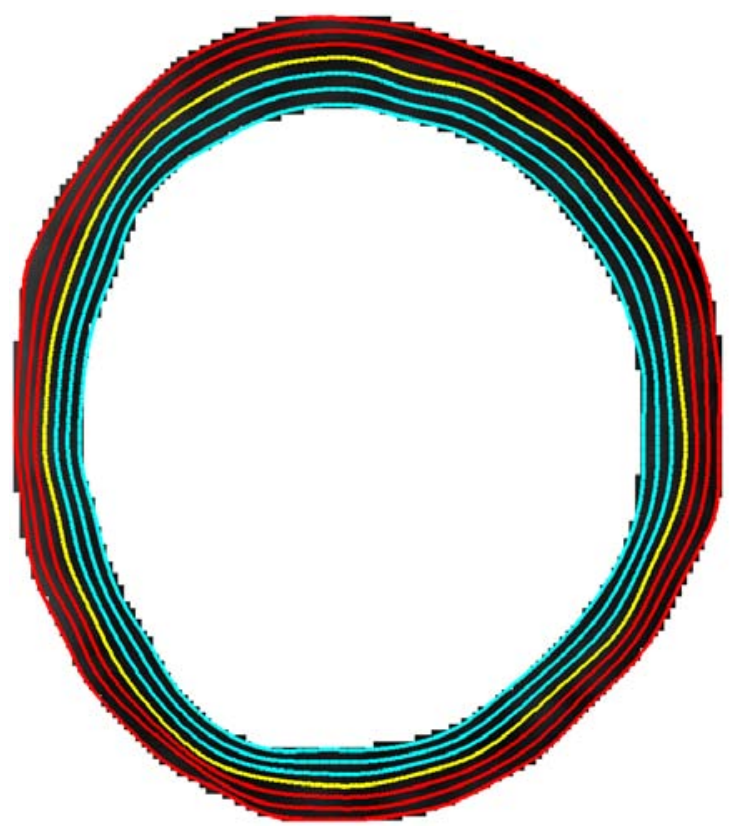

(a)

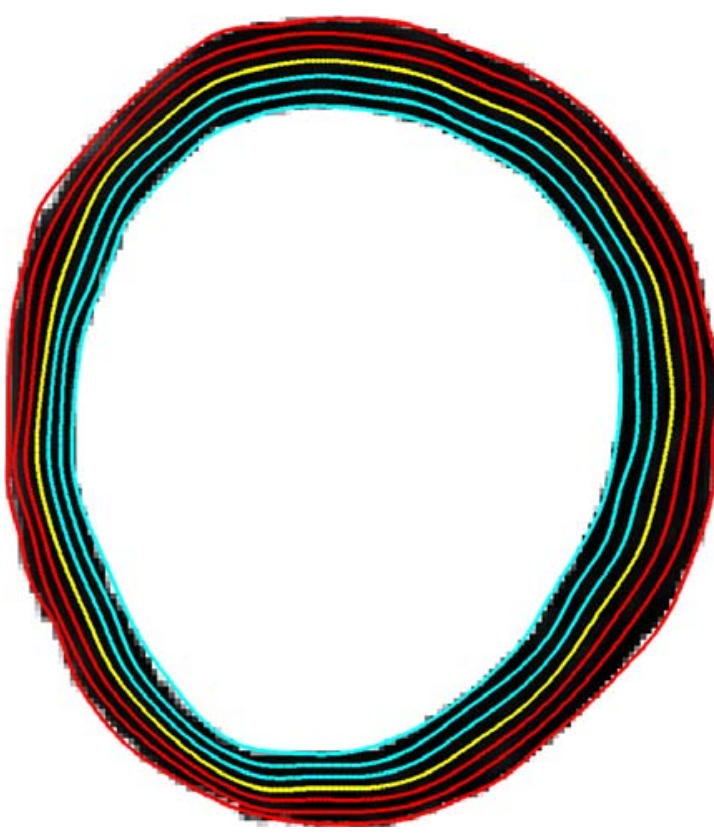

(b)

FIGURE 69: A typical example of the generated iso-contours for the reference $(a)$ and target $(b) \mathrm{LV}$ walls. The extracted centerline of the LV wall is shown in yellow.

\section{E. PERFUSION TIME SERIES AND PARAMETERS}

The ultimate goal of the proposed analysis framework is to improve regional and transmural analysis and visualization of cardiac perfusion of a given patient. Thus, the final step of the proposed framework is to construct agent kinetic (time-intensity) curves, by calculating the average intensities of the LV wall for each image of the time series, and to derive perfusion indexes from these curves (see Figure 70). As described in Section III.C.3, the agent delivery is characterized using both the transient phase indexes (time-to-peak, peak signal intensity, and initial up-slope [81]) and the tissue phase signal change index or the average plateau index, which is less dependent on temporal sampling [87]. 


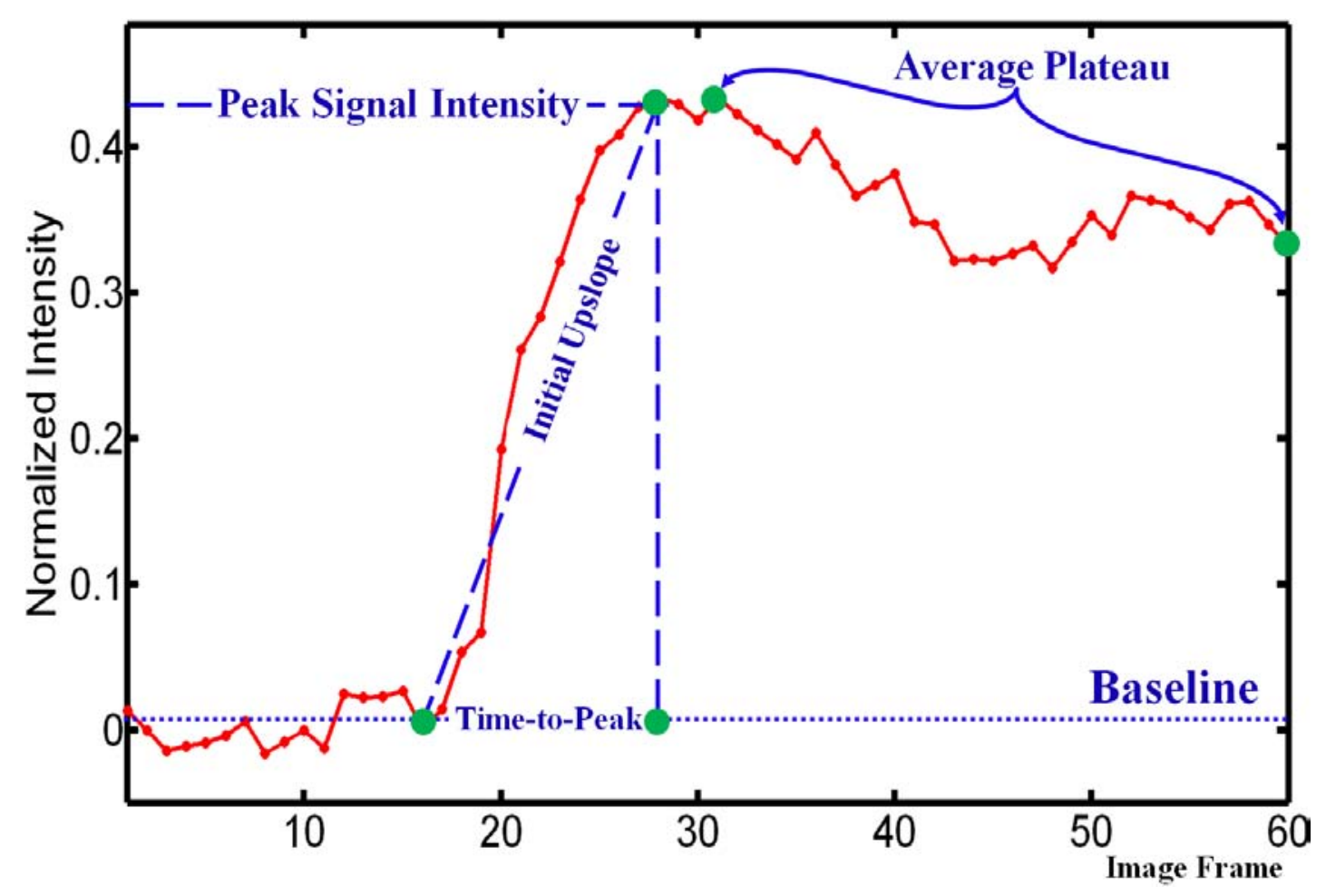

FIGURE 70: A typical agent kinetic curve showing the time points within the cardiac perfusion MRI data used to estimate the perfusion indexes. Image frames are acquired at an interval of approximately one second.

\section{F. PERFORMANCE EVALUATION}

In order to evaluate the accuracy of the proposed segmentation approach, the Dice similarity coefficient (DSC) [382], described in Section III.D.1, was used to characterize the agreement between the segmented and ground truth regions. Second, the accuracy of the proposed nonrigid registration is evaluated using realistic synthetic phantoms [5] and a Euclidean distance error metric, which is calculated for each point on the borders of the reference wall and its corresponding point on the target wall's borders. The corresponding pair of points (460 points) were selected by solving the Laplace equation between the borders of the reference and all target walls. Finally, the overall performance of the proposed framework was evaluated using a segment-wise comparisons of the perfusion indexes for the pre- and post treatment using the 17-segment model of the heart [6] and by parametric perfusion maps. Since ischemic heart disease has both regional and transmural manifes- 
tations, pixel-by-pixel maps are created as a method of regional display of the computed perfusion indexes (Figure 70). These parametric maps (see e.g., Figure 80) will be used by radiologists for visual identification of pathological tissues and assessment of the response to the myoregeneration therapy.

\section{G. EXPERIMENTAL RESULTS}

\section{Clinical Data}

The proposed framework has been tested on 24 independent FP-MRI data sets. These data sets were obtained from eight patients with prior myocardial infarctions, documented by viability MRI, and who were undergoing a novel myoregneration therapy [65]. Short-axis images were obtained using a 1.5 T Espree system-Siemens Medical Solutions, USA Inc.-with phased array wrap-around reception coils. Saturation recovery GRE imaging $\left(T_{R^{-}}\right.$minimum $/ T_{E^{-}} 1.2 \mathrm{msec} / T_{I^{-}} 100 \mathrm{msec} /$ Flip angle- $\left.12^{\circ}\right)$ was performed with cardiac gating, and during a typical 20 second breath-hold followed by free (shallow) breathing acquisition. Slice thickness is $10 \mathrm{~mm}$ and in-plane resolution is $1.87 \times 1.87 \mathrm{~mm}^{2}$. Typically, 60 time frames were obtained with a time frame acquired in an R-R, heart interval.

\section{Registration Results and Evaluation}

The proposed nonrigid registration using the first-and second-order NMI is evaluated using realistic synthetic phantoms [5]. The visual appearance of the phantom images (Figure 71) were generated to mimic the varying contrast-enhanced intensity of the agent transit based on the integration of the Gibbs sampler and the maximum A posteriori (MAP) estimates [21] for a pairwise energy function of a 3D Generalized Gauss-Markov random field (GGMRF) probabilistic model [418]. The phantom images were constructed from a manually segmented LV wall using a descriptive mathematical model that accounts for physical features of the LV and physiological LV responses as the heart progresses through the cardiac cycle [5]. The simulated phantom contains images with different types of de- 
formations: namely, small, moderate, and large deformations, as shown Figure 72.

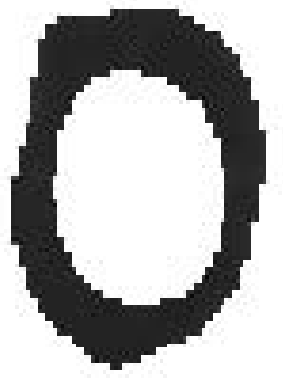

(a)

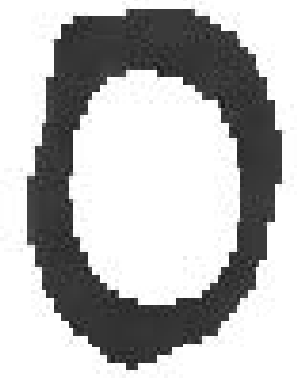

(b)

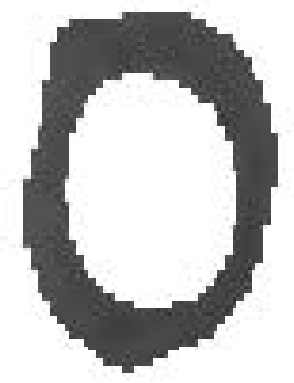

(c)

FIGURE 71: The realistic synthetic phantom images [5] at different myocardial signal intensities: $(a)$ baseline, $(b)$ peak intensity, and $(c)$ tissue distribution phases. The images were generated based on the integration of the Gibbs sampler and the MAP estimates [21].

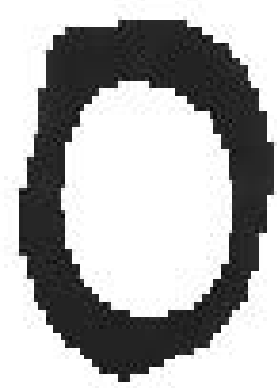

(a)

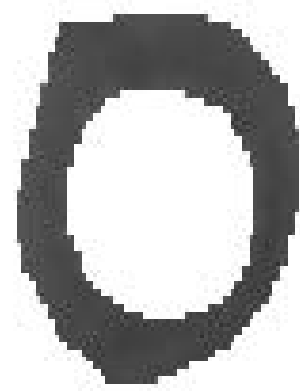

(b)

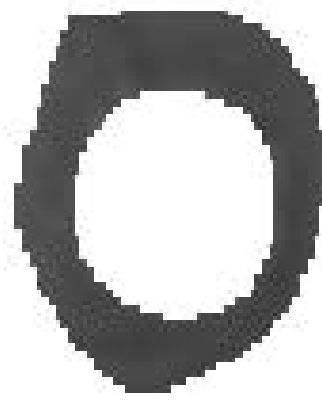

(c)

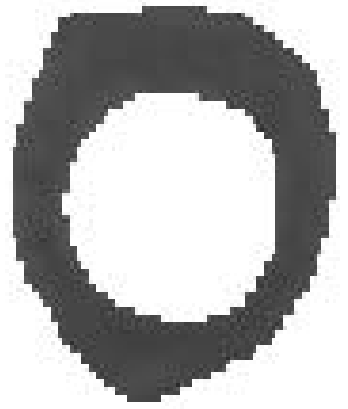

$(d)$

FIGURE 72: Three different types of deformations of the realistic synthetic phantom [5] for the tissue distribution phase: (a) reference image; and target images with $(b)$ small, $(c)$ moderate, and $(d)$ large deformations.

To assess the accuracy of the proposed registration approach, the deformed images were registered to the reference phantom image (Figure $71(a)$ ) using $(i)$ the B-splines model using the first-order NMI only $\left(\mathrm{BS}_{1}\right)$, and $(i i)$ the B-splines model using the proposed first- and second-order NMI $\left(\mathrm{BS}_{2}\right)$. The accuracy of registration is assessed by calculating the Euclidian distances between a set of 460 points on the border of the reference (Figure $71(a)$ ) and all target walls. The average error statistics are summarized in Table 12, and the registration results for one of the phantom images (Figure $71(c)$ ) for the three types of deformations are shown in Figure 73. 


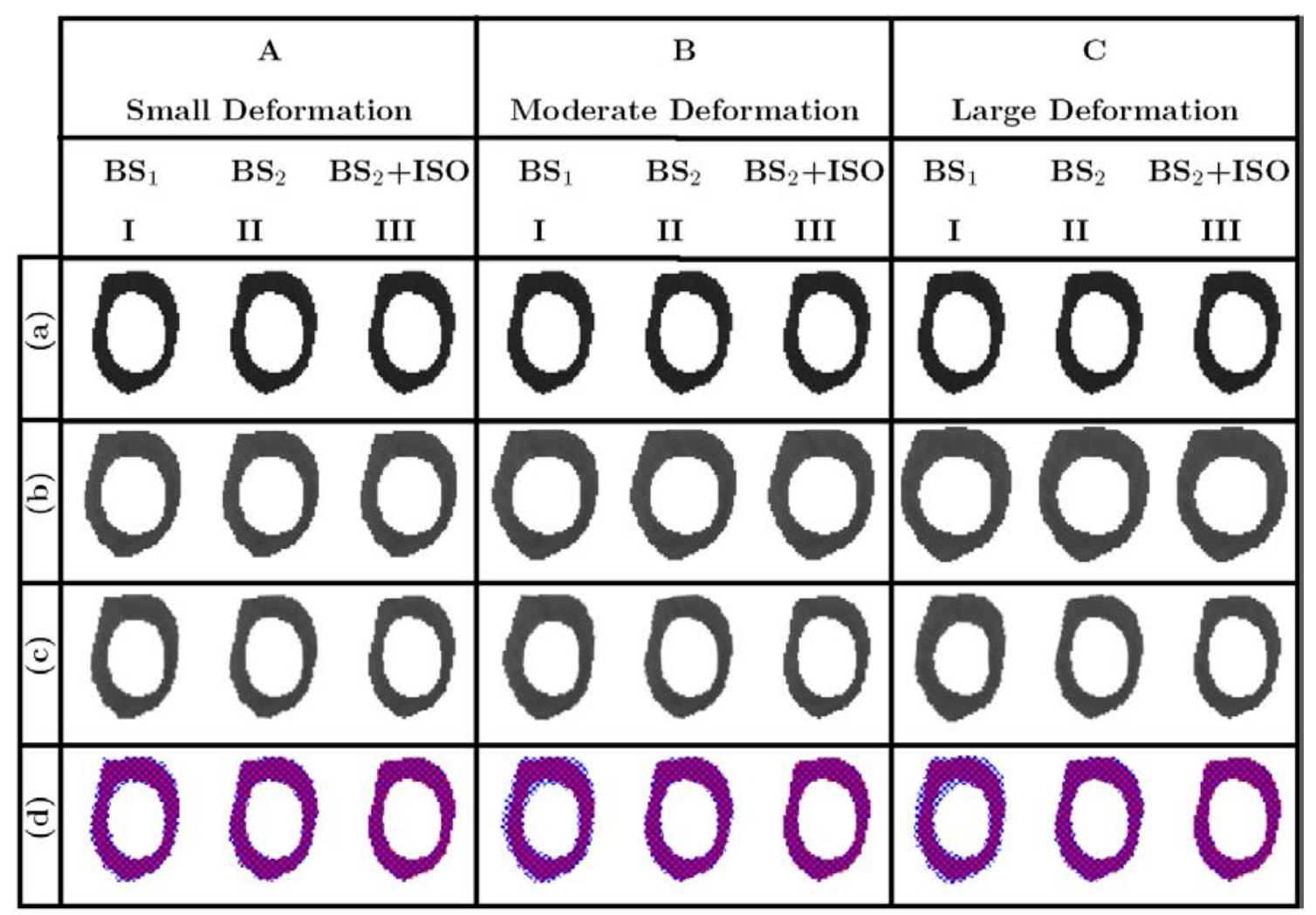

FIGURE 73: Registration accuracy of the synthetic phantom shown in Figure $71(c)$ for the (A) small, (B) moderate, and (C) large deformations: (a) reference image, $(b)$ deformed image, $(c)$ registered image, and $(d)$ checkerboard visualization of the superimposed reference and target images after alignment using (I) the B-splines model with first-order NMI only $\left(\mathrm{BS}_{1}\right)$, (II) B-splines model with the proposed first- and second-order NMI $\left(\mathrm{BS}_{2}\right)$, and (III) the proposed approach $\mathrm{BS}_{2}$ followed by iso-contours registration $\left(\mathrm{BS}_{2}+\mathrm{ISO}\right)$.

In addition to the evaluation of the proposed registration methodology on synthetic phantoms, the accuracy of the proposed registration was also qualitatively assessed using physiological data. A typical agent kinetic curve for one data set before and after registration is shown in Figure 74. As is readily seen, the proposed registration methodology effectively reduces frame-to-frame signal intensity variability related primarily to motion effects. The reduction of signal variability over the time series produces more smoothed time-intensity curves that allows for accurate estimation of perfusion-related parameters. 
TABLE 12: Accuracy of the proposed nonrigid registration (in $\mathrm{mm}$ ) on the realistic synthetic phantoms [5]. Note that the total number of points is 460 ("SD"-standard deviation).

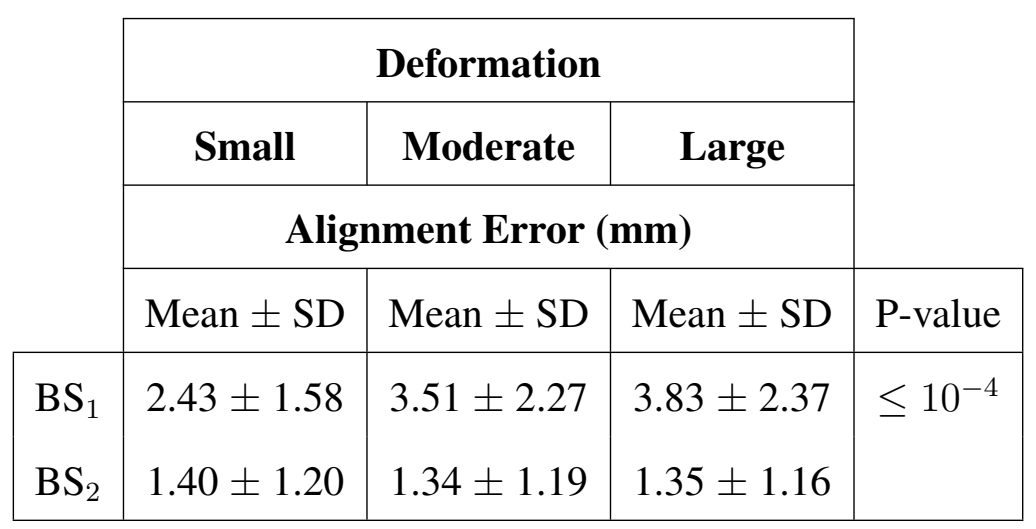

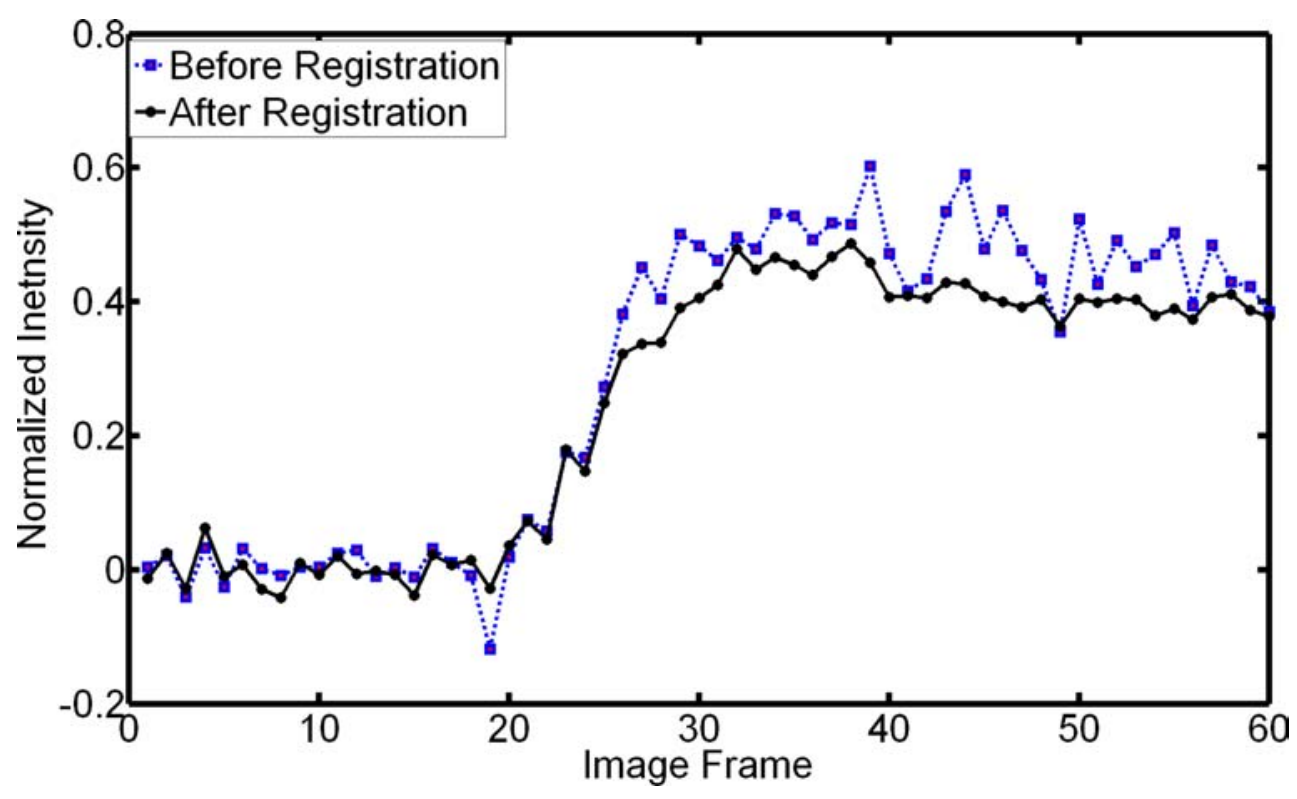

FIGURE 74: Typical time-intensity curves, averaged over the entire myocardium, for one patient before and after registration.

3. Segmentation Results and Evaluation

One of the image frames and its step-wise segmentation of the myocardial wall is shown in Figure 75. Additional segmentation results for three independent data sets are demonstrated in Figure 76. The accuracy of the proposed segmentation approach is evaluated using the DSC [382], and the statistics are summarized in Table 13. The ground truth was obtained by manual contouring of the LV borders by an MR imaging expert. 


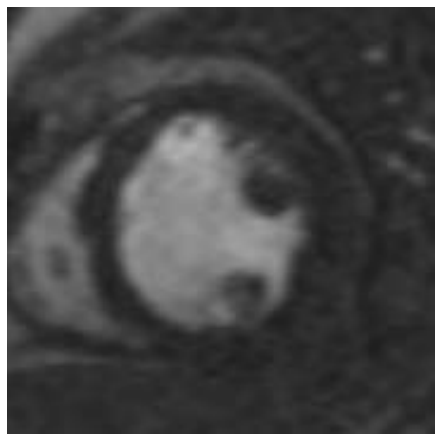

(a)

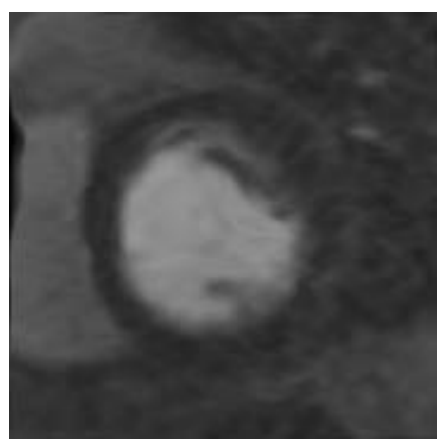

(d)

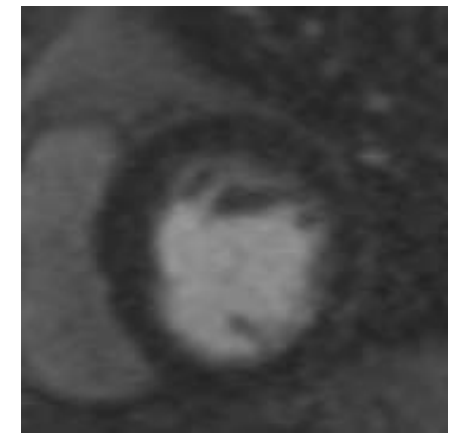

(b)

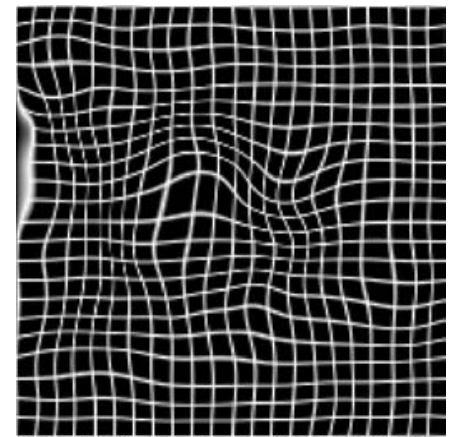

$(e)$

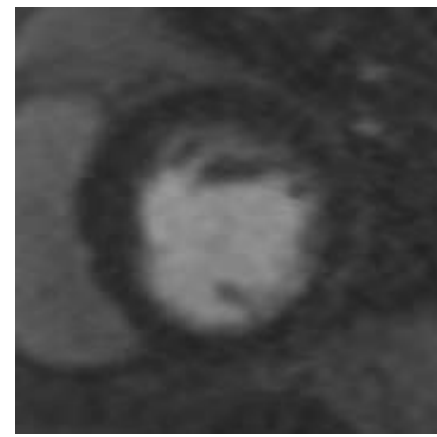

$(c)$

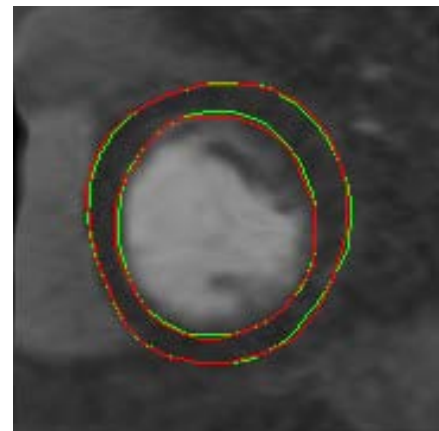

$(f)$

FIGURE 75: Step-by-step segmentation: (a) reference image, $(b)$ target image, $(c)$ affine registration, $(d) \mathrm{B}$-splines registration, $(e)$ deformation field used for B-splines registration, and $(f)$ final borders' segmentation (red) with respect to the ground truth (green).
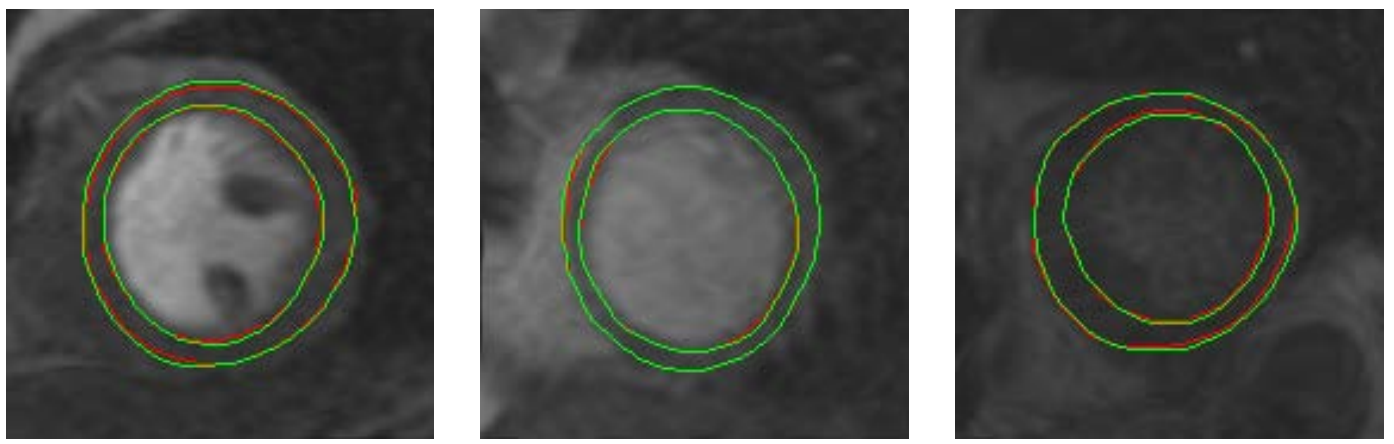

FIGURE 76: More segmentation results of the proposed segmentation approach for one image frame in three different patients showing reliable determination of the wall borders (red) with respect to the ground truth (green), at different myocardial signal intensities.

To highlight the advantage of the proposed level-set segmentation approach, its performance was compared against two other shape-based segmentation techniques; namely, 
the shape-based segmentation approach proposed by Tsai et al. [1] that uses a linear combination of signed distance maps to build the shape prior, and its modified version that was proposed by Abdelmunim and Farag [2] and uses a linear combination of signed vector level set functions to construct the shape prior. To illustrate the comparative qualitative accuracy of the proposed segmentation approach versus Tsai et al. [1] and Abdelmunim and Farag [2], representative examples for two different data sets are shown in Figure 77. Table 13 compares the DSC statistics for the proposed, Tsai et al. [1], and Abdelmunim and Farag [2] approaches. Differences between the mean DSC value for the proposed segmentation, and Tsai et al. [1] and Abdelmunim and Farag [2] are statistically significant by the paired $t$-test. As demonstrated in Table 13, the proposed approach yields higher segmentation accuracy compared to other approaches. This is due to that the proposed approach accounts for both global and local motions of the heart; while in the shape-based approach [1] and the vector level set approach [2], only the global motion of the heart is handled during image alignment.

TABLE 13: Performance of the proposed segmentation versus the shape-based approach [1] and the vector level set approach [2] in terms of the DSC compared with the expert manual ground truth. Note that "SD" stands for standard deviation.

\begin{tabular}{|l|c|l|}
\cline { 2 - 3 } \multicolumn{1}{c|}{} & \multicolumn{2}{c|}{ DSC } \\
\hline Algorithm & Mean \pm SD & P-value \\
\hline Proposed approach & $\mathbf{0 . 9 1 0} \pm \mathbf{0 . 0 3 7}$ & \\
Shape-based approach [1] & $0.862 \pm 0.045$ & $\leq 10^{-4}$ \\
Vector level set approach [2] & $0.844 \pm 0.047$ & $\leq 10^{-4}$ \\
\hline
\end{tabular}

To evaluate the effect of intra- and inter-observer variability, one temporal data set comprising of 50 images was manually segmented by two experienced observers. The DSC values comparing the proposed automatic segmentation results are summarized in Table 14. A paired $t$-test was performed between the DSCs of the proposed segmentation approach with respect to each of the observer's contouring. The $P$ values of 0.638 (intra-observer), 

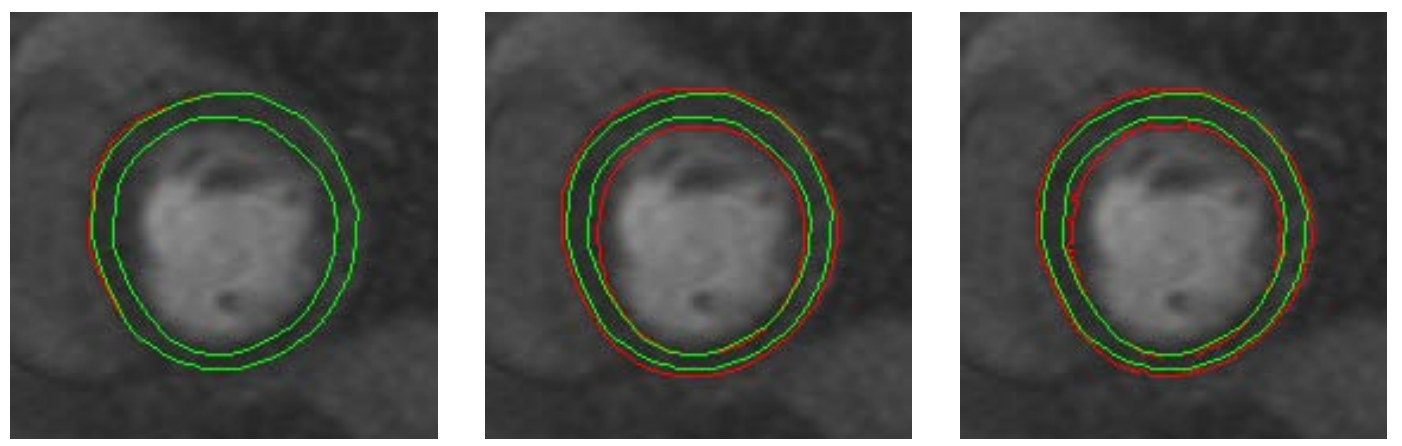

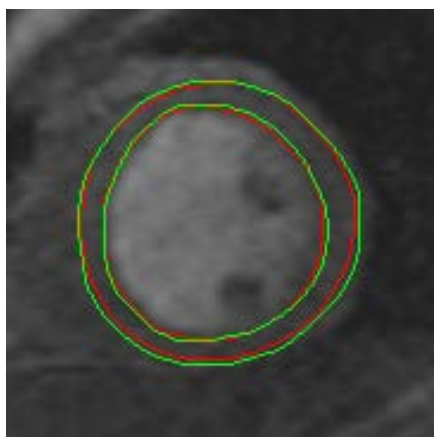

(a)

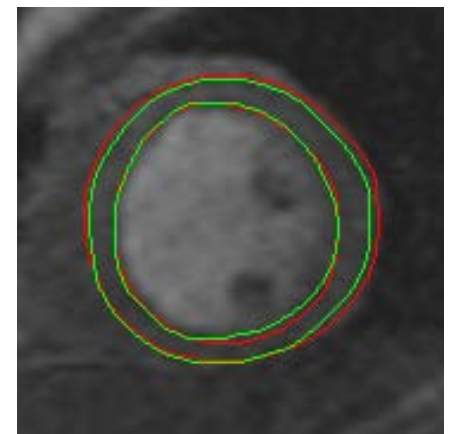

(b)

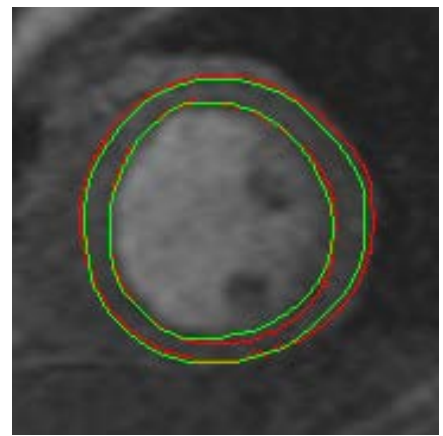

(c)

FIGURE 77: Comparative segmentation results for $(a)$ the proposed approach, $(b)$ the shape-based approach [1], and (c) the vector level set approach [2] for different image frames from different data sets.

TABLE 14: Inter-observer performance of the segmentation of 50 agent-enhanced image frames. DSC between the segmented myocardium and expert ground truth obtained using both intra- and inter-observers. Note that "SD" stands for standard deviation.

\begin{tabular}{|l|c|c|c|}
\cline { 2 - 4 } \multicolumn{1}{c|}{} & \multicolumn{3}{c|}{ DSC } \\
\cline { 2 - 4 } \multicolumn{1}{c|}{} & Observer & intra-observer & inter-observer \\
\cline { 2 - 4 } & 1 & 2 & 3 \\
\hline Mean \pm SD & $0.890 \pm 0.022$ & $0.887 \pm 0.020$ & $0.885 \pm 0.023$ \\
\hline P-value & $(1,2): 0.638$ & $(2,3): 0.673$ & $(1,3): 0.065$ \\
\hline
\end{tabular}

and 0.673 and 0.065 (inter-observers) are not statistically significant and support strong agreement. Moreover, to evaluate the sensitivity of the proposed segmentation approach to selection of the training images, the eight manually segmented independent patient time series data sets were divided into three groups and performed the 3-fold cross validation. 
Table 15 shows that such a dependence is statistically insignificant. To further test the robustness of the proposed segmentation approach, the receiver operating characteristic (ROC) [380] was employed. The ROC for segmentation was computed using $(i)$ the intensity information only; (ii) the intensity information and spatial interactions, and (iii) the intensity information and shape features. The results shown in Figure 78 clearly demonstrate that the full features combination results in the greatest area under the ROC curve $\left(A_{z}=0.915\right)$

TABLE 15: Sensitivity to the selection of the training data, divided into three groups. Note that "SD" stands for standard deviation.

\begin{tabular}{|l|c|c|c|}
\cline { 2 - 4 } \multicolumn{1}{c|}{} & \multicolumn{3}{c|}{ DSC } \\
\cline { 2 - 4 } \multicolumn{1}{c|}{} & Group \#1 & Group \#2 & Group \#3 \\
\cline { 2 - 4 } \multicolumn{1}{c|}{} & 1 & 2 & 3 \\
\hline Mean \pm SD & $0.904 \pm 0.031$ & $0.910 \pm 0.031$ & $0.910 \pm 0.028$ \\
\hline P-value & $(1,2): 0.252$ & $(2,3): 0.812$ & $(1,3): 0.443$ \\
\hline
\end{tabular}

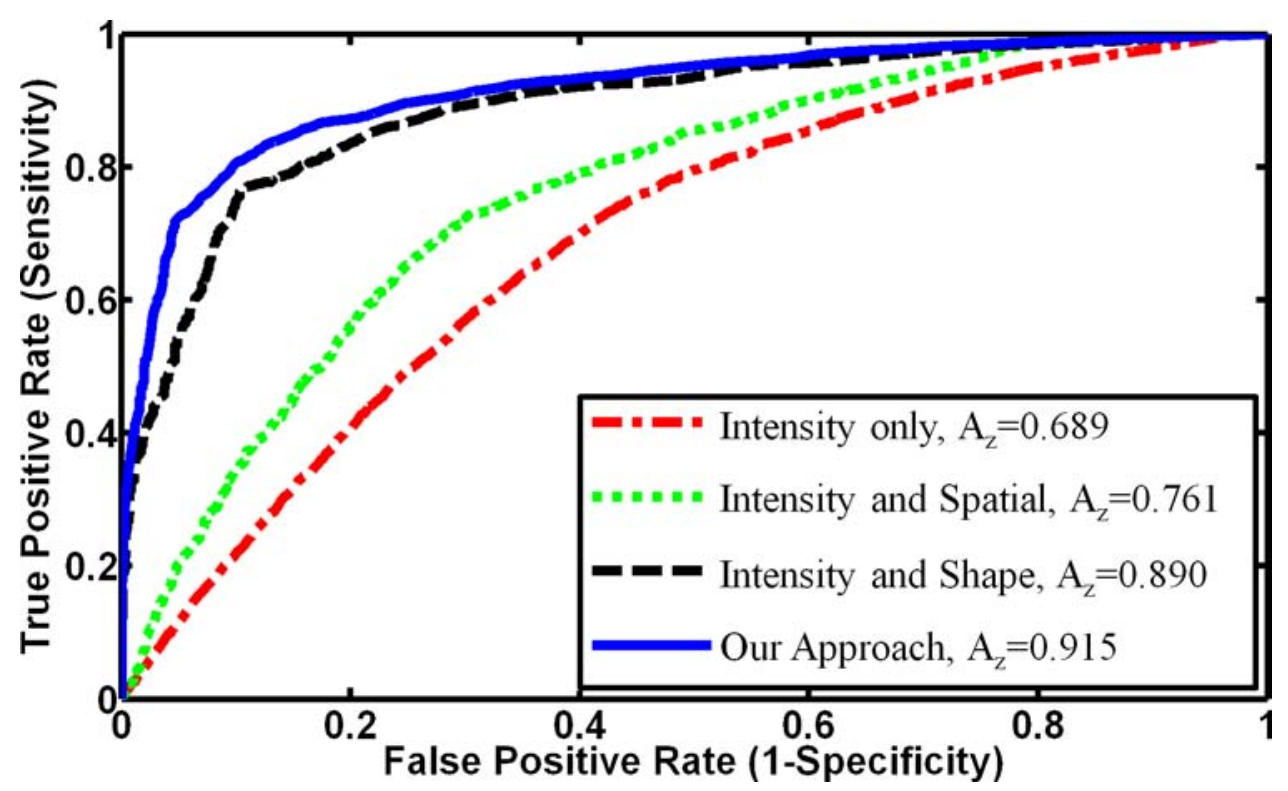

FIGURE 78: The ROC curves for different feature combinations for level set guidance: the proposed approach (blue); intensity feature only (red), combined intensity and spatial interactions features (green), and combined intensity and shape features (black). 


\section{Iso-Contours Refinement}

The ultimate goal of the proposed framework is to compute perfusion parameters on pixel-wise basis for the evaluation of myocardial perfusion. Thus, to account for any misregistration residual of the LV wall after the segmentation step, a final Laplace-based registration refinement step was employed to ensure that there is a one-on-one pixel match of all segmented walls, i.e., the same pixels are being evaluated between the image frames in given data set. Figure 79 demonstrates a typical example of the final Laplace-based registration step of one image frame. For visual assessment of the registration adequacy, a checkerboard display before and after the registration refinement step is shown in Figure 79 (d) and (e), respectively. The reference and target objects are in red and blue, respectively. It is evident based on the figure that the application of the Laplace-based local alignment of contours shows near ideal matching of the target and reference contours; therefore it ensures pixel-on-pixel matches of all segmented frames.

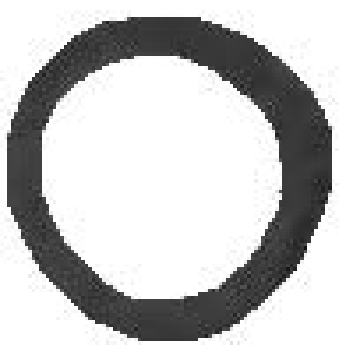

(a)

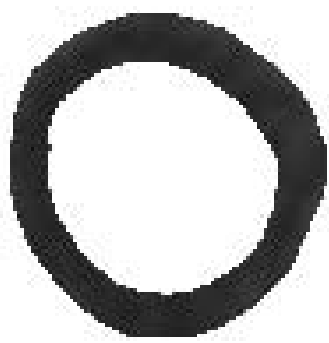

(b)

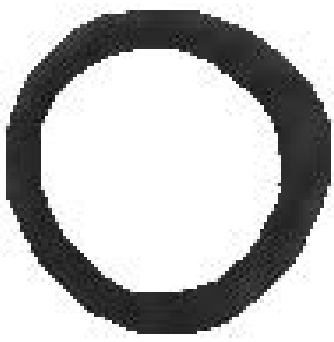

(d)

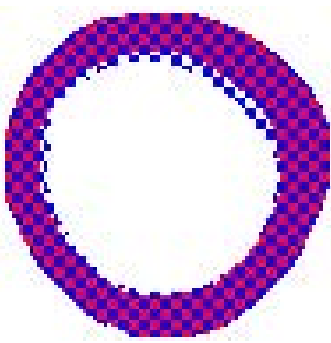

(c)

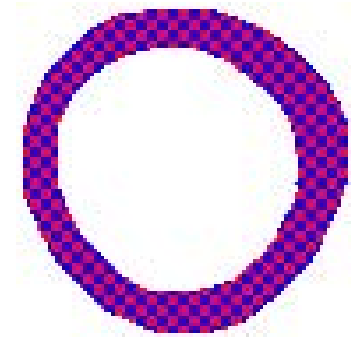

(e)

FIGURE 79: An example of the final iso-contours-based adjustment: $(a)$ reference image, (b) target image, $(c)$ checkerboard visualization of the superimposed target and reference walls before iso-contours registration, $(d)$ Laplace-based registration, and $(e)$ checkerboard visualization after iso-contours registration showing nearly ideal match of the boundaries. 
5. Perfusion Indexing and Physiological Assessment

The effectiveness of the proposed framework is illustrated using parametric map displays, which were developed to aid in visual assessment of patient responses to therapy. Following iso-contours registration, agent transit curves of registered data are constructed and the perfusion indexes (see Section IV.E) are estimated on a per-pixel basis. Then, the computed indexes are displayed using pixel-wise parametric maps. An example for one image section of a patient with heart damage before and after treatment using the four indexes is shown in Figure 80. The figure clearly demonstrates that the peak signal intensity (Figure $80(a)$ ) and the average plateau indexes (Figure $80(d)$ ) are the most uniform across regions. Moreover, a transmural gradient of perfusion is not evident before treatment; while after treatment the perfusion indexes not only show an improvement, but also one can discern a gradient of perfusion across the wall (bottom row in Figure 80). This was consistent for data corresponding to the treated patients. Thus, these functional maps hold promise for clinicians to visualize treatment effects in patients.

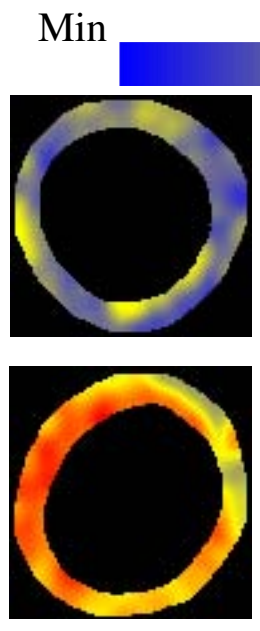

(a)
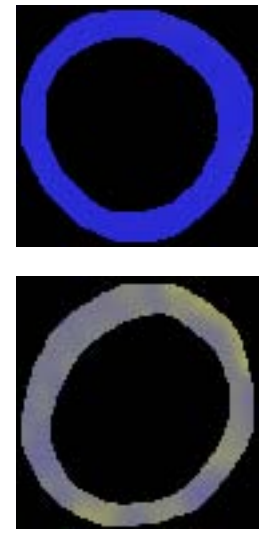

(b)
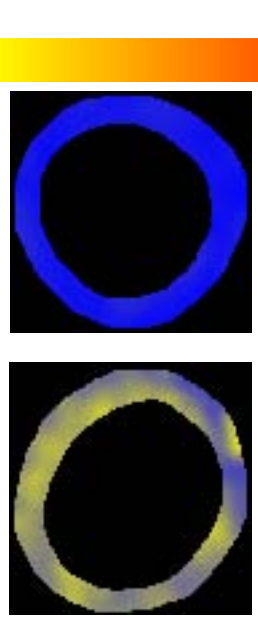

(c)
$\operatorname{Max}$
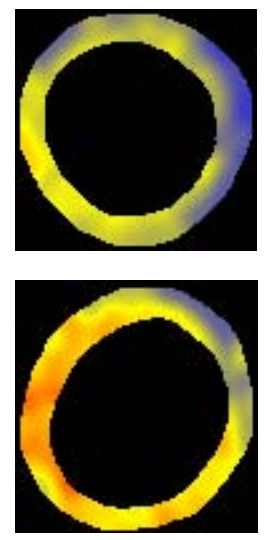

(d)

FIGURE 80: Parametric maps for the perfusion indexes: (a) peak signal intensity, $(b)$ time-to-peak, $(c)$ initial up-slope, and $(d)$ average of tissue distribution (plateau) phase for one subject. The red and blue hues of the color scale relate to highest and lowest values, respectively. The top and bottom rows represent pre- and post-therapy, respectively. Note that there is an apparent improvement in the myocardial wall after therapy. 
In order to quantify the perfusion, the estimated values of the perfusion indexes are assessed using the standardized myocardial segment model [6] in which the LV wall is divided into 17 circumferential segments: six segments at the basal and mid-cavity levels, four segments at the apical level, and one at the the extreme tip of the ventricle. A schematic illustration of the locations of segments corresponding to the mid-cavity section of the heart is shown in Figure 81. Table 16 summarizes the average values of the four estimated perfusion-related parameters, for the six sectors of the mid-cavity level of the heart corresponding to the patient data in Figure 80. From the presented values, after undergoing treatment these perfusion indexes have show an improvement as compared with those before treatment. Moreover, the $95 \%$ confidence interval statistic was used to compare the four perfusion indexes before and after treatment. Figure 82 shows the box plots of the perfusion indexes' values for pre- and post-treatment for all patients in this study, averaged over the six sectors of the mid-cavity level. Based on the groups' separation, shown in Figure 82 , it can be concluded that, on average, there is an improvement of the estimated indexes after undergoing treatment compared with that before treatment.
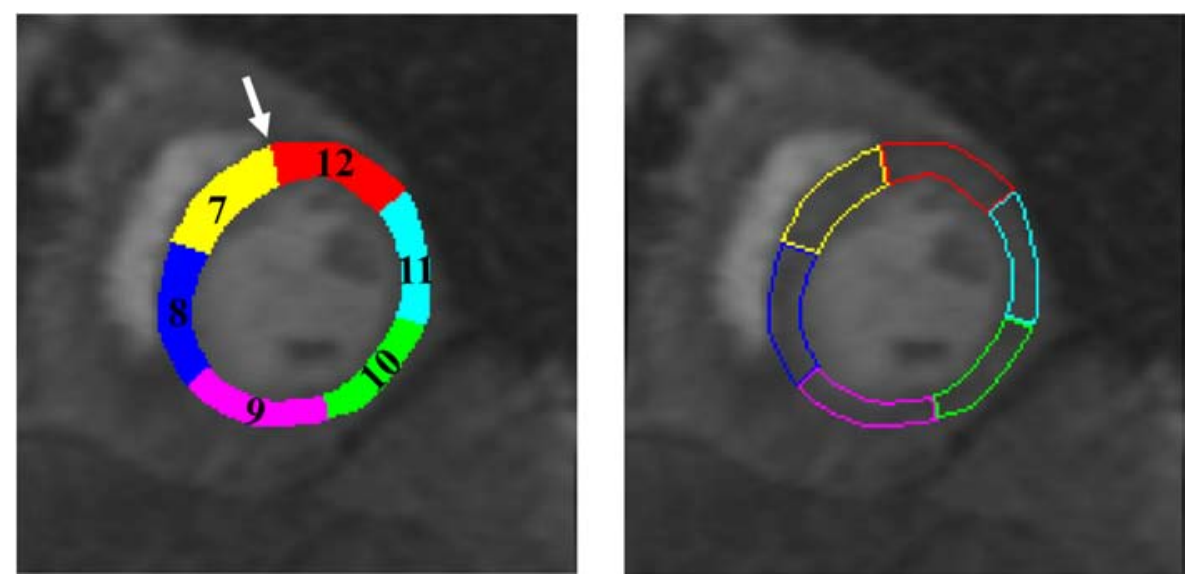

FIGURE 81: Corresponding segment locations for the mid-cavity image section of the myocardial 17-segment model [6]. The segment numbering starts counter-clockwise from the anatomical landmark indicated by the white arrow. Please note that the coloring is simply to indicate different segments and provide visibility of the segment numbering. 
TABLE 16: Average perfusion parameter values for the mid-cavity level sectors of the 17-segment model [6] for the results shown in Figure 80.

\begin{tabular}{|l|c|c|c|c|c|c|c|c|}
\cline { 2 - 9 } \multicolumn{1}{c|}{} & \multicolumn{2}{c|}{ Peak Value } & \multicolumn{2}{c|}{ Time-to-Peak } & \multicolumn{2}{c|}{ Up-Slope } & \multicolumn{2}{c|}{ Average Plateau } \\
\hline Sector Number & Pre & Post & Pre & Post & Pre & Post & Pre & Post \\
\hline Sector \#7 & 0.314 & 0.523 & 38.59 & 13.50 & 0.008 & 0.038 & 0.396 & 0.411 \\
Sector \#8 & 0.246 & 0.678 & 39.12 & 14.72 & 0.006 & 0.046 & 0.401 & 0.602 \\
Sector \#9 & 0.312 & 0.689 & 39.92 & 15.35 & 0.008 & 0.045 & 0.516 & 0.674 \\
Sector \#10 & 0.280 & 0.603 & 40.47 & 13.68 & 0.007 & 0.044 & 0.445 & 0.604 \\
Sector \#11 & 0.260 & 0.599 & 39.93 & 14.17 & 0.007 & 0.043 & 0.316 & 0.582 \\
Sector \#12 & 0.203 & 0.462 & 39.15 & 13.83 & 0.005 & 0.036 & 0.384 & 0.570 \\
\hline
\end{tabular}

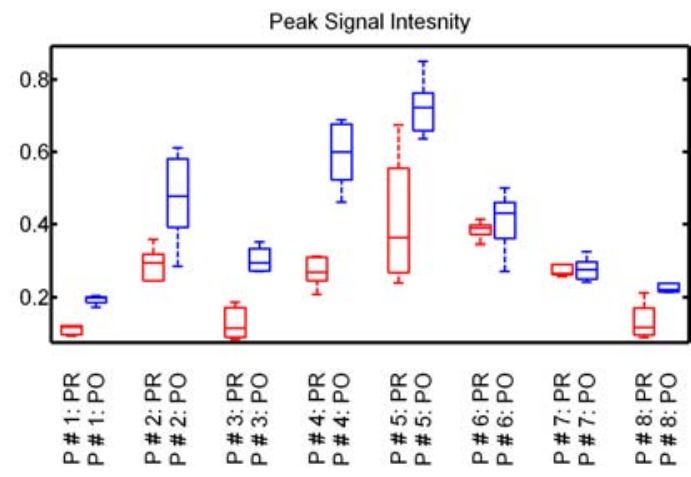

(a)

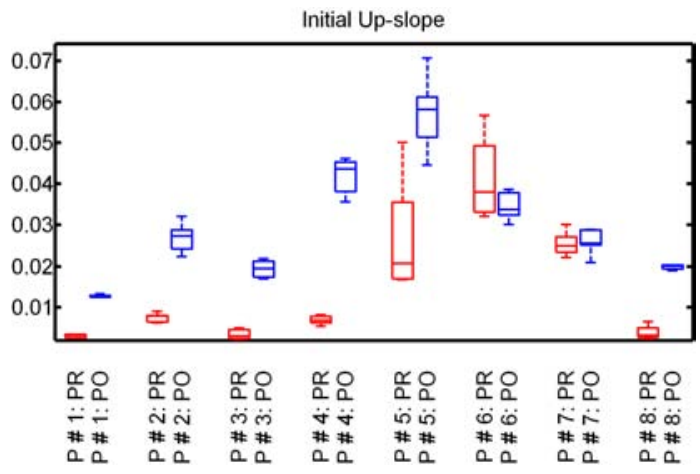

(c)

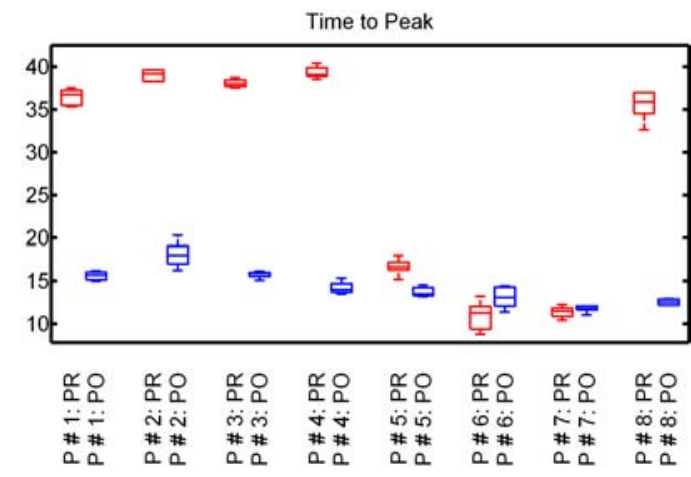

(b)

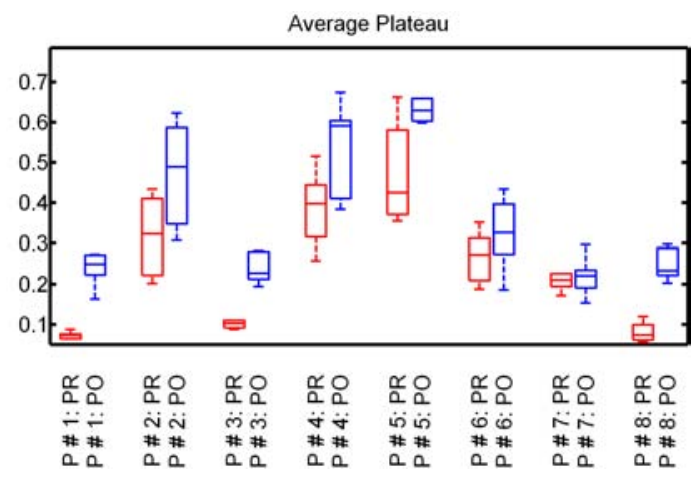

$(d)$

FIGURE 82: Box plots of the perfusion indexes (average over all heart sectors) for the pre-treatment (PR,red) and post-treatment (PO,blue) for all patients enrolled in this study: peak signal intensity $(a)$, time-to-peak $(b)$, initial up-slope $(c)$, and average plateau $(d)$. 


\section{H. SUMMARY}

Detection of heart failure in its earliest forms when there is an optimal opportunity to intervene using existing clinical strategies to restore damaged heart tissue may represent an important advance in the personalized management for individual patients to prevent progression to end-stage disease. In this chapter, a novel framework for improved analysis of cardiac first-pass perfusion MRI has been introduced. The proposed framework demonstrated the documented ability to reveal regional perfusion improvements with therapy, and transmural perfusion differences across the myocardial wall in a preliminary cohort of eight total participants who are undergoing a novel myoregeneration therapy. Essential contributions of this chapter are: $(i)$ a two-step registration methodology (affine-based registration followed by a local B-splines alignment) that accounts for heart deformations due to respiratory motion and heart contraction based on maximizing a new similarity function, which accounts for the first- and second-order normalized mutual information; (ii) deformable model-based segmentation combining a weighted probabilistic shape prior, first-order image intensities, and a second-order spatial interactions model; (iii) a Laplace-based registration refinement method for ultimate pixel-on-pixel matches of the segmented myocardium for accurate analysis of CA transit into the heart wall. The presented work in this chapter has been published in the International Conference on Image Processing (ICIP) [419], the International Conference on Pattern Recognition (ICPR) [97], and the International Symposium on Biomedical Imaging (ISBI) conference [96, 420]. 


\section{CHAPTER V EARLY DETECTION OF PROSTATE CANCER}

This chapter presents a noninvasive approach for early diagnosis of prostate cancer from dynamic contrast-enhanced magnetic resonance imaging (DCE-MRI). In order to precisely analyze the complex 3D DCE-MRI of the prostate, a novel processing framework that consists of four basic steps is proposed. The first step isolates the prostate region from the surrounding anatomical tissue based on a maximum a posteriori (MAP) estimate of a log-likelihood function that accounts for three image descriptors: the shape priori of the prostate, the spatial interaction between the prostate tissue, and the current appearance of the prostate tissue and its background. In the second step, a nonrigid registration approach based on the solution of the Laplace equation is employed to account for any local deformations that could occur in the prostate during the scanning process due to patient breathing and local motion. In the third step, the contrast agent kinetics are obtained from the segmented prostate of the whole image sequence of the patient. Then, two perfusionrelated features are collected from these curves and a $k_{n}$-nearest neighbor classifier is used to distinguish between malignant and benign detected tumors. Finally, parametric perfusion maps that illustrate the propagation of the contrast agent (CA) into the prostate tissues are constructed. This is achieved based on the analysis of the 3D spatial interaction of the change of the gray-level values of prostate voxels using a generalized Gauss-Markov random field (GGMRF) image model. Moreover, the tumor boundaries are determined using a level set deformable model controlled by the perfusion information and the spatial interaction between the prostate voxels. Experimental results on 30 clinical prostate DCE-MRI data sets yielded promising diagnostic results. 


\section{A. PROSTATE: ANATOMY, FUNCTION, AND DISEASES}

The prostate is the largest accessory gland in the male urinary and reproductive system. It is a cone-shaped organ and is about the size of a walnut that weighs approximately 15-20 grams and measures approximately $4 \times 2 \times 3$ centimeters in a mature male [421]. In this section, brief descriptions of the prostate anatomy and function, as well as the diseases that affect the prostate, are provided.

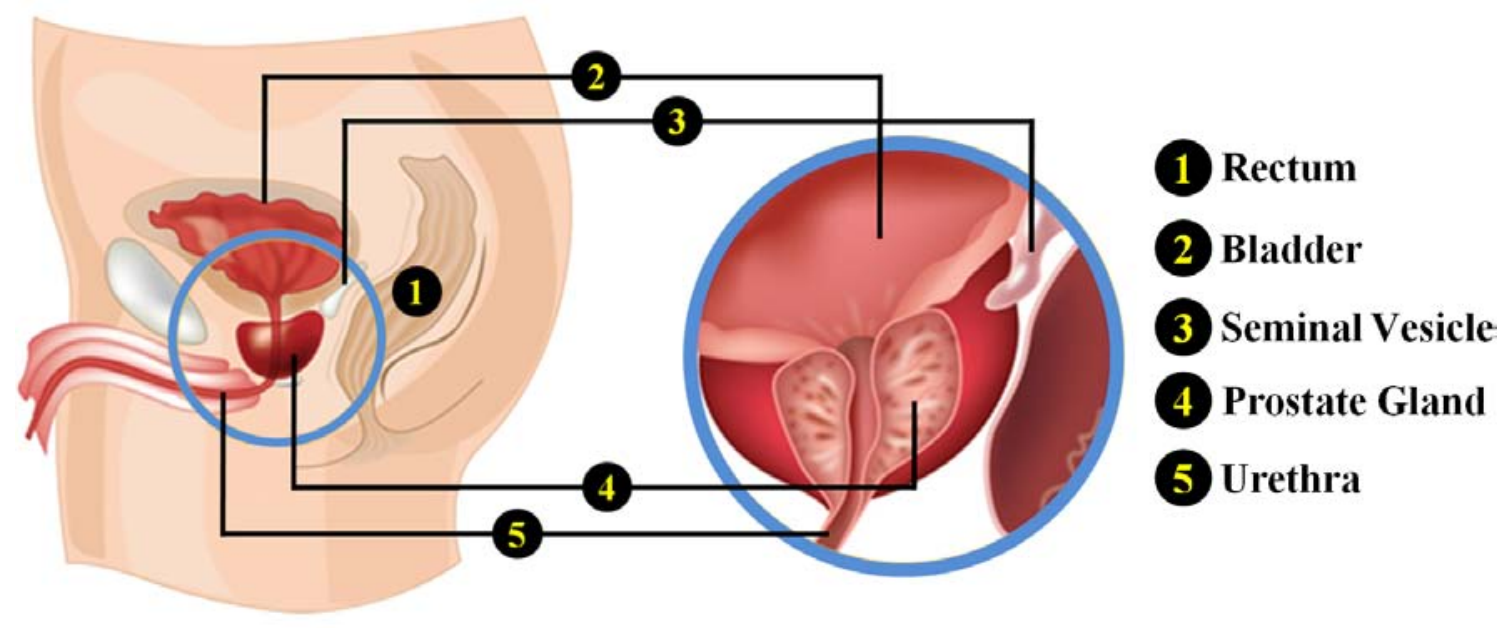

FIGURE 83: Schematic illustration of an anatomical view of the lower abdomen area that contains the prostate [22].

The prostate is located deep in the pelvis just below the urinary bladder and in front of the rectum, see Figure 83. It surrounds the urethra as it exits the bottom of the bladder. The prostate has two main functions. First, it stores, secretes, and controls the flow of the milky fluid, which constitutes $30 \%$ of the volume of the semen and is injected into the urethra along with sperm when a male is sexually aroused. Second, it controls the diameter of the urethra, thereby controlling the flow of urine [422]. To accomplish these functions, the prostate contains three main cell types: (i) gland cells that excrete seminal fluid, (ii) muscles cells that control the diameter of the urethra for urine flow and ejaculation, and (iii) fibrous cells that make up the supportive structure of the prostate [422]. 
In pathology, the prostate region is divided into different segments or zones. Each of these zones consists of different cell types and is susceptible to different types of diseases. Figure 84 illustrates the main glandular zones of the prostate: the central zone $(\mathrm{CZ})$, the transition zone (TZ), and the peripheral zone (PZ). The PZ is the sub-capsular portion of the prostate gland that surrounds the distal urethra and constitutes up to $70 \%$ of a normal prostate gland. The $\mathrm{CZ}$ is the second largest region of the prostate that surrounds the ejaculatory ducts and constitutes about $25 \%$ of a normal prostate gland. The third zone is the $\mathrm{TZ}$ that surrounds the urethra and is the region of the prostate that grows through-out life. The TZ comprises about $5 \%$ of a normal prostate gland [423].

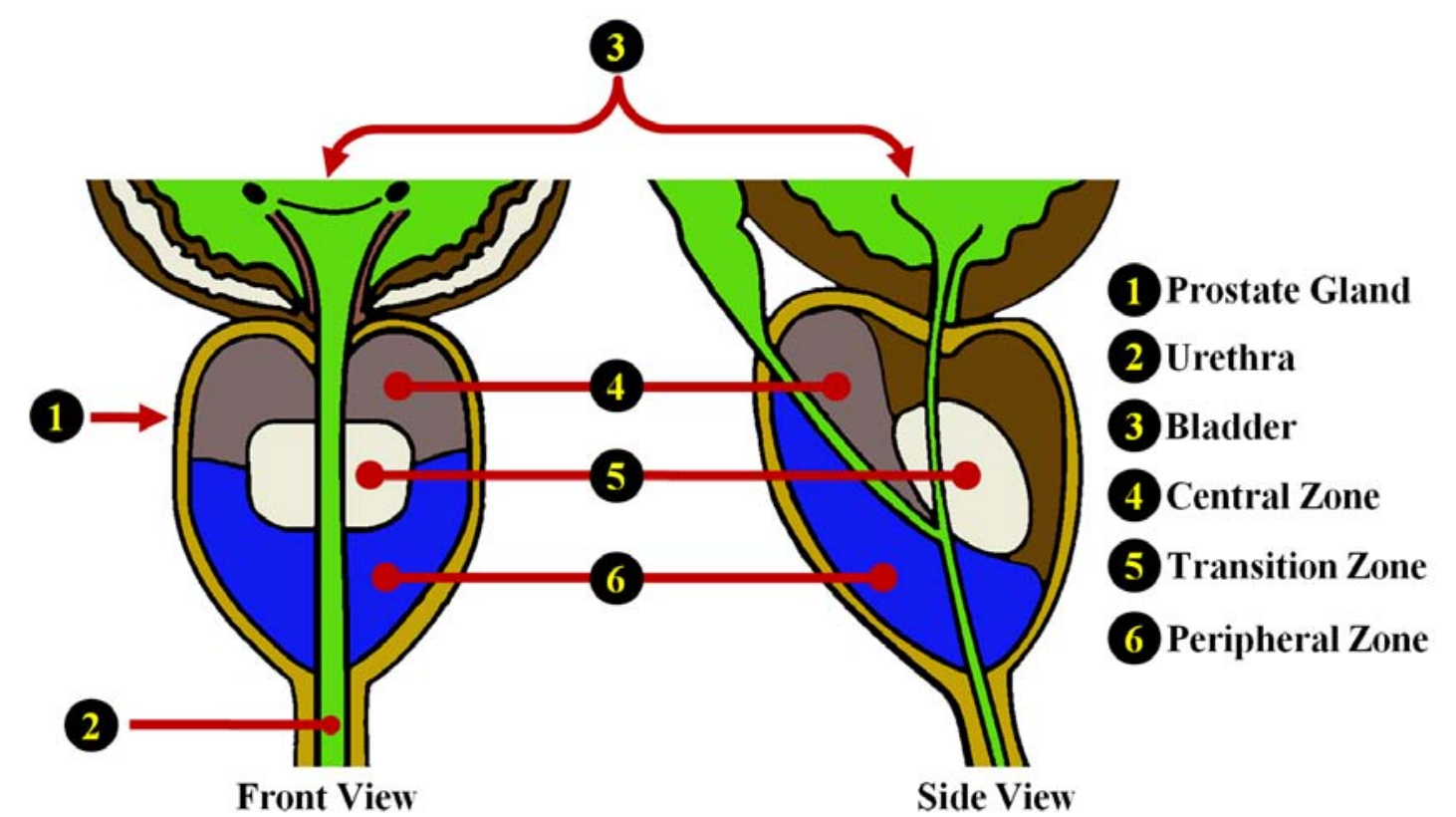

FIGURE 84: Schematic illustration of the front and side cross-sectional views of the prostate showing different prostate zones [23].

The function of the prostate can be affected by various types of medical complications. The three most common prostate problems are the prostatitis, benign prostatic hyperplasia (prostate enlargement), and prostate cancer. Prostatitis is microscopic inflammation (swelling) of the tissue of the prostate gland, which affects mostly young and middle-aged men [424]. This inflammatory disease is usually caused by bacteria and is categorized into four groups based on the chronicity of symptoms [425]: Type I-acute bacterial prostatitis, 
Type II-chronic bacterial prostatitis, Type III-chronic abacterial prostatitis (chronic pelvic pain syndrome), and Type IV-asymptomatic inflammatory prostatitis. Type I is less likely to occur; however, it is potentially life-threatening. Type II is also relatively uncommon and occurs when bacteria find a spot on the prostate where they can survive. On the other hand, Type III is the most common and can be found in men of any age; nevertheless, it is the least understood form of prostatitis [426]. The incidence of Type IV is unknown and diagnosis is made after a biopsy and is usually correlated with the higher PSA values [427]. Benign prostatic hyperplasia is the second medical complication that affects the prostate, which is caused by non-cancerous enlargement of the prostate gland and is associated with aging. This condition can cause men to have some troubles, such as the frequent need to urinate, and the difficulty to start urination or to fully empty the bladder [428].

The last type of prostate disease is prostate cancer, which is one of the most common cancers among males. In most cases, prostate cancer has no symptoms and is only found due to screening. When symptoms are present they include pain, difficulty in urinating, and problems during sexual intercourse. Prostate cancer is a slow growing cancer that may invade other organs if untreated. In addition, it has different occurrence rates at different zones of the prostate, due to the difference in cell types that compromise each zone. Particularly, the vast majority (70\%) of prostate cancer originate in the PZ, while only $25 \%$ occur in the $\mathrm{TZ}$ and $5 \%$ in the $\mathrm{CZ}$, which tends to be the more aggressive type [429]. In order to determine how far the cancer has spread (within the prostate or to other body parts), a staging test is performed. Staging of the prostate cancer plays an important role in the choice and the success of treatment. Prostate cancer has four stages (Stages I through IV). Stage I is the earliest cancer stage in which the cancer cells are confined to a microscopic area and are too small to be either felt by a physician or seen in imaging tests. Stage II is more advanced than stage I and the cancer can be felt by a physician. Like stage I, however, the cancer is still confined only to the prostate gland. In stage III, the cancer spreads outside the prostate to nearby tissues, e.g., seminal vesicles. The final stage of prostate cancer is stage IV in which the cancer cells have spread beyond the outer layer of the prostate to the nearby organs, such as the lymph nodes, bones, rectum, or bladder. 
Prostate cancer is the sixth leading cause of cancer-related death in men worldwide and is the second in the U.S. [430]. Therefore, it is crucial to detect/diagnosis prostate cancer in its earliest stage in order to improve the effectiveness of treatment and increase the patient's chance of survival. In recent years, both the diagnosis and the treatment of prostate cancer continue to evolve using different techniques and methods. The next section overviews different techniques that are used for early diagnosis of prostate cancer as well as the related work on prostate cancer detection and diagnosis in the context of the proposed framework.

\section{B. CURRENT DIAGNOSTIC METHODS OF PROSTATE CANCER}

Prostate cancer is the most frequently diagnosed male malignancy and the second leading cause (after lung cancer) of cancer-related death in the USA, with more than 238,000 new cases and a mortality rate of about 30,000 in 2013 [71]. Early diagnosis improves the effectiveness of the treatment and increases the patient's chances of survival. Compared to other types of cancers, such as lung cancer, prostate cancer, when treated by removing the prostate gland, has a zero chance of recurrence. There are many techniques that are used for the diagnosis of prostate cancer. The main diagnostic tools for prostate cancer are digital rectal exam (DRE), serum concentration using prostate specific antigen (PSA) blood test, and needle biopsy. The DRE test is carried out by a skilled physician who manually feels for any abnormalities in the prostate gland through the rectum. The DRE is inexpensive and easy to perform. However, the accuracy of a DRE examination is not high enough and depends on the physician experience. Also,it can only detect tumors with sufficient volumes. Another screen test for the diagnosis of prostate cancer is performed using PSA- an enzyme that is secreted by the prostatic cells. The higher the values of PSA, the more likely the prostate gland is to have cancer. However, PSA is associated with a high-risk of over diagnosis of prostate cancer as higher PSA levels may reflect other conditions, such as an enlarged or inflamed prostate [431]. In addition, PSA screening lacks the ability to provide accurate information about the location and the extent of the 
cancer. If either the DRE or PSA tests raise any concern, the needle biopsy is performed to collect tissue samples from the prostate, which are analyzed in a lab to determine if cancer cells are present or not. Biopsy remains the gold standard for diagnosis of prostate cancer, but it is the last resort because of its invasive nature, high costs, and potential morbidity rates. Additionally, the relatively small needle biopsy samples have a higher possibility of producing false positive diagnosis.

To overcome these limitations, noninvasive evaluation of prostate cancer has been clinically explored with several medical imaging tools, such as ultrasound, computed tomography (CT), and magnetic resonance imaging (MRI). These imaging methods are favorable due to their ability to provide reliable information about the size and shape of prostate gland and can localize the cancer foci, which would improve the accuracy of diagnosis and enable more efficient treatment. Transrectal ultrasound (TRUS) is the most commonly used technique for prostate imaging. TRUS is used in estimating the volume of the prostate gland in PSA screening, and is often used in planning and guiding needle biopsies [432]. TRUS is often chosen due its inexpensive cost, ease of use, portability, and real-time nature. However, it does have several disadvantages, including low contrast and a low signal-to-noise ratio (SNR) [433]. As a result, it can be difficult to accurately detect and locate cancerous cells using TRUS images. Traditional CT imaging modality is widely used for post-therapy evaluation by physicians to assess the effectiveness of treatment [432]. However, it uses radiation and has poor soft-tissue contrast resolution, which does not allow precise distinction of the internal or external anatomy of the prostate. As an alternative for ultrasound and CT, magnetic resonance (MR) imaging techniques are becoming increasingly attractive as new diagnostic tools for prostate cancer. Over the past years, these MRI-based techniques have shown varying degrees of success for improved visualization and localization of prostate cancer $[75,77,79,80,132,134-140,434,435]$. The key advantage of MRI is that it provides higher contrast of soft tissues, which allows for better detection of cancerous tissues. In addition to anatomical information, MRI can provide valuable functional information about the tissue by using functional acquisition techniques, such as MR spectroscopy (MRS), DCE-MRI, and diffusion-weighted imaging (DWI) [77]. 
In this chapter, the focus is on using DCE-MRI to develop a computer-aided diagnostic (CAD) system for early detection of prostate cancer. The the lack of ionizing radiation, increased spatial resolution, and the ability to provide both anatomical and functional information are the key motivation of using DCE-MRI. In general, early diagnosis of prostate cancer using DCE-MRI requires intermediate image processing steps, such as prostate registration, segmentation, and classification. The next sections introduce the related work on prostate segmentation and registration, and the state-of-the-art CAD systems for early detection of prostate cancer.

\section{Related Work in Prostate Segmentation and Registration}

Prostate segmentation, i.e., the delineation of prostate borders from the surrounding tissues, is a basic step in any noninvasive CAD system for early detection of prostate cancer. However, accurate delineation of prostate borders in MR images is a challenge due to: large variations of prostate shapes within a specific time series as well as across subjects; the lack of strong edges and diffused prostate boundaries; and the similar intensity profile of the prostate and surrounding tissues. Although manual outlining of the prostate border enables the prostate volume to be determined, it is time consuming and observer dependent. Moreover, traditional edge detection methods [436] are unable to extract the correct boundaries of the prostate since the graylevel distributions of the prostate and the surrounding organs are hardly distinguishable. To overcome this limitation, most successful known approaches have addressed the segmentation challenges by incorporating the prostate appearances and shapes into their segmentation techniques.

In particular, an automated framework by Allen et al. [437] was proposed for 3D prostate segmentation that consists of two steps. voxel classification is performed based on Gaussian probabilities of grey level. Then, a statistical shape model is used to segment the prostate region. A hybrid 2D/3D active shape model (ASM)-based methodology for global optimal segmentation of the 3D MRI prostate data was proposed by Zhu et al. [438]. Iterative segmentation was performed by a 2D ASM search on each slice, then the final surface 
is reconstructed from the $2 \mathrm{D}$ search results and updated by re-estimating the parameters of the 3D probabilistic shape model. Klein et al. $[439,440]$ presented an atlas-based segmentation approach that utilized a localized version of mutual information (MI) to extract the prostate from MR images. The segmentation of the prostate is obtained as the average of the best-matched registered atlas set to the test image (image to be segmented). Flores-Tapia et al. [441] proposed a semi-automated edge detection technique for MRI prostate segmentation. In their framework, the prostate borders were detected by tracing four manually-selected reference points on the edge of the prostate using a static Wavelet transform [442] to locate the prostate edges. Toth et al. [443] presented an algorithm for the automatic segmentation of the prostate in multi-modal MRI. Their algorithm starts by isolating the region of interest (ROI) from MRS data. Then, an ASM within the ROI is used to obtain the final segmentation. A semi-automated approach by Vikal et al. [444] used a priori knowledge of prostate shape to detect the contour in each slice and then refined them to form a 3D prostate surface. An unsupervised segmentation method was proposed by Liu et al. [445] for the segmentation of MR prostate images. A level set deformable model was employed and was guided by an elliptical prostate shape prior and intensity gradient was employed to refine the initial results obtained by Otsu thresholding [446]. A maximum A posteriori (MAP)-based framework was proposed by Makni et al. [447] to perform automated 3D MRI prostate segmentation. Their framework combined graylevel, contextual information regarding voxels' neighborhoods using MRF, and statistical shape information to find optimum segmentation based on Bayesian a posteriori classification, estimated with the iterative conditional mode (ICM) algorithm. Liu et al. [227] proposed an automated approach that utilized fuzzy MRF modeling for prostate segmentation from multi-parametric MRI. Their framework exploited $T_{2}$-weighted image intensities, pharmacokinetic (PK) parameter $k_{\mathrm{ep}}$, and apparent diffusion coefficient (ADC) values in a Bayesian approach to label prostate pixels as cancerous or non-cancerous. The labeled pixels are then clustered using the k-means algorithm. The system had a specificity of $89.58 \%$, sensitivity of $87.50 \%$, accuracy of $89.38 \%$, and a DSC of $62.2 \%$. A similar approach was developed by Artan et al. [228] and located cancerous regions using cost-sensitive support vector ma- 
chine (SVM). Prostate segmentation was performed using a conditional random field and the same three features in [227] were utilized for classification. The DSC for prostate localization and segmentation was $0.46 \pm 0.26$, and the area under the receiver operator characteristic (ROC) curves $\left(A_{z}\right)$ of the classification was $0.79 \pm 0.12$. Ozer et al. [448] also developed a technique that directly segmented prostate cancers using the same three features in [227, 228]. Both the SVM and relevance vector machine (RVM) [449] classifiers were used and the system showed a specificity of 0.78 and a sensitivity of 0.74 for RVM and 0.74 and 0.79 for SVM. Gao et al. [450] proposed a shape-based technique that utilized point cloud registration of the MR images before segmenting the prostate. The final prostate border is obtained by minimizing a cost functional that incorporated both the local image statistics as well as the learned shape prior. Martin et al. [451] developed an atlas-based approach for segmenting the prostate from 3D MR images by mapping a probabilistic anatomical atlas to the test image. The resulting map is used to constrain a deformable model-based segmentation framework. Firjani et al. [452] proposed a MAPbased framework that combines a graph-cut approach and three image features (grey-level intensities, spatial interactions between the prostate pixels, and a prior shape model) for 2D DCE-MRI prostate segmentation. Their method was later extended in [76] to allow for 3D segmentation from DCE-MRI volumes. It utilized both a 3D MRF to model the spatial interaction between the prostate voxels and a 3D shape prior. Recently, Dowling et al. [453] proposed an automated framework that combined dynamic multi-atlas label fusion methods. They employed the diffeomorphic demons method for the nonrigid registration using the selective and iterative method for performance level estimation (SIMPLE) technique [454]. In their framework, a pre-processing step for bias field correction, histogram equalization, and anisotropic diffusion smoothing was employed. Ghose et al. [455] proposed a probabilistic graph-cut-based framework for prostate segmentation based on the fusion of the posterior probabilities determined with a probabilistic atlas and a supervised random forest learning framework. An automated technique that first applied global registration to the prostate MRI data followed by an active appearance model (AAM) based segmentation of the prostate tissue was proposed by Ghose et al. [456]. Table 17 sum- 
marizes the reviewed methodologies for prostate segmentation and registration with the validation data sets and achieved performance for each study.

In summary, a tremendous number of studies have been developed for the segmentation and registration of prostate MRI data. However, in most of these approaches the segmentation and registration reliability is not very high due to the following reasons: $(i)$ parametric shape models fail in the presence of large gray-level variability across subjects and time; (ii) edge detection methods are not suitable for discontinued objects; (iii) deformable models tend to fail in the case of excessive noise, poor image resolution, diffused boundaries or occluded objects if they do not incorporate a priori models (e.g., shape and appearance). In addition, most of the motion correction models account only for the global motion and do not take into account the local motion of the prostate due to transmitted respiratory and peristaltic effects. Furthermore, the existing local motion correction methods are intensity-based techniques, which are prone to nonlinear intensity variations over the time series and perform poorly in pre-contrast images. Also, local motion correction methods register the original grey level data without any prior segmentation; therefore, they do not guarantee voxel-on-voxel matches of the registered perfusion data.

TABLE 17: Summary of the discussed prostate segmentation and registration techniques and their experimental performance. Note that DIM and AL stand for data dimension (i.e., 2D, 3D, or 4D) and the automation level (i.e., automated or semi-automated), respectively.

\begin{tabular}{|c|c|c|}
\hline Study & DIM, AL, and Methods & Data and Performance \\
\hline $\begin{array}{l}\text { Allen et } \\
\text { al. [437] }\end{array}$ & $\begin{array}{l}\text { - } 3 \mathrm{D} \\
\text { - Automated } \\
\text { - ASM-based segmentation }\end{array}$ & $\begin{array}{l}\text { - } 22 \text { data sets } \\
\text { - MAD: } 2.8 \pm 0.82\end{array}$ \\
\hline $\begin{array}{l}\text { Klein et } \\
\text { al. [439] }\end{array}$ & $\begin{array}{l}\text { - } 3 \mathrm{D} \\
\text { - Automated } \\
\text { - Affine + B-Splines Registration } \\
\text { - Atlas-based segmentation }\end{array}$ & $\begin{array}{l}\text { - } 38 \text { data sets } \\
\text { - Median DSC: } 0.82\end{array}$ \\
\hline $\begin{array}{l}\text { Zhu et } \\
\text { al. [438] }\end{array}$ & $\begin{array}{l}\text { - } 3 \mathrm{D} \\
\text { - Automated } \\
\text { - Hybrid 2D+3D ASM }\end{array}$ & $\begin{array}{l}\text { - } 26 \text { data sets (288 slices) } \\
\text { - RMSD: } 5.481 \pm 2.91\end{array}$ \\
\hline $\begin{array}{l}\text { Flores-Tapia } \\
\text { et al. [441] }\end{array}$ & $\begin{array}{l}\text { - } 3 \mathrm{D} \\
\text { - Semi-automated } \\
\text { - Edge detection } \\
\end{array}$ & $\begin{array}{l}\text { - } 1 \text { data set (19 slices) } \\
\text { - DSC: } 0.93 \pm 0.005\end{array}$ \\
\hline \multicolumn{3}{|c|}{ continued on the next page ... } \\
\hline
\end{tabular}


TABLE 17 - continued from the previous page

\begin{tabular}{|c|c|c|}
\hline Study & DIM, AL, and Methods & Data and Performance \\
\hline $\begin{array}{ll}\text { Toth } & \text { et } \\
\text { al. }[443] & \end{array}$ & $\begin{array}{l}\text { - } 3 \mathrm{D} \\
\text { - Automated } \\
\text { - Unsupervised spectral clustering } \\
\text { - ASM-based segmentation }\end{array}$ & $\begin{array}{l}\text { - } 150 \text { slices } \\
\text { - Average OR: } 0.83 \text {, average } \\
\text { SEN: 0.89, average SPE: } \\
\text { 0.86, and average PPV: } 0.93\end{array}$ \\
\hline $\begin{array}{l}\text { Klein et } \\
\text { al. [440] }\end{array}$ & $\begin{array}{l}\text { - } 3 \mathrm{D} \\
\text { - Automated } \\
\text { - Affine + B-splines registration } \\
\text { - Atlas matching }\end{array}$ & $\begin{array}{l}\text { - } 50 \text { slices } \\
\text { - Median DSC: } 0.85\end{array}$ \\
\hline $\begin{array}{ll}\text { Vikal et } \\
\text { al. [444] }\end{array}$ & $\begin{array}{l}\text { - } 3 \mathrm{D} \\
\text { - Semi-automated } \\
\text { - } \mathrm{ASM}\end{array}$ & $\begin{array}{l}\text { - } 3 \text { data sets (39 slices) } \\
\text { - DSC: } 0.93 \pm 0.3 \text { and MAD: } \\
2.00 \pm 0.6(\mathrm{~mm})\end{array}$ \\
\hline $\begin{array}{l}\text { Makni et } \\
\text { al. [447] }\end{array}$ & $\begin{array}{l}\text { - } 3 \mathrm{D} \\
\text { - Automated } \\
\text { - } \text { ASM }\end{array}$ & $\begin{array}{l}\text { - } 12 \text { data sets } \\
\text { - Mean HD: } 9.62(\mathrm{~mm}), \mathrm{GD} \text { : } \\
2.39(\mathrm{~mm}), \text { OR: } 0.84, \mathrm{VPC} \text { : } \\
\text { 0.90, and DSC: } 0.91\end{array}$ \\
\hline $\begin{array}{l}\text { Liu et } \\
\text { al. [445] }\end{array}$ & $\begin{array}{l}\text { - } 2 \mathrm{D} \\
\text { - Automated } \\
\text { - Level-set-based segmentation }\end{array}$ & $\begin{array}{l}\text { - } 10 \text { data sets } \\
\text { - DSC: } 0.91 \pm 0.03\end{array}$ \\
\hline $\begin{array}{l}\text { Ozer et al. } \\
{[448]}\end{array}$ & $\begin{array}{l}\text { - } 2 \mathrm{D} \\
\text { - } \text { Automated } \\
\text { - SVM and RVM }\end{array}$ & $\begin{array}{l}\text { - } 20 \text { data sets } \\
\text { - SPE: } 0.78, \text { SEN: } 0.74 \text {, and } \\
\text { DSC: } 0.51 \text { for RVM } \\
\text { - SPE: } 0.74 \text {, SEN: } 0.79 \text { and } \\
\text { DSC: } 0.52 \text { for SVM }\end{array}$ \\
\hline $\begin{array}{ll}\text { Artan } & \text { et } \\
\text { al. }[228] & \end{array}$ & $\begin{array}{l}\text { - } 2 \mathrm{D} \\
\text { - Automated } \\
\text { - Cost-sensitive conditional ran- } \\
\text { dom field }\end{array}$ & $\begin{array}{l}\text { - } 21 \text { data sets } \\
\text { - } A_{z}: 0.790 \pm 0.12 \text { and DSC: } \\
0.46 \pm 0.26\end{array}$ \\
\hline $\begin{array}{ll}\text { Liu } & \text { et } \\
\text { al. [227] } & \end{array}$ & $\begin{array}{l}\text { - } 3 \mathrm{D} \\
\text { - Automated } \\
\text { - fuzzy MRFs }\end{array}$ & $\begin{array}{l}\text { - } 11 \text { data sets } \\
\text { - SPE: } 0.896, \text { SEN: } 0.894 \text {, and } \\
\text { DSC: } 0.622\end{array}$ \\
\hline $\begin{array}{ll}\text { Gao } & \text { et } \\
\text { al. }[450] & \end{array}$ & $\begin{array}{l}\text { - } 3 \mathrm{D} \\
\text { - Automated } \\
\text { - Affine registration } \\
\text { - Level set segmentation }\end{array}$ & $\begin{array}{l}\text { - } 48 \text { data sets } \\
\text { - DSC: } 0.84 \pm 0.03 \text { and } 95 \% \\
\text { HD: } 8.10 \pm 1.50(\mathrm{~mm}) \quad(33 \\
\text { data sets) } \\
\text { - DSC: } 0.82 \pm 0.03 \text { and } 95 \% \\
\text { HD: } 10.22 \pm 4.03 \text { (mm) }(15 \\
\text { public data sets) }\end{array}$ \\
\hline $\begin{array}{l}\text { Martin et } \\
\text { al. [451] }\end{array}$ & $\begin{array}{l}\text { - } 3 \mathrm{D} \\
\text { - Automated } \\
\text { - Affine + Multi-resolution } \\
\text { Demons registration } \\
\text { - } \text { ASM }\end{array}$ & $\begin{array}{l}\text { - } 36 \text { data sets } \\
\text { - Median DSC: } 0.86 \text { and Aver- } \\
\text { age surface error: } 2.41 \mathrm{~mm}\end{array}$ \\
\hline \multicolumn{3}{|c|}{ continued on the next page ... } \\
\hline
\end{tabular}


TABLE 17 - continued from the previous page

\begin{tabular}{|c|c|c|}
\hline Study & DIM, AL, and Methods & Data and Performance \\
\hline $\begin{array}{ll}\text { Firjani } & \text { et } \\
\text { al. }[452] & \end{array}$ & $\begin{array}{l}\text { - } 2 \mathrm{D} \\
\text { - Automated } \\
\text { - Affine registration } \\
\text { - Graph-cut segmentation }\end{array}$ & $\begin{array}{l}\text { - } 10 \text { Subjects (180 Volumes) } \\
\text { - MD: } 5.2 \pm 1.2\end{array}$ \\
\hline $\begin{array}{l}\text { Firjani } \\
\text { al. }[76]\end{array}$ & $\begin{array}{l}\text { - } 3 \mathrm{D} \\
\text { - Automated } \\
\text { - Affine registration } \\
\text { - Graph-cut segmentation }\end{array}$ & $\begin{array}{l}\text { - } 15 \text { data sets } \\
\text { - OAE: 0.6 } \pm 0.80, \quad \text { DSC: } \\
0.92 \pm 0.004, \\
0.98 \pm 0.004, \quad \text { and } \text { SEV: } \\
0.85 \pm 0.004\end{array}$ \\
\hline $\begin{array}{l}\text { Dowling et } \\
\text { al. [453] }\end{array}$ & $\begin{array}{l}\text { - } 3 \mathrm{D} \\
\text { - Automated } \\
\text { - Diffeomorphic demons registra- } \\
\text { tion } \\
\text { - Atlas-based segmentation }\end{array}$ & $\begin{array}{l}\text { - } 50 \text { data sets } \\
\text { - Median DSC: } 0.86 \text { and Aver- } \\
\text { age surface error: } 2.0(\mathrm{~mm})\end{array}$ \\
\hline $\begin{array}{l}\text { Ghose et } \\
\text { al. [455] }\end{array}$ & $\begin{array}{l}\text { - } 3 \mathrm{D} \\
\text { - Semi-automated } \\
\text { - Affine + nonrigid Demon regis- } \\
\text { tration } \\
\text { - Graph-cut segmentation }\end{array}$ & $\begin{array}{l}\text { - } 15 \text { data sets } \\
\text { - DSC: } \quad 0.91 \pm 0.04 \quad \text { and } \\
\text { 95\%HD: } 4.69 \pm 2.62(\mathrm{~mm})\end{array}$ \\
\hline $\begin{array}{l}\text { Ghose et } \\
\text { al. [456] }\end{array}$ & $\begin{array}{l}\text { - } 2 \mathrm{D} \\
\text { - Automated } \\
\text { - Affine registration } \\
\text { - AAM-based segmentation }\end{array}$ & $\begin{array}{l}-15 \text { data sets } \\
\text { - DSC: } 0.88 \pm 0.11 ; \quad \text { HD: } \\
3.38 \pm 2.81(\mathrm{~mm}) \text {, and MAD: } \\
1.32 \pm 1.53\end{array}$ \\
\hline AMM: & \multicolumn{2}{|l|}{ Active appearance model. } \\
\hline ASM: & \multicolumn{2}{|l|}{ Active shape model. } \\
\hline DSC: & \multicolumn{2}{|c|}{$\begin{array}{l}\text { Dice similarity coefficient: } \mathrm{DSC}=\frac{2 \cdot \mathrm{TP}}{2 \cdot \mathrm{TP}+\mathrm{FP}+\mathrm{FN}} \\
\text { where, TP: true positive, } \mathrm{FP}: \text { false positive, FN: false negative. }\end{array}$} \\
\hline GD: & \multicolumn{2}{|l|}{ Gravity distance. } \\
\hline HD: & \multicolumn{2}{|l|}{ Hausdorff distance. } \\
\hline MD: & \multicolumn{2}{|l|}{ Mean distance. } \\
\hline MAD: & \multicolumn{2}{|l|}{ Mean absolute distance. } \\
\hline N/A: & \multicolumn{2}{|l|}{ Not applicable. } \\
\hline OR: & \multicolumn{2}{|l|}{ Overlap ratio. } \\
\hline OAE: & \multicolumn{2}{|l|}{ Overlapping area error; $\mathrm{OAE}=\frac{\mathrm{FP}+\mathrm{FN}}{\mathrm{TP}+\mathrm{FN}} \%$} \\
\hline PPV: & \multicolumn{2}{|l|}{ Positive predictive value; $\mathrm{PPV}=\frac{\mathrm{TP}}{\mathrm{TP}+\mathrm{FP}}$. } \\
\hline RMSD: & \multicolumn{2}{|l|}{ Root mean squared distance. } \\
\hline RVM: & \multicolumn{2}{|l|}{ Relevance vector machine. } \\
\hline SEN: & \multicolumn{2}{|l|}{ Sensitivity; $\mathrm{SEN}=\frac{\mathrm{TP}}{\mathrm{TP}+\mathrm{FN}}$. } \\
\hline SPE: & \multicolumn{2}{|c|}{ Specificity; $\mathrm{SPE}=\frac{\mathrm{TN}}{\mathrm{TN}+\mathrm{FP}}$; where $\mathrm{TN}$ : true negative. } \\
\hline VPC: & \multicolumn{2}{|l|}{ Volume properly contoured. } \\
\hline
\end{tabular}




\section{Related Work in CAD Systems for Prostate Cancer}

The development of CAD systems for detecting prostate cancer using MR image modalities is an ongoing area of research. Current medical studies suggest that $T_{2}$-weighted MRI and DCE-MRI hold promise for improving prostate cancer detection, thereby reducing the need for prostate biopsy [130, 133, 134, 241, 251, 435, 457-465]. In this section, the state-of-the-art CAD systems developed for prostate cancer detection will be discussed. A summary of the discussed systems along with their computational methods, validation data sets, and validation accuracy are given in Table 18 .

To the best of our knowledge, the first semi-automated computerized MRI-based CAD system for prostate cancer diagnosis was developed by Chan et al [457]. In their study, multi-modal MRI ( $T_{2}$-weighted, $T_{2}$-mapping, and line scan diffusion imaging) were used to estimate malignancy likelihood in the $\mathrm{PZ}$ of the prostate. Both statistical maps and textural features were obtained and a SVM and a linear discriminant analysis (LDA) classifiers were employed for the classification. Their systems resulted in an $A_{z}$ of $0.761 \pm 0.043$ and $0.839 \pm 0.064$, for SVM and LDA respectively. Madabhushi et al [458] proposed an automated CAD system for detecting prostatic adenocarcinoma from MR prostate images. In their method, multiple image features, including gray levels statistics (intensity values, mean, and standard deviation), intensity gradient, and Gabor filter features, were used for classifying groups of pixels as tumors. A $k_{n}$-nearest neighbor classifier and Bayesian conditional densities were used for classification, and the system achieved an $A_{z}$ of 0.957. A study by Engelbrecht et al. [130] evaluated which MRI parameters would result in optimal discrimination of prostatic carcinoma from normal $\mathrm{PZ}$ and $\mathrm{CZ}$ of the prostate. Using the ROC curves, their study concluded that the relative peak enhancement was the most accurate perfusion parameter for cancer detection in the $\mathrm{PZ}$ and $\mathrm{CZ}$ of the gland. A semiautomated CAD system by Kim et al. [459] demonstrated that parametric imaging of the wash-in rate was more accurate for the detection of prostate cancer in the PZ than was $T_{2}$-weighted imaging alone. However, they also observed significant overlap between the wash-in rate for cancer and normal tissue in the TZ. Fütterer et al. [435] developed a CAD 
system to compare the accuracies of $T_{2}$-weighted MRI, DCE-MRI, and MRS imaging for prostate cancer localization. The results showed higher accuracy in DCE-MRI than were achieved with $T_{2}$-weighted MRI in prostate cancer localization. A similar study was conducted by Rouvière et al. [460] for the detection of postradiotherapy recurrence of prostate cancer. Their study also concluded that DCE-MRI possesses the ability to depict the intraprostatic distribution of recurrent cancer after therapy more accurately and with less inter-observer variability than $T_{2}$-weighted MRI. Puech et al. $[133,134]$ developed a semiautomated dynamic MRI-based CAD system for the detection of prostate cancer. Candidate lesion ROIs were selected either manually or by using a region growing technique initiated by a user-selected seed point. Lesions are classified as benign, malignant or indeterminate based on the analysis of the median wash-in and wash-out values. Their CAD system demonstrated a sensitivity and specificity of $100 \%$ and $45 \%$ for the PZ, and sensitivity and specificity of $100 \%$ and $40 \%$ for the TZ. Ocak et al. [241] developed a CAD system using PK analysis for prostate cancer diagnostics in patients with biopsy-proven lesions. In their framework, four PK parameters $\left(K^{\text {trans }}, k_{\mathrm{ep}}, v_{\mathrm{e}}\right.$, and the area under the gadolinium concentration curve) were determined and compared for cancer, inflammation, and healthy peripheral. Their results showed improvement in prostate cancer specificity using the $K^{\text {trans }}$ and $k_{\text {ep }}$ parameters over that obtained using conventional $T_{2}$-weighted MRI. An automated DCE-MRI CAD system for prostate cancer detection was proposed by Viswanath et al. [461]. Prostate borders were segmented using an ASM, and a nonrigid registration scheme (affine and thin plate spline) was employed to map the whole mount histological sections onto corresponding 2D DCE-MRI. In order to classify prostate tissue, a local linear embedding approach [466] was used to create a feature vector using local neighborhood intensities. Then, a k-means clustering approach was used for the classification and the system achieved an accuracy of about 77\%. Their framework was later extended in [462] by combining $T_{2}$-weighted features and DCE-MRI functional features. The system validation showed that the integration of both modalities $\left(A_{z}\right.$ of 0.815$)$ has a better performance of either individual modalities ( 0.704 for $T_{2}$-weighted MRI and 0.682 for DCE-MRI). A semi-automated framework by Vos et al. [463] classified prostate le- 
sions using quantitative PK maps and $T_{1}$ estimates. PK features were extracted from a user-defined ROI around the prostate and a SVM was used to estimate the likelihood of malignancy. Based on the ROC analysis, the reported results showed that the system had an accuracy of $83 \%$ in the classification of the ROIs with abnormal enhancement patterns in the PZ. Ampeliotis et al. [464] proposed a semi-automated multi-parametric CAD system that used $T_{2}$-weighted and DCE-MRI. The $T_{2}$-weighted pixel intensities and the four low-frequency coefficients of the discrete cosine transform were used as features and probabilistic neural networks were employed as the classifier. Based on the ROC analysis $\left(A_{z}\right.$ of 0.898 ), their study concluded that the fused $T_{2}$-weighted and dynamic MRI features outperform that of either modality's features alone. A similar CAD system was proposed by Litjens et al. [465] that employed an ASM to segment the prostate. In order to classify the segmented prostate voxels, the $\mathrm{ADC}, K^{\text {trans }}$, and $k_{\text {ep }}$ parameters were estimated and a SVM classifier with a radial basis function kernel was used. The validation results showed a sensitivity of $74.7 \%$ and $83.4 \%$ with seven and nine FPs per patient, respectively. Vos et al. [251] utilized an automated CAD system for the detection of prostate cancer. Just as in [465], the prostate was segmented using an ASM-based technique. Then, multiple ROIs were located within the segmented prostate using peak and mean neighborhood intensity and ADC values. These values and the differences between the peak and the mean were again used as features for ROI classification. In addition, the 25 percentile $T_{2}, 25$ percentile ADC, 25 percentile wash-out, 50 percentile $T_{1}, 75$ percentile $K^{\text {trans }}$, and 75 percentile $v_{\mathrm{e}}$ were used as features. The resulting feature vector was classified using an LDA classifier. This system had an $A_{z}$ of $0.83 \pm 0.20$. A maximum $A_{z}$ of 0.88 was reported for high-grade tumors, but the system had difficulty classifying lower grade tumors, achieving a maximum $A_{z}$ of 0.74. Another semi-automated multi-parametric system by Peng et al. [467] utilized $T_{2}$-weighted, DCE-MRI and DWI. Candidate features, including the $T_{2}$-weighted intensity skew, the $K^{\text {trans }}$, and the average and $10^{\text {th }}$ percentile ADC, were calculated from a manually-selected ROI. Then, an LDA classifier was used to differentiate prostate cancer from normal tissue in those ROIs. Their CAD system concluded that the best diagnostic performance ( $A_{z}$ of $0.95 \pm 0.02$, SEN of 0.82 , and SPE of 0.953 ) is obtained by combining 
the $10^{\text {th }}$ percentile ADC, average ADC, and $T_{2}$-weighted intensity skewness features.

In summary, developing noninvasive CAD systems for the detection and diagnosis of prostate cancer is an area of research interest. Current CAD systems focus mainly on the initial voxel classification stage by obtaining likelihood maps that combine information from MR images using mathematical descriptors. State-of-the art studies showed that voxel basis discrimination between benign and malignant tissue is feasible with good performances. However, the majority of these studies were performed by radiologists who selected an ROI (small window) around the prostate and followed signal changes within these ROIs. In addition, the final diagnosis and patient management is left to the radiologist. Unfortunately, such approaches not only require manual interaction of the operators, but also ROI selection biases the final decision and brings up the same issue of over- or under-estimating the problem in the entire gland, just as with biopsy. Moreover, manual ROI selection and function curve generation from these ROIs assume that the prostates (prostate contours) remain exactly the same from scan to scan. Nonetheless, prostate contours may not always exactly match due to patient movement or breathing effects; therefore, motion correction techniques should be applied first before ROI selection. Also, to automate the algorithm and to cancel ROI dependency, segmentation approaches that can separate the prostate from the surrounding structures are needed.

TABLE 18: Summary of MRI-based CAD systems for prostate cancer detection and diagnosis, including segmentation method, selected features, classifier, and experimental performance.

\begin{tabular}{|c|c|c|}
\hline Study & Segmentation (s), Features (f) \& Classifier (c) & Data and Performance \\
\hline $\begin{array}{ll}\text { Chan et } & \text { et } \\
\text { al. [457] } & \end{array}$ & $\begin{array}{l}\text { (s) Manual ROIs } \\
\text { (f) } T_{2} \text { intensities, 3D } T_{2} \text { intensity gradient, and } \\
\text { Gabor filter } \\
\text { (c) LDA and SVM }\end{array}$ & $\begin{array}{l}\text { - } 5 \text { Subjects (33 images) } \\
\text { - } A_{z}: \quad 0.839 \pm 0.064 \\
\text { (LDA) } \\
0.761 \pm 0.043(\mathrm{SVM})\end{array}$ \\
\hline $\begin{array}{lr}\text { Litjens } & \text { et } \\
\text { al. [465] } & \end{array}$ & $\begin{array}{l}\text { (s) } \mathrm{ASM} \\
\text { (f) } T_{2} \text {-weighted intensities, } K^{\text {trans }}, k_{\mathrm{ep}} \text {, and } \\
\mathrm{ADC} \\
\text { (c) } \mathrm{SVM}\end{array}$ & $\begin{array}{l}\text { - } 188 \text { subjects } \\
\text { - SEN: } 0.747 \text { (at } 7 \text { FP per } \\
\text { patient) and } 0.834 \text { (at } 9 \\
\text { FP per patient) }\end{array}$ \\
\hline & & tinued on the next page \\
\hline
\end{tabular}


TABLE 18 - continued from the previous page

\begin{tabular}{|c|c|c|}
\hline Study & Segmentation (s), Features (f) \& Classifier (c) & Data and Performance \\
\hline $\begin{array}{l}\text { Engelbrecht } \\
\text { et al. [130] }\end{array}$ & $\begin{array}{l}\text { (s) Manual ROIs } \\
\text { (f) Onset time, time to peak, peak enhance- } \\
\text { ment, washout, and } T_{2} \text { relaxation rate } \\
\text { (c) N/A }\end{array}$ & $\begin{array}{l}\text { - } 36 \text { subjects } \\
\text { - } A_{z} \text { PZ: } 0.79 \text { (Onset } \\
\text { time), } 0.60 \text { (time to } \\
\text { peak), } 0.89 \quad \text { (peak } \\
\text { enhancement), } 0.93 \\
\text { (relative peak enhance- } \\
\text { ment), } 0.82 \text { (wash-out), } \\
\text { and } 0.64 \text { ( } T_{2} \text { relaxation } \\
\text { rate) } \\
\text { - } A_{z} \text { CZ: } 0.70 \text { (onset } \\
\text { time), } 0.60 \text { (time to } \\
\text { peak), } 0.79 \quad \text { (peak } \\
\text { enhancement), } 0.83 \\
\text { (relative peak enhance- } \\
\text { ment), } 0.82 \text { (wash-out), } \\
\text { and } 0.51 \text { ( } T_{2} \text { relaxation } \\
\text { rate) }\end{array}$ \\
\hline $\begin{array}{l}\text { Rouvière et } \\
\text { al. [460] }\end{array}$ & $\begin{array}{l}\text { (s) Manual ROIs } \\
\text { (f) N/A } \\
\text { (c) Evaluation and scoring by three indepen- } \\
\text { dent readers. The MRI scoring results } \\
\text { were correlated against biopsy results in } 10 \\
\text { prostate sectors }\end{array}$ & $\begin{array}{l}\text { - } 22 \text { subjects } \\
\text { - ACC: 0.59 }\left(T_{2}{ }^{-}\right. \\
\text {weighted MRI) } \\
\text { - ACC: } 0.75 \text { (DCE-MRI) }\end{array}$ \\
\hline $\begin{array}{ll}\text { Kim } & \text { et } \\
\text { al. [459] } & \end{array}$ & $\begin{array}{l}\text { (s) Manual ROIs } \\
\text { (f) Wash-in rate } \\
\text { (c) A cut-off threshold selected by a radiologist } \\
\text { differentiate cancer from normal tissue }\end{array}$ & $\begin{array}{l}\text { - } 53 \text { subjects } \\
\text { - ACC: } 0.62 \quad\left(T_{2}-\right. \\
\text { weighted MRI) } \\
\text { - ACC: } 0.88 \text { (DCE-MRI) }\end{array}$ \\
\hline $\begin{array}{l}\text { Madabhushi } \\
\text { et al. [458] }\end{array}$ & $\begin{array}{l}\text { (s) N/A } \\
\text { (f) } T_{2} \text { intensities, 3D } T_{2} \text { Intensity gradient, and } \\
\text { Gabor filter } \\
\text { (c) Bayes classifier }\end{array}$ & $\begin{array}{l}\text { - } 5 \text { Subjects (33 images) } \\
\text { - SEN: } 0.42, \text { SPE: } 0.97 \\
\text { and PPV: } 0.43\end{array}$ \\
\hline $\begin{array}{l}\text { Madabhushi } \\
\text { et al. [468] }\end{array}$ & $\begin{array}{l}\text { (s) N/A } \\
\text { (f) } T_{2} \text { intensities, 3D } T_{2} \text { intensity gradient, and } \\
\text { Gabor filter } \\
\text { (c) Bayes and } k_{n} \text {-nearest neighbor classifiers }\end{array}$ & $\begin{array}{l}\text { - } 5 \text { Subjects (33 images) } \\
\text { - } A_{z}: 0.957\end{array}$ \\
\hline $\begin{array}{l}\text { Fütterer et } \\
\text { al. [435] }\end{array}$ & $\begin{array}{l}\text { (s) Manual ROIs } \\
\text { (f) } v_{\mathrm{e}}, k_{\mathrm{ep}}, K^{\text {trans }} \text {, and wash-out slopes } \\
\text { (c) Prospectively evaluation and scoring of the } \\
\text { selected features by two independent radi- } \\
\text { ologists }\end{array}$ & $\begin{array}{l}\text { - } 34 \text { subjects } \\
\text { - } A_{z}: 0.68\left(T_{2} \text {-weighted }\right. \\
\text { MRI), } 0.91 \quad \text { (DCE- } \\
\text { MRI), and } 0.80 \text { (MRS) }\end{array}$ \\
\hline $\begin{array}{l}\text { Viswanath } \\
\text { et al. [461] }\end{array}$ & $\begin{array}{l}\text { (s) ASM } \\
\text { (f) Ratio of the wash-in and wash-out rates } \\
\text { (c) K-means clustering }\end{array}$ & $\begin{array}{l}\text { - } 21 \text { subjects } \\
\text { - SEN: 60.72\%, SPE: } \\
83.24 \% \text {, and ACC: } \\
77.2 \%\end{array}$ \\
\hline
\end{tabular}


TABLE 18 - continued from the previous page

\begin{tabular}{|c|c|c|}
\hline Study & Segmentation (s), Features (f) \& Classifier (c) & Data and Performance \\
\hline $\begin{array}{ll}\text { Ocak et } \\
\text { al. }[241]\end{array}$ & $\begin{array}{l}\text { (s) Manual ROIs } \\
\text { (f) } K^{\text {trans }}, k_{\mathrm{ep}}, v_{\mathrm{e}} \text {, and the area under the } \\
\text { gadolinium concentration curve } \\
\text { (c) Logistic regression modeling }\end{array}$ & $\begin{array}{l}\text { - } 50 \text { subjects } \\
\text { - For } T_{2} \text {-weighted MRI } \\
\text { SEN: 0.94, SPE: } 0.37 \text {, } \\
\text { PPV: 50, and NPV: } 0.89 \\
\text { - For DCE-MRI SEN: } \\
\text { 0.73, SPE: 0.88, PPV: } \\
\text { 75, and NPV: } 0.75\end{array}$ \\
\hline $\begin{array}{l}\text { Puech et } \\
\text { al. }[133, \\
134]\end{array}$ & $\begin{array}{l}\text { (s) Manual ROIs/region growing } \\
\text { (f) Wash-in and wash-out slopes } \\
\text { (c) Automatic scoring algorithm of the selected } \\
\text { features }\end{array}$ & $\begin{array}{l}\text { - } 84 \text { subjects } \\
\text { - for PZ SEN: } 100 \% \text { and } \\
\text { SPE: } 45 \% \\
\text { - for TZ SEN: } 100 \% \text { and } \\
\text { SPE: } 40 \%\end{array}$ \\
\hline $\begin{array}{ll}\text { Vos } & \text { et } \\
\text { al. [463] } & \end{array}$ & $\begin{array}{l}\text { (s) Manual ROIs } \\
\text { (f) } 50 \% T_{1} \text { static, } 75 \% v_{e}, 75 \% k_{\mathrm{ep}}, 75 \% \\
K^{\text {trans }} \text {, and } 25 \% \text { wash-out } \\
\text { (c) SVM }\end{array}$ & $\begin{array}{l}\text { - } 34 \text { subjects } \\
\text { - } A_{z}: 0.83\end{array}$ \\
\hline $\begin{array}{l}\text { Ampeliotis } \\
\text { et al. [464] }\end{array}$ & $\begin{array}{l}\text { (s) Manual ROIs } \\
\text { (f) } T_{2} \text {-weighted features (intensity, and the in- } \\
\text { tensity variance and kurtosis for a } 3 \times 3 \text { win- } \\
\text { dow) and } S(t) \text { of DCE-MRI sequence } \\
\text { (c) Probabilistic neural networks }\end{array}$ & 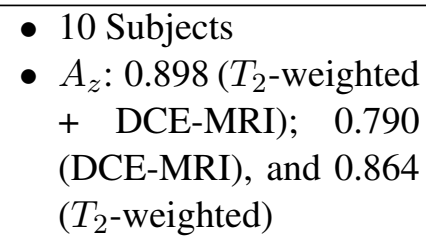 \\
\hline $\begin{array}{r}\text { Viswanath } \\
\text { et al. [462] }\end{array}$ & $\begin{array}{l}\text { (s) ASM } \\
\text { (f) } T_{2} \text { features (standard deviation, gradient, } \\
\text { Haralick feature), and DCE features (wash- } \\
\text { in and wash-out) } \\
\text { (c) Bayes classifier and random forest }\end{array}$ & $\begin{array}{l}\text { - } 6 \text { subjects (18 Images) } \\
\text { - } A_{z}: 0.815 \pm 0.029 \\
\text { - ACC: } 0.861 \pm 0.004\end{array}$ \\
\hline $\begin{array}{ll}\text { Lopes } & \text { et } \\
\text { al. [469] } & \end{array}$ & $\begin{array}{l}\text { (s) Manual ROIs } \\
\text { (f) Fractal dimension, and Multi-fractional } \\
\text { Brownian motion } \\
\text { (c) SVM and AdaBoost }\end{array}$ & $\begin{array}{l}\text { - } 17 \text { subjects } \\
\text { - SEN: } 0.83 \text { and SPE: } \\
0.91 \text { for AdaBoost } \\
\text { - SEN: } 0.85 \text { and SPE: } \\
0.93 \text { for SVM }\end{array}$ \\
\hline $\begin{array}{ll}\text { Shah } & \text { et } \\
\text { al. [470] } & \end{array}$ & $\begin{array}{l}\text { (s) K-means } \\
\text { (f) } T_{2} \text {-weighted intensities, } K_{\mathrm{ep}}, k_{\mathrm{el}}, T_{1} \text {, and } \\
\mathrm{ADC} \\
\text { (c) } \mathrm{SVM}\end{array}$ & 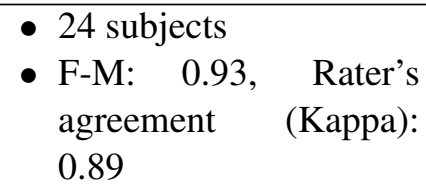 \\
\hline $\begin{array}{ll}\text { Sung } & \text { et } \\
\text { al. [229] } & \end{array}$ & $\begin{array}{l}\text { (s) Manual ROIs } \\
\text { (f) baseline and peak signal intensities, initial } \\
\text { up-slope, wash-in and washout rates, time } \\
\text { to peak, percentage of relative enhance- } \\
\text { ment, percentage enhancement ratio, time } \\
\text { of arrival, } K_{\mathrm{ep}}, k_{\mathrm{el}} \text {, and } A^{\text {Brix }} \\
\text { (c) SVM }\end{array}$ & $\begin{array}{l}\text { - } 42 \text { subjects } \\
\text { - SEN: } 0.77 \text {, SPE: } 0.77 \text {, } \\
\text { and ACC: } 0.83\end{array}$ \\
\hline $\begin{array}{ll}\text { Vos } & \text { et } \\
\text { al. }[251] & \end{array}$ & $\begin{array}{l}\text { (s) ASM } \\
\text { (f) } T_{2} \text {-weighted intensities, } T_{2} \text { relaxation time, } \\
\text { wash-out rate, } K^{\text {trans }}, v_{\mathrm{e}} \text {, and ADC } \\
\text { (c) LDA }\end{array}$ & $\begin{array}{l}\text { - } 200 \text { subjects } \\
\text { - SEN: } 0.41 \text { (at } 1 \text { FPs), } \\
0.65 \text { (at } 3 \text { FPs), and } \\
0.74 \text { (at } 5 \text { FPs) }\end{array}$ \\
\hline
\end{tabular}


TABLE 18 - continued from the previous page

\begin{tabular}{|c|c|c|}
\hline Study & Segmentation (s), Features (f) \& Classifier (c) & Data and Performance \\
\hline $\begin{array}{ll}\text { Niaf et } \\
\text { al. [139] }\end{array}$ & $\begin{array}{l}\text { (s) Manual ROIs } \\
\left.\text { (f) PK features ( } K^{\text {trans }}, k_{\mathrm{ep}} \text {, and } v_{\mathrm{e}}\right) \text {, nonpara- } \\
\text { metric features (Image intensity, texture, } \\
\text { gradient, peak intensity, time-t-peak, } T_{\max } \text {, } \\
\text { wash-in and wash-out slopes, AUC) } \\
\text { (c) Nonlinear SVM, LDA, } k_{n} \text {-nearest neigh- } \\
\text { bor, and naïve Bayes classifiers }\end{array}$ & $\begin{array}{l}\text { - } 30 \text { subjects } \\
\text { - } A_{z}: 0.89\end{array}$ \\
\hline $\begin{array}{ll}\text { Peng } & \text { et } \\
\text { al. [467] } & \end{array}$ & $\begin{array}{l}\text { (s) Manual ROIs } \\
\text { (f) } T_{2} \text {-weighted weighted skewness, } K^{\text {trans }} \text {, } \\
\text { average ADC, and } 10^{\text {th }} \text { percentile } \\
\text { (c) LDA }\end{array}$ & $\begin{array}{l}\text { - } 48 \text { Subjects } \\
\text { - } A_{z}: 0.95 \pm 0.02, \text { SEN: } \\
0.820 \text {, and SPE:0.953 }\end{array}$ \\
\hline $\begin{array}{l}\text { Hambrock } \\
\text { et al. [471] }\end{array}$ & $\begin{array}{l}\text { (s) Manual ROIs } \\
\text { (f) } 25 \text { percentile of } T_{2} \text { wash-out, } 25 \text { percentile } \\
\mathrm{ADC} \text {, and } 75 \text { percentile of } K^{\text {trans }} \text { and } v_{\mathrm{e}} \\
\text { (c) LDA }\end{array}$ & $\begin{array}{l}\text { - } 34 \text { subjects } \\
\text { - } A_{z}: 0.90,0.92 \text {, and } 0.87 \\
\text { for whole prostate, } \mathrm{PZ} \text {, } \\
\text { and } \mathrm{TZ} \text {, respectively. }\end{array}$ \\
\hline ACC: & \multicolumn{2}{|c|}{$\begin{array}{l}\text { Accuracy; } \mathrm{ACC}=\frac{\mathrm{TP}+\mathrm{TN}}{\mathrm{TP}+\mathrm{FP}+\mathrm{FN}+\mathrm{TN}} \text {; where } \mathrm{TP}: \text { true positive, } \\
\text { FP: false positive, } \mathrm{FN} \text { : false negative, and } \mathrm{TN} \text { : true negative. }\end{array}$} \\
\hline ADC: & \multicolumn{2}{|l|}{ Apparent diffusion coefficient. } \\
\hline DSC: & \multicolumn{2}{|l|}{ Dice similarity coefficient: $\mathrm{DSC}=\frac{2 \cdot \mathrm{TP}}{2 \cdot \mathrm{TP}+\mathrm{FP}+\mathrm{FN}}$. } \\
\hline F-M: & \multicolumn{2}{|l|}{ F-Measure. } \\
\hline IAUC & \multicolumn{2}{|l|}{ Initial area under the time-concentration curve. } \\
\hline LDA: & \multicolumn{2}{|l|}{ Linear discriminate analysis. } \\
\hline N/A: & \multicolumn{2}{|l|}{ Not applicable. } \\
\hline PPV: & \multicolumn{2}{|l|}{ Positive predictive value; $\mathrm{PPV}=\frac{\mathrm{TP}}{\mathrm{TP}+\mathrm{FP}}}$. \\
\hline RVM: & \multicolumn{2}{|l|}{ Relevance vector machine. } \\
\hline ROC: & \multicolumn{2}{|l|}{ Receiver operating characteristic. } \\
\hline SEN: & \multicolumn{2}{|l|}{ Sensitivity; $\mathrm{SEN}=\frac{\mathrm{TP}}{\mathrm{TP}+\mathrm{FN}}$. } \\
\hline SPE: & \multicolumn{2}{|l|}{ Specificity; $\mathrm{SPE}=\frac{\mathrm{TN}}{\mathrm{TN}+\mathrm{FP}}$; where $\mathrm{TN}$ : true negative. } \\
\hline SVM: & \multicolumn{2}{|l|}{ Support vector machine. } \\
\hline
\end{tabular}

To overcome the limitations of the existing work on prostate cancer diagnosis using MR images, a novel noninvasive DCE-MRI-based framework (Figure 85) for early diagnosis of prostate cancer is proposed. The proposed framework performs sequentially the following processing steps. First, the prostate is segmented from the surrounding anatomical tissues based on a MAP estimate of a new likelihood function. To handle the object inhomogeneities and variability and overcome image noise, the proposed likelihood function accounts for the visual appearances of the prostate and background, 3D spatial interaction between the prostate voxels, and a learned 3D shape model. Second, in order to account for any local prostate deformations that could occur during the scanning process, a nonrigid registration algorithm is employed, which is based on deforming a target prostate object 
over evolving iso-contours to match a reference object. The correspondences between the target and reference objects are found by the solution of the Laplace equation as described in Section III.C.2. In the third step, agent kinetic curves that show the contrast agent (CA) propagation into the tissue are obtained, and then used to collect two features to distinguish between malignant and benign detected tumors using a $k_{n}$-nearest neighbor classifier. Finally, parametric map displays that illustrate the propagation of the CA into the prostate tissue are constructed for visual assessment and characterization of the physiological data. Details of the proposed framework are described in the following sections.

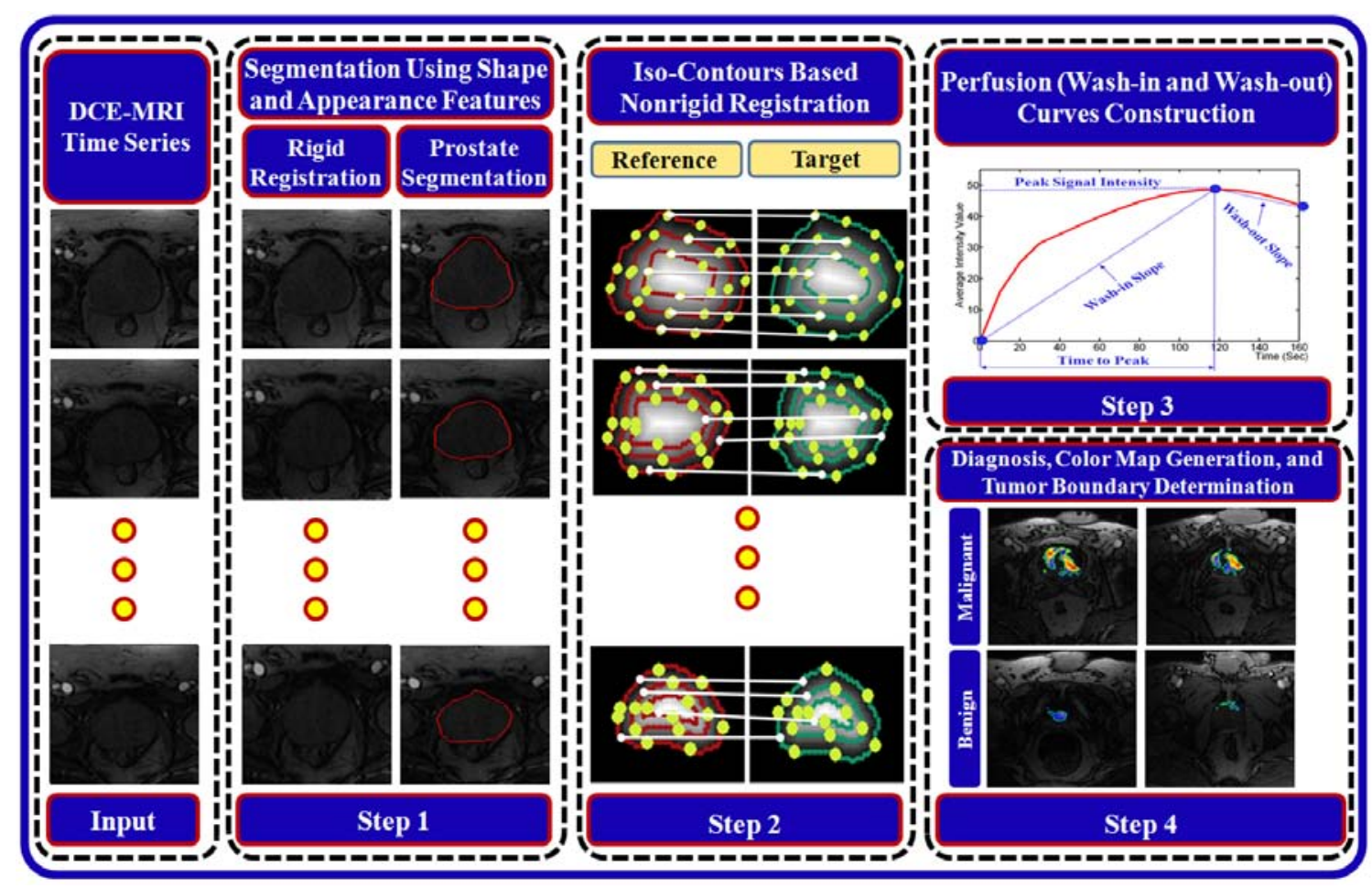

FIGURE 85: The proposed CAD system for early detection of prostate cancer.

\section{DELINEATION OF THE PROSTATE BORDERS}

The segmentation of the prostate from DCE-MRI is a challenge due to the anatomical complexity of the prostate and the undistinguishable gray-level distribution of the prostate and surrounding organs. To account for these challenges, a MAP-based approach 
based on a learned shape model and an identifiable joint Markov-Gibbs random field (MGRF) model (Figure 86) is proposed. The proposed MGRF image model relates the joint probability of an image and its object- background region segmentation map, to geometric structure and to the energy of repeated patterns within the image [472]. The basic theory behind such models is that they assume that the signals associated with each voxel depend on the signals of the neighboring voxel, and thus explicitly take into account their spatial interaction, and other features, such as shape.

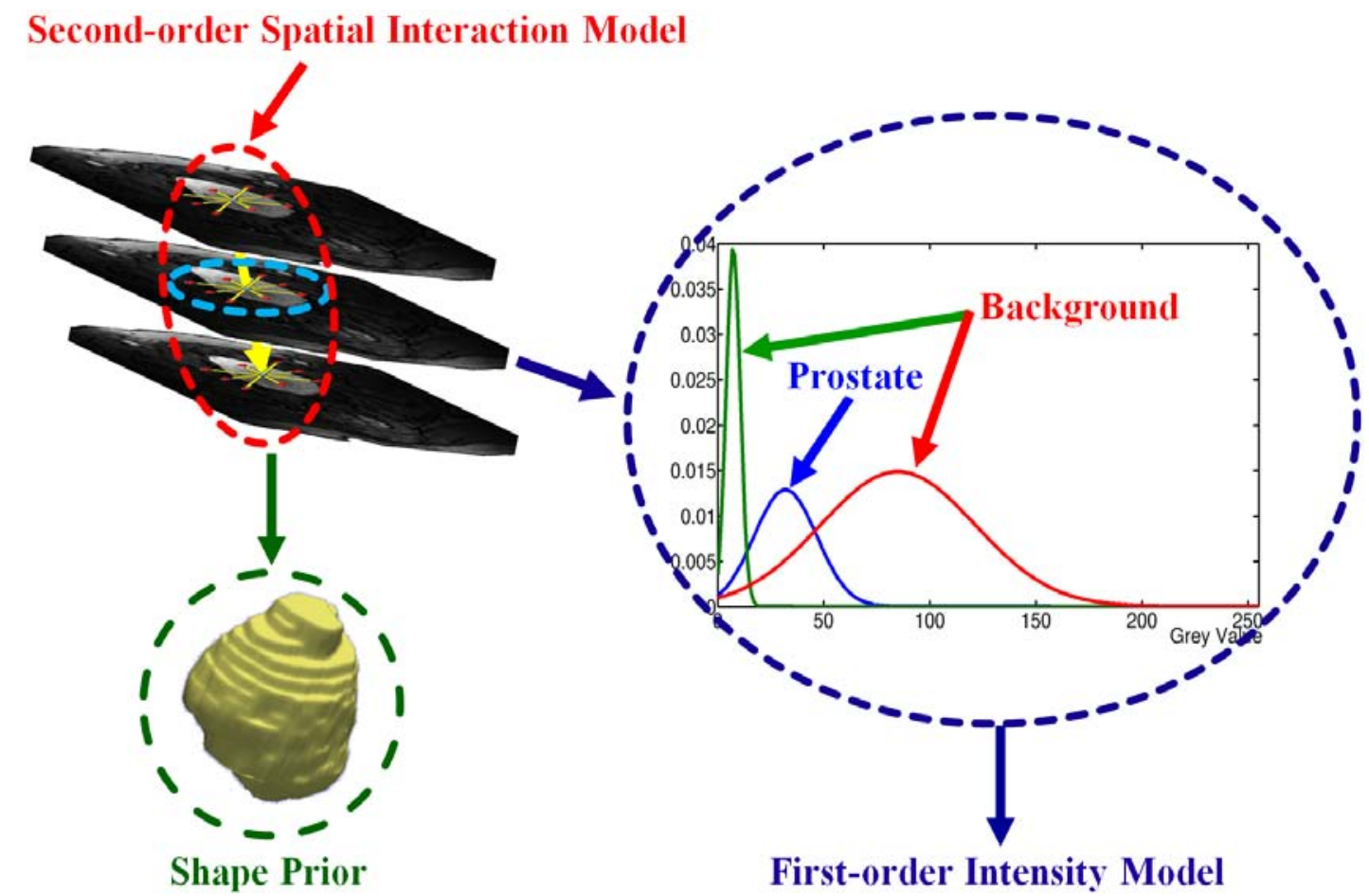

FIGURE 86: Illustration of the Joint Markov-Gibbs random field (MGRF) image model of the prostate DCE-MRI.

The input 3D DCE-MRI data $\mathbf{g}$ and its region map $\mathbf{m}$ are described by the joint MGRF model as outlined in Section III.C.1.b, Equation (45). The Bayesian MAP estimate of the map, given $\mathbf{g}, \mathbf{m}^{*}=\arg \max _{\mathbf{m}} L(\mathbf{g}, \mathbf{m})$ maximize the log-likelihood function:

$$
L(\mathbf{g}, \mathbf{m})=\log (P(\mathbf{g} \mid \mathbf{m}))+\log (P(\mathbf{m}))
$$

where $P(\mathbf{g} \mid \mathbf{m})$ is a conditional distribution of the images given the map $\mathbf{m}$ and $P(\mathbf{m})=$ $P_{\mathrm{sp}}(\mathbf{m}) P_{\mathbf{V}}(\mathbf{m})$ is an unconditional probability distribution of maps. Here, $P_{\mathrm{sp}}(\mathbf{m})$ denotes 
the prostate shape prior, and $P_{\mathbf{V}}(\mathbf{m})$ is a Gibbs probability distribution with potentials $\mathbf{V}$, which specifies a MGRF model of spatially homogeneous maps $\mathbf{m}$.

The specific visual appearance of the prostate in each data set to be segmented is taken into account by modeling the marginal gray level distribution with the LCDG model [348-350] as described in Section III.C.1.c. To overcome noise effect and to ensure the homogeneity of the segmentation, the spatial voxel interactions between the region labels of a given map $\mathbf{m}$ are also taken into account using the pairwise MGRF spatial model as described in Section III.C.1.b and the nearest voxel 26-neighbors shown in Figure 87. In addition to voxel-wise image intensities and their pairwise spatial interaction, additional constraints based on the expected shape of the prostate are introduced by co-aligning each given DCE-MRI data to a training database and using probabilistic 3D prostate shape model $P_{\mathrm{sp}}(\mathbf{m})$, see Figure 88.

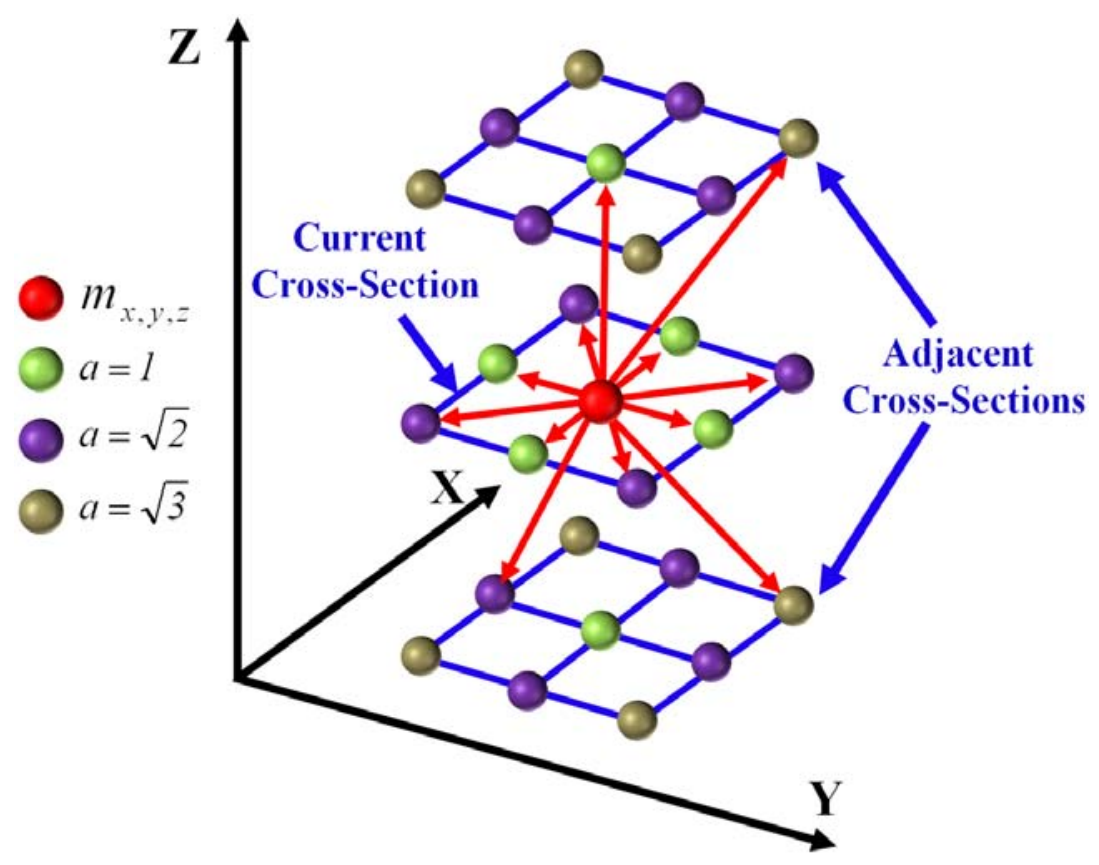

FIGURE 87: 3D second-order MGRF neighborhood system. Note that the reference voxel is shown in red and $a$ represents the absolute distance between two voxels in the same and adjacent MRI slices, or cross-sections.

To perform initial prostate segmentation, a given 3D DCE-MRI is aligned to one of the training data. The shape model provides the voxel-wise object and background proba- 

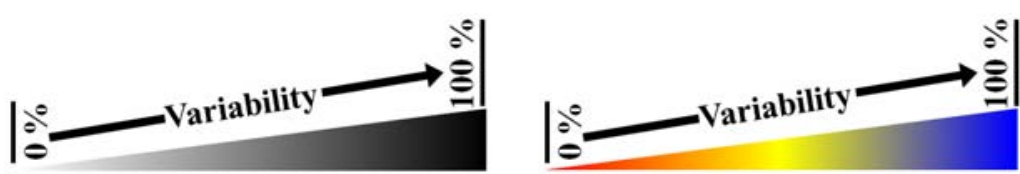

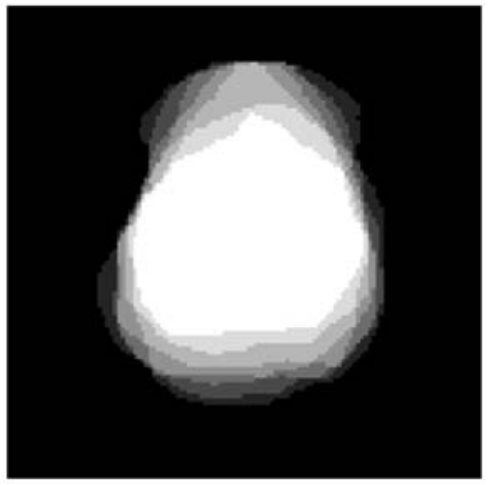

(a)

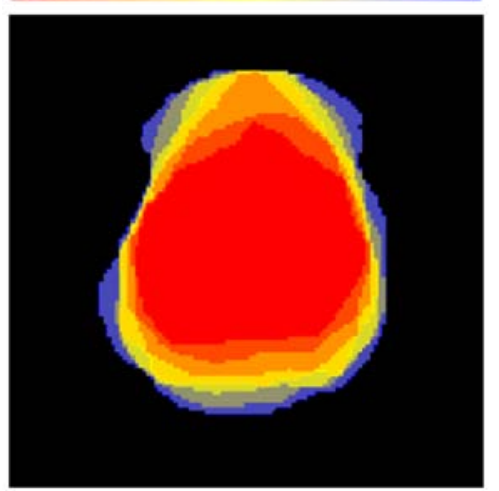

(b)

FIGURE 88: Gray-coded $(a)$ and color-coded $(b)$ axial view of the prostate shape prior.

bilities being used, together with the conditional image intensity model $P(\mathbf{g} \mid \mathbf{m})$, to build an initial region map. The final Bayesian segmentation is performed using the identified joint MGRF model of the DCE-MRI data and its region maps. In total, the proposed prostate segmentation approach involves the steps summarized in Algorithm 5.

\section{Nonrigid Registration}

Due to patient breathing and local movement, accurate registration is a main issue in DCE-MRI time series. After affine registration and prostate segmentation, the Laplacebased nonrigid registration demonstrated in Section III.C.2 is used for local motion correction of the prostate over the time frame of image acquisition. For completeness, the main steps of the Laplace-based nonrigid registration is summarized in Figure 89 as follows:

1. Generation of the distance maps inside the prostate regions (Figure $89(a, b))$.

2. Generation of the iso-contours using distance maps in Step 1 (Figure $89(c, d)$ ).

3. Solution of the Laplace equation between respective reference and target iso-contours to co-allocate the corresponding points. 
Algorithm 5 Prostate Segmentation Approach

Input: 3D MRI prostate data $\mathrm{g}$ to be segmented.

Output: segmented prostate border (the final estimate $\mathbf{m}$ ).

\section{- Training Phase:}

1. Co-alignment of the 3D DCE-MRI training sets using a 3D affine registration in a way that maximizes their MI [366].

2. Manual delineation of the prostate borders from the co-aligned data.

3. Estimation of the voxel-wise probabilities by counting how many times each voxel $(x, y, z)$ was segmented as the prostate.

\section{- Testing Phase:}

1. Perform a 3D affine alignment of a given MRI to an arbitrary prototype prostate from the training set using MI [366] as a matching metric.

2. Estimate the conditional intensity model $P(\mathbf{g} \mid \mathbf{m})$ by identifying the bimodal LCDG.

3. Use the intensity model found in Step 2 and the learned probabilistic shape model to perform an initial segmentation (region map $\mathbf{m}$ ) of the prostate.

4. Use $\mathbf{m}$ to estimate the potential for the Potts MGRF model and to identify the MGRF model $P(\mathbf{m})$ of region maps.

5. Improve m using voxel-wise stochastic relaxation (ICM [473]) through successive iterations to maximize Equation (59) until the log-likelihood remains almost the same for two successive iterations.

6. Update the Shape Prior: by adding the current segmented 3D prostate data to the prior calculated shape model. 


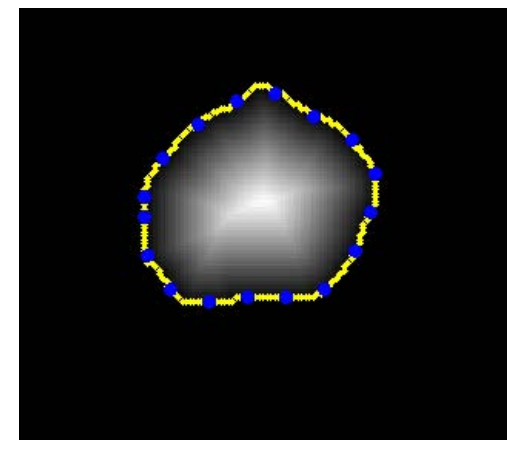

(a)

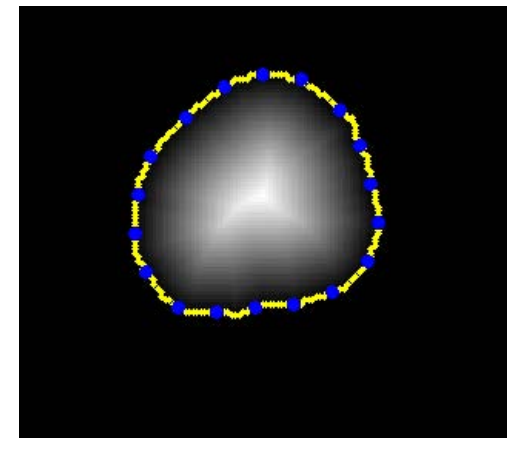

(c)

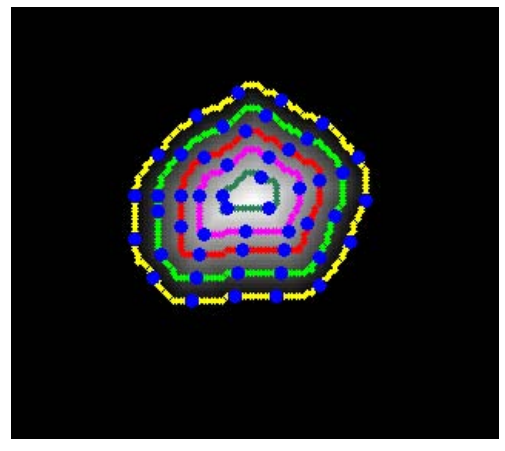

(b)

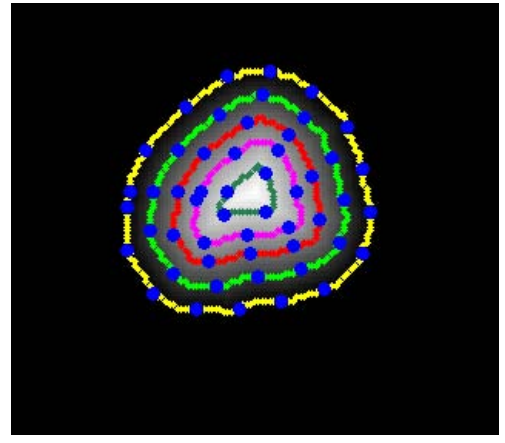

(d)

FIGURE 89: Generation of the iso-contours: the reference and target distance maps $(a, b)$, and their iso-contours $(c, d)$.

\section{Perfusion Characterization and Tumor Boundary Determination}

After the nonrigid alignment, the time-intensity or agent kinetic curves are constructed by calculating the average intensities of prostate regions for each time sequence. These curves show the response of the prostate tissue to the transient of the CA perfusing into each image section (see Figure 90). To characterize the physiological data, parametric map displays are constructed to illustrate the propagation of the CA into the prostate tis-

sue. To construct the initial color maps, the changes in image signals $\Delta_{x, y, z}$ due to the CA transient are estimated (see Figure 90) from the constructed kinetic curves as the difference between the signals of image sequences at peak time $\left(T_{p}\right)$ and initial time $\left(T_{0}\right)$. To preserve continuity (remove inconsistencies), the initial estimated $\Delta_{x, y, z}$ values are considered as samples from a Generalized Gauss-Markov random field (GGMRF) image model [418] of measurements with the 26-voxel neighborhood shown in Figure 87. Continuity of the con- 
structed 3D volume (see Figure 91) is amplified by using their MAP estimates as shown in [418]:

$$
\widehat{\Delta}_{\mathbf{P}}=\arg \min _{\widetilde{\Delta}_{\mathbf{P}}}\left\{\left|\Delta_{\mathbf{P}}-\widetilde{\Delta}_{\mathbf{P}}\right|^{a}+\alpha^{a} \chi^{b} \sum_{\mathbf{P}^{\prime} \in \nu_{\mathbf{P}}} \Gamma_{\mathbf{P}, \mathbf{P}^{\prime}}\left|\widetilde{\Delta}_{\mathbf{P}}-\Delta_{\mathbf{P}^{\prime}}\right|^{b}\right\}
$$

where $\Delta_{x, y, z}$ and $\widetilde{\Delta}_{x, y, z}$ denote the original values and their expected estimates at the observed 3D location, $\mathbf{P}=(x, y, z), \nu_{\mathbf{P}}$ is the neighborhood system (Figure 87$), \Gamma_{\mathbf{P}, \mathbf{P}^{\prime}}$ is the GGMRF potential, and $\alpha$ and $\chi$ are scaling factors. The parameter $b \in[1.01,2.0]$ controls the smoothing level (e.g., smooth, $b=2$, vs. relatively abrupt edges, $b=1.01$ ). The parameter $a \in\{1,2\}$ determines the Gaussian, $a=2$, or Laplace, $a=1$, prior distribution of the estimator. Then, the color maps are generated based on the final estimated $\widehat{\Delta}$ ( Figure 95).

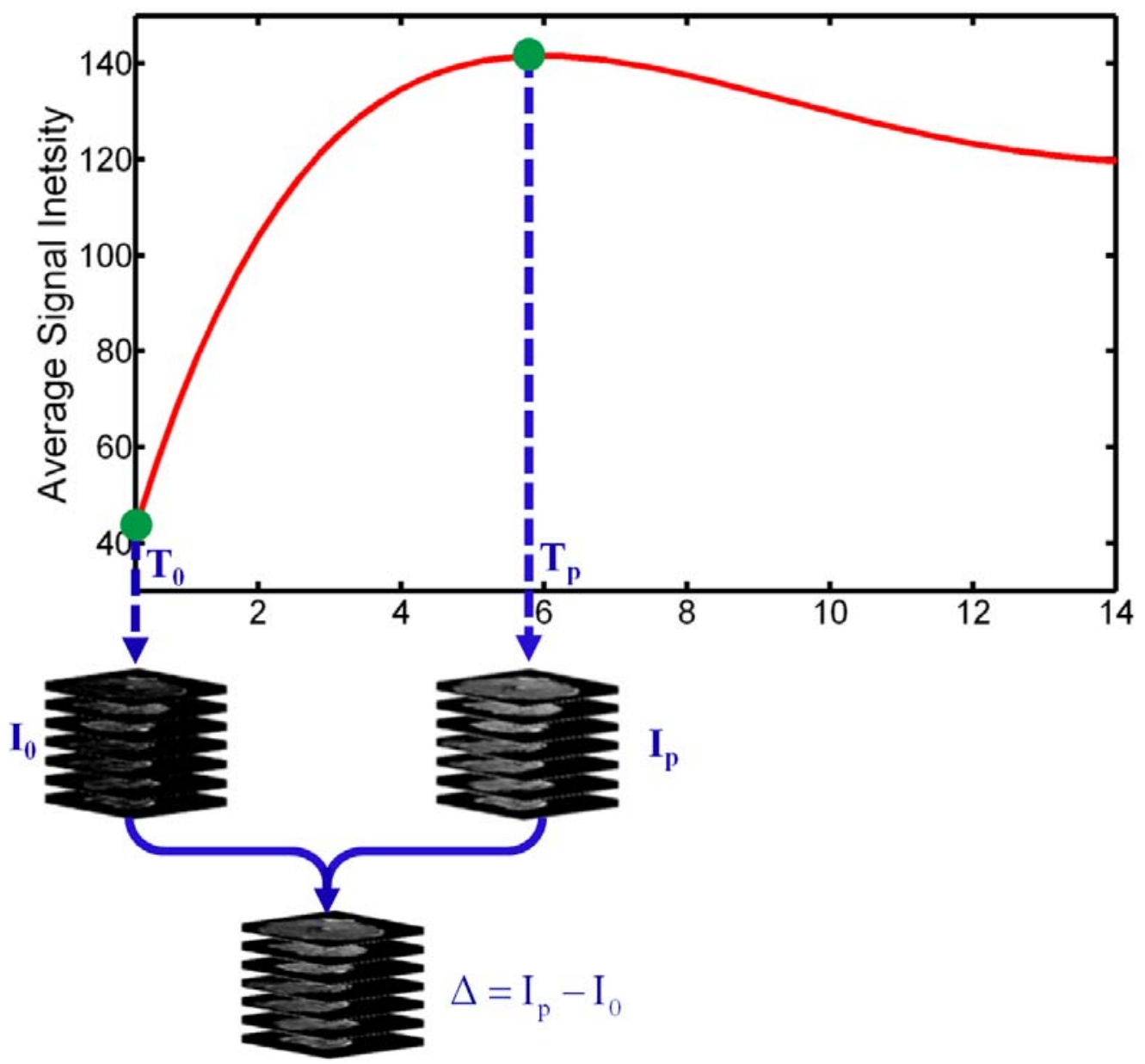

FIGURE 90: Estimating the changes in image signals $\Delta$ from the kinetic curve as the difference between the peak $\left(I_{p}\right)$ and initial $\left(I_{0}\right)$ signals of the image sequences. 


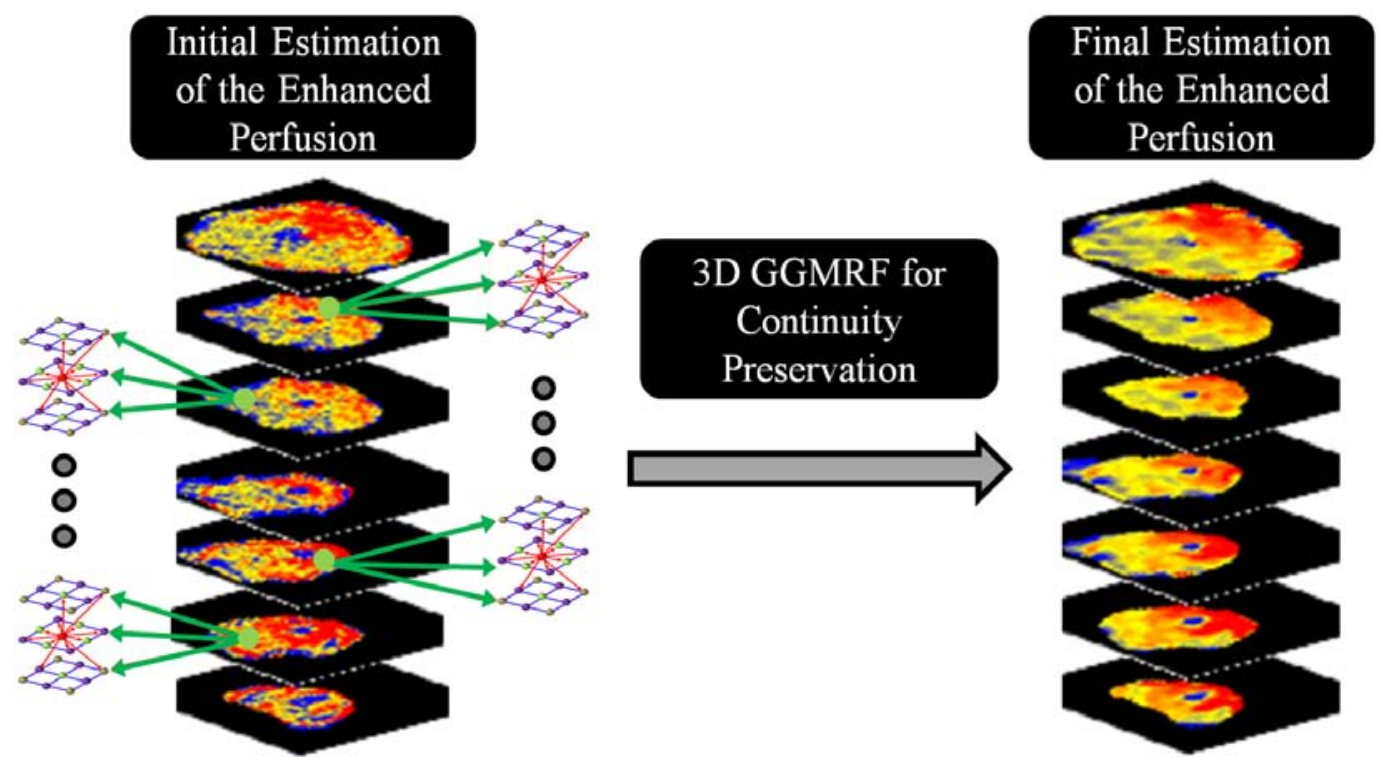

FIGURE 91: Enhanced perfusion estimation and continuity analysis using the 3D GGMRF image model.

The final step after the 3D GGMRF smoothing is the delineation of the detected tumor boundary, which is important to determine the cancer stage in case of malignancy. To achieve this, the level set deformable model presented in Section III.C.1 is applied again. The evolution of the level set is controlled by a stochastic speed function that accounts for the perfusion information and spatial interactions between the prostate voxels.

\section{EXPERIMENTAL RESULTS}

1. Patients and Data Acquisition

The performance of the proposed framework has been evaluated by applying it on DCE-MRI prostate data that has been collected from 30 patients. These patients had biopsy-proven prostate cancer. DCE-MRI was obtained at $1.5 \mathrm{~T}$ using a gradient-echo $T_{2}$ imaging (SIGNA Horizon, General Electric Medical Systems, Milwaukee, WIS) using an additional pelvic coil. Images were taken at a $7 \mathrm{~mm}$ thickness with an interslice gap of 0.5 $\mathrm{mm} ; T_{R}$ was $50 \mathrm{msec}$; $T_{E}$ was minimum; flip angle at $60^{\circ}$; band width was 31.25 kilohertz 
(kHz); field-of-view (FOV) was $28 \mathrm{~cm}$; and the number of slices was 7. The DCE-MRI process started with a series of MRI scans which were used to establish a baseline in image intensity. These scans were performed without the administration of the CA so that the tissue's non-enhanced image intensity could be established. In the next stage, 10 cubic centimeter $(c c)$ of gadoteric acid (Dotarem 0.5 millimole/milliliter $(\mathrm{mmol} / \mathrm{mL})$; Guerbet, France) was administered intravenously at a rate of $3 \mathrm{~mL} / \mathrm{sec}$. At this point, a series of MRI scans was performed every $10 \mathrm{sec}$ for approximately $3 \mathrm{~min}$, and every series contained 7 slices. Note that all the subjects were diagnosed using a biopsy (ground truth).

\section{Segmentation Results}

The proposed segmentation approach has been tested on DCE-MRI sequences for 30 independent subjects. Figure 92 shows some segmentation results of the prostate region at selected image sections for different subjects and their associated false positive (FP) and false negative (FN) segmentation errors, with respect to the ground truth segmentation. The ground truths were obtained by manual delineation of the prostate borders by an MR imaging expert. The positive predictive value (PPV), sensitivity (SEN), and Dice similarity coefficient (DSC) statistics for the proposed approach are summarized in Table 19.

TABLE 19: Error statistics of the proposed segmentation approach. Note that "SD", stands for standard deviation.

\begin{tabular}{|c|c|c|c|}
\cline { 2 - 4 } \multicolumn{1}{c|}{} & \multicolumn{3}{|c|}{ Performance Metric } \\
\cline { 2 - 4 } \multicolumn{1}{c|}{ PPV } & SEN & DSC \\
\hline Mean \pm SD & $0.982 \pm 0.004$ & $0.846 \pm 0.004$ & $0.923 \pm 0.004$ \\
\hline
\end{tabular}

To highlight the advantage of the proposed segmentation approach, all time series images have been segmented using the shape-based (SB) approach proposed by Tsai et al. [1]. The comparative results for a few of them are shown in Figure 93 and Table 20 summarizes the segmentation error statistics of the proposed approach and the SB approach 

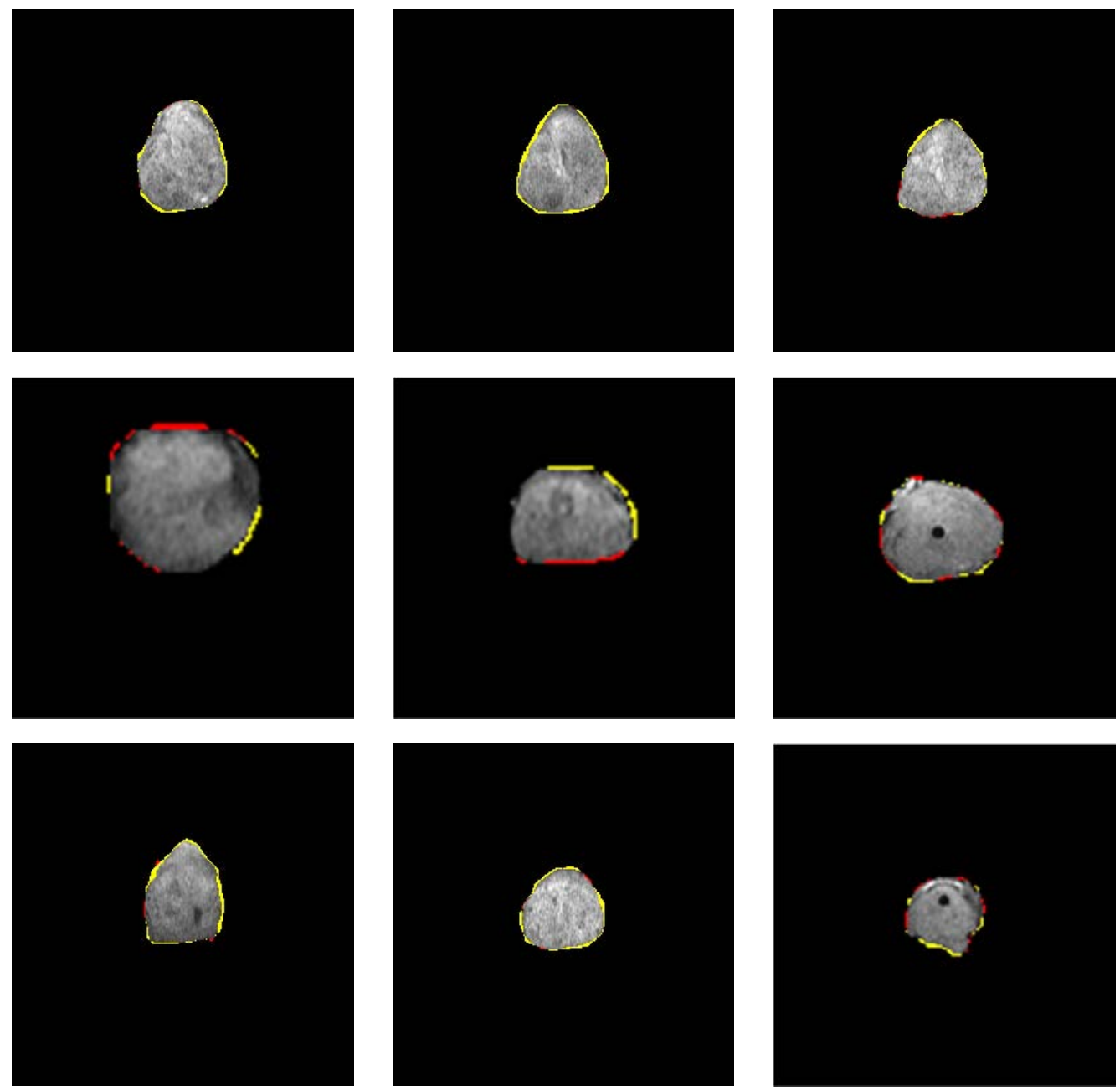

FIGURE 92: Sample segmentation results of the proposed segmentation approach for different subjects at different cross-sections. The false negative (FN) and false positive (FP) error referenced to the ground truth $\mathrm{G}$ are shown in yellow and red, respectively.

with respect to ground truth. The differences between the mean errors of the proposed approach and the SB approach are shown to be statistically significant by the unpaired $t$-test (the two-tailed value $\mathrm{P}$ is less than 0.0001 ).

\section{Diagnostic Results}

The ultimate goal of the proposed framework is to successfully distinguish between malignant and benign detected tumors by constructing the time-intensity curves from the DCE-MRI sequences. The curves show the response of the prostate tissues as the CA 

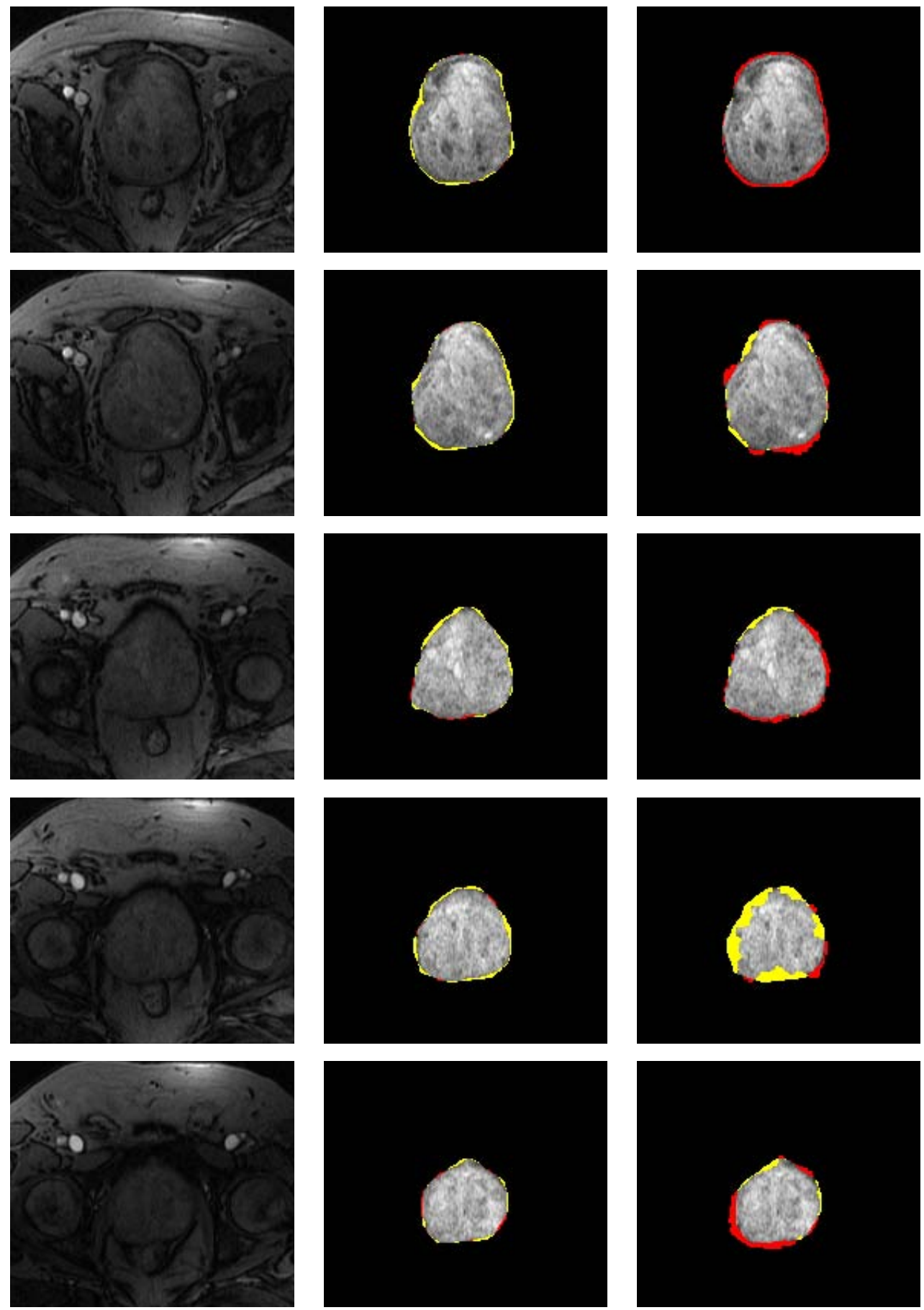

(a)

(b)

(c)

FIGURE 93: Comparative segmentation results: (a) different prostate dynamic MR images,

(b) proposed approach segmentation, and (c) segmentation with [1]. The false negative $(\mathrm{FN})$ and false positive (FP) segmentation errors referenced to the ground truth $\mathrm{G}$ are shown in yellow and red, respectively 
TABLE 20: Comparative segmentation accuracies of the proposed prostate segmentation against the shape-based (SB) approach [1] in comparison to the expert's ground truth ("SD" - standard deviation).

\begin{tabular}{|l|c|c|}
\cline { 2 - 3 } \multicolumn{1}{c|}{} & \multicolumn{2}{c|}{ Segmentation Technique } \\
\cline { 2 - 3 } \multicolumn{1}{c|}{} & Proposed Approach & SB Approach [1] \\
\hline Mean \pm SD $(\%)$ & $0.53 \pm 0.33$ & $5.91 \pm 4.44$ \\
\hline Two-tailed P-value & \multicolumn{2}{|c|}{0.0001} \\
\hline
\end{tabular}

perfuses. The malignant subjects show an abrupt increase of the signal intensity and the benign subjects show a delay in reaching their peak (see Figure 94). From these curves, it is conclude that the peak signal value and the wash-in slope are the two major features that can be extracted for the classification of prostate cancer. To distinguish between benign and malignant cases, a $k_{n}$-nearest neighbor classifier is used to learn the statistical characteristics of both benign and malignant cases from the time-intensity curves of the training sets. Nine data sets were used for the training and the other 21 data sets were used for testing. The diagnostic accuracy of the $k_{n}$ classifier was $100 \%$ for all training and testing data sets.

Following the classification, a visual assessment is performed using color-coded maps. Figure 95 presents the color-coded maps over all image sections before and after applying the 3D GGMRF smoothing for three independent subjects. Figures 96 show two examples of the tumor contours determination for one benign and one malignant subject.

\section{E. SUMMARY}

In this chapter, a noninvasive framework for detecting prostate cancer using DCEMRI is proposed. The framework includes delineation of the prostate region, local motion correction, and $k_{n}$-classification. The proposed framework has the documented ability to reliably distinguish benign from malignant prostate cancer, in a biopsy proven preliminary cohort of 30 patients. To extract the prostate region, a new 3D approach that is based 


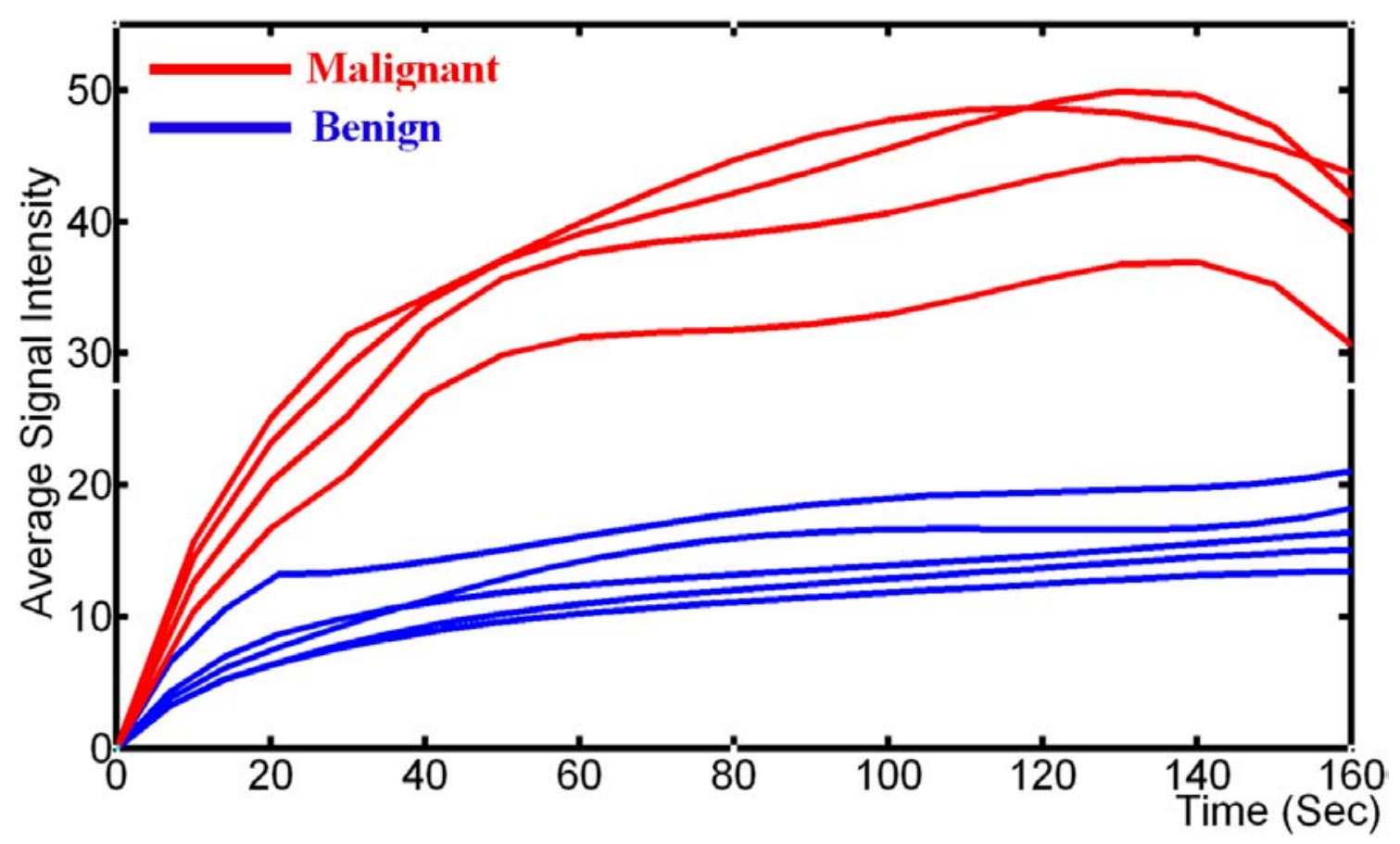

FIGURE 94: Selected normalized signal intensity, averaged over the entire prostate, with respect to the timing of contrast agent (CA) delivery for malignant (red) and benign (blue) subject.

on a MAP estimate of a new log-likelihood function that accounts for a priori shape, the spatial interactions between the prostate voxels, and the current appearance of the prostate tissues and its background is proposed. Following segmentation, a nonrigid registration approach is introduced. The proposed approach deforms the prostate object on iso-contours instead of a square lattice, which provides more degrees of freedom to obtain accurate deformation. The agent kinetic curves of the co-aligned prostate regions are constructed and the two features extracted from these curves undergo $k_{n}$-classification. Applications of the proposed framework yield promising results that would, in the near future, represent a supplement of the current technologies to determine the type of prostate cancer. The work presented in this chapter has been published in the IEEE International Symposium on Signal Processing and Information Technology (ISSPIT) conference [474], the IEEE International Symposium on Biomedical Imaging (ISBI) conference [76], the International Conference on Developments in eSystems Engineering (DeSE) [75], Journal of Biomed- 


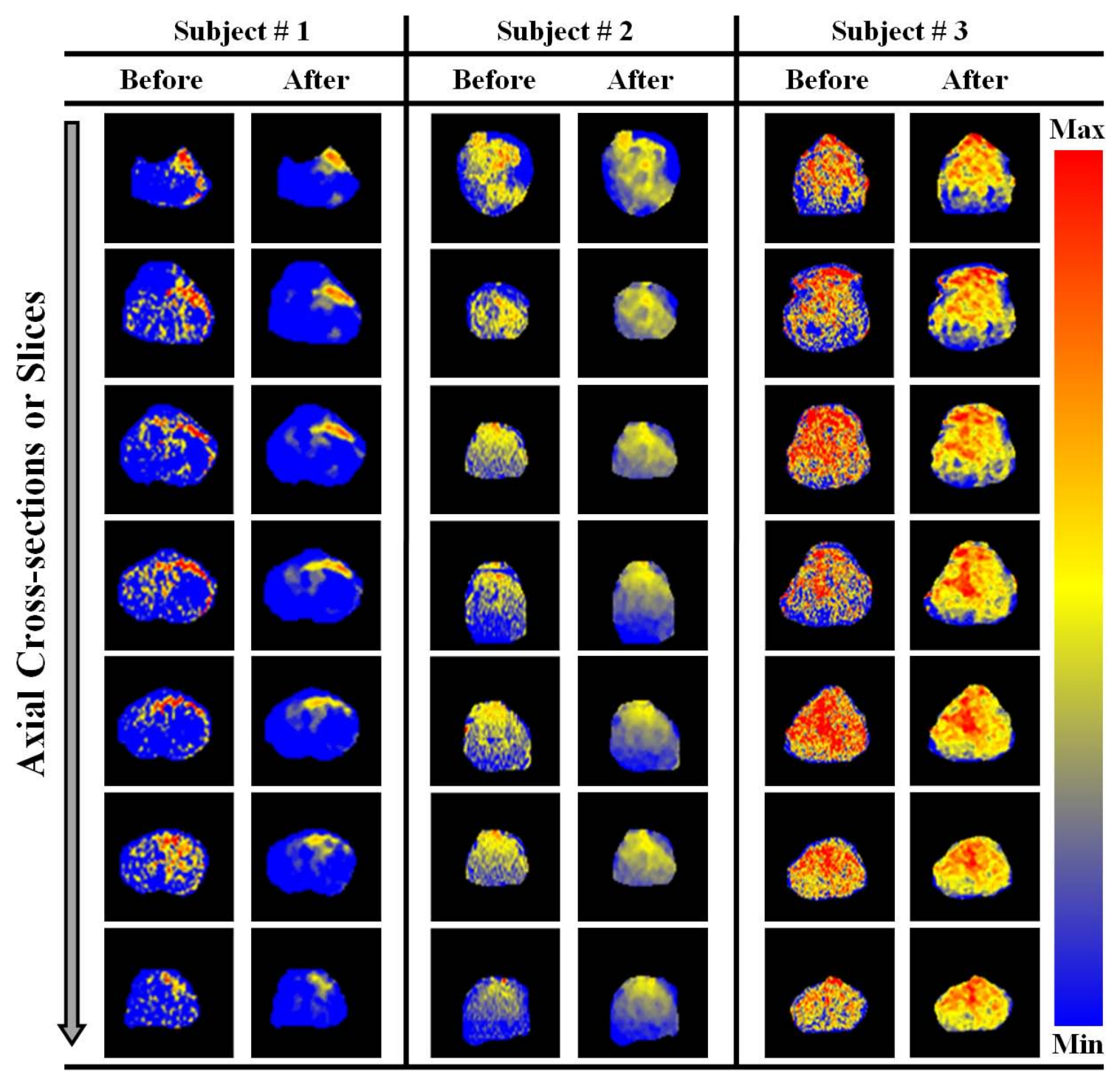

FIGURE 95: Color-coded maps for three subjects before and after the 3D GGMRF smoothing using $\alpha=1, \chi=5, b=1.01, a=2$, and $\Gamma_{\mathbf{P}, \mathbf{P}^{\prime}}=\sqrt{2}$. The red and blue ends of the color scale relate to the maximum and minimum changes, respectively.

ical Science and Engineering [80], and the Handbook of Computational Intelligence in Biomedical Imaging [77]. 

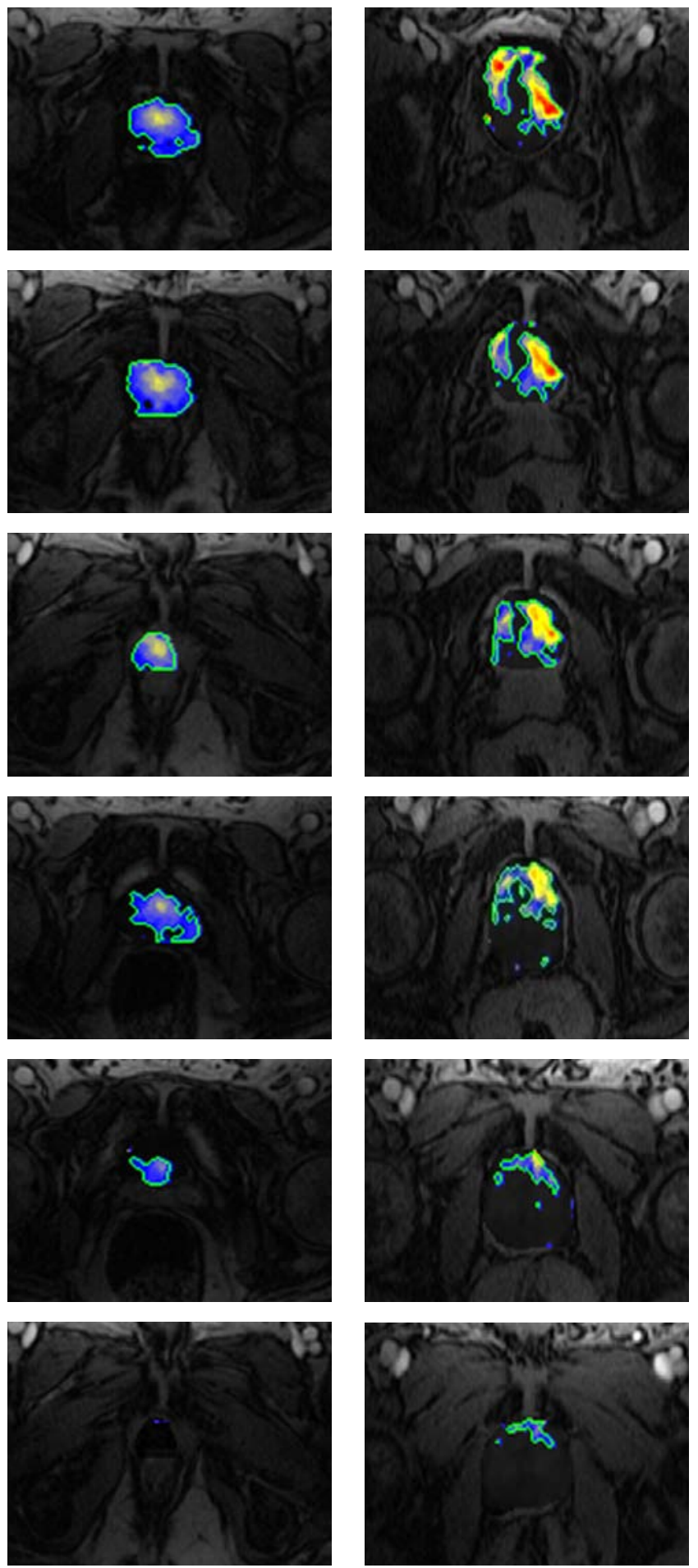

(a)

(b)

FIGURE 96: Color-coded maps of local tumor progression overlaid on anatomic DCE-MRI data for one benign $(a)$ and one malignant $(b)$ subject. The determined tumor contours are shown in green. 


\section{CHAPTER VI CONCLUSIONS AND FUTURE WORK}

The work presented in this dissertation documents the ability of contrast-enhanced (CE) medical images for the evaluation of human organ functionality. The effectiveness of $\mathrm{CE}$ images have been successfully tested for efficient analysis of CE images in three case studies ( $i$ ) early detection of acute renal transplant rejection using dynamic contrastenhanced MRI (DCE-MRI); (ii) evaluation of myocardial perfusion in patients with heart failure using cardiac perfusion first-pass MRI (FP-MRI); and (iii) early detection of prostate cancer using DCE-MRI. The key main advanatge of the proposed computer-aided diagnostic (CAD) system for the evaluation of human organ functionality is the ability of the developed motion correction model to provide accurate pixel-on-pixel matches of the registered images for generating and displaying parametric maps. These capabilities are of great importance for the radiologists to help investigate, in the case of organ dysfunction, which local regions need attention and follow-up with appropriate treatment. Comparison results with other motion correction models using synthetic and in-vivo data showed the higher accuracy of the proposed model. In addition, summary of the main contributions of this dissertation are as follows:

- A novel stochastic speed, instead of traditional edge-based geometric speed functions, is proposed to guide the evolution of a geometric (level set-based) deformable model to segment/extract the object of interest from medical imaging. The proposed speed function accounts for three image features: the higher-order spatial-interaction between the image pixels, prior probabilistic shape model, and first-order visual appearance of the object-of-interest. These three features are integrated into a twolevel joint Markov-Gibbs random field (MGRF) model of the object-of-interest and its background. 
- An unsupervised probabilistic model to learn the second- and higher-order spatial interactions between the object pixels (voxels) from medical images is proposed. The model possesses the ability to account for the appearance features to learn the large inhomogeneity in the object-of-interest (e.g., the kidney). The proposed model adds to the pairwise the higher-order spatial interactions between region labels of a given segmentation, namely, the families of the triple and quad cliques.

- An adaptive probabilistic shape model that has the ability to learn both the shape of the object-of-interest and the subject-to-subject variability is proposed. The main advantage of the proposed shape prior model is its ability to impose temporal constraints on the segmentation of dynamic MRI data.

- A novel global alignment scheme is developed for the alignment of medical objects using a new similarity function that accounts for both the first- and second-order normalized mutual information (NMI). The incorporation of the second-order NMI similarity term increases the ability of the NMI to capture more features from the images, and therefore can improve the registration accuracy.

- A new nonrigid registration to compensate for local object deformations caused by physiological effects is proposed. The proposed nonrigid registration deforms each pixel of the target object over a set of nested, equi-spaced contours (i.e., iso-contours) to closely match the reference object, by exploiting the geometric features, rather than image intensities to avoid problems associated with nonlinear intensity variations in medical images.

Several possibilities for the future work of this dissertation include, but are not limited to, the following:

- Extending the proposed 3D (2D+time) CAD sytem for the detection of acute renal rejection to deal with 4D (3D+time) DCE-MRI in order to more accurately localize the pathology in the whole kidney. 
- Testing the proposed mathematical models and learning techniques in other clinical studies, such as detection of lung cancer using CE computed tomography (CE-CT) images [21,475-506], brain cancer using DCE-MRI, and colon cancer using CE-CT images.

- The focus of this dissertation for the analysis of CE images was on using nonparametric perfusion techniques, future work will investigate the fusion of the nonparametric indexes and pharmacokinetic (PK) parameters, such as the volume of the extra-vascular extracellular space (ESS), capillary permeability. This fusion is expected to give critical insight into the functionality of the transplanted kidney.

- A future work of this dissertation is to investigate the integration of the proposed work with the BioImaging lab work for the detection of other brain disorders such as autism [507-522] and dyslexia [523-531]. 


\section{REFERENCES}

[1] A. Tsai, A. J. Yezzi, W. M. Wells III, C. M. Tempany, D. Tucker, A. C. Fan, W. E. L. Grimson, and A. S. Willsky. A shape-based approach to the segmentation of medical imagery using level sets. IEEE Trans. Med. Imaging, 22(2):137-154, 2003.

[2] H. E. Abd El Munim and A. A. Farag. Curve/surface representation and evolution using vector level sets with application to the shape-based segmentation problem. IEEE Trans. Pattern Anal. Mach. Intell., 29(6):945-958, 2007.

[3] F. Khalifa, A. El-Baz, G. Gimel'farb, and M. Abo El-Ghar. Non-invasive imagebased approach for early detection of acute renal rejection. In Proc. Med. Image Comput. Comput.-Assist. Interv., (MICCAI'10), pages 10-18, 2010.

[4] D. Rueckert, L. I. Sonoda, C. Hayes, D. L. G. Hill, M. O. Leach, and D. J. Hawkes. Non-rigid registration using free-form deformations: Application to breast MR images. IEEE Trans. Med. Imaging, 18(8):712-721, 1999.

[5] T. Arts, W. C. Hunter, A. Douglas, A. M. Muijtjens, and R. S. Reneman. Description of the deformation of the left ventricle by a kinematic model. J. Biomechan., 25(10):1119-1127, 1992.

[6] M. D. Cerqueira, N. J. Weissman, V. Dilsizian, A. K. Jacobs, S. Kaul, W. K. Laskey, D. J. Pennell, J. A. Rumberger, T. Ryan, and M. S. Verani. Standardized myocardial segmentation and nomenclature for tomographic imaging of the heart. Circulation, 105(4):539-542, 2002.

[7] M. Abou El-Ghar and T. El-Diasty. Ectopic insertion of the ureter into the seminal vesicle. World J. Radiol., 5(9):349-351, 2013.

[8] A. Naum, N. Kleven-Madsen, M. Biermann, B. Johnsen, B. A. Tvedt, S. Rotevatn, J. E. Nordrehaug, and T. Bach-Gansmo. Quantitative comparison of myocardial perfusion defects using different reconstruction algorithms. J. Clin. Exper. Cardiol., pages S5:002. doi:10.4172/2155-9880.S5-002, 2011.

[9] R. H. Baroni, M. I. Novis, Â. H. M. Caiado, L. M. d. O. Cerri, C. d. C. Leite, and G. G. Cerri. Magnetic resonance imaging of the prostate: An overview for radiologists. Radiol. Brasileira, 42(3):185-192, 2009.

[10] C. Scotti, E. M. Marone, L. E. Brasca, G. M. Peretti, R. Chiesa, A. Del Maschio, G. Fraschini, and F. Camnasio. Pseudoaneurysm overlying an osteochondroma: A noteworthy complication. J. Orthop. Traumatol., 11(4):251-255, 2010.

[11] M. S. Beauchamp. Human Brain Mechanisms for Multisensory Integration and Perception [Online]. Available. http://nba.uth.tmc.edu/resources/ faculty/members/beauchamp.htm. 
[12] MAYO Foundation for Medical Education and Research. Kidney Biopsy [Online]. Available. http://www.mayoclinic.com/health/medical/ IM0 $4229 \mathrm{~m}$.

[13] P. S. Tofts and A. G. Kermode. Measurement of the blood-brain barrier permeability and leakage space using dynamic MR imaging. 1. Fundamental concepts. Magn. Reson. Med., 17(2):357-367, 1991.

[14] G. J. M. Parker, C. Roberts, A. Macdonald, G. A. Buonaccorsi, S. Cheung, D. L. Buckley, A. Jackson, Y. Watson, K. Davies, and G. C. Jayson. Experimentallyderived functional form for a population-averaged high-temporal-resolution arterial input function for dynamic contrast-enhanced MRI. Magn. Reson. Med., 56(5):9931000, 2006.

[15] Arizona Transplant Associates, PC. About the Kidneys [Online]. Available. http: //www.arizonatransplant.com/healthtopics/.

[16] Addison Wesley Longman, Inc. Human Analtomy: Nephron Diagram Labeled [Online]. Available. http://www.dedeep.com/ nephron-diagram-labeled/.

[17] Pearson Eduaction, Inc. Kidney Physiology: Mechanisms of Urine Formation [Online]. Available. http://classes.midlandstech.edu/carterp/ Courses/bio211/chap25/chap25.htm.

[18] S. Lee, G. Wolberg, and S. Y. Shin. Scattered data interpolation with multilevel B-splines. IEEE Trans. Vis. Comput. Graph., 3(3):228-244, 1997.

[19] WebMD: Heart Disease Health Center. How the Healthy Heart Works [Online]. Available. http://www.webmd.com/heart-disease/ healthy-heart-works.

[20] Kaiser Permanente. Heart Function: Basic Anatomy and Function of the Heart [Online]. Available. http: / / www . permanente. net/homepage/kaiser/ pages/f63363.html.

[21] A. Soliman, F. Khalifa, A. Alansary, G. Gimel'farb, and A. El-Baz. Segmentation of lung region based on using parallel implementation of joint MGRF: Validation on 3D realistic lung phantoms. In Proc. IEEE Int. Symp. Biomed. Imaging: From Nano to Macro, (ISBI'13), pages 852-855, 2013.

[22] HealthNews Texas. PSA Test Reduces Prostate Cancer Deaths [Online]. Available. http://healthnewstexas.com/5262/ psa-test-reduces-prostate-cancer-deaths/.

[23] SIU School of Medicine. Enlarged Prostate [Online]. Available. http: //www . siumed.edu/surgery/urology/bph.html.

[24] P. Mansfield. Imaging by nuclear magnetic resonance. J. Phys. E: Sci. Instrument., 21:18-30, 1984. 
[25] P. Mansfield. Snapshot magnetic resonance imaging (nobel lecture). Angew. Chem. Int. Ed. Engl., 43(41):5456-5464, 2004.

[26] E. M. Haacke. Magnetic Resonance Imaging: Physical Principles and Sequence Design. J. Wiley \& Sons, New York, USA, 1999.

[27] H. Michaely, K. Herrmann, K. Nael, N. Oesingmann, M. Reiser, and S. Schoenberg. Functional renal imaging: Nonvascular renal disease. Abdom. Imaging, 32(1):1-16, 2007.

[28] D. J. Collins and A. R. Padhani. Dynamic magnetic resonance imaging of tumor perfusion. IEEE Eng. Med. Biol. Mag., 23(5):65-83, 2004.

[29] B. Sitharaman, K. R. Kissell, K. B. Hartman, L. A. Tran, A. Baikalov, et al. Superparamagnetic gadonanotubes are high-performance MRI contrast agents. Chem. Commun., 1(31):3915-13917, 2006.

[30] C. Sato, S. Naganawa, T. Nakamura, H. Kumada, S. Miura, O. Takizawa, and T. Ishigaki. Differentiation of noncancerous tissue and cancer lesions by apparent diffusion coefficient values in transition and peripheral zones of the prostate. Magn. Reson. Imaging, 21(10):258-262, 2005.

[31] R. Bammer. Basic principles of diffusion-weighted imaging. Eur. J. Radiol., 45(23):169-184, 2003.

[32] P. C. Sundgren, Q. Dong, D. Gómez-Hassan, S. K. Mukherji, P. Maly, and R. Welsh. Diffusion tensor imaging of the brain: Review of clinical applications. Neuroradiol., 46(5):339-250, 2004.

[33] V. J. Wedeen, R. P. Wang, J. D. Schmahmann, T. Benner, W. Y. Tseng, G. Dai, D. N. Pandya, P. Hagmann, H. D'Arceuil, and A. J. de Crespigny. Diffusion spectrum magnetic resonance imaging (DSI) tractography of crossing fibers. Neuroimage, 41(4):1267-1277, 2008.

[34] P. Hagmann, L. Jonasson, P. Maeder, J.-P. Thiran, V. J. Wedeen, and R. Meuli. Understanding diffusion MR imaging techniques: From scalar diffusion-weighted imaging to diffusion tensor imaging and beyond. Radiographics, 26(1):205-223, 2006.

[35] S. Ogawa, T. M. Lee, A. S. Nayak, and P. Glynn. Oxygenation-sensitive contrast in magnetic resonance image of rodent brain at high magnetic fields. Magn. Reson. Med., 14(1):68-78, 1990.

[36] X. Yang and K. Murase. Tagged cardiac MR image segmentation by contrast enhancement and texture analysis. In Proc. Int. Conf. Electron. Measurement Instruments, (ICEMI'09), pages 210-214, 2009.

[37] X. Liu and J. L. Prince. Shortest path refinement for motion estimation from tagged MR images. IEEE Trans. Med. Imaging, 29(8):1560-1572, 2010.

[38] L. Axel and L. Dougherty. Heart wall motion: Improved method of spatial modulation of magnetization for MR imaging. Radiol., 172(8):349-350, 1989. 
[39] E. L. Barbier, L. Lamalle, and M. Décorps. Methodology of brain perfusion imaging. J. Magn. Reson. Imaging, 13(4):496-520, 2001.

[40] E. S. Paulson and K. M. Schmainda. Comparison of dynamic susceptibilityweighted contrast-enhanced MR Methods: Recommendations for measuring relative cerebral blood volume in brain tumors. Radiol., 249(2):601-613, 2008.

[41] L. Mechtler. Neuroimaging in neuro-oncology. Neurol. Clin., 27(1):171-201, 2009.

[42] A. Elnakib, G. M. Beache, G. Gimel'farb, and A. El-Baz. A new framework for automated segmentation of left ventricle wall from contrast enhanced cardiac magnetic resonance images. In Proc. IEEE Int. Conf. Image Process., (ICIP'11), pages 2289-2292, 2011.

[43] A. Elnakib, G. M. Beache, M. Nitzken, G. Gimel'farb, and A. El-Baz. A new framework for automated identification of pathological tissues in contrast enhanced cardiac magnetic resonance images. In Proc. IEEE Int. Symp. Biomed. Imaging: From Nano to Macro, (ISBI'11), pages 1272-1275, 2011.

[44] A. Elnakib, G. M. Beache, G. Gimelfarb, and A. El-Baz. New automated MarkovGibbs random field based framework for myocardial wall viability quantification on agent enhanced cardiac magnetic resonance images. Int. J. Cardiovasc. Imaging, 28(7):1683-1698, 2012.

[45] C. Andersen and F. T. Jensen. Differences in blood-tumour-barrier leakage of human intracranial tumours: Quantitative monitoring of vasogenic oedema and its response to glucocorticoid treatment. Acta Neurochir., 140(9):919-924, 1998.

[46] X. P. Zhu, K. L. Li, I. D. Kamaly-Asl, D. R. Checkley, J. J. L. Tessier, J. C. Waterton, and A. Jackson. Quantification of endothelial permeability, leakage space, and blood volume in brain tumors using combined $\mathrm{T} 1$ and $\mathrm{T} 2 *$ contrast-enhanced dynamic MR imaging. J. Magn. Reson. Imaging, 11(6):575-585, 2000.

[47] M. Haris, R. K. Gupta, A. Singh, N. Husain, M. Husain, C. M. Pandey, C. Srivastava, S. Behari, and R. K. S. Rathore. Differentiation of infective from neoplastic brain lesions by dynamic contrast-enhanced MRI. Neuroradiol., 50(6):531-540, 2008.

[48] S. Bisdas, T. Naegele, R. Ritz, A. Dimostheni, C. Pfannenberg, M. Reimold, T. S. Koh, and U. Ernemann. Distinguishing recurrent high-grade gliomas from radiation injury: A pilot study using dynamic contrast-enhanced MR imaging. Acad. Radiol., 18(5):575-583, 2011.

[49] S. Mussurakis, D. L. Buckley, S. Bowsley, P. J. Carleton, J. N. Fox, L. W. Turnbull, and A. Horsman. Dynamic contrast-enhanced magnetic resonance imaging of the breast combined with pharmacokinetic analysis of gadolinium-DTPA uptake in the diagnosis of local recurrence of early stage breast carcinoma. Invest. Radiol., 30(11):650-662, 1995.

[50] S. Mussurakis, D. L. Buckley, P. J. Drew, J. N. Fox, P. J. Carleton, L. W. Turnbull, and A. Horsman. Dynamic MR imaging of the breast combined with analysis of contrast agent kinetics in the differentiation of primary breast tumours. Clin. Radiol., 52(7):516-526, 1997. 
[51] E. Furman-Haran, E. Schechtman, F. Kelcz, K. Kirshenbaum, and H. Degani. Magnetic resonance imaging reveals functional diversity of the vasculature in benign and malignant breast lesions. Cancer, 104(4):708-718, 2005.

[52] F. Sardanelli, F. Podo, G. D’Agnolo, A. Verdecchia, M. Santaquilani, et al. Multicenter comparative multimodality surveillance of women at genetic-familial high risk for breast cancer (hibcrit study): Interim results. Radiol., 242(3):698-715, 2007.

[53] D. Vincensini, V. Dedieu, P. A. Eliat, C. Vincent, C. Bailly, J. de Certaines, and F. Joffre. Magnetic resonance imaging measurements of vascular permeability and extracellular volume fraction of breast tumors by dynamic Gd-DTPA-enhanced relaxometry. Magn. Reson. Imaging, 25(3):293-302, 2007.

[54] M.-L. W. Ah-See, A. Makris, N. J. Taylor, M. Harrison, P. I. Richman, et al. Early changes in functional dynamic magnetic resonance imaging predict for pathologic response to neoadjuvant chemotherapy in primary breast cancer. Clin. Cancer Res., 14(20):6580-6589, 2008.

[55] M. C. Schabel, G. R. Morrell, K. Y. Oh, C. A. Walczak, R. B. Barlow, and L. A. Neumayer. Pharmacokinetic mapping for lesion classification in dynamic breast MRI. J. Magn. Reson. Imaging, 31(6):1371-1378, 2010.

[56] R. Johansen, L. R. Jensen, J. Rydland, P. E. Goa, K. A. Kvistad, T. F. Bathen, D. E. Axelson, S. Lundgren, and I. S. Gribbestad. Predicting survival and early clinical response to primary chemotherapy for patients with locally advanced breast cancer using DCE-MRI. J. Magn. Reson. Imaging, 29(6):1300-1307, 2009.

[57] Q. G. de Lussanet, W. H. Backes, A. W. Griffioen, A. R. Padhani, C. I. Baeten, A. van Baardwijk, P. Lambin, G. L. Beets, J. van Engelshoven, and R. G. H. BeetsTan. Dynamic contrast-enhanced magnetic resonance imaging of radiation therapyinduced microcirculation changes in rectal cancer. Int. J. Radiat. Oncol. Biol. Phys., 63(5):1309-1315, 2005.

[58] A. R. Padhani, C. Hayes, L. Assersohn, T. Powles, A. Makris, J. Suckling, M. O. Leach, and J. E. Husband. Prediction of clinicopathologic response of breast cancer to primary chemotherapy at contrast-enhanced MR imaging: Initial clinical results. Radiol., 239(2):361-374, 2006.

[59] M. F. Akisik, K. Sandrasegaran, G. Bu, C. Lin, G. D. Hutchins, and E. G. Chiorean. Pancreatic cancer: Utility of dynamic contrast-enhanced MR imaging in assessment of antiangiogenic therapy. Radiol., 256(2):441-449, 2010.

[60] T. Chikui, E. Kitamoto, S. Kawano, T. Sugiura, M. Obara, et al. Pharmacokinetic analysis based on dynamic contrast-enhanced MRI for evaluating tumor response to preoperative therapy for oral cancer. J. Magn. Reson. Imaging, 36(3):589-597, 2012.

[61] B. J. Nankivell and S. I. Alexander. Rejection of the kidney allograft. N. Eng. J. Med., 363(15):1451-1562, 2010. 
[62] USRDS. US Renal Data System Annual Data Report 2011: Atlas of Chronic Kidney Disease and End-Stage Renal Disease in the United States, National Institutes of Health, National Institute of Diabetes and Digestive and Kidney Diseases, Bethesda, MD, 2011.

[63] A. Kalogeropoulos, V. Georgiopoulou, S. B. Kritchevsky, B. M. Psaty, N. L. Smith, et al. Epidemiology of incident heart failure in a contemporary elderly cohort: The health, aging, and body composition study. Arch. Intern. Med., 169(7):708, 2009.

[64] A. R. Chugh, G. M. Beache, J. H. Loughran, N. Mewton, J. B. Elmore, et al. Administration of cardiac stem cells in patients with ischemic cardiomyopathy: The SCIPIO trial surgical aspects and interim analysis of myocardial function and viability by magnetic resonance. Circulation, 126(11 suppl 1):S54-S64, 2012.

[65] R. Bolli, A. R. Chugh, D. D’Amario, J. H. Loughran, M. F. Stoddard, et al. Cardiac stem cells in patients with ischaemic cardiomyopathy (SCIPIO): Initial results of a randomised phase 1 trial. Lancet, 378(9806):1847-1857, 2011.

[66] O. I. Soliman, S. W. Kirschbaum, B. M. van Dalen, H. B. van der Zwaan, B. M. Delavary, W. B. Vletter, R.-J. M. van Geuns, F. J. Ten Cate, and M. L. Geleijnse. Accuracy and reproducibility of quantitation of left ventricular function by real-time three-dimensional echocardiography versus cardiac magnetic resonance. Am. J. Cardiol., 102(6):778-783, 2008.

[67] F. Khalifa, G. M. Beache, G. Gimel'farb, G. A. Giridharan, and A. El-Baz. Accurate automatic analysis of cardiac cine images. IEEE Trans. Biomed. Eng., 59(2):445455, 2012.

[68] F. Khalifa, G. M. Beache, M. Nitzken, G. Gimel'farb, G. A. Giridharan, and A. ElBaz. Automatic analysis of left ventricle wall thickness using short-axis cine CMR images. In Proc. IEEE Int. Symp. Biomed. Imaging: From Nano to Macro, (ISBI'11), pages 1306-1309, 2011.

[69] A. Elnakib, G. M. Beache, H. Sliman, G. Gimel'farb, T. Inanc, and A. El-Baz. A novel laplace-based method to estimate the strain from cine cardiac magnetic resonance images. In Proc. IEEE Int. Conf. Image Process., (ICIP'13), pages 690-694, 2013.

[70] S. P. Sourborn and D. L. Buckley. Tracer kinetic modeling in MRI: Estimating perfusion and capillary permeability. Phys. Med. Biol., 57(2):R1-R33, 2012.

[71] American Cancer Society. Cancer Facts and Figures, 2013.

[72] R. Etzioni, R. Gulati, M. R. Cooperberg, D. M. Penson, N. S. Weiss, and I. M. Thompson. Limitations of basing screening policies on screening trials: The US preventive services task force and prostate cancer screening. Med. Care, 51(4):295300, 2013.

[73] U.-H. Stenman, J. Leinonen, W.-M. Zhang, and P. Finne. Prostate-specific antigen. In Seminars in Cancer Biol., volume 9, pages 83-93, 1999. 
[74] Y. J. Choi, J. K. Kim, N. Kim, K. W. Kim, E. K. Choi, and K.-S. Cho. Functional MR imaging of prostate cancer. Radiographics, 27(1):63-75, 2007.

[75] A. Firjani, F. Khalifa, A. Elnakib, G. Gimel'farb, M. Abo El-Ghar, A. Elmaghraby, and A. El-Baz. Non-invasive image-based approach for early detection of prostate cancer. In Proc. Int. Conf. Dev. eSys. Eng., (DeSE'11), pages 172-177, 2011.

[76] A. Firjani, A. Elnakib, F. Khalifa, G. Gimel'farb, M. Abo El-Ghar, J. Suri, A. Elmaghraby, and A. El-Baz. A new 3D automatic segmentation framework for accurate extraction of prostate from DCE-MRI. In Proc. IEEE Int. Symp. Biomed. Imaging: From Nano to Macro, (ISBI'11), pages 1476-1479, 2011.

[77] A. Firjani, F. Khalifa, A. Elnakib, G. Gimel'farb, M. Abo El-Ghar, A. Elmaghraby, and A. El-Baz. A novel image-based approach for early detection of prostate cancer using DCE-MRI. In K. Suzuki, editor, Computational Intelligence in Biomedical Imaging, chapter 3, pages 55-85. Springer Science and Business Media, 2014.

[78] A. Firjani, F. Khalifa, A. Elnakib, G. Gimel'farb, M. Abo El-Ghar, A. Elmaghraby, and A. El-Baz. 3D automatic approach for precise segmentation of the prostate from diffusion-weighted magnetic resonance imaging. In Proc. IEEE Int. Conf. Image Process., (ICIP'11), pages 2285-2288, 2011.

[79] A. Firjani, F. Khalifa, A. Elnakib, G. Gimel'farb, A. Elmaghraby, and A. El-Baz. A novel image-based approach for early detection of prostate cancer. In Proc. IEEE Int. Conf. Image Process., (ICIP'12), pages 2849-2852, 2012.

[80] A. Firjani, A. Elnakib, F. Khalifa, G. Gimel'farb, M. Abou El-Ghar, A. Elmaghraby, and A. El-Baz. A diffusion-weighted imaging based diagnostic system for early detection of prostate cancer. J. Biomed. Sci. Eng., 6(3A):346-356, 2013.

[81] E. Nagel, C. Klein, I. Paetsch, S. Hettwer, B. Schnackenburg, K. Wegscheider, and E. Fleck. Magnetic resonance perfusion measurements for the non-invasive detection of coronary artery disease. Circulation, 108(4):423-437, 2003.

[82] P. L. Choyke, J. A. Frank, M. E. Girton, S. W. Inscoe, M. J. Carvlin, J. L. Black, H. A. Austin, and A. J. Dwyer. Dynamic Gd-DTPA-enhanced MR imaging of the kidney: Experimental results. Radiol., 170(3):713-720, 1989.

[83] L. Bokacheva, H. Rusinek, J. L. Zhang, Q. Chen, and V. S. Lee. Estimates of glomerular filtration rate from MR renography and tracer kinetic models. J. Magn. Reson. Imaging, 29(2):371-382, 2009.

[84] D. L. Buckley. Uncertainty in the analysis of tracer kinetics using dynamic contrastenhanced T1-weighted MRI. Magn. Reson. Med., 47(3):601-606, 2002.

[85] G. J. M. Parker, J. Suckling, S. F. Tanner, A. R. Padhani, J. E. Husband, and M. O. Leach. MRIW: Parametric analysis software for contrast-enhanced dynamic MR imaging in cancer. Radiographics, 18(2):497-506, 1998.

[86] P. Gibbs, G. P. Liney, M. Lowry, P. J. Kneeshaw, and L. W. Turnbull. Differentiation of benign and malignant sub-1 cm breast lesions using dynamic contrast enhanced MRI. Breast, 13(2):115-121, 2004. 
[87] G. M. Beache, S. Kulke, H. Kantor, P. N. T. Campbell, D. Chesler, H. Gerwirtz, B. Rosen, T. Brady, and T. Weisskoff. Imaging perfusion deficits in ischemic heart disease with susceptibility-enhanced T2-weighted MRI: Preliminary human studies. Magn. Reson. Imaging, 16(1):19-27, 1998.

[88] M. J. Paldino and D. P. Barboriak. Fundamentals of quantitative dynamic contrastenhanced MR imaging. Magn. Reson. Imaging Clin. N. Am., 17(2):277-289, 2009.

[89] A. Semiz Oysu, E. Ayanoglu, N. Kodalli, C. Oysu, C. Uneri, and C. Erzen. Dynamic contrast-enhanced MRI in the differentiation of posttreatment fibrosis from recurrent carcinoma of the head and neck. Clin. Imaging, 29(5):307-312, 2005.

[90] J. Schwitter, D. Nanz, S. Kneifel, K. Bertschinger, M. Büchi, P. R. Knüsel, B. Marincek, T. F. Lüscher, and G. K. Von Schulthess. Assessment of myocardial perfusion in coronary artery disease by magnetic resonance: A comparison with positron emission tomography and coronary angiography. Circulation, 103(18):2230-2235, 2001.

[91] T. Ibrahim, S. G. Nekolla, K. Schreiber, K. Odaka, S. Volz, J. Mehilli, M. Güthlin, W. Delius, and M. Schwaiger. Assessment of coronary flow reserve: Comparison between contrast-enhanced magnetic resonance imaging and positron emission tomography. J. Am. College Cardiol., 39(5):864-870, 2002.

[92] V. Positano, M. F. Santarelli, and L. Landini. Automatic characterization of myocardial perfusion in contrast-enhanced MRI. EURASIP J. Appl. Signal Process., 5(1):413-421, 2003.

[93] H. Ólafsdóttir, M. B. Stegmann, and H. B. W. Larsson. Automatic assessment of cardiac perfusion MRI. In Proc. Med. Image Comput. Comput.-Assist. Interv., (MICCAI'04), pages 1060-1061, 2004.

[94] H. Xue, S. Zuehlsdorff, P. Kellman, A. Arai, S. Nielles-Vallespin, C. Chefdhotel, C. H. Lorenz, and J. Guehring. Unsupervised inline analysis of cardiac perfusion MRI. In Proc. Med. Image Comput. Comput.-Assist. Interv., (MICCAI'09), pages 741-749, 2009.

[95] M.-Y. M. Su, K.-C. Yang, C.-C. Wu, Y.-W. Wu, H.-Y. Yu, R.-Y. Tseng, and W.Y. I. Tseng. First-pass myocardial perfusion cardiovascular magnetic resonance at 3 Tesla. J. Cardiovasc. Magn. Reson., 9(4):633-644, 2007.

[96] F. Khalifa, G. M. Beache, A. Elnakib, H. Sliman, G. Gimel'farb, K. C. Welch, and A. El-Baz. A new nonrigid registration framework for improved visualization of transmural perfusion gradients on cardiac first-pass perfusion MRI. In Proc. IEEE Int. Symp. Biomed. Imaging: From Nano to Macro, (ISBI'12), pages 828-831, 2012.

[97] F. Khalifa, G. M. Beache, G. Gimel'farb, and A. El-Baz. A novel CAD system for analyzing cardiac first-pass MRI images. In Proc. IEEE Int. Conf. Pattern Recogni., (ICPR'12), pages 77-80, 2012.

[98] G. Tarroni, C. Corsi, P. F. Antkowiak, F. Veronesi, C. M. Kramer, et al. Myocardial perfusion: Near-automated evaluation from contrast-enhanced MR images obtained at rest and during vasodilator stress. Radiol., 265(2):576-583, 2012. 
[99] J. M. Hawnaur, X. P. Zhu, and C. E. Hutchinson. Quantitative dynamic contrast enhanced MRI of recurrent pelvic masses in patients treated for cancer. Br. J. Radiol., 71(851):1136-1142, 1998.

[100] X. M. Zhang, D. Yu, H. L. Zhang, Y. Dai, D. Bi, Z. Liu, M. R. Prince, and C. Li. 3D dynamic contrast-enhanced MRI of rectal carcinoma at 3T: Correlation with microvascular density and vascular endothelial growth factor markers of tumor angiogenesis. J. Magn. Reson. Imaging, 27(6):1309-1316, 2008.

[101] K. Bol, J. C. Haeck, H. C. Groen, W. J. Niessen, M. R. Bernsen, M. de Jong, and J. F. Veenland. Can DCE-MRI explain the heterogeneity in radiopeptide uptake imaged by SPECT in a pancreatic neuroendocrine tumor model? PloS one, 8(10):e77076, 2013

[102] J. Scharf, A. Kemmling, T. Hess, A. Mehrabi, G. Kauffmann, C. Groden, and G. Brix. Assessment of hepatic perfusion in transplanted livers by pharmacokinetic analysis of dynamic magnetic resonance measurements. Invest. Radiol., 42(4):224229, 2007.

[103] Y. Ohno, M. Nogami, T. Higashino, D. Takenaka, S. Matsumoto, H. Hatabu, and K. Sugimura. Prognostic value of dynamic MR imaging for non-small-cell lung cancer patients after chemoradiotherapy. J. Magn. Reson. Imaging, 21(6):775-783, 2005.

[104] M. Medved, G. Karczmar, C. Yang, J. Dignam, T. F. Gajewski, H. Kindler, E. Vokes, P. MacEneany, M. T. Mitchell, and W. M. Stadler. Semiquantitative analysis of dynamic contrast enhanced MRI in cancer patients: Variability and changes in tumor tissue over time. J. Magn. Reson. Imaging, 20(1):122-128, 2004.

[105] S. Glaßer, U. Preim, K. Tönnies, and B. Preim. A visual analytics approach to diagnosis of breast DCE-MRI data. Comput. Graph., 34(5):602-611, 2010.

[106] A. Karahaliou, K. Vassiou, N. S. Arikidis, S. Skiadopoulos, T. Kanavou, and L. Costaridou. Assessing heterogeneity of lesion enhancement kinetics in dynamic contrast-enhanced MRI for breast cancer diagnosis. Br. J. Radiol., 83(988):296-309, 2010.

[107] R. G. Abramson, X. Li, T. L. Hoyt, P.-F. Su, L. R. Arlinghaus, K. J. Wilson, V. G. Abramson, A. B. Chakravarthy, and T. E. Yankeelov. Early assessment of breast cancer response to neoadjuvant chemotherapy by semi-quantitative analysis of high-temporal resolution DCE-MRI: Preliminary results. Magn. Reson. Imaging, 31(9):1457-1464, 2013.

[108] R. Fusco, S. Filice, V. Granata, Y. Mandato, A. Porto, et al. Can semi-quantitative evaluation of uncertain (type II) time-intensity curves improve diagnosis in breast DCE-MRI? J. Biomed. Sci. Eng., 6(3A):418-425, 2013.

[109] L. Martincich, F. Montemurro, G. De Rosa, V. Marra, R. Ponzone, et al. Monitoring response to primary chemotherapy in breast cancer using dynamic contrast-enhanced magnetic resonance imaging. Breast Cancer Res. Treat., 83(1):67-76, 2004. 
[110] C. El Khoury, V. Servois, F. Thibault, A. Tardivon, L. Ollivier, M. Meunier, C. Allonier, and S. Neuenschwander. MR quantification of the washout changes in breast tumors under preoperative chemotherapy: Feasibility and preliminary results. Am. J. Roentgenol., 184(5):1499-1504, 2005.

[111] N. Tuncbilek, F. Tokatli, S. Altaner, A. Sezer, M. Türe, I. K. Omurlu, and O. Temizoz. Prognostic value DCE-MRI parameters in predicting factor disease free survival and overall survival for breast cancer patients. Eur. J. Radiol., 81(5):863-867, 2012.

[112] P. C. Stomper, J. S. Winston, S. Herman, D. L. Klippenstein, M. A. Arredondo, and L. E. Blumenson. Angiogenesis and dynamic MR imaging gadolinium enhancement of malignant and benign breast lesions. Breast Cancer Res. Treat., 45(1):39-46, 1997.

[113] L. Esserman, N. Hylton, T. George, and N. Weidner. Contrast-enhanced magnetic resonance imaging to assess tumor histopathology and angiogenesis in breast carcinoma. Breast J., 5(1):13-21, 1999.

[114] C. K. Kuhl, P. Mielcareck, S. Klaschik, C. Leutner, E. Wardelmann, J. Gieseke, and H. H. Schild. Dynamic breast MR imaging: Are signal intensity time course data useful for differential diagnosis of enhancing lesions? Radiol., 211(1):101-110, 1999.

[115] R. Matsubayashi, Y. Matsuo, G. Edakuni, T. Satoh, O. Tokunaga, and S. Kudo. Breast masses with peripheral rim enhancement on dynamic contrast-enhanced MR images: Correlation of MR findings with histologic features and expression of growth factors. Radiol., 217(3):841-848, 2000.

[116] C. E. Loo, H. J. Teertstra, S. Rodenhuis, M. J. van de Vijver, J. Hannemann, S. H. Muller, M.-J. V. Peeters, and K. G. A. Gilhuijs. Dynamic contrast-enhanced MRI for prediction of breast cancer response to neoadjuvant chemotherapy: Initial results. Am. J. Roentgenol., 191(5):1331-1338, 2008.

[117] M. Rakoczy, D. McGaughey, M. J. Korenberg, J. Levman, and A. L. Martel. Feature selection in computer-aided breast cancer diagnosis via dynamic contrast-enhanced magnetic resonance images. J. Digital Imaging, 26(1):198-208, 2013.

[118] V. B. Ho, S. F. Allen, M. N. Hood, and P. L. Choyke. Renal masses: Quantitative assessment of enhancement with dynamic MR imaging. Radiol., 224(3):695-700, 2002.

[119] H. J. Michaely, S. O. Schoenberg, N. Oesingmann, C. Ittrich, C. Buhlig, et al. Renal artery stenosis: Functional assessment with dynamic MR perfusion measurementsfeasibility study. Radiol., 238(2):586-596, 2006.

[120] V. Positano, I. Bernardeschi, V. Zampa, M. Marinelli, L. Landini, and M. F. Santarelli. Automatic 2D registration of renal perfusion image sequences by mutual information and adaptive prediction. Magn. Reson. Mat. Phys., Biol. Med., 26(3):325-335, 2013. 
[121] J. A. de Priester, J. A. den Boer, M. H. Christiaans, A. G. Kessels, E. L. Giele, A. Hasman, H. P. van Hooff, and J. van Engelshoven. Automated quantitative evaluation of diseased and nondiseased renal transplants with MR renography. J. Magn. Reson. Imaging, 17(1):95-103, 2003.

[122] A. Farag, A. El-Baz, S. Yuksel, M. Abou El-Ghar, and T. Eldiasty. A framework for the detection of acute rejection with dynamic contrast enhanced magnetic resonance imaging. In Proc. IEEE Int. Symp. Biomed. Imaging: From Nano to Macro, (ISBI'06), pages 418-421, 2006.

[123] A. El-Baz, A. Farag, R. Fahmi, S. Yuksel, W. Miller, M. Abou El-Ghar, T. El-Diasty, and M. Ghoneim. A new CAD system for the evaluation of kidney diseases using DCE-MRI. In Proc. Med. Image Comput. Comput.-Assist. Interv., (MICCAI'08), pages 446-453, 2006.

[124] A. El-Baz, A. Farag, R. Fahmi, S. Yuksel, M. Abo El-Ghar, and T. Eldiasty. Image analysis of renal DCE MRI for the detection of acute renal rejection. In Proc. IEEE Int. Conf. Pattern Recogni., (ICPR'06), pages 822-825, 2006.

[125] A. El-Baz, G. Gimel'farb, and M. Abou El-Ghar. New motion correction models for automatic identification of renal transplant rejection. In Proc. Med. Image Comput. Comput.-Assist. Interv., (MICCAI’07), pages 235-243, 2007.

[126] A. El-Baz, G. Gimel'farb, and M. Abo El-Ghar. A novel image analysis approach for accurate identification of acute renal rejection. In Proc. IEEE Int. Conf. Image Process., (ICIP'08), pages 1812-1815, 2008.

[127] A. El-Baz, G. Gimel'farb, and M. Abo El-Ghar. Image analysis approach for identification of renal transplant rejection. In Proc. IEEE Int. Conf. Pattern Recogni., (ICPR'08), pages 1-4, 2008.

[128] F. Khalifa, G. M. Beache, M. Abou El-Ghar, T. El-Diasty, G. Gimel'farb, M. Kong, and A. El-Baz. Dynamic contrast-enhanced MRI-based early detection of acute renal transplant rejection. IEEE Trans. Med. Imaging, 32(10):1910-1927, 2013.

[129] F. Khalifa, M. Abou El-Ghar, B. Abdollahi, H. B. Frieboes, T. El-Diasty, and A. ElBaz. A comprehensive non-invasive framework for automated evaluation of acute renal transplant rejection using DCE-MRI. NMR Biomed., 26(11):1460-1470, 2013.

[130] M. R. Engelbrecht, H. J. Huisman, R. J. Laheij, G. J. Jager, G. J. van Leenders, C. A. Hulsbergen-Van De Kaa, J. J. de la Rosette, J. G. Blickman, and J. O. Barentsz. Discrimination of prostate cancer from normal peripheral zone and central gland tissue by using dynamic contrast-enhanced MR imaging. Radiol., 229(1):248-254, 2003.

[131] S. M. Noworolski, R. G. Henry, D. B. Vigneron, and J. Kurhanewicz. Dynamic contrast-enhanced MRI in normal and abnormal prostate tissues as defined by biopsy, MRI, and 3D MRSI. Magn. Reson. Med., 53(2):249-255, 2005.

[132] J. Ren, Y. Huan, H. Wang, Y.-J. Chang, H.-T. Zhao, Y.-L. Ge, Y. Liu, and Y. Yang. Dynamic contrast-enhanced MRI of benign prostatic hyperplasia and prostatic carcinoma: Correlation with angiogenesis. Clin. Radiol., 63(2):153-159, 2008. 
[133] P. Puech, N. Betrouni, R. Viard, A. Villers, X. Leroy, and L. Lemaitre. Prostate cancer computer-assisted diagnosis software using dynamic contrast-enhanced MRI. In Proc. Annu. IEEE Conf. Eng. Med. Biol. Soc., (EMBS'07), pages 5567-5570, 2007.

[134] P. Puech, N. Betrouni, N. Makni, A.-S. Dewalle, A. Villers, and L. Lemaitre. Computer-assisted diagnosis of prostate cancer using DCE-MRI data: Design, implementation and preliminary results. Int. J. Comput. Assist. Radiol. Surg., 4(1):1$10,2009$.

[135] M. A. Haider, P. Chung, J. Sweet, A. Toi, K. Jhaveri, C. Ménard, P. Warde, J. Trachtenberg, G. Lockwood, and M. Milosevic. Dynamic contrast-enhanced magnetic resonance imaging for localization of recurrent prostate cancer after external beam radiotherapy. Int. J. Radiat. Oncol. Biol. Phys., 70(2):425-430, 2008.

[136] E. Casciani, E. Polettini, E. Carmenini, I. Floriani, G. Masselli, L. Bertini, and G. F. Gualdi. Endorectal and dynamic contrast-enhanced MRI for detection of local recurrence after radical prostatectomy. Am. J. Roentgenol., 190(5):1187-1192, 2008.

[137] M. Valerio, V. Panebianco, A. Sciarra, M. Osimani, S. Salsiccia, et al. Classification of prostatic diseases by means of multivariate analysis on in vivo proton MRSI and DCE-MRI data. NMR Biomed., 22(10):1036-1046, 2009.

[138] S. Isebaert, F. De Keyzer, K. Haustermans, E. Lerut, T. Roskams, I. Roebben, H. Van Poppel, S. Joniau, and R. Oyen. Evaluation of semi-quantitative dynamic contrast-enhanced MRI parameters for prostate cancer in correlation to whole-mount histopathology. Eur. J. Radiol., 81(3):217-222, 2012.

[139] E. Niaf, O. Rouvière, F. Mège-Lechevallier, F. Bratan, and C. Lartizien. Computeraided diagnosis of prostate cancer in the peripheral zone using multiparametric MRI. Phys. Med. Biol., 57(12):3833-3851, 2012.

[140] J. J. Fütterer, M. R. Engelbrecht, H. J. Huisman, G. J. Jager, C. A. Hulsbergen-van De Kaa, J. A. Witjes, and J. O. Barentsz. Staging prostate cancer with dynamic contrast-enhanced endorectal MR imaging prior to radical prostatectomy: Experienced versus less experienced readers. Radiol., 237(2):541-549, 2005.

[141] T. Lindeberg. Edge detection and ridge detection with automatic scale selection. Int. J. Comput. Vis., 30(2):117-156, 1998.

[142] M. Kaufmann, G. Von Minckwitz, H. Bear, A. Buzdar, P. McGale, et al. Recommendations from an international expert panel on the use of neoadjuvant (primary) systemic treatment of operable breast cancer: New perspectives 2006. Anna. Oncol., 18(12):1927-1934, 2007.

[143] H. K. Thompson, C. F. Starmer, R. E. Whalen, and H. D. McIntosh. Indicator transit time considered as a gamma variate. Circ. Res., 14(6):502-515, 1964.

[144] C. Cobelli, G. Toffolo, and D. M. Foster. A simplified formulation of the gamma variate function. Phys. Med. Biol., 37(7):1597-1600, 1992. 
[145] H. B. W. Larsson, M. Stubgaard, J. L. Frederiksen, M. Jensen, O. Henriksen, and O. B. Paulson. Quantitation of blood-brain barrier defect by magnetic resonance imaging and gadolinium-DTPA in patients with multiple sclerosis and brain tumors. Magn. Reson. Med., 16(1):117-131, 1990.

[146] G. Brix, W. Semmler, R. Port, L. Schad, G. Layer, and W. Lorenz. Pharmacokinetic parameters in CNS Gd-DTPA enhanced MR imaging. J. Comput. Assist. Tomogr., 15(4):621-628, 1991.

[147] T. S. Koh, S. Bisdas, D. M. Koh, and C. H. Thng. Fundamentals of tracer kinetics for dynamic contrast-enhanced MRI. J. Magn. Reson. Imaging, 34(6):1262-1276, 2011.

[148] S. S. Kety. The theory and applications of the exchange of inert gas at the lungs and tissues. Pharmacol. Rev., 3(1):1-41, 1951.

[149] C. Cobelli, G. Toffolo, and D. M. Foster. Tracer-to-tracee ratio for analysis of stable isotope tracer data: Link with radioactive kinetic formalism. Am. J. Phys., 262(6):968-975, 1992.

[150] J. A. Johnson and T. A. Wilson. A model for capillary exchange. Am. J. Physiol., 210(6):1299-1303, 1966.

[151] K. S. St Lawrence and T.-Y. Lee. An adiabatic approximation to the tissue homogeneity model for water exchange in the brain: I. Theoretical derivation. J. Cereb. Blood Flow Metab., 18(12):1365-1377, 1998.

[152] L. E. Kershaw and H.-L. M. Cheng. Temporal resolution and SNR requirements for accurate DCE-MRI data analysis using the AATH model. Magn. Reson. Med., 64(6):1772-1780, 2010.

[153] T. E. Yankeelov and J. C. Gore. Dynamic contrast enhanced magnetic resonance imaging in oncology: Theory, data acquisition, analysis, and examples. Curr. Med. Imaging Rev., 3(2):91, 2009.

[154] H. J. Weinmann, M. Laniado, and W. Mutzel. Pharmacokinetics of GdDTPA/ dimeglumine after intravenous injection into healthy volunteers. Physiol. Chem. Phys. Med. NMR, 16(2):167-172, 1984.

[155] D. De Naeyer. Algorithm development and protocol optimization for pharmacokinetic modeling of dynamic contrast-enhanced magnetic resonance imaging. $\mathrm{PhD}$ thesis, Ghent University, Ghent, Belgium, 2011.

[156] T. Fritz-Hansen, E. Rostrup, H. B. W. Larsson, L. Søndergaard, P. Ring, and O. Henriksen. Measurement of the arterial concentration of Gd-DTPA using MRI: A step toward quantitative perfusion imaging. Magn. Reson. Med., 36(2):225-231, 1996.

[157] M. J. P. van Osch, E.-J. Vonken, M. A. Viergever, J. van der Grond, and C. J. G. Bakker. Measuring the arterial input function with gradient echo sequences. Magn. Reson. Med., 49(6):1067-1076, 2003. 
[158] G. O. Cron, C. Foottit, T. E. Yankeelov, L. I. Avruch, M. E. Schweitzer, and I. Cameron. Arterial input functions determined from MR signal magnitude and phase for quantitative dynamic contrast-enhanced MRI in the human pelvis. Magn. Reson. Med., 66(2):498-504, 2011.

[159] D. A. Kovar, M. Lewis, and G. S. Karczmar. A new method for imaging perfusion and contrast extraction fraction: Input functions derived from reference tissues. $J$. Magn. Reson. Imaging, 8(5):1126-1134, 1998.

[160] T. E. Yankeelov, G. O. Cron, C. L. Addison, J. C. Wallace, R. C. Wilkins, B. A. Pappas, G. E. Santyr, and J. C. Gore. Comparison of a reference region model with direct measurement of an AIF in the analysis of DCE-MRI data. Magn. Reson. Med., 57(2):353-361, 2007.

[161] S. Walker-Samuel, C. C. Parker, M. O. Leach, and D. J. Collins. Reproducibility of reference tissue quantification of dynamic contrast-enhanced data: Comparison with a fixed vascular input function. Phys. Med. Biol., 52(1):75, 2007.

[162] M. Heisen, X. Fan, J. Buurman, N. A. W. van Riel, G. S. Karczmar, and B. M. ter Haar Romeny. The use of a reference tissue arterial input function with lowtemporal-resolution DCE-MRI data. Phys. Med. Biol., 55(16):4871, 2010.

[163] A. R. Padhani, C. Hayes, S. Landau, and M. O. Leach. Reproducibility of quantitative dynamic MRI of normal human tissues. NMR Biomed., 15(2):143-153, 2002.

[164] T. E. Yankeelov, J. J. Luci, M. Lepage, R. Li, L. Debusk, P. C. Lin, R. R. Price, and J. C. Gore. Quantitative pharmacokinetic analysis of DCE-MRI data without an arterial input function: A reference region model. Magn. Reson. Med., 23(4):519$529,2005$.

[165] C. Yang, G. S. Karczmar, M. Medved, and W. M. Stadler. Estimating the arterial input function using two reference tissues in dynamic contrast-enhanced MRI studies: Fundamental concepts and simulations. Magn. Reson. Med., 52(5):1110-1117, 2004.

[166] C. Yang, G. S. Karczmar, M. Medved, A. Oto, M. Zamora, and W. M. Stadler. Reproducibility assessment of a multiple reference tissue method for quantitative DCE-MRI analysis. Magn. Reson. Med., 61(4):851-859, 2009.

[167] C. Yang, W. M. Stadler, G. S. Karczmar, M. Milosevic, I. Yeung, and M. A. Haider. Comparison of quantitative parameters in cervix cancer measured by dynamic contrast-enhanced MRI and CT. Magn. Reson. Med., 63(6):1601-1609, 2010 .

[168] J. U. Fluckiger, M. C. Schabel, and E. V. R. DiBella. Model-based blind estimation of kinetic parameters in dynamic contrast enhanced (DCE)-MRI. Magn. Reson. Med., 62(6):1477-1486, 2009.

[169] M. C. Schabel, J. U. Fluckiger, and E. V. R. DiBella. A model-constrained Monte Carlo method for blind arterial input function estimation in dynamic contrastenhanced MRI: I. Simulations. Phys. Med. Biol., 55(16):4783-4806, 2010. 
[170] U. Hoffmann, G. Brix, M. V. Knopp, T. He $\beta$, and W. J. Lorenz. Pharmacokinetic mapping of the breast: A new method for dynamic MR mammography. Magn. Reson. Med., 33(4):506-514, 1995.

[171] P. S. Tofts. Modeling tracer kinetics in dynamic Gd-DTPA MR imaging. J. Magn. Reson. Imaging, 7(1):91-101, 1997.

[172] D. Gadian, J. Payne, D. Bryant, I. Young, D. Carr, and G. Bydder. GadoliniumDTPA as a contrast agent in mr imaging-theoretical projections and practical observations. Journal of computer assisted tomography, 9(2):242-251, 1985.

[173] F. Bloch. Nuclear induction. Phys. Rev., 70(7-8):460-474, 1946.

[174] D. W. McRobbie, E. A. Moore, M. J. Graves, and M. R. Prince. MRI from Picture to Proton. Cambridge University Press, Cambridge, UK, 2006.

[175] G. J. Stanisz and R. M. Henkelman. Gd-DTPA relaxivity depends on macromolecular content. Magn. Reson. Med., 44(5):665-667, 2000.

[176] H. Z. Wang, S. J. Riederer, and J. N. Lee. Optimizing the precision in T1 relaxation estimation using limited flip angles. Magn. Reson. Med., 5(5):399-416, 1987.

[177] B. Whitcher and V. J. Schmid. Quantitative analysis of dynamic contrast-enhanced and diffusion-weighted magnetic resonance imaging for oncology in R. J. Stat. Software, 44(5):1-29, 2011.

[178] H.-L. M. Cheng and G. A. Wright. Rapid high-resolution T1 mapping by variable flip angles: Accurate and precise measurements in the presence of radiofrequency field inhomogeneity. Magn. Reson. Med., 55(3):566-574, 2006.

[179] M. S. C. T. J. F. O. H. H. B. W. Larsson, P. Christiansen. In vivo calculation of the unidirectional inflow constant across the blood-brain barrier using MRI. In Proc. Annu. Meeting Soc. Magn. Reson. Med., page 752, 1990.

[180] B. M. Kelm, B. H. Menze, O. Nix, C. M. Zechmann, and F. A. Hamprecht. Estimating kinetic parameter maps from dynamic contrast-enhanced MRI using spatial prior knowledge. IEEE Trans. Med. Imaging, 28(10):1534-1547, 2009.

[181] G. Brix, J. Griebel, F. Kiessling, and F. Wenz. Tracer kinetic modelling of tumour angiogenesis based on dynamic contrast-enhanced CT and MRI measurements. Eur. J. Nucl. Med. Mol. Imaging, 37(1):30-51, 2010.

[182] H. T. Ma, J. F. Griffith, D. K. Yeung, and P. C. Leung. Modified Brix model analysis of bone perfusion in subjects of varying bone mineral density. J. Magn. Reson. Imaging, 31(5):1169-1175, 2010.

[183] P. S. Tofts, G. Brix, D. L. Buckley, J. L. Evelhoch, E. Henderson, M. V. Knopp, H. B. W. Larsson, T. Lee, N. A. Mayr, and G. J. M. Parker. Estimating kinetics parameters from dynamic contrast-enhanced T1-weighted MRI of a diffusible tracer: Standardized quantities and symbols. J. Magn. Reson. Imaging, 10(3):223-232, 1999. 
[184] C. S. Patlak, R. G. Blasberg, and J. D. Fenstermacher. Graphical evaluation of bloodto-brain transfer constants from multiple-time uptake data. J. Cereb. Blood Flow Metab., 3(1):1-7, 1983.

[185] N. Hackstein, J. Heckrodt, and W. S. Rau. Measurement of single-kidney glomerular filtration rate using a contrast-enhanced dynamic gradient-echo sequence and the Rutland-Patlak plot technique. J. Magn. Reson. Imaging, 18(6):714-725, 2003.

[186] N. Hackstein, H. Kooijman, S. Tomaselli, and W. S. Rau. Glomerular filtration rate measured using the Patlak plot technique and contrast-enhanced dynamic MRI with different amounts of gadolinium-DTPA. J. Magn. Reson. Imaging, 22(3):406-414, 2005 .

[187] D. L. Buckley, A. E. Shurrab, C. M. Cheung, A. P. Jones, H. Mamtora, and P. A. Kalra. Measurement of single kidney function using dynamic contrast-enhanced MRI: Comparison of two models in human subjects. J. Magn. Reson. Imaging, 24(5):1117-1123, 2006.

[188] S. P. Sourbron, H. J. Michaely, M. F. Reiser, and S. O. Schoenberg. MRI- measurement of perfusion and glomerular filtration in the human kidney with a separable compartment model. Invest. Radiol., 43(1):40-48, 2008.

[189] H. Bagher-Ebadian, R. Jain, S. P. Nejad-Davarani, T. Mikkelsen, M. Lu, et al. Model selection for DCE-T1 studies in glioblastoma. Magn. Reson. Med., 68(1):241-251, 2012 .

[190] H. Chen, F. Li, X. Zhao, C. Yuan, B. Rutt, and W. S. Kerwin. Extended graphical model for analysis of dynamic contrast-enhanced MRI. Magn. Reson. Med., 66(3):868-878, 2011.

[191] M. E. Gaens, W. H. Backes, S. Rozel, M. Lipperts, S. N. Sanders, et al. Dynamic contrast-enhanced MR imaging of carotid atherosclerotic plaque: Model selection, reproducibility, and validation. Radiol., 266(1):271-279, 2013.

[192] G. Brix, F. Kiessling, R. Lucht, S. Darai, K. Wasser, S. Delorme, and J. Griebel. Microcirculation and microvasculature in breast tumors: Pharmacokinetic analysis of dynamic MR image series. Magn. Reson. Med., 52(2):420-429, 2004.

[193] S. Sourbron, M. Ingrisch, A. Siefert, M. Reiser, and K. Herrmann. Quantification of cerebral blood flow, cerebral blood volume, and blood-brain-barrier leakage with DCE-MRI. Magn. Reson. Med., 62(1):205-217, 2009.

[194] S. B. Donaldson, C. M. L. West, S. E. Davidson, B. M. Carrington, G. Hutchison, A. P. Jones, S. P. Sourbron, and D. L. Buckley. A comparison of tracer kinetic models for T1-weighted dynamic contrast-enhanced MRI: Application in carcinoma of the cervix. Magn. Reson. Med., 63(3):691-700, 2010.

[195] H. B. W. Larsson, F. Courivaud, E. Rostrup, and A. E. Hansen. Measurement of brain perfusion, blood volume, and blood-brain barrier permeability, using dynamic contrast-enhanced T1-weighted MRI at 3 tesla. Magn. Reson. Med., 62(5):1270$1281,2009$. 
[196] W. Huang, L. A. Tudorica, X. Li, S. B. Thakur, Y. Chen, et al. Discrimination of benign and malignant breast lesions by using shutter-speed dynamic contrastenhanced MR imaging. Radiol., 261(2):394-403, 2011.

[197] X. Li, E. B. Welch, A. B. Chakravarthy, L. Xu, L. R. Arlinghaus, et al. Statistical comparison of dynamic contrast-enhanced MRI pharmacokinetic models in human breast cancer. Magn. Reson. Med., 68(1):261-271, 2012.

[198] C. S. Landis, X. Li, F. W. Telang, J. A. Coderre, P. L. Micca, W. D. Rooney, L. L. Latour, G. Vétek, I. Pályka, and C. S. Springer. Determination of the MRI contrast agent concentration time course in vivo following bolus injection: Effect of equilibrium transcytolemmal water exchange. Magn. Reson. Med., 44(4):563-574, 2000.

[199] S. Kim, H. Quon, L. A. Loevner, M. A. Rosen, L. Dougherty, A. M. Kilger, J. D. Glickson, and H. Poptani. Transcytolemmal water exchange in pharmacokinetic analysis of dynamic contrast-enhanced MRI data in squamous cell carcinoma of the head and neck. J. Magn. Reson. Imaging, 26(6):1607-1617, 2007.

[200] X. Li, W. Huang, E. A. Morris, L. A. Tudorica, V. E. Seshan, W. D. Rooney, I. Tagge, Y. Wang, J. Xu, and C. S. Springer. Dynamic NMR effects in breast cancer dynamiccontrast-enhanced MRI. Proc. Natl. Acad. Sci., 105(46):17937-17942, 2008.

[201] K. B. Larson, J. Markham, and M. E. Raichle. Tracer-kinetic models for measuring cerebral blood flow using externally detected radiotracers. J. Cereb. Blood Flow Metab., 7(4):443-463, 1987.

[202] Y. Sawada, C. S. Patlak, and R. G. Blasberg. Kinetic analysis of cerebrovascular transport based on indicator diffusion technique. Am. J. Physiol., 256(3):H794H812, 1989.

[203] W. C. Sangren and C. W. Sheppard. A mathematical derivation of the exchange of a labeled substance between a liquid flowing in a vessel and an external compartment. Bull. Math. Biophys., 15(4):387-394, 1953.

[204] W. H. Press, B. P. Flannery, S. A. Teukolsky, , and W. T. Vetterling. Numerical Recipes in FORTRAN: The Art of Scientific Computing. Cambridge University Press, Cambridge, England, 1992.

[205] G. R. Moran and F. S. Prato. Modeling tissue contrast agent concentration: A solution to the tissue homogeneity model using a simulated arterial input function. Magn. Reson. Med., 45(1):42-45, 2001.

[206] A. Garpebring, N. Ostlund, and M. Karlsson. A novel estimation method for physiological parameters in dynamic contrast-enhanced MRI: Application of a distributed parameter model using Fourier-domain calculations. IEEE Trans. Med. Imaging, 28(9):1375-1383, 2009.

[207] L. E. Kershaw and D. L. Buckley. Precision in measurements of perfusion and microvascular permeability with T1-weighted dynamic contrast-enhanced MRI. Magn. Reson. Med., 56(5):986-992, 2006. 
[208] T. Fritz-Hansen, E. Rostrup, L. Sørndergaard, P. B. Ring, O. Amtorp, and H. B. W. Larsson. Capillary transfer constant of Gd-DTPA in the myocardium at rest and during vasodilation assessed by MRI. Magn. Reson. Med., 40(6):922-929, 1998.

[209] J.-P. M. Vallée, H. D. Sostman, J. R. Macfall, T. R. Degrado, J. Zhang, et al. Quantification of myocardial perfusion by MRI after coronary occlusion. Magn. Reson. Med., 40(2):287-297, 1998.

[210] J. H. S. Cullen, M. A. Horsfield, C. R. Reek, G. R. Cherryman, D. B. Barnett, and N. J. Samani. A myocardial perfusion reserve index in humans using first-pass contrast-enhanced magnetic resonance imaging. J. Am. Coll. Cardiol., 33(5):13861394, 1999.

[211] G. Nielsen, T. Fritz-Hansen, C. G. Dirks, G. B. Jensen, and H. B. W. Larsson. Evaluation of heart perfusion in patients with acute myocardial infarction using dynamic contrast-enhanced magnetic resonance imaging. J. Magn. Reson. Imaging, 20(3):403-410, 2004.

[212] J. P. Pärkkä, P. Niemi, A. Saraste, J. W. Koskenvuo, M. Komu, et al. Comparison of MRI and positron emission tomography for measuring myocardial perfusion reserve in healthy humans. Magn. Reson. Med., 55(4):772-779, 2006.

[213] T. Fritz-Hansen, J. D. Hove, K. F. Kofoed, H. Kelbaek, and H. B. W. Larsson. Quantification of MRI measured myocardial perfusion reserve in healthy humans: A comparison with positron emission tomography. J. Magn. Reson. Imaging, 27(4):818824, 2008.

[214] C. A. Hulka, B. L. Smith, D. C. Sgroi, L. Tan, W. B. Edmister, J. P. Semple, T. Campbell, D. B. Kopans, T. J. Brady, and R. M. Weisskoff. Benign and malignant breast lesions: Differentiation with echo-planar MR imaging. Radiol., 197(1):33-38, 1995.

[215] C. A. Hulka, W. B. Edmister, B. L. Smith, L. Tan, D. C. Sgroi, T. Campbell, D. B. Kopans, and R. M. Weisskoff. Dynamic echo-planar imaging of the breast: Experience in diagnosing breast carcinoma and correlation with tumor angiogenesis. Radiol., 205(3):837-842, 1997.

[216] E. K. Andersen, K. H. Hole, K. V. Lund, K. Sundfør, G. B. Kristensen, H. Lyng, and E. Malinen. Pharmacokinetic parameters derived from dynamic contrast enhanced MRI of cervical cancers predict chemoradiotherapy outcome. Radiother. Oncol., 2013.

[217] M. V. Knopp, E. Weiss, H. P. Sinn, J. Mattern, H. Junkermann, et al. Pathophysiologic basis of contrast enhancement in breast tumors. J. Magn. Reson. Imaging, 10(3):260-266, 1999.

[218] P. Armitage, C. Behrenbruch, M. Brady, and N. Moore. Extracting and visualizing physiological parameters using dynamic contrast-enhanced magnetic resonance imaging of the breast. Med. Image Anal., 9(4):315-329, 2005. 
[219] M. D. Pickles, M. Lowry, D. J. Manton, P. Gibbs, and L. W. Turnbull. Role of dynamic contrast enhanced MRI in monitoring early response of locally advanced breast cancer to neoadjuvant chemotherapy. Breast Cancer Res. Treat., 91(1):1-10, 2005 .

[220] R. Fusco, M. Sansone, S. Maffei, N. Raiano, and A. Petrillo. Dynamic contrastenhanced MRI in breast cancer: A comparison between distributed and compartmental tracer kinetic models. J. Biomed. Graph. Comput., 2(2):23-36, 2012.

[221] H. Hawighorst, R. Engenhart, M. V. Knopp, G. Brix, M. Grandy, M. Essig, P. Miltner, I. Zuna, M. Fuss, and G. van Kaick. Intracranial meningeomas: Time-and dosedependent effects of irradiation on tumor microcirculation monitored by dynamic MR imaging. Magn. Reson. Imaging, 15(4):423-432, 1997.

[222] F. L. Giesel, P. L. Choyke, A. Mehndiratta, C. M. Zechmann, H. von Tengg-Kobligk, et al. Pharmacokinetic analysis of malignant pleural mesotheliomainitial results of tumor microcirculation and its correlation to microvessel density (CD-34). Acad. Radiol., 15(5):563-570, 2008.

[223] J. A. Loncaster, B. M. Carrington, J. R. Sykes, A. P. Jones, S. M. Todd, et al. Prediction of radiotherapy outcome using dynamic contrast enhanced MRI of carcinoma of the cervix. Int. J. Radiat. Oncol. Biol. Phys., 54(3):759-767, 2002.

[224] M. Müller-Schimpfle, G. Brix, G. Layer, P. Schlag, R. Engenhart, S. Frohmuller, T. Hess, I. Zuna, W. Semmler, and G. van Kaick. Recurrent rectal cancer: Diagnosis with dynamic MR imaging. Radiol., 189(3):881-889, 1993.

[225] X. Yang, J. Liang, J. T. Heverhagen, G. Jia, P. Schmalbrock, S. Sammet, R. Koch, and M. V. Knopp. Improving the pharmacokinetic parameter measurement in dynamic contrast-enhanced MRI by use of the arterial input function: Theory and clinical application. Magn. Reson. Med., 59(6):1448-1456, 2008.

[226] F. Kiessling, M. Lichy, R. Grobholz, M. Heilmann, N. Farhan, et al. Simple models improve the discrimination of prostate cancers from the peripheral gland by T1weighted dynamic MRI. Eur. Radiol., 14(10):1793-1801, 2004.

[227] X. Liu, D. L. Langer, M. A. Haider, Y. Yang, M. N. Wernick, and I. S. Yetik. Prostate cancer segmentation with simultaneous estimation of Markov random field parameters and class. IEEE Trans. Med. Imaging, 28(6):906-915, 2009.

[228] Y. Artan, M. A. Haider, D. L. Langer, T. H. van der Kwast, A. J. Evans, Y. Yang, M. N. Wernick, J. Trachtenberg, and I. S. Yetik. Prostate cancer localization with multispectral MRI using cost-sensitive support vector machines and conditional random fields. IEEE Trans. Image Process., 19(9):2444-2455, 2010.

[229] Y. S. Sung, H.-J. Kwon, B.-W. Park, G. Cho, C. K. Lee, K.-S. Cho, and J. K. Kim. Prostate cancer detection on dynamic contrast-enhanced MRI: Computer-aided diagnosis versus single perfusion parameter maps. Am. J. Roentgenol., 197(5):11221129, 2011. 
[230] J. U. Harrer, G. J. M. Parker, H. A. Haroon, D. L. Buckley, K. Embelton, C. Roberts, D. Balériaux, and A. Jackson. Comparative study of methods for determining vascular permeability and blood volume in human gliomas. J. Magn. Reson. Imaging, 20(5):748-757, 2004.

[231] M. Haris, R. K. Gupta, A. Singh, N. Husain, M. Husain, C. M. Pandey, C. Srivastava, S. Behari, and R. K. S. Rathore. Differentiation of infective from neoplastic brain lesions by dynamic contrast-enhanced MRI. Neuroradiol., 50(6):531-540, 2008.

[232] M. Haris, N. Husain, A. Singh, R. Awasthi, R. K. Singh Rathore, M. Husain, and R. K. Gupta. Dynamic contrast-enhanced (DCE) derived transfer coefficient $\left(k^{\text {trans }}\right)$ is a surrogate marker of matrix metalloproteinase 9 (MMP-9) expression in brain tuberculomas. J. Magn. Reson. Imaging, 28(3):588-597, 2008.

[233] M. Bergamino, L. Saitta, L. Barletta, L. Bonzano, G. L. Mancardi, L. Castellan, J. L. Ravetti, and L. Roccatagliata. Measurement of blood-brain barrier permeability with $\mathrm{T}_{1}$-Weighted dynamic contrast-enhanced MRI in brain tumors: A comparative study with two different algorithms. ISRN Neuroscience, 2013:1-6, 2013.

[234] G. J. Hunter, L. M. Hamberg, N. Choi, R. K. Jain, T. McCloud, and A. J. Fischman. Dynamic T1-weighted magnetic resonance imaging and positron emission tomography in patients with lung cancer: Correlating vascular physiology with glucose metabolism. Clin. Cancer Res., 4(4):949-955, 1998.

[235] J. H. Naish, L. E. Kershaw, D. L. Buckley, A. Jackson, J. C. Waterton, and G. J. M. Parker. Modeling of contrast agent kinetics in the lung using T1-weighted dynamic contrast-enhanced MRI. Magn. Reson. Med., 61(6):1507-1514, 2009.

[236] C. Hayes, A. R. Padhani, and M. O. Leach. Assessing changes in tumour vascular function using dynamic contrast-enhanced magnetic resonance imaging. NMR Biomed., 15(2):154-163, 2002.

[237] J. Veltman, M. Stoutjesdijk, R. Mann, H. J. Huisman, J. O. Barentsz, J. G. Blickman, and $\mathrm{C}$. Boetes. Contrast-enhanced magnetic resonance imaging of the breast: The value of pharmacokinetic parameters derived from fast dynamic imaging during initial enhancement in classifying lesions. Eur. Radiol., 18(6):1123-1133, 2008.

[238] R. H. El Khouli, K. J. Macura, I. R. Kamel, M. A. Jacobs, and D. A. Bluemke. 3-T dynamic contrast-enhanced MRI of the breast: Pharmacokinetic parameters versus conventional kinetic curve analysis. Am. J. Roentgenol., 197(6):1498-1505, 2011.

[239] X. Li, L. R. Arlinghaus, G. D. Ayers, A. B. Chakravarthy, R. G. Abramson, et al. DCE-MRI analysis methods for predicting the response of breast cancer to neoadjuvant chemotherapy: Pilot study findings. Magn. Reson. Med., 71(4):1592-1602, 2014.

[240] A. Jena, S. B. Mehta, and S. Taneja. Optimizing MRI scan time in the computation of pharmacokinetic parameters $\left(k^{\text {trans }}\right)$ in breast cancer diagnosis. J. Magn. Reson. Imaging, 38(3):573-579, 2013. 
[241] I. Ocak, M. Bernardo, G. Metzger, T. Barrett, P. Pinto, P. S. Albert, and P. L. Choyke. Dynamic contrast-enhanced MRI of prostate cancer at 3T: A study of pharmacokinetic parameters. Am. J. Roentgenol., 189(4):W192-W201, 2007.

[242] A. S. N. Jackson, S. A. Reinsberg, S. A. Sohaib, E. M. Charles-Edwards, S. Jhavar, et al. Dynamic contrast-enhanced MRI for prostate cancer localization. Br. J. Radiol., 82(974):148-156, 2009.

[243] M. Lowry, B. Zelhof, G. P. Liney, P. Gibbs, M. D. Pickles, and L. W. Turnbull. Analysis of prostate DCE-MRI: Comparison of fast exchange limit and fast exchange regimen pharmacokinetic models in the discrimination of malignant from normal tissue. Invest. Radiol., 44(9):577-584, 2009.

[244] D. L. Langer, T. H. van der Kwast, A. J. Evans, J. Trachtenberg, B. C. Wilson, and M. A. Haider. Prostate cancer detection with multi-parametric MRI: Logistic regression analysis of quantitative T2, diffusion-weighted imaging, and dynamic contrast-enhanced MRI. J. Magn. Reson. Imaging, 30(2):327-334, 2009.

[245] S. F. Riches, G. S. Payne, V. A. Morgan, S. Sandhu, C. Fisher, M. Germuska, D. J. Collins, A. Thompson, and N. M. desouza. MRI in the detection of prostate cancer: combined apparent diffusion coefficient, metabolite ratio, and vascular parameters. Am. J. Roentgenol., 193(6):1583-1591, 2009.

[246] P. C. Vos, T. Hambrock, J. O. Barenstz, and H. J. Huisman. Computer-assisted analysis of peripheral zone prostate lesions using T2-weighted and dynamic contrast enhanced T1-weighted MRI. Phys. Med. Biol., 55(6):1719-1734, 2010.

[247] D. L. Langer, T. H. van der Kwast, A. J. Evans, A. Plotkin, J. Trachtenberg, B. C. Wilson, and M. A. Haider. Prostate tissue composition and MR measurements: Investigating the relationships between $\mathrm{ADC}, T_{2}, K^{\text {trans }}$, $v_{e}$, and corresponding histologic features. Radiol., 255(2):485-494, 2010.

[248] A. Oto, C. Yang, A. Kayhan, M. Tretiakova, T. Antic, C. Schmid-Tannwald, S. Eggener, G. S. Karczmar, and W. M. Stadler. Diffusion-weighted and dynamic contrast-enhanced MRI of prostate cancer: Correlation of quantitative MR parameters with gleason score and tumor angiogenesis. Am. J. Roentgenol., 197(6):13821390, 2011.

[249] Y.-J. Chen, W.-C. Chu, Y.-S. Pu, S.-C. Chueh, C.-T. Shun, and W.-Y. I. Tseng. Washout gradient in dynamic contrast-enhanced MRI is associated with tumor aggressiveness of prostate cancer. J. Magn. Reson. Imaging, 36(4):912-919, 2012.

[250] K. Røe, L. T. G. Mikalsen, A. J. van der Kogel, J. Bussink, H. Lyng, A. H. Ree, L. Marignol, and D. R. Olsen. Vascular responses to radiotherapy and androgendeprivation therapy in experimental prostate cancer. Radiat. Oncol., 7(1):75-87, 2012.

[251] P. C. Vos, J. O. Barentsz, N. Karssemeijer, and H. J. Huisman. Automatic computeraided detection of prostate cancer based on multiparametric magnetic resonance image analysis. Phys. Med. Biol., 57(6):1527-1542, 2012. 
[252] E. K. Vos, G. Litjens, T. Kobus, T. Hambrock, C. A. Kaa, J. O. Barentsz, H. Huisman, and T. W. J. Scheenen. Assessment of prostate cancer aggressiveness using dynamic contrast-enhanced magnetic resonance imaging at 3T. Eur. Urol., 64(3):448-455, 2013.

[253] C. Li, M. Chen, S. Li, X. Zhao, C. Zhang, X. Luo, and C. Zhou. Detection of prostate cancer in peripheral zone: Comparison of MR diffusion tensor imaging, quantitative dynamic contrast-enhanced MRI, and the two techniques combined at 3.0 T. Acta. Radiol., 55(2):239-247, 2014.

[254] A. B. Rosenkrantz, A. Sabach, J. S. Babb, B. W. Matza, S. S. Taneja, and F.-M. Deng. Prostate cancer: Comparison of dynamic contrast-enhanced MRI techniques for localization of peripheral zone tumor. Am. J. Roentgenol., 201(3):471-478, 2013.

[255] G. Atkin, N. J. Taylor, F. M. Daley, J. J. Stirling, P. Richman, R. Glynne-Jones, J. A. d'Arcy, D. J. Collins, and A. R. Padhani. Dynamic contrast-enhanced magnetic resonance imaging is a poor measure of rectal cancer angiogenesis. Br. J. Surg., 93(8):992-1000, 2006.

[256] K. Mross, U. Fasol, A. Frost, R. Benkelmann, J. Kuhlmann, et al. DCE-MRI assessment of the effect of vandetanib on tumor vasculature in patients with advanced colorectal cancer and liver metastases: A randomized phase I study. J. Angiogenes. Res., 1(5):1-12, 2009.

[257] J. Gu, P.-L. Khong, S. Wang, Q. Chan, E. X. Wu, W. Law, R. K. Liu, and J. Zhang. Dynamic contrast-enhanced MRI of primary rectal cancer: Quantitative correlation with positron emission tomography/computed tomography. J. Magn. Reson. Imaging, 33(2):340-347, 2011.

[258] O. M. Hahn, C. Yang, M. Medved, G. Karczmar, E. Kistner, T. Karrison, E. Manchen, M. Mitchell, M. J. Ratain, and W. M. Stadler. Dynamic contrastenhanced magnetic resonance imaging pharmacodynamic biomarker study of sorafenib in metastatic renal carcinoma. J. Clin. Oncol., 26(28):4572-4578, 2008.

[259] R. J. Hodgson, T. Barnes, S. Connolly, B. Eyes, R. S. D. Campbell, and R. Moots. Changes underlying the dynamic contrast-enhanced MRI response to treatment in rheumatoid arthritis. Skeletal Radiol., 37(3):201-207, 2008.

[260] R. Hodgson, P. OConnor, and R. Moots. MRI of rheumatoid arthritis-image quantitation for the assessment of disease activity, progression and response to therapy. Rheumatology, 47(1):13-21, 2008.

[261] G. Adluru, E. V. DiBella, and M. C. Schabel. Model-based registration for dynamic cardiac perfusion MRI. J. Magn. Reson. Imaging, 24(5):1062-1070, 2006.

[262] N. A. Pack and E. V. R. DiBella. Comparison of myocardial perfusion estimates from dynamic contrast-enhanced magnetic resonance imaging with four quantitative analysis methods. Magn. Reson. Med., 64(1):125-137, 2010. 
[263] B. Huang, C.-S. Wong, B. Whitcher, D. L.-W. Kwong, V. Lai, Q. Chan, and P.-L. Khong. Dynamic contrast-enhanced magnetic resonance imaging for characterising nasopharyngeal carcinoma: Comparison of semiquantitative and quantitative parameters and correlation with tumour stage. Eur. Radiol., 10(1):1-8, 2013.

[264] Q. G. de Lussanet, J. C. G. van Golde, R. G. H. Beets-Tan, M. J. Post, M. S. Huijberts, N. C. Schaper, A. G. H. Kessels, J. van Engelshoven, and W. H. Backes. Dynamic contrast-enhanced MRI of muscle perfusion combined with MR angiography of collateral artery growth in a femoral artery ligation model. NMR Biomed., 20(8):717-725, 2007.

[265] L. Dong, W. S. Kerwin, M. S. Ferguson, R. Li, J. Wang, H. Chen, G. Canton, T. S. Hatsukami, and C. Yuan. Cardiovascular magnetic resonance in carotid atherosclerotic disease. J. Cardiovasc. Magn. Reson., 15(1):11-53, 2009.

[266] N. Michoux, L. Huwart, J. Abarca-Quinones, M. Dorvillius, L. Annet, F. Peeters, and B. E. Van Beers. Transvascular and interstitial transport in rat hepatocellular carcinomas: Dynamic contrast-enhanced MRI assessment with low-and high-molecular weight agents. J. Magn. Reson. Imaging, 28(4):906-914, 2008.

[267] D. Checkley, J. J. Tessier, J. Kendrew, J. C. Waterton, and S. R. Wedge. Use of dynamic contrast-enhanced MRI to evaluate acute treatment with ZD6474, a VEGF signalling inhibitor, in PC-3 prostate tumours. Br. J. Cancer, 89(10):1889-1895, 2003.

[268] M. A. Zahra, L. T. Tan, A. N. Priest, M. J. Graves, M. Arends, R. A. F. Crawford, J. D. Brenton, D. J. Lomas, and E. Sala. Semiquantitative and quantitative dynamic contrast-enhanced magnetic resonance imaging measurements predict radiation response in cervix cancer. Int. J. Radiat. Oncol. Biol. Phys., 74(3):766-773, 2009.

[269] S. I. Semple, V. N. Harry, D. E. Parkin, and F. J. Gilbert. A combined pharmacokinetic and radiologic assessment of dynamic contrast-enhanced magnetic resonance imaging predicts response to chemoradiation in locally advanced cervical cancer. Int. J. Radiat. Oncol. Biol. Phys., 75(2):611-617, 2009.

[270] S. Kim, L. A. Loevner, H. Quon, A. Kilger, E. Sherman, G. Weinstein, A. Chalian, and H. Poptani. Prediction of response to chemoradiation therapy in squamous cell carcinomas of the head and neck using dynamic contrast-enhanced MR imaging. Am. J. Neuroradiol., 31(2):262-268, 2010.

[271] C. Roberts, G. J. M. Parker, C. J. Rose, Y. Watson, J. P. O'Connor, S. M. Stivaros, A. Jackson, and V. E. Rushton. Glandular function in sjögren syndrome: Assessment with dynamic contrast-enhanced MR imaging and tracer kinetic modelinginitial experience1. Radiol., 246(3):845-853, 2008.

[272] F. K.-H. Lee, A. D. King, M. K.-M. Kam, B. B.-Y. Ma, and D. K.-W. Yeung. Radiation injury of the parotid glands during treatment for head and neck cancer: Assessment using dynamic contrast-enhanced MR imaging. Radiat. Res., 175(3):291-296, 2011 . 
[273] S. Agrawal, R. Awasthi, A. Singh, M. Haris, R. K. Gupta, and R. K. S. Rathore. An exploratory study into the role of dynamic contrast-enhanced (DCE) MRI metrics as predictors of response in head and neck cancers. Clin. Radiol., 67(9):e1-e5, 2012.

[274] F. K.-H. Lee, A. D. King, B. B.-Y. Ma, and D. K.-W. Yeung. Dynamic contrast enhancement magnetic resonance imaging (DCE-MRI) for differential diagnosis in head and neck cancers. Eur. J. Radiol., 81(4):784-788, 2012.

[275] W. Kerwin, A. Hooker, M. Spilker, P. Vicini, M. Ferguson, T. Hatsukami, and C. Yuan. Quantitative magnetic resonance imaging analysis of neovasculature volume in carotid atherosclerotic plaque. Circulation, 107(6):851-856, 2003.

[276] W. S. Kerwin, K. D. O’Brien, M. S. Ferguson, N. Polissar, T. S. Hatsukami, and C. Yuan. Inflammation in carotid atherosclerotic plaque: A dynamic contrastenhanced MR imaging study. Radiol., 241(2):459-468, 2006.

[277] W. S. Kerwin, M. Oikawa, C. Yuan, G. P. Jarvik, and T. S. Hatsukami. MR imaging of adventitial vasa vasorum in carotid atherosclerosis. Magn. Reson. Med., 59(3):507-514, 2008.

[278] T. Kurita, H. Sakuma, K. Onishi, M. Ishida, K. Kitagawa, T. Yamanaka, T. Tanigawa, T. Kitamura, K. Takeda, and M. Ito. Regional myocardial perfusion reserve determined using myocardial perfusion magnetic resonance imaging showed a direct correlation with coronary flow velocity reserve by Doppler flow wire. Eur. Heart J., 30(4):444-452, 2009.

[279] T. Ichihara, M. Ishida, K. Kitagawa, Y. Ichikawa, T. Natsume, N. Yamaki, H. Maeda, K. Takeda, and H. Sakuma. Quantitative analysis of first-pass contrast-enhanced myocardial perfusion MRI using a Patlak plot method and blood saturation correction. Magn. Reson. Med., 62(2):373-383, 2009.

[280] B. D. de Senneville, I. A. Mendichovszky, S. Roujol, I. Gordon, C. Moonen, and N. Grenier. Improvement of MRI-functional measurement with automatic movement correction in native and transplanted kidneys. J. Magn. Reson. Imaging, 28(4):970-978, 2008.

[281] Q. Jiang, J. R. Ewing, G. L. Ding, L. Zhang, Z. G. Zhang, et al. Quantitative evaluation of BBB permeability after embolic stroke in rat using MRI. J. Cereb. Blood Flow Metab., 25(5):583-592, 2005.

[282] V. L. Nguyen, M. E. Kooi, W. H. Backes, R. H. M. van Hoof, A. E. C. M. Saris, et al. Suitability of pharmacokinetic models for dynamic contrast-enhanced MRI of abdominal aortic aneurysm vessel wall: A comparison. PloS one, 8(10):e75173, 2013.

[283] J. A. Jacquez. Compartmental analysis in biology and medicine. University of Michigan Press, Ann Arbor, MI, USA, 1985.

[284] G. Brix, M. L. Bahner, U. Hoffmann, A. Horvath, and W. Schreiber. Regional blood flow, capillary permeability, and compartmental volumes: Measurement with dynamic CT-initial experience. Radiol., 210(1):269-276, 1999. 
[285] G. Brix, M. Salehi Ravesh, S. Zwick, J. Griebel, and S. Delorme. On impulse response functions computed from dynamic contrast-enhanced image data by algebraic deconvolution and compartmental modeling. Phys. Med., 28(2):119-128, 2012.

[286] L. H. D. Cheong, C. C. T. Lim, and T. San Koh. Dynamic contrast-enhanced CT of intracranial meningioma: Comparison of distributed and compartmental tracer kinetic modelsinitial results. Radiol., 232(3):921-930, 2004.

[287] T. S. Koh, C. K. Tan, L. H. Cheong, and C. C. Lim. Cerebral perfusion mapping using a robust and efficient method for deconvolution analysis of dynamic contrastenhanced images. Neuroimage, 32(2):643-653, 2006.

[288] S. Metz, C. Ganter, S. Lorenzen, S. van Marwick, K. Herrmann, et al. Phenotyping of tumor biology in patients by multimodality multiparametric imaging: Relationship of microcirculation, $\alpha \mathrm{v} \beta 3$ expression, and glucose metabolism. J. Nucl. Med., 51(11):1691-1698, 2010.

[289] I. Thomassin-Naggara, D. Balvay, C. A. Cuenod, E. Daraï, C. Marsault, and M. Bazot. Dynamic contrast-enhanced MR imaging to assess physiologic variations of myometrial perfusion. Eur. Radiol., 20(4):984-994, 2010.

[290] L. J. Bains, D. M. McGrath, J. H. Naish, S. Cheung, Y. Watson, M. B. Taylor, J. P. Logue, G. J. M. Parker, J. C. Waterton, and D. L. Buckley. Tracer kinetic analysis of dynamic contrast-enhanced MRI and CT bladder cancer data: A preliminary comparison to assess the magnitude of water exchange effects. Magn. Reson. Med., 64(2):595-603, 2010.

[291] S. B. Donaldson, G. Betts, S. C. Bonington, J. J. Homer, N. J. Slevin, L. E. Kershaw, H. Valentine, C. M. L. West, and D. L. Buckley. Perfusion estimated with rapid dynamic contrast-enhanced magnetic resonance imaging correlates inversely with vascular endothelial growth factor expression and pimonidazole staining in headand-neck cancer: A pilot study. Int. J. Radiat. Oncol. Biol. Phys., 81(4):1176-1183, 2011 .

[292] S. Bisdas, M. Baghi, J. Wagenblast, T. J. Vogl, C. H. Thng, and T. S. Koh. Gadolinium-enhanced echo-planar T2-weighted MRI of tumors in the extracranial head and neck: Feasibility study and preliminary results using a distributedparameter tracer kinetic analysis. J. Magn. Reson. Imaging, 27(5):963-969, 2008.

[293] T. Koh, C. Thng, S. Hartono, J. Kwek, J. Khoo, et al. Dynamic contrast-enhanced MRI of neuroendocrine hepatic metastases: A feasibility study using a dual-input two-compartment model. Magn. Reson. Med., 65(1):250-260, 2011.

[294] E. Henderson, J. Sykes, D. Drost, H.-J. Weinmann, B. K. Rutt, and T.-Y. Lee. Simultaneous MRI measurement of blood flow, blood volume, and capillary permeability in mammary tumors using two different contrast agents. J. Magn. Reson. Imaging, 12(6):991-1003, 2000. 
[295] G. R. Moran, R. E. Thornhill, J. Sykes, and F. S. Prato. Myocardial viability imaging using Gd-DTPA: Physiological modeling of infarcted myocardium, and impact on injection strategy and imaging time. Magn. Reson. Med., 48(5):791-800, 2002.

[296] V. J. Schmid, B. Whitcher, A. R. Padhani, and G.-Z. Yang. Quantitative analysis of dynamic contrast-enhanced MR images based on Bayesian P-splines. IEEE Trans. Med. Imaging, 28(6):789-798, 2009.

[297] L. E. Kershaw, C. E. Hutchinson, and D. L. Buckley. Benign prostatic hyperplasia: Evaluation of T1, T2, and microvascular characteristics with T1-weighted dynamic contrast-enhanced MRI. J. Magn. Reson. Imaging, 29(3):641-648, 2009.

[298] D. L. Buckley, C. Roberts, G. J. M. Parker, J. P. Logue, and C. E. Hutchinson. Prostate cancer: Evaluation of vascular characteristics with dynamic contrastenhanced T1-weighted MR imaginginitial experience. Radiol., 233(3):709-715, 2004.

[299] J. G. Korporaal, M. van Vulpen, C. A. van den Berg, and U. A. van der Heide. Tracer kinetic model selection for dynamic contrast-enhanced computed tomography imaging of prostate cancer. Invest. Radiol., 47(1):41-48, 2012.

[300] K.-L. Li, X. P. Zhu, . Waterton, and A. Jackson. Improved 3D quantitative mapping of blood volume and endothelial permeability in brain tumors. J. Magn. Reson. Imaging, 12(2):347-357, 2000.

[301] C. Chih-Feng, H. Ling-Wei, L. Chun-Chung, L. Chen-Chang, W. Hsu-Huei, T. Yuan-Hsiung, and L. Ho-Ling. In vivo correlation between semi-quantitative hemodynamic parameters and $k^{\text {trans }}$ derived from DCE-MRI of brain tumors. Int. J. Imaging Sys. Technol., 22(2):132-136, 2012.

[302] A. Radjenovic, B. Dall, J. P. Ridgway, and M. A. Smith. Measurement of pharmacokinetic parameters in histologically graded invasive breast tumours using dynamic contrast-enhanced MRI. Br. J. Radiol., 81(962):120-128, 2008.

[303] A. Anderlik, A. Z. Munthe-Kaas, O. K. Oye, E. Eikefjord, J. Rorvik, D. M. Ulvang, F. G. Zollner, and A. Lundervold. Quantitative assessment of kidney function using dynamic contrast enhanced MRI-Steps towards an integrated software prototype. In Proc. Int. Symp. Image, Signal Process. Anal., pages 575-581, 2009.

[304] F. A. van Dorsten, M. van der Graaf, M. R. W. Engelbrecht, G. J. L. H. van Leenders, A. Verhofstad, M. Rijpkema, J. J. M. C. H. de la Rosette, J. O. Barentsz, and A. Heerschap. Combined quantitative dynamic contrast-enhanced MR imaging and ${ }^{1} \mathrm{H}$ MR spectroscopic imaging of human prostate cancer. J. Magn. Reson. Imaging, 20(2):279-287, 2004.

[305] L. Tabar, H.-H. T. Chen, S. W. Duffy, and U. B. Krusemo. Primary and adjuvant therapy, prognostic factors and survival in 1053 breast cancers diagnosed in a trial of mammography screening. Japanese J. Clin. Oncol., 29(12):608-616, 1999.

[306] S. Sourbron. Technical aspects of MR perfusion. Eur. J. Radiol., 76(3):304-313, 2010. 
[307] M. Pellerin, T. E. Yankeelov, and M. Lepage. Incorporating contrast agent diffusion into the analysis of DCE-MRI data. Magn. Reson. Med., 58(6):1124-1134, 2007.

[308] G. Jia, C. O’Dell, J. T. Heverhagen, X. Yang, J. Liang, R. V. Jacko, S. Sammet, T. Pellas, P. Cole, and M. V. Knopp. Colorectal liver metastases: Contrast agent diffusion coefficient for quantification of contrast enhancement heterogeneity at MR imaging. Radiol., 248(3):901-909, 2008.

[309] D. L. Buckley, L. E. Kershaw, and G. J. Stanisz. Cellular-interstitial water exchange and its effect on the determination of contrast agent concentration in vivo: Dynamic contrast-enhanced MRI of human internal obturator muscle. Magn. Reson. Med., 60(5):1011-1019, 2008.

[310] T. Franiel, B. Hamm, and H. Hricak. Dynamic contrast-enhanced magnetic resonance imaging and pharmacokinetic models in prostate cancer. Eur. Radiol., 21(3):616-626, 2011.

[311] H. B. W. Larsson, S. Rosenbaum, and T. Fritz-Hansen. Quantification of the effect of water exchange in dynamic contrast MRI perfusion measurements in the brain and heart. Magn. Reson. Med., 46(2):272-281, 2001.

[312] D. C. Kimber, C. E. Gray, C. E. Stackpole, M. A. Miller, A. B. Drakontides, and L. C. Leavell. Kimber-Gray-Stackpole's Anatomy and Physiology. Macmillan, New York, 1977.

[313] W. Kapit, R. Macey, and E. Meisami. The Physiology Coloring Book. HarperCollins \& Row, 1987.

[314] S. B. Park, J. K. Kim, and K.-S. Cho. Complications of renal transplantation ultrasonographic evaluation. J. Ultrasound Med., 26(5):615-633, 2007.

[315] S. A. Akbar, S. Z. H. Jafri, M. A. Amendola, B. L. Madrazo, R. Salem, and K. G. Bis. Complications of renal transplantation. Radiographics, 25(5):1335-1356, 2005.

[316] C. Höhnke, D. Abendroth, S. Schleibner, and W. Land. Vascular complications in 1,200 kidney transplantations. Transplantat. Proc., 19(5):36-91, 1987.

[317] M. H. Scott and R. A. Sells. Primary adenocarcinoma in a transplanted cadaveric kidney. Transplantation, 46(1):157-158, 1988.

[318] T. H. Mathew. Recurrence of disease following renal transplantation. Am. J. Kidney Dis., 12(2):85-96, 1988.

[319] J. R. Chapman, P. J. OConnell, and B. J. Nankivell. Chronic renal allograft dysfunction. J. Am. Soc. Nephrol., 16(10):3015-3026, 2005.

[320] E. D. Brown, M. Y. M. Chen, N. T. Wolfman, D. J. Ott, and N. E. Watson. Complications of renal transplantation: Evaluation with US and radionuclide imaging. Radiographics, 20(3):607-622, 2000.

[321] G. M. Baxter. Ultrasound of renal transplantation. Clin. Radiol., 56(10):802-818, 2001. 
[322] H. M. Isoniemi, L. Krogerus, E. von Willebrand, E. Taskinen, J. Ahonen, and P. Häyry. Histopathological findings in well-functioning, long-term renal allografts. Kidney International, 41(1):155-160, 1992.

[323] J. D. Pirsch, R. J. Ploeg, S. Gange, A. M. D’Alessandro, S. J. Knechtle, H. W. Sollinger, M. Kalayoglu, and F. O. Belzer. Determinants of graft survival after renal transplantation. Transplantation, 61(11):1581-1586, 1996.

[324] H. Bennett and D. Li. MR imaging of renal function. Magn. Reson. Imaging Clin. N. Am., 5(1):5-18, 1997.

[325] G. L. Myers, W. G. Miller, J. Coresh, J. Fleming, N. Greenberg, et al. Recommendations for improving serum creatinine measurement: A report from the laboratory working group of the national kidney disease education program. Clin. Chem., 52(1):5-18, 2006.

[326] D. Yang, Q. Ye, M. Williams, Y. Sun, T. C.-C. Hu, D. S. Williams, J. M. F. Moura, and C. Ho. USPIO enhanced dynamic MRI: Evaluation of normal and transplanted rat kidneys. Magn. Reson. Med., 46(6):1152-1163, 2001.

[327] E. Giele. Computer methods for semi-automatic MR renogram determination. $\mathrm{PhD}$ thesis, Eindhoven University of Technology, Eindhoven, 2002.

[328] A. Taylor and J. V. Nally. Clinical applications of renal scintigraphy. Am. J. Roentgenol., 164(1):31-41, 1995.

[329] J. G. Heaf and J. Iversen. Uses and limitations of renal scintigraphy in renal transplantation monitoring. Eur. J. Nucl. Med., 27(7):871-879, 2000.

[330] C. Kolofousi, K. Stefanidis, D. D. Cokkinos, D. Karakitsos, E. Antypa, and P. Piperopoulos. Ultrasonographic features of kidney transplants and their complications: An imaging review. ISRN Radiol., 2013:1-12, 2013.

[331] C. Sebastià, S. Quiroga, R. Boyé, C. Cantarell, M. Fernandez-Planas, and A. Alvarez. Helical CT in renal transplantation: Normal findings and early and late complications. Radiographics, 21(5):1103-1117, 2001.

[332] A. Grabner, D. Kentrup, U. Schnöckel, M. Schäfers, and S. Reuter. Non-invasive diagnosis of acute renal allograft rejection- Special focus on gamma scintigraphy and positron emission tomography. In T. Rath, editor, Current Issues and Future Direction in Kidney Transplantation, chapter 4, pages 89-104. InTech, 2013.

[333] G. K. von Schulthess, W. Kuoni, G. Gerig, S. Duewell, and G. Krestin. Semiautomated ROI analysis in dynamic MRI studies-Part II: Application to renal function examination first experiences. J. Comput. Assist. Tomogr., 15(5):733-741, 1991.

[334] G. Gerig, R. Kikinis, W. Kuon, G. K. van Schulthess, and O. Kübler. Semiautomated ROI analysis in dynamic MRI studies-Part I: Image analysis tools for automatic correction of organ displacements. IEEE Trans. Image Process., 11(2):221-232, 1992. 
[335] P. J. Yim, H. B. Marcos, P. L. Choyke, M. McAuliffe, D. McGarry, and I. Heaton. Registration of time-series contrast enhanced magnetic resonance images for renography. In IEEE Symp. Comput.-Based Med. Syst., (CMBS'01), volume 1, pages 516-520, 2001.

[336] J. A. de Priester, A. G. Kessels, E. L. Giele, J. A. Den Boer, M. L. Christiaans, A. Hasman, and J. A. Van Engelshoven. MR renography by semiautomated image analysis: Performance in renal transplant recipients. J. Magn. Reson. Imaging, 14(2):134-140, 2001.

[337] E. W. Giele, J. de Priester, J. A. Blom, J. A. den Boer, A. H. J. A. van Engelshoven, and M. Geerlings. Movement correction of the kidney in dynamic MRI scans using FFT phase difference movement detection. J. Magn. Reson. Imaging, 14(6):741749, 2001.

[338] H. K. Koh, W. Shen, B. Shuter, and A. A. Kassim. Segmentation of kidney cortex in MRI studies using a constrained morphological 3D H-maxima transform. In Proc. Int. Conf. Control Autom. Robot. Vis. (ICARCV'06), pages 1-5, 2006.

[339] Y. Sun, J. M. Moura, D. Yang, Q. Ye, and C. Ho. Kidney segmentation in MRI sequences using temporal dynamics. In Proc. IEEE Int. Symp. Biomed. Imaging: From Nano to Macro, (ISBI'02), pages 98-101, 2002.

[340] Y. Sun, D. Yang, Q. Ye, M. Williams, J. M. F. Moura, F. Boada, Z.-P. Liang, and C. Ho. Improving spatiotemporal resolution of USPIO-enhanced dynamic imaging of rat kidneys. Magn. Reson. Imaging, 21(6):593-598, 2003.

[341] Y. Sun, M. P. Jolly, and J. M. F. Moura. Integrated registration of dynamic renal perfusion MR images. In Proc. IEEE Int. Conf. Image Process., (ICIP'04), volume 3, pages 1923-1926, 2004.

[342] Y. Sun, J. M. F. Moura, and C. Ho. Subpixel registration in renal perfusion MR image sequence. In Proc. IEEE Int. Symp. Biomed. Imaging: From Nano to Macro, (ISBI'04), pages 700-703, 2004.

[343] Y. Sun. Registration and segmentation in perfusion MRI: Kidneys and hearts. PhD thesis, Carnegie Mellon University, Pittsburg, 2004.

[344] T. F. Chan and L. A. Vese. Active contours without edges. IEEE Trans. Image Process., 10(2):266-277, 2001.

[345] H. E. Abdelmunim, A. A. Farag, W. Miller, and M. AboelGhar. A kidney segmentation approach from DCE-MRI using level sets. In Computer Vision and Pattern Recognition Workshops, (CVPRW'08), volume 1, pages 1-6, 2008.

[346] S. E. Yuksel, A. El-Baz, A. A. Farag, M. Abou El-Ghar, T. Eldiasty, and M. A. Ghoneim. A kidney segmentation framework for dynamic contrast enhanced magnetic resonance imaging. J. Vibration and Control, 13(9-10):1505-1516, 2007.

[347] S. E. Yuksel, A. El-Baz, and A. A. Farag. A kidney segmentation framework for dynamic contrast enhanced magnetic resonance imaging. In Proc. Int. Symp. Math. Methods Eng., (MME'06), pages 55-64, 2006. 
[348] A. El-Baz. Novel stochastic models for medical image analysis. $\mathrm{PhD}$ thesis, University of Louisville, Louisville, KY, USA, 2006.

[349] A. El-Baz and G. Gimel'farb. EM-based approximation of empirical distributions with linear combinations of discrete Gaussians. In Proc. IEEE Int. Conf. Image Process., (ICIP'07), volume 4, pages 373-376, 2007.

[350] A. El-Baz, A. Elnakib, F. Khalifa, M. Abou El-Ghar, P. McClure, A. Soliman, and G. Gimel'farb. Precise segmentation of 3-D magnetic resonance angiography. IEEE Trans. Biomed. Eng., 59(7):2019-2029, 2012.

[351] A. El-Baz and G. Gimel'farb. Image segmentation with a parametric deformable model using shape and appearance priors. In Proc. IEEE Int. Conf. Comput. Vis. Pattern Recogni. (CVPR'08), pages 1-8, 2008.

[352] A. El-Baz and G. Gimel'farb. Robust medical images segmentation using learned shape and appearance models. In Proc. Med. Image Comput. Comput.-Assist. Interv., (MICCAI'09), pages 281-288, 2009.

[353] O. Gloger, K. D. Tönnies, V. Liebscher, B. Kugelmann, R. Laqua, and H. Völzke. Prior shape level set segmentation on multistep generated probability maps of MR datasets for fully automatic kidney parenchyma volumetry. IEEE Trans. Med. Imaging, 31(2):312-325, 2012.

[354] Y. Boykov and G. Funka-Lea. Graph cuts and efficient N-D image segmentation. Int. J. Comput. Vis., 70(2):109-131, 2006.

[355] H. Rusinek, Y. Boykov, M. Kaur, S. Wong, L. Bokacheva, J. B. Sajous, A. J. Huang, S. Heller, and V. S. Lee. Performance of an automated segmentation algorithm for 3D MR renography. Magn. Reson. Med., 57(6):1159-1167, 2007.

[356] A. M. Ali, A. A. Farag, and A. El-Baz. Graph cuts framework for kidney segmentation with prior shape constraints. In Proc. Med. Image Comput. Comput.-Assist. Interv., (MICCAI'07), volume 1, pages 384-392, 2007.

[357] B. Chevaillier, Y. Ponvianne, J.-L. Collette, D. Mandry, M. Claudon, and O. Pietquin. Functional semi-automated segmentation of renal DCE-MRI sequences. In Proc. IEEE Int. Conf. Acoust., Speech, and Signal Process., (ICASSP'08), pages 525-528, 2008.

[358] B. Chevaillier, D. Mandry, J.-L. Collette, M. Claudon, M.-A. Galloy, and O. Pietquin. Functional segmentation of renal DCE-MRI sequences using vector quantization algorithms. Neural Process. Lett., 34(1):71-85, 2011.

[359] T. Song, V. S. Lee, H. Rusinek, S. Wong, and A. F. Laine. Four dimensional MR image analysis of dynamic renography. In Proc. Annu. IEEE Conf. Eng. Med. Biol. Soc., (EMBS'06), pages 3134-3137, 2006.

[360] F. Zöllner, R. Sance, P. Rogelj, M. J. Ledesma-Carbayo, J. Rørvik, A. Santos, and A. Lundervold. Assessment of 3D DCE-MRI of the kidneys using non-rigid image registration and segmentation of voxel time courses. Comput. Med. Imaging Graph., 33(3):171-181, 2009. 
[361] J. B. MacQueen. Some methods for classification and analysis of multivariate observations. In Proc. fifth Berkeley Symp. Math. Stat. Probab., pages 281-297, 1967.

[362] S. Li, F. G. Zöllner, A. D. Merrem, Y. Peng, J. Roervik, A. Lundervold, and L. R. Schad. Wavelet-based segmentation of renal compartments in DCE-MRI of human kidney: Initial results in patients and healthy volunteers. Comput. Med. Imaging Graph., 36(1):108-118, 2012.

[363] X. Yang, P. Ghafourian, P. Sharma, K. Salman, D. Martin, and B. Fei. Nonrigid registration and classification of the kidneys in 3D dynamic contrast enhanced (DCE) MR images. In Proc. SPIE Med. Imaging: Image Process., volume 8314, pages 1-9, 2012.

[364] H. Wang, L. Dong, J. O’Daniel, R. Mohan, A. S. Garden, K. K. Ang, D. A. Kuban, M. Bonnen, J. Y. Chang, and R. Cheung. Validation of an accelerated demons algorithm for deformable image registration in radiation therapy. Phys. Med. Biol., 50(12):2887-2905, 2005.

[365] S. Osher and R. Fedkiw. Level Set Methods and Dynamic Implicit Surfaces. Springer Verlag, New York, USA, 2006.

[366] P. A. Viola and W. M. W. III. Alignment by maximization of mutual information. Int. J. Comput. Vis., 24(2):137-154, 1997.

[367] A. Farag, A. El-Baz, and G. Gimel'farb. Precise segmentation of multimodal images. IEEE Trans. Image Process., 15(4):952-968, 2006.

[368] A. Elnakib, M. A. El-Ghar, G. Gimel'farb, R. Falk, J. Suri, and A. El-Baz. Modified Akaike information criterion for selecting the numbers of mixture components: An application to initial lung segmentation. In L. Saba and J. Suri, editors, MultiDetector CT Imaging: Abdomen, Pelvis, and CAD Applications, chapter 28, pages 609-620. CRC Press: Taylor and Francis Group, 2014.

[369] D. L. Wilson and J. A. Noble. An adaptive segmentation algorithm for time-of-flight MRA data. IEEE Trans. Med. Imaging, 18(10):938-945, 1999.

[370] G. L. Gimel'farb, A. A. Farag, and A. El-Baz. Expectation-maximization for a linear combination of gaussians. In Proc. IEEE Int. Conf. Pattern Recogni., (ICPR'04), pages 422-425, 2004.

[371] A. Farag, A. El-Baz, and G. Gimel'farb. Density estimation using modified expectation maximization for a linear combination of Gaussians. In Proc. IEEE Int. Conf. Image Process., (ICIP'04), volume 3, pages 1871-1874, 2004.

[372] A. A. Farag, A. El-Baz, and R. M. Mohamed. Density estimation using generalized linear model and a linear combination of Gaussians. Int. J. Signal Process., 1:76-79, 2005 .

[373] A. El-Baz, A. A. Farag, and G. Gimel'farb. Iterative approximation of empirical grey-level distributions for precise segmentation of multimodal images. EURASIP J. Applied Signal Processing, 2005:1969-1983, 2005. 
[374] A. Farag, A. El-Baz, and G. Gimel'farb. Precise image segmentation by iterative EM-based approximation of empirical grey level distributions with linear combinations of Gaussians. In Comput. Vis. Pattern Recogni. Workshop (CVPRW'2004), pages 121-129, 2004.

[375] A. El-Baz, R. M. Mohamed, A. A. Farag, and G. Gimel'farb. Unsupervised segmentation of multi-modal images by a precise approximation of individual modes with linear combinations of discrete Gaussians. In IEEE Comput. Soc. Conf. Comput. Vis. Pattern Recogni. Workshops (CVPRW'2005), pages 54-54, 2005.

[376] S. Haker, S. Angenent, A. Tannenbaum, and R. Kikinis. Nondistorting flattening maps and the 3D visualization of colon CT images. IEEE Trans. Med. Imaging, 19(7):665-670, 2000.

[377] M. Prasad, A. Ramesh, P. Kavanagh, B. K. Tamarappoo, R. N. J. Gerlach, V. Cheng, L. E. Thomson, D. S. Berman, G. Germano, and P. J. Slomka. Automated quantification of 3D regional myocardial wall thickening from gated magnetic resonance images. J. Magn. Reson. Imaging, 31(2):317-327, 2010.

[378] L. te Strake, L. J. SchultzéKool, L. C. Paul, A. M. Tegzess, J. J. Weenings, J. Hermans, J. Doornbos, R. G. Bluemm, and J. L. Bloem. Magnetic resonance imaging of renal transplants: Its value in the differentiation of acute rejection and cyclosporin a nephrotoxicity. Clin. Radiol., 39(3):220-228, 1988.

[379] E. Elbeltagi, T. Hegazy, and D. Grierson. Comparison among five evolutionarybased optimization algorithms. Adv. Eng. Inf., 19(1):43-53, 2005.

[380] T. Fawcett. An introduction to ROC analysis. Pattern Recogni. Lett., 27(8):861-874, 2006.

[381] H. Skalská and V. Freylich. Web-bootstrap estimate of area under ROC curve. Austrian J. Stat., 35(2-3):325-330, 2006.

[382] K. H. Zou, S. K. Warfield, A. Bharatha, C. M. C. Tempany, M. R. Kaus, S. J. Haker, W. M. Wells III, F. A. Jolesz, and R. Kikinis. Statistical validation of image segmentation quality based on a spatial overlap index. Acad. Radiol., 11(2):178-189, 2004.

[383] J. M. Bland and D. G. Altman. Statistical methods for assessing agreement between two methods of clinical measurement. Lancet, 1:307-310, 1986.

[384] V. Kumar, A. K. Abbas, N. Fausto, and J. C. Aster. Robbins \& Cotran Pathologic Basis of Disease. Elsevier Saunders, 2009.

[385] F. H. Martini, M. J. Timmons, and R. B. Tallitsch. Human Anatomy. Pearson Benjamin Cummings, 2008.

[386] WebMD: Heart Disease Health Center. Heart and Cardiovascular Diseases [Online]. Available. http://www.webmd.com/heart-disease/guide/ diseases-cardiovascular. 
[387] Encyclopedia of Science. Congenital Heart Defects [Online]. Available. http://www.daviddarling.info/encyclopedia/C/congenital_ heart_defects.html.

[388] W. J. Manning, W. Li, and R. R. Edelman. A preliminary report comparing magnetic resonance coronary angiography with conventional angiography. New Engl. J. Med., 328(12):828-832, 1993.

[389] O. M. Muehling, P. Panse, M. Jerosch-Herold, B. V. Wilson, R. F. Wilson, N. M. Wilke, and L. W. Miller. Cardiac magnetic resonance perfusion imaging identifies transplant arteriopathy by a reduced endomyocardial resting perfusion. J. Heart Lung Transplant., 28(4):1122-1123, 2005.

[390] N. Merkle, J. Wohrle, O. Grebe, T. Nusser, M. Kunze, H. A. Kestler, M. Kochs, and V. Hombach. Assessment of myocardial perfusion for detection of coronary artery stenoses by steady-state, free-precession magnetic resonance first-pass imaging. Heart, 93(11):1381-1385, 2007.

[391] T. Kurita, H. Sakuma, K. Onishi, M. Ishida, K. Kitagawa, T. Yamanaka, T. Tanigawa, T. Kitamura, K. Takeda, and M. Ito. Regional myocardial perfusion reserve determined using myocardial perfusion magnetic resonance imaging showed a direct correlation with coronary flow velocity reserve by doppler flow wire. European Heart Journal, 30(4):444-452, 2009.

[392] J. P. Greenwood, N. Maredia, J. F. Younger, J. M. Brown, J. Nixon, et al. Cardiovascular magnetic resonance and single-photon emission computed tomography for diagnosis of coronary heart disease (CE-MARC): A prospective trial. The Lancet, 379(9814):453-460, 2012.

[393] J. Schwitter, C. M. Wacker, N. Wilke, N. Al-Saadi, E. Sauer, et al. MR-IMPACT II: magnetic resonance imaging for myocardial perfusion assessment in coronary artery disease trial: perfusion-cardiac magnetic resonance vs. single-photon emission computed tomography for the detection of coronary artery disease: a comparative multicentre, multivendor trial. Eur. Heart J., 34(10):775-781, 2013.

[394] J. Schulz-Menger, D. A. Bluemke, J. Bremerich, S. D. Flamm, M. A. Fogel, et al. Standardized image interpretation and post processing in cardiovascular magnetic resonance: Society for cardiovascular magnetic resonance (SCMR) board of trustees task force on standardized post processing. J. Cardiovasc. Magn. Reson., 15:35, 2013.

[395] L. J. Spreeuwers and M. Breeuwer. Automatic detection of myocardial boundaries in MR cardio perfusion images. In Proc. Med. Image Comput. Comput.-Assist. Interv., (MICCAI'01), pages 1228-1231, 1999.

[396] M. F. Santarelli, V. Positano, C. Michelassi, M. Lombardi, and L. L. Automated cardiac MRI segmentation: Theory and measurement evaluation. Med. Eng. Phys., 25(2):149-159, 2003. 
[397] C. Pluempitiwiriyawej and S. Sotthivirat. Active contours with automatic initialization for myocardial perfusion analysis. In Proc. Annu. IEEE Conf. Eng. Med. Biol. Soc., (EMBS'05), pages 3332-3335, 2005.

[398] G. Adluru, E. V. R. DiBella, and R. T. Whitaker. Automatic segmentation of cardiac short axis slices in perfusion MRI. In Proc. IEEE Int. Symp. Biomed. Imaging: From Nano to Macro, (ISBI'06), pages 133-136, 2006.

[399] M. Stegmann, H. Olafsdottir, and H. Larsson. Unsupervised motion-compensation of multi-slice cardiac perfusion MRI. Med. Image Anal., 9(4):394-410, 2005.

[400] V. Gupta, E. A. Hendriks, J. Milles, R. J. van der Geest, M. Jerosch-Herold, J. H. C. Reiber, and B. P. Lelieveldt. Fully automatic registration and segmentation of firstpass myocardial perfusion MR image sequences. Acad. Radiol., 17(11):1375-1385, 2010.

[401] N. Baka, J. Milles, E. A. Hendriks, A. Suinesiaputra, M. J. Herold, J. H. C. Reiber, and B. P. F. Lelieveldt. Segmentation of myocardial perfusion MR sequences with multi-band active appearance models driven by spatial and temporal features. In Proc. SPIE Med. Imaging: Image Process., volume 6914, pages 1-10, 2008.

[402] D. Mahapatra and Y. Sun. Rigid registration of renal perfusion images using a neurobiology-based visual saliency model. EURASIP J. Appl. Signal Process., 2010:1-16, 2010.

[403] D. Mahapatra. Joint segmentation and groupwise registration of cardiac perfusion images using temporal information. J. Digital Imaging, 26(2):173-182, 2012.

[404] M. Breeuwer, L. Spreeuwers, and M. Quist. Automatic quantitative analysis of cardiac MR perfusion images. In Proc. SPIE Med. Imaging: Image Process., volume 4322, pages 733-742, 2001.

[405] K. K. Wong, E. S. Yang, E. X. Wu, H.-F. Tse, and S. T. Wong. First-pass myocardial perfusion image registration by maximization of normalized mutual information. $J$. Magn. Reson. Imaging, 27(3):529-537, 2008.

[406] L. M. Bidaut and J. P. Valle. Automated registration of dynamic MR images for the quantification of myocardial perfusion. J. Magn. Reson. Imaging, 13(4):648-655, 2001.

[407] C. Dornier, M. K. Ivancevic, P. Thevenaz, and J. P. Vallee. Improvement in the quantification of myocardial perfusion using an automatic spline-based registration algorithm. J. Magn. Reson. Imaging, 18(2):160-168, 2003.

[408] S. N. Gupta, M. Solaiyappan, G. M. Beache, Q. E. Arai, and T. K. Foo. Fast method for correcting image misregistration due to organ motion in time-series MRI data. Magn. Reson. Med., 49(3):506-514, 2003.

[409] C. M. Gallippi, C. M. Kramer, Y. L. Hu, D. A. Vido, N. Reichek, and W. J. Rogers. Fully automated registration and warping of contrast-enhanced first pass perfusion images. J. Cardiovasc. Magn. Reson., 4(4):459-469, 2002. 
[410] J. Milles, R. J. van der Geest, M. Jerosch-Herold, J. H. C. Reiber, and B. P. F. Lelieveldt. Fully automated motion correction in first-pass myocardial perfusion MR image sequences. IEEE Trans. Med. Imaging, 27(11):1611-1621, 2008.

[411] G. Wollny, P. Kellman, A. Santos, and M. J. Ledesma-Carbayo. Automatic motion compensation of free breathing acquired myocardial perfusion data by using independent component analysis. Med. Image Anal., 16(5):1015-1028, 2012.

[412] G. Wollny, M. J. Ledesma-Carbayo, P. Kellman, and A. Santos. Exploiting quasiperiodicity in motion correction of free-breathing myocardial perfusion MRI. IEEE Trans. Med. Imaging, 29(8):1516-1527, 2010.

[413] G. Tarroni, A. R. Patel, F. Veronesi, J. Walter, C. Lamberti, R. M. Lang, V. MorAvi, and C. Corsi. MRI-based quantification of myocardial perfusion at rest and stress using automated frame-by-frame segmentation and non-rigid registration. In Comput. Cardiol., pages 1-4, 2010.

[414] C. Li, Y. Sun, and P. Chai. Pseudo ground truth based nonrigid registration of myocardial perfusion MRI. Med. Image Anal., 15(4):449-459, 2011.

[415] L. Tautz, A. Hennemuth, M. Andersson, A. Seeger, H. Knutsson, and O. Friman. Phase-based non-rigid registration of myocardial perfusion MRI image sequences. In Proc. IEEE Int. Symp. Biomed. Imaging: From Nano to Macro, (ISBI'10), pages 516-519, 2010.

[416] J. P. Thirion. Image matching as a diffusion process: An analogy with Maxwell's demons. Med. Image Anal., 2(3):243-260, 1998.

[417] F. Y. Wu. The Potts model. Rev. Mod Phys., 54(1):235-268, 1982.

[418] C. Bouman and K. Sauer. A generalized Gaussian image model for edge-preserving MAP estimation. IEEE Trans. Image Process., 2(3):296-310, 1993.

[419] F. Khalifa, G. M. Beache, A. Firjani, K. C. Welch, G. Gimel'farb, and A. El-Baz. A new nonrigid registration approach for motion correction of cardiac first-pass perfusion MRI. In Proc. IEEE Int. Conf. Image Process., (ICIP'12), pages 1665-1668, 2012.

[420] F. Khalifa, G. M. Beache, A. Elnakib, H. Sliman, G. Gimel'farb, K. C. Welch, and A. El-Baz. A new shape-based framework for the left ventricle wall segmentation from cardiac first-pass perfusion MRI. In Proc. IEEE Int. Symp. Biomed. Imaging: From Nano to Macro, (ISBI'13), pages 41-44, 2013.

[421] H. Gray. Anatomy of the Human Body. Lea \& Febiger, 2000.

[422] D. Shier, J. Butler, and R. Lewis. Human Anatomy and Physiology. McGraw-Hill, 2001.

[423] J. E. McNeal. The zonal anatomy of the prostate. The Prostate, 2(1):35-49, 1981.

[424] J. J. Stevermer and S. K. Easley. Treatment of prostatitis. Am. Fam. Physician, 61(10):3015-3034, 2000. 
[425] E. Meares and T. A. Stamey. Bacteriologic localization patterns in bacterial prostatitis and urethritis. Invest. Urol., 5(5):492, 1968.

[426] T. Hedayati and C. Stehman. Prostatitis. emedicine from webmd website, 2011.

[427] A. C. Piovesan, G. d. C. Freire, F. C. M. Torricelli, P. Cordeiro, R. Yamada, and M. Srougi. Incidence of histological prostatitis and its correlation with PSA density. Clinics, 64(11):1049-1051, 2009.

[428] Urology Care Foundation. BPH: Management [Online]. Available. http: / / www . urologyhealth.org/urology/index.cfm?article=144.

[429] C. H. Lee, O. Akin-Olugbade, and A. Kirschenbaum. Overview of prostate anatomy, histology, and pathology. Endocrinol. Metab. Clin. N. Am., 40(3):565-575, 2011.

[430] P. D. Baade, D. R. Youlden, and L. J. Krnjacki. International epidemiology of prostate cancer: geographical distribution and secular trends. Mol. Nutr. Food Res., 53(2):171-184, 2009.

[431] J. Hugosson, S. Carlsson, G. Aus, S. Bergdahl, A. Khatami, P. Lodding, C.G. Pihl, J. Stranne, E. Holmberg, and H. Lilja. Mortality results from the göteborg randomised population-based prostate-cancer screening trial. Lancet Oncol., 11(8):725-732, 2010.

[432] H. Hricak, P. L. Choyke, S. C. Eberhardt, S. A. Leibel, and P. T. Scardino. Imaging prostate cancer: A multidisciplinary perspective. Radiol., 243(1):28-53, 2007.

[433] J. C. Applewhite, B. Matlaga, D. McCullough, M. Hall, et al. Transrectal ultrasound and biopsy in the early diagnosis of prostate cancer. Cancer Control, 8(2):141-150, 2001 .

[434] C. R. Loiselle, P. R. Eby, W. B. DeMartini, S. Peacock, N. Bittner, C. D. Lehman, and J. N. Kim. Dynamic contrast-enhanced MRI kinetics of invasive breast cancer: A potential prognostic marker for radiation therapy. Int. J. Radiat. Oncol. Biol. Phys., 76(5):1314-1319, 2010.

[435] J. J. Fütterer, S. W. T. P. J. Heijmink, T. W. J. Scheenen, J. Veltman, H. J. Huisman, et al. Prostate cancer localization with dynamic contrast-enhanced MR imaging and proton MR spectroscopic imaging. Radiol., 241(2):449-458, 2006.

[436] R. Zwiggelaar, Y. Zhu, and S. Williams. Semi-automatic segmentation of the prostate. In Proc. First Iberian Conf. Pattern Recogni. Image Anal., pages 11081116, 2003.

[437] P. D. Allen, J. Graham, D. C. Williamson, and C. E. Hutchinson. Differential segmentation of the prostate in MR images using combined 3D shape modelling and voxel classification. In Proc. IEEE Int. Symp. Biomed. Imaging: From Nano to Macro, (ISBI'06), pages 410-413, 2006.

[438] Y. Zhu, S. Williams, and R. Zwiggelaar. A hybrid ASM approach for sparse volumetric data segmentation. Pattern Recogni. Image Anal., 17(2):252-258, 2007. 
[439] S. Klein, U. A. van der Heide, B. W. Raaymakers, A. N. Kotte, M. Staring, and J. P. Pluim. Segmentation of the prostate in MR images by atlas matching. In Proc. IEEE Int. Symp. Biomed. Imaging: From Nano to Macro, (ISBI'07), pages 1300-1303, 2007.

[440] S. Klein, U. A. van der Heide, I. M. Lips, M. van Vulpen, M. Staring, and J. P. Pluim. Automatic segmentation of the prostate in 3D MR images by atlas matching using localized mutual information. Med. Phys., 35(5):1407-1417, 2008.

[441] D. Flores-Tapia, G. Thomas, N. Venugopal, B. McCurdy, and S. Pistorius. Semi automatic MRI prostate segmentation based on wavelet multiscale products. In Proc. Annu. IEEE Conf. Eng. Med. Biol. Soc., (EMBS'08), pages 3020-3023, 2008.

[442] J.-C. Pesquet, H. Krim, and H. Carfantan. Time-invariant orthonormal wavelet representations. IEEE Trans. Image Process., 44(8):1964-1970, 1996.

[443] R. Toth, P. Tiwari, M. Rosen, A. Kalyanpur, S. Pungavkar, and A. Madabhushi. A multimodal prostate segmentation scheme by combining spectral clustering and active shape models. In Proc. SPIE Med. Imaging: Image Process., pages 1-12, 2008.

[444] S. Vikal, S. Haker, C. Tempany, and G. Fichtinger. Prostate contouring in MRI guided biopsy. In Proc. SPIE Med. Imaging: Image Process., pages 1-11, 2009.

[445] X. Liu, D. Langer, M. Haider, T. Van der Kwast, A. Evans, M. Wernick, and I. Yetik. Unsupervised segmentation of the prostate using MR images based on level set with a shape prior. In Proc. Annu. IEEE Conf. Eng. Med. Biol. Soc., (EMBS'09), pages 3613-3616, 2009.

[446] N. Otsu. A threshold selection method from gray-level histograms. IEEE Trans. Syst. Man Cybern., 9(1):62-66, 1979.

[447] N. Makni, P. Puech, R. Lopes, A.-S. Dewalle, O. Colot, and N. Betrouni. Combining a deformable model and a probabilistic framework for an automatic 3D segmentation of prostate on MRI. Int. J. Comput. Assist. Radiol. Surg., 4(2):181-188, 2009.

[448] S. Ozer, M. A. Haider, D. L. Langer, T. H. van der Kwast, A. J. Evans, M. N. Wernick, J. Trachtenberg, and I. S. Yetik. Prostate cancer localization with mltispectral MRI based on relevance vector machines. In Proc. IEEE Int. Symp. Biomed. Imaging: From Nano to Macro, (ISBI'09), pages 73-76, 2009.

[449] M. E. Tipping. Sparse Bayesian learning and the relevance vector machine. J. Mach. Learning Res., 1(9):211-244, 2001.

[450] Y. Gao, R. Sandhu, G. Fichtinger, and A. R. Tannenbaum. A coupled global registration and segmentation framework with application to magnetic resonance prostate imagery. IEEE Trans. Med. Imaging, 29(10):1781-1794, 2010.

[451] S. Martin, J. Troccaz, and V. Daanen. Automated segmentation of the prostate in 3D MR images using a probabilistic atlas and a spatially constrained deformable model. Med. Phys., 37:1579-1590, 2010. 
[452] A. Firjany, A. Elnakib, A. El-Baz, G. Gimel'farb, M. Abo El-Ghar, and A. Elmagharby. Novel stochastic framework for accurate segemntation of prostate in dynamic contrast-enhanced MRI. In Proc. Int. Wokshop Prostate Cancer Imaging: Comput.-Aided Diagno. Progno. Interv., pages 121-130, 2010.

[453] J. A. Dowling, J. Fripp, S. Chandra, J. P. W. Pluim, J. Lambert, J. Parker, J. Denham, P. B. Greer, and O. Salvado. Fast automatic multi-atlas segmentation of the prostate from 3D MR images. In Proc. Int. Workshop on Prostate Cancer Imaging: Image Anal. Image-guided Interv., pages 10-21, 2011.

[454] T. R. Langerak, U. A. van der Heide, A. N. Kotte, M. A. Viergever, M. van Vulpen, and J. P. Pluim. Label fusion in atlas-based segmentation using a selective and iterative method for performance level estimation (SIMPLE). IEEE Trans. Med. Imaging, 29(12):2000-2008, 2010.

[455] S. Ghose, J. Mitra, A. Oliver, R. Marti, X. Llado, J. Freixenet, J. C. Vilanova, D. Sidibé, and F. Mériaudeau. Graph cut energy minimization in a probabilistic learning framework for 3D prostate segmentation in MRI. In Proc. IEEE Int. Conf. Pattern Recogni., (ICPR'12), pages 125-128, 2012.

[456] S. Ghose, A. Oliver, R. Martí, X. Lladó, J. Freixenet, J. Mitra, J. C. Vilanova, and F. Meriaudeau. A hybrid framework of multiple active appearance models and global registration for 3D prostate segmentation in MRI. In Proc. SPIE Med. Imaging: Image Process., pages 83140S-83140S, 2012.

[457] I. Chan, W. Wells III, R. V. Mulkern, S. Haker, J. Zhang, K. H. Zou, S. E. Maier, and C. M. Tempany. Detection of prostate cancer by integration of line-scan diffusion, T2-mapping and T2-weighted magnetic resonance imaging; a multichannel statistical classifier. Med. Phys., 30(9):2390-2398, 2003.

[458] A. Madabhushi, M. D. Feldman, D. N. Metaxas, J. Tomaszeweski, and D. Chute. Automated detection of prostatic adenocarcinoma from high-resolution ex vivo MRI. IEEE Trans. Med. Imaging, 24(12):1611-1625, 2005.

[459] J. K. Kim, S. S. Hong, Y. J. Choi, S. H. Park, H. Ahn, C.-S. Kim, and K.-S. Cho. Wash-in rate on the basis of dynamic contrast-enhanced MRI: Usefulness for prostate cancer detection and localization. J. Magn. Reson. Imaging, 22(5):639-646, 2005 .

[460] O. Rouvière, O. Valette, S. Grivolat, C. Colin-Pangaud, R. Bouvier, J. Y. Chapelon, A. Gelet, and D. Lyonnet. Recurrent prostate cancer after external beam radiotherapy: Value of contrast-enhanced dynamic MRI in localizing intraprostatic tumorcorrelation with biopsy findings. Urol., 63(5):922-927, 2004.

[461] S. Viswanath, B. N. Bloch, E. Genega, N. Rofsky, R. Lenkinski, J. Chappelow, R. Toth, and A. Madabhushi. A comprehensive segmentation, registration, and cancer detection scheme on 3 tesla in vivo prostate DCE-MRI. In Proc. Med. Image Comput. Comput.-Assist. Interv., (MICCAI'08), pages 662-669, 2008. 
[462] S. Viswanath, B. N. Bloch, M. Rosen, J. Chappelow, R. Toth, N. Rofsky, R. Lenkinski, E. Genega, A. Kalyanpur, and A. Madabhushi. Integrating structural and functional imaging for computer assisted detection of prostate cancer on multi-protocol in vivo 3 Tesla MRI. In Proc. SPIE Med. Imaging: Image Process., pages 72603I72603I, 2009.

[463] P. C. Vos, T. Hambrock, C. A. Hulsbergen-van de Kaa, J. J. Fütterer, J. O. Barentsz, and H. J. Huisman. Computerized analysis of prostate lesions in the peripheral zone using dynamic contrast enhanced MRI. Med. Phys., 35(3):888-899, 2008.

[464] D. Ampeliotis, A. Antonakoudi, K. Berberidis, E. Psarakis, and A. Kounoudes. A computer-aided system for the detection of prostate cancer based on magnetic resonance image analysis. In Proc. 3rd Int. Symp. Commun., Control, Signal Process, (ISCCSP'08), pages 1372-1377, 2008.

[465] G. Litjens, P. Vos, J. Barentsz, N. Karssemeijer, and H. Huisman. Automatic computer aided detection of abnormalities in multi-parametric prostate MRI. In Proc. SPIE Med. Imaging: Computer-Aided Diagno., pages 79630T-79630T, 2011.

[466] S. T. Roweis and L. K. Saul. Nonlinear dimensionality reduction by locally linear embedding. Science, 290(5500):2323-2326, 2000.

[467] Y. Peng, Y. Jiang, C. Yang, J. B. Brown, T. Antic, I. Sethi, C. Schmid-Tannwald, M. L. Giger, S. E. Eggener, and A. Oto. Quantitative analysis of multiparametric prostate MR images: Differentiation between prostate cancer and normal tissue and correlation with Gleason score. Radiol., 267(3):787-796, 2013.

[468] A. Madabhushi, J. Shi, M. Feldman, M. Rosen, and J. Tomaszewski. Comparing ensembles of learners: Detecting prostate cancer from high resolution MRI. In Proc. Int. Workshop Comput. Vis. Approaches Med. Image Anal., pages 25-36, 2006.

[469] R. Lopes, A. Ayache, N. Makni, P. Puech, A. Villers, S. Mordon, and N. Betrouni. Prostate cancer characterization on MR images using fractal features. Med. Phys., 38(1):83-95, 2011.

[470] V. Shah, B. Turkbey, H. Mani, Y. Pang, T. Pohida, M. J. Merino, P. A. Pinto, P. L. Choyke, and M. Bernardo. Decision support system for localizing prostate cancer based on multiparametric magnetic resonance imaging. Med. Phys., 39(7):40934103, 2012.

[471] T. Hambrock, P. C. Vos, C. A. Hulsbergen-van de Kaa, J. O. Barentsz, and H. J. Huisman. Prostate cancer: Computer-aided diagnosis with multiparametric 3-T MR Imaging-Effect on observer performance. Radiol., 266(2):521-530, 2013.

[472] G. Gimel'farb. Image textures and Gibbs random fields. Kluwer, Dordrecht, The Netherlands, 1999.

[473] J. Besag. On the statistical analysis of dirty pictures. J. Royal Stat. Soc. Series B, 48(3):259-302, 1986. 
[474] A. Firjani, A. Elnakib, F. Khalifa, A. El-Baz, G. Gimel'farb, M. Abou El-Ghar, and A. Elmaghraby. A novel 3D segmentation approach for segmenting the prostate from dynamic contrast enhanced MRI using current appearance and learned shape prior. In Proc. IEEE Int. Symp. Signal Process. Inf. Technol., (ISSPIT'10), pages 137-143, 2010 .

[475] A. El-Baz, A. A. Farag, R. Falk, and R. La Rocca. Automatic identification of lung abnormalities in chest spiral CT scans. In Proc. IEEE Int. Conf. Acoust. Speech Sig. Process., (ICASSP'03), volume 2, pages 261-264, 2003.

[476] A. El-Baz, A. A. Farag, R. Falk, and R. La Rocca. A unified approach for detection, visualization, and identification of lung abnormalities in chest spiral CT scans. In International Congress Series, volume 1256, pages 998-1004, 2003.

[477] A. El-Baz, A. A. Farag, R. Falk, and R. La Rocca. Detection, visualization and identification of lung abnormalities in chest spiral CT scan: Phase-I. In Proc. Int. Conf. Biomed. Eng., pages 38-42, 2002.

[478] A. A. Farag, A. El-Baz, G. Gimel'farb, and R. Falk. Detection and recognition of lung abnormalities using deformable templates. In Proc. Int. Conf. Pattern Recogni., (ICPR'04), volume 3, pages 738-741, 2004.

[479] A. A. Farag, A. El-Baz, G. G. Gimelfarb, R. Falk, and S. G. Hushek. Automatic detection and recognition of lung abnormalities in helical CT images using deformable templates. In Proc. Medical Image Comput. Comput.-Assis. Interv., (MICCAI'04), pages 856-864. 2004.

[480] A. El-Baz, S. E. Yuksel, S. Elshazly, and A. A. Farag. Non-rigid registration techniques for automatic follow-up of lung nodules. In Proc. Comput. Assist. Radiol. Surg., (CARS'05), volume 1281, pages 1115-1120, 2005.

[481] A. A. Farag, A. El-Baz, G. Gimelfarb, M. A. El-Ghar, and T. Eldiasty. Quantitative nodule detection in low dose chest CT scans: new template modeling and evaluation for cad system design. In Proc. Medical Image Comput. Comput.-Assist. Interv., (MICCAI'05), pages 720-728. 2005.

[482] A. El-Baz, A. Farag, G. Gimel'farb, R. Falk, M. A. El-Ghar, and T. Eldiasty. A framework for automatic segmentation of lung nodules from low dose chest CT scans. In Proc. Int. Conf. Pattern Recogni., (ICPR'06), pages 611-614, 2006.

[483] A. M. Ali, A. S. El-Baz, and A. A. Farag. A novel framework for accurate lung segmentation using graph cuts. In Proc. IEEE Int. Symp. Biomed. Imaging: From Nano to Macro, (ISBI'07), pages 908-911, 2007.

[484] A. El-Baz, G. Gimel'farb, R. Falk, and M. A. El-Ghar. A novel approach for automatic follow-up of detected lung nodules. In Proc. IEEE Int. Conf. Image Process., (ICIP'07), volume 5, pages V-501, 2007.

[485] A. A. Farag, A. El-Baz, G. Gimelfarb, R. Falk, M. A. El-Ghar, T. Eldiasty, and S. Elshazly. Appearance models for robust segmentation of pulmonary nodules in 3d ldct chest images. In Proc. Medical Image Comput. Comput.-Assis. Interv., (MICCAI'06), pages 662-670. 2006. 
[486] A. M. Ali and A. A. Farag. Automatic lung segmentation of volumetric low-dose CT scans using graph cuts. In Advances in Visual Computing, pages 258-267. 2008.

[487] A. El-Baz, G. L. Gimel'farb, R. Falk, M. Abou El-Ghar, T. Holland, and T. Shaffer. A new stochastic framework for accurate lung segmentation. In Proc. Med. Image Comput. Comput.-Assist. Interv., (MICCAI'08), pages 322-330, 2008.

[488] A. El-Baz, G. L. Gimel'farb, R. Falk, D. Heredis, and M. Abou El-Ghar. A novel approach for accurate estimation of the growth rate of the detected lung nodules. In Proc. Int. Workshop Pulmonary Image Analysis, pages 33-42, 2008.

[489] A. El-Baz, G. L. Gimel'farb, R. Falk, T. Holland, and T. Shaffer. A framework for unsupervised segmentation of lung tissues from low dose computed tomography images. In Proc. British Machine Vision, (BMVC'08), pages 1-10, 2008.

[490] A. El-Baz, G. Gimel'farb, R. Falk, and M. A. El-Ghar. A new approach for automatic analysis of 3D low dose CT images for accurate monitoring the detected lung nodules. In Proc. IEEE Int. Conf. Pattern Recogni., (ICPR'08), pages 1-4, 2008.

[491] A. El-Baz, G. Gimel'farb, R. Falk, M. A. El-Ghar, and H. Refaie. Promising results for early diagnosis of lung cancer. In Proc. IEEE Int. Symp. Biomed. Imaging: From Nano to Macro, (ISBI'08), pages 1151-1154, 2008.

[492] A. El-Baz, G. Gimel'farb, R. Falk, and M. Abo El-Ghar. Automatic analysis of 3D low dose CT images for early diagnosis of lung cancer. Pattern Recogni., 42(6):1041-1051, 2009.

[493] A. El-Baz, G. Gimelfarb, R. Falk, M. A. El-Ghar, S. Rainey, D. Heredia, and T. Shaffer. Toward early diagnosis of lung cancer. In Proc. Medical Image Comput. Comput.-Assis. Interv., (MICCAI'09), pages 682-689. 2009.

[494] A. El-Baz, G. Gimel'farb, R. Falk, and M. El-Ghar. Appearance analysis for diagnosing malignant lung nodules. In Proc. IEEE Int. Symp. Biomed. Imaging: From Nano to Macro ,(ISBI'10), pages 193-196, 2010.

[495] A. El-Baz, P. Sethu, G. Gimel'farb, F. Khalifa, A. Elnakib, R. Falk, and M. A. El-Ghar. Elastic phantoms generated by microfluidics technology: Validation of an imaged-based approach for accurate measurement of the growth rate of lung nodules. Biotech. J., 6(2):195-203, 2011.

[496] A. El-Baz, G. Gimelfarb, R. Falk, M. A. El-Ghar, and J. Suri. Appearance analysis for the early assessment of detected lung nodules. In Lung Imaging and Computer Aided Diagnosis, chapter 17, pages 395-404. 2011.

[497] A. El-Baz, P. Sethu, G. Gimelfarb, F. Khalifa, A. Elnakib, R. Falk, M. A. El-Ghar, and J. Suri. Validation of a new imaged-based approach for the accurate estimating of the growth rate of detected lung nodules using real computed tomography images and elastic phantoms generated by state-of-theart microfluidics technology. In Lung Imaging and Computer Aided Diagnosis, chapter 18, pages 405-420. 2011. 
[498] A. El-Baz, M. Nitzken, G. Gimelfarb, E. Van Bogaert, R. Falk, M. A. El-Ghar, and J. Suri. Three-dimensional shape analysis using spherical harmonics for early assessment of detected lung nodules. In Lung Imaging and Computer Aided Diagnosis, chapter 19, pages 421-438. 2011.

[499] A. El-Baz, G. Gimelfarb, R. Falk, and M. A. El-Ghar. 3D MGRF-based appearance modeling for robust segmentation of pulmonary nodules in 3D LDCT chest images. In Lung Imaging and Computer Aided Diagnosis, chapter 3, pages 51-63. 2011.

[500] B. Abdollahi, A. Soliman, A. Civelek, X.-F. Li, G. Gimelfarb, and A. El-Baz. A novel 3D joint MGRF framework for precise lung segmentation. In Machine Learning in Medical Imaging, pages 86-93. 2012.

[501] A. El-Baz, F. Khalifa, A. Elnakib, M. Nitzken, A. Soliman, P. McClure, M. A. ElGhar, and G. Gimelfarb. A novel approach for global lung registration using 3D Markov-Gibbs appearance model. In Proc. Medical Image Comput. Comput.-Assis. Interv., (MICCAI'12), pages 114-121, 2012.

[502] A. El-Baz, G. Gimel'farb, M. Abou El-Ghar, and R. Falk. Appearance-based diagnostic system for early assessment of malignant lung nodules. In Proc. IEEE Int. Conf. Image Process., (ICIP'12), pages 533-536, 2012.

[503] A. El-Baz, A. Soliman, P. McClure, G. Gimel'farb, M. A. El-Ghar, and R. Falk. Early assessment of malignant lung nodules based on the spatial analysis of detected lung nodules. In Proc. IEEE Int. Symp. Biomed. Imaging: From Nano to Macro, (ISBI'12), pages 1463-1466, 2012.

[504] A. El-Baz, A. Elnakib, M. Abou El-Ghar, G. Gimel'farb, R. Falk, and A. Farag. Automatic detection of $2 \mathrm{D}$ and 3D lung nodules in chest spiral CT scans. Int. J. Biomed. Imaging, 2013, 2013.

[505] A. El-Baz, G. M. Beache, G. Gimel'farb, K. Suzuki, K. Okada, A. Elnakib, A. Soliman, and B. Abdollahi. Computer-aided diagnosis systems for lung cancer: Challenges and methodologies. Int. J. Biomed. Imaging, 2013:1-46, 2013.

[506] A. Soliman, F. Khalifa, A. Alansary, G. Gimel'farb, and A. El-Baz. Performance evaluation of an automatic MGRF-based lung segmentation approach. In Proc. Int. Symp. Comput. Models for Life Sci., (CMLS'13), pages 323-332, 2013.

[507] M. Nitzken, M. F. Casanova, F. Khalifa, G. Sokhadze, and A. El-Baz. Shape-based detection of cortex variability for more accurate discrimination between autistic and normal brains. In A. El-Baz, R. Acharya, A. Laine, and J. Suri, editors, Handbook of Multi Modality State-of-the-Art Medical Image Segmentation and Registration Methodologies, chapter 7, pages 161-185. Springer, 2011.

[508] M. Nitzken, M. F. Casanova, G. Gimel'farb, F. Khalifa, A. Elnakib, A. E. Switala, and A. El-Baz. 3D shape analysis of the brain cortex with application to autism. In Proc. IEEE Int. Symp. Biomed. Imaging: From Nano to Macro, (ISBI'11), pages 1847-1850, 2011. 
[509] A. Elnakib, M. F. Casanova, G. Gimel'farb, and A. El-Baz. Autism diagnostics by 3D shape analysis of the corpus callosum. In K. Suzuki, editor, Machine Learning in Computer-aided Diagnosis: Medical Imaging Intelligence and Analysis, chapter 15, pages 315-335. IGI Global, Berlin, 2012.

[510] A. Elnakib, M. F. Casanova, G. Gimel'farb, A. E. Switala, and A. El-Baz. Autism diagnostics by centerline-based shape analysis of the corpus callosum. In Proc. IEEE Int. Symp. Biomed. Imaging: From Nano to Macro, (ISBI'11), pages 18431846, 2011.

[511] A. El-Baz, A. Elnakib, M. F. Casanova, G. Gimel'farb, A. E. Switala, D. Jordan, and S. Rainey. Accurate automated detection of autism related corpus callosum abnormalities. J. Med. Sys., 35(5):929-939, 2011.

[512] M. F. Casanova, A. El-Baz, A. Elnakib, A. E. Switala, E. L. Williams, D. L. Williams, N. J. Minshew, and T. E. Conturo. Quantitative analysis of the shape of the corpus callosum in patients with autism and comparison individuals. Autism, 15(2):223-238, 2011.

[513] A. Elnakib, A. El-Baz, M. F. Casanova, G. Gimel'farb, and A. E. Switala. Imagebased detection of corpus callosum variability for more accurate discrimination between autistic and normal brains. In Proc. IEEE Int. Conf. Image Process., (ICIP'10), pages 4337-4340, 2010.

[514] M. F. Casanova, A. Farag, E.-B. Ayman, M. Meghan, H. Hassan, R. Fahmi, and A. E. Switala. Abnormalities of the gyral window in autism: A macroscopic correlate to a putative minicolumnopathy. J. Spec. Edu. Rehabilitat., 7(1-2):77-81, 2006.

[515] R. Fahmi, A. S. El-Baz, H. Abd El Munim, A. A. Farag, and M. F. Casanova. Classification techniques for autistic vs. typically developing brain using MRI data. In Proc. IEEE Int. Symp. Biomed. Imaging: From Nano to Macro (ISBI'07), pages 1348-1351, 2007.

[516] A. El-Baz, M. F. Casanova, G. Gimel'farb, M. Mott, and A. E. Switwala. A new image analysis approach for automatic classification of autistic brains. In Proc. IEEE Int. Symp. Biomed. Imaging: From Nano to Macro (ISBI'07), pages 352-355, 2007.

[517] A. A. Farag, R. Fahmi, M. F. Casanova, A. E. Abdel-Hakim, H. A. El-Munim, and A. El-Baz. Robust neuroimaging-based classification techniques of autistic vs. typically developing brain. In A. A. Farag and J. S. Suri, editors, Deformable Models, volume 1, chapter 16, pages 535-566. 2007.

[518] R. Fahmi, A. Elbaz, H. Hassan, A. A. Farag, and M. F. Casanova. Structural MRIbased discrimination between autistic and typically developing brain. In Proc. Comput. Assi. Radiol. Surg., (CARS'07), pages 24-26, 2007.

[519] A. El-Baz, M. F. Casanova, G. Gimel'farb, M. Mott, and A. E. Switala. Autism diagnostics by 3D texture analysis of cerebral white matter gyrifications. In Proc. Medical Image Comput. Comput.-Assis. Interv., (MICCAI'07), pages 882-890, 2007. 
[520] M. F. Casanova, A. El-Baz, M. Mott, G. Mannheim, H. Hassan, R. Fahmi, J. Giedd, J. M. Rumsey, A. E. Switala, and A. Farag. Reduced gyral window and corpus callosum size in autism: Possible macroscopic correlates of a minicolumnopathy. $J$. Autism Dev. Disord., 39(5):751-764, 2009.

[521] M. F. Casanova, A. El-Baz, E. Vanbogaert, P. Narahari, and A. Switala. A topographic study of minicolumnar core width by lamina comparison between autistic subjects and controls: Possible minicolumnar disruption due to an anatomical element in-common to multiple laminae. Brain Pathology, 20(2):451-458, 2010.

[522] M. F. Casanova, A. El-Baz, S. S. Kamat, B. A. Dombroski, F. Khalifa, A. Elnakib, A. Soliman, A. Allison-McNutt, and A. E. Switala. Focal cortical dysplasias in autism spectrum disorders. Acta Neuropathol. Commun., 1(1):67, 2013.

[523] A. Elnakib, M. F. Casanova, G. Gimel'farb, A. E. Switala, and A. El-Baz. Dyslexia diagnostics by 3-D shape analysis of the corpus callosum. IEEE Trans. Info. Technol. Biomed., 16(4):700-708, 2012.

[524] E. L. Williams, A. El-Baz, M. Nitzken, A. E. Switala, and M. F. Casanova. Spherical harmonic analysis of cortical complexity in autism and dyslexia. Translat. Neurosci., 3(1):36-40, 2012.

[525] M. Nitzken, M. F. Casanova, G. Gimel'farb, A. Elnakib, F. Khalifa, A. Switala, and A. El-Baz. 3D shape analysis of the brain cortex with application to dyslexia. In Proc. IEEE Int. Conf. Image Process., (ICIP'11), pages 2657-2660, 2011.

[526] M. F. Casanova, A. El-Baz, A. Elnakib, J. Giedd, J. M. Rumsey, E. L. Williams, and A. E. Switala. Corpus callosum shape analysis with application to dyslexia. Translat. Neurosci., 1(2):124-130, 2010.

[527] A. Elnakib, A. El-Baz, M. F. Casanova, G. Gimel'farb, and A. E. Switala. Imagebased detection of corpus callosum variability for more accurate discrimination between dyslexic and normal brains. In Proc. IEEE Int. Symp. Biomed. Imaging: From Nano to Macro, (ISBI'10), pages 109-112, 2010.

[528] A. Elnakib, A. El-Baz, M. F. Casanova, and A. E. Switala. Dyslexia diagnostics by centerline-based shape analysis of the corpus callosum. In Proc. Int. Conf. Pattern Recogni., (ICPR'10), pages 261-264, 2010.

[529] A. El-Baz, M. Casanova, G. Gimel'farb, M. Mott, A. Switala, E. Vanbogaert, and R. McCracken. Dyslexia diagnostics by 3D texture analysis of cerebral white matter gyrifications. In Proc. Int. Conf. Pattern Recogni., (ICPR'08), pages 1-4, 2008.

[530] A. El-Baz, M. Casanova, G. Gimel'farb, M. Mott, A. Switala, E. Vanbogaert, and R. McCracken. A new CAD system for early diagnosis of dyslexic brains. In Proc. Int. Conf. Image Process., (ICIP'08), pages 1820-1823, 2008.

[531] A. El-Baz, M. Casanova, G. Gimel'farb, M. Mott, and A. Switala. An MRI-based diagnostic framework for early diagnosis of dyslexia. Int. J. Comput. Assist. Radiol. Surg., 3(3-4):181-189, 2008. 


\section{APPENDIX I}

\section{ANALYTICAL ESTIMATION OF GIBBS POTENTIALS}

Let $\mathbf{L}=\{0,1\}$ and $\mathbf{R}=\{(x, y): 0 \leq x \leq X-1,0 \leq y \leq Y-1\}$ denote a binary set of object ("1") and background ("0") labels, and a finite arithmetic lattice supporting binary maps $\mathbf{m}: \mathbf{R} \rightarrow \mathbf{L}$, respectively. Let $\mathbf{C}_{a}$ be a family of $s$-order cliques of interaction graph with nodes in the lattice sites $(x, y)$ and edges connecting the interacting (interdependent) sites. Let $A$ clique families describe the spatial geometry of interactions between the region labels for an MGRF model of binary maps:

$$
P_{\mathbf{V}}(\mathbf{m})=\frac{1}{Z_{\mathbf{V}}} \exp \left(\sum_{a=1}^{A} \sum_{\mathbf{c} \in \mathbf{C}_{a}} V_{a}(\mathbf{m}(x, y):(x, y) \in \mathbf{c})\right)
$$

where $\mathbf{V}=\left[V_{a}:\{0,1\} \rightarrow(-\infty, \infty): a=1, \ldots, A\right]$ is a collection of potential functions for the families $\mathbf{C}_{a}$ and $Z_{\mathbf{V}}$ is the partition function

$$
Z_{\mathbf{V}}=\sum_{\mathbf{m} \in \mathbb{M}} \exp \left(\sum_{a=1}^{A} \sum_{\mathbf{c} \in \mathbf{C}_{a}} V_{a}(\mathbf{m}(x, y):(x, y) \in \mathbf{c})\right)
$$

normalising the probabilities over the population $\mathbb{M}=\{0,1\}^{X Y}$ of the maps.

Let $\mathbf{F}\left(\mathbf{m}^{\circ}\right)=\left[\rho_{a} F_{a}\left(\mu_{1}, \ldots, \mu_{s} \mid \mathbf{m}^{\circ}\right):\left(\mu_{1}, \ldots, \mu_{s}\right) \in\{0,1\}^{s} ; a=1, \ldots, A\right]$ and $\mathbf{P}_{\mathbf{V}}=$ $\left[\rho_{a} P_{a}\left(\mu_{1}, \ldots, \mu_{s} \mid \mathbf{V}\right):\left(\mu_{1}, \ldots, \mu_{s}\right) \in\{0,1\}^{s} ; a=1, \ldots, A\right]$ where $\rho_{a}=\frac{1}{X Y}\left|\mathbf{C}_{a}\right|$ is the relative cardinality of the clique family with respect to the lattice cardinality, denote the collection of scaled relative frequencies $F_{a}\left(\mu_{1}, \ldots, \mu_{s} \mid \mathbf{m}^{\circ}\right)$ of co-occurrences of configurations $\left(\mu_{1}, \ldots, \mu_{s}\right)$ of binary labels in the cliques of each family $\mathbf{C}_{a}$ over a given training map $\mathbf{m}^{\circ}$ and the corresponding scaled marginal probabilities $P_{a}\left(\mu_{1}, \ldots, \mu_{s} \mid \mathbf{V}\right)$ of these configurations for the MGRF model, respectively.

The normalized $\log$-likelihood $L\left(\mathbf{V} \mid \mathbf{m}^{\circ}\right)=\frac{1}{X Y} \log \mathbf{P}_{\mathbf{V}}\left(\mathbf{m}^{\circ}\right)$ of the map $\mathbf{m}^{\circ}$ has the gradient

$$
\nabla L\left(\mathbf{V} \mid \mathbf{m}^{\circ}\right) \equiv \frac{\partial}{\partial \mathbf{V}} L\left(\mathbf{V} \mid \mathbf{m}^{\circ}\right)=\mathbf{F}\left(\mathbf{m}^{\circ}\right)-\mathbf{P}_{\mathbf{V}}
$$


and its Hessian matrix of the second derivatives is equal to the negated covariance matrix of the marginal probabilities of signal co-occurrences in the cliques (due to non-negative definiteness of the covariance matrix, the log-likelihood is unimodal over the potential space).

The analytical potential estimate (i.e., the approximate maximum likelihood estimate) is specified as scaled gradient vector at the origin in the potential space:

$$
\mathbf{V}^{*}=\lambda^{*}\left(\mathbf{F}\left(\mathbf{m}^{\circ}\right)-\mathbf{P}_{\mathbf{0}}\right)
$$

where the factor $\lambda^{*}$ maximizes the truncated (to the first three terms) Taylor series decomposition of the $\log$-likelihood $L\left(\mathbf{V} \mid \mathbf{m}^{\circ}\right)$ about the origin:

$$
\begin{aligned}
L\left(\mathbf{V} \mid \mathbf{m}^{\circ}\right) \approx & L\left(\mathbf{0} \mid \mathbf{m}^{\circ}\right)+\lambda\left(\mathbf{F}\left(\mathbf{m}^{\circ}\right)-\mathbf{P}_{\mathbf{0}}\right)^{\top}\left(\mathbf{F}\left(\mathbf{m}^{\circ}\right)-\mathbf{P}_{\mathbf{0}}\right) \\
& -\frac{\lambda^{2}}{2}\left(\mathbf{F}\left(\mathbf{m}^{\circ}\right)-\mathbf{P}_{\mathbf{0}}\right)^{\top} \mathbf{D}_{\mathbf{0}}\left(\mathbf{F}\left(\mathbf{m}^{\circ}\right)-\mathbf{P}_{\mathbf{0}}\right)
\end{aligned}
$$

where $\mathbf{D}_{0}$ denotes the covariance matrix for marginal clique-wise probabilities at the origin. Generally,

$$
\lambda^{*}=\frac{\left(\mathbf{F}\left(\mathbf{m}^{\circ}\right)-\mathbf{P}_{\mathbf{0}}\right)^{\top}\left(\mathbf{F}\left(\mathbf{m}^{\circ}\right)-\mathbf{P}_{\mathbf{0}}\right)}{\left(\mathbf{F}\left(\mathbf{m}^{\circ}\right)-\mathbf{P}_{\mathbf{0}}\right)^{\top} \mathbf{D}_{\mathbf{0}}\left(\mathbf{F}\left(\mathbf{m}^{\circ}\right)-\mathbf{P}_{\mathbf{0}}\right)}
$$

The origin, $\mathbf{V}=\mathbf{0}$ (zero potentials), corresponds to an independent random field (IRF) of equiprobable labels, so that the covariance matrix is closely approximated with the diagonal matrix of variances.

For the binary labels, $m(x, y) \in\{0,1\}$, the marginal co-occurrence probabilities over cliques of second, third, and fourth order are $\frac{1}{4}, \frac{1}{8}$, and $\frac{1}{16}$, respectively. If for the symmetry sake, only equality and inequality of the labels are taken into account, then the combinations of co-occurrences and their probabilities are summarized in Table 21.

Provided the cardinalities of the clique families are close to the lattice cardinality (so that $\rho_{a} \approx 1$ ) for all the families $a=1, \ldots, A$, the resulting potential estimates for the second- and third-order models are independent of the number $A$ of the clique families:

Second-order family: $\quad V_{2: a: \text { eq }}=-V_{2: a: \mathrm{ne}}=4\left(F_{a: \mathrm{eq}}\left(\mathbf{m}^{\circ}\right)-\frac{1}{2}\right)$

Third-order family: $\quad V_{3: a: \mathrm{eq}_{3}}=-V_{3: a: \mathrm{eq}_{2}}=\frac{16}{3}\left(F_{a: \mathrm{eq}_{3}}\left(\mathbf{m}^{\circ}\right)-\frac{1}{4}\right)$ 
TABLE 21: Label combinations and their marginal probabilities for second-, third-, and fourth-order cliques. Here, $p$ denote the probability, "eq" and "ne" denote two equal or non-equal labels for a second-order clique, and "eq," denote $i$ equal labels for a third- or fourth-order clique.

\begin{tabular}{|l|c|c|c|c|c|c|c|}
\hline Clique order & \multicolumn{2}{|c|}{2} & \multicolumn{2}{c|}{3} & \multicolumn{3}{c|}{4} \\
\hline Label combinations & $\mathrm{eq}$ & $\mathrm{ne}$ & $\mathrm{eq}_{3}$ & $\mathrm{eq}_{2}$ & $\mathrm{eq}_{4}$ & $\mathrm{eq}_{3}$ & $\mathrm{eq}_{2}$ \\
\hline Marginal $p$ for the IRF & $1 / 2$ & $1 / 2$ & $1 / 4$ & $3 / 4$ & $1 / 8$ & $1 / 2$ & $3 / 8$ \\
\hline Its variance $p(1-p)$ & $1 / 4$ & $1 / 4$ & $3 / 16$ & $3 / 16$ & $7 / 64$ & $1 / 4$ & $15 / 64$ \\
\hline
\end{tabular}

But for the fourth-order model the factor depends on the number of clique families and the marginal probabilities of label combinations for these families on the training map $\mathbf{m}^{\circ}$ :

$$
\begin{aligned}
V_{4: a: \mathrm{eq}_{4}} & =\lambda^{*}\left(F_{a: \mathrm{eq}_{4}}\left(\mathbf{m}^{\circ}\right)-\frac{1}{8}\right) \\
V_{4: a: \mathrm{eq}_{3}} & =\lambda^{*}\left(F_{a: \mathrm{eq}_{3}}\left(\mathbf{m}^{\circ}\right)-\frac{1}{2}\right) \\
V_{4: a: \mathrm{eq}_{2}} & =\lambda^{*}\left(F_{a: \mathrm{eq}_{2}}\left(\mathbf{m}^{\circ}\right)-\frac{3}{8}\right)=-\left(V_{4: a: \mathrm{eq}_{4}}+V_{4: a: \mathrm{eq}_{3}}\right)
\end{aligned}
$$

where

$$
\lambda^{*}=\frac{\sum_{a=1}^{A}\left(\left(F_{a: \mathrm{eq}_{4}}\left(\mathbf{m}^{\circ}\right)-\frac{1}{8}\right)^{2}+\left(F_{a: \mathrm{eq}_{3}}\left(\mathbf{m}^{\circ}\right)-\frac{1}{2}\right)^{2}+\left(F_{a: \mathrm{eq}_{2}}\left(\mathbf{m}^{\circ}\right)-\frac{3}{8}\right)^{2}\right)}{\sum_{a=1}^{A}\left(\frac{7}{64}\left(F_{a: \mathrm{eq}_{4}}\left(\mathbf{m}^{\circ}\right)-\frac{1}{8}\right)^{2}+\frac{1}{4}\left(F_{a: \mathrm{eq}_{3}}\left(\mathbf{m}^{\circ}\right)-\frac{1}{2}\right)^{2}+\frac{15}{64}\left(F_{a: \mathrm{eq}_{2}}\left(\mathbf{m}^{\circ}\right)-\frac{3}{8}\right)^{2}\right)}
$$




\section{APPENDIX II NOMENCLATURE}

The following convention is used throughout the dissertation

- $T_{1}$ is the spin-lattice (longitudinal) relaxation time of an MRI scan.

- $T_{2}$ is the spin-spin (transverse) relaxation time of an MRI scan.

- $C_{\mathrm{a}}(t), C_{\mathrm{p}}(t)$, and $C_{\mathrm{t}}(t)$ are the concentration of the contrast agent in the feeding artery, blood plasma, and the tissue of interest, respectively.

- $K^{\text {trans }}$ is the volume transfer constant of the contrast agent from the blood plasma to the interstitial space. and

- $k_{\mathrm{ep}}$ is the reverse transfer constat of the contrast agent from the interstitial space to the blood plasma.

- $k_{\mathrm{el}}$ denotes the elimination rate of the constant constat from blood plasma.

- $V_{\mathrm{t}}, V_{\mathrm{p}}$, and $V_{\mathrm{e}}$ are the total tissue, plasma, and interstitial space volumes in $\mathrm{ml}$, respectively.

- $v_{\mathrm{p}}$ and $v_{\mathrm{e}}$ are the blood plasma and interstitial space volume fractions, respectively.

- $F_{\mathrm{p}}$ is the blood plasma flow per unit mass of tissue fraction.

- $P S$ is the Permeability-surface area product of the tissue.

- $\rho$ is the tissue density.

- $T_{10}$ is the native longitudinal relaxation time (i.e., $T_{1}$ before a contrast agent injection). 
- $R_{1}=1 / T_{1}$ is the longitudinal relaxation rate.

- $r_{1}$ is relaxivity constant of the contrast agent.

- $(x, y)$ denotes the cartesian coordinates of points (pixels) in the image plane.

- $\mathbf{Q}=\{0, \ldots, Q-1\}$ denotes sets of gray levels $q$.

- $\mathbf{L}=\{1, \ldots, l\}$ denotes sets of region labels $l$.

- $\mathbf{R}=\{(x, y): 0 \leq x \leq X-1,0 \leq y \leq Y-1\}$ denotes a finite 2D arithmetic lattice supporting gray level images $\mathbf{g}: \mathbf{R} \rightarrow \mathbf{Q}$ and their region (segmentation) maps $\mathbf{m}: \mathbf{R} \rightarrow \mathbf{L}$

- $\mathbf{g}=\left\{g_{x, y}:(x, y) \in \mathbf{R} ; g_{x, y} \in \mathbf{Q}\right\}$ denotes a gray level digital image taking gray values from the finite set $\mathbf{Q}=\{0,1, \ldots, Q-1\}$.

- $\mathbf{m}=\left\{m_{x, y}:(x, y) \in \mathbf{R} ; m_{x, y} \in \mathbf{L}\right\}$ denotes region map taking labels from a finite set $\mathbf{L}=\{1, \ldots, l\}$.

- $P(\mathbf{g}, \mathbf{m})=P(\mathbf{g} \mid \mathbf{m}) P(\mathbf{m})$ is a joint two-level Markov-Gibbs random field probability model of a gray scale image $\mathbf{g}$ and its region map $\mathbf{m}$.

- $P(\mathbf{g} \mid \mathbf{m})$ is a conditional distribution of images, given the map (the lower level of the joint MGRF model).

- $P(\mathbf{m})=P_{s p}(\mathbf{m}) P_{\mathbf{V}}(\mathbf{m})$ is an unconditional Gibbs probability distribution of maps with shape prior (the higher level of the joint MGRF model).

- $P_{\mathrm{sp}}(\mathbf{m})$ is the shape prior probability of the region maps.

- $P_{\mathbf{V}}(\mathbf{m})$ is an unconditional Gibbs probability distribution of the region maps.

- $\phi(x, y)$ denotes the level set function.

- $t$ denotes the continuous time.

- $\tau$ denotes a time step. 
- $n$ denotes a discrete time instant.

- $\nabla$ is the differential operator $\nabla=\left[\frac{\partial}{\partial x}, \frac{\partial}{\partial y}, \frac{\partial}{\partial z}\right]$.

- $\vartheta(x, y)$ denotes the deformable model evolution magnitude and direction at each pixel location.

- $\Omega_{1: x, y}$ and $\Omega_{0: x, y}$ are the combined shape, spatial, and intensity probabilities of the object and background, respectively.

- $\kappa$ is the curvature of the deformable contour.

- $F(x, y)=\kappa \vartheta(x, y)$ is the deformable model speed function at each pixel location.

- $\eta$ denotes the normalized cross-correlation.

- $\alpha$ is the normalization factor of the weighted shape prior.

- $A$ is the clique families that describe the geometry of spatial interactions over the nearest 8-neighborhoods of pixels.

- $\mathbf{V}=\left[V_{a}:\{0,1\} \rightarrow(-\infty, \infty): a=1, \ldots, A\right]$ is a collection of Gibbs potential functions $V_{a}$ for the families $\mathbf{C}_{a}$.

- $f_{a, \text { eq }}($.$) denotes the relative frequency of co-occurrences of the labels in the cliques$ of each family $\mathbf{C}_{a}$

- $Z$ is the partition function that normalizes the Gibbs probability distribution.

- $\theta \equiv\left(\mu, \sigma^{2}\right)$ is a shorthand notation for the mean, $\mu$, and variance, $\sigma^{2}$ of a Gaussian distribution.

- $\psi(q \mid \theta)$ is a Gaussian density of the grey level value with $\theta$ denoting its mean and variance.

- $\Phi_{\theta}(q)=(\psi(q \mid \theta): q \in \mathbf{Q})$ is a discrete Gaussian density distribution; where $\psi(q \mid \theta)=\Phi_{\theta}(q+0.5)-\Phi_{\theta}(q-0.5)$ for $q=1, \ldots, Q-2, \psi(0 \mid \theta)=\Phi_{\theta}(0.5)$, and $\psi(Q-1 \mid \theta)=1-\Phi_{\theta}(Q-1.5)$. 
- $M_{p}$ and $M_{n}$ are the number of positive and negative Gaussian kernels, respectively, for the estimated mixed density.

- $w_{\mathrm{p}, .}$ and $w_{\mathrm{n}, .}$ are the mixed weight of positive and negative Gaussian kernels, respectively.

- $P_{\mathrm{w}, \Theta}(q)$ is the estimated density for the mixed gray level distribution.

- $\gamma$ denotes a scalar field.

- $\mathrm{G}$ denotes the expert, ground truth segmented region.

- $\mathbf{S}$ denotes the model segmented region.

- $e$ denotes the relative error.

- $A_{z}$ denotes the area under the receiver operating characteristic (ROC) curve.

- $\Pi$ denotes a lattice of $u \times v$ control points $\Pi_{u, v}, u=0, \ldots, U-1 ; v=0, \ldots, V-1$.

- $\beta_{j}(),. j=-1, \ldots, 2$, is the uniform cubic B-spline basis function.

- $\lfloor\varepsilon\rfloor$ denotes the integer part of a real-valued number $\varepsilon$.

- $(s, h): s=x-\lfloor x\rfloor \in[0,1)$ and $h=y-\lfloor y\rfloor \in[0,1)$, is the relative position of the cartesian point $(x, y)$ with respect to the four nearest lattice points $(u, v),(u+$ $1, v),(u, v+1),(u+1, v+1)$

- $\Upsilon(.,$.$) is the similarity metric between the reference and target images.$

- $H($.$) is the Shannon's entropy of the image signals.$

- $H(.,$.$) is the joint entropy of the image signals.$

- $P_{I}($.$) and P_{I}(.,$.$) denote the marginal and joint probability distributions of the gray$ levels, respectively.

- $P_{S}($.$) and P_{S}(.,$.$) denote the marginal and joint MGRF probability distributions of$ gray scale images, respectively. 
- $d \in \mathbf{D}=\{1, \sqrt{2}, \sqrt{3}\}$ is the Euclidean distance between the center location of a $3 \times 3 \times 3$ neighborhood and the surrounding 26 pixels.

- $\mathbf{N}=\left\{\mathbf{N}_{d}, d \in \mathbf{D}\right\}$ denote the family of the neighboring pixel pairs supporting the Gibbs potentials for a $3 \times 3 \times 3$ neighborhood system.

- $\left|\mathbf{C}_{\mathbf{N}}\right|$ is the cardinality of the neighborhood system.

- $\xi$ and $\zeta$ are the shifts in $x$ - and $y$-directions, respectively. 


\section{APPENDIX III LIST OF ABBREVIATIONS}

1D One-Dimensional.

2CXM Two-Compartment Exchange Model.

2D Two-Dimensional.

3D Three-Dimensional.

4D Four-Dimensional.

AAM Active Appearance Model.

AATH Adiabatic Approximation of the Tissue Homogeneity.

ACC Accuracy.

ADC Apparent Diffusion Coefficient.

AIF Arterial Input Function.

AAM Active Shape Model.

ATN Acute Tubular Necrosis.

AUC Area Under the Curve.

BM Brix Model.

BS B-Splines.

CA Contrast Agent.

CAD Computer-Aided Diagnostic.

CC Cross-Correlation.

CE Contrast-Enhanced.

CFR Coronary Flow Reserve.

CT Computed Tomography.

CZ Central Zone.

DCE-MRI Dynamic Contrast-Enhanced Magnetic Resonance Imaging. 


\begin{tabular}{|c|c|}
\hline DG & Discrete-Gaussian. \\
\hline DP & Distributed-Parameter. \\
\hline DRE & Digital Rectal Exam. \\
\hline DSC & Dice Similarity Coefficient. \\
\hline DSI & Diffusion Spectrum Imaging. \\
\hline DTI & Diffusion Tensor Imaging. \\
\hline DWI & Diffusion-Weighted Imaging. \\
\hline EES & Extracellular and Extravascular Space. \\
\hline EM & Expectation Maximization. \\
\hline ETK & Extended Tofts and Kermode. \\
\hline fMRI & Functional Magnetic Resonance Imaging. \\
\hline FN & False Negative. \\
\hline FOV & Field of View. \\
\hline FP & False Positive. \\
\hline FP-MRI & First-Pass Magnetic Resonance Imaging. \\
\hline FXL & Fast Exchange Limit. \\
\hline GD & Gravity Distance. \\
\hline Gd-DTAP & Gadolinium-Diethylene Triamine Pentacetate. \\
\hline GGMRF & Generalized Gauss-Markov Random Field. \\
\hline GFR & Glomerular Filtration Rate. \\
\hline HD & Hausdorff Distance. \\
\hline IAUC & Initial Area Under the Curve. \\
\hline ICA & Principal Component Analysis. \\
\hline LCDG & Linear Combinations of Discrete Gaussians. \\
\hline LDA & Linear Discriminant Analysis. \\
\hline $\mathrm{LM}$ & Larsson Model. \\
\hline LV & Left Ventricle. \\
\hline MAD & Mean Absolute Distance. \\
\hline
\end{tabular}




\begin{tabular}{|c|c|}
\hline MAP & Maximum A Posteriori. \\
\hline MBF & Myocardial Blood Flow. \\
\hline MD & Mean Distance. \\
\hline MGRF & Markov-Gibbs Random Field. \\
\hline MI & Mutual Information. \\
\hline MITR & Maximum Intensity Time Ratio. \\
\hline MR & Magnetic Resonance. \\
\hline MRF & Markov Random Field. \\
\hline MRI & Magnetic Resonance Imaging. \\
\hline MRS & Magnetic Resonance Spectroscopy. \\
\hline MTT & Mean Transit Time. \\
\hline NAC & Neoadjuvant Chemotherapy. \\
\hline NCC & Normalized Cross-Correlation. \\
\hline NMI & Normalized Mutual Information. \\
\hline OAE & Overlapping Area Error. \\
\hline OB & Observer. \\
\hline OR & Overlap Ratio. \\
\hline PDE & Partial Differential Equation. \\
\hline PDMD & Phase Difference Movement Detection. \\
\hline PER & Peak Enhancement Ratio. \\
\hline PET & Positron Emission Tomography. \\
\hline PK & Pharmacokinetic. \\
\hline PM & Patlak Model. \\
\hline PPV & Positive Predictive Value. \\
\hline PSA & Prostate Specific Antigen. \\
\hline PWI & Perfusion-Weighted Imaging. \\
\hline PZ & Peripheral Zone. \\
\hline RF & Radio Frequency. \\
\hline
\end{tabular}


RMSD Root Mean Squared Distance.

ROC Receiver Operating Characteristic.

ROI Region of Interest.

RSI Relative Signal Intensity.

RVM Relevance Vector Machine.

SB Shape-Based.

SD Standard Deviation.

SEN Sensitivity.

SNR Signal-to-Noise Ratio.

SPE Specificity.

SPGRE Spoiled Gradient Echo.

SVM Support Vector Machine.

TH Tissue Homogeneity.

TN True Negative.

TP True Positive.

TRUS Transrectal Ultrasound.

TZ Transition Zone.

VLS Vector Level Set.

VPC Volume Properly Contoured. 


\title{
CURRICULUM VITAE
}

\author{
Fahmi Abdallah Khalifa \\ Paul C. Lutz Hall, Room \# 304 \\ BioImaging Laboratory \\ Department of Bioengineering \\ University of Louisville, Louisville, KY, USA \\ E-mail:fakhal01@louisville.edu \\ Tel: (502) 852-4032
}

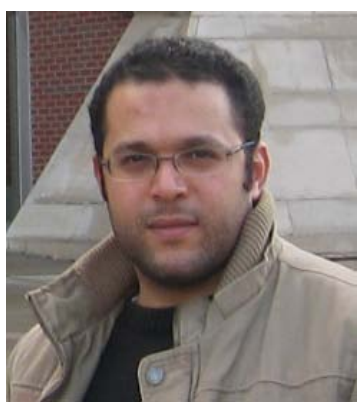

\section{Education}

2014 Ph.D., Electrical and Computer Engineering Department, University of Louisville, Louisville, KY 40292, USA

Ph.D. Dissertation: Analysis of Contrast-Enhanced Medical Images- Dr. Ayman S. El-Baz and Dr. Karla Conn Welch, Dissertation Advisors.

2007 M.Sc., Electrical and Communications Engineering, Mansoura University, Mansoura 35516, Egypt.

M.Sc. Thesis: A Study of Interference in Mobile Multitier CDMA Communication Systems- Dr. Fayez W. Zaki and Dr. Sherif Kishk, Thesis Advisors.

2003 B.Sc., Electronics and Communications Engineering, Mansoura University, Mansoura 35516, Egypt.

Sr. Project: Design of a Slow Frequency Hopping Spread Spectrum (FH/SS) Communication System.

\section{Experience}

2009-2014 Graduate Research Assistant, BioImaging laboratory, Department of Bioengineering, University of Louisville, Louisville, KY 40292, USA.

2004-2008 Graduate Research Assistant, Department of Electronics and Communications Engineering, Mansoura University, Mansoura 35516, Egypt.

\section{Teaching Experience}

Assist in teaching, laboratory demonstration, conducting tutorials, grading, and senior graduation projects of the following Electrical Engineering undergraduate courses: 


\begin{tabular}{|c|c|c|}
\hline Place & Course Subject & Semester/Year \\
\hline Mansoura University & $\begin{array}{c}\text { Wireless Communications Systems } \\
\text { Electronic Circuits Laboratory II } \\
\text { Advanced Electronics III }\end{array}$ & 2007-2008 \\
\hline Mansoura University & $\begin{array}{c}\text { Linear Network Analysis and } \\
\text { Synthesis } \\
\text { Communication Theory 2 } \\
\text { Advanced Electronics II }\end{array}$ & $2005-2006$ \\
\hline Mansoura University & $\begin{array}{c}\text { Electronic Circuits Laboratory I } \\
\text { Communication Theory 1 } \\
\text { Basic Electronics } \\
\text { Circuits Theory }\end{array}$ & $2003-2004$ \\
& C & \\
\hline
\end{tabular}

\section{Professional Affiliations and Training}

- Member, Institute of Electrical and Electronics Engineers (IEEE).

- Member, IEEE Signal Processing (SPS) Society.

- Member, Egyptian Engineers Syndicate, Electrical Engineering, License \#07552/50.

\section{Awards and Recognition}

- John M. Houchens Prize for the outstanding dissertation for May 2014 graduation, University of Louisville, 2014.

- Graduate Dean's Citation Prize in recognition of excellent achievement as a candidate for advanced degree in the University of Louisville, April 2014.

- Outstanding Graduate Student in the Electrical and Computer Engineering Department, Speed School of Engineering, University of Louisville, April 2014.

- Best paper runner-up in the International Symposium on Computational Models for Life Science, Novemeber 2013, http: / / cmls-conf .org/2013/index. php?page=prizes.

- Participant in the BioImaging Lab team that ranked the first place award in The International MRBrainS Challenge at MICCAI Conference among 56 teams submitted their results and 12 teams invited for the onsite competition, September 2013, http://mrbrains13.isi.uu.nl/.

- Theobald Scholarship Award in the Electrical and Computer Engineering Department, Speed School of Engineering, University of Louisville, April 2013.

- Outstanding Graduate Student in the Electrical and Computer Engineering Department, Speed School of Engineering, University of Louisville, April 2012.

- Citation Paper Award from the Society of UroRadiology (SUR), 2011.

- Who's Who Among Students in American Universities \& College Award 2011.

- University of Louisville Travel Awards 2011 and 2013.

- NSF Travel Award 2011 to attend the IEEE International Symposium on Biomedical Imaging: From Nano to Macro 2011 (ISBI'11).

- Sponsored Research Tuition Award from Summer 2012 through Spring 2014.

- Reviewer, IEEE Transactions on Biomedical Engineering, Computer Methods and Programs in Biomedicine, International Journal of Biomedical Imaging, and Journal Electronic Imaging. 


\section{Class Work Grade}

A total of 30 credit hours in Electrical Engineering, Mathematics, and Computer Science subjects- cumulative GPA is 3.925. Particular course concentration has been in statistical modeling, medical image analysis, and machine learning. Relevant courses include: introduction to medical imaging, digital image processing, medical image analysis, variational approaches for medical image analysis, introduction to biometrics, physiology for engineers, and probability and random variables.

\section{$\underline{\text { Research Activities }}$}

- Image modeling, 2D and 3D image segmentation and registration, and texture and shape analysis.

- Development of computer-aided diagnostic (CAD) system using dynamic contrastenhanced MR abdominal images for early detection of acute renal transplant rejection.

- Development of a CAD system for early for functional assessment of myocardial perfusion to follow-up on treatment of cardiac diseases using cardiac perfusion MRI and non-contrast cine MRI.

- Development of a CAD system for the analysis of dynamic contrast-enhanced MRI of prostate for early diagnosis of prostate cancer.

- Assisted in grants writing and preparing primary results for the BioImaging Laboratory, University of Louisville, Louisville, KY, USA.

\section{Publications}

During my Ph.D. (Summer 2009-Spring 2014), I have authored or co-authored more than 50 technical publications that have appeared in world-renown journals (including IEEE Transactions on Medical Imaging, Medical Physics, NMR in Biomedicine, IEEE Transactions on Biomedical Engineering) book chapters, top-rank international conferences and workshops (including MICCAI, ISBI, ICPR, ICIP), and abstracts.

Summary of 5-yr Impact Factor (IF) for Peer-Reviewed Journal Articles

\begin{tabular}{|l|c|l|}
\hline \multicolumn{1}{|c|}{ Journal } & IF & \multicolumn{1}{c|}{ Note } \\
\hline IEEE Transactions on Medical Imaging & 4.268 & Most Prestigious Journal in the Field \\
\hline NMR in Biomedicine & 3.513 & \\
\hline Biotechnology Journal & 3.446 & \\
\hline Medical Physics & 3.138 & One of the Best Journals in the Field \\
\hline $\begin{array}{l}\text { IEEE Transactions on Biomedical Engineer- } \\
\text { ing }\end{array}$ & 2.525 & \\
\hline Pattern Recognition Letters & 1.529 & \\
\hline Translational Neuroscience & 0.482 & \\
\hline $\begin{array}{l}\text { Journal of Biomedical Science and Engineer- } \\
\text { ing }\end{array}$ & - & \\
\hline Acta Neuropathologica Communications & - & \\
\hline
\end{tabular}


Summary of Top Peer International Conferences

\begin{tabular}{|l|c|c|}
\hline \multicolumn{1}{|c|}{ Conference } & Acceptance Rate & Note \\
\hline $\begin{array}{l}\text { Information Processing in Medical } \\
\text { Imaging (IPMI) }\end{array}$ & $\begin{array}{c}10 \% \text { (total } \\
\text { submission 500) }\end{array}$ & $\begin{array}{c}\text { Top-tier Conference with } \\
\text { Unlimited-time Questions }\end{array}$ \\
\hline $\begin{array}{l}\text { Medical Image Computing and } \\
\text { Computer-Assisted Intervention } \\
\text { (MICCAI) }\end{array}$ & $\begin{array}{c}32 \% \text { (total } \\
\text { submission } \sim 800)\end{array}$ & $\begin{array}{c}\text { Most Prestigious Conference in } \\
\text { the Field }\end{array}$ \\
\hline $\begin{array}{l}\text { IEEE International Symposium on } \\
\text { Biomedical Imaging (ISBI) }\end{array}$ & $\begin{array}{c}54 \% \text { (total } \\
\text { submission } \sim 700)\end{array}$ & $\begin{array}{c}\text { One of the Best Conferences in } \\
\text { the Field }\end{array}$ \\
\hline $\begin{array}{l}\text { IEEE International Conference on } \\
\text { Pattern Recognition (ICPR) }\end{array}$ & $\begin{array}{c}49 \%(\text { total } \\
\text { submission } \sim 2000)\end{array}$ & \\
\hline $\begin{array}{l}\text { IEEE International Conference on } \\
\text { Image Processing (ICIP) }\end{array}$ & $\begin{array}{c}52 \%(\text { total } \\
\text { submission } \sim 1000)\end{array}$ & \\
\hline
\end{tabular}

\section{- Journal Articles $($ Total = 12)}

1. B. Dombroski, M. Nitzken, A. Elnakib, F. Khalifa, A. El-Baz, and M. F. Casanova "Cortical surface complexity in a population-based normative sample," Translational Neuroscience, vol. 5, no. 1, pp.1-8, 2014.

2. A. Alansary, A. Soliman, F. Khalifa, A. Elnakib, M. Mostapha, W. Nitzken, M. Casanova, A. El-Baz, "MAPBased Framework for Segmentation of MR Brain Images Based on Visual Appearance and Prior Shape," MIDAS Journal, 2013 [Online]. Available: http://hdl.handle.net/10380/3440.

3. M. F. Casanova, A. El-Baz, S. S. Kamat, B. A. Dombroski, F. Khalifa, A. Elnakib, A. Soliman, A. Allison-McNutt, and A. E. Switala, "Focal cortical dysplasias in autism spectrum disorders," Acta Neuropathologica Communications, vol. 1, no. 1, pp. 67, 2013.

4. F. Khalifa, G. M. Beache, M. Abou El-Ghar, T. El-Diasty, G. Gimel'farb, M. Kong, and A. El-Baz, "Dynamic contrast-enhanced MRI-based early detection of acute renal transplant rejection," IEEE Transactions on Medical Imaging, vol. 32, no. 10, pp. 1910-1927, 2013.

5. F. Khalifa, M. Abou El-Ghar, B. Abdollahi, H. B. Frieboes, T. El-Diasty and A. ElBaz, "A comprehensive non-invasive framework for automated evaluation of acute renal transplant rejection using DCE-MRI," NMR in Biomedicine, vol. 26, no. 11, pp. 1460-1470, 2013.

6. H. Sliman, F. Khalifa, A. Elnakib, A. Soliman, G. M. Beache, A. Elmaghraby, G. Gimel'farb, and A. El-Baz,"Myocardial borders segmentation from cine MR images using bi-directional coupled parametric deformable models," Medical Physics, vol. 40, no. 9, pp. 1-13, 2013.

7. A. Rudra, A. Chowdhury, A. Elnakib, F. Khalifa, A. Soliman, G. M. Beache, and A. El-Baz, "Kidney segmentation using graph cuts and pixel connectivity," Pattern Recognition Letters, vol. 34, no. 13, pp. 1470-1475, 2013.

8. A. Firjani, A. Elnakib, F. Khalifa, G. Gimel'farb, M. Abou El-Ghar, A. Elmaghraby, and A. El-Baz, "A diffusion-weighted imaging based diagnostic system for early detection of prostate cancer," Journal of Biomedical Science and Engineering, vol. 6, no. 3A, pp. 346-356, 2013.

9. A. El-Baz, A. Elnakib, F. Khalifa, M. Abou El-Ghar, P. McClure, A. Soliman, 
and G. Gimel'farb "Precise segmentation of 3D magnetic resonance angiography," IEEE Transactions on Biomedical Engineering, vol. 59, no. 7, pp. 2019-2029, 2012.

10. F. Khalifa, G. M. Beache, G. Gimelfarb, G. A. Giridharan, and A. El-Baz, "Accurate automatic analysis of cardiac cine images," IEEE Transactions on Biomedical Engineering, vol. 59, no. 2, pp. 445-455, 2012.

11. A. El-Baz, P. Sethu, G. Gimel'farb, F. Khalifa, A. Elnakib, R. Falk, and M. Abo El-Ghar, "Elastic phantoms generated by microfluidics technology: Validation of an imaged-based approach for accurate measurement of the growth rate of lung nodules," Biotechnology Journal, vol. 6, no. 2, pp. 195-203, 2011.

12. F. Khalifa, S. Kishk, F. W. Zaki, "Performance study and capacity analysis of two tier-CDMA system," Al-Azhar Engineering Journal, vol. 2, no. 5, pp. 114-121, 2007.

\section{- Book Chapters (Total = 6)}

1. A. Firjani, F. Khalifa, A. Elnakib, G. Gimel'farb, M. Abo El-Ghar, A. Elmaghraby, and A. El-Baz,"A novel image-based approach for early detection of prostate cancer using DCE-MRI," Computational Intelligence in Biomedical Imaging, K. Suzuki Ed, Springer-Verlag, New York, 2014, ch. 3, pp. 55-82.

2. M. Mostapha, F. Khalifa, A. Alansary, A. Soliman, J. Suri, and A. El-Baz,"Computer aided diagnosis systems for acute renal transplant rejection: Challenges and methodologies," Handbook Abdomen and Thoracic Imaging: An Engineering and Clinical Perspective, A. El-Baz, Luca Saba, and J. Suri Eds, Springer-Verlag, New York, 2014, ch. 1, pp. 1-36.

3. F. Khalifa, G. M. Beache, G. Gimel'farb, J. S. Suri, and A. El-Baz, "State-of-the-art medical image registration methodologies: A survey," Handbook of Multi Modality State-of-the-Art Medical Image Segmentation and Registration Methodologies, A. El-Baz, U. R. Acharya, M. Mirmedhdi, and J. S. Suri, Eds., Springer-Verlag, New York, 2011, vol. 1, ch. 9, pp. 235-280.

4. F. Khalifa, G. M. Beache, G. Gimel'farb, G. A. Giridharan, and A. El-Baz, "A new image-based framework for analyzing cine images," Handbook of Multi Modality State-of-the-Art Medical Image Segmentation and Registration Methodologies, A. El-Baz, U. R. Acharya, M. Mirmedhdi, and J. S. Suri, Eds., Springer-Verlag, New York, 2011, vol. 2, ch. 3, pp. 69-99.

5. M. Nitzken, M. F. Casanova, F. Khalifa, G. Sokhadze, and A. El-Baz, "ShapeBased Detection of Cortex Variability for More Accurate Discrimination Between Autistic and Normal Brains," Handbook of Multi Modality State-of-the-Art Medical Image Segmentation and Registration Methodologies, A. El-Baz, U. R. Acharya, M. Mirmedhdi, and J. S. Suri, Eds., Springer-Verlag, New York, 2011, vol. 2, ch. 7, pp. $157-182$.

6. A. El-Baz, P. Sethu, G. Gimel'farb, F. Khalifa, A. Elnakib, R. Falk, M. Abo ElGhar, and J. Suri, "Validation of a New Imaged-Based Approach for the Accurate Estimating of the Growth Rate of Detected Lung Nodules Using Real CT images and Elastic Phantoms Generated by State-of-the-Art Microfluidics Technology," Handbook of Lung Imaging and Computer Aided Diagnosis, A. El-Baz, and J. S. Suri, Eds., Taylor \& Francis, 2011, ch. 18, pp. 405-420. 


\section{- Peer-Reviewed Conference Proceedings $($ Total = 34)}

1. M. Mostapha, A. Alansary, A. Soliman, F. Khalifa, R. Khodeir, M. Nitzken, M. Casanova, and A. El-Baz, "Atlas-based approach for the segmentation of infant DTI MR brain images," In: Proceedings of IEEE International Symposium on Biomedical Imaging: From Nano to Macro (ISBI'14), Beijing, China, April 28-May 2, 2014 (in press).

2. A. Soliman, F. Khalifa, A. Alansary, G. Gimel'farb, and A. El-Baz, "Performance evaluation of an automatic MGRF-based lung segmentation approach," In: Proceedings of International Symposium on Computational Models for Life Science (CMLS'13), Sydney, Australia, November 27-29, 2013, vol. 1559, pp. 323-332.

3. H. Sliman, F. Khalifa, A. Elnakib, A. Soliman, G. M. Beache, G. Gimel'farb, A. Emam, A. Elmaghraby, and A. El-Baz, "Accurate segmentation framework for the left ventricle wall from cardiac cine MRI," In: Proceedings of International Symposium on Computational Models for Life Science (CMLS'13), Sydney, Australia, November 27-29, vol. 1559, pp. 287-296. (Best paper runner-up).

4. M. Mostapha, F. Khalifa, A. Alansary, A. Soliman, G. Gimel'farb, and A. El-Baz, "Dynamic MRI-based computer aided diagnostic systems for early detection of kidney transplant rejection: A survey," In: Proceedings of International Symposium on Computational Models for Life Science (CMLS'13), Sydney, Australia, November 27-29, vol. 1559, pp. 297-306.

5. H. Sliman, F. Khalifa, A. Elnakib, A. Soliman, G. M. Beache, A. Elmaghraby, and A. El-Baz, "A new segmentation-based tracking framework for extracting the left ventricle cavity from cine cardiac MRI," In: Proceedings of IEEE International Conference on Image Processing (ICIP'13), Melbourne, Australia, September 1518, 2013, pp. 685-689. (Selected for oral presentation).

6. F. Khalifa, G. M. Beache, A. Elnakib, H. Sliman, G. Gimel'farb, K. C. Welch, and A. El-Baz, "A new shape-based Framework for the left ventricle wall segmentation from cardiac first-pass perfusion MRI," In: Proceedings of IEEE International Symposium on Biomedical Imaging: From Nano to Macro (ISBI'13), San Francisco, CA, April 7-11, 2013, pp. 41-44.

7. A. Soliman, F. Khalifa, A. Alansary, G. Gimel'farb, and A. El-Baz, "Segmentation of lung region based on using parallel implementation of joint MGRF: Validation on 3D realistic lung phantoms," In: Proceedings of IEEE International Symposium on Biomedical Imaging: From Nano to Macro (ISBI'13), San Francisco, CA, April 7-11, 2013, pp. 852-855.

8. F. Khalifa, G. M. Beache, G. Gimel'farb, and A. El-Baz, "A novel CAD system for analyzing cardiac first-pass MR images," In: Proceedings of IEEE International Conference on Pattern Recognition (ICPR'12), Tsukuba, Japan, November 11-15, 2012, pp. 77-80.

9. A. El-Baz, F. Khalifa, A. Elnakib, M. Nitkzen, A. Soliman, P. McClure, G. Gimel' farb, and M. Abou El-Ghar, "A Novel Approach for global lung registration using 3D Markov-Gibbs appearance model," In: Proceedings of International Conference on Medical Image Computing and Computer-Assisted Intervention (MICCAI'12), Nice, France, October 1-5, 2012, pp. 114-121.

10. F. Khalifa, G. M. Beache, A. Firjani, K. C. Welch, G. Gimel'farb, and A. ElBaz, "A new nonrigid registration approach for motion correction of cardiac firstpass perfusion MRI," In: Proceedings of IEEE International Conference on Image 
Processing (ICIP'12), Orlando, FL, September 30-October 3, 2012, pp. 16651668. (Selected for oral presentation).

11. A. Firjani, F. Khalifa, A. Elnakib, G. Gimel'farb, M. Abo El-Ghar, A. Elmaghraby, and A. El-Baz, "A novel image-based approach for early detection of prostate cancer," In: Proceedings of IEEE International Conference on Image Processing (ICIP'12), Orlando, FL, September 30-October 3, 2012, pp. 2849-2852.

12. M. Nitkzen, A. Elnakib, F. Khalifa, G. M. Beache, G. Gimel'farb, and A. El-Baz, "Accurate modeling of tagged CMR 3D image appearance characteristics to improve cardiac cycle strain estimation," In: Proceedings of IEEE International Conference on Image Processing (ICIP'12), Orlando, FL, September 30-October 3, 2012, pp. 521-524.

13. F. Khalifa, G. M. Beache, G. Gimel'farb, and A. El-Baz, "A new nonrigid registration framework for improved visualization of transmural perfusion gradients on cardiac first-pass perfusion MRI," In: Proceedings of IEEE International Symposium on Biomedical Imaging: From Nano to Macro (ISBI'12), Barcelona, Spain, May 2-5, 2012, pp. 828-831.

14. M. Nitkzen, A. Elnakib,F. Khalifa, G. M. Beache, G. Gimel'farb, and A. ElBaz, "Improving full-cardiac cycle strain estimation from tagged CMR by accurate modeling of 3D image appearance characteristics," In: Proceedings of IEEE International Symposium on Biomedical Imaging: From Nano to Macro (ISBI'12), Barcelona, Spain, May 2-5, 2012, pp. $462-465$ (Selected for oral presentation).

15. A. S. Chowdhury, R. Roy, S. Bose, F. Khalifa, A. Elnakib, and A. El-Baz, "Nonrigid biomedical image registration using graph cuts with a novel data term," In: Proceedings of IEEE International Symposium on Biomedical Imaging: From Nano to Macro (ISBI'12), Barcelona, Spain, May 2-5, 2012, pp. 446-449. (Selected for oral presentation).

16. A. Firjani, F. Khalifa, A. Elnakib, G. Gimel'farb, M. Abo El-Ghar, A. Elmaghraby, and A. El-Baz, "Non-invasive image-based approach for early detection of prostate cancer," In: Proceedings of the International Conference on Developments in eSystems Engineering (DeSE'11), Dubai, UAE, December 6-8, 2011, pp. 172-177.

17. F. Khalifa, A. Elnakib, G. M. Beache, G. Gimel'farb, M. Abo El-Ghar, G. Sokhadze, S. Manning, P. McClure, and A. El-Baz, "3D kidney segmentation from CT images using a level set approach guided by a novel stochastic speed function," In: Proceedings of International Conference on Medical Image Computing and ComputerAssisted Intervention (MICCAI'11), Toronto, Canada, September 18-22, pp. 587594.

18. A. El-Baz, M. Nitzken, A. Elnakib, F. Khalifa, G. Gimel'farb, R. Falk, and M. Abo El-Ghar, "3D shape analysis for early diagnosis of malignant lung nodules," In: Proceedings of International Conference on Medical Image Computing and Computer-Assisted Intervention (MICCAI'11), Toronto, Canada, September 18-22, pp. 172-177.

19. F. Khalifa, G. Gimel'farb, M. Abo El-Ghar, G. Sokhadze, S. Manning, P. McClure, R. Ouseph, and A. El-Baz, "A novel approach for accurate estimation of left ventricle global indexes from short-axis cine MRI," In: Proceedings of IEEE International Conference on Image Processing (ICIP'11), Brussels, Belgium, September 11-14, pp. 2697-2700. (Selected for oral presentation).

20. F. Khalifa, G. M. Beache, G. Gimel'farb, and A. El-Baz, “A new deformable 
model-based segmentation approach for accurate extraction of the kidney from abdominal CT images," In: Proceedings of IEEE International Conference on Image Processing (ICIP'11), Brussels, Belgium, September 11-14, pp. 3454-3457.

21. A. Firjani, F. Khalifa, A. Elnakib, G. Gimel'farb, M. Abo El-Ghar, A. Elmaghraby, and A. El-Baz, "A new 3D automatic segmentation framework for early diagnosis of prostate cancer," In: Proceedings of IEEE International Conference on Image Processing (ICIP'11), Brussels, Belgium, September 11-14, pp. 2333-2336.

22. M. Nitzken, M. F. Casanova, G. Gimel'farb, A. Elnakib, F. Khalifa, A. Switala, and A. El-Baz, "3D shape analysis of the brain cortex with application to dyslexia," In: Proceedings of IEEE International Conference on Image Processing (ICIP'11), Brussels, Belgium, September 11-14, pp. 2713-2716. (Selected for oral presentation).

23. A. El-Baz, M. Nitzken, F. Khalifa, A. Elnakib, G. Gimel'farb, R. Falk, and M. Abo El-Ghar, "3D shape analysis for early diagnosis of malignant lung nodules," In: Proceedings of International Conference on Information Processing in Medical Imaging (IPMI'11), Monastery Irsee, Germany (Bavaria), July 3-8, 2011, pp. 772783.

24. A. Firjani, A. Elnakib, F. Khalifa, G. Gimel'farb, M. Abo El-Ghar, A. Elmaghraby, and A. El-Baz, "A new 3D automatic segmentation framework for accurate extraction of prostate from diffusion imaging," In: Proceedings of Biomedical Science and Engineering Conference-Image Informatics and Analytics in Biomedicine (BSEC'11), Knoxville, TN, March 15-17, 2011, pp. 1-4.

25. F. Khalifa, G. M. Beache, M. Nitzken, G. Gimel'farb, G. Giridharan, and A. ElBaz, "Automatic analysis of left ventricle wall thickness using short-axis cine CMR images," In: Proceedings of IEEE International Symposium on Biomedical Imaging: From Nano to Macro (ISBI'11), Chicago, IL, March 30- April 2, 2011, pp. 1306-1309.

26. A. Firjani, A. Elnakib, F. Khalifa, G. Gimel'farb, M. Abo El-Ghar, J. Suri, A. Elmaghraby, and A. El-Baz, "A new 3D automatic segmentation framework for accurate extraction of prostate from DCE-MRI," In: Proceedings of IEEE International Symposium on Biomedical Imaging: From Nano to Macro (ISBI'11), Chicago, IL, March 30- April 2, 2011, pp. 1476-1479.

27. M. Nitzken, M. Casanova, G. Gimel'farb, F. Khalifa, A. Elnakib, A. Switala, and A. El-Baz, "3D shape analysis of the brain cortex with application to autism," In: Proceedings of IEEE International Symposium on Biomedical Imaging: From Nano to Macro (ISBI'11), Chicago, IL, March 30- April 2, 2011, pp. 1847-1850.

28. A. Firjani, A. Elnakib, F. Khalifa, A. El-Baz, G. Gimel'farb, M. Abo El-Ghar, and A. Elmaghraby, "A novel 3D segmentation approach for segmenting the prostate from dynamic contrast enhanced MRI using current appearance and learned shape prior," In: Proceedings of IEEE International Symposium on Signal Processing and Information Technology (ISSPIT'10), Luxor, Egypt, December 15-18, 2010, pp 137-143. 137-143.

29. F. Khalifa, A. El-Baz, G. Gimel'farb, and M. Abu El-Ghar, "Non-Invasive imagebased approach for early detection of acute renal rejection," In: Proceedings of International Conference on Medical Image Computing and Computer-Assisted Intervention (MICCAI'10), Beijing, China, September 20-24, 2010, pp. 10-18, 2010. (Selected for oral presentation). 
30. A. El-Baz, P. Sethu, G. Gimel'farb, F. Khalifa, A. Elnakib, R. Falk, M. Abo ElGhar, "A new validation approach for the growth rate measurement using elastic phantoms generated by state-of-the-art microfluidics technology,"In: Proceedings of IEEE International Conference on Image Processing (ICIP'10), Hong Kong, September 26-29, 2010, pp. 4381-4383.

31. F. Khalifa, G. M. Beache, A. El-Baz, and G. Gimel'farb, "Deformable model guided by stochastic speed with application in cine images segmentation," In: Proceedings of IEEE International Conference on Image Processing (ICIP'10), Hong Kong, September 26-29, 2010, pp. 1725-1728. (Selected for oral presentation).

32. F. Khalifa, A. El-Baz, G. Gimel'farb, R. Ouseph, and M. Abu El-Ghar "ShapeAppearance guided level-set deformable model for image segmentation," In: Proceedings of International Conference on Pattern Recognition (ICPR'10), Istanbul, Turkey, August 23-26, 2010, pp. 4581-4584.

33. F. Khalifa, and A. T. Alouani, "Survey of watershed modeling and sensor data fusion," In: Proceedings of $41^{\text {st }}$ Southeastern Symposium on System Theory (SSST'09), Tullahoma, TN, March 15-17, 2009, pp. 39-43. (Selected for oral presentation).

34. F. Khalifa, S. Kishk, and F. W. Zaki, "SIR-based uplink admission scheme for a two-tier CDMA system under mobility incorporation," In: Proceedings of IEEE International Conference on Computer Engineering and Systems (ICCES'07), Cairo, Egypt, November. 27-29, 2007, pp. 353-358. (Selected for oral presentation).

\section{- Abstracts Published in Proceedings (Total = 2)}

1. F. Khalifa, M. Abou El-Ghar, B. Abdollahi, H. B. Frieboes, T. El-Diasty, and A. ElBaz, "Dynamic contrast-enhanced MRI-based early detection of acute renal transplant rejection," In: Proceedings of Annual Scientific Meeting and Educational Course Brochure of the Society of Abdominal Radiology (SAR'14), Boca Raton, Florida, March 23-28, 2014. Abstract CID: 1855912M.

2. M. Abou El-Ghar, A. El-Baz, F. Khalifa, A. Elnakib, A. Firjani, and T. El-Diasty "Non-invasive image-based approach for early diagnosis of prostate cancer," In: Proceedings of the European Society of Urogenital Radiology Symposium (ESUR'11), Dubrovnik, Croatia, October 13-16, 2011. (Citation paper award).

\section{- Patents and Disclosures $($ Total $=1)$}

1. A. El-Baz and F. Khalifa, "Computer aided diagnostic system for classifying kidneys," US Provisional Patent Application \# 61/974,134..

\section{Graduate Advisors}

Dr. Ayman S. El-Baz, Dissertation Director, Department of Bioengineering; and Dr. Karla Conn Welch, Dissertation Co-advisor, Electrical and Computer Engineering Department, Speed School of Engineering, University of Louisville, Louisville, KY. 